\title{
Computational Study of Tandem C-C Coupling and C-H Activation Reaction of a Flexible Substrate
}

Davuluri Yogeswara Rao, Anakuthil Anoop

Submitted date: 06/05/2019 - Posted date: 06/05/2019

Licence: CC BY-NC-ND 4.0

Citation information: Rao, Davuluri Yogeswara; Anoop, Anakuthil (2019): Computational Study of Tandem C-C Coupling and C-H Activation Reaction of a Flexible Substrate. ChemRxiv. Preprint.

We carried out an extensive density functional theory calculations on the Pd catalyzed intramolecular Heck reaction followed by $\mathrm{C}-\mathrm{H}$ activation of a flexible substrate like Stilbene system

File list (2) 


\title{
Computational Study of tandem C-C
}

\section{Coupling and C-H Activation Reaction of a Flexible Substrate}

\author{
Davuluri Yogeswara Rao, Anakuthil Anoop* \\ Department of Chemistry, Indian Institute of Technology Kharagpur, INDIA. \\ E-mail: anoop@chem.iitkgp.ac.in
}

Keywords: Intramolecular Heck reaction, C-H Activation, Computational study, Reaction mechanism

\begin{abstract}
We carried out an extensive density functional theory calculations on the Pd catalyzed intramolecular Heck reaction followed by $\mathrm{C}-\mathrm{H}$ activation of a brominated diene, ( $Z$ )-1-bromo5-methyl-1,2-diphenylhexa-1,5-dien-3-ol, that yields fused cyclopentane. Accounting for the flexibility of the substrate, we have selected eight conformations using a combination of conformation search and modeling. Coordination of the catalyst $\left(\mathrm{PdPPh}_{3}\right)$ on the re and si faces of substrate generated 16 catalyst-substrate complexes. The bidentate complexes that has coordination from both the double bonds of the substrate is more stable than the corresponding monodentate complexes. The barriers for oxidative addition are 7.6-15.2 kcal
\end{abstract}


$\mathrm{mol}^{-1}$; the monodentate complexes are in general more reactive with lower activation free energies. The next step, migratory insertion, is regioselective. The selectivity is correlated with the unique geometric features of the intermediates. In the twisted orientation, $\mathrm{Pd}-\mathrm{C} 1$ and $\mathrm{C} 5-\mathrm{C} 6$ bonds are perpendicular to each other while these bonds are parallel in eclipsed ones. The twisted orientations facilitate 5-exo and 6-endo cyclization during the migratory insertion, whereas the eclipsed orientation allows only 5-exo cyclization. The 5-exo route have much lower activation free energies, in agreement with the experiments. To identify the most favourable pathways among the many proposed mechanisms for the $\mathrm{C}-\mathrm{H}$ activations in similar tandem reactions, we have analyzed these pathways. The next step in 5-exo pathways is the $\mathrm{C}-\mathrm{H}$ activation where ortho-H of phenyl group moves to $\mathrm{Pd}$. This may happen directly or after the exchange of $\mathrm{Br}^{-}$with $\mathrm{HCO}_{3}{ }^{-}$. Among the four conformations analyzed, the latter pathway has lower activation free energies for three of them. The other way to compare the path is to compare the energies of the transition states. In this way, the former direct pathway is more favourable for three conformations. The next important step is the second C-C bond formation. Here also we analyzed three pathways different in the oxidation state and coordination at $\mathrm{Pd}$ centre. Comparing these pathways on stepwise barriers, each of the conformations have different favourable pathways. But on comparing the energies of the transition states, the most favourable path is the base-assisted pathway for three conformations. The major takeaway from this extensive analysis is that various conformations of a flexible substrate have different characteristic reaction profiles which makes it difficult to conclude on the choice of pathways. It is crucial to take care of the conformational flexibility. 


\section{Introduction}

Heck reaction or Mizoroki-Heck reaction, the palladium catalyzed vinylation of organic halides (or triflates), is one of the most versatile and convenient method for forming carbon-carbon bonds at unsubstituted vinylic positions ${ }^{1-5}$. The intramolecular version of Heck reaction is specifically attractive due to their entropy economy and the carbocyclic product scope ${ }^{6,7}$. In recent years, tandem Heck coupling and $\mathrm{C}-\mathrm{H}$ activation gained interest due to their atom economy and step economy. ${ }^{8-10}$ Understanding the mechanism of these cascade reactions is crucial for the future development of rational design.

Recently, J. K Ray and coworkers have reported Pd-catalyzed intramolecular Heck cyclization on halogenated diene scaffolds undergoing various mode of cyclization and termination leading to the formation of structurally differing fused cyclopenta(e)none and aromatic analogue ${ }^{11}$. Although we have provided our preliminary computational results, a detailed computational study was necessary to appreciate the complexity involved in the mechanism of these tandem reactions. Among the reported reactions, some of the substrates can have highly complex reaction mechanisms. The conformational flexibility of the substrate with rotatable phenyl rings and flexible side-chain creates the first level of complexity. In addition, the tandem reactions have enhanced complexity with the possibility of many intertwined pathways in the catalytic cycle.

Computational studies on tandem Heck coupling and C-H activation reactions is rare ${ }^{12,13}$

although there are numerous experimental studies and theoretical studies for selected steps. ${ }^{14}$ While the studies on the selected steps are intended to solve specific problems and can bring in certain insights, the study of the whole is expected to give a comprehensive understanding. In the current article, we are presenting the detailed study on the Pd catalyzed tandem cyclisation reaction of (Z)-1-bromo-5-methyl-1,2-diphenylhexa-1,5-dien-3-ol, $\mathbf{1} \rightarrow \mathbf{2}$ (Figure 1) using the Density Functional Theory based calculations. We analyzed the pathways for the formation of the experimentally found product as well as the other possible product. This 
is one of the most comprehensive studies comprising four pathways, and more importantly considering many conformations of the substrate for the large part of the catalytic cycle.

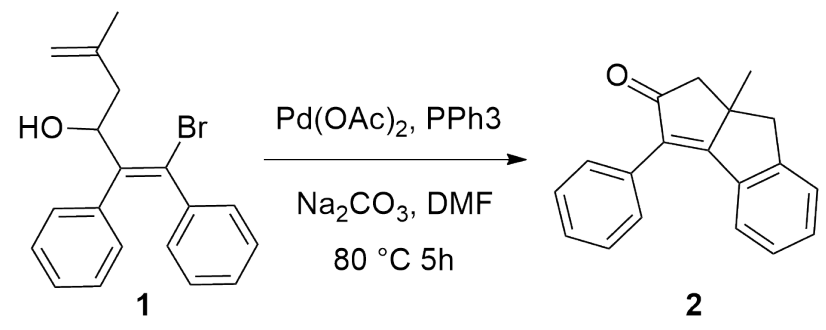

Figure 1: The Reaction considered in this study

\section{Methods}

One of the highlights of this study is the exploration of the conformational space of the substrate. For this, we carried out an initial conformational search using RDKit ${ }^{15}$ with MMFF94 ${ }^{16}$ forcefield for $S$ isomer of the substrate. The geometries generated from RDKit was optimized with Turbomole program package employing BP86 ${ }^{17-22}$ functional and def2-SVP23 basis set along with the Resolution-of-the-Identity ${ }^{24}$ approximation and third generation of dispersion correction with Beck-Johnson damping ${ }^{25,26}$. This method, RI-BP86-D3BJ/def2SVP, is sufficiently accurate and highly efficient. After selecting the important conformations of $S$ isomers as described below in the results, we have modelled the $R$ isomers of the selected conformations by changing the chirality at $\mathrm{C} 3$. These selected isomers were used for the study of the remaining steps in the cycle, rather than choosing one or a couple of conformations by chemical intution.

We used Turbomole program for optimizations. We characterised all minima (zero imaginary mode) and transition states (one imaginary mode) using normal mode analysis with aoforce module. The Gibbs free energy corrections were taken from these calculations. The mode of the transition state was further confirmed by intrinsic reaction coordinate ${ }^{27}$ using DRC module of Turbomole. We used relaxed surface scan to go from the reactant or intermediate 
geometry to the product or next intermediate geometry in each step. The end point of the scan was fully optimized. Using these two geometries as the end points, we have optimized the minimum energy path (MEP) followed by transition state search using Climbing Image Nudged Elastic Band ${ }^{28,29}$ (CI-NEB). We used DL-find ${ }^{30}$ module in Chemshell ${ }^{31}$ program for relaxed surface scan and NEB with Turbomole interface. With help of this method we study all intermediate steps in the catalytic cycle for finding proper transition states. Further, we also performed individual transition state optimization with Turbomole using the TS geometry from CI-NEB.

\section{Results and Discussions}

The catalytic cycle of general Heck coupling reactions consists of mainly three steps; oxidative addition (OA), migratory insertion (MI) and $\beta$-hydride elimination (Figure 2). The reaction $\mathbf{1} \rightarrow \mathbf{2}$ shown in Figure 1 involves a tandem intramolecular Heck reaction and $\mathrm{C}-\mathrm{H}$ activation. A Scheme of the pathways considered in our study is shown in Figure 3. The overall catalytic cycle starts with oxidative addition $(\mathbf{B})$, followed by migratory insertion for the first C-C bond formation (D). The steps $\mathbf{A}$ to $\mathbf{D}$ are common for all Heck reactions.

There are many more possibilities in the tandem intramolecular Heck coupling followed by $\mathrm{CH}$ activation. There are two possibilities for the migratory insertion (step D in Figure 3) - 5-exo or 6-endo cyclization. We have studied the 5-exo-cyclization pathway that gives experimentally observed product as well as the other 6-endo pathway. The next step in the 5-exo pathway is $\mathrm{C}-\mathrm{H}$ activation and a second $\mathrm{C}-\mathrm{C}$ bond formation. We have studied the $\mathrm{C}-\mathrm{H}$ activation occuring directly after the 5-exo-cyclization (E) and the other possibility where $\mathrm{Br}^{-}$is replaced by $\mathrm{H}_{2} \mathrm{CO}_{3}{ }^{-}$( $\mathbf{E}^{\prime}$ also known as base-assisted pathway). Similarly for the second C-C bond formation through reductive elimination, we have studied three possibilities, F, F', and $\mathbf{F}^{\prime \prime}$, the pathways differing in the oxidation state and coordination at $\mathrm{Pd}$. In the 6-endo 


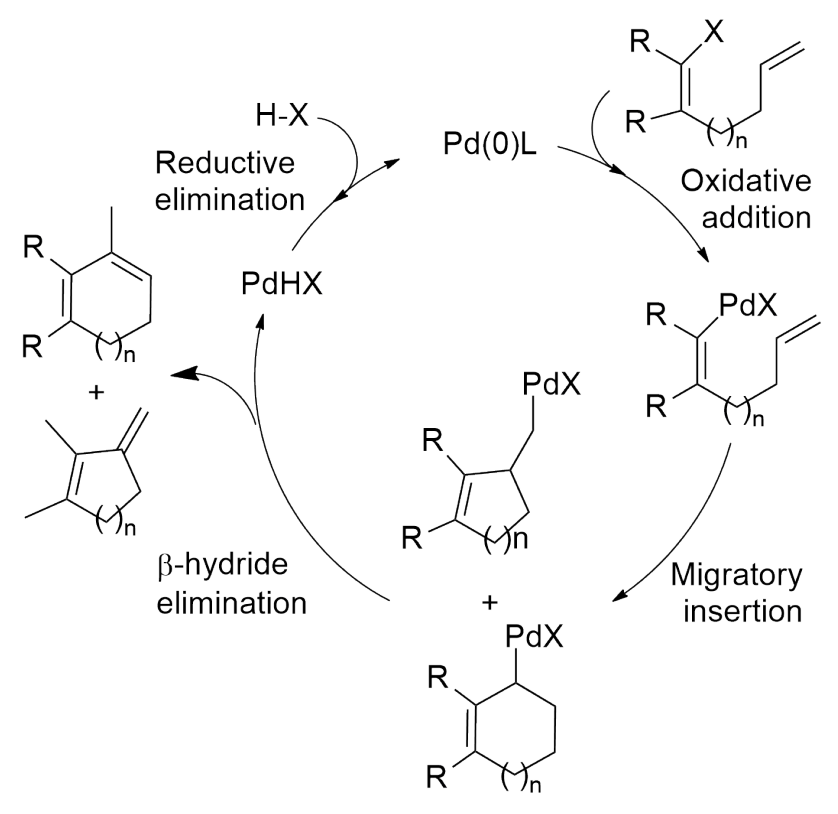

Figure 2: A General Catalytic cycle for Intramolecular Heck coupling

pathway, similar to the general Heck coupling in Figure 2, the $\beta$-hydride elimination is the next step.

Considering the flexibility of the substrate, we have selected a few conformations of the substrate with conformational analysis and modelling as described in the section below. We have computed the intermediates and transition states for all the selected conformations for the first two common steps, oxidative addition and migratory insertion. Thereafter, some of these conformations were filtered out based on factors such as the feasibility for continuing the reaction, similarity of intermediates of different pathways, large differences in the activation barriers for the some steps. At least two $R$ isomers and two $S$ isomers were included in the analyses of the remaining steps.

\section{Conformational Analysis of Substrate}

The effect of considering more than one conformation in the study of catalytic cycle is one of the major aspect of this article. Three rotatable bonds generate the conformational flexibility in our substrate. We carried out an initial conformational search using RDKit to understand 


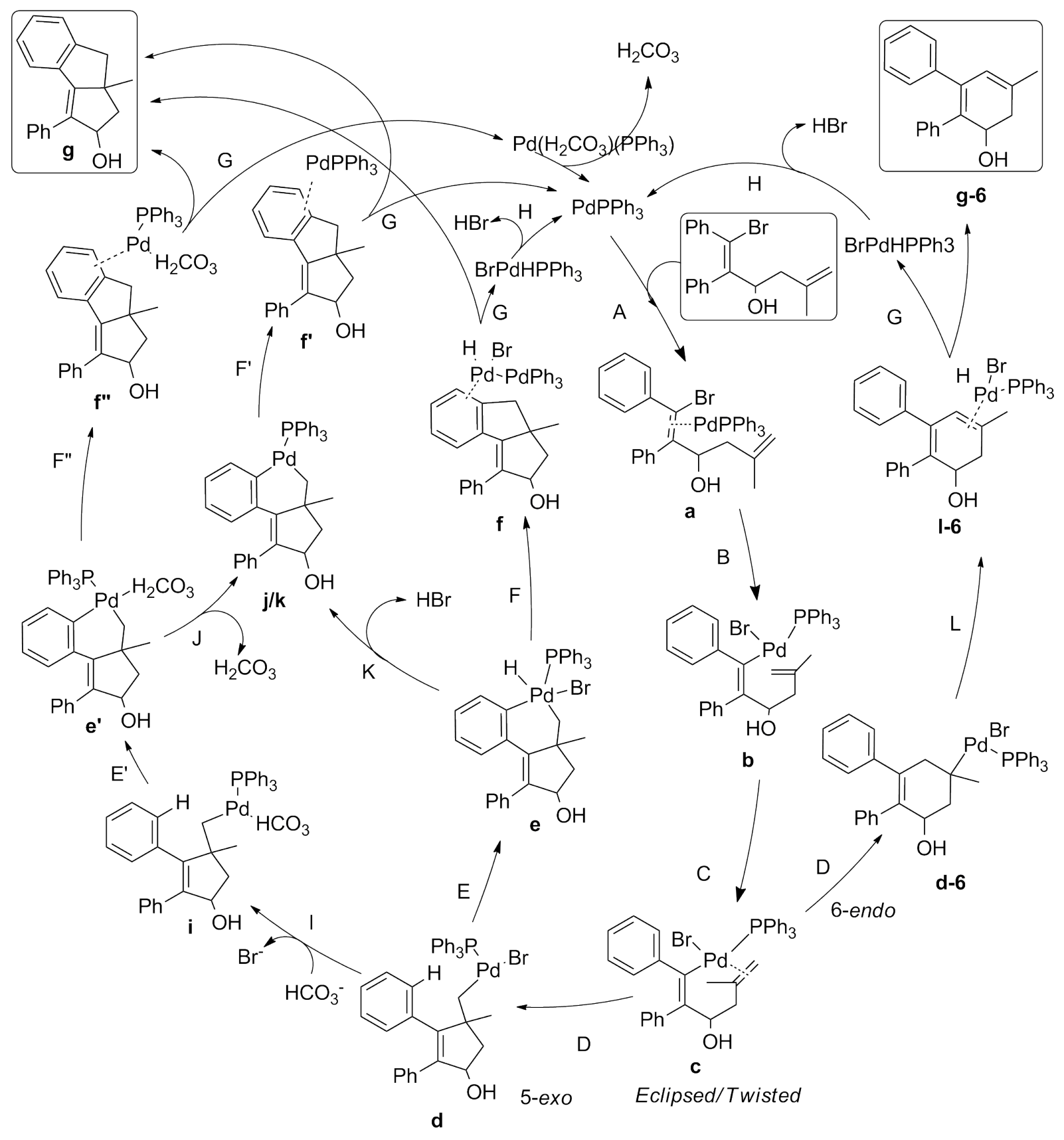

A) Complexation, B) Oxidative Addition, C) Coordination (Eclipsed/Twisted), D) Migratory Insertion(5-exo/6-endo), E) $\mathrm{C}-\mathrm{H}$ Activation, $\left.\mathrm{F}, \mathrm{F}^{\prime}, \mathrm{F}^{\prime \prime}\right) \mathrm{C}-\mathrm{C}$ bond formation, G) Product Formation, $\mathrm{H}$ ) Reductive Elimination, I) Replace $\mathrm{Br}^{-}$by $\mathrm{HCO}_{3}{ }^{-}$, J) $\mathrm{H}_{2} \mathrm{CO}_{3}$ assisted $\mathrm{CH}$ activation, $\left.\mathrm{K}\right) \mathrm{H}_{2} \mathrm{CO}_{3}$ Elimination, $\left.\mathrm{L}\right) \mathrm{HBr}$ Elimination, $\mathrm{M}$ ) $\beta$-hydride Elimination

Figure 3: The pathways analyzed in this study for the reaction $\mathbf{1} \rightarrow \mathbf{2}$ (Figure 1) 
the conformational space. We generated 50 conformations of $S$ isomer and the geometries were further optimized at RI-BP86-D3BJ/def2-SVP level using Turbomole. These optimized geometries were analyzed for their similarity for finding the unique conformations.

These conformations can be grouped into a few clusters of similar geometries based on a few geometric parameters, the rotatable single bonds, C2-C3, C3-C4 and C4-C5. See Figure 4 for a schematic representation of the clustering. The rotation around $\mathrm{C} 2-\mathrm{C} 3$ generated two types of conformations differing in the orientation of the side-chain $\mathbf{R}\left(\mathbf{R}=\mathrm{CH}(\mathrm{OH}) \mathrm{CH}_{2}\left(=\mathrm{CH}_{2}\right) \mathrm{CH}_{3}\right)$, above or below the plane of stilbene double-bond $(\mathrm{C} 1=\mathrm{C} 2)$. As the $R$ isomer of one set and the $S$ isomer of the other set will have similar environment for the reaction, we have taken only one of them to reduce computation.

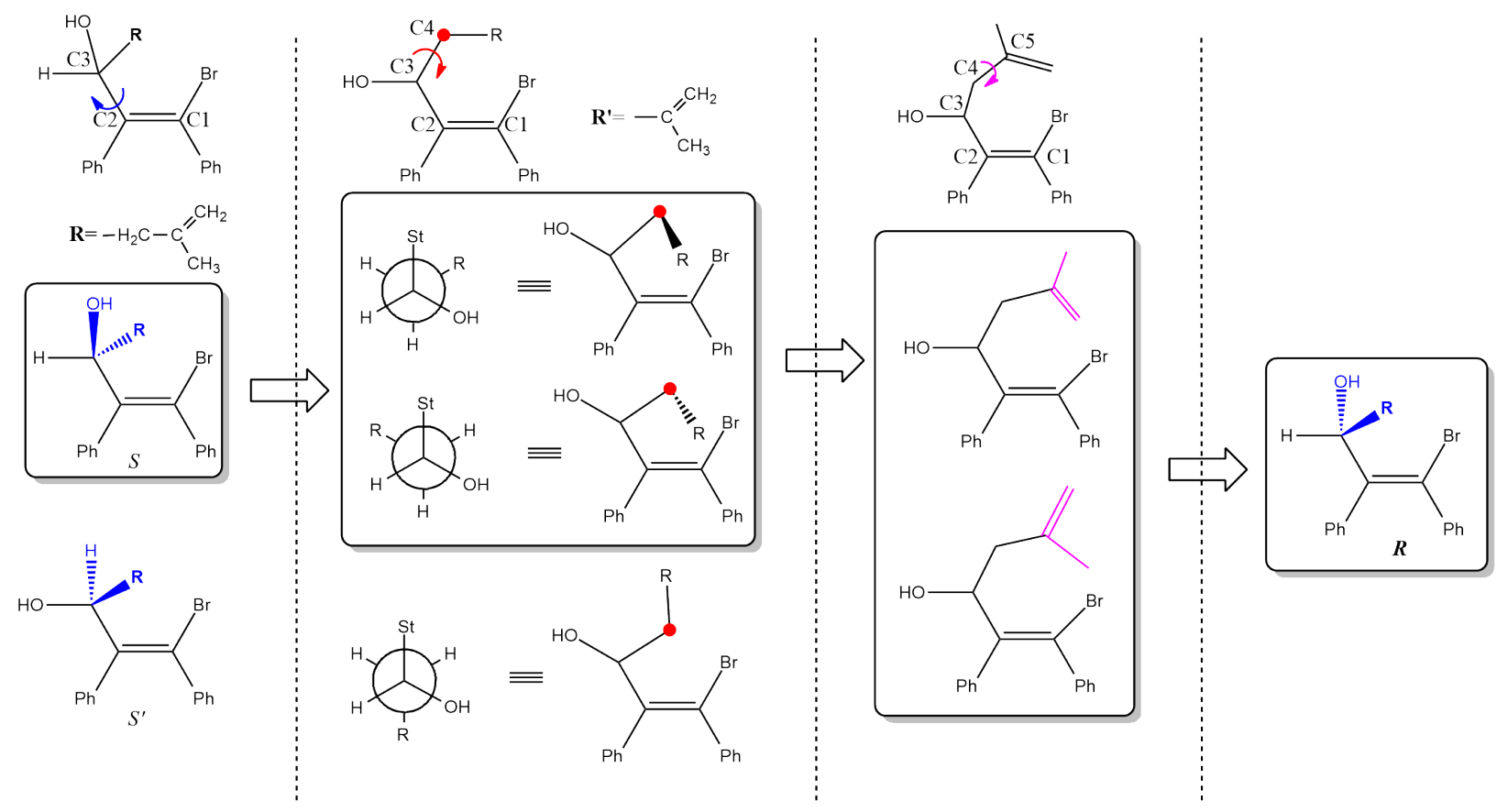

Figure 4: A schematic representation of the selection of conformations for the study. The geometries generated by RDKit was clustered based on the $\mathrm{C}-\mathrm{C}$ bond rotations. The selected sets are shown in the frames. In the final step, the enantiomers of the selected geometries were modelled.

The remaining conformations consists of three sets based on the rotation around C3-C4. One of these sets that has the $\mathbf{R}^{\prime}\left(\mathbf{R}^{\prime}=\mathrm{CH}_{2}\left(=\mathrm{CH}_{2}\right) \mathrm{CH}_{3}\right)$ pointing away from $\mathrm{C} 1=\mathrm{C} 2$ was excluded in our study because $\mathbf{R}^{\prime}$ and $\mathrm{C} 1=\mathrm{C} 2$ are in anti arrangement and therefore is not 
suitable for the subsequent $\mathrm{C}-\mathrm{C}$ bond formation. The final basis for clustering is the rotation around C4-C5. There are two sets of geometries based on this parameter. We have included both sets. This hierarchical clustering $-1 / 2$ from first step, $2 / 3$ from second step, $2 / 2$ in third step - results in four sets $(2 \times 2)$.

We selected one conformation from each of this clusters and arrived at four $S$ conformations (S1-S4). We need to consider the $R$ and $S$ isomers, as the experiment was done with the racemic substrate. With the selected unique conformations of $S$ isomer, we have modelled the $R$ isomers by flipping the chirality at C3 carbon. These geometries were optimized at the same level, RI-BP86-D3BJ/def2-SVP to create four $R$ isomers (R1-R4). Thus, we have identified eight substrate conformations as the unique conformations for our study. The relevant torsion angles and the relative energies are given in Table 1. We assume that these eight conformations cover the possible range of conformational space and used them as the substrates for studying the catalytic cycle.

Table 1: Relative energies $\left(\Delta E^{a} ; \mathrm{kcal} \mathrm{mol}^{-1}\right)$ of the selected substrate isomers and their relevant torsion angles (in degrees), $\alpha \leftarrow(C 1-C 2-C 3-C 4) ; \beta \leftarrow(C 2-C 3-C 4-C 5)$; $\gamma \leftarrow(C 3-C 4-C 5-C 6)$; and $\theta \leftarrow(C 1-C 2-C 5-C 6)$.

\begin{tabular}{lrrrrr}
\hline $\mathrm{Xn}$ & $\Delta E^{a}$ & $\alpha$ & $\beta$ & $\gamma$ & $\theta$ \\
\hline $\mathrm{R} 1$ & 0.0 & 80.2 & 61.2 & 78.2 & 173.8 \\
$\mathrm{R} 2$ & 0.3 & 80.1 & 50.2 & -113.4 & 30.6 \\
$\mathrm{R} 3$ & 1.7 & 101.9 & -69.3 & -94.3 & -67.9 \\
$\mathrm{R} 4$ & 0.7 & 100.1 & -68.1 & 100.2 & 67.1 \\
$\mathrm{~S} 1$ & 2.1 & 76.7 & 50.2 & 76.9 & 157.2 \\
$\mathrm{~S} 2$ & 0.5 & 76.8 & 51.2 & -110.9 & 29.3 \\
$\mathrm{~S} 3$ & 1.8 & 102.6 & -73.0 & -85.8 & -70.0 \\
$\mathrm{~S} 4$ & 2.4 & 96.2 & -65.9 & 96.4 & 61.6 \\
\hline
\end{tabular}

To simplify our discussion, we will reduce the dimensionality by showing the differences 
between the isomers by one representative dihedral angle $(\theta=\angle \mathrm{C} 1-\mathrm{C} 2-\mathrm{C} 5-\mathrm{C} 6)$ which shows the angle between the two double bonds in the substrate. The double bonds point away from each other $\left(\theta=173.8^{\circ}\right.$ and $\left.157.2^{\circ}\right)$ in $\mathbf{R} 1$ and $\mathbf{S 1}$. In $\mathbf{R 2}$ and $\mathbf{S 2}$, the orientation of double bonds are similar to each other $\left(\theta=30.6^{\circ}\right.$ and $\left.29.3^{\circ}\right)$. In the others, R3, S3, R4, and S4 the angle between the double bonds are around 60 degrees.

The energies of all the conformations $\left(\mathbf{X n} ; \mathbf{X}=R\right.$ or $S, \mathbf{n}=\mathbf{1 - 4}$ ) are within $\sim 2 \mathrm{kcal} \mathrm{mol}^{-1}$. The difference in their energies can be attributed to various noncovalent interactions such as, $\mathrm{CH} \cdots \pi, \mathrm{CH} \cdots \mathrm{O}$ and $\mathrm{O}-\mathrm{H} \cdots \pi$ interactions, among the phenyl, $-\mathrm{CH}$, and $-\mathrm{OH}$ groups. These interactions are schematically shown in Figure 5, the detailed analysis using non-covalent index (NCI) is given in the supporting information. Choosing any one among these energetically similar conformations is difficult and non-intuitive. Thus we included all eight conformations in the study.

\section{Complexes of the Active Catalyst with Substrates (A)}

We proceeded to the catalytic cycle with these eight selected substrate isomers. The catalyst will form a $\pi$-complex with the substrate through the double bond $(\mathrm{C} 1=\mathrm{C} 2)$ prior to the oxidative addition (OA) step. Thus, we first modelled the substrate-catalyst complexes (A). The mostly recommended ${ }^{32-36}$ active catalyst model $\mathrm{PdL}(\mathrm{L}=$ tryphenylphosphine), mono ligated palladium catalyst was chosen as the catalyst in this work. We have considered the two possibilities of $\pi$-complexes where $\mathrm{PdPh}_{3}$ is coordinated to one of the two faces (re and si) of $\mathrm{C} 1=\mathrm{C} 2$ double bond.

In the optimized geometries where $\mathrm{Pd}$ and the side-chain are in the same side of the olefinic plane, a bidentate complex is formed by the coordination of both the double bonds. We will include $\mathbf{B}$ to the name of these substrate conformation to indicate the bidentate coordination of substrate to the complex (e.g. BR1 is the catalyst-substrate complex with PdL coorindated with the substrate R1). The optimized geometries are shown in Figure 6. In the other set of 

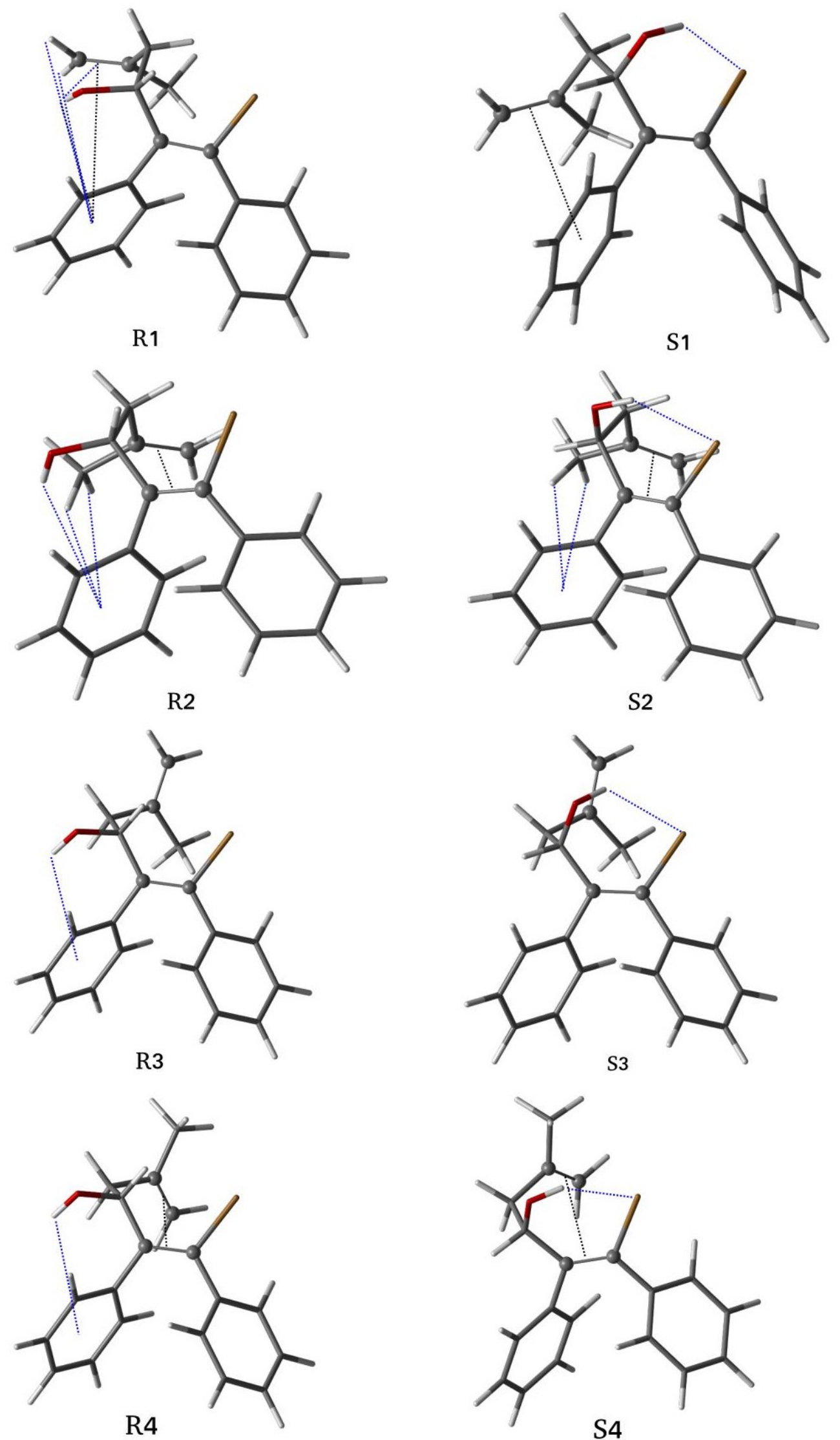

Figure 5: Optimized geometries of the selected conformations of the substrate, $[\mathbf{R}, \mathbf{S}][\mathbf{1}-\mathbf{4}]$ 
complexes, the Pd and the side-chain are on the opposite side of the olefinic plane. Therefore $\mathrm{PdL}$ is coordinated monodentatively to $\mathrm{C} 1=\mathrm{C} 2$. These complexes are named with $\mathbf{M}$ to indicate the monodentate coordination (e. g., MR1).

The complexation (Step A in Figure 3) is exergonic for both monodentate $\left(\Delta G^{A}=-29.7\right.$ to $\left.-32.2 \mathrm{kcal} \mathrm{mol}^{-1}\right)$ and bidentate $\left(-36.8\right.$ to $\left.-43.2 \mathrm{kcal} \mathrm{mol}^{-1}\right)$ coordination (Table 2$)$. The complexation energy $\left(\Delta G^{A}\right)$ is calculated as the energy difference between the complex and the sum of the energies of the free substrate and PdL. The bidentate complexes $\mathbf{B X n}(\mathbf{X}=$ $\mathbf{R}$ or $\mathbf{S}, \mathrm{n}=1-4)$ are more stable with the stabilization from an additional coordination and extra stabilization from chelation compared to monodentate complexes.

Table 2: Relative energies $\left(\Delta E^{a} ; \mathrm{kcal} \mathrm{mol}^{-1}\right)$, complexation energies $\left(\Delta G^{A}\right)$ and the relevant torsion angles $(\theta \leftarrow(C 1-C 2-C 5-C 6)$; in degrees) of bindentate (BXn) and monodenatate (MXn) complexes. The $\Delta E^{a}$ 's are calculated with reference to MR1a for monodentate complexes and BR1a bidentate complexes.

\begin{tabular}{lrrrrrrr}
\hline MXna & $\theta$ & $\Delta E^{a}$ & $\Delta G^{A}$ & BXna & $\theta$ & $\Delta E^{a}$ & $\Delta G^{A}$ \\
\hline R1 & 166.4 & 0.00 & -29.73 & R1 & 125.0 & 0.00 & -37.25 \\
S1 & 159.2 & -0.64 & -31.63 & S1 & 122.8 & -2.80 & -42.44 \\
R2 & 31.2 & 1.01 & -29.95 & R2 & 15.6 & -1.00 & -36.79 \\
S2 & 27.4 & -2.30 & -32.19 & S2 & 16.2 & -5.01 & -41.25 \\
R3 & -73.1 & 1.16 & -30.07 & R3 & -22.5 & -3.63 & -41.01 \\
S3 & -74.1 & -0.30 & -31.39 & S3 & -28.3 & -3.14 & -41.39 \\
R4 & 64.5 & -0.33 & -30.47 & R4 & 87.0 & -6.63 & -43.17 \\
S4 & 60.4 & -0.6 & -31.88 & S4 & 181.3 & -2.05 & -41.67 \\
\hline
\end{tabular}

The range of relative energies among the $\mathbf{M X n}$ is $\sim 2.5 \mathrm{kcal} \mathrm{mol}^{-1}$, slightly more then their spread in substrate conformations $\left(\sim 2 \mathrm{kcal} \mathrm{mol}^{-1}\right)$. The order of relative energies has changed - while MS2a is the most stable complex, R1 is the most stable substrate. Such variations are expected as the interactions of substrate functional groups with the phenyl groups of the 


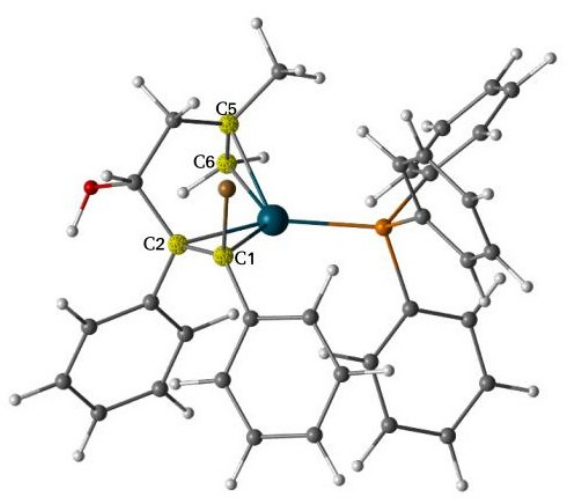

BR1a

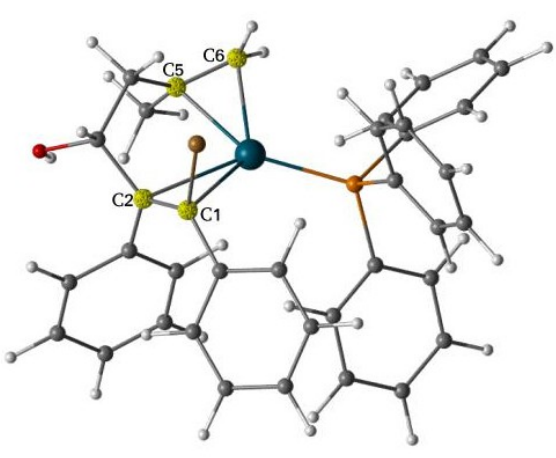

BR2a

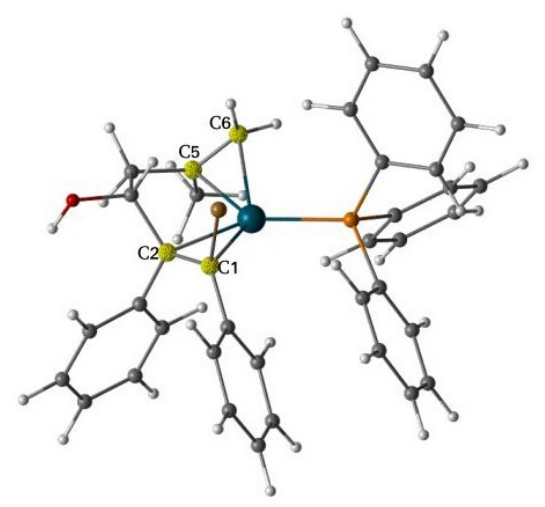

BR3a

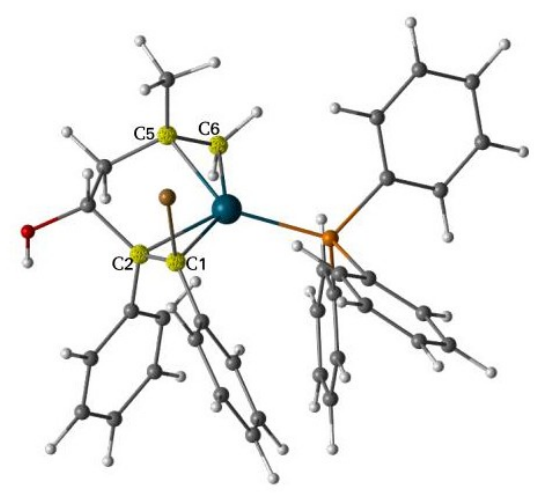

BR4a

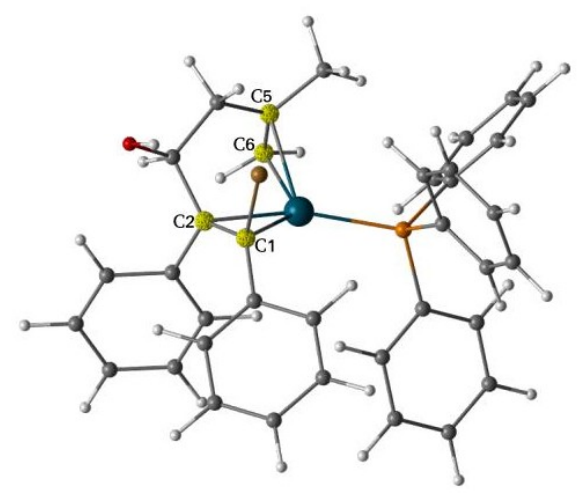

BS1a

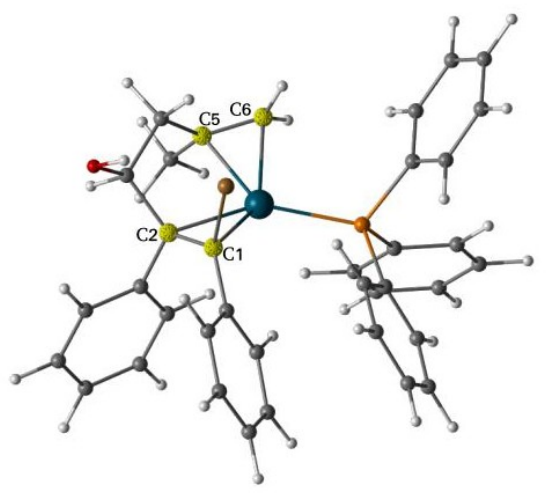

BS2a

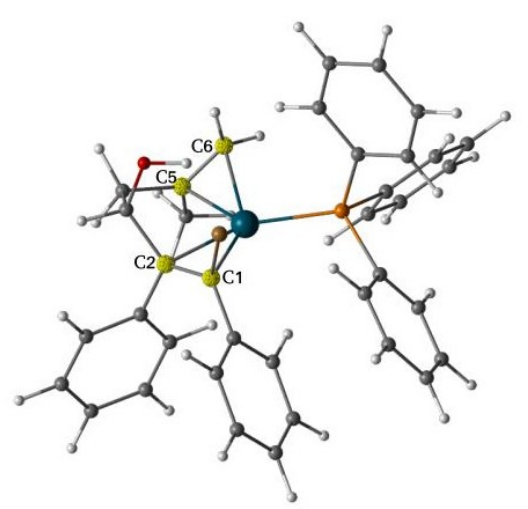

BS3a

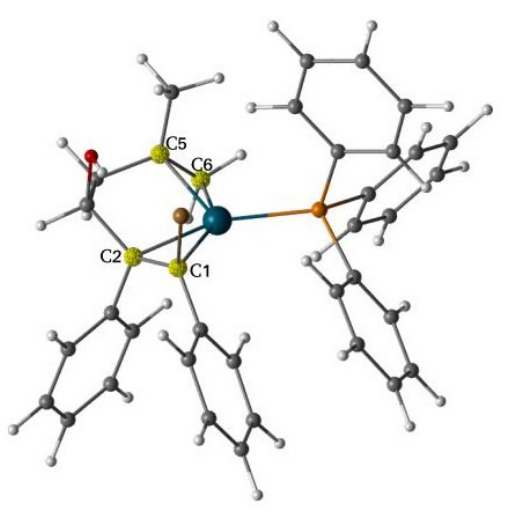

BS4a

Figure 6: Optimized geometries of bidentate catalyst-substrate complexes $\mathbf{B}[\mathbf{R}, \mathbf{S}][\mathbf{1}-\mathbf{4}] \mathbf{a}$ 
ligand may stabilize some conformations more than the others.

The spread of energies in $\mathbf{B X n}$ are more, $6.4 \mathrm{kcal} \mathrm{mol}^{-1}$, than in $\mathbf{M X n}$ and in substrates. The magnitude of interactions among the functional groups, attractive or repulsive, are higher as the groups come closer in the bidentate coordination. The difference in ring-strain for different cyclic arrangements also may contribute the variations in relative energies. These variations also changes the ordering of energies. The most stable substrate $\mathbf{R} \mathbf{1}$ lead to the least stable bidentate complex BR1a. There are various geometric factors that affect the relative energies, however, none of these takes a dominant role as we observe no particular trends in the relative energies.

The bidentate complexes of 1 and 4 conformations (BR1a, BS1a, BR4a, and BS4a) have the si face of $\mathrm{C} 5=\mathrm{C} 6$ coordinated with $\mathrm{Pd}$. The angle $(\theta)$ that represent the relative orientation of double bonds in $\mathbf{1}$ and $\mathbf{4}$ conformations are $>81.3^{\circ}$. We refer to this as twisted complexes considering the nearly perpendicular relative orientations of double bonds. In contrast, the complexes of $\mathbf{2}$ and $\mathbf{3}$ conformations have a re face coordination of $\mathrm{C} 5=\mathrm{C} 6$ with $\mathrm{Pd}$. The $\theta$ in $\mathbf{2}$ and $\mathbf{3}$ are $<22.5^{\circ}$. We refer to them as eclipsed complexes. Coordination of $s i$ face gives twisted and the coordination of re face gives eclipsed complexes.

Generally, the perpendicular orientation of trans olefins are more stable than the parallel orientation in $\pi$-coordinated bis-olefinic metal complexes. ${ }^{37}$ The twisted complex BR4a with $\theta=87.0^{\circ}$ is most stable. The stabilization from this factor, however, is not found in the similar BS4a with $\theta=81.250^{\circ}$ that is less stable than BR4a by $\sim 4 \mathrm{kcal} \mathrm{mol}^{-1}$.

Another difference between the complexes is the chirality at $\mathrm{C} 3$ carbon containing the $\mathrm{OH}$ group. In two of the $S$ complexes, BS3a and BS4a, we can see the $\mathrm{OH}-\mathrm{Br}$ interactions that will give some extra stability. The varying stabilizations from the interactions among the aromatic groups in stilbene and triphenylphosphine also bring variations in the relative stabilities.

Although the MXna complexes are at least $7 \mathrm{kcal} \mathrm{mol}^{-1}$ less stable than the corresponding 
BXna complexes we included both types because of their high high complexation energies $\left(>30 \mathrm{kcal} \mathrm{mol}^{-1}\right)$. We studied the oxidative addition with these 16 geometries.

\section{Oxidative Addition (B)}

We have studied the oxidative addition (OA; step B in Figure 3) with all the 16 substratecatalyst complexes, $[\mathbf{M}, \mathbf{B}][\mathbf{R}, \mathbf{S}][\mathbf{1 - 4}] \mathbf{a}$. In this step, the $\mathrm{Pd}$ is inserted into the $\mathrm{Pd}-\mathrm{C} 1$ bond. The associated changes in the geometry are: the Pd moves from the $\pi$-coordination with $\mathrm{C} 1=\mathrm{C} 2$ bond to form the $\mathrm{Pd}-\mathrm{C} 1$ bond, Br migrates from $\mathrm{C} 1$ to $\mathrm{Pd}$, and the $\mathrm{C} 5=\mathrm{C} 6$ (the double-bond of the side chain) slightly moved away from the Pd weakening the $\pi$-coordination of $\mathrm{Pd}$ with $\mathrm{C} 5=\mathrm{C} 6$.

The activation free energies $\left(\Delta^{\ddagger} G^{B}\right.$; Table 3$)$ vary between $8.7-15.2 \mathrm{kcal} \mathrm{mol}^{-1}$ for $\mathbf{B X n}$ and 7.6-9.5 kcal mol ${ }^{-1}$ for $\mathbf{M X n}$. The lower step-wise barriers for MXn compared to those for BXn are due to higher relative energies of MXna. The transition states corresponding to BXn are more stable than those of MXn. Thus, the relatively less stable MXna substratecatalyst complexes are more reactive with a relatively lower barriers compared to the BXna complexes.

Table 3: Free energies of activation $\left(\Delta^{\ddagger} G^{B}\right)$ and reaction $\left(\Delta^{r} G^{B}\right)$ for oxidative addition (a $\rightarrow \mathbf{b}$; in kcal mol$\left.{ }^{-1}\right)$.

\begin{tabular}{lrrllr}
\hline MXna & $\Delta^{\ddagger} G^{B}$ & $\Delta^{r} G^{B}$ & BXna & $\Delta^{\ddagger} G^{B}$ & $\Delta^{r} G^{B}$ \\
\hline R1 & 9.27 & -21.06 & R1 & 14.19 & -8.64 \\
S1 & 9.52 & -22.82 & S1 & 15.22 & -9.29 \\
R2 & 9.08 & -20.58 & R2 & 14.43 & -2.50 \\
S2 & 8.79 & -22.11 & S2 & 13.02 & -5.79 \\
R3 & 9.31 & -21.07 & R3 & 10.41 & -6.06 \\
S3 & 7.58 & -22.61 & S3 & 10.29 & 4.67
\end{tabular}




\begin{tabular}{lrrlrr}
\hline MXna & $\Delta^{\ddagger} G^{B}$ & $\Delta^{r} G^{B}$ & BXna & $\Delta^{\ddagger} G^{B}$ & $\Delta^{r} G^{B}$ \\
\hline R4 & 8.17 & -19.89 & R4 & 9.82 & -6.44 \\
S4 & 8.39 & -20.80 & S4 & 8.71 & 1.25 \\
\hline
\end{tabular}

The smallest step-wise barrier for the OA step is for MS3a $\left(7.58 \mathrm{kcal} \mathrm{mol}^{-1}\right)$ and the most stable TS geometry is that of BR4a. The largest step-wise barrier is for BS1a (15.22 kcal $\mathrm{mol}^{-1}$ ) and the least stable TS is that of BR1a (10.2 kcal mol-1 higher). The large range of values $\left(\sim 6 \mathrm{kcal} \mathrm{mol}^{-1}\right)$ for the activation free energies further justify the importance of conformational sampling for estimating the accurate rate of the reaction for substrates with large conformational space.

After the oxidative addition in bidentate complexes $(\mathbf{B X n})$, the geometries of intermediates corresponding to the isomers $\mathbf{R} 1, \mathbf{R} 4, \mathbf{S} 3$ and, $\mathbf{S} 4$ have retained the $\pi$-coordination from $\mathrm{C} 5=\mathrm{C} 6$, the olefin of the side-chain, while the $\mathrm{C} 5=\mathrm{C} 6$ moved away from Pd-centre and resulted in a T-shaped geometry, i. e., square-planar with a vacant site at $\mathrm{Pd}(\mathrm{II})$-centre. The other notable changes are in the intermediates BS3b and RS4b that has $\mathrm{OH} \cdots \mathrm{Br}$ interaction, the Br moved away from $\mathrm{Pd}$. This intermediate (b) will be rearranged to facilitate the migratory insertion.

\section{Coordination of the Second Double Bond to Pd (C)}

The second double bond, $\mathrm{C} 5=\mathrm{C} 6$, has to be coordinated with $\mathrm{Pd}$ to facilitate migratory insertion. This rearrangement (step $\mathbf{C} ; \mathbf{b} \rightarrow \mathbf{c}$ in Figure 3 ) is a minor change in geometry for BR1, BR4, BS3 and, BS4 where the $\pi$-coordination was preserved in oxidative addition step. The Pd-C5 and Pd-C6 distances before $\left(d^{1 b}\right.$ and $\left.d^{2 b}\right)$ and after $\left(d^{1 c}\right.$ and $\left.d^{2 c}\right)$ the rearrangement $(\mathrm{C})$ is given in Table 4 . In the other $\mathbf{B X n b}$ intermediates, the rearrangement will bring back the $\pi$-coordination from $\mathrm{C} 5=\mathrm{C} 6$. This rearrangement is exergonic in all cases. 
Although the $\mathrm{C} 5=\mathrm{C} 6$ are relatively farther away from $\mathrm{Pd}$ in the monodentate intermediates, the coordination can be achieved in MXnb $(n=3,4)$. The major difference between the BXnc and MXnc is in the orientation of ligands around $\mathrm{Pd} . \mathrm{PPh}_{3}$ is in cis position with $\mathrm{C} 1$ in $\mathbf{M X n c}$ while $\mathrm{PPh}_{3}$ is in trans position with $\mathrm{C} 1$ in BXnc.

Table 4: The Pd-C distances (in $\AA$ ) before and after the rearrangement $(\mathrm{C} ; \mathbf{X n b} \rightarrow \mathbf{X n c}$ ). $d^{1 b} \leftarrow(P d-C 5)$ in $\mathbf{B X n b}, d^{2 b} \leftarrow(P d-C 6)$ in $\mathbf{X n b}, d^{1 c} \leftarrow(P d-C 5), d^{2 c} \leftarrow(P d-C 6)$ in Xnc.

\begin{tabular}{lcccccc}
\hline Xnb & $d^{1 b}$ & $d^{2 b}$ & $\Delta E^{b}$ & Xnc & $d^{1 c}$ & $d^{2 c}$ \\
\hline BR1 & 2.12 & 2.11 & 6.09 & BR1 & 2.16 & 2.17 \\
BR2 & 4.54 & 4.79 & 13.20 & BR2 & 2.31 & 2.17 \\
BR3 & 3.63 & 3.38 & 7.26 & BR3 & 2.36 & 2.17 \\
BR4 & 2.23 & 2.18 & 3.77 & BR4 & 2.24 & 2.17 \\
BS1 & 4.14 & 4.90 & 2.39 & BS1 & 2.23 & 2.16 \\
BS2 & 3.74 & 3.78 & 4.24 & BS2 & 2.37 & 2.17 \\
BS3 & 2.64 & 2.27 & 17.35 & BS3 & 2.37 & 2.17 \\
BS4 & 2.41 & 2.24 & 13.95 & BS4 & 2.27 & 2.17 \\
MR1 & 4.66 & 5.88 & 2.15 & MR1 & - & - \\
MR2 & 4.78 & 4.97 & 3.04 & MR2 & - & - \\
MR3 & 3.59 & 3.64 & 3.85 & MR3 & 2.54 & 2.28 \\
MR4 & 3.92 & 3.40 & 3.58 & MR4 & 2.37 & 2.23 \\
MS1 & 5.64 & 6.51 & 13.02 & MS1 & - & - \\
MS2 & 3.98 & 4.18 & 11.03 & MS2 & - & - \\
MS3 & 4.34 & 4.67 & 0.00 & MS3 & 2.55 & 2.28 \\
MS4 & 3.98 & 4.18 & 2.41 & MS4 & 2.41 & 2.24 \\
\hline
\end{tabular}

The resulting complexes, $[\mathbf{B}, \mathbf{M}] \mathbf{X n c}$, are tetra coordinated $\operatorname{Pd}(\mathrm{II})$ complexes. We will now discuss the migratory insertion (MI; D in Figure 3) in the next section. 

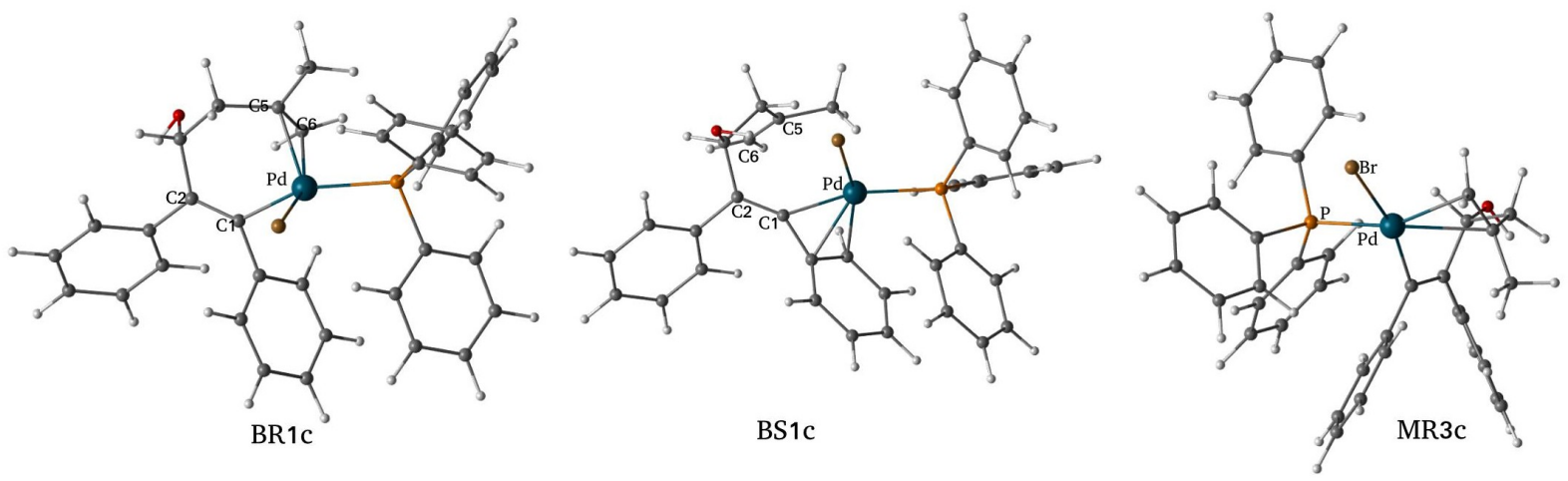

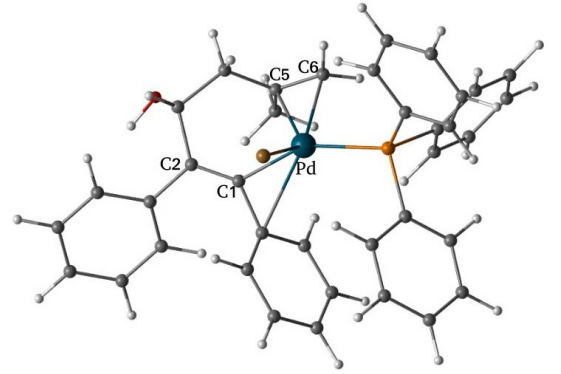

BR2c

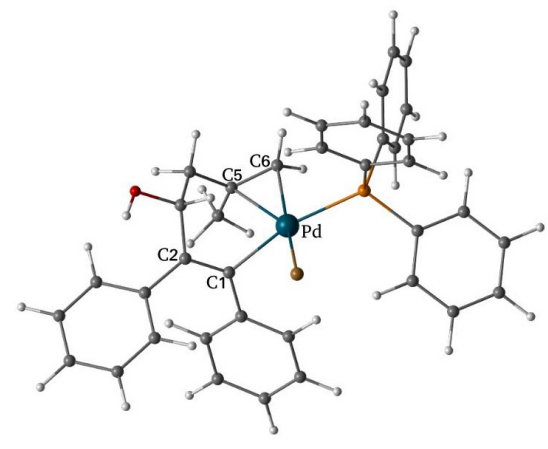

BR3c

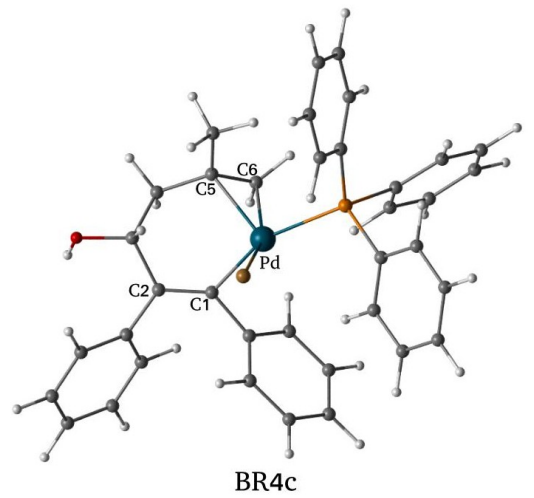

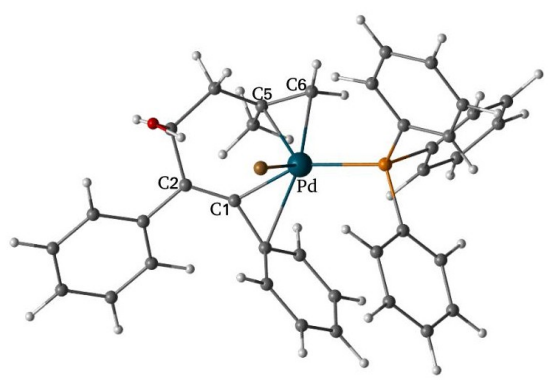

BS2c
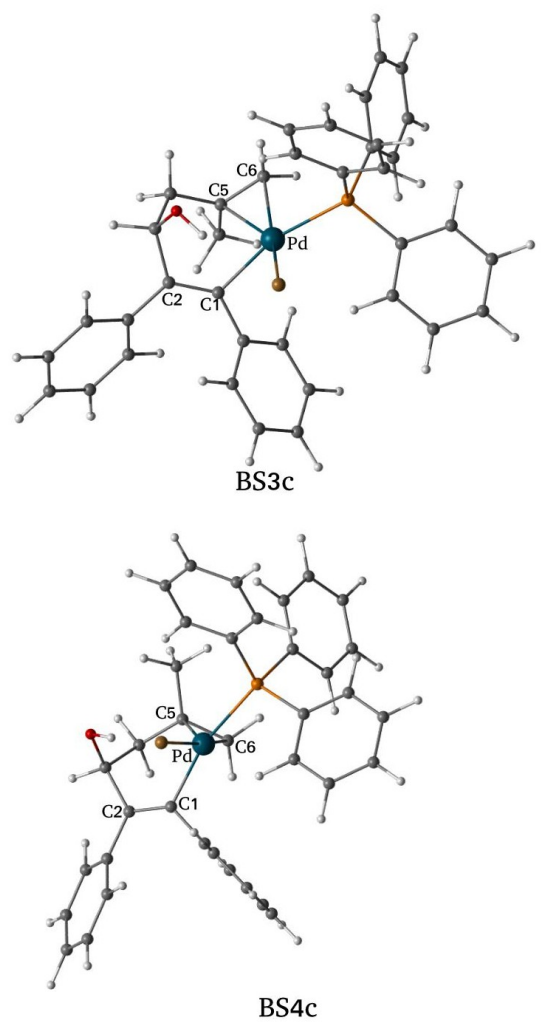
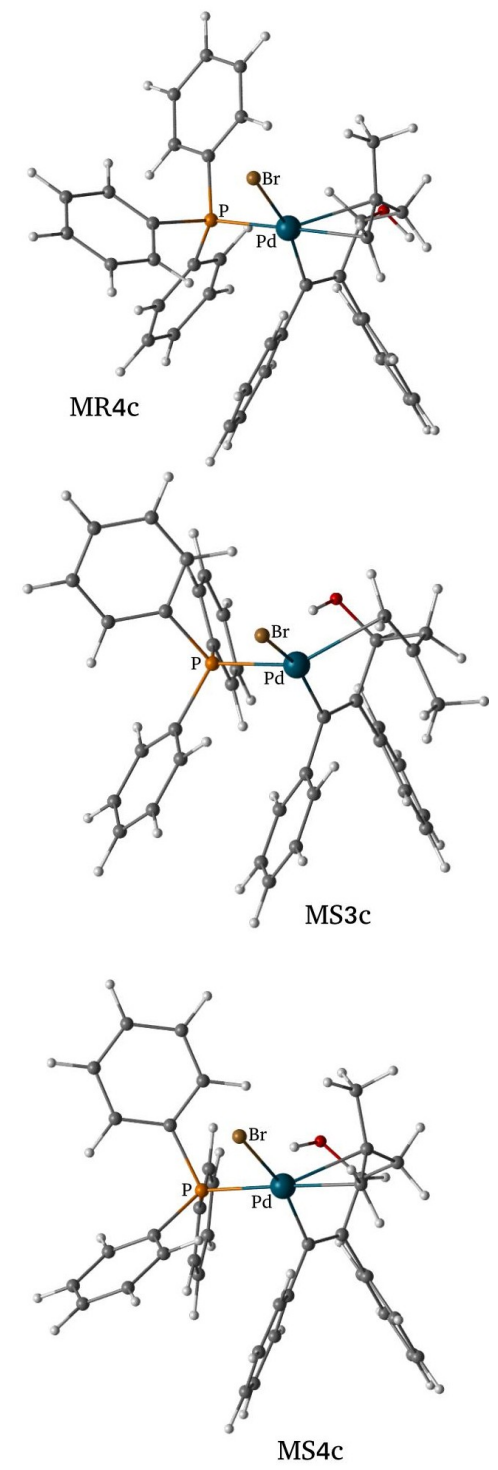

Figure 7: Optimized geometries of the intermediates, $\mathbf{B}[\mathbf{R}, \mathbf{S}][\mathbf{1}-\mathbf{4}] \mathbf{c}$, resulting from the oxidative addition (B) followed by rearrangements (C) 


\section{Migratory Insertion aka Carbopalladation (D)}

The migratory insertion is the most important regioselective step that determine the products. This is the first diverging step in the catalytic cycle that can produce either 5-exo or 6-endo products. We calculated the reactions from $12 \mathbf{c}$ intermediates - eight BXnc and four MXnc - leading to $18 \mathbf{d}$ intermediates - 12 exo and 6 endo. Among all the stepwise activation free energies $\left(\Delta^{\ddagger} G^{D}\right.$; Table 5$)$, the lowest barriers are for the exo cyclizations $\left(\sim 4-6 \mathrm{kcal} \mathrm{mol}^{-1}\right)$. The lowest barrier for endo cyclization is $20.8 \mathrm{kcal} \mathrm{mol}^{-1}$ showing a clear preference for the products based on exo-cyclization, in support of the experimental observation.

Table 5: Free energy of activation $\left(\Delta^{\ddagger} G^{D}\right)$ and reaction $\left(\Delta^{r} G^{D}\right)$ of migratory insertion step in kcal $\mathrm{mol}^{-1}$.

\begin{tabular}{|c|c|c|c|c|}
\hline Xnc & product & $\Delta^{\ddagger} G^{D}$ & $\Delta E^{D(T S)}$ & $\Delta^{r} G^{D}$ \\
\hline BR1 & exo & 24.80 & 18.82 & -8.67 \\
\hline BR1 & endo & 26.17 & 23.15 & -13.78 \\
\hline BS1 & exo & 35.03 & 27.06 & -2.56 \\
\hline BS1 & endo & 22.99 & 13.55 & -6.01 \\
\hline BR2 & exo & 4.77 & 10.06 & -19.74 \\
\hline $\mathrm{BS} 2$ & exo & 6.03 & 3.42 & -26.19 \\
\hline BR3 & exo & 4.00 & 9.15 & -16.57 \\
\hline BS3 & exo & 5.86 & 3.44 & -23.07 \\
\hline BR4 & exo & 29.28 & 27.27 & -5.95 \\
\hline BR4 & endo & 24.58 & 22.03 & -8.84 \\
\hline $\mathrm{BS} 4$ & exo & 34.19 & 28.93 & -3.02 \\
\hline $\mathrm{BS} 4$ & endo & 20.75 & 14.17 & -5.15 \\
\hline MR3 & exo & 17.60 & 9.01 & -2.97 \\
\hline MS3 & exo & 14.92 & 0.00 & -2.53 \\
\hline MR4 & exo & 42.01 & 28.13 & 10.29 \\
\hline
\end{tabular}




\begin{tabular}{lcrrr}
\hline Xnc & product & $\Delta^{\ddagger} G^{D}$ & $\Delta E^{D(T S)}$ & $\Delta^{r} G^{D}$ \\
\hline MR4 & endo & 29.76 & 14.62 & 1.26 \\
MS4 & exo & 47.98 & 29.07 & 15.87 \\
MS4 & endo & 25.71 & 6.69 & 2.73 \\
\hline
\end{tabular}

What makes exo pathways special? We can look at the geometries of the $\mathbf{c}$ intermediates of the low barrier paths, $\mathbf{B X n c ;} \mathbf{X}=R, S ; \mathbf{n}=2$, 3. All these low barrier paths are from bidentate-eclipsed intermediates (Figure 8). Only exo cyclizations are possible for them. The similar paths from monodentate-eclipsed paths, MX3, the $\Delta^{\ddagger} G^{D}$ for 5-exo-cyclization are 17.6 and $14.9 \mathrm{kcal} \mathrm{mol}^{-1}$ for MR3 and MS3, much higher than the BXn counterparts (4.0 and $5.9 \mathrm{kcal} \mathrm{mol}^{-1}$ ). Although MS3 has $14.9 \mathrm{kcal} \mathrm{mol}^{-1}$ of step-wise barrier, it has the most stable transition state geometry among all the transition states in migratory insertion paths.

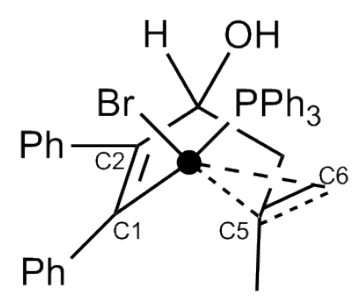

Eclipsed

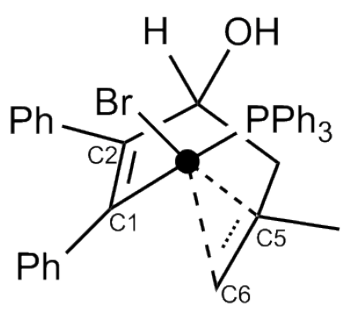

Twisted

Figure 8: The orientation of $\mathrm{C} 5=\mathrm{C} 6$ in twisted and eclipsed Xnc-complexes

The twisted isomers of $\mathbf{1}$ and $\mathbf{4}$ conformations can undergo both cyclizations. Between the two possible cyclizations, the 6-endo cyclization is more favoured in all the cases except for BR1c where 5-exo pathway is slightly more favourable. The barriers $\Delta^{\ddagger} G^{D}$ for 6 -endo cyclizations are in the range $21-26 \mathrm{kcal} \mathrm{mol}^{-1}$. The exo cyclizations from twisted intermediates have much higher activation barriers ranging between $25-35 \mathrm{kcal} \mathrm{mol}^{-1}$ for $\mathbf{B}$ pathways and 42 and $48 \mathrm{kcal} \mathrm{mol}^{-1}$ for $\mathbf{M}$ pathways. Another observation is that the $S$ pathways have higher barriers than $R$ pathways in these exo cyclizations from twisted intermediates.

Some of the gometrical features of the $\mathbf{c}$ intermediates (Figure 7) can contribute to the 
selectivity. A major reaction coordinate in this step is the approach of $\mathrm{C} 5$ to $\mathrm{C} 1$ for 5-exo and $\mathrm{C} 6$ to $\mathrm{C} 1$ for 6-endo cyclization. The distances, $d^{3 c}$ between in $\mathrm{C} 1$ and $\mathrm{C} 5$ and $d^{4 c}$ between $\mathrm{C} 1$ and $\mathrm{C} 6$ are shown in Table 6. A representative geometric parameter that helps to identify the twisted versus eclipsed intermediates are the dihedral angles in $\theta^{1 c} \leftarrow(C 1-C 2-C 5-C 6)$ and $\theta^{2 c} \leftarrow(C 1-P d-C 5-C 6)$.

Table 6: Bond distances between the coupling carbon atoms, $d^{3 c} \leftarrow(C 1-C 5), d^{4 c} \leftarrow$ $(C 1-C 6), \theta^{1 c} \leftarrow(C 1-C 2-C 5-C 6), \theta^{2 c} \leftarrow(C 1-P d-C 5-C 6)$ in Xnc. The distances $(d)$ are in $\AA$ and angle $(\theta)$ in degrees.

\begin{tabular}{lrrrr}
\hline Xnc & $d^{3 c}$ & $d^{4 c}$ & $\theta^{1 c}$ & $\theta^{2 c}$ \\
\hline BR1 & 3.00 & 2.88 & 66.06 & -81.05 \\
BR2 & 2.67 & 3.48 & -32.99 & -169.05 \\
BR3 & 2.64 & 3.42 & -56.63 & -169.06 \\
BR4 & 3.01 & 2.99 & 39.58 & -92.17 \\
BS1 & 3.00 & 2.99 & 39.68 & -93.29 \\
BS2 & 2.69 & 3.46 & -54.86 & -173.91 \\
BS3 & 2.69 & 3.46 & -54.84 & -173.86 \\
BS4 & 2.98 & 3.00 & 40.97 & -94.76 \\
MR3 & 2.83 & 3.50 & -54.94 & -176.65 \\
MR4 & 3.09 & 3.03 & 32.52 & -92.57 \\
MS3 & 2.81 & 3.48 & -53.33 & -177.91 \\
MS4 & 3.11 & 3.00 & 38.80 & -90.70 \\
\hline
\end{tabular}


The relative orientation of $\mathrm{C} 5=\mathrm{C} 6$ with $\mathrm{Pd}-\mathrm{C} 1$ bond is nearly perpendicular in twisted intermediates of $\mathbf{1}$ and $\mathbf{4}$ conformations as can be seen from the dihedral angle C1-Pd-C5-C6 $\theta^{2 c}=81^{\circ}-95^{\circ}$. Hence the $\mathrm{C} 5$ and $\mathrm{C} 6$ atoms, the carbon atoms that are going to couple with $\mathrm{C} 1$, are in similar distances from $\mathrm{C} 1(\sim 3 \AA)$. Thus, both 5-exo and 6-endo cyclization are possible from these intermediates. On the other hand in the eclipsed intermediates of $\mathbf{2}$ and 3 conformations, the $\mathrm{C} 5=\mathrm{C} 6$ bond is parallel to $\mathrm{Pd}-\mathrm{C} 1$ bond $\left(\theta=169^{\circ}-178^{\circ}\right)$. Thus in these cases, the $\mathrm{C} 1$ is close to $\mathrm{C} 5(\sim 2.6-2.8 \AA)$, but $\mathrm{C} 6$ is farther from $\mathrm{C} 1(\sim 3.4-3.5 \AA)$. This way, $\mathrm{C} 5$ flanks the approach of $\mathrm{C} 1$ to $\mathrm{C} 6$, and therefore only $\mathrm{C} 1-\mathrm{C} 5$ coupling is expected from these orientations. For these intermediates, the 6-endo cyclization is practically impossible without a major rearrangement of the complex.

All the MI steps from the BXnc intermediates are exergonic, while MXnc intemediates have endergonic MI steps. In the BXnd intermediates formed by the MI, the Pd orients such that the vacant coordination site generated by the MI step is satisfied by coordinating to an adjacent double bond, oxygen or bromine. In $\mathbf{B S 2 d ^ { \prime }}$ and $\mathbf{B S 3 d} \mathbf{d}^{\prime}$, Pd gets coordinated to $\mathrm{C} 1=\mathrm{C} 2$ and have a $\mathrm{OH} \cdots \mathrm{Br}$ interaction as the $\mathrm{OH}$ and $\mathrm{Br}$ are on the same side of the ring. These are also the most stable among the four $\mathbf{d}$ intermediates formed from eclipsed $-\mathbf{c}$ intermediates. Similarly, BR2d and BR3d have similar geometry around the newly formed 5-membered-cycle. The methyl and hydroxyl groups are on the same side of the ring, both are on the opposite side of the $\mathrm{CH}_{2} \mathrm{PdL}$. Among the 5-exo interemdiates formed from eclipsed c intermediates, 1 and $\mathbf{4}$ are similar.

The reaction profile for all the conformations for the steps upto the migratory insertion is shown in Figure 9. Comparing the geometries and energies of the intermediates formed by MI, we can find that the pathways corresponding to $\mathbf{2}$ and $\mathbf{3}$ have converged and similarly 1 and 4 pathways have converged. For the remaining part of the catalytic cycle, we chose BR3d' $^{\prime}, \mathbf{B S 3 d} \mathbf{d}^{\prime}, \mathbf{B R 4 d}$, and BS4d intermediates. We discuss the 5-exo pathways first, the 6-endo pathway will be discussed at the end. 


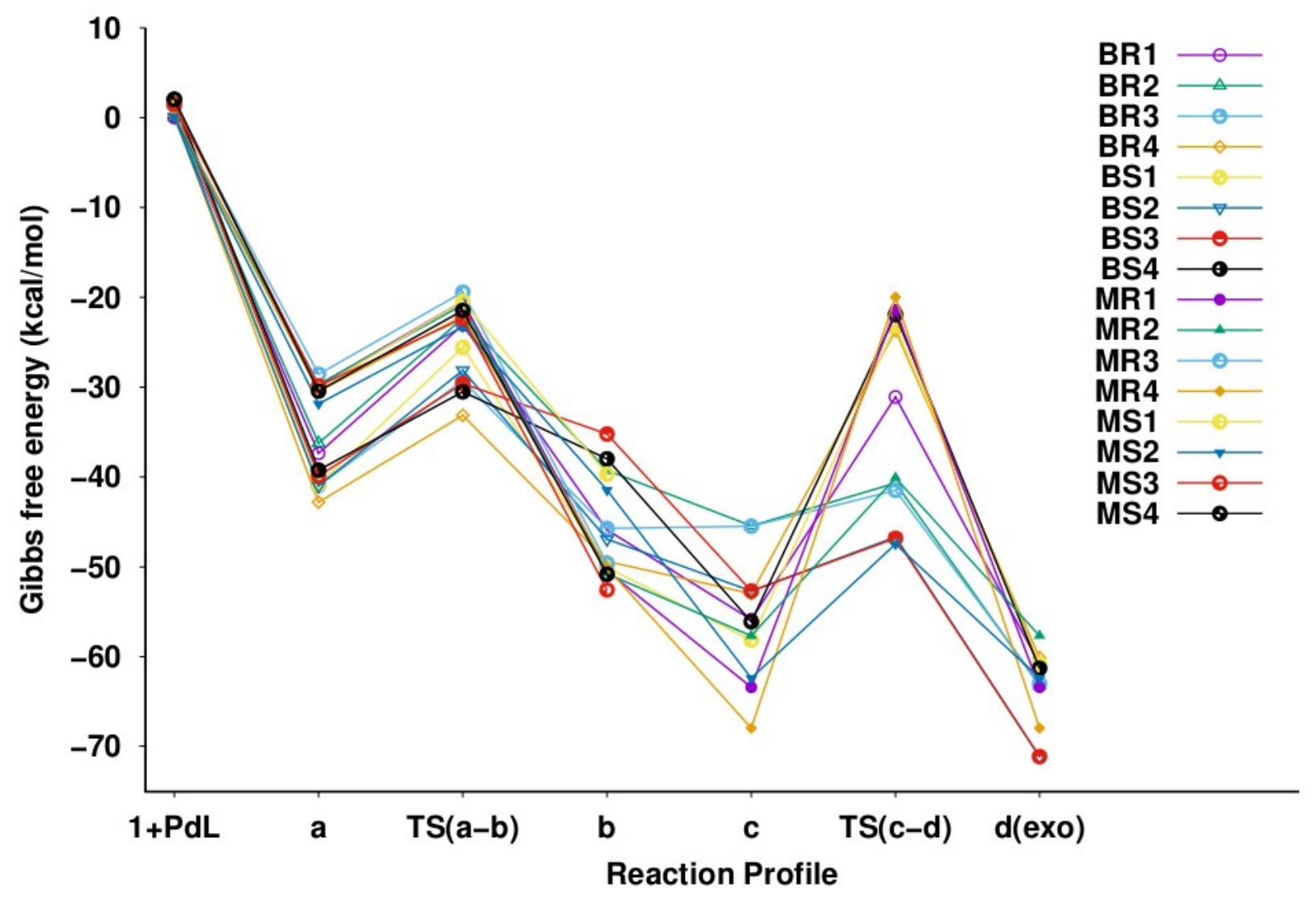

Figure 9: Reaction profile for all conformations for steps A-D 


\section{C-H Activation}

The next step in the pathways for the formation of $\mathbf{2}$ (Figure 1 ) is the activation of C'-H in the ortho-position of the phenyl group that is adjacent to $\mathrm{Pd}$. This can occur directly from the $\mathbf{d}$ intermediates formed by the 5-exo cyclization. The other possibility is the base assisted (or concerted metalation-deprotonation $)^{38-40}$ pathway. We discuss these two possibilites below.

\section{Direct C-H Activation from d (E)}

In the four intermediates we considered for the subsequent steps, the $\mathrm{Pd}(\mathrm{II})$ is coordinated weakly with the adjacent phenyl ring in $\mathbf{B R} \mathbf{4 d}$ and $\mathbf{B S 4 d}$, that is a suitable orientation for C-H activation. But, in $\mathbf{B R 3 d} \mathbf{d}^{\prime}$ and $\mathbf{B S 3 d} \mathbf{d}^{\prime}$, the $\mathrm{Pd}$ is coordinated with stilbene $(\mathrm{C} 1=\mathrm{C} 2)$ and would require a rearrangement before the $\mathrm{C}-\mathrm{H}$ activation. This rearrangement is endothermic by $2.2 \mathrm{kcal} \mathrm{mol}^{-1}$ for $\mathbf{B R 3 d ^ { \prime }}$ and $10 \mathrm{kcal} \mathrm{mol}^{-1}$ for $\mathbf{B S 3 d ^ { \prime }}$. The optimized structures of C-H coordinated complexes BR4d, BS4d, BR3d, and BS3d are presented in Figure 10 with their structural parameters. In all the $\mathbf{d}$ intermediates, the $\mathrm{Pd}(\mathrm{II})$ is coordinated to the nearest phenyl ring of the stilbene.

In the $\mathrm{C}-\mathrm{H}$ activation, hydrogen migrates from carbon $\left(\mathrm{C}^{\prime}\right)$ to $\mathrm{Pd}(\mathrm{II})$ and forms the eintermediates that has $\mathrm{Pd}(\mathrm{IV})$ centre with higher coordination number and oxidation state. The $\mathrm{Pd}(\mathrm{II})$ activates the C'-H bond in the ortho-position of the phenyl ring to which $\mathrm{Pd}$ is coordinated. The $\mathrm{C}-\mathrm{H}$ activation is the important and rate determing step in the catalytic cycle. ${ }^{38-42}$ In our calculations also this step (step E in Figure 3) has the highest step-wise activation barriers, $\sim 27 \mathrm{kcal} \mathrm{mol}^{-1}$, among all the other steps calculated so far (Table 7). 

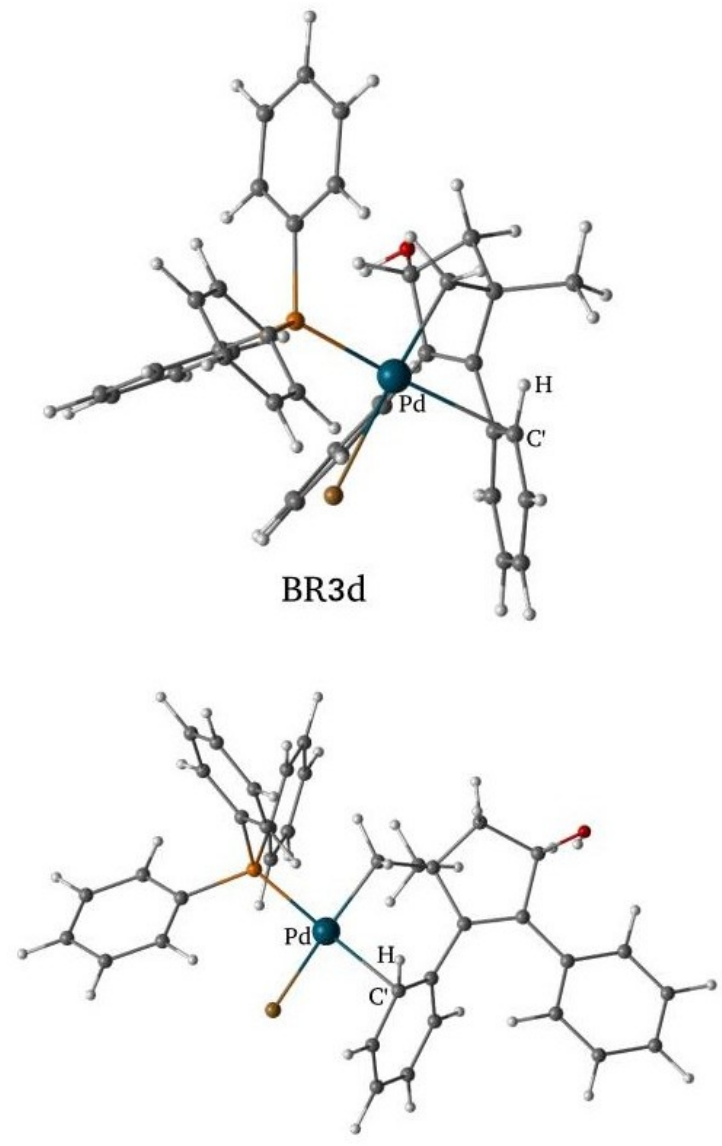

BR4d
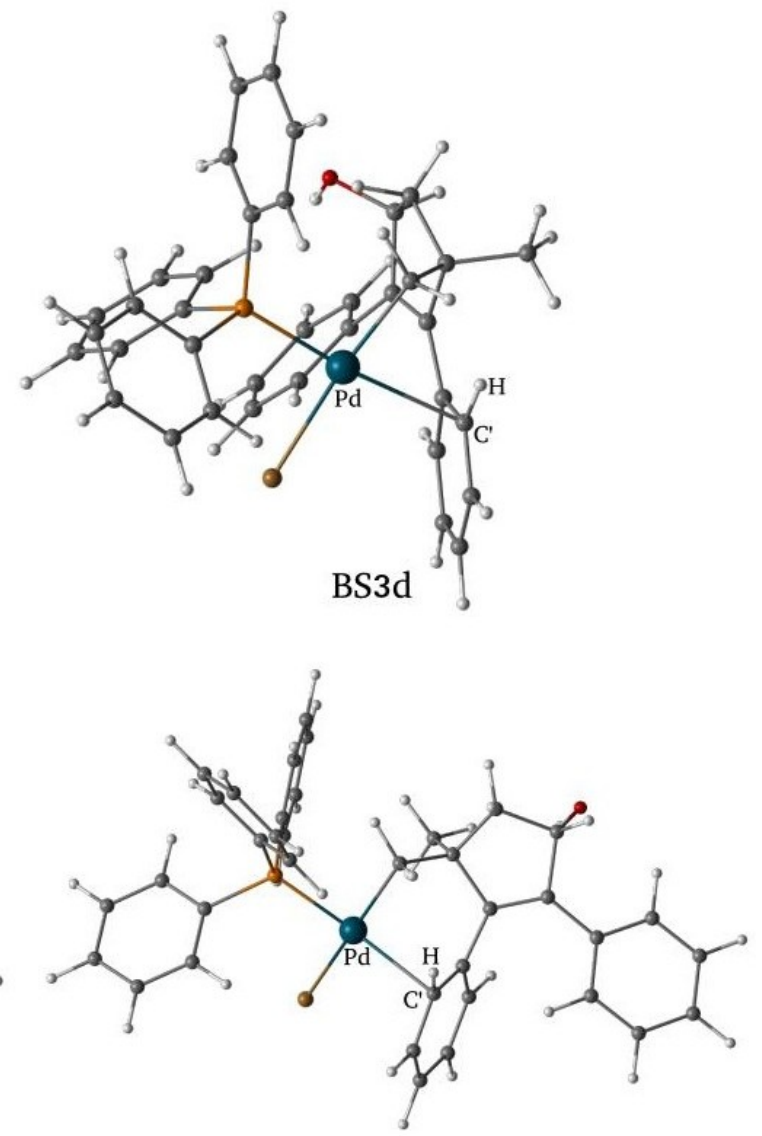

BS4d

Figure 10: Optimized geometries of $\mathbf{d}$ intermediates formed by migratory insertion, followed by a rearrangement. The $\mathrm{C}^{\prime}-\mathrm{H}$ is coordinated to $\mathrm{Pd}$ in these complexes 
Table 7: Free energies of activation and reaction $\left(\Delta^{\ddagger} G^{E}\right.$ and $\Delta^{r} G^{E}$; in kcal mol $\left.{ }^{-1}\right)$ for C-H activation.

\begin{tabular}{lrr}
\hline BXnd & $\Delta^{\ddagger} G^{E}$ & $\Delta^{r} G^{E}$ \\
\hline R3 & 27.63 & 23.36 \\
S3 & 27.50 & 25.83 \\
R4 & 27.51 & 24.92 \\
S4 & 26.43 & 23.69 \\
\hline
\end{tabular}

This step is highly endergonic. The optimized geometries of $\mathrm{Pd}(\mathrm{IV})$ complexes of BR3e, BR4e, and BS4e are presented in the Figure 11. The intermediates after the $\mathrm{C}-\mathrm{H}$ activation of isomers BR3e, BR4e, and BS4e are octahedral Pd(IV)-complexes with one vacant site. The BS3e intermediate has a different geometry in which the fourth site of $\mathrm{Pd}(\mathrm{IV})$ is coordinated with oxygen of the $\mathrm{OH}$ group.

\section{Base Assisted C-H Activation ( $\left.\mathrm{E}^{\prime}\right)$}

Base Assisted C-H Activation, also known as Concerted Metalation-Deprotonation (CMD), occurs after the bromide in the $\mathbf{d}$ intermediate is replaced by $\mathrm{HCO}_{3}^{-}$(step I in Figure 3). This ligand exchange process (I) is endergonic by 11.2-20.6 kcal mol-1 (Table 8).

Table 8: Free energy of reaction for Ligand exchange reaction $\left(\Delta^{r} G^{I}\right)$, free energy of activation $\left(\Delta^{\ddagger} G^{E^{\prime}}\right)$ and reaction $\left(\Delta^{r} G^{E^{\prime}}\right)$ for base $\left(\mathrm{H}_{2} \mathrm{CO}_{3}\right)$ assisted $\mathrm{C}-\mathrm{H}$ bond activation. All Energies are in $\mathrm{kcal} \mathrm{mol}^{-1}$.

\begin{tabular}{lrrr}
\hline BXnd & $\Delta^{r} G^{I}$ & $\Delta^{\ddagger} G^{E^{\prime}}$ & $\Delta^{r} G^{E^{\prime}}$ \\
\hline R3 & 20.58 & 19.22 & 8.66 \\
S3 & 13.04 & 30.12 & 17.97 \\
R4 & 12.95 & 14.96 & 3.87 \\
S4 & 11.27 & 17.34 & 10.45
\end{tabular}




\begin{tabular}{|c|c|c|c|}
\hline BXnd & $\Delta^{r} G^{I}$ & $\Delta^{\ddagger} G^{E^{\prime}}$ & $\Delta^{r} G^{E}$ \\
\hline
\end{tabular}

The $\mathrm{C}-\mathrm{H}$ activation in this pathways is achieved by a different mechanism. The oxygen of the bicarbonate base takes the hydrogen from ortho position to form $\mathrm{H}_{2} \mathrm{CO}_{3}$ coordinated Pd(II) complex. Compared to the direct C-H activation (E) discussed above, this step (E') have significantly lower activation free energies $\left(\Delta^{\ddagger} G^{E^{\prime}}\right)$ except for BS3d. This step is much less endergonic compared to E (Table 8).

Although the barriers are lower for $\mathrm{E}^{\prime}$ in three cases, this pathway involves an endergonic ligand exchange step, I. Thus it is worthy to compare the energies of transition states ( $\mathbf{e}+$ $\mathrm{H}_{2} \mathrm{CO}_{3}$ versus $\mathbf{e}^{\prime}+\mathrm{HBR}$ ). There is slight advantage for base-assisted $\mathrm{E}^{\prime}$ path in $\mathbf{R} 4$, while for the other conformations, the direct $\mathrm{E}$ paths are more favourable than the $\mathrm{E}^{\prime}$ path.

\section{$\mathrm{C}-\mathrm{C}$ reductive elimination}

The $\mathrm{C}-\mathrm{C}$ reductive elimination is the second $\mathrm{C}-\mathrm{C}$ bond formation step that facilitate the formation of the second ring. We studied this step from three different types of intermediates, i) $\mathbf{e}$ in the direct path, ii) $\mathbf{e}^{\prime}$ in which $\mathrm{HBr}$ is replaced by $\mathrm{H}_{2} \mathrm{CO}_{3}$, and iii) $\mathbf{j} / \mathbf{k}$ where $\mathrm{HBr}$ or $\mathrm{H}_{2} \mathrm{CO}_{3}$ is eliminated. The $\mathrm{Pd}$ is coordinated, in addition to the substrate, by only $\mathrm{PPh}_{3}$. The $\mathbf{e}$ is formed by direct $\mathrm{C}-\mathrm{H}$ activation, whereas $\mathbf{e}^{\prime}$ is formed by $\mathrm{C}-\mathrm{H}$ activation after the replacement of $\mathrm{HBr}$ by $\mathrm{H}_{2} \mathrm{CO}_{3}$, and $\mathbf{j} / \mathbf{k}$ is formed by the elimination of $\mathrm{HBr}$ from $\mathbf{e}(\mathrm{J})$, or elimination of $\mathrm{H}_{2} \mathrm{CO}_{3}(\mathrm{~K})$ from $\mathbf{e}^{\prime}$.

\section{The direct path $(\mathrm{F})$}

The activation free energies $\left(\Delta^{\ddagger} G^{F}\right)$ for this step is given in Table 9 . The $\Delta^{\ddagger} G^{F}$ is considerably low for BR3e pathway $\left(8.78 \mathrm{kcal} \mathrm{mol}^{-1}\right)$ among the corresponding barriers for the other isomers $\left(17.20-22.33 \mathrm{kcal} \mathrm{mol}^{-1}\right)$. A closer look at the path using IRC shows a two step 


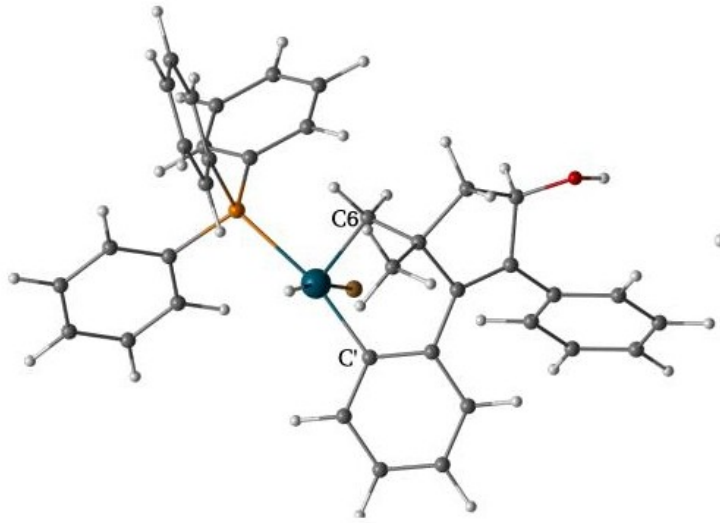

BR3e

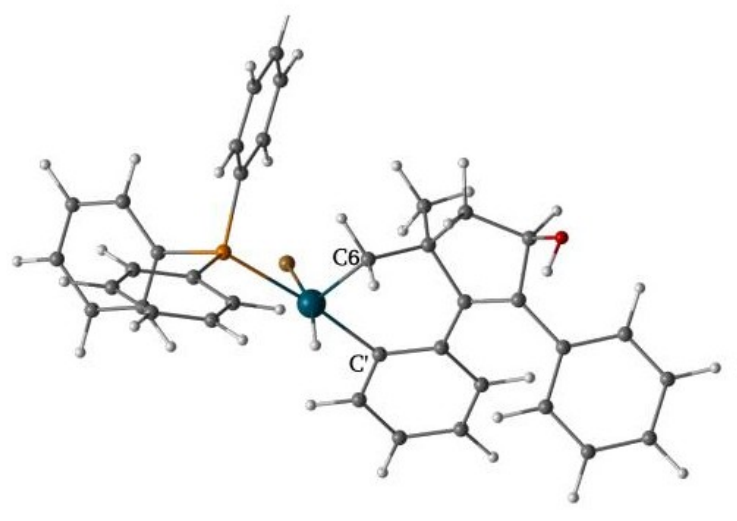

BR4e
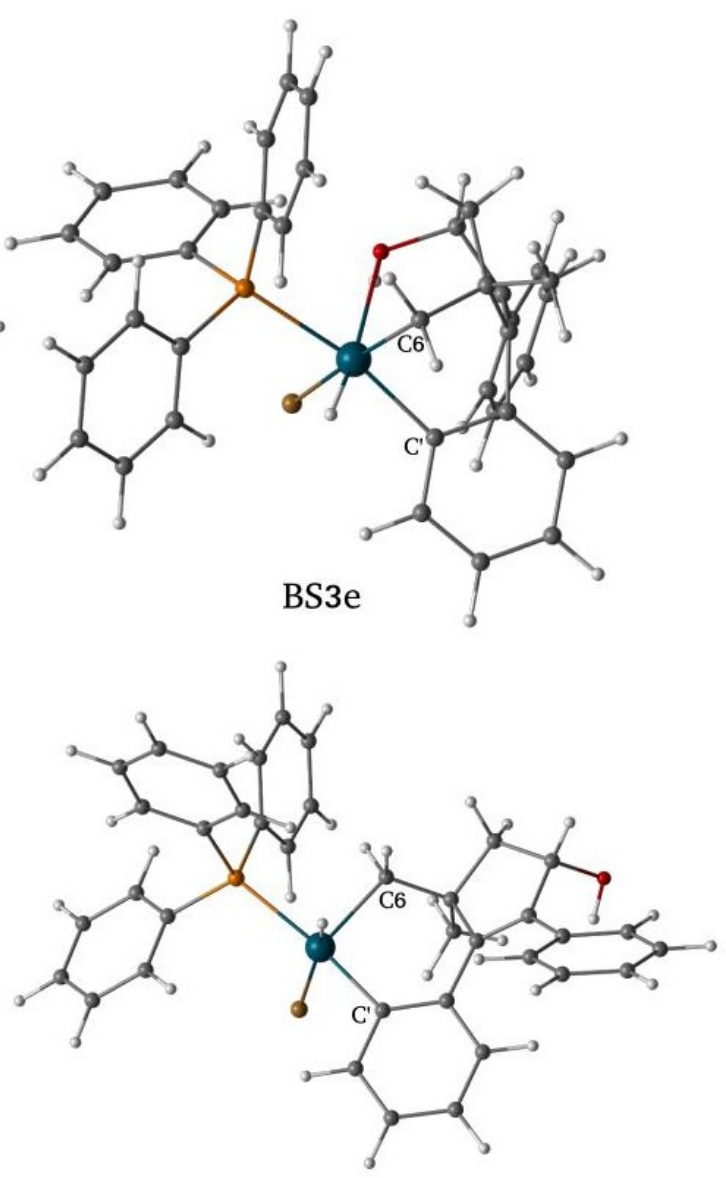

BS4e

Figure 11: Optimized geometries of e intermediates formed by $\mathrm{CH}$ Activation. 
process, an initial movement of the $\mathrm{PPh}_{3}$ ligand from a cis position with respect to $s p^{3}$ carbon (C6) to the trans position. This rearrangement from BR3e to BR3e' has a a free energy barrier of $7.15 \mathrm{kcal} \mathrm{mol}^{-1}$ and is endergonic by $1.37 \mathrm{kcal} \mathrm{mol}^{-1}$. In $\mathbf{B R} 3 \mathbf{e}^{\prime}$, the $\mathrm{PPh}_{3}$ have stabilising interactions with phenyl groups of stilbene that lower the activation energy. The highest barrier among the pathways is for BS3e that can be attributed to the interaction with $-\mathrm{OH}$ and $\mathrm{Pd}$ which are in the same side of the ring that increases the strain in the transition state and hence increases the activation free energy $\left(22.33 \mathrm{kcal} \mathrm{mol}^{-1}\right)$. The C-C reductive elimination is exergonic in all the cases (Table 9).

Table 9: Free energy of Activation $\left(\Delta^{\ddagger} G^{F}\right)$ and reaction $\left(\Delta^{r} G^{F}\right)$ for C-C reductive elimination.

\begin{tabular}{lrr}
\hline BXne & $\Delta^{\ddagger} G^{F}$ & $\Delta^{r} G^{F}$ \\
\hline R3 & 8.78 & -28.89 \\
S3 & 22.33 & -33.29 \\
R4 & 17.20 & -28.61 \\
S4 & 19.48 & -27.11 \\
\hline
\end{tabular}

\section{C-C Bond Formation (F') after $\mathrm{HBr} / \mathrm{H}_{2} \mathrm{CO}_{3}$ Elimination $(\mathrm{J} / \mathrm{K})$}

In contrast to the direct pathway (F) discussed above where the $\mathrm{HBr}$ is removed after the C-C reductive elimination, the $\mathrm{HBr}$ or $\mathrm{H}_{2} \mathrm{CO}_{3}$ is eliminated in the $\mathrm{F}^{\prime}$ path prior to the $\mathrm{C}-\mathrm{C}$ reductive elimination. In the $\mathrm{HBr}$ elimination from $\mathbf{e}$, first the $\mathrm{H}-\mathrm{Br}$ bond is formed and the $\mathrm{HBr}$ remain coordinated with $\mathrm{Pd}$. The dissociation of this complex in free $\mathbf{k}$ and $\mathrm{HBr}$ is endothermic with a dissociation energy $\left(\Delta^{\text {diss. }} G^{K}\right.$; Table 10) of $6.9-10.6 \mathrm{kcal} \mathrm{mol}^{-1}$. The elimination of $\mathrm{HBr}$ is fairly easy for BS3 with a free energy of activation $\left(\Delta^{\ddagger} G^{K}\right)$ of $6.7 \mathrm{kcal}$ mol $^{-1}$, while $\Delta^{\ddagger} G^{K}$ is rather high for BS4 $\left(19.5 \mathrm{kcal} \mathrm{mol}^{-1}\right)$. Similarly we have calculated the $\Delta^{\text {diss. }} G^{J}$ for the dissociation of $\mathrm{H}_{2} \mathrm{CO}_{3}$ from $\mathbf{e}^{\prime}$. The dissociation energy for $\mathrm{H}_{2} \mathrm{CO}_{3}$ is lower for R3, and higher for others. 
Table 10: Free energies of activation $\left(\Delta^{\ddagger} G^{K}\right)$ and reaction $\left(\Delta^{r} G^{K}\right)$ for the elimination of $\mathrm{HBr}$. The last column shows the $\Delta^{\text {diss. }} G$ for the dissociation into $\mathbf{j}+\mathrm{HBr}$.

\begin{tabular}{lrrrr}
\hline BXne & $\Delta^{\ddagger} G^{K}$ & $\Delta^{r} G^{K}$ & $\Delta^{\text {diss. }} G^{K}$ & $\Delta^{\text {diss. }} G^{J}$ \\
\hline R3 & 7.73 & 0.38 & 10.64 & 8.34 \\
S3 & 6.71 & 0.06 & 6.91 & 11.54 \\
R4 & 10.16 & -0.84 & 7.38 & 17.01 \\
S4 & 19.46 & -0.05 & 6.85 & 10.72 \\
\hline
\end{tabular}

Table 11: Free energies of activation for C-C reductive elimination $\left(\Delta^{\ddagger} G^{F^{\prime}}\right)$, and free energies of reaction for the C-C reductive elimination $\left(\Delta^{r} G^{F^{\prime}}\right)$ in $\mathbf{R 3}, \mathbf{S 3}, \mathbf{R} 4$, and $\mathbf{S} 4$ pathways. All energies in $\mathrm{kcal} \mathrm{mol}^{-1}$.

\begin{tabular}{lrr}
\hline BXnl & $\Delta^{\ddagger} G^{F^{\prime}}$ & $\Delta^{r} G^{F^{\prime}}$ \\
\hline R3 & 13.80 & -24.56 \\
S3 & 10.63 & -24.63 \\
R4 & 15.05 & -24.01 \\
S4 & 17.43 & -22.54 \\
\hline
\end{tabular}

The free energies of activation $\left(\Delta^{\ddagger} G^{F^{\prime}}\right.$; Table 11$)$ are 10.6 - $17.4 \mathrm{kcal} \mathrm{mol}^{-1}$.

\section{Base assisted C-C bond formation $\left(\mathrm{F}^{\prime \prime}\right)$}

In this pathway, $\mathrm{F}^{\prime \prime}$, the $\mathrm{C}-\mathrm{C}$ bond formation occurs from $\mathbf{e}^{\prime \prime}$ before the elimination of $\mathrm{H}_{2} \mathrm{CO}_{3}$. This step has a barrier of $8.23 \mathrm{kcal} \mathrm{mol}^{-1}$ for $\mathbf{S 3}$ and $\sim 17 \mathrm{kcal} \mathrm{mol}^{-1}$ for the others. 
Table 12: Free energy of Activation $\left(\Delta^{\ddagger} G^{K}\right)$ and reaction $\left(\Delta^{r} G^{K}\right)$ for base $\left(\mathrm{H}_{2} \mathrm{CO}_{3}\right)$ assisted $\mathrm{C}-\mathrm{C}$ reductive elimination.

\begin{tabular}{lrr}
\hline BXne' & $\Delta^{\ddagger} G^{F^{\prime \prime}}$ & $\Delta^{r} G^{F^{\prime \prime}}$ \\
\hline R3 & 16.31 & -16.14 \\
S3 & 8.23 & -21.75 \\
R4 & 17.53 & -12.58 \\
S4 & 17.42 & -16.93 \\
\hline
\end{tabular}

Comparing all the three pathways for C-C bond formation, the most favourable path for R3 is $\mathrm{F}, \mathrm{F}^{\prime \prime}$ for $\mathbf{S 3}$, and $\mathrm{F}^{\prime}$ for $\mathbf{R} 4$. The pathways $\mathrm{F}^{\prime}$ and $\mathrm{F}^{\prime \prime}$ have similar barriers for $\mathbf{S} 4$. The conformations of 3 have lower barriers $\left(\sim 8 \mathrm{kcal} \mathrm{mol}^{-1}\right)$ than $4\left(\sim 17 \mathrm{kcal} \mathrm{mol}^{-1}\right)$. On directly comparing the free energies of transition states $-\mathbf{f}+\mathrm{H}_{2} \mathrm{CO}_{3}, \mathbf{f}^{\prime}+\mathrm{HBr}+\mathrm{H}_{2} \mathrm{CO}_{3}$, and $\mathbf{f}^{\prime \prime}+$ $\mathrm{HBr}$ - the most favourable path is the base-assisted $\mathrm{F}^{\prime \prime}$ for $\mathbf{R} 4, \mathbf{S 3}$, and $\mathbf{S} 4$. The direct path $\mathrm{F}$ is most favorable for $\mathbf{R 3}$.

All the three routes of $\mathrm{C}-\mathrm{C}$ formation results in the generation of the product scaffold coordinated with $\mathrm{PdL}, \mathrm{PdL}(\mathrm{H})(\mathrm{Br})$, or $\mathrm{PdL}\left(\mathrm{H}_{2} \mathrm{CO}_{3}\right)$. These product-complexes span all the conceivable isomeric product geometries. The BS3 and BR4 has the $-\mathrm{CH}_{3}$ and $-\mathrm{OH}$ groups in the opposite sides of the newly formed five membered ring, while they are in the same side in BR3 and BS4. The products are released $(\mathrm{G})$ from these intermediates.

\section{$\beta$-hydride elimination in the 6-endo-cyclization pathway(M)}

All mechanisms discussed so far lead to the product 2 (Figure 1) through a 5-exo cyclization followed by other steps. Another possible route is through a 6-endo cyclization (D) instead of 5-exo cyclization, after the oxidative addition (C). This pathway leads to the product g-6 after the $\beta$-hydride elimination (L). The $\beta$-hydride elimination is endergonic by 7.74 kcal $\mathrm{mol}^{-1}$ for BR4 and $7.58 \mathrm{kcal} \mathrm{mol}^{-1}$ for BS4. The free energies of activation $\left(\Delta^{\ddagger} G^{L}\right)$ barriers 

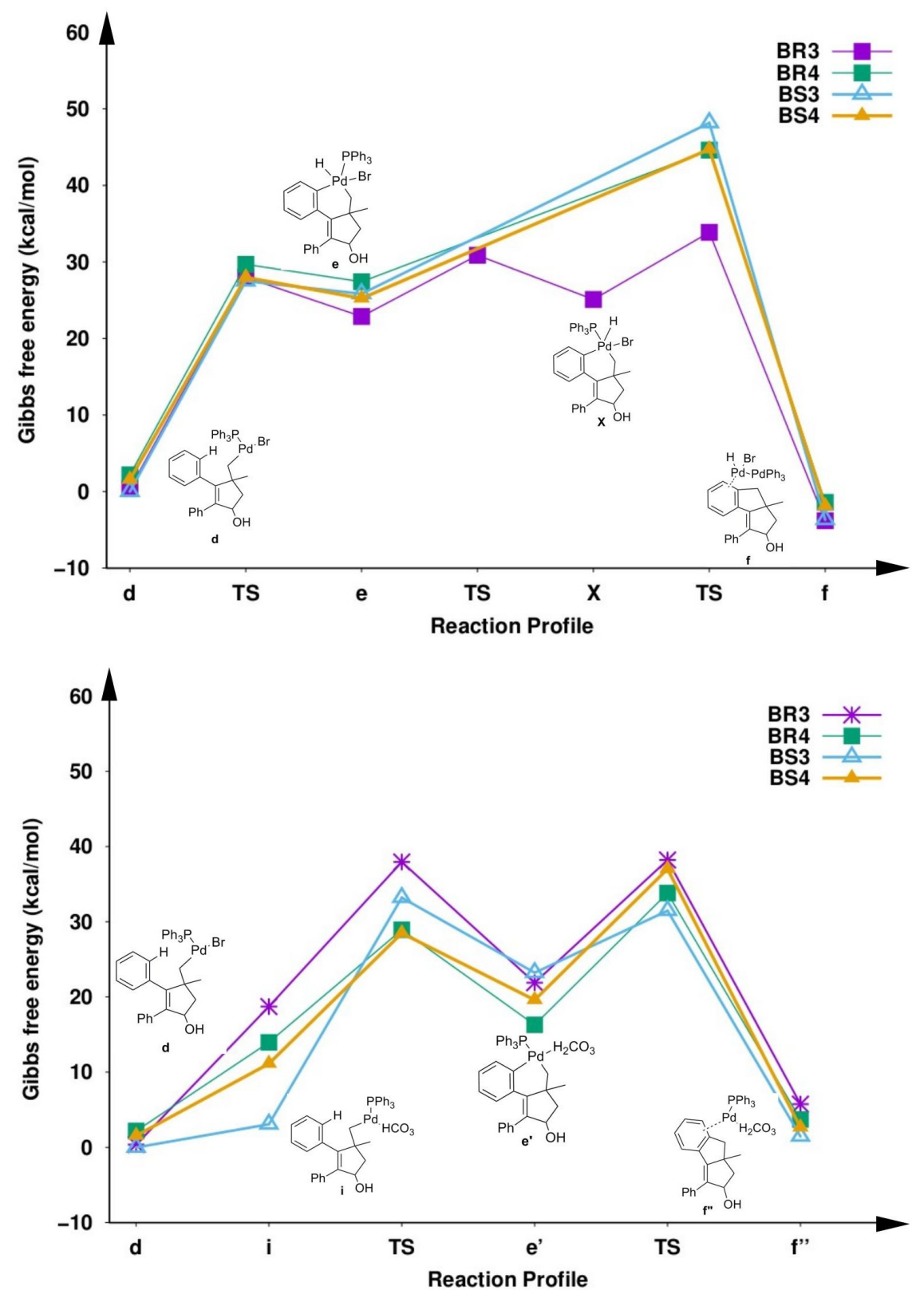

Figure 12: Reaction profiles for $\mathrm{C}-\mathrm{H}$ activation process through two pathways: Top) direct path (d to f), and Bottom) base assisted path (d to $\mathrm{f}^{\prime \prime}$ ) 
are similar, $8.07 \mathrm{kcal} \mathrm{mol}^{-1}$ (BR4c) and $7.46 \mathrm{kcal} \mathrm{mol}^{-1}(\mathbf{B S 4 c})$. The transition states and the intermediates (1-6) are nearly isoenergetic. For BS4c, the Gibbs free energy of the TS is lower by $0.12 \mathrm{kcal} \mathrm{mol}^{-1}$ than the intermediates due to higher entropy, but the total energy $(E)$ of the TS is higher by $0.44 \mathrm{kcal} \mathrm{mol}^{-1}$.

\section{Conclusion}

We have studied all the steps in the catalytic cycle of tandem Heck reaction/C-H activation reaction, $\mathbf{1} \rightarrow \mathbf{2}$ (Figure 1) using the Density Functional Theory based calculations. We analyzed the pathways for the formation of two possible products and many possible pathways. This study consider many conformations of the substrate for the large part of the catalytic cycle.

To account for the conformational flexibility of the substrate, a series of steps based on i) a molecular mechanics (MMFF94) based conformational search using RDKit, ii) clustering of the RI-BP86-D3BJ/def2-SVP optimized geometries, and iii) modeling and optimization of enantiomer used. Eight different conformations selected this way were used for the analysis of catalytic cycle.

The coordination of PdL to re and si faces of the substrates produce $16 \pi$-complexes with the complexation energies ranging between $30-43 \mathrm{kcal} \mathrm{mol}^{-1}$. The complexes where one of the double bond is coordinated (MXn) is less stable than the bidentate complexes with coordination from both the double bonds (BXn). The MXn's are more reactive with lower activation energies for oxidative addition compared to BXn's. But the BXn transition states for oxidative addition are more stable.

Migratory insertion is the regioselective step. The unique geometries features of the intermediates after the oxidative addition and coordination of the second $\mathrm{C}=\mathrm{C}$ bond, $\mathrm{Xnc}$, has a crucial role in determining the course of migratory insertion. The twisted orientation 
facilitate 5-exo and 6-endo cyclization during the migratory insertion, whereas the eclipsed orientation allows only 5-exo cyclization. The barriers show that the 5-exo cyclization is far more favourable, in agreement with the experiments.

The next step in 5-exo pathways is the $\mathrm{C}$-H activation where ortho- $\mathrm{H}$ of phenyl group moves to $\mathrm{Pd}$. This may happen directly or after the exchange of $\mathrm{Br}^{-}$with $\mathrm{HCO}_{3}^{-}$. Among the four conformations analyzed, the latter pathway has lower activation free energies for three of them. The other way to compare the path is to compare the energies of the transition states. In this way, the former direct pathway is more favourable for three conformations.

The next important step is the second C-C bond formation. Here also we analyzed three pathways. Comparing these pathways on stepwise barriers, each of the conformations have different favourable pathways. But on comparing the energies of the transition states, the most favourable path is the base-assisted pathway for three conformations.

\section{Conflicts of interest}

The authors declare no conflict of interests

\section{Acknowledgements}

We thank computational facilities from DST FIST grants (SR/FST/CSII-026/2013 and SR/FST/CSII-011/2005). DYR thank UGC for fellowship.

\section{References}

(1) Heck, R. F. Mechanism of Arylation and Carbomethoxylation of Olefins with Organopalladium Compounds. Journal of the American Chemical Society 1969, 91 (24), 6707-6714. 
https://doi.org/10.1021/ja01052a029.

(2) Mizoroki, T.; Mori, K.; Ozaki, A. Arylation of Olefin with Aryl Iodide Catalyzed by Palladium. Bulletin of the Chemical Society of Japan 1971, 44 (2), 581-581. https: //doi.org/10.1246/bcsj.44.581.

(3) Heck, R. F.; Nolley, J. P. Palladium-Catalyzed Vinylic Hydrogen Substitution Reactions with Aryl, Benzyl, and Styryl Halides. The Journal of Organic Chemistry 1972, 37 (14), 2320-2322. https://doi.org/10.1021/jo00979a024.

(4) Dieck, H. A.; Heck, R. F. Organophosphinepalladium Complexes as Catalysts for Vinylic Hydrogen Substitution Reactions. Journal of the American Chemical Society 1974, 96 (4), 1133-1136. https://doi.org/10.1021/ja00811a029.

(5) Frech, C. The Mizoroki-Heck Reaction. Edited by Martin Oestreich. Angewandte Chemie International Edition 2009, 48 (38), 6947-6947. https://doi.org/10.1002/anie.200903122.

(6) Donde, Y.; Overman, L. E. Asymmetric Carbon-Carbon Bond-Forming Reactions: Asymmetric Intramolecular Heck Reactions. In Catalytic asymmetric synthesis; John Wiley \& Sons, Inc., 2005; pp 675-697. https://doi.org/10.1002/0471721506.ch21.

(7) Link, J. T. The Intramolecular Heck Reaction. In Organic reactions; American Cancer Society, 2004; pp 157-561. https://doi.org/10.1002/0471264180.or060.02.

(8) Döndaş, H. A.; Gracia Retamosa, M. de; Sansano, J. M. Recent Development in PalladiumCatalyzed Domino Reactions: Access to Materials and Biologically Important Carbo- and Heterocycles. Organometallics 2019. https://doi.org/10.1021/acs.organomet.9b00110.

(9) Baudoin, O. Ring Construction by Palladium(0)-Catalyzed c(sp3)H Activation. Accounts of Chemical Research 2017, 50 (4), 1114-1123. https://doi.org/10.1021/acs.accounts. $7 \mathrm{~b} 00099$.

(10) Mehta, V. P.; García-López, J.-A. $\sigma$-Alkyl-PdII Species for Remote c-H Functionalization. 
ChemCatChem 2017, 9 (7), 1149-1156. https://doi.org/10.1002/cctc.201601624.

(11) Ray, J. K.; Singha, R.; Ray, D.; Ray, P.; Rao, D. Y.; Anoop, A. Palladium-Catalyzed Expedient Heck Annulations in 1-Bromo-1,5-Dien-3-Ols: Exceptional Formation of Fused Bicycles. Tetrahedron Letters 2019, 60 (13), 931-935. https://doi.org/10.1016/j.tetlet.2019. 02.043 .

(12) Davies, D. L.; Macgregor, S. A.; McMullin, C. L. Computational Studies of CarboxylateAssisted c-H Activation and Functionalization at Group 8-10 Transition Metal Centers. Chemical Reviews 2017, 117 (13), 8649-8709. https://doi.org/10.1021/acs.chemrev.6b00839.

(13) Gandeepan, P.; Muller, T.; Zell, D.; Cera, G.; Warratz, S.; Ackermann, L. 3d Transition Metals for c-H Activation. Chemical Reviews 2019, 119 (4), 2192-2452. https://doi.org/10. 1021/acs.chemrev.8b00507.

(14) Sperger, T.; Fisher, H. C.; Schönebeck, F. Computationally Deciphering PalladiumCatalyzed Reaction Mechanisms. Wiley Interdisciplinary Reviews: Computational Molecular Science 2016, 6 (3), 226-242. https://doi.org/10.1002/wcms.1244.

(15) Landrum, G. RDKit: Open-source cheminformatics http://www.rdkit.org.

(16) Halgren, T. A.; Nachbar, R. B. Merck Molecular Force Field. IV. Conformational Energies and Geometries for MMFF94. Journal of Computational Chemistry 1996, 17 (5-6), 587-615. https://doi.org/10.1002/(sici)1096-987x(199604)17:5/6<587::aid-jcc4>3.0.co;2-q.

(17) Dirac, P. A. M.; Fowler, R. H. Quantum Mechanics of Many-Electron Systems. Proceedings of the Royal Society of London. Series A, Containing Papers of a Mathematical and Physical Character 1929, 123 (792), 714-733. https://doi.org/10.1098/rspa.1929.0094.

(18) Slater, J. C. A Simplification of the Hartree-Fock Method. Phys. Rev. 1951, 81 (3), 385-390. https://doi.org/10.1103/PhysRev.81.385.

(19) Vosko, S. H.; Wilk, L.; Nusair, M. Accurate Spin-Dependent Electron Liquid Correlation 
Energies for Local Spin Density Calculations: A Critical Analysis. Canadian Journal of Physics 1980, 58 (8), 1200-1211. https://doi.org/10.1139/p80-159.

(20) Perdew, J. P. Density-Functional Approximation for the Correlation Energy of the Inhomogeneous Electron Gas. Phys. Rev. B 1986, 33 (0, 12), 8822-8824. https://doi.org/ 10.1103/PhysRevB.33.8822.

(21) Perdew, J. P. Erratum: Density-Functional Approximation for the Correlation Energy of the Inhomogeneous Electron Gas. Phys. Rev. B 1986, 34 (0, 10), 7406-7406. https: //doi.org/10.1103/PhysRevB.34.7406.

(22) Becke, A. D. Density-Functional Exchange-Energy Approximation with Correct Asymptotic Behavior. Phys. Rev. A 1988, 38 (0, 6), 3098-3100. https://doi.org/10.1103/PhysRevA. 38.3098 .

(23) Schäfer, A.; Horn, H.; Ahlrichs, R. Fully Optimized Contracted Gaussian Basis Sets for Atoms Li to Kr. J. Chem. Phys. 1992, 97 (4), 2571-2577. https://doi.org/10.1063/1.463096.

(24) Eichkorn, K.; Weigend, F.; Treutler, O.; Ahlrichs, R. Auxiliary Basis Sets for Main Row Atoms and Transition Metals and Their Use to Approximate Coulomb Potentials. Theoretical Chemistry Accounts 1997, 97 (1), 119-124. https://doi.org/10.1007/s002140050244.

(25) Grimme, S.; Antony, J.; Ehrlich, S.; Krieg, H. A Consistent and Accurate Ab Initio Parametrization of Density Functional Dispersion Correction (DFT-d) for the 94 Elements H-Pu. J. Chem. Phys. 2010, 132 (15), 154104. https://doi.org/10.1063/1.3382344.

(26) Grimme, S.; Ehrlich, S.; Goerigk, L. Effect of the Damping Function in Dispersion Corrected Density Functional Theory. Journal of Computational Chemistry 2011, 32 (7), 1456-1465. https://doi.org/10.1002/jcc.21759.

(27) Kenneth, D., M; James, P., J. Calculation of Vibrational Frequencies Using Molecular Trajectories. Journal of Molecular Structure: THEOCHEM 1988, 163, 143-149. https: 
//doi.org/https://doi.org/10.1016/0166-1280(88)80386-5.

(28) Henkelman, G.; Uberuaga, P., Blas; Jonsson, H. A Climbing Image Nudged Elastic Band Method for Finding Saddle Points and Minimum Energy Paths. J. Chem. Phys. 2000, 113 (22), 9901-9904. https://doi.org/10.1063/1.1329672.

(29) Henkelman, G.; Jónsson, H. Improved Tangent Estimate in the Nudged Elastic Band Method for Finding Minimum Energy Paths and Saddle Points. The Journal of Chemical Physics 2000, 113 (22), 9978-9985. https://doi.org/10.1063/1.1323224.

(30) Kästner, J.; Carr, J. M.; Keal, T. W.; Thiel, W.; Wander, A.; Sherwood, P. DL-FIND: An Open-Source Geometry Optimizer for Atomistic Simulations. The Journal of Physical Chemistry A 2009, 113 (43), 11856-11865. https://doi.org/10.1021/jp9028968.

(31) ChemShell, a Computational Chemistry Shell.

(32) Lam, K. C.; Marder, T. B.; Lin, Z. DFT Studies on the Effect of the Nature of the Aryl Halide Y-C6H4-X on the Mechanism of Its Oxidative Addition to Pd0L Versus Pd0L2. Organometallics 2007, 26 (3), 758-760. https://doi.org/10.1021/om060784a.

(33) Timea R. Kegl, T. K., Laszlo Kollar. DFT Study on the Oxidative Addition of 4Substituted Iodobenzenes on Pd(0)-Phosphine Complexes. Advances in Physical Chemistry 2015, 2015 (6), 1-6. https://doi.org/10.1155/2015/985268.

(34) Xue, L.; Lin, Z. Theoretical Aspects of Palladium-Catalysed Carbon-carbon CrossCoupling Reactions. Chem. Soc. Rev. 2010, 39 (5), 1692-1705. https://doi.org/10.1039/ B814973A.

(35) Ahlquist, M.; Norrby, P.-O. Oxidative Addition of Aryl Chlorides to Monoligated Palladium(0) a DFT-SCRF Study. Organometallics 2007, 26 (3), 550-553. https://doi.org/ 10.1021/om0604932.

(36) Li, Z.; Fu, Y.; Guo, Q.-X.; Liu, L. Theoretical Study on Monoligated Pd-Catalyzed 
Cross-Coupling Reactions of Aryl Chlorides and Bromides. Organometallics 2008, 27 (16), 4043-4049. https://doi.org/10.1021/om701065f.

(37) Grüber, R.; Fleurat-Lessard, P. Density Functional Study of Indole Formation by an Intramolecular Heck Reaction. Organometallics 2014, 33 (8), 1996-2003. https://doi.org/ 10.1021/om5001309.

(38) Xie, H.; Fan, T.; Lei, Q.; Fang, W. New Progress in Theoretical Studies on PalladiumCatalyzed c-c Bond-Forming Reaction Mechanisms. Science China Chemistry 2016, 59 (11), 1432-1447. https://doi.org/10.1007/s11426-016-0018-2.

(39) Yang, Y.-F.; She, Y. Computational Exploration of Pd-Catalyzed c-H Bond Activation Reactions. International Journal of Quantum Chemistry 2018, 118 (21), e25723. https: //doi.org/10.1002/qua.25723.

(40) Franzoni, I.; Yoon, H.; García-López, J.-A.; Poblador-Bahamonde, A. I.; Lautens, M. Exploring the Mechanism of the Pd-Catalyzed Spirocyclization Reaction: A Combined DFT and Experimental Study. Chem. Sci. 2018, 9 (6), 1496-1509. https://doi.org/10.1039/ C7SC04709F.

(41) Qiao, Y.; Zhao, J.; Chang, J.; Wei, D. Insights into the Oxidative Palladium-Catalyzed Regioselective Synthesis of 3-Arylindoles from N-Ts-Anilines and Styrenes: A Computational Study. ChemCatChem 2019, 11 (2), 780-789. https://doi.org/10.1002/cctc.201801531.

(42) Dang, Y.; Qu, S.; Nelson, J. W.; Pham, H. D.; Wang, Z.-X.; Wang, X. The Mechanism of a Ligand-Promoted c(sp3)H Activation and Arylation Reaction via Palladium Catalysis: Theoretical Demonstration of a $\mathrm{Pd}(\mathrm{II}) / \mathrm{Pd}(\mathrm{IV})$ Redox Manifold. Journal of the American Chemical Society 2015, 137 (5), 2006-2014. https://doi.org/10.1021/ja512374g. 


\title{
Computational Study of Tandem C-C Coupling and $\mathrm{C}-\mathrm{H}$ Activation Reaction of a Flexible Substrate
}

\author{
Davuluri Yogeswara Rao, Anakuthil Anoop*
}

\section{Supporting Information}




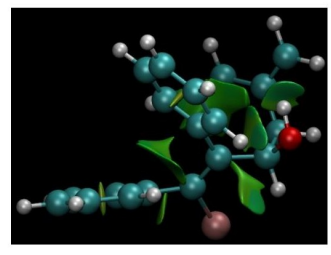

R1; 0.00

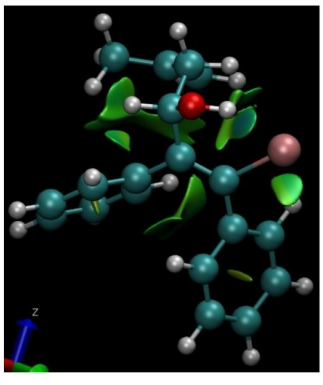

S3; 0.12

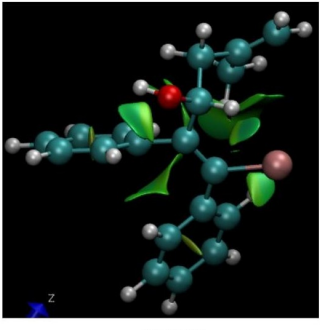

R5; 1.36

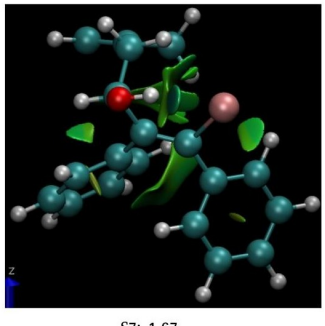

S7; 1.67
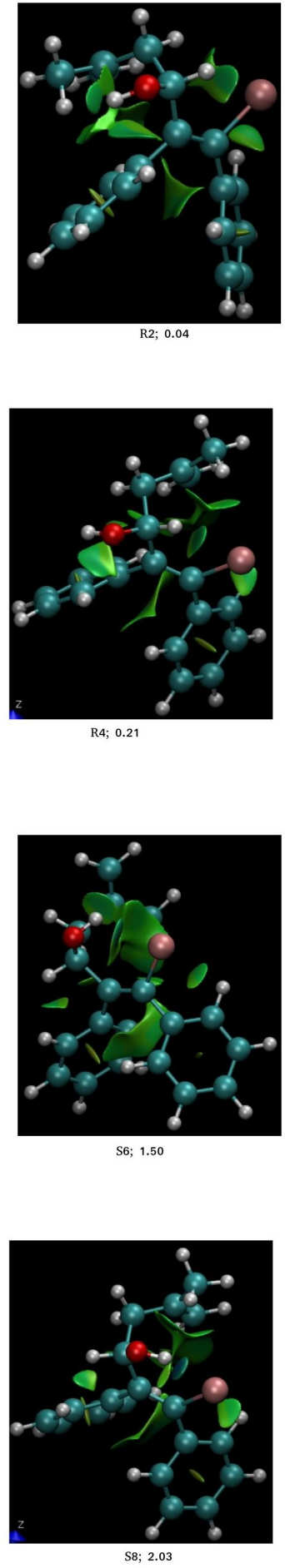

Figure 1: NCI-plot for substrates 


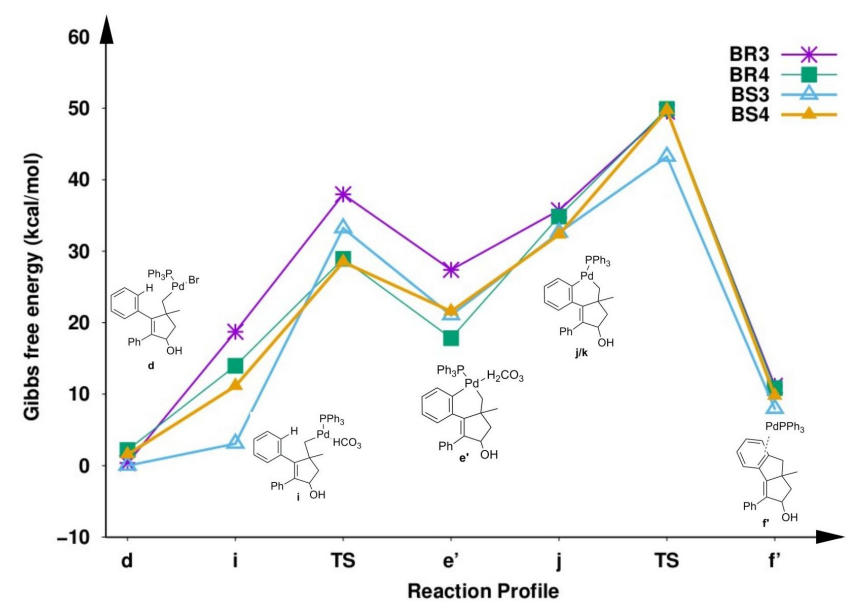

Figure 2: Reaction Profile for $\mathrm{C}-\mathrm{H}$ activation process by base assist mechanism(d to f')

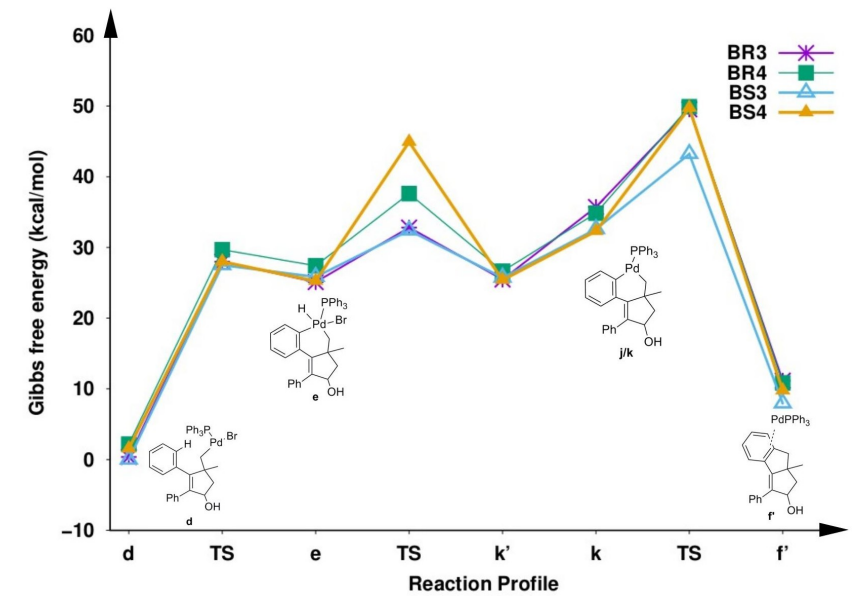

Figure 3: Reaction Profile for $\mathrm{C}-\mathrm{H}$ activation process by $\mathrm{HBr}$ elimination mecha$\operatorname{nism}\left(d\right.$ to $\left.f^{\prime}\right)$ 


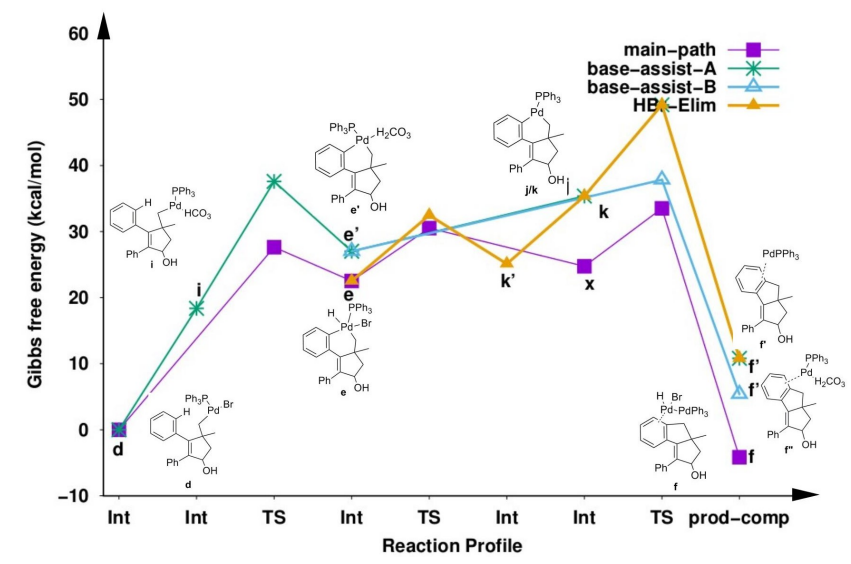

Figure 4: Reaction Profile for C-H activation process in BR3 path

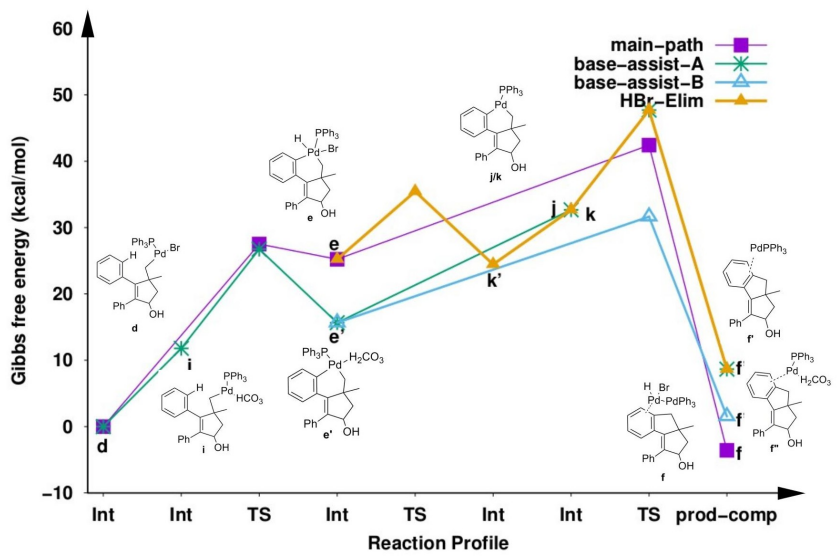

Figure 5: Reaction Profile for C-H activation process in BR4 path 


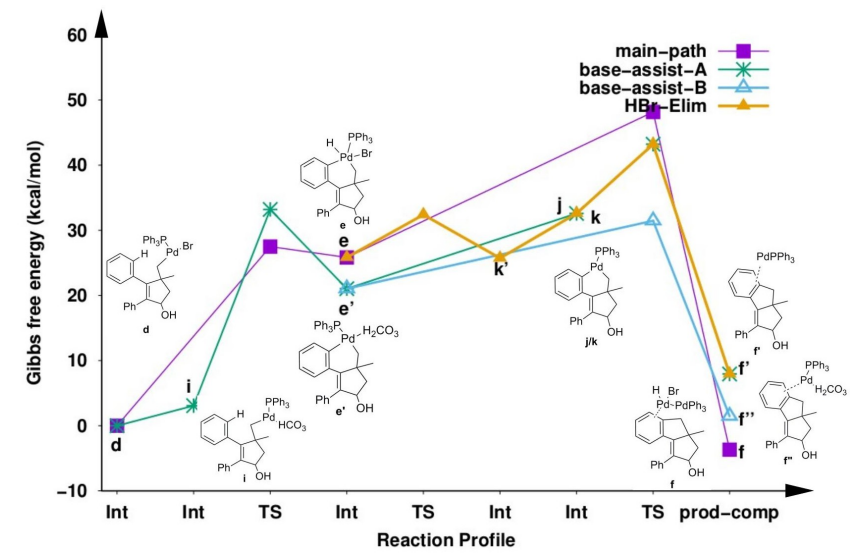

Figure 6: Reaction Profile for C-H activation process in BS3 path

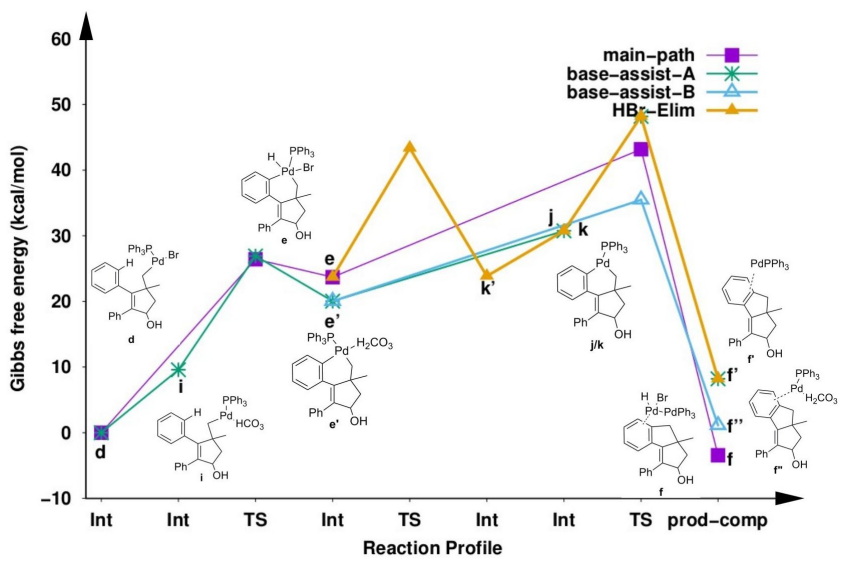

Figure 7: Reaction Profile for $\mathrm{C}-\mathrm{H}$ activation process in BS4 path 


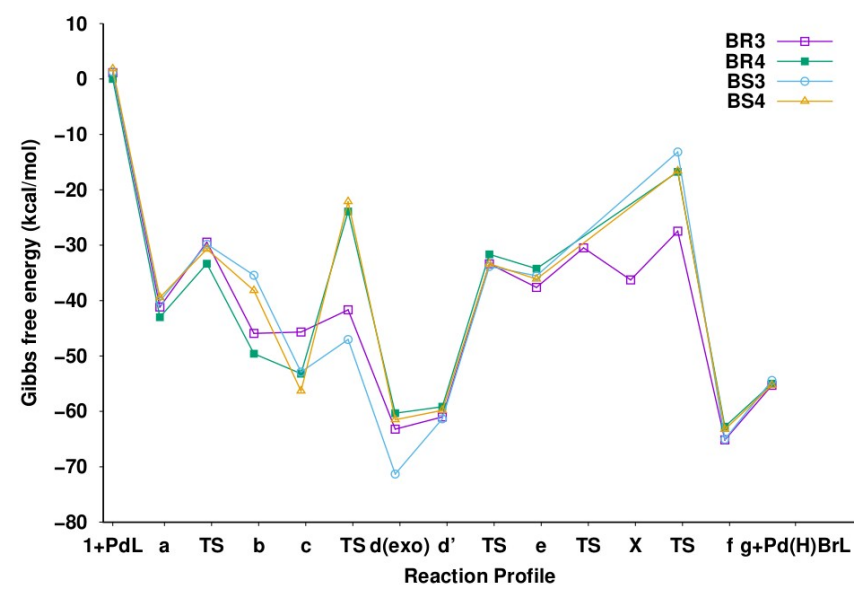

Figure 8: Reaction Profile of complete catalytic cycle (1+PdPPh3, A, B, C, $\mathrm{D}($ exo $), \mathrm{E}, \mathrm{F}, \mathrm{X}$, and $\mathrm{g}+\mathrm{PPh} 3 \mathrm{Pd}(\mathrm{H}) \mathrm{Br}$ ) for $\mathrm{R} 3, \mathrm{R} 4, \mathrm{~S} 3$, and $\mathrm{S} 4$ 


\section{Cartesian coordinates}

\section{Optimized Substrate Geometries(Xn)}

$\begin{array}{lrrr}\text { R1 } & & & \\ \text { 40 } & & & \\ \text { SCF } & \text { Energy }=-3384.324630463 & \\ \text { C } & 0.1641519 & 3.8596021 & -2.6943423 \\ \text { C } & -0.4793661 & 3.8600671 & -1.4352201 \\ \text { C } & -1.8912192 & 3.8318333 & -1.3930792 \\ \text { C } & -2.6387050 & 3.7727622 & -2.5775052 \\ \text { C } & -1.9892860 & 3.7566998 & -3.8233897 \\ \text { C } & -0.5858776 & 3.8066351 & -3.8761968 \\ \text { C } & 0.3280151 & 3.8752638 & -0.1941455 \\ \text { C } & 1.3904422 & 3.0778083 & 0.1028056 \\ \text { C } & 1.7513999 & 1.9720853 & -0.8333983 \\ \text { C } & 0.7836993 & 1.0215640 & -1.2336978 \\ \text { C } & 1.1094655 & -0.0083205 & -2.1244640 \\ \text { C } & 2.4119245 & -0.1095417 & -2.6419141 \\ \text { C } & 3.3804911 & 0.8335950 & -2.2633209 \\ \text { C } & 3.0578720 & 1.8653378 & -1.3681604 \\ \text { Br } & -0.2445710 & 5.2259051 & 1.0740407 \\ \text { C } & 1.3485381 & 1.1180836 & 2.4830670 \\ \text { C } & -0.0309632 & 0.6573982 & 2.0914425 \\ \text { C } & 2.3785148 & 0.2584178 & 2.6622365 \\ \text { C } & 1.5352102 & 2.6104435 & 2.6390008 \\ \text { C } & 2.2164413 & 3.2524038 & 1.3920922 \\ \text { O } & 3.5430539 & 2.7871887 & 1.2616967 \\ \text { H } & 2.2929380 & 4.3416467 & 1.5895058 \\ \text { H } & 0.5653210 & 3.1156756 & 2.8175848 \\ \text { H } & 2.1985841 & 2.8400688 & 3.4975708 \\ \text { H } & 3.3572996 & 0.6042431 & 3.0321331 \\ \text { H } & 2.2607921 & -0.8231180 & 2.4900132 \\ \text { H } & -0.4448638 & 1.2841321 & 1.2762921 \\ \text { H } & -0.7241461 & 0.7780416 & 2.9529759 \\ \text { H } & -0.0503795 & -0.4029256 & 1.7748949 \\ \text { H } & 3.4877952 & 1.8060405 & 1.2317946 \\ \text { H } & -0.2413424 & 1.1035538 & -0.8477842 \\ \text { H } & 0.3384499 & -0.7361556 & -2.4194176 \\ \text { H } & 2.6687674 & -0.9179023 & -3.3432226 \\ \text { H } & 4.3997599 & 0.7728037 & -2.6746040 \\ \text { H } & 3.8126379 & 2.6095064 & -1.0844745 \\ \text { H } & -2.3991865 & 3.8533944 & -0.4174667 \\ \text { H } & -3.7376483 & 3.7407656 & -2.5270163\end{array}$




\begin{tabular}{|c|c|c|c|}
\hline & -2.5768882 & 3.7141620 & -4.7529011 \\
\hline & -0.0690934 & 3.8089275 & -4.8477572 \\
\hline & 1.2614712 & 3.8963074 & -2.7360684 \\
\hline \\
\hline \multicolumn{4}{|c|}{ bing } \\
\hline \multicolumn{4}{|c|}{ CF Energy $=-3384.324152143$} \\
\hline & 0.0730715 & 3.7876234 & -2.6634649 \\
\hline & -0.5563034 & 3.7700971 & -1.3974210 \\
\hline & -1.9629395 & 3.6512945 & -1.3394788 \\
\hline & -2.7164376 & 3.5228488 & -2.5143226 \\
\hline & -2.0798888 & 3.5256185 & -3.7670486 \\
\hline & -0.6833994 & 3.6641967 & -3.8361983 \\
\hline & 0.2601932 & 3.8553427 & -0.1653707 \\
\hline & 1.3745508 & 3.1312160 & 0.1202012 \\
\hline & 1.7875576 & 2.0339571 & 22363 \\
\hline & 0.8950429 & 0.9771634 & 9696 \\
\hline & 1.28 & $-0.0^{\circ}$ & 5405 \\
\hline & 2.567 & -0.0 & -2. \\
\hline & 3.460 & 0.9 & -2.2 \\
\hline & 3.077 & 2.0 & -1.3 \\
\hline $3 r$ & -0.3976235 & 5.15 & 9276 \\
\hline & 1.1534386 & 1.084 & 29915 \\
\hline & 2.2914954 & 0.118 & 2.0963834 \\
\hline & -0.1350794 & 0.6848878 & 2.2730204 \\
\hline & 1.5008520 & 2.5317435 & 2.5926649 \\
\hline & 2.1711725 & 3.3045822 & 1.4204453 \\
\hline & 3.5506035 & 3.0000738 & 1.2826475 \\
\hline & 2.1427346 & 4.3801186 & 1.6898766 \\
\hline & 0.5802011 & 3.0808662 & 2.8727829 \\
\hline & 2.2070649 & 2.6076175 & 3.4489526 \\
\hline & -0.9599122 & 1.3985791 & 2.4258785 \\
\hline & -0.4044392 & -0.3654266 & 2.0773793 \\
\hline & 3.0730024 & 0.2189909 & 2.8785215 \\
\hline & 2.7826111 & 0.2972709 & 1.1130227 \\
\hline & 1.9413736 & -0.9319863 & 2.0811883 \\
\hline & 3.6248463 & 2.0558110 & 1.0430240 \\
\hline & -0.1036380 & 0.9870903 & -0.6358567 \\
\hline & 0.5733809 & -0.8875038 & -2.1490553 \\
\hline & 2.8701841 & -0.9145151 & -3.1646404 \\
\hline & 4.4646083 & 0.9615281 & -2.6795359 \\
\hline & 3.7687613 & 2.8428522 & -1.1799220 \\
\hline & -2.4611222 & 3.6557834 & -0.3588034 \\
\hline & -3.8104369 & 3.4207554 & -2.4510542 \\
\hline & -2.6728128 & 3.4284953 & -4.6890710 \\
\hline & -0.1773238 & 3.6820610 & -4.813248 \\
\hline
\end{tabular}


40

$\mathrm{SCF}$ Energy $=-3384.321867227$

\begin{tabular}{lrrr}
$\mathrm{C}$ & 0.0944895 & 3.7664430 & -2.6750965 \\
$\mathrm{C}$ & -0.5579408 & 3.7847326 & -1.4211781 \\
$\mathrm{C}$ & -1.9660148 & 3.6705831 & -1.3857285 \\
$\mathrm{C}$ & -2.6982451 & 3.5107801 & -2.5700896 \\
$\mathrm{C}$ & -2.0388757 & 3.4795861 & -3.8105765 \\
$\mathrm{C}$ & -0.6412360 & 3.6144417 & -3.8579343 \\
$\mathrm{C}$ & 0.2321647 & 3.8918916 & -0.1726593 \\
$\mathrm{C}$ & 1.3177058 & 3.1411163 & 0.1507657 \\
$\mathrm{C}$ & 1.7455395 & 2.0353324 & -0.7581960 \\
$\mathrm{C}$ & 0.8939972 & 0.9321460 & -0.9894798 \\
$\mathrm{C}$ & 1.2942708 & -0.1178479 & -1.8270310 \\
$\mathrm{C}$ & 2.5546560 & -0.0867101 & -2.4476979 \\
$\mathrm{C}$ & 3.4097817 & 1.0048540 & -2.2275819 \\
$\mathrm{C}$ & 3.0115882 & 2.0592056 & -1.3894974 \\
$\mathrm{Br}$ & -0.4536179 & 5.2059445 & 1.0691155 \\
$\mathrm{C}$ & 0.3662480 & 2.2302161 & 3.0866175 \\
$\mathrm{C}$ & -0.7645994 & 1.5830657 & 2.3277231 \\
$\mathrm{C}$ & 0.1667109 & 2.8511440 & 4.2678682 \\
$\mathrm{C}$ & 1.7542500 & 2.1657375 & 2.4784403 \\
$\mathrm{C}$ & 2.1040837 & 3.2786475 & 1.4541425 \\
$\mathrm{O}$ & 3.5089986 & 3.3004640 & 1.2195487 \\
$\mathrm{H}$ & 1.8632039 & 4.2602743 & 1.9062582 \\
$\mathrm{H}$ & -0.1175590 & 3.6063286 & -4.8257783 \\
$\mathrm{H}$ & 2.5171007 & 2.2585269 & 3.2777317 \\
$\mathrm{H}$ & 1.9126196 & 1.1801096 & 1.9836684 \\
$\mathrm{H}$ & 0.9991935 & 3.3147592 & 4.8210791 \\
$\mathrm{H}$ & -0.8367910 & 2.9247530 & 4.7158655 \\
$\mathrm{H}$ & -0.5414382 & 0.5141116 & 2.1207769 \\
$\mathrm{H}$ & -0.9167540 & 2.0712808 & 1.3429732 \\
$\mathrm{H}$ & -1.7178405 & 1.6394229 & 2.8870913 \\
$\mathrm{H}$ & 3.7630802 & 2.4050563 & 0.9173970 \\
$\mathrm{H}$ & -0.0951189 & 0.9117986 & -0.5111576 \\
$\mathrm{H}$ & 0.6151491 & -0.9667582 & -1.9984643 \\
$\mathrm{H}$ & 2.8670692 & -0.9103069 & -3.1073237 \\
$\mathrm{H}$ & 4.3925993 & 1.0444121 & -2.7216737 \\
\hline & -2.6639956 & 2.9316411 & -1.2429111 \\
$\mathrm{H}$ & -3.7934228 & 3.4126014 & -2.5240228 \\
$\mathrm{H}$ & 1.6151112 & 3.3593927 & -4.7404218 \\
$\mathrm{H}$ & 3.8742874 & -2.7141171
\end{tabular}


40

SCF Energy $=-3384.323553539$

0.2120622
-0.4610486
-1.8724498
-2.5899889
-1.9112382
-0.5088748
0.3105813
1.3448882
1.7126543
0.7753360
1.1159869
2.4016861

3. 9021169

$-2.7155913$

3. 9128342

$-1.4730441$

$$
\text { C }
$$

C

C

C

西

C

3.3410529

3.3410529

$3.8508872-1.4614693$

$3.7524540-2.6613534$

$3.7296646-3.8918358$

$3.8102604-3.9138784$

$3.9580401-0.2092624$

$3.1432395 \quad 0.1223140$

$2.0216807-0.7923625$

$1.0062086-1.0869813$

$-0.0603753-1.9294544$

$-0.1340723-2.4925417$

$0.8706121-2.2113444$

$-0.2976450 \quad 5.3303414 \quad 1.0098006$

$\begin{array}{llll}\text { C } & 0.1867391 & 2.2861927 & 2.9188965\end{array}$

$\begin{array}{llll}\text { C } & 0.0069592 & 3.2770807 & 4.0410422\end{array}$

C $\quad-0.8474563 \quad 1.6080671 \quad 2.3795840$

C $\quad 1.6006322 \quad 2.0986324 \quad 2.4166317$

C $\quad 2.1016585 \quad 3.2064281 \quad 1.4482210$

$\begin{array}{llll}0 & 3.5109257 & 3.1116209 & 1.2767077\end{array}$

$\mathrm{H} \quad 1.9278676 \quad 4.2019192 \quad 1.9013077$

$\begin{array}{llll}\mathrm{H} & 2.3153491 & 2.0952799 & 3.2675482\end{array}$

$\mathrm{H} \quad 1.6984921 \quad 1.1214850 \quad 1.8958724$

$\mathrm{H} \quad-0.6902298 \quad 0.8693373 \quad 1.5784660$

$\mathrm{H} \quad-1.8810992 \quad 1.7642379 \quad 2.7261887$

$\mathrm{H} \quad 0.4307335 \quad 4.2680128 \quad 3.7743621$

$\mathrm{H} \quad 0.5434777 \quad 2.9357018 \quad 4.9530917$

$\mathrm{H} \quad-1.0598059 \quad 3.4215658 \quad 4.2979926$

$\mathrm{H} \quad 3.7009884 \quad 2.2021497 \quad 0.9695635$

$\mathrm{H} \quad-0.2339853 \quad 1.0747296 \quad-0.6567296$

$\mathrm{H} \quad 0.3708525 \quad-0.8395658 \quad-2.1506504$

$\mathrm{H} \quad 2.6681930 \quad-0.9709605 \quad-3.1556101$

$\mathrm{H} \quad 4.3447241 \quad 0.8291777 \quad-2.6614730$

$\mathrm{H} \quad 3.7238174 \quad 2.7487415 \quad-1.1759141$

$\mathrm{H} \quad-2.4007031 \quad 3.8738496 \quad-0.4968899$

$\mathrm{H} \quad-3.6887047 \quad 3.6937337 \quad-2.6357893$

$\mathrm{H} \quad-2.4760265 \quad 3.6567799 \quad-4.8335672$

$\mathrm{H} \quad 0.0300842 \quad 3.8060875 \quad-4.8733494$

$\mathrm{H} \quad 1.3087539 \quad 3.9656522 \quad-2.7326192$

S1

40 


$\begin{array}{lrrr}\text { SCF } & \text { Energy }=-3384.321343614 & \\ \text { C } & 0.0357325 & 3.7618557 & -2.6233111 \\ \text { C } & -0.5718494 & 3.6905107 & -1.3485636 \\ \text { C } & -1.9682511 & 3.4914840 & -1.2687032 \\ \text { C } & -2.7325034 & 3.3357947 & -2.4333409 \\ \text { C } & -2.1171981 & 3.3916049 & -3.6954196 \\ \text { C } & -0.7319983 & 3.6110674 & -3.7850817 \\ \text { C } & 0.2615382 & 3.8045827 & -0.1322275 \\ \text { C } & 1.4166669 & 3.1416668 & 0.1371300 \\ \text { C } & 1.8626223 & 2.0538381 & -0.7788961 \\ \text { C } & 0.9890562 & 1.0122242 & -1.1633865 \\ \text { C } & 1.4272324 & -0.0110808 & -2.0126239 \\ \text { C } & 2.7449993 & -0.0106353 & -2.5017285 \\ \text { C } & 3.6229709 & 1.0199746 & -2.1277105 \\ \text { C } & 3.1886168 & 2.0395419 & -1.2679848 \\ \text { Br } & -0.4463582 & 5.1048166 & 1.1390943 \\ \text { C } & 1.3395007 & 1.3089393 & 2.4817307 \\ \text { C } & -0.1298382 & 1.0347508 & 2.2889130 \\ \text { C } & 2.2671024 & 0.3298086 & 2.4618721 \\ \text { C } & 1.7365075 & 2.7635718 & 2.6563313 \\ \text { C } & 2.3195772 & 3.3903263 & 1.3588281 \\ \text { O } & 2.7044624 & 4.7382334 & 1.5316529 \\ \text { H } & 3.2627751 & 2.8467831 & 1.1499074 \\ \text { H } & 0.8632349 & 3.3663658 & 2.9788032 \\ \text { H } & 2.5181257 & 2.8770634 & 3.4348035 \\ \text { H } & 3.3367081 & 0.5476767 & 2.6135202 \\ \text { H } & 1.9909838 & -0.7215089 & 2.2854728 \\ \text { H } & -0.5428807 & 1.6531711 & 1.4638206 \\ \text { H } & -0.7027063 & 1.3170588 & 3.1991041 \\ \text { H } & -0.3306216 & -0.0309679 & 2.0666283 \\ \text { H } & 1.8837435 & 5.2519642 & 1.6872941 \\ \text { H } & -0.0412635 & 1.0079815 & -0.7820559 \\ \text { H } & 0.7343672 & -0.8186652 & -2.2935377 \\ \text { H } & 3.0878549 & -0.8142501 & -3.1707995 \\ \text { H } & 4.6554655 & 1.0318959 & -2.5087637 \\ \text { H } & 3.8774576 & 2.8513293 & -0.9886383 \\ \text { H } & -2.4510735 & 3.4541836 & -0.2807511 \\ \text { H } & -3.8176653 & 3.1704008 & -2.3547675 \\ \text { H } & -2.7187083 & 3.2729214 & -4.6092592 \\ \text { H } & -0.2444378 & 3.6702934 & -4.7698062 \\ \text { H } & 1.1193516 & 3.9312267 & -2.6916495 \\ & & & \\ \text { S2 } & & & \\ \text { SO } & & & \\ \text { C } & 0.0394431 & 3.8325224 & -2.6596784\end{array}$




$\begin{array}{lrrr}\text { C } & -0.5805059 & 3.7453379 & -1.3918910 \\ \text { C } & -1.9808352 & 3.5692860 & -1.3287761 \\ \text { C } & -2.7370019 & 3.4527398 & -2.5030977 \\ \text { C } & -2.1096100 & 3.5241685 & -3.7585169 \\ \text { C } & -0.7199785 & 3.7196326 & -3.8313353 \\ \text { C } & 0.2436717 & 3.8190761 & -0.1660401 \\ \text { C } & 1.3858532 & 3.1320071 & 0.0922459 \\ \text { C } & 1.8090026 & 2.0501503 & -0.8419192 \\ \text { C } & 0.9198132 & 1.0143619 & -1.2042298 \\ \text { C } & 1.3362534 & -0.0168900 & -2.0551096 \\ \text { C } & 2.6475938 & -0.0321148 & -2.5619764 \\ \text { C } & 3.5418031 & 0.9909840 & -2.2061072 \\ \text { C } & 3.1286899 & 2.0204679 & -1.3466865 \\ \text { Br } & -0.4584357 & 5.0939721 & 1.1330630 \\ \text { C } & 1.2222004 & 1.1572626 & 2.3102643 \\ \text { C } & 2.3367310 & 0.1678922 & 2.0807574 \\ \text { C } & -0.0790874 & 0.8019950 & 2.2575611 \\ \text { C } & 1.6248804 & 2.5923097 & 2.5641676 \\ \text { C } & 2.2712737 & 3.2991098 & 1.3376990 \\ \text { O } & 2.6548860 & 4.6266016 & 1.6258222 \\ \text { H } & 3.2219600 & 2.7756630 & 1.1109742 \\ \text { H } & 0.7386909 & 3.1819295 & 2.8727095 \\ \text { H } & 2.3695046 & 2.6550851 & 3.3873831 \\ \text { H } & -0.8795431 & 1.5422317 & 2.4147273 \\ \text { H } & -0.3830815 & -0.2372845 & 2.0545683 \\ \text { H } & 3.0547209 & 0.1724182 & 2.9289943 \\ \text { H } & 2.9186291 & 0.4241115 & 1.1692731 \\ \text { H } & 1.9523330 & -0.8618354 & 1.9512351 \\ \text { H } & 1.8317848 & 5.1288578 & 1.8052905 \\ \text { H } & -0.0981394 & 1.0203219 & -0.7910919 \\ \text { H } & 0.6328557 & -0.8204576 & -2.3211021 \\ \text { H } & 2.9735114 & -0.8436259 & -3.2300276 \\ \text { H } & 4.5694166 & 0.9889311 & -2.6002521 \\ \text { H } & 3.8291043 & 2.8264870 & -1.0787495 \\ \text { H } & -2.4724704 & 3.5168111 & -0.3460236 \\ \text { H } & -3.8255700 & 3.3048600 & -2.4374366 \\ \text { H } & -2.7049428 & 3.4360588 & -4.6798498 \\ \text { H } & -0.2223110 & 3.7905708 & -4.8102492 \\ \text { H } & 1.1262062 & 3.9854933 & -2.7151893 \\ & & & \\ \text { S3 } & & & \\ \text { 40 } & & & \\ \text { SCF } & \text { Energy }=-3384.321804377 & \\ \text { C } & 0.0689516 & 3.8254595 & -2.7153852 \\ & -0.5981952 & 3.8183095 & -1.4691640 \\ & -1.9982082 & 3.6294080 & -1.4488626\end{array}$




$\begin{array}{lrrr}\text { C } & -2.7064506 & 3.4201708 & -2.6402105 \\ \text { C } & -2.0312804 & 3.4126411 & -3.8725841 \\ \text { C } & -0.6422401 & 3.6222238 & -3.9051339 \\ \text { C } & 0.1768109 & 3.9872913 & -0.2168117 \\ \text { C } & 1.2999727 & 3.2968601 & 0.1157316 \\ \text { C } & 1.7040071 & 2.1379122 & -0.7369797 \\ \text { C } & 0.7999870 & 1.0806067 & -0.9860173 \\ \text { C } & 1.1780206 & -0.0162124 & -1.7710845 \\ \text { C } & 2.4688714 & -0.0792463 & -2.3232536 \\ \text { C } & 3.3797335 & 0.9611460 & -2.0775670 \\ \text { C } & 3.0032880 & 2.0569491 & -1.2862023 \\ \text { Br } & -0.6106726 & 5.3118312 & 0.9617394 \\ \text { C } & 0.6528970 & 2.6365427 & 3.1691724 \\ \text { C } & -0.5698152 & 1.9388391 & 2.6307492 \\ \text { C } & 0.5999851 & 3.4644897 & 4.2371548 \\ \text { C } & 1.9553181 & 2.4206995 & 2.4300707 \\ \text { C } & 2.2175328 & 3.5016768 & 1.3307745 \\ \text { O } & 2.2754504 & 4.7973067 & 1.8605382 \\ \text { H } & 3.2418929 & 3.2980638 & 0.9508754 \\ \text { H } & 2.8076947 & 2.4829482 & 3.1356222 \\ \text { H } & 1.9819304 & 1.4180435 & 1.9551106 \\ \text { H } & 1.5077778 & 3.9462033 & 4.6336841 \\ \text { H } & -0.3486540 & 3.6691758 & 4.7583872 \\ \text { H } & -0.4233241 & 0.8371765 & 2.6339418 \\ \text { H } & -0.7581870 & 2.2281366 & 1.5761676 \\ \text { H } & -1.4754412 & 2.1749823 & 3.2212889 \\ \text { H } & 1.4268758 & 4.9561622 & 2.3277749 \\ \text { H } & -0.2113315 & 1.1313809 & -0.5580284 \\ \text { H } & 0.4588662 & -0.8290567 & -1.9535487 \\ \text { H } & 2.7648884 & -0.9391135 & -2.9428866 \\ \text { H } & 4.3911521 & 0.9232350 & -2.5099641 \\ \text { H } & 3.7173603 & 2.8776701 & -1.1178286 \\ \text { H } & -2.5275047 & 3.6435312 & -0.4845491 \\ \text { H } & -3.7951801 & 3.2628819 & -2.6061004 \\ \text { H } & -2.5888802 & 3.2525548 & -4.8077577 \\ \text { H } & -0.1072694 & 3.6328666 & -4.8667569 \\ \text { H } & 1.1551697 & 3.9893523 & -2.7401062 \\ & & & \\ \text { S4 } & & & \\ \text { 40 } & & & \\ \text { SCF } & \text { Energy }=-3384.320789836 & \\ \text { C } & 0.0802156 & 3.8154798 & -2.6531116 \\ \text { C } & -0.5386066 & 3.8057451 & -1.3827714 \\ \text { C } & -1.9305189 & 3.5786960 & -1.3028236 \\ \text { C } & -2.6790537 & 3.3383628 & -2.4631486 \\ & -2.0524003 & 3.3346622 & -3.7211341\end{array}$




$\begin{array}{lrrr}\text { C } & -0.6716897 & 3.5787879 & -3.8114342 \\ \text { C } & 0.2760305 & 4.0095590 & -0.1625766 \\ \text { C } & 1.4156206 & 3.3446523 & 0.1613112 \\ \text { C } & 1.8118582 & 2.1743398 & -0.6804866 \\ \text { C } & 0.9036624 & 1.1193643 & -0.9227457 \\ \text { C } & 1.2786523 & 0.0159929 & -1.7001665 \\ \text { C } & 2.5689118 & -0.0540832 & -2.2527621 \\ \text { C } & 3.4828126 & 0.9857130 & -2.0153640 \\ \text { C } & 3.1102793 & 2.0873403 & -1.2302955 \\ \mathrm{Br} & -0.4663314 & 5.4009599 & 0.9796834 \\ \mathrm{C} & 0.5685243 & 2.7234177 & 3.0997080 \\ \mathrm{C} & 0.4497868 & 3.7590485 & 4.1895748 \\ \mathrm{C} & -0.4872113 & 2.0032030 & 2.6644987 \\ \mathrm{C} & 1.9438769 & 2.5372023 & 2.5010185 \\ \mathrm{C} & 2.3232109 & 3.5586817 & 1.3765589 \\ \mathrm{O} & 2.4803414 & 4.8828870 & 1.8312720 \\ \mathrm{H} & 3.3383316 & 3.2500602 & 1.0463177 \\ \mathrm{H} & 2.7243043 & 2.6371561 & 3.2846842 \\ \mathrm{H} & 2.0364980 & 1.5214550 & 2.0648842 \\ \mathrm{H} & -0.3704393 & 1.2324156 & 1.8868296 \\ \mathrm{H} & -1.4979192 & 2.1585448 & 3.0738003 \\ \mathrm{H} & 0.9061106 & 4.7186436 & 3.8731500 \\ \mathrm{H} & 1.0155896 & 3.4369962 & 5.0913015 \\ \mathrm{H} & -0.6018449 & 3.9393904 & 4.4841643 \\ \mathrm{H} & 1.5956222 & 5.3074975 & 1.8491845 \\ \mathrm{H} & -0.1078552 & 1.1790642 & -0.4968892 \\ \mathrm{H} & 0.5575743 & -0.7966264 & -1.8757483 \\ \mathrm{H} & 2.8626341 & -0.9195580 & -2.8656658 \\ \mathrm{H} & 4.4939087 & 0.9419699 & -2.4479165 \\ \mathrm{H} & 3.8267299 & 2.9064539 & -1.0641744 \\ \mathrm{H} & -2.4190939 & 3.5824294 & -0.3171127 \\ \mathrm{H} & -3.7607851 & 3.1515292 & -2.3847470 \\ \mathrm{H} & -2.6419413 & 3.1489328 & -4.6316316 \\ \mathrm{H} & -0.1753046 & 3.5896097 & -4.7935009 \\ \mathrm{H} & 1.1606088 & 4.0050235 & -2.7211346\end{array}$

\section{Catalyst}

$\begin{array}{lrrr}\text { Pd-PPh3 } & & \\ \text { 35 } & & & \\ \text { SCF } & \text { Energy }=-1163.896646601 & \\ \text { Pd } & 0.0014905 & -0.0069045 & 0.3903040 \\ \text { P } & 0.0006561 & -0.0032558 & 2.5559726 \\ \text { C } & 1.6577943 & 0.1152833 & 3.3623163\end{array}$




$\begin{array}{lrrr}\text { C } & -0.9293459 & 1.3756807 & 3.3585243 \\ \text { C } & -0.7278530 & -1.4956092 & 3.3637236 \\ \text { C } & 1.8917176 & 0.8978056 & 4.5110888 \\ \text { C } & 3.1681654 & 0.9220924 & 5.0980853 \\ \text { C } & 4.2137238 & 0.1618046 & 4.5498492 \\ \text { C } & 3.9836175 & -0.6207764 & 3.4038480 \\ \text { C } & 2.7150921 & -0.6369513 & 2.8074890 \\ \text { H } & 1.0733741 & 1.4896176 & 4.9470021 \\ \text { H } & 3.3448833 & 1.5402798 & 5.9916490 \\ \text { H } & 5.2127362 & 0.1838455 & 5.0113272 \\ \text { H } & 4.8018619 & -1.2127849 & 2.9661292 \\ \text { H } & 2.5281324 & -1.2263022 & 1.8950282 \\ \text { C } & -1.7334824 & 1.1894043 & 4.5009768 \\ \text { C } & -2.3906406 & 2.2854259 & 5.0852805 \\ \text { C } & -2.2435862 & 3.5712864 & 4.5406372 \\ \text { C } & -1.4414350 & 3.7608148 & 3.4007542 \\ \text { C } & -0.7951853 & 2.6677756 & 2.8070341 \\ \text { H } & -1.8461729 & 0.1845790 & 4.9340894 \\ \text { H } & -3.0217077 & 2.1313001 & 5.9740256 \\ \text { H } & -2.7603936 & 4.4275741 & 5.0001502 \\ \text { H } & -1.3286151 & 4.7656770 & 2.9658721 \\ \text { H } & -0.1836000 & 2.7988786 & 1.8995371 \\ \text { C } & -0.1648235 & -2.0936480 & 4.5091909 \\ \text { C } & -0.7846990 & -3.2091292 & 5.0974892 \\ \text { C } & -1.9708827 & -3.7281416 & 4.5539545 \\ \text { C } & -2.5358037 & -3.1335313 & 3.4110712 \\ \text { C } & -1.9128939 & -2.0290984 & 2.8133257 \\ \text { H } & 0.7607999 & -1.6859284 & 4.9413877 \\ \text { H } & -0.3358952 & -3.6745375 & 5.9884457 \\ \text { H } & -2.4535587 & -4.6024109 & 5.0166932 \\ \text { H } & -3.4616595 & -3.5409700 & 2.9770846 \\ \text { H } & -2.3317600 & -1.5691458 & 1.9034888\end{array}$

\section{Optimized Geometries(BXna)}

$\begin{array}{lccc}\text { R1 } & & & \\ 75 & & & \\ \text { SCF } & \text { Energy }=-4548.306560953 & \\ \text { C } & 2.5130515 & 1.1784604 & -2.3455717 \\ \text { C } & 1.5879454 & 1.5168222 & -1.3280432 \\ \text { C } & 0.4369107 & 2.2530947 & -1.7156954 \\ \text { C } & 0.1870472 & 2.5725413 & -3.0517763 \\ \text { C } & 1.0896945 & 2.1831225 & -4.0585269 \\ \text { C } & 2.2560381 & 1.4984396 & -3.6902204\end{array}$




\begin{tabular}{lrrr} 
C & 1.7252891 & 1.0980053 & 0.1021446 \\
$\mathrm{C}$ & 2.5000569 & 0.0339998 & 0.6750709 \\
$\mathrm{C}$ & 3.3573483 & -0.7899594 & -0.2476563 \\
$\mathrm{C}$ & 2.8293965 & -1.8475975 & -1.0157623 \\
$\mathrm{C}$ & 3.6430437 & -2.5694417 & -1.9008310 \\
$\mathrm{C}$ & 5.0102367 & -2.2646671 & -2.0144158 \\
$\mathrm{C}$ & 5.5574318 & -1.2299439 & -1.2373517 \\
$\mathrm{C}$ & 4.7372297 & -0.4950033 & -0.3651142 \\
$\mathrm{Br}$ & 1.2996832 & 2.6166951 & 1.3089286 \\
$\mathrm{C}$ & 0.6966314 & -1.1563226 & 2.8011533 \\
$\mathrm{C}$ & -0.6189252 & -0.9188852 & 3.5085494 \\
$\mathrm{C}$ & 0.9279626 & -2.3377424 & 2.1118355 \\
$\mathrm{C}$ & 1.7994336 & -0.1633069 & 3.1670635 \\
$\mathrm{C}$ & 2.9620679 & -0.0292221 & 2.1698984 \\
$\mathrm{O}$ & 3.8005793 & -1.1552569 & 2.4060573 \\
$\mathrm{H}$ & 3.5068144 & 0.9202116 & 2.4095057 \\
$\mathrm{H}$ & 1.3360283 & 0.8303060 & 3.3112072 \\
$\mathrm{H}$ & 2.2389529 & -0.4500053 & 4.1496183 \\
$\mathrm{H}$ & 1.9487145 & -2.6277352 & 1.8253593 \\
$\mathrm{H}$ & 0.1322651 & -3.0955351 & 2.0505041 \\
$\mathrm{H}$ & -0.9880262 & 0.1129333 & 3.3357499 \\
$\mathrm{H}$ & -0.4815124 & -1.0292113 & 4.6075526 \\
$\mathrm{H}$ & -1.3999455 & -1.6307688 & 3.1806715 \\
$\mathrm{H}$ & 4.4390456 & -1.2185748 & 1.6669927 \\
$\mathrm{H}$ & 1.7561356 & -2.0826175 & -0.9143468 \\
$\mathrm{H}$ & 3.2054765 & -3.3798028 & -2.5033658 \\
$\mathrm{H}$ & 5.6497715 & -2.8342530 & -2.7053979 \\
$\mathrm{H}$ & 6.6261271 & -0.9805852 & -1.3222675 \\
$\mathrm{H}$ & 5.1583697 & 0.3535951 & 0.1980701 \\
$\mathrm{H}$ & -0.2854911 & 2.5507418 & -0.9458051 \\
$\mathrm{H}$ & -0.7326747 & 3.1199950 & -3.3067252 \\
$\mathrm{H}$ & 0.8902288 & 2.4233638 & -5.1135923 \\
$\mathrm{H}$ & 2.9939214 & 1.2099769 & -4.4547380 \\
$\mathrm{H}$ & 3.4431459 & 0.6583509 & -2.1001621 \\
$\mathrm{Pd}$ & 0.4525849 & -0.5817030 & 0.6039883 \\
$\mathrm{P}$ & -1.6814500 & -0.5388674 & -0.2245870 \\
$\mathrm{C}$ & -2.4429216 & 1.1304542 & -0.3449170 \\
$\mathrm{C}$ & -2.2969187 & 1.9909031 & 0.7648468 \\
$\mathrm{H}$ & -1.7744824 & 1.6296623 & 1.6636933 \\
$\mathrm{C}$ & -2.7673980 & 3.3097725 & 0.7050000 \\
$\mathrm{H}$ & -2.6387601 & 3.9753982 & 1.5716169 \\
$\mathrm{C}$ & -3.3726768 & 3.7877189 & -0.4708590 \\
$\mathrm{C}$ & -3.7244499 & 4.8290788 & -0.5256473 \\
\hline & -3.5178187 & 2.9361812 & -1.5787774 \\
$\mathrm{H}$ & -3.0592652 & 1.6101476 & -1.5174967
\end{tabular}




$\begin{array}{lrrr}\text { H } & -3.1598266 & 0.9499372 & -2.3913044 \\ \text { C } & -1.7313514 & -1.1629522 & -1.9560124 \\ \text { C } & -2.8499687 & -1.8321074 & -2.4940314 \\ \text { H } & -3.7303116 & -2.0211786 & -1.8613191 \\ \text { C } & -2.8371895 & -2.2578535 & -3.8321958 \\ \text { H } & -3.7117729 & -2.7826389 & -4.2464403 \\ \text { C } & -1.7134964 & -2.0140589 & -4.6409880 \\ \text { H } & -1.7081985 & -2.3480944 & -5.6898705 \\ \text { C } & -0.5970288 & -1.3476502 & -4.1075753 \\ \text { H } & 0.2876923 & -1.1489029 & -4.7305266 \\ \text { C } & -0.6012119 & -0.9292644 & -2.7690794 \\ \text { H } & 0.2731962 & -0.4099024 & -2.3503439 \\ \text { C } & -2.9722984 & -1.5415043 & 0.6224178 \\ \text { C } & -2.6744555 & -2.9022959 & 0.8593896 \\ \text { H } & -1.7227851 & -3.3174193 & 0.4904291 \\ \text { C } & -3.5776007 & -3.7153422 & 1.5570554 \\ \text { H } & -3.3389631 & -4.7757584 & 1.7305847 \\ \text { C } & -4.7815827 & -3.1734959 & 2.0440567 \\ \text { H } & -5.4862166 & -3.8084122 & 2.6021926 \\ \text { C } & -5.0794867 & -1.8204713 & 1.8172284 \\ \text { H } & -6.0208024 & -1.3925401 & 2.1948489 \\ \text { C } & -4.1824612 & -1.0062626 & 1.1041782 \\ \text { H } & -4.4211325 & 0.0522031 & 0.9226674\end{array}$

R2

75

SCF Energy $=-4548.309140807$

C $\quad 1.7114328 \quad 1.9703644$

C $\quad 1.0407422 \quad 1.9087080$

$-1.9918753$

$-0.1183367$

2.7118151

$-0.7438095$

$-0.6158886$

3.4907713

$-0.5978610$

3.5151614

$-1.6477786$

0.0445630

2.7607774

$-2.8883707$

$-3.0408626$

1.2177682

1.0577908

0.3941694

0.4356373

C $\quad 2.5111371$

0.0499031

$-0.4272620$

$-0.8651589$

C $\quad 2.3625646$

$-1.2573736$

$-1.7399397$

C 2.8829553

$-1.6387560$

$-2.9838757$

C $\quad 4.1606045$

$-1.2072476$

$-3.3754668$

C $\quad 4.9121837$

$-0.3953091$

$-2.5088008$

C $\quad 4.3893901$

$-0.0074929$

$-1.2653498$

$\mathrm{Br}$

1.1504506

1.9420460

2.1473606

1.9048271

1.8176355

1.0552165

2.5827495

$-3.2477659$

2. 2463571

0.4825238
C $\quad 2.6443741$

$-2.5152699$

2.6695492 


\begin{tabular}{lrrr} 
C & 3.3791277 & -0.2374542 & 1.7253094 \\
$\mathrm{O}$ & 4.6933044 & -0.6753484 & 1.4189164 \\
$\mathrm{H}$ & 3.5257734 & 0.7253494 & 2.2552562 \\
$\mathrm{H}$ & 1.9701715 & -0.6414291 & 3.3302506 \\
$\mathrm{H}$ & 3.4066213 & -1.7072399 & 3.3213977 \\
$\mathrm{H}$ & 0.0293588 & -2.0363157 & 3.1301116 \\
$\mathrm{H}$ & -0.0128845 & -3.4294496 & 1.8829924 \\
$\mathrm{H}$ & 3.2424194 & -3.8665481 & 1.7035374 \\
$\mathrm{H}$ & 3.2235064 & -2.7677662 & 0.2885842 \\
$\mathrm{H}$ & 1.8977654 & -3.9285342 & 0.5132769 \\
$\mathrm{H}$ & 4.6226122 & -1.4856023 & 0.8780329 \\
$\mathrm{H}$ & 1.3578573 & -1.5871280 & -1.4293904 \\
$\mathrm{H}$ & 2.2764476 & -2.2717386 & -3.6472720 \\
$\mathrm{H}$ & 4.5719675 & -1.5037095 & -4.3524460 \\
$\mathrm{H}$ & 5.9136365 & -0.0487837 & -2.8067389 \\
$\mathrm{H}$ & 4.9721570 & 0.6442321 & -0.6025362 \\
$\mathrm{H}$ & -0.6432184 & 2.7186550 & 0.3627664 \\
$\mathrm{H}$ & -1.5326902 & 4.0774735 & -1.4881902 \\
$\mathrm{H}$ & -0.3421451 & 4.1256715 & -3.7181763 \\
$\mathrm{H}$ & 1.7712741 & 2.7825106 & -3.9920786 \\
$\mathrm{H}$ & 2.6325552 & 1.4066349 & -2.1562936 \\
$\mathrm{Pd}$ & 0.5049263 & -0.8612515 & 0.7644273 \\
$\mathrm{P}$ & -1.5936653 & -0.7748629 & -0.1863214 \\
$\mathrm{C}$ & -2.8783373 & -2.0381500 & 0.2337243 \\
$\mathrm{C}$ & -2.6475920 & 0.7352270 & -0.1106128 \\
$\mathrm{C}$ & -1.2576359 & -1.0698016 & -1.9665204 \\
$\mathrm{C}$ & -3.8588610 & -2.4535867 & -0.6917889 \\
$\mathrm{C}$ & -4.8563121 & -3.3601538 & -0.3037418 \\
$\mathrm{C}$ & -4.8933350 & -3.8494201 & 1.0140851 \\
$\mathrm{C}$ & -3.9286975 & -3.4277729 & 1.9431746 \\
$\mathrm{C}$ & -2.9225182 & -2.5290819 & 1.5527748 \\
$\mathrm{H}$ & -3.8391727 & -2.0679155 & -1.7222062 \\
$\mathrm{H}$ & -5.6140719 & -3.6837872 & -1.0337011 \\
$\mathrm{H}$ & -5.6779250 & -4.5596940 & 1.3162858 \\
$\mathrm{H}$ & -3.9554264 & -3.8038350 & 2.9772621 \\
$\mathrm{H}$ & -2.1608043 & -2.1954935 & 2.2718778 \\
$\mathrm{C}$ & -3.4638085 & 1.1768830 & -1.1697006 \\
$\mathrm{C}$ & -4.2496486 & 2.3295504 & -1.0178399 \\
$\mathrm{C}$ & -4.2411477 & 3.0372037 & 0.1965083 \\
$\mathrm{C}$ & -3.4463943 & 2.5856042 & 1.2640836 \\
$\mathrm{C}$ & -2.6490414 & 1.4419189 & 1.1095167 \\
$\mathrm{H}$ & -3.4757116 & 0.6275653 & -2.1224966 \\
$\mathrm{H}$ & -4.8742129 & 2.6779701 & -1.8546097 \\
& -4.8567815 & 3.9422730 & 0.3113693 \\
\hline & -1.4344861 & 3.1361605 & 2.2168777 \\
& & 1.1054745 & 1.9264963
\end{tabular}




$\begin{array}{lrrr}\text { C } & -1.1104528 & -2.3960265 & -2.4340612 \\ \text { C } & -0.6691820 & -2.6414281 & -3.7427564 \\ \text { C } & -0.3565760 & -1.5691800 & -4.5969151 \\ \text { C } & -0.4813516 & -0.2511214 & -4.1298264 \\ \text { C } & -0.9267263 & 0.0011066 & -2.8240444 \\ \text { H } & -1.3408263 & -3.2401042 & -1.7665594 \\ \text { H } & -0.5662542 & -3.6787816 & -4.0963685 \\ \text { H } & -0.0069158 & -1.7627805 & -5.6222730 \\ \text { H } & -0.2210312 & 0.5980269 & -4.7786789 \\ \text { H } & -1.0034139 & 1.0372771 & -2.4692284\end{array}$

R3

75

SCF Energy $=-4548.312347916$

$\begin{array}{lll}\text { C } & 1.6984538 & 2.0322427\end{array}$

$\begin{array}{lll}\text { C } & 1.3557625 & 0.8979659\end{array}$

$-2.3649320$

$-1.5996090$

C $\quad 1.2083853 \quad-0.3400153$

$-2.2640082$

$\begin{array}{lll}\text { C } & 1.4199797 & -0.4480991\end{array}$

$-3.6427991$

1.7634993

0.6874939

$-4.3942444$

1.8982976

1.9278845

$-3.7494319$

1.1842521

0.9689440

$-0.1194700$

2.0712701

0.3273845

0.8023588

$\begin{array}{ll}-0.4488604 & 0.2092810\end{array}$

$-1.8271729$

$-0.0704058$

$\quad 4.2260776$

$-2.5144643$

$-0.6238389$

C $\quad 5.4195294$

$-1.8296147$

$-0.9072344$

C $\quad 5.5067808$

$-0.4522768$

$-0.6480771$

C 4.4114823

0.2347570

$-0.0994279$

$\mathrm{Br} \quad 0.2724285 \quad 2.6346739$

0.4697674

$-1.4293772$

2.9092351

1.0619615

$-2.9040338$

2.8181078

$-0.2682620$

$-1.0459694$

3.1000836

2.2488907

$-0.4961007$

3.1735832

2.2759709

0.7592288

2.2733185

3.4493970

1.5362697

2.4809339

2.5430709

1.4294897

4.2400102

2. 2852229

$-0.1846384$

$-1.0624352$

2.9803808

$-0.510101$

$-0.0480052$

3.5041645

$-1.0540626$

$-1.8109183$

3.1986786

$-3.1042367$

2.1014202

2.2152959

$-3.5106889$

2.5339448

$\mathrm{H} \quad 1.7502641$

$-3.2635471$

3.8106234

4.2079216

0.9591299

2.2581366

H 2.1864218

$-2.3446219$

0.1326096

$\mathrm{H}$

4.1407452

$-3.5899772$

$-0.8423070$ 


\begin{tabular}{lrrr} 
H & 6.2756868 & -2.3661828 & -1.3434944 \\
$\mathrm{H}$ & 6.4294239 & 0.0972920 & -0.8900301 \\
$\mathrm{H}$ & 4.4623210 & 1.3213798 & 0.0645619 \\
$\mathrm{H}$ & 0.8906512 & -1.2133816 & -1.6774050 \\
$\mathrm{H}$ & 1.2849029 & -1.4213116 & -4.1369339 \\
$\mathrm{H}$ & 1.9114832 & 0.6089225 & -5.4820071 \\
$\mathrm{H}$ & 2.1632263 & 2.8255907 & -4.3289775 \\
$\mathrm{H}$ & 1.7995196 & 3.0052821 & -1.8624071 \\
$\mathrm{Pd}$ & 0.1165938 & -0.5360865 & 0.9677509 \\
$\mathrm{P}$ & -1.9655185 & -0.7222912 & -0.0054665 \\
$\mathrm{C}$ & -2.0075474 & -0.4590643 & -1.8262809 \\
$\mathrm{C}$ & -1.7525074 & 0.8471676 & -2.3023869 \\
$\mathrm{H}$ & -1.5938169 & 1.6683022 & -1.5864442 \\
$\mathrm{C}$ & -1.6773847 & 1.0936826 & -3.6779556 \\
$\mathrm{H}$ & -1.4654695 & 2.1116470 & -4.0362775 \\
$\mathrm{C}$ & -1.8371756 & 0.0393734 & -4.5941937 \\
$\mathrm{H}$ & -1.7616239 & 0.2323157 & -5.6748851 \\
$\mathrm{C}$ & -2.0823997 & -1.2607217 & -4.1262324 \\
$\mathrm{H}$ & -2.2091160 & -2.0898016 & -4.8393128 \\
$\mathrm{C}$ & -2.1708652 & -1.5121780 & -2.7461389 \\
$\mathrm{H}$ & -2.3672477 & -2.5322954 & -2.3840436 \\
$\mathrm{C}$ & -3.3070420 & 0.3984616 & 0.5683593 \\
$\mathrm{C}$ & -3.1939215 & 0.9782688 & 1.8474122 \\
$\mathrm{H}$ & -2.3000144 & 0.7662600 & 2.4510249 \\
$\mathrm{C}$ & -4.1987972 & 1.8309913 & 2.3297111 \\
$\mathrm{H}$ & -4.0996868 & 2.2832911 & 3.3280930 \\
$\mathrm{C}$ & -5.3198869 & 2.1177262 & 1.5333237 \\
$\mathrm{H}$ & -6.1046328 & 2.7923295 & 1.9082662 \\
$\mathrm{C}$ & -5.4321669 & 1.5528454 & 0.2509667 \\
$\mathrm{H}$ & -6.3040972 & 1.7844342 & -0.3796955 \\
$\mathrm{C}$ & -4.4295169 & 0.6985222 & -0.2329041 \\
$\mathrm{H}$ & -4.5072931 & 0.2722224 & -1.2445220 \\
$\mathrm{C}$ & -2.6577507 & -2.4187252 & 0.1926908 \\
$\mathrm{C}$ & -4.0329254 & -2.6982474 & 0.3113876 \\
$\mathrm{H}$ & -4.7632547 & -1.8758331 & 0.2917616 \\
$\mathrm{C}$ & -4.4712014 & -4.0238699 & 0.4670775 \\
$\mathrm{H}$ & -5.5470202 & -4.2336669 & 0.5684483 \\
$\mathrm{C}$ & -3.5436276 & -5.0785294 & 0.4950561 \\
$\mathrm{H}$ & -3.8913552 & -6.1156056 & 0.6171796 \\
$\mathrm{C}$ & -2.1694867 & -4.8056660 & 0.3759624 \\
$\mathrm{H}$ & -1.4376238 & -5.6273452 & 0.4031958 \\
$\mathrm{H}$ & -1.7290599 & -3.4813620 & 0.2365714 \\
& -0.6529094 & -3.2484753 & 0.1695088 \\
& & & \\
\hline & & & \\
\hline
\end{tabular}




$\begin{array}{lrrr}\text { SCF } & \text { Energy }=-4548.317163648 & \\ \text { C } & 3.9379688 & -0.4285305 & -1.3283509 \\ \text { C } & 2.8711307 & -0.7844483 & -0.4732299 \\ \text { C } & 2.2605977 & -2.0458165 & -0.6390460 \\ \text { C } & 2.6867903 & -2.9208375 & -1.6491768 \\ \text { C } & 3.7416316 & -2.5533950 & -2.5010889 \\ \text { C } & 4.3687851 & -1.3073879 & -2.3347201 \\ \text { C } & 2.4288596 & 0.1955040 & 0.5848327 \\ \text { Pd } & 0.3968722 & -0.1583903 & 1.1777411 \\ \text { P } & -1.6459817 & -0.4127529 & 0.1079239 \\ \text { C } & 1.6841986 & 1.3301637 & 0.1511424 \\ \text { Br } & 1.5561043 & 2.8521077 & 1.4018745 \\ \text { C } & 1.4152296 & 1.7102536 & -1.2670573 \\ \text { C } & 1.6022358 & 3.0280173 & -1.7392523 \\ \text { C } & 1.3134085 & 3.3692882 & -3.0697464 \\ \text { C } & 0.8400397 & 2.4005773 & -3.9667290 \\ \text { C } & 0.6686903 & 1.0811447 & -3.5152503 \\ \text { C } & 0.9445254 & 0.7435907 & -2.1870582 \\ \text { C } & 3.2902049 & 0.1135068 & 1.8726673 \\ \text { O } & 4.6781233 & 0.0733826 & 1.5876948 \\ \text { C } & 2.7914456 & -1.0728671 & 2.7208020 \\ \text { C } & 1.3243888 & -0.8605242 & 3.0800949 \\ \text { C } & 0.3063684 & -1.7598597 & 2.7241228 \\ \text { C } & 1.0615154 & 0.1755390 & 4.1567206 \\ \text { H } & 3.1377406 & 1.0532932 & 2.4377789 \\ \text { H } & 3.4159910 & -1.1615913 & 3.6369872 \\ \text { H } & 2.9172296 & -2.0114812 & 2.1397007 \\ \text { H } & 0.5525744 & -2.6993234 & 2.2022184 \\ \text { H } & -0.6615758 & -1.7395734 & 3.2492078 \\ \text { H } & 1.4587593 & -0.1905437 & 5.1303512 \\ \text { H } & -0.0213291 & 0.3698081 & 4.2811285 \\ \text { H } & 1.5565331 & 1.1427296 & 3.9430946 \\ \text { H } & 4.8283929 & -0.7059496 & 1.0141680 \\ \text { H } & 1.4056697 & -2.3105619 & 0.0005963 \\ \text { H } & 2.1751307 & -3.8862049 & -1.7767329 \\ \text { H } & 4.0717555 & -3.2355580 & -3.2993339 \\ \text { H } & 5.1915541 & -1.0095007 & -3.0023744 \\ \text { H } & 4.4065824 & 0.5593886 & -1.2117258 \\ \text { H } & 0.7688022 & -0.2815494 & -1.8419667 \\ \text { H } & 0.2962266 & 0.3053589 & -4.2015542 \\ \text { H } & 0.6004415 & 2.6705709 & -5.0060663 \\ \text { H } & 1.4583577 & 4.4083574 & -3.4031129 \\ \text { H } & 1.9635348 & 3.7997212 & -1.0456466 \\ \text { C } & -2.2720622 & 0.8705648 & -1.0532775 \\ \text { C } & -3.1194908 & -0.7798521 & 1.1539247 \\ & -1.4577664 & -1.9337769 & -0.9108637\end{array}$




$\begin{array}{lrrr}\text { C } & -1.2172216 & -1.8935101 & -2.2998211 \\ \text { C } & -0.8688988 & -3.0629684 & -2.9948009 \\ \text { C } & -0.7663197 & -4.2877244 & -2.3170583 \\ \text { C } & -1.0205120 & -4.3393014 & -0.9343259 \\ \text { C } & -1.3593664 & -3.1727014 & -0.2356440 \\ \text { H } & -1.2956303 & -0.9400636 & -2.8397163 \\ \text { H } & -0.6753280 & -3.0127890 & -4.0770212 \\ \text { H } & -0.4930184 & -5.2026291 & -2.8641036 \\ \text { H } & -0.9505349 & -5.2961980 & -0.3949038 \\ \text { H } & -1.5459111 & -3.2162893 & 0.8478836 \\ \text { C } & -3.2526527 & 0.6150261 & -2.0341776 \\ \text { C } & -3.6378056 & 1.6300309 & -2.9225593 \\ \text { C } & -3.0565803 & 2.9072920 & -2.8292928 \\ \text { C } & -2.1026817 & 3.1736067 & -1.8345304 \\ \text { C } & -1.7130025 & 2.1590301 & -0.9477474 \\ \text { H } & -3.7089954 & -0.3832412 & -2.1126510 \\ \text { H } & -4.3955106 & 1.4234747 & -3.6939250 \\ \text { H } & -3.3526314 & 3.6980933 & -3.5353439 \\ \text { H } & -1.6438367 & 4.1698198 & -1.7549477 \\ \text { H } & -0.9562121 & 2.3549574 & -0.1740553 \\ \text { C } & -4.1746620 & -1.6157014 & 0.7349272 \\ \text { C } & -5.2788439 & -1.8302510 & 1.5742957 \\ \text { C } & -5.3411801 & -1.2083136 & 2.8333870 \\ \text { C } & -4.2922867 & -0.3741763 & 3.2556002 \\ \text { C } & -3.1823172 & -0.1668277 & 2.4220285 \\ \text { H } & -4.1246104 & -2.1120576 & -0.2460758 \\ \text { H } & -6.0970312 & -2.4882686 & 1.2435441 \\ \text { H } & -6.2080761 & -1.3793328 & 3.4895646 \\ \text { H } & -4.3344799 & 0.1105901 & 4.2428006 \\ \text { H } & -2.3452719 & 0.4717752 & 2.7480129\end{array}$

S1

75

SCF Energy $=-4548 \cdot 311018803$

$\begin{array}{lrrr}\text { C } & 2.7343295 & 1.6797997 & -2.3146356 \\ \text { C } & 1.5696692 & 1.5953677 & -1.5200093 \\ \text { C } & 0.3400121 & 2.0126571 & -2.0817472 \\ \text { C } & 0.2677043 & 2.4598892 & -3.4052818 \\ \text { C } & 1.4271831 & 2.5010901 & -4.2015227 \\ \text { C } & 2.6578767 & 2.1160767 & -3.6470764 \\ \text { C } & 1.6076929 & 1.1048238 & -0.1119198 \\ \text { C } & 2.4693315 & 0.0967440 & 0.4344260 \\ \text { C } & 3.3021240 & -0.7050485 & -0.5260695 \\ \text { C } & 2.7202301 & -1.4965150 & -1.5406958 \\ \text { C } & 3.5180317 & -2.2141011 & -2.4421099 \\ \text { C } & 4.9187886 & -2.1598702 & -2.3430363\end{array}$




\begin{tabular}{lrrr} 
C & 5.5106489 & -1.3876245 & -1.3290785 \\
$\mathrm{C}$ & 4.7122822 & -0.6683744 & -0.4263186 \\
$\mathrm{Br}$ & 1.1277487 & 2.6432191 & 1.0978106 \\
$\mathrm{C}$ & 0.8377351 & -1.1801333 & 2.6657862 \\
$\mathrm{C}$ & -0.4433143 & -1.0287542 & 3.4538558 \\
$\mathrm{C}$ & 1.0793761 & -2.3293607 & 1.9294862 \\
$\mathrm{C}$ & 1.8979448 & -0.1274214 & 2.9635859 \\
$\mathrm{C}$ & 2.9942820 & 0.0570093 & 1.8916435 \\
$\mathrm{O}$ & 3.8565994 & 1.1456705 & 2.2010478 \\
$\mathrm{H}$ & 3.6472184 & -0.8379720 & 1.9348993 \\
$\mathrm{H}$ & 1.3898665 & 0.8445789 & 3.1225655 \\
$\mathrm{H}$ & 2.3950860 & -0.3689976 & 3.9309497 \\
$\mathrm{H}$ & 2.0730541 & -2.5684304 & 1.5210066 \\
$\mathrm{H}$ & 0.3266250 & -3.1314079 & 1.9134303 \\
$\mathrm{H}$ & -0.8956728 & -0.0292260 & 3.2906328 \\
$\mathrm{H}$ & -0.2283266 & -1.1086059 & 4.5426768 \\
$\mathrm{H}$ & -1.1906599 & -1.7989425 & 3.1861572 \\
$\mathrm{H}$ & 3.3205024 & 1.9557403 & 2.0560635 \\
$\mathrm{H}$ & 1.6210401 & -1.5457745 & -1.6098036 \\
$\mathrm{H}$ & 3.0401287 & -2.8239881 & -3.2238639 \\
$\mathrm{H}$ & 5.5473609 & -2.7226895 & -3.0497640 \\
$\mathrm{H}$ & 6.6071264 & -1.3385937 & -1.2424506 \\
$\mathrm{H}$ & 5.1743768 & -0.0399372 & 0.3501693 \\
$\mathrm{H}$ & -0.5668540 & 1.9717046 & -1.4624551 \\
$\mathrm{H}$ & -0.7041204 & 2.7672824 & -3.8202494 \\
$\mathrm{H}$ & 1.3714916 & 2.8416043 & -5.2467184 \\
$\mathrm{H}$ & 3.5754289 & 2.1604329 & -4.2535275 \\
$\mathrm{H}$ & 3.7048950 & 1.3935595 & -1.8898361 \\
$\mathrm{Pd}$ & 0.4400732 & -0.5932475 & 0.4516287 \\
$\mathrm{P}$ & -1.7489011 & -0.6819697 & -0.2030209 \\
$\mathrm{C}$ & -2.6376029 & 0.9266124 & -0.2990195 \\
$\mathrm{C}$ & -2.3911832 & 1.8659588 & 0.7251115 \\
$\mathrm{H}$ & -1.6919269 & 1.6097603 & 1.5354025 \\
$\mathrm{C}$ & -2.9953116 & 3.1304421 & 0.6855164 \\
$\mathrm{H}$ & -4.0079988 & -3.2936090 & -3.8682407 \\
$\mathrm{H}$ & -2.7896202 & 3.8586325 & 1.4843423 \\
$\mathrm{C}$ & -3.8366581 & 3.4744212 & -0.3866118 \\
$\mathrm{H}$ & -4.2973998 & 4.4730566 & -0.4273065 \\
$\mathrm{C}$ & -4.0788636 & 2.5455463 & -1.4127075 \\
$\mathrm{H}$ & -4.7323892 & 2.8142725 & -2.2567282 \\
$\mathrm{C}$ & -3.4865265 & 1.2730443 & -1.3696173 \\
$\mathrm{H}$ & -3.6723525 & 0.5494110 & -2.1770672 \\
\hline & -1.9483476 & -1.3819995 & -1.8946935 \\
$\mathrm{H}$ & -3.8215245 & -2.4825744 & -2.2688231 \\
& -3.1497961 & -2.6657181 & -3.5835807 \\
& & & \\
$\mathrm{H}$ & &
\end{tabular}




$\begin{array}{llll}\text { C } & -2.1637443 & -2.3483446 & -4.5333782 \\ \text { H } & -2.2481072 & -2.7292128 & -5.5626246 \\ \text { C } & -1.0693993 & -1.5467344 & -4.1655481 \\ \text { H } & -0.2929354 & -1.2906804 & -4.9019695 \\ \text { C } & -0.9577994 & -1.0710105 & -2.8509859 \\ \text { H } & -0.1047073 & -0.4394676 & -2.5618451 \\ \text { C } & -2.8682324 & -1.7278551 & 0.8183938 \\ \text { C } & -2.5040604 & -3.0821053 & 0.9945952 \\ \text { H } & -1.6239203 & -3.4784098 & 0.4634793 \\ \text { C } & -3.2530927 & -3.9144744 & 1.8368612 \\ \text { H } & -2.9650965 & -4.9695333 & 1.9610902 \\ \text { C } & -4.3643081 & -3.3992244 & 2.5299112 \\ \text { H } & -4.9458927 & -4.0491548 & 3.2010101 \\ \text { C } & -4.7278167 & -2.0538710 & 2.3614611 \\ \text { H } & -5.5982159 & -1.6466837 & 2.8984231 \\ \text { C } & -3.9882813 & -1.2205098 & 1.5042969 \\ \text { H } & -4.2797910 & -0.1682693 & 1.3707013\end{array}$

S2

75

SCF Energy $=-4548.314520697$

$\begin{array}{lrrr}\text { C } & 2.2465450 & 2.4120246 & -1.9710228 \\ \text { C } & 1.6761574 & 1.2360421 & -1.4399272 \\ \text { C } & 1.1927231 & 0.2602156 & -2.3383132 \\ \text { C } & 1.2847522 & 0.4468723 & -3.7222329 \\ \text { C } & 1.8477508 & 1.6243560 & -4.2395786 \\ \text { C } & 2.3279726 & 2.6059946 & -3.3574543 \\ \text { C } & 1.6192057 & 0.9948100 & 0.0317991 \\ \text { C } & 2.4512192 & 0.0626253 & 0.7241482 \\ \text { C } & 3.2789197 & -0.8307536 & -0.1580739 \\ \text { C } & 2.7828123 & -2.0308782 & -0.7010144 \\ \text { C } & 3.5839329 & -2.8288061 & -1.5323166 \\ \text { C } & 4.8988704 & -2.4352606 & -1.8277300 \\ \text { C } & 5.4083787 & -1.2448901 & -1.2799854 \\ \text { C } & 4.6070554 & -0.4492303 & -0.4483106 \\ \text { Br } & 1.1226383 & 2.6729323 & 1.0368194 \\ \text { C } & 1.1561163 & -1.2540703 & 2.8570783 \\ \text { C } & 1.8826362 & -2.5784619 & 2.7188060 \\ \text { C } & -0.2440005 & -1.2326795 & 2.9475798 \\ \text { C } & 2.0091718 & -0.0504407 & 3.2536251 \\ \text { C } & 3.0614829 & 0.2369262 & 2.1574459 \\ \text { O } & 3.7793262 & 1.4421767 & 2.3327151 \\ \text { H } & 3.8352900 & -0.5543692 & 2.2257206 \\ \text { H } & 1.3651495 & 0.8428875 & 3.3850772 \\ \text { H } & 2.5305221 & -0.2286347 & 4.2221671 \\ \text { H } & -0.7700199 & -0.3726129 & 3.3928678\end{array}$




\begin{tabular}{|c|c|c|c|}
\hline & -0.8065494 & -2.1793100 & 2.9028423 \\
\hline & 2.2771677 & -2.8918300 & 3.7114519 \\
\hline & 2.7445810 & -2.5312848 & 2.0260039 \\
\hline & 1.2032401 & -3.3746390 & 2.3571909 \\
\hline & 3.1339710 & 2.1682626 & 2.1869680 \\
\hline & 1.7491441 & -2.3227611 & -0.4570170 \\
\hline & 3.1776002 & -3.7617167 & -1.9520544 \\
\hline & 5.5283565 & -3.0561956 & -2.4831163 \\
\hline & 6.4384237 & -0.9302087 & -1.5076308 \\
\hline & 4.9973642 & 0.4848738 & -0.0164203 \\
\hline & 0.7146158 & -0.6385441 & -1.9281015 \\
\hline & 0.8873579 & -0.3234715 & -4.3991152 \\
\hline & 1.9030701 & 1.7813360 & -5.3274424 \\
\hline & 2.7726657 & 3.5329235 & -3.7508001 \\
\hline & 2.6197988 & 3.1849477 & -1.2835854 \\
\hline d & 0.3366795 & -0.41 & \\
\hline & -1.7565327 & -0.2437720 & -0.03 \\
\hline & -1.869 & 0.66 & -1.62 \\
\hline & -1.366 & 1.980 & -1.6720927 \\
\hline $\mathrm{H}$ & -0.9 & 426 & -0.75 \\
\hline & -1.3310959 & 2.6884116 & -2.87 \\
\hline $\mathrm{H}$ & -0.922 & 3.70 & -2.8987587 \\
\hline C & -1.774 & 2.07 & -4.0657582 \\
\hline $\mathrm{H}$ & -1.7246613 & 2.62 & -5.0188382 \\
\hline $\mathrm{C}$ & -2.2666940 & 0.76 & -4.0328622 \\
\hline $\mathrm{H}$ & -2.609 & 0.28 & -4.95 \\
\hline $\mathrm{C}$ & -2.318 & 0.05 & -2.82 \\
\hline $\mathrm{H}$ & -2.6 & -0.9 & -2 \\
\hline C & -2.4 & -1.9 & -0 . \\
\hline C & -3.7 & -2.1 & -0.6 \\
\hline & -4.5 & -1.3 & -0 . \\
\hline & -4.2 & -3.4 & -0 . \\
\hline$H$ & -5.3 & -3.6 & -1 \\
\hline & -3.3 & -4 & -1 \\
\hline $\mathrm{H}$ & -3.6 & -5 & -1 . \\
\hline $\mathrm{C}$ & -1.9 & -4 & \\
\hline $\mathrm{H}$ & -1.2 & -5.0 & \\
\hline $\mathrm{C}$ & -1.509 & $-2.97 \varsigma$ & -0.5531 \\
\hline $\mathrm{H}$ & -0.4406036 & -2.7828563 & -0.365841 \\
\hline C & -3.1182171 & 0.4817906 & 0.979453 \\
\hline C & -3.4844206 & -0.1966214 & 2.163812 \\
\hline $\mathrm{H}$ & -2.9945276 & -1.1476640 & 2.418994 \\
\hline & -4.4790214 & 0.3246845 & 3.002008 \\
\hline $\mathrm{H}$ & -4.7600979 & -0.2179649 & 3.917496 \\
\hline C & -5.1132644 & 1.5369096 & 2.676458 \\
\hline & -5.8900049 & 1.9494861 & 3.337929 \\
\hline
\end{tabular}




\begin{tabular}{lrrr} 
C & -4.7535618 & 2.2146984 & 1.5013465 \\
H & -5.2507910 & 3.1598716 & 1.2347202 \\
C & -3.7641488 & 1.6899352 & 0.6525740 \\
H & -3.5000207 & 2.2208077 & -0.2732424 \\
& & & \\
S3 & \multicolumn{3}{r}{} \\
75 & \multicolumn{3}{r}{} \\
SCF & Energy $=-4548.311549220$ & \\
C & 2.5533822 & 0.8889044 & -2.8135050 \\
C & 1.7557431 & 0.2377579 & -1.8475666 \\
C & 1.2362972 & -1.0369041 & -2.1527786 \\
C & 1.5069305 & -1.6515653 & -3.3827903 \\
C & 2.2985510 & -0.9938445 & -4.3362778 \\
C & 2.8230595 & 0.2783719 & -4.0457760 \\
C & 1.4846256 & 0.8608479 & -0.5148853 \\
C & 2.3058091 & 0.6211613 & 0.6416231 \\
C & 3.4856416 & -0.2859087 & 0.4304832 \\
C & 3.3444630 & -1.6646622 & 0.1664694 \\
C & 4.4627780 & -2.4724312 & -0.0836054 \\
C & 5.7531500 & -1.9183025 & -0.0631236 \\
C & 5.9109338 & -0.5479000 & 0.1997328 \\
C & 4.7885425 & 0.2601445 & 0.4389140 \\
Br & 0.7424390 & 2.7264802 & -0.8164354 \\
C & 1.3112865 & -0.6029370 & 3.0124577 \\
C & 1.8041464 & -2.0260827 & 3.1741299 \\
C & -0.0552031 & -0.3381145 & 3.1461478 \\
C & 2.4086133 & 0.4779684 & 3.1178802 \\
C & 2.3975764 & 1.4734784 & 1.9459222 \\
O & 1.3089457 & 2.3509995 & 2.1794919 \\
H & 3.3543597 & 2.0570533 & 1.9419620 \\
H & 2.3109909 & 1.0512368 & 4.0644912 \\
H & 3.3949541 & -0.0248717 & 3.1442738 \\
H & -0.4133718 & 0.6780105 & 3.3681941 \\
H & -0.7537731 & -1.1635166 & 3.3561506 \\
H & 2.6461486 & -2.2557616 & 2.4928687 \\
H & 0.9948927 & -2.7637374 & 3.0070467 \\
H & 2.1837347 & -2.1681211 & 4.2112184 \\
H & 1.1419085 & 2.8531030 & 1.3491122 \\
H & 2.3324376 & -2.0937662 & 0.1614774 \\
H & 4.3237728 & -3.5439204 & -0.2934481 \\
H & 6.6326902 & -2.5513615 & -0.2549545 \\
H & 6.9161952 & -0.0992684 & 0.2083123 \\
H & 4.9252599 & 1.3373900 & 0.6162018 \\
H & 0.6009247 & -1.5311367 & -1.4038474 \\
& 1.0782197 & -2.6408882 & -3.6001810 \\
& 2.5050804 & -1.4677828 & -5.3080744
\end{tabular}




$\begin{array}{lrrr}\text { H } & 3.4499820 & 0.7986135 & -4.7860893 \\ \text { H } & 2.9580388 & 1.8872684 & -2.5902800 \\ \text { Pd } & 0.3498993 & -0.1666283 & 0.9502752 \\ \text { P } & -1.8050647 & -0.3189658 & 0.1594983 \\ \text { C } & -1.9817129 & -0.6759615 & -1.6382562 \\ \text { C } & -1.6101672 & 0.3377690 & -2.5498493 \\ \text { H } & -1.3226834 & 1.3315200 & -2.1744551 \\ \text { C } & -1.5765644 & 0.0736429 & -3.9244800 \\ \text { H } & -1.2717734 & 0.8680354 & -4.6218567 \\ \text { C } & -1.9009584 & -1.2058227 & -4.4070648 \\ \text { H } & -1.8605979 & -1.4157225 & -5.4865049 \\ \text { C } & -2.2714808 & -2.2164481 & -3.5055342 \\ \text { H } & -2.5333487 & -3.2191570 & -3.8770110 \\ \text { C } & -2.3152682 & -1.9549738 & -2.1254375 \\ \text { H } & -2.6081822 & -2.7509752 & -1.4246692 \\ \text { C } & -2.7599764 & 1.2376553 & 0.3520836 \\ \text { C } & -2.2617517 & 2.2158275 & 1.2365284 \\ \text { H } & -1.3066734 & 2.0353925 & 1.7536644 \\ \text { C } & -2.9548423 & 3.4237060 & 1.4122613 \\ \text { H } & -2.5559260 & 4.1855744 & 2.0988882 \\ \text { C } & -4.1418648 & 3.6646947 & 0.7012352 \\ \text { H } & -4.6817297 & 4.6145429 & 0.8353846 \\ \text { C } & -4.6326377 & 2.6993836 & -0.1958852 \\ \text { H } & -5.5542655 & 2.8932673 & -0.7656397 \\ \text { C } & -3.9431479 & 1.4905236 & -0.3745997 \\ \text { H } & -4.3118849 & 0.7452509 & -1.0958318 \\ \text { C } & -2.8195683 & -1.6304200 & 0.9606706 \\ \text { C } & -4.1816341 & -1.4877775 & 1.2883886 \\ \text { H } & -4.7046794 & -0.5468009 & 1.0637317 \\ \text { C } & -4.8689483 & -2.5398360 & 1.9162989 \\ \text { H } & -5.9309921 & -2.4165551 & 2.1780364 \\ \text { C } & -4.2071436 & -3.7429626 & 2.2117529 \\ \text { H } & -4.7496450 & -4.5647465 & 2.7033012 \\ \text { C } & -2.8466412 & -3.8903773 & 1.8893609 \\ \text { H } & -2.3199071 & -4.8270622 & 2.1272903 \\ \text { C } & -2.1536886 & -2.8346351 & 1.2791009 \\ \text { H } & -1.0782004 & -2.9257191 & 1.0529615 \\ & & & \\ \text { S4 } & & & \\ 75 & & & \\ \text { SCF } & \text { Energy }=-4548.309813955 & \\ \text { C } & 3.4156585 & 1.0632876 & -2.2920271 \\ \text { C } & 2.2200587 & 0.5026587 & -1.7914643 \\ \text { C } & 1.5624757 & -0.4860389 & -2.5484558 \\ \text { C } & 2.0873797 & -0.9130226 & -3.7782432 \\ \text { C } & 3.2811688 & -0.3571639 & -4.2634913\end{array}$




$\begin{array}{lrrr}\text { C } & 3.9451316 & 0.6320670 & -3.5148684 \\ \text { C } & 1.6928661 & 0.9285470 & -0.4551222 \\ \text { C } & 2.3191426 & 0.5048992 & 0.7672914 \\ \text { C } & 3.4954253 & -0.4220752 & 0.6106261 \\ \text { C } & 3.3485364 & -1.7336595 & 0.1085057 \\ \text { C } & 4.4610434 & -2.5629159 & -0.0889554 \\ \text { C } & 5.7504790 & -2.1020734 & 0.2249429 \\ \text { C } & 5.9135100 & -0.8019141 & 0.7293926 \\ \text { C } & 4.7985285 & 0.0302489 & 0.9156008 \\ \text { Br } & 0.9933053 & 2.8283601 & -0.6628729 \\ \text { C } & 0.7160305 & -0.6722179 & 3.1056955 \\ \text { C } & -0.4930444 & -0.0459858 & 3.7564465 \\ \text { C } & 0.6560732 & -1.9267612 & 2.5503953 \\ \text { C } & 2.0378424 & 0.0849048 & 3.2367997 \\ \text { C } & 2.2422366 & 1.1800016 & 2.1624562 \\ \text { O } & 1.2059752 & 2.1292837 & 2.3325356 \\ \text { H } & 3.2310655 & 1.6673761 & 2.3639189 \\ \text { H } & 2.0813640 & 0.5755525 & 4.2321506 \\ \text { H } & 2.8876795 & -0.6217900 & 3.1742809 \\ \text { H } & 1.5685807 & -2.4417426 & 2.2116929 \\ \text { H } & -0.2861820 & -2.4972274 & 2.5479601 \\ \text { H } & -0.5703657 & 1.0237098 & 3.4880357 \\ \text { H } & -0.3784431 & -0.0908757 & 4.8624241 \\ \text { H } & -1.4293981 & -0.5691818 & 3.4814155 \\ \text { H } & 1.1896415 & 2.7095254 & 1.5369276 \\ \text { H } & 2.3349496 & -2.0875033 & -0.1367847 \\ \text { H } & 4.3185269 & -3.5777994 & -0.4903377 \\ \text { H } & 6.6252484 & -2.7527159 & 0.0746392 \\ \text { H } & 6.9195425 & -0.4255073 & 0.9707634 \\ \text { H } & 4.9471831 & 1.0560553 & 1.2848217 \\ \text { H } & 0.6377879 & -0.9234336 & -2.1449081 \\ \text { H } & 1.5572975 & -1.6852795 & -4.3558465 \\ \text { H } & 3.6981311 & -0.6934013 & -5.2251720 \\ \text { H } & 4.8852065 & 1.0671635 & -3.8870348 \\ \text { H } & 3.9324259 & 1.8366612 & -1.7044438 \\ \text { Pd } & 0.3526980 & -0.3481847 & 0.5470225 \\ \text { P } & -1.8292811 & -0.4285301 & -0.1045413 \\ \text { C } & -2.7157674 & 1.1372510 & 0.2757856 \\ \text { C } & -2.0999613 & 2.0647153 & 1.1388027 \\ \text { H } & -1.0944816 & 1.8480087 & 1.5335526 \\ \text { C } & -2.7504522 & 3.2680490 & 1.4553119 \\ \text { H } & -2.2606324 & 3.9906175 & 2.1254303 \\ \text { C } & -4.0110660 & 3.5531651 & 0.9064068 \\ \text { H } & -4.5179252 & 4.4993734 & 1.1505699 \\ \text { H } & -4.6216546 & 2.6357298 & 0.0319343 \\ & -5.6042286 & 2.8636152 & -0.4089248\end{array}$




$\begin{array}{lrrr}\text { C } & -3.9758137 & 1.4320961 & -0.2868352 \\ \text { H } & -4.4448246 & 0.7192721 & -0.9826123 \\ \text { C } & -2.8230097 & -1.7348886 & 0.7294695 \\ \text { C } & -3.9671699 & -1.4686788 & 1.5071545 \\ \text { H } & -4.3526752 & -0.4411672 & 1.5783289 \\ \text { C } & -4.6100258 & -2.5104907 & 2.1977691 \\ \text { H } & -5.4991087 & -2.2904552 & 2.8083653 \\ \text { C } & -4.1247081 & -3.8248857 & 2.1109428 \\ \text { H } & -4.6315929 & -4.6382482 & 2.6518707 \\ \text { C } & -2.9814007 & -4.0972911 & 1.3378510 \\ \text { H } & -2.5906007 & -5.1241263 & 1.2716514 \\ \text { C } & -2.3265897 & -3.0571804 & 0.6637670 \\ \text { H } & -1.4102338 & -3.2616374 & 0.0864583 \\ \text { C } & -2.2031691 & -0.6404766 & -1.8953208 \\ \text { C } & -2.9509758 & -1.7093746 & -2.4252719 \\ \text { H } & -3.3955888 & -2.4564881 & -1.7512150 \\ \text { C } & -3.1320141 & -1.8195977 & -3.8151096 \\ \text { H } & -3.7168072 & -2.6585717 & -4.2226086 \\ \text { C } & -2.5758651 & -0.8633700 & -4.6797328 \\ \text { H } & -2.7180780 & -0.9536775 & -5.7673756 \\ \text { C } & -1.8356395 & 0.2103595 & -4.1518845 \\ \text { H } & -1.3925944 & 0.9615181 & -4.8229042 \\ \text { C } & -1.6448354 & 0.3207052 & -2.7686878 \\ \text { H } & -1.0506641 & 1.1518637 & -2.3561129\end{array}$

\section{Optimized Geometries(MXna)}

\begin{tabular}{lrrr} 
R1 & & & \\
75 & \multicolumn{4}{l}{} \\
SCF & Energy $=-4548.292050107$ & \\
C & -2.6846013 & -1.4370333 & -0.8410769 \\
C & -2.2592360 & -2.1821057 & 0.2839907 \\
C & -3.0304906 & -3.2970843 & 0.6797397 \\
C & -4.1752743 & -3.6688130 & -0.0410003 \\
C & -4.5779804 & -2.9295927 & -1.1655217 \\
C & -3.8278997 & -1.8083984 & -1.5592231 \\
C & -0.9907085 & -1.8189482 & 0.9820318 \\
C & 0.3053591 & -1.7924689 & 0.3846939 \\
C & 0.4674222 & -2.0525754 & -1.0911550 \\
C & -0.1992548 & -3.1069907 & -1.7539578 \\
C & -0.0053125 & -3.3366857 & -3.1230636 \\
C & 0.8622963 & -2.5207191 & -3.8669300 \\
C & 1.5260813 & -1.4622304 & -3.2261632 \\
C & 1.3245673 & -1.2273417 & -1.8598648
\end{tabular}




\begin{tabular}{|c|c|c|c|}
\hline $3 r$ & -1.1168954 & -2.0418960 & 2.9401766 \\
\hline C & 1.9284371 & -4.4429039 & 0.5878985 \\
\hline C & 0.7438970 & -5.3595522 & 0.4233496 \\
\hline & 3.0181828 & -4.5146513 & -0.2089499 \\
\hline & 1.8301775 & -3.4369114 & 1.7134123 \\
\hline & 1.5815318 & -1.9715310 & 1.2495227 \\
\hline & 2.7399685 & -1.4575262 & 0.6151987 \\
\hline & 1.4511938 & -1.3675509 & 2.1679737 \\
\hline & 1.0237284 & -3.7205668 & 2.4178641 \\
\hline & 2.7757449 & -3.3992427 & 2.2923851 \\
\hline & 3.9099660 & -3.8960511 & -0.0195356 \\
\hline & 3.0630901 & -5.2131243 & -1.05 \\
\hline & -0.2124388 & -4.80 & 0.4878721 \\
\hline & 0.7233 & -6.0 & 562 \\
\hline & 0.7678 & -5.9 & -0.5 \\
\hline & 2.8 & -1.9 & -0 . \\
\hline & -0.8 & -3.7 & -1 \\
\hline & -0.5 & -4.1 & -3 \\
\hline & 1.0 & -2.7 & -4.5 \\
\hline $\mathrm{H}$ & 2.185 & $-0.7 s$ & -3.7 \\
\hline $\mathrm{H}$ & 1.8034640 & -0.36 & -1.3 \\
\hline $\mathrm{H}$ & -2.7233223 & -3.8740523 & 1.5642136 \\
\hline & -4.7587358 & -4.5445458 & 0.2819533 \\
\hline & -5.4788493 & -3.2203425 & -1.7268729 \\
\hline & -4.1373443 & -1.2135690 & -2.43 \\
\hline & -2.0838081 & -0.5633084 & -1.14 \\
\hline $\mathrm{Pd}$ & -0.421 & 0.21 & $0.66 c$ \\
\hline & 0.37 & 2.19 & -0.1274927 \\
\hline & -0.71 & 3.66 & 0.0 \\
\hline & -0.8607099 & 4.64 & -0.9 \\
\hline & -0.3279064 & 4.53 & -1.887 \\
\hline & -1.6904300 & 5.75 & -0.7102830 \\
\hline$n$ & -1.8054486 & 6.51 & -1.4973838 \\
\hline & -2.3720844 & 5.8984582 & 0.5101533 \\
\hline & -3.0235855 & 6.7691351 & 0.6798343 \\
\hline & -2.2281185 & 4.9218498 & 1.5110794 \\
\hline & -2.7665613 & 5.0247475 & 2.4653590 \\
\hline & -1.4067117 & 3.8065348 & 1.2902067 \\
\hline & -1.3005905 & 3.0288969 & 2.0642971 \\
\hline & 2.0106113 & 2.7758553 & 0.4729532 \\
\hline & 2.9828508 & 1.7982280 & 0.7766225 \\
\hline & 2.7458326 & 0.7246191 & 0.6834778 \\
\hline & 4.2615956 & 2.1947910 & 1.1977587 \\
\hline & 5.0148423 & 1.4266085 & 1.4299292 \\
\hline & 4.5732003 & 3.5584938 & 1.334886 \\
\hline & 5.5741212 & 3.8651510 & 1.6753707 \\
\hline
\end{tabular}




$\begin{array}{lrrr}\text { C } & 3.6015384 & 4.5309058 & 1.0441142 \\ \text { H } & 3.8387817 & 5.6000205 & 1.1559443 \\ \text { C } & 2.3231868 & 4.1435295 & 0.6108624 \\ \text { H } & 1.5642498 & 4.9067042 & 0.3826885 \\ \text { C } & 0.6048056 & 2.0922531 & -1.9546279 \\ \text { C } & -0.4734027 & 1.6012123 & -2.7221468 \\ \text { H } & -1.4127905 & 1.3258753 & -2.2172974 \\ \text { C } & -0.3466180 & 1.4496166 & -4.1080912 \\ \text { H } & -1.1916683 & 1.0602371 & -4.6952383 \\ \text { C } & 0.8667470 & 1.7708934 & -4.7428009 \\ \text { H } & 0.9724378 & 1.6375579 & -5.8300683 \\ \text { C } & 1.9459497 & 2.2497801 & -3.9835777 \\ \text { H } & 2.8991560 & 2.4987213 & -4.4746716 \\ \text { C } & 1.8172577 & 2.4153169 & -2.5929959 \\ \text { H } & 2.6645989 & 2.7904257 & -2.0004390 \\ & & & \\ \text { R2 } & & & \\ 75 & & & \\ \text { SCF } & \text { Energy }=-4548.290435118 & \\ \text { C } & -2.1914992 & -1.4356451 & -2.0633908 \\ \text { C } & -2.4281646 & -1.9926086 & -0.7807170 \\ \text { C } & -3.5230198 & -2.8708171 & -0.6282051 \\ \text { C } & -4.3423479 & -3.1952315 & -1.7202610 \\ \text { C } & -4.0906181 & -2.6450709 & -2.9870934 \\ \text { C } & -3.0102259 & -1.7609316 & -3.1511518 \\ \text { C } & -1.5084695 & -1.6628986 & 0.3463300 \\ \text { C } & -0.0881075 & -1.7419169 & 0.2934212 \\ \text { C } & 0.5708083 & -2.2370816 & -0.9637081 \\ \text { C } & 0.2029853 & -3.4762224 & -1.5313374 \\ \text { C } & 0.8771114 & -3.9744450 & -2.6536622 \\ \text { C } & 1.9336523 & -3.2476418 & -3.2309838 \\ \text { C } & 2.2983319 & -2.0065079 & -2.6862330 \\ \text { C } & 1.6177717 & -1.5030004 & -1.5655888 \\ \text { Br } & -2.3775494 & -1.6906735 & 2.1129836 \\ \text { C } & 1.3235095 & -4.4372430 & 1.3069754 \\ \text { C } & 2.7227723 & -4.3445507 & 0.7479880 \\ \text { C } & 0.5493906 & -5.5228777 & 1.0930553 \\ \text { C } & 0.8190433 & -3.2957633 & 2.1605454 \\ \text { C } & 0.7760037 & -1.8599366 & 1.5672547 \\ \text { O } & 2.0751051 & -1.3098968 & 1.3938476 \\ \text { H } & 0.2956370 & -1.2332884 & 2.3435964 \\ \text { H } & -0.2006333 & -3.5339445 & 2.5246110 \\ \text { H } & 1.4703077 & -3.2068368 & 3.0594160 \\ \text { H } & -0.4743245 & -5.5836321 & 1.4956674 \\ \text { H } & 0.9109072 & -6.3794839 & 0.5017271 \\ \text { H } & 3.4437065 & -3.9973277 & 1.5166629\end{array}$




\begin{tabular}{|c|c|c|c|}
\hline J & 2.7708363 & -3.6172959 & -0.0915606 \\
\hline & 3.0661576 & -5.3184620 & 0.3486901 \\
\hline & 2.4242549 & -1.6318188 & 0.5376831 \\
\hline & -0.6123324 & -4.0533516 & -1.0773931 \\
\hline & 0.5760538 & -4.9431414 & -3.0806276 \\
\hline & 2.4616406 & -3.6444682 & -4.1114801 \\
\hline & 3.1063193 & -1.4100647 & -3.1354557 \\
\hline & 1.8658754 & -0.5080169 & -1.1755715 \\
\hline & -3.7282691 & -3.3024929 & 0.3617018 \\
\hline & -5.1873204 & -3.8857724 & -1.5760248 \\
\hline & -4.7373163 & -2.8973865 & -3.8410939 \\
\hline & -2.8035998 & -1.3144698 & -4.1356442 \\
\hline & -1.3469892 & -0.7400067 & -2.1888124 \\
\hline d & -0.7264129 & 0.3214128 & 0.1605031 \\
\hline & 0.4365941 & 2.2468227 & -0.1111120 \\
\hline & 0.7372433 & 3.2768107 & 1.3830222 \\
\hline & 1.8583639 & 4.1217795 & 1.5217902 \\
\hline & 2.6224112 & 4.15 & \\
\hline & 2.0108577 & 4.9039923 & 2.67 \\
\hline & 2.8898389 & 5.5 & 2.7 \\
\hline & 1.0480 & 4.8 & 3.6 \\
\hline & 1.172 & 5.4 & 4. \\
\hline & -0.06 & 4.0 & 8486 \\
\hline & -0.814 & 3.9 & 4. \\
\hline & -0.2 & 3.2 & 2.4 \\
\hline & -1.0716821 & 2.5238142 & 2.3 \\
\hline & 2.1282467 & 1.9454887 & -0.7675279 \\
\hline & 2.4351567 & 2.1317874 & -2.1302597 \\
\hline & 1.6902810 & 2.5826835 & -2.8025113 \\
\hline & 3.6843272 & 1.7297556 & -2.6321119 \\
\hline & 3.9139804 & 1.8744346 & -3.6988053 \\
\hline & 4.6352318 & 1.1468715 & -1.7786353 \\
\hline & 5.6122563 & 0.8303426 & -2.1740604 \\
\hline & 4.3330945 & 0.9662009 & -0.4165257 \\
\hline & 5.070 & 0.503 & 0.2569285 \\
\hline & 3.0851718 & 1.3559274 & 0.0890001 \\
\hline & 2.8344599 & 1.1613173 & 1.1412791 \\
\hline & -0.2870364 & 3.4229726 & -1.3340478 \\
\hline & -1.2372271 & 2.9125819 & -2.2429831 \\
\hline & -1.5336165 & 1.8530606 & -2.1632720 \\
\hline & -1.7981424 & 3.7457486 & -3.2240237 \\
\hline & -2.5393784 & 3.3392092 & -3.9286145 \\
\hline & -1.4202556 & 5.0965866 & -3.2961512 \\
\hline & -1.8637772 & 5.7530763 & -4.0602298 \\
\hline & -0.4817570 & 5.6127912 & -2.3850825 \\
\hline & -0.1910509 & 6.6732498 & -2.434806 \\
\hline
\end{tabular}




\begin{tabular}{lrrr} 
C & 0.0844306 & 4.7808326 & -1.4074258 \\
H & 0.8123027 & 5.1912072 & -0.6918685 \\
& & & \\
R3 & & & \\
75 & \multicolumn{3}{r}{} \\
SCF & Energy $=-4548.290223003$ & \\
C & -1.2690978 & 2.5226400 & -0.9456155 \\
C & -2.1995438 & 2.0646399 & 0.0192992 \\
C & -3.3165250 & 2.8800267 & 0.3079599 \\
C & -3.5107711 & 4.0958499 & -0.3652192 \\
C & -2.5876027 & 4.5304802 & -1.3304388 \\
C & -1.4612450 & 3.7385297 & -1.6118868 \\
C & -2.0049900 & 0.7334734 & 0.6701485 \\
C & -1.8361730 & -0.5008910 & -0.0233524 \\
C & -1.8231738 & -0.5282571 & -1.5277234 \\
C & -2.8348192 & 0.0944617 & -2.2914100 \\
C & -2.8274898 & 0.0200639 & -3.6920598 \\
C & -1.8192378 & -0.6917263 & -4.3634916 \\
C & -0.8127751 & -1.3269621 & -3.6179528 \\
C & -0.8123270 & -1.2407591 & -2.2169674 \\
Br & -2.6104913 & 0.6928211 & 2.5423481 \\
C & -4.7561791 & -1.6851601 & 0.9240139 \\
C & -5.3171579 & -0.4587034 & 0.2523609 \\
C & -5.2718884 & -2.1609655 & 2.0767989 \\
C & -3.5869127 & -2.3842396 & 0.2598924 \\
C & -2.1656561 & -1.8631129 & 0.6192583 \\
O & -1.2079272 & -2.8617183 & 0.2798998 \\
H & -2.1016203 & -1.7531280 & 1.7177184 \\
H & -3.5747842 & -3.4491729 & 0.5666295 \\
H & -3.6988006 & -2.3630442 & -0.8470757 \\
H & -4.8651370 & -3.0650257 & 2.5578057 \\
H & -6.1091823 & -1.6555613 & 2.5836169 \\
H & -5.6318875 & -0.6870529 & -0.7885647 \\
H & -4.5572913 & 0.3457034 & 0.1810867 \\
H & -6.1879764 & -0.0523012 & 0.8012978 \\
H & -1.2016323 & -2.9393582 & -0.6962090 \\
H & -3.6281360 & 0.6550091 & -1.7810519 \\
H & -3.6201060 & 0.5260133 & -4.2641025 \\
H & -1.8160629 & -0.7460500 & -5.4626544 \\
H & -0.0045480 & -1.8772942 & -4.1230736 \\
H & 0.0179479 & -1.6789148 & -1.6466540 \\
H & -4.0346403 & 2.5550192 & 1.0742315 \\
H & -4.3915064 & 4.7113686 & -0.1264838 \\
H & -2.7383666 & 5.4887354 & -1.8504005 \\
H & -0.7140683 & 4.0694220 & -2.3490800 \\
& -0.3798343 & 1.9104094 & -1.1560994
\end{tabular}




$\begin{array}{lrrr}\text { Pd } & 0.0590760 & 0.2269188 & 0.7378367 \\ \text { P } & 2.1976568 & -0.1875147 & 0.1071065 \\ \text { C } & 3.6053217 & -0.1516094 & 1.2917995 \\ \text { C } & 3.4281289 & 0.5520979 & 2.5002074 \\ \text { H } & 2.4509556 & 1.0162859 & 2.7130765 \\ \text { C } & 4.4822351 & 0.6498539 & 3.4221164 \\ \text { H } & 4.3378041 & 1.2015240 & 4.3633346 \\ \text { C } & 5.7139678 & 0.0322664 & 3.1470653 \\ \text { H } & 6.5381855 & 0.1002686 & 3.8733179 \\ \text { C } & 5.8909362 & -0.6809834 & 1.9483820 \\ \text { H } & 6.8532783 & -1.1711404 & 1.7355976 \\ \text { C } & 4.8417770 & -0.7725112 & 1.0209270 \\ \text { H } & 4.9790407 & -1.3323052 & 0.0833246 \\ \text { C } & 2.4369359 & -1.7739988 & -0.7948466 \\ \text { C } & 1.8663366 & -2.9317882 & -0.2235930 \\ \text { H } & 1.2879803 & -2.8492995 & 0.7082049 \\ \text { C } & 1.9794879 & -4.1665922 & -0.8785772 \\ \text { H } & 1.5218080 & -5.0617746 & -0.4312066 \\ \text { C } & 2.6523286 & -4.2545604 & -2.1102421 \\ \text { H } & 2.7284277 & -5.2220711 & -2.6294999 \\ \text { C } & 3.2206521 & -3.1036715 & -2.6812805 \\ \text { H } & 3.7478302 & -3.1680412 & -3.6455007 \\ \text { C } & 3.1180829 & -1.8652623 & -2.0256713 \\ \text { H } & 3.5569039 & -0.9637889 & -2.4783033 \\ \text { C } & 2.6602498 & 1.0919835 & -1.1392034 \\ \text { C } & 3.7230087 & 1.9951751 & -0.9450874 \\ \text { H } & 4.3630939 & 1.9050635 & -0.0551263 \\ \text { C } & 3.9623408 & 3.0149374 & -1.8828898 \\ \text { H } & 4.7915007 & 3.7200232 & -1.7186839 \\ \text { C } & 3.1512120 & 3.1359074 & -3.0218084 \\ \text { H } & 3.3413246 & 3.9361187 & -3.7530699 \\ \text { C } & 2.0936635 & 2.2299467 & -3.2244126 \\ \text { H } & 1.4521641 & 2.3133919 & -4.1148989 \\ \text { C } & 1.8437385 & 1.2189927 & -2.2873523 \\ \text { H } & 1.0083571 & 0.5202445 & -2.4462719 \\ & & & \\ \text { R4 } & & & \\ 75 & & & \\ \text { SCF } & \text { Energy }=-4548.292500606 & \\ \text { C } & -1.4434597 & 0.3573793 & -2.6568184 \\ \text { C } & -2.3706907 & 0.8504278 & -1.7117899 \\ \text { C } & -3.6493207 & 1.2486041 & -2.1611973 \\ \text { C } & -3.9969311 & 1.1293440 & -3.5141943 \\ \text { C } & -3.0723863 & 0.6206976 & -4.4437186 \\ \text { C } & -1.7923994 & 0.2399037 & -4.0092285 \\ \text { C } & -2.0262716 & 0.8803327 & -0.2590292\end{array}$




$\begin{array}{lrrr}\text { C } & -1.7972808 & -0.2687066 & 0.5494574 \\ \text { C } & -1.7894868 & -1.6377770 & -0.0766395 \\ \text { C } & -2.8024532 & -2.0656952 & -0.9633307 \\ \text { C } & -2.7884027 & -3.3615902 & -1.5006670 \\ \text { C } & -1.7724958 & -4.2680201 & -1.1540119 \\ \text { C } & -0.7644066 & -3.8622439 & -0.2642096 \\ \text { C } & -0.7708088 & -2.5617176 & 0.2627337 \\ \text { Br } & -2.5440974 & 2.5806574 & 0.5918626 \\ \text { C } & -4.6362138 & 0.0841390 & 1.9812392 \\ \text { C } & -4.9163438 & 1.2336456 & 2.9166604 \\ \text { C } & -5.3709904 & -0.1353551 & 0.8706406 \\ \text { C } & -3.4926452 & -0.8246042 & 2.3729745 \\ \text { C } & -2.0704404 & -0.2729609 & 2.0661803 \\ \text { O } & -1.1021309 & -1.0040096 & 2.8079297 \\ \text { H } & -1.9923820 & 0.7683445 & 2.4309902 \\ \text { H } & -3.5076875 & -0.9973594 & 3.4703801 \\ \text { H } & -3.5964575 & -1.8145613 & 1.8812296 \\ \text { H } & -5.1798327 & -1.0016992 & 0.2198321 \\ \text { H } & -6.1942647 & 0.5385197 & 0.5851070 \\ \text { H } & -4.0016370 & 1.8326177 & 3.1062524 \\ \text { H } & -5.2595299 & 0.8532406 & 3.9037263 \\ \text { H } & -5.6924120 & 1.9137253 & 2.5164234 \\ \text { H } & -1.1012622 & -1.9198913 & 2.4618006 \\ \text { H } & -3.6009337 & -1.3683667 & -1.2461293 \\ \text { H } & -3.5830533 & -3.6642947 & -2.1995541 \\ \text { H } & -1.7642021 & -5.2827173 & -1.5800170 \\ \text { H } & 0.0521207 & -4.5476448 & 0.0088811 \\ \text { H } & 0.0631950 & -2.2317353 & 0.8960853 \\ \text { H } & -4.3720568 & 1.6415855 & -1.4315327 \\ \text { H } & -5.0007197 & 1.4359493 & -3.8461423 \\ \text { H } & -3.3478917 & 0.5315075 & -5.5056349 \\ \text { H } & -1.0516818 & -0.1477146 & -4.7250321 \\ \text { H } & -0.4418725 & 0.0610735 & -2.3070804 \\ \text { Pd } & 0.0633933 & 0.7396903 & 0.1117240 \\ \text { P } & 2.2071295 & -0.0029281 & 0.1227525 \\ \text { C } & 3.6507183 & 1.0604364 & 0.5357157 \\ \text { C } & 4.8844377 & 0.5310681 & 0.9666312 \\ \text { H } & 4.9973877 & -0.5561681 & 1.0953511 \\ \text { C } & 5.9612774 & 1.3900823 & 1.2352326 \\ \text { H } & 6.9214401 & 0.9734660 & 1.5760688 \\ \text { C } & 5.8146735 & 2.7787213 & 1.0718067 \\ \text { H } & 6.6604075 & 3.4499804 & 1.2853016 \\ \text { C } & 4.5857992 & 3.3103928 & 0.6453273 \\ \text { H } & 4.4655704 & 4.3976816 & 0.5249210 \\ \text { C } & 3.5043519 & 2.4543003 & 0.3850961 \\ \text { H } & 2.5297329 & 2.8613391 & 0.0689458\end{array}$




$\begin{array}{lrrr}\text { C } & 2.4627367 & -1.4930304 & 1.1729995 \\ \text { C } & 1.9905393 & -1.4331671 & 2.5020562 \\ \text { H } & 1.4908792 & -0.5214808 & 2.8607788 \\ \text { C } & 2.0991492 & -2.5538843 & 3.3373647 \\ \text { H } & 1.7169506 & -2.5014921 & 4.3679051 \\ \text { C } & 2.6678341 & -3.7450092 & 2.8516088 \\ \text { H } & 2.7380735 & -4.6288079 & 3.5038509 \\ \text { C } & 3.1391299 & -3.8072779 & 1.5297987 \\ \text { H } & 3.5852050 & -4.7373920 & 1.1455081 \\ \text { C } & 3.0420682 & -2.6840349 & 0.6910672 \\ \text { H } & 3.4034869 & -2.7372711 & -0.3463584 \\ \text { C } & 2.5929466 & -0.6026299 & -1.5790487 \\ \text { C } & 1.6861556 & -1.5126268 & -2.1715704 \\ \text { H } & 0.8211951 & -1.8761485 & -1.5959448 \\ \text { C } & 1.8862487 & -1.9541942 & -3.4860316 \\ \text { H } & 1.1748172 & -2.6657491 & -3.9319373 \\ \text { C } & 2.9827491 & -1.4820064 & -4.2309166 \\ \text { H } & 3.1338892 & -1.8207622 & -5.2671225 \\ \text { C } & 3.8823367 & -0.5758161 & -3.6481026 \\ \text { H } & 4.7414741 & -0.2023686 & -4.2261066 \\ \text { C } & 3.6934029 & -0.1387966 & -2.3251313 \\ \text { H } & 4.4023657 & 0.5704072 & -1.8732305 \\ & & & \\ \text { S1 } & & & \\ 75 & & & \\ \text { SCF } & \text { Energy = }-4548.293023754 & \\ \text { C } & -1.5648398 & 2.1185703 & -1.8476853 \\ \text { C } & -2.4292154 & 1.9321089 & -0.7432711 \\ \text { C } & -3.7233299 & 2.4964733 & -0.8014567 \\ \text { C } & -4.1500916 & 3.1933249 & -1.9414350 \\ \text { C } & -3.2903454 & 3.3490516 & -3.0421522 \\ \text { C } & -1.9929671 & 2.8127828 & -2.9867919 \\ \text { C } & -1.9850971 & 1.1091022 & 0.4225485 \\ \text { C } & -1.7354926 & -0.3003362 & 0.4308820 \\ \text { C } & -1.8208098 & -1.1161774 & -0.8328552 \\ \text { C } & -2.7186323 & -0.8363084 & -1.8879020 \\ \text { C } & -2.7500748 & -1.6348815 & -3.0409572 \\ \text { C } & -1.8903573 & -2.7363238 & -3.1740475 \\ \text { C } & -1.0005341 & -3.0368469 & -2.1281718 \\ \text { C } & -0.9706957 & -2.2412964 & -0.9787264 \\ \text { Br } & -2.5135291 & 1.9892518 & 2.1365042 \\ \text { C } & -4.1371744 & -2.0345020 & 1.0914453 \\ \text { C } & -5.1038446 & -1.0590962 & 0.4728426 \\ \text { C } & -4.0488847 & -3.3201520 & 0.6945652 \\ \text { C } & -3.2376538 & -1.4794319 & 2.1783220 \\ \text { C } & -1.7857507 & -1.1672498 & 1.7165575\end{array}$




\begin{tabular}{lrrr} 
O & -1.0101313 & -0.7004247 & 2.8072520 \\
H & -1.3226669 & -2.1327734 & 1.4379002 \\
H & -3.6741236 & -0.5509553 & 2.5990408 \\
H & -3.1364131 & -2.1995200 & 3.0155915 \\
H & -3.3392592 & -4.0191041 & 1.1659562 \\
H & -4.6659593 & -3.7124991 & -0.1285943 \\
H & -4.5815788 & -0.1380383 & 0.1396591 \\
H & -5.8566946 & -0.7296526 & 1.2222251 \\
H & -5.6375099 & -1.4897417 & -0.3958993 \\
H & -1.3442493 & 0.1941511 & 3.0377434 \\
H & -3.4155566 & 0.0049715 & -1.8098723 \\
H & -3.4637895 & -1.3904460 & -3.8425341 \\
H & -1.9152695 & -3.3571744 & -4.0823626 \\
H & -0.3101317 & -3.8898281 & -2.2082494 \\
H & -0.2450886 & -2.4780411 & -0.1907869 \\
H & -4.3991107 & 2.3833583 & 0.0589146 \\
H & -5.1649413 & 3.6185968 & -1.9690289 \\
H & -3.6273279 & 3.8974428 & -3.9348701 \\
H & -1.3027000 & 2.9378642 & -3.8346827 \\
H & -0.5498977 & 1.6884443 & -1.7937135 \\
C & 3.8378079 & 1.9428628 & 0.8187829 \\
C & 3.7149151 & 0.5434219 & 0.6881756 \\
C & 4.7773771 & -0.2882293 & 1.0954027 \\
C & 5.9518699 & 0.2768886 & 1.6187464 \\
C & 6.0748066 & 1.6715297 & 1.7338203 \\
C & 5.0159937 & 2.5046812 & 1.3316165 \\
P & 2.1534257 & -0.1106893 & -0.0323442 \\
C & 2.5024723 & -0.1274661 & -1.8434259 \\
C & 3.7929111 & 0.0508752 & -2.3801351 \\
C & 3.9870404 & 0.0114282 & -3.7715123 \\
C & 2.8995771 & -0.2128547 & -4.6319142 \\
C & 1.6104862 & -0.3919813 & -4.0991701 \\
C & 1.4096486 & -0.3405777 & -2.7129948 \\
Pd & 0.1159959 & 0.7966902 & 0.4169406 \\
C & 2.1817638 & -1.8964163 & 0.4150807 \\
C & 2.6696850 & -2.8901213 & -0.4562437 \\
C & 2.5805825 & -4.2450265 & -0.0946641 \\
C & 2.0123979 & -4.6117337 & 1.1365090 \\
C & 1.5322051 & -3.6194505 & 2.0101919 \\
C & 1.6083539 & -2.2656233 & 1.6517628 \\
H & 3.0995935 & -2.6041779 & -1.4275482 \\
H & 2.9521173 & -5.0188754 & -0.7837385 \\
H & 1.9349367 & -5.6744086 & 1.4124638 \\
& 1.0734452 & -3.9007615 & 2.9702144 \\
& 1.1659800 & -1.4947563 & 2.3035242 \\
H & & & -1.7085664 \\
\hline
\end{tabular}




$\begin{array}{rrrr}\text { H } & 4.9968445 & 0.1570549 & -4.1849168 \\ \text { H } & 3.0564252 & -0.2440126 & -5.7209663 \\ \text { H } & 0.7509373 & -0.5681312 & -4.7631328 \\ \text { H } & 0.4017333 & -0.4769607 & -2.2938664 \\ \text { H } & 4.6809462 & -1.3806382 & 1.0049918 \\ \text { H } & 6.7765254 & -0.3778485 & 1.9394825 \\ \text { H } & 6.9957733 & 2.1114493 & 2.1456127 \\ \text { H } & 5.1051036 & 3.5973649 & 1.4283816 \\ \text { H } & 2.9972235 & 2.5899704 & 0.5189321\end{array}$

S2

75

SCF Energy $=-4548.295735503$

C $\quad-2.0212436 \quad-1.6529027$

$\begin{array}{lll}\text { C } & -1.9174326 & -2.4516900\end{array}$

C $\quad-2.5306096 \quad-3.7239689$

$-1.9382687$

$-0.7752098$

$-0.7767388$

C $\quad-3.2004077-4.1938250$

$-1.9156625$

C $\quad-3.2778330-3.3997731$

$-3.0727104$

C $\quad-2.6881794-2.1247555$

$-3.0764495$

$\begin{array}{lll}-1.1229527 & -1.9664785 & 0.3922854\end{array}$

$0.2849956-1.7296826$

C $\quad 1.0991316-1.8962125$

C $\quad 0.8845974 \quad-2.9436126$

C $\quad 1.6863631 \quad-3.0681934$

0.4125855

$-0.8434766$

$-1.7653522$

$-2.9092459$

$\begin{array}{llll}\text { C } & 2.7274602 & -2.1580569 & -3.1565534\end{array}$

$\begin{array}{llll}\text { C } & 2.9666847 & -1.1229687 & -2.2364536\end{array}$

$\begin{array}{llll}\text { C } & 2.1651430 & -0.9987255 & -1.0944004\end{array}$

$\begin{array}{llll}\mathrm{Br} & -2.0505930 & -2.3673727 & 2.1106941\end{array}$

$\begin{array}{llll}\text { C } & 1.8048645 & -4.2385876 & 1.1776097\end{array}$

$\begin{array}{llll}\text { C } & 3.2084289 & -4.0970571 & 0.6474553\end{array}$

$\begin{array}{llll}\text { C } & 0.9684989 & -5.2124874 & 0.7600455\end{array}$

$\begin{array}{llll}\text { C } & 1.3536224 & -3.2281571 & 2.2059904\end{array}$

$\begin{array}{llll}\text { C } & 1.1538812 & -1.7714787 & 1.6951162\end{array}$

$\begin{array}{llll}0 & 0.7279131 & -0.9429648 & 2.7644017\end{array}$

$\begin{array}{llll}\mathrm{H} & 2.1393218 & -1.3607866 & 1.4000641\end{array}$

H $\quad 0.3954701 \quad-3.5593841 \quad 2.6517683$

$\mathrm{H} \quad 2.0931695 \quad-3.1582528 \quad 3.0332026$

$\begin{array}{llll}\mathrm{H} & -0.0539927 & -5.2959313 & 1.1611027\end{array}$

$\begin{array}{llll}\mathrm{H} & 1.2760676 & -5.9484666 & 0.0003373\end{array}$

$\begin{array}{llll}\mathrm{H} & 3.9490504 & -4.1412303 & 1.4752787\end{array}$

$\begin{array}{llll}\mathrm{H} & 3.3505387 & -3.1170625 & 0.1443796\end{array}$

$\begin{array}{llll}\mathrm{H} & 3.4537079 & -4.8887237 & -0.0858664\end{array}$

$\mathrm{H} \quad-0.1849051 \quad-1.2215072 \quad 2.9967738$

$\mathrm{H} \quad 0.0979494 \quad-3.6823111-1.5735650$

$\mathrm{H} \quad 1.4970966-3.8943033 \quad-3.6116035$

H $\quad 3.3525251 \quad-2.2588664 \quad-4.0568020$ 


$\begin{array}{lrrr}\text { H } & 3.7757349 & -0.3971222 & -2.4080194 \\ \text { H } & 2.3484465 & -0.1712769 & -0.3961538 \\ \text { H } & -2.4712721 & -4.3500445 & 0.1253612 \\ \text { H } & -3.6650415 & -5.1915419 & -1.8988676 \\ \text { H } & -3.8055976 & -3.7703960 & -3.9645518 \\ \text { H } & -2.7510680 & -1.4856321 & -3.9701016 \\ \text { H } & -1.5539799 & -0.6534244 & -1.9293011 \\ \text { Pd } & -0.7960328 & 0.1309051 & 0.3301157 \\ \text { P } & 0.0952349 & 2.1813277 & -0.0814313 \\ \text { C } & 1.8901538 & 2.2248593 & 0.3287302 \\ \text { C } & 2.8555370 & 2.7657981 & -0.5436128 \\ \text { H } & 2.5411841 & 3.2210094 & -1.4945036 \\ \text { C } & 4.2185145 & 2.7018039 & -0.2078467 \\ \text { H } & 4.9700334 & 3.1163777 & -0.8971199 \\ \text { C } & 4.6213272 & 2.1058631 & 0.9986424 \\ \text { H } & 5.6902894 & 2.0495801 & 1.2548920 \\ \text { C } & 3.6574494 & 1.5714078 & 1.8724267 \\ \text { H } & 3.9675630 & 1.0915418 & 2.8131210 \\ \text { C } & 2.2957781 & 1.6221126 & 1.5399262 \\ \text { H } & 1.5478857 & 1.1420054 & 2.1922943 \\ \text { C } & -0.5663260 & 3.7075420 & 0.7048049 \\ \text { C } & 0.2548562 & 4.7835651 & 1.0975026 \\ \text { H } & 1.3442194 & 4.7189176 & 0.9561653 \\ \text { C } & -0.3165132 & 5.9306888 & 1.6719400 \\ \text { H } & 0.3300938 & 6.7660057 & 1.9813992 \\ \text { C } & -1.7072861 & 6.0125118 & 1.8530775 \\ \text { H } & -2.1520179 & 6.9120328 & 2.3051674 \\ \text { C } & -2.5296783 & 4.9399904 & 1.4657590 \\ \text { H } & -3.6186745 & 4.9967065 & 1.6143563 \\ \text { C } & -1.9609012 & 3.7887909 & 0.9015282 \\ \text { H } & -2.5979568 & 2.9366276 & 0.6131074 \\ \text { C } & 0.0709093 & 2.5887626 & -1.8798733 \\ \text { C } & 0.3340109 & 1.5354158 & -2.7841755 \\ \text { H } & 0.5379609 & 0.5255386 & -2.3960735 \\ \text { C } & 0.3480000 & 1.7776113 & -4.1645207 \\ \text { H } & 0.5623408 & 0.9501617 & -4.8576583 \\ \text { C } & 0.0810209 & 3.0679154 & -4.6568963 \\ \text { H } & 0.0819644 & 3.2562519 & -5.7413558 \\ \text { C } & -0.1921047 & 4.1150196 & -3.7617799 \\ \text { H } & -0.4058165 & 5.1252997 & -4.1431048 \\ \text { C } & -0.1937523 & 3.8802528 & -2.3758932 \\ \text { H } & -0.4048429 & 4.7032533 & -1.6770036 \\ \text { S3 } & & & \\ \text { SCF } & \text { Energy }=-4548.292490475 & \\ & & & \end{array}$




\begin{tabular}{|c|c|c|c|}
\hline & -1.2292298 & -1.7603029 & -2.3919935 \\
\hline & -1.6422387 & -2.3949630 & -1.1934217 \\
\hline & -2.5208745 & -3.4960380 & -1.2919280 \\
\hline & -2.9559627 & -3.9598311 & -2.5431260 \\
\hline & -2.5287872 & -3.3300732 & -3.7226270 \\
\hline & -1.6644254 & -2.2245706 & -3.6384220 \\
\hline & -1.1095855 & -1.9160089 & 0.1180855 \\
\hline & 0.2751750 & -1.7494901 & \\
\hline & 1.3037851 & -2.0988165 & -0.6219757 \\
\hline & 1.2692899 & -3.3264059 & -1.3181701 \\
\hline & 2.2653608 & -3.6481771 & -2.2517271 \\
\hline & 3.3195515 & -2.7539189 & -2.5008549 \\
\hline & 3.3744138 & -1.5371414 & -1.8001709 \\
\hline & 2.3771466 & -1.2161784 & -0.8699065 \\
\hline & -2.4241989 & -2.1538073 & 1.5856205 \\
\hline & 0.3398923 & -4.0 & 2. \\
\hline & -0.1802222 & -5.0 & 8672 \\
\hline & -0.151 & -4.0 & 3. \\
\hline & 1.431 & -3.0 & 2.3 \\
\hline & 0.9 & -1.6 & $1 . \varepsilon$ \\
\hline & 0.2 & -1.0 & $2 . \varepsilon$ \\
\hline & 1.8 & -1.0 & 1. \\
\hline & 2.08 & -2.8 & 3. \\
\hline & 2.06 & -3.53 & 1.5 \\
\hline & 0.24 & -3.3472513 & 4.7 \\
\hline & -0.9685400 & -4.7087679 & 4.2 \\
\hline & 0.6420972 & -5.630 & 1.2 \\
\hline & -0.6030586 & -4.448 & 0.8 \\
\hline & -0.9704618 & -5.6666457 & 2.0 \\
\hline & -0.6575602 & -1.4660546 & 2.9 \\
\hline & 0.449 & -4.03 & -1.1 \\
\hline & 2.21 & $-4.6 c$ & -2.7 \\
\hline & 4.0966 & -3.0 & -3.2 \\
\hline & $4.187 !$ & -0.82 & -1.9 \\
\hline & 2.407 & -0.248 & -0.3 \\
\hline & -2.8658005 & -3.9922768 & -0.3736224 \\
\hline & -3.6378466 & -4.8224543 & -2.5928229 \\
\hline & -2.8749081 & -3.6920164 & -4.7025973 \\
\hline & -1.3283746 & -1.7131431 & -4.5531034 \\
\hline & -0.5540906 & -0.8931250 & -2.3198273 \\
\hline & -0.6759959 & 0.1607372 & 0.0620652 \\
\hline & 0.2560333 & 2.2165567 & -0.1468140 \\
\hline & -0.7882325 & 3.5825584 & -0.811986 \\
\hline & -0.3162608 & 4.5355298 & -1.7373513 \\
\hline & 0.7142085 & 4.4659517 & -2.1171687 \\
\hline & -1.1627577 & 5.5645868 & -2.181490 \\
\hline
\end{tabular}




$\begin{array}{lrrr}\text { H } & -0.7888653 & 6.3039446 & -2.9062509 \\ \mathrm{C} & -2.4812866 & 5.6504408 & -1.7048704 \\ \mathrm{H} & -3.1430616 & 6.4564703 & -2.0564815 \\ \mathrm{C} & -2.9573086 & 4.6999323 & -0.7848206 \\ \mathrm{H} & -3.9922487 & 4.7586065 & -0.4151576 \\ \mathrm{C} & -2.1171159 & 3.6668253 & -0.3447998 \\ \mathrm{H} & -2.4888684 & 2.9107249 & 0.3660404 \\ \mathrm{C} & 0.9889803 & 2.9551424 & 1.3702966 \\ \mathrm{C} & 1.1358622 & 2.1321610 & 2.5058447 \\ \mathrm{H} & 0.7670368 & 1.0920424 & 2.4868934 \\ \mathrm{C} & 1.7249590 & 2.6524309 & 3.6707923 \\ \mathrm{H} & 1.8345075 & 2.0061627 & 4.5548668 \\ \mathrm{C} & 2.1584675 & 3.9874272 & 3.7105795 \\ \mathrm{H} & 2.6154089 & 4.3926781 & 4.6264899 \\ \mathrm{C} & 1.9971541 & 4.8128266 & 2.5829028 \\ \mathrm{H} & 2.3246790 & 5.8632586 & 2.6156573 \\ \mathrm{C} & 1.4119003 & 4.3007131 & 1.4156220 \\ \mathrm{H} & 1.2763002 & 4.9500328 & 0.5370797 \\ \mathrm{C} & 1.6588935 & 2.1540574 & -1.3398439 \\ \mathrm{C} & 1.3823555 & 1.6737719 & -2.6395232 \\ \mathrm{H} & 0.3476935 & 1.4086822 & -2.9092082 \\ \mathrm{C} & 2.4136678 & 1.5235736 & -3.5742511 \\ \mathrm{H} & 2.1865007 & 1.1483728 & -4.5833492 \\ \mathrm{C} & 3.7393166 & 1.8308534 & -3.2166976 \\ \mathrm{H} & 4.5519158 & 1.7006082 & -3.9472712 \\ \mathrm{C} & 4.0227193 & 2.2913019 & -1.9216767 \\ \mathrm{H} & 5.0587559 & 2.5261527 & -1.6333640 \\ \mathrm{C} & 2.9869591 & 2.4577487 & -0.9846742 \\ \mathrm{H} & 3.2140249 & 2.8192537 & 0.0288597\end{array}$

S4

75

SCF Energy $=-4548.292218918$

C $\quad-1.4173632-1.7593368$

$\begin{array}{lll}\text { C } & -1.6559522 & -2.4452917\end{array}$

C $\quad-2.3535109-3.6722633$

C $\quad-2.7804975-4.2062391$

C $\quad-2.5245581-3.5230886$

C $\quad-1.8428932-2.2952319$

$\begin{array}{lll}-1.1203385 & -1.9009441\end{array}$

$-2.3587005$

$-1.1454015$

$-1.1901978$

$-2.4145927$

$-3.6157654$

$-3.5812295$

0.1376084

$\begin{array}{lllr}\text { C } & 0.2614637 & -1.7236258 & 0.4444959 \\ \text { C } & 1.2989427 & -2.0323732 & -0.6049865\end{array}$

C $\quad 1.2520147-3.1985751 \quad-1.3992938$

$\begin{array}{llll}\text { C } & 2.2599951 & -3.4679075 & -2.3375233\end{array}$

$\begin{array}{llll}\text { C } & 3.3396438 & -2.5842407 & -2.4948661\end{array}$

$\begin{array}{llll}\text { C } & 3.4080886 & -1.4297613 & -1.6963952\end{array}$ 


$\begin{array}{lrrr}\text { C } & 2.3995119 & -1.1601386 & -0.7635388 \\ \mathrm{Br} & -2.4523928 & -2.0902542 & 1.6025430 \\ \mathrm{C} & 0.2086449 & -4.1350467 & 2.5221225 \\ \mathrm{C} & -0.4579938 & -4.0766765 & 3.8738909 \\ \mathrm{C} & -0.1508591 & -5.0291035 & 1.5765771 \\ \mathrm{C} & 1.3277105 & -3.1456553 & 2.2850868 \\ \mathrm{C} & 0.8976751 & -1.7093686 & 1.8513807 \\ \mathrm{O} & 0.1960802 & -1.0105917 & 2.8637153 \\ \mathrm{H} & 1.8482782 & -1.1492492 & 1.7271888 \\ \mathrm{H} & 1.9213779 & -3.0164558 & 3.2151391 \\ \mathrm{H} & 2.0157048 & -3.5290462 & 1.5050676 \\ \mathrm{H} & 0.3737713 & -5.0821974 & 0.6106395 \\ \mathrm{H} & -0.9778861 & -5.7378205 & 1.7418950 \\ \mathrm{H} & -0.7450590 & -3.0374690 & 4.1301653 \\ \mathrm{H} & 0.2535583 & -4.4009539 & 4.6651457 \\ \mathrm{H} & -1.3551737 & -4.7228548 & 3.9270185 \\ \mathrm{H} & -0.7616676 & -1.2146671 & 2.7672795 \\ \mathrm{H} & 0.4152189 & -3.8995200 & -1.2863973 \\ \mathrm{H} & 2.1983821 & -4.3810176 & -2.9490189 \\ \mathrm{H} & 4.1259928 & -2.7946423 & -3.2356666 \\ \mathrm{H} & 4.2410136 & -0.7207455 & -1.8088825 \\ \mathrm{H} & 2.4437233 & -0.2380172 & -0.1669580 \\ \mathrm{H} & -2.5461444 & -4.2122907 & -0.2519014 \\ \mathrm{H} & -3.3161835 & -5.1677245 & -2.4300060 \\ \mathrm{H} & -2.8627437 & -3.9434251 & -4.5750374 \\ \mathrm{H} & -1.6436207 & -1.7456673 & -4.5136571 \\ \mathrm{H} & -0.8756224 & -0.7998149 & -2.3149556 \\ \mathrm{Pd} & -0.6748880 & 0.1757365 & 0.0478546 \\ \mathrm{P} & 0.2850180 & 2.2226577 & -0.1653717 \\ \mathrm{C} & 1.0315383 & 2.9519920 & 1.3493235 \\ \mathrm{C} & 1.1972933 & 2.1207188 & 2.4763047 \\ \mathrm{H} & 0.8301190 & 1.0796765 & 2.4568386 \\ \mathrm{C} & 1.7997122 & 2.6342524 & 3.6372890 \\ \mathrm{H} & 1.9229989 & 1.9815657 & 4.5148158 \\ \mathrm{C} & 2.2279366 & 3.9708559 & 3.6821167 \\ \mathrm{H} & 2.6951941 & 4.3708009 & 4.5951369 \\ \mathrm{C} & 2.0473184 & 4.8045831 & 2.5636498 \\ \mathrm{H} & 3.7509019 & 1.8196958 & -3.2543847 \\ \mathrm{C} & 1.4701110 & 5.8563361 & 2.6005848 \\ \mathrm{H} & 1.2977153 & 4.2992325 & 1.4001148 \\ \mathrm{C} & 1.6842863 & 2.1393456 & -1.3622236 \\ \mathrm{C} & 1.4063338 & 1.6162527 & -2.6445229 \\ \mathrm{C} & 0.3764284 & 1.3152707 & -2.8938921 \\ & 2.4304183 & 1.4673023 & -3.5873506 \\ & 2.2020231 & 1.0567638 & -4.5822267 \\ & & & \\ \mathrm{H} & & \end{array}$




$\begin{array}{lrrr}\text { H } & 4.5585333 & 1.6904541 & -3.9906238 \\ \mathrm{C} & 4.0368060 & 2.3220453 & -1.9754935 \\ \mathrm{H} & 5.0695507 & 2.5912841 & -1.7059855 \\ \mathrm{C} & 3.0078680 & 2.4861138 & -1.0307417 \\ \mathrm{H} & 3.2370056 & 2.8806760 & -0.0300954 \\ \mathrm{C} & -0.7388854 & 3.6042922 & -0.8300353 \\ \mathrm{C} & -0.2603484 & 4.5350845 & -1.7743110 \\ \mathrm{H} & 0.7629317 & 4.4388111 & -2.1674185 \\ \mathrm{C} & -1.0912131 & 5.5766250 & -2.2191052 \\ \mathrm{H} & -0.7123488 & 6.2985993 & -2.9586530 \\ \mathrm{C} & -2.4002493 & 5.6970405 & -1.7243233 \\ \mathrm{H} & -3.0497205 & 6.5128155 & -2.0763928 \\ \mathrm{C} & -2.8827944 & 4.7686048 & -0.7852273 \\ \mathrm{H} & -3.9105327 & 4.8544969 & -0.4010618 \\ \mathrm{C} & -2.0586336 & 3.7230239 & -0.3445383 \\ \mathrm{H} & -2.4359739 & 2.9841541 & 0.3812931\end{array}$

\section{Optimized Geometries(BXn)(TS_ab)}

\begin{tabular}{lrrr} 
R1 & & & \\
75 & \multicolumn{4}{l}{} \\
SCF & Energy $=-4548.282020320$ & \\
C & -0.0033402 & 3.1504773 & -2.4617977 \\
C & -0.6039805 & 3.0507396 & -1.1798409 \\
C & -1.9951350 & 3.3013134 & -1.0900147 \\
C & -2.7666073 & 3.5684467 & -2.2249633 \\
C & -2.1663982 & 3.6006180 & -3.4952775 \\
C & -0.7789448 & 3.4018530 & -3.6011342 \\
C & 0.2029966 & 2.7173994 & 0.0104529 \\
C & 1.4840137 & 2.2086311 & 0.0672392 \\
C & 1.8870636 & 1.2973325 & -1.0450021 \\
C & 1.0494015 & 0.2075806 & -1.3686888 \\
C & 1.3714436 & -0.6612951 & -2.4205118 \\
C & 2.5501861 & -0.4636081 & -3.1573137 \\
C & 3.4111242 & 0.5979400 & -2.8203117 \\
C & 3.0900389 & 1.4668666 & -1.7694206 \\
Br & -0.4885563 & 4.4980925 & 1.5251241 \\
C & 1.1069495 & 1.9539362 & 3.2408426 \\
C & 0.6250275 & 2.5323557 & 4.5561282 \\
C & 0.8017463 & 0.6325887 & 2.9122706 \\
C & 2.1616378 & 2.7994342 & 2.5510804 \\
C & 2.5859618 & 2.4007908 & 1.1339902 \\
D & 3.4830527 & 1.2924201 & 1.1644381 \\
H & 3.2150340 & 3.2362470 & 0.7466031
\end{tabular}




$\begin{array}{lrrr}\text { H } & 1.8120963 & 3.8513628 & 2.5472442 \\ \mathrm{H} & 3.0926073 & 2.7875059 & 3.1632782 \\ \mathrm{H} & 1.3070697 & 0.0763614 & 2.1061853 \\ \mathrm{H} & 0.2293265 & 0.0134566 & 3.6206234 \\ \mathrm{H} & 0.2473033 & 3.5657415 & 4.4191992 \\ \mathrm{H} & 1.4665013 & 2.5843898 & 5.2829040 \\ \mathrm{H} & -0.1805349 & 1.9203116 & 5.0060067 \\ \mathrm{H} & 2.9968194 & 0.5375895 & 1.5458174 \\ \mathrm{H} & 0.1385890 & 0.0493915 & -0.7744234 \\ \mathrm{H} & 0.6947529 & -1.4964167 & -2.6574952 \\ \mathrm{H} & 2.8083943 & -1.1414167 & -3.9851083 \\ \mathrm{H} & 4.3438184 & 0.7496480 & -3.3850227 \\ \mathrm{H} & 3.7668322 & 2.2958088 & -1.5171280 \\ \mathrm{H} & -2.4686770 & 3.2934393 & -0.1042425 \\ \mathrm{H} & -3.8463485 & 3.7447713 & -2.1085981 \\ \mathrm{H} & -2.7700975 & 3.7990097 & -4.3937918 \\ \mathrm{H} & -0.2863818 & 3.4517778 & -4.5841316 \\ \mathrm{H} & 1.0784662 & 3.0084727 & -2.5686730 \\ \mathrm{Pd} & -0.6516427 & 1.8983741 & 1.7276946 \\ \mathrm{P} & -2.5858638 & 0.7202718 & 1.2007791 \\ \mathrm{C} & -4.1208426 & 1.7226909 & 1.0037005 \\ \mathrm{C} & -4.1874869 & 2.9521114 & 1.6944289 \\ \mathrm{H} & -3.3249411 & 3.2763772 & 2.3000502 \\ \mathrm{C} & -5.3213693 & 3.7686111 & 1.5743570 \\ \mathrm{H} & -5.3625720 & 4.7275550 & 2.1125564 \\ \mathrm{C} & -6.3900595 & 3.3719765 & 0.7512838 \\ \mathrm{H} & -7.2741060 & 4.0190462 & 0.6456628 \\ \mathrm{C} & -6.3230398 & 2.1531967 & 0.0552953 \\ \mathrm{H} & -7.1557773 & 1.8424647 & -0.5941142 \\ \mathrm{C} & -5.1943958 & 1.3269530 & 0.1822387 \\ \mathrm{H} & -5.1420971 & 0.3743349 & -0.3652436 \\ \mathrm{C} & -2.7054446 & -0.4810207 & -0.1992584 \\ \mathrm{C} & -3.1978267 & -1.7935780 & -0.0367383 \\ \mathrm{H} & -3.5318512 & -2.1362926 & 0.9528735 \\ \mathrm{C} & -3.2666070 & -2.6660236 & -1.1351685 \\ \mathrm{H} & -3.6502070 & -3.6880012 & -0.9931662 \\ \mathrm{C} & -2.8546017 & -2.2379807 & -2.4080515 \\ \mathrm{H} & -2.9090771 & -2.9246428 & -3.2665259 \\ \mathrm{C} & -2.3763089 & -0.9279212 & -2.5788775 \\ \mathrm{H} & -2.0523557 & -0.5757398 & -3.5695816 \\ \mathrm{C} & -2.2981668 & -0.0561420 & -1.4831500 \\ \mathrm{H} & -1.9245133 & 0.9651199 & -1.6286040 \\ & -2.9818227 & -0.3316328 & 2.6645151 \\ \mathrm{C} & -2.0659927 & -1.3505132 & 3.0149582 \\ & -2868936 & -2.1467859 & 4.1464008\end{array}$




$\begin{array}{lrrr}\text { H } & -1.5715928 & -2.9435020 & 4.4016502 \\ \text { C } & -3.4147485 & -1.9228204 & 4.9574299 \\ \text { H } & -3.5826189 & -2.5402694 & 5.8527205 \\ \text { C } & -4.3241520 & -0.9089686 & 4.6182319 \\ \text { H } & -5.2106954 & -0.7305638 & 5.2457938 \\ \text { C } & -4.1154774 & -0.1201672 & 3.4737572 \\ \text { H } & -4.8380042 & 0.6649022 & 3.2081187\end{array}$

\begin{tabular}{lrrr} 
R2 & \multicolumn{3}{l}{} \\
75 & \multicolumn{4}{l}{} \\
SCF & Energy $=-4548.283554630$ & \\
C & -0.1164826 & 2.6324972 & -3.0528838 \\
C & -0.9264759 & 2.9692057 & -1.9406951 \\
C & -2.2938703 & 3.2526055 & -2.1831190 \\
C & -2.8495785 & 3.1334591 & -3.4616731 \\
C & -2.0441194 & 2.7454753 & -4.5463932 \\
C & -0.6747581 & 2.5134880 & -4.3341407 \\
C & -0.3967881 & 2.9798166 & -0.5553651 \\
C & 0.7801419 & 2.4714631 & -0.0491718 \\
C & 1.5976004 & 1.5015507 & -0.8341550 \\
C & 1.1071812 & 0.2088938 & -1.1198121 \\
C & 1.9100082 & -0.7212022 & -1.7928564 \\
C & 3.2131490 & -0.3797339 & -2.1939963 \\
C & 3.7080773 & 0.9066570 & -1.9258372 \\
C & 2.9088850 & 1.8424344 & -1.2478635 \\
Br & -1.2150934 & 4.6507703 & 0.5665223 \\
C & 1.1254125 & 0.2657652 & 2.1664651 \\
C & 2.5789536 & -0.1445763 & 2.1179675 \\
C & 0.1360492 & -0.6351615 & 1.9919589 \\
C & 0.8133966 & 1.7248846 & 2.4184928 \\
C & 1.3007229 & 2.7630144 & 1.3707596 \\
O & 2.7084365 & 2.9522290 & 1.4083131 \\
H & 0.8770913 & 3.7399345 & 1.6751402 \\
H & -0.2967372 & 1.8415113 & 2.5029275 \\
H & 1.2547380 & 2.0400824 & 3.3910045 \\
H & -0.9200228 & -0.3228909 & 1.9916986 \\
H & 0.3531452 & -1.7003321 & 1.8153911 \\
H & 3.1663910 & 0.3213278 & 2.9362221 \\
H & 3.0477292 & 0.1617397 & 1.1548879 \\
H & 2.6943626 & -1.2438234 & 2.1821984 \\
H & 3.1284741 & 2.1096156 & 1.1461889 \\
H & 0.0956552 & -0.0591109 & -0.7919562 \\
H & 1.5082974 & -1.7232937 & -1.9991073 \\
H & 3.8412442 & -1.1155638 & -2.7189712 \\
H & 4.7218232 & 1.1889771 & -2.2489291 \\
H & 3.2835298 & 2.8565485 & -1.0515488
\end{tabular}




\begin{tabular}{|c|c|c|c|}
\hline $\mathrm{H}$ & -2.9310336 & 3.5478036 & -1.3408785 \\
\hline $\mathrm{H}$ & -3.9232821 & 3.3301583 & -3.6004569 \\
\hline & -2.4774506 & 2.6380735 & -5.5522537 \\
\hline & -0.0240103 & 2.2352807 & -5.1770496 \\
\hline & 0.9539528 & 2.4457129 & -2.9152356 \\
\hline $\mathrm{Pd}$ & -1.8530482 & 2.1543931 & 0.7767512 \\
\hline & -3.2672119 & 0.5336555 & 0.1076065 \\
\hline & -4.0917325 & -0.5170669 & 1.3800778 \\
\hline & -4.6884045 & 1.1511756 & -0.8999535 \\
\hline & -2.4709545 & -0.6991073 & -1.0011198 \\
\hline & -5.0393193 & -1.5045963 & 1.0383016 \\
\hline & -5.6365542 & -2.2826445 & 2.0402230 \\
\hline & -5.2941392 & -2.0802829 & 3.3898770 \\
\hline & -4.3557859 & -1.0951405 & 3.7360441 \\
\hline & -3.7586721 & -0.3134247 & 2.7331207 \\
\hline & -5.3108642 & -1.6631547 & -0.0162366 \\
\hline & -6.3761310 & -3.0512366 & 1.7684656 \\
\hline & -5.7651306 & -2.69 & 9927 \\
\hline & -4.0880736 & -0.9309202 & 4.79 \\
\hline & -3.0 & 7347 & 2.9880271 \\
\hline & -5.2 & 0.4432995 & -1.9886989 \\
\hline $\mathrm{C}$ & -6.2948028 & 0.9945867 & -2.7283886 \\
\hline $\mathrm{C}$ & -6.8210688 & 2.2502900 & -2.3829585 \\
\hline $\mathrm{C}$ & -6.2873913 & 2.9546413 & -1.2892809 \\
\hline $\mathrm{C}$ & -5.223 & 2.4100117 & -0.5558837 \\
\hline $\mathrm{H}$ & -4.8196114 & -0.5332648 & -2.2764093 \\
\hline $\mathrm{H}$ & -6.7080854 & 0.4395960 & -3.5844693 \\
\hline $\mathrm{H}$ & -7.6 & 2.68 & -2.96 \\
\hline $\mathrm{H}$ & -6.6 & 2885 & -1.0147244 \\
\hline $\mathrm{H}$ & -4.7 & 915 & 0028 \\
\hline & -2.0 & -1.9 & -0 . \\
\hline C & -1.3 & $-2 . \varepsilon$ & -1 . \\
\hline C & -1.0 & -2.4 & -2 . \\
\hline C & -1.4 & -1.2 & \\
\hline $\mathrm{C}$ & -2.1 & -0. & -2 . \\
\hline $\mathrm{H}$ & -2.3 & -2. & \\
\hline $\mathrm{H}$ & -1.0 & -3.8 & -0.9 \\
\hline $\mathrm{H}$ & -0.4921026 & -3.164 & -3.324792 \\
\hline $\mathrm{H}$ & -1.1848667 & -0.9003177 & -4.177575 \\
\hline $\mathrm{H}$ & -2.4268141 & 0.6639870 & -2.7054372 \\
\hline \multicolumn{4}{|l|}{ R3 } \\
\hline \multicolumn{4}{|l|}{75} \\
\hline \\
\hline 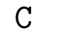 & 0.5898813 & 5.7216184 & -1.385 \\
\hline & 0.0544613 & 4.6394315 & -0.656723 \\
\hline
\end{tabular}




\begin{tabular}{|c|c|c|c|}
\hline & -1.2147736 & 4.1419668 & -1.0352551 \\
\hline & -1.9113773 & 4.6883472 & -2.1171463 \\
\hline & -1.3663833 & 5.7611260 & -2.8405173 \\
\hline & -0.1138417 & 6.2744963 & -2.4690746 \\
\hline & 0.7966134 & 3.9611885 & 0.4377392 \\
\hline & 1.1251077 & 2.6204475 & 0.4557197 \\
\hline & 0.6615439 & 1.7420949 & -0.6558721 \\
\hline & -0.0669032 & 0.5520293 & -0.4100893 \\
\hline & -0.4832744 & -0.2747826 & -1.4668997 \\
\hline & -0.1825076 & 0.0681791 & -2.7935581 \\
\hline & 0.5528416 & & -3.0529400 \\
\hline & 0.9745415 & 2.059 & -2.0 \\
\hline & 2.2325058 & 5.3153856 & 21088 \\
\hline & 0.7080 & 2.2 & 3.7 \\
\hline & -0.5200 & 1.65 & 4.4 \\
\hline & 1.36 & & 4.1 \\
\hline & 1.31 & & 2.6 \\
\hline & 2.03 & & 1.4 \\
\hline & 2.91 & & 0.8 \\
\hline & 2.698 & 2.7 & 1.8 \\
\hline & 2.0769244 & 0.6 & 3.1 \\
\hline & $0.539^{\circ}$ & $0.5 \mathrm{~s}$ & 2.3 \\
\hline & 2.3642187 & 3.6005499 & 3.8292257 \\
\hline & 0.9827756 & 3.8729793 & 5.06 \\
\hline & -1.3485417 & 1.4777783 & 3.7122609 \\
\hline & -0.8839983 & 2.3369058 & 5.2221415 \\
\hline & -0.2806814 & 0.6802308 & 4.89 \\
\hline & 2.3597305 & 0.4385862 & 87532 \\
\hline & -0.3382339 & 0.2814482 & 0.6200379 \\
\hline & -1.0561774 & -1.1889929 & -1.2478832 \\
\hline & -0.5138743 & -0.5 & -3.6223968 \\
\hline & 0.8063095 & 1.512 & -4.0884923 \\
\hline & 1.5569956 & 2.9675088 & -2.2082382 \\
\hline & -1.6562129 & 3.3097460 & -0.4743268 \\
\hline & -2.8983479 & 4.2830012 & -2.3831284 \\
\hline & -1.9212117 & 6.2014619 & -3.6822975 \\
\hline & 0.3247035 & 7.1173764 & -3.0247792 \\
\hline & 1.5714013 & 6.1243230 & -1.1061779 \\
\hline & 0.0067040 & 4.3356766 & 2.4170860 \\
\hline & -2.2093178 & 4.7989331 & 2.2928305 \\
\hline & -2.8536286 & 5.8969065 & 0.9628846 \\
\hline & -2.0126009 & 6.9538403 & 0.5573732 \\
\hline & -1.0105581 & 7.0425534 & 1.0069367 \\
\hline & -2.4371399 & 7.8514881 & -0.4309810 \\
\hline & -1.7707599 & 8.6664790 & -0.7499052 \\
\hline & -3.6982335 & 7.6932482 & -1.031356 \\
\hline
\end{tabular}




$\begin{array}{lrr}-4.0253439 & 8.3905368 & -1.8175684 \\ -4.5333614 & 6.6347443 & -0.6384135 \\ -5.5175905 & 6.5025324 & -1.1135011 \\ -4.1150836 & 5.7379830 & 0.3577365 \\ -4.7677398 & 4.9048108 & 0.6568617 \\ -2.8670291 & 5.6157797 & 3.8122231 \\ -2.5720317 & 5.0162064 & 5.0568274 \\ -1.9771734 & 4.0906775 & 5.0792901 \\ -3.0308624 & 5.5895486 & 6.2496441 \\ -2.7991369 & 5.1085982 & 7.2122617 \\ -3.7769105 & 6.7819274 & 6.2169651 \\ -4.1304449 & 7.2382418 & 7.1539393 \\ -4.0639686 & 7.3889563 & 4.9842968 \\ -4.6456283 & 8.3229936 & 4.9515973 \\ -3.6163208 & 6.8091277 & 3.7847781 \\ -3.8504616 & 7.2858956 & 2.8220257 \\ -3.2746990 & 3.2943802 & 2.1820646 \\ -4.5884219 & 3.2467542 & 2.6924069 \\ -5.0104068 & 4.1299789 & 3.1958477 \\ -5.3499199 & 2.0726892 & 2.5718298 \\ -6.3730705 & 2.0417138 & 2.9767494 \\ -4.8097638 & 0.9398437 & 1.9387696 \\ -5.4106565 & 0.0226075 & 1.8443372 \\ -3.4968993 & 0.9775949 & 1.4384739 \\ -3.0621371 & 0.0931994 & 0.9484492 \\ -2.7305591 & 2.1448282 & 1.5715284 \\ -1.6864577 & 2.1716314 & 1.2226991\end{array}$

R4

75

SCF Energy $=-4548.298302432$

$\begin{array}{lrrr}\text { C } & 2.9293950 & 1.3541867 & -1.5684726 \\ \text { C } & 1.8146257 & 1.4417901 & -0.7027362 \\ \text { C } & 1.2449034 & 0.2484512 & -0.2050207 \\ \text { C } & 1.7603652 & -1.0015353 & -0.5785467 \\ \text { C } & 2.8614440 & -1.0780155 & -1.4475704 \\ \text { C } & 3.4449240 & 0.1017336 & -1.9388052 \\ \text { C } & 1.2774027 & 2.7712214 & -0.2929429 \\ \text { Pd } & -1.2407137 & 3.6365003 & 0.9827891 \\ \text { P } & -3.5303151 & 3.3912175 & 0.5962788 \\ \text { C } & 0.0397021 & 3.1436157 & -0.7200193 \\ \text { Br } & -0.3628336 & 5.3245685 & -0.8082220 \\ \text { C } & -0.7355412 & 2.4739001 & -1.7906154 \\ \text { C } & -0.6258239 & 2.9017187 & -3.1330495 \\ \text { C } & -1.3645690 & 2.2677996 & -4.1417373 \\ \text { C } & -2.2198861 & 1.1960448 & -3.8269095\end{array}$




\begin{tabular}{|c|c|c|c|}
\hline & -2.3202851 & 0.7515162 & -2.4990377 \\
\hline & -1.5839783 & 1.3871575 & -1.4895887 \\
\hline & 2.0987366 & 3.5512608 & 0.7276434 \\
\hline & 3.4963493 & 3.4014686 & 0.5301364 \\
\hline & 1.6721652 & 3.1306842 & 2.1592902 \\
\hline & 0.3362755 & 3.6921572 & 2.6033930 \\
\hline & -0.7360807 & 2.8433457 & 2.9419811 \\
\hline & 0.3693193 & 5.1317860 & 3.0875131 \\
\hline & 1.8894467 & 4.6318494 & 0.5891258 \\
\hline & 2.4729915 & 3.4887923 & 2.8435970 \\
\hline & 1.6676969 & 2.0206521 & 2.2139143 \\
\hline & -0.5993181 & 1.7495255 & 2.9038482 \\
\hline & -1.5469025 & 3.1979896 & 3.6012284 \\
\hline & 1.0020354 & 5.220 & 3.9998637 \\
\hline & -0.6430033 & 5.504 & 3.3372126 \\
\hline & 0.8014470 & 5.81 & 2.3 \\
\hline & 3.692 & 2.44 & $0.5 \varepsilon$ \\
\hline & 0.3874132 & 0.316 & 0.4 \\
\hline & 1.2991196 & -1.921 & -0.1 \\
\hline & 3.2650392 & -2.0583368 & -1.7 \\
\hline & 4.3052599 & 0.0477657 & -2.62 \\
\hline & 3.3765885 & 2.2805844 & -1.9558091 \\
\hline & -1.6774600 & 1.0573300 & -0.4480414 \\
\hline & -2.9871661 & -0.0821169 & -2.2358098 \\
\hline & -2.8089037 & 0.7095264 & -4.6190619 \\
\hline & -1.2757535 & 2.6153064 & -5.1822632 \\
\hline & 0.0297416 & 3.7522624 & -3.3684373 \\
\hline & -4.4002967 & 4.2613302 & -0.7728029 \\
\hline & -4.3748202 & 4.0612199 & 2.0935449 \\
\hline & -4.2016938 & 1.6801278 & 0.4797099 \\
\hline & -5.1531695 & 1.2917148 & -0.4827985 \\
\hline & -5.5639942 & -0.0496312 & -0.5676860 \\
\hline & -5.0342897 & -1.0101837 & 0.3087009 \\
\hline & -4.0887871 & -0.6252680 & 1.2775374 \\
\hline & -3.6694245 & 0.7095534 & 1.3575930 \\
\hline & -5.5641612 & 2.0399727 & -1.1757205 \\
\hline & -6.3031207 & -0.3448287 & -1.3281575 \\
\hline & -5.3552646 & -2.0604978 & 0.2375897 \\
\hline & -3.6688348 & -1.3734570 & 1.9670730 \\
\hline & -2.9081873 & 1.0127948 & 2.0935028 \\
\hline & -5.6431256 & 4.9049600 & -0.5982590 \\
\hline & -6.2704889 & 5.5308702 & -1.6872788 \\
\hline & -5.6660438 & 5.5150694 & -2.9560701 \\
\hline & -4.4263629 & 4.8772519 & -3.1318394 \\
\hline & -3.7905607 & 4.2604867 & -2.0447163 \\
\hline & -6.1203775 & 4.9148413 & 0.393013 \\
\hline
\end{tabular}




$\begin{array}{lllr}\mathrm{H} & -7.2392070 & 6.0336485 & -1.5434304 \\ \mathrm{H} & -6.1604842 & 6.0068456 & -3.8077876 \\ \mathrm{H} & -3.9409544 & 4.8652068 & -4.1193746 \\ \mathrm{H} & -2.8156512 & 3.7759602 & -2.1819147 \\ \mathrm{C} & -5.4117464 & 3.3905461 & 2.7710758 \\ \mathrm{C} & -5.9795756 & 3.9544998 & 3.9267622 \\ \mathrm{C} & -5.5257423 & 5.1934744 & 4.4067005 \\ \mathrm{C} & -4.4956720 & 5.8710141 & 3.7291433 \\ \mathrm{C} & -3.9175116 & 5.3046650 & 2.5847318 \\ \mathrm{H} & -5.7736610 & 2.4223699 & 2.3946861 \\ \mathrm{H} & -6.7853398 & 3.4206479 & 4.4536499 \\ \mathrm{H} & -5.9719412 & 5.6317477 & 5.3122847 \\ \mathrm{H} & -4.1342285 & 6.8418007 & 4.1010728 \\ \mathrm{H} & -3.0957363 & 5.8197748 & 2.0605189\end{array}$

S1

75

SCF Energy $=-4548.290184978$

$$
-0.4540655
$$

2.8039101

C $\quad-0.7702249$

3.0166202

$-2.0323300$

3.5864127

$-2.9686133$

3.8630088

$-2.6648560$

$-1.3970589$

3.5856988

3.0703632

0.1731775

2.6730505

2.0874097

1.1459010

1.7346626

0.0415430

$-2.4599764$

$-1.0929274$

$-0.7956342$

$-1.7968645$

$-3.1409170$

$-3.4621059$

$-0.0094466$

$-0.0630211$

$-1.1727352$

$-1.4394878$

C $\quad 0.8932432$

$-0.8660217$

$-2.4574203$

2.3831657

$-0.6901513$

$-3.2216382$

2.3831657
3.2335734

0.3971950

$-2.9532087$

C $\quad 2.9190945$

1.3060491

$-1.9329043$

4.5222324

1.4998380

Br 0.1004671

1.4379353

3.0941849

4.5420278

2.5359037

0.2725068

2.4030668

2.3935583

2. 3299398

0.8695296

C $\quad 2.6248568$

3.5074065

0.4209770

3. 3046069

1.5003934

0.6475641

3. 3250961

3.4280533

2.6736742

2. 1638049

2.2640978

2.8309388

3.4771269

$-0.0644593$

1.5331570

0.4704871

$-0.4451384$

3.1430009

0.8144294

2.8012848

4.6398025

H 2.1744536

1.7607765

5.1340179 


\begin{tabular}{|c|c|c|c|}
\hline & 0.5281252 & 1.0614376 & 5.0070997 \\
\hline $\mathrm{H}$ & 2.6507443 & 4.2319174 & 0.5335493 \\
\hline & -0.0168801 & -0.0954059 & -0.8391937 \\
\hline & 0.5499023 & -1.7187861 & -2.6466940 \\
\hline & 2.6337769 & -1.4037231 & -4.0213083 \\
\hline & 4.1495618 & 0.5412595 & -3.5467633 \\
\hline & 3.5681004 & 2.1692979 & -1.7225074 \\
\hline & -2.2675229 & 3.8179881 & 0.2478513 \\
\hline & -3.9446350 & 4.2860321 & -1.5153661 \\
\hline & -3.4012511 & 3.7872150 & -3.9334726 \\
\hline & -1.1294143 & 2.8754141 & -4.5117817 \\
\hline & 0.5288620 & 2.410 & -2.7407969 \\
\hline & -0.6482890 & 1.9793074 & 1.7725777 \\
\hline & -2.6565934 & 0.962 & 1.3472961 \\
\hline & -4.1765515 & 1.962 & 1.027079 \\
\hline & -4.2684576 & 3.205 & $1.686740 s$ \\
\hline & -3.4330968 & 3.533 & 2.327849 \\
\hline & -5.3908764 & 4.0256239 & $1.502958 s$ \\
\hline & -5.4488202 & 4.9966333 & 2.017565 \\
\hline & -6.4299960 & 3.6138744 & 0.6498758 \\
\hline & -7.3054078 & 4.2622987 & 0.4934711 \\
\hline & -6.3460465 & 2.3739488 & -0.0051589 \\
\hline & -7.1575104 & 2.0470802 & -0.6732987 \\
\hline & -5.2270040 & 1.5471998 & 0.1852783 \\
\hline & -5.1649398 & 0.5825965 & -0.3393081 \\
\hline & -2.5726229 & -0.2107190 & -0.0640730 \\
\hline & -2.1806449 & -1.5494023 & 0.1580200 \\
\hline & -2.0322245 & -1.9119854 & 1.1859917 \\
\hline & -1.9807260 & -2.4186626 & -0.9250843 \\
\hline & -1.6815061 & -3.4610372 & -0.7372599 \\
\hline & -2.1617973 & -1.9623203 & -2.2420940 \\
\hline & -2.0096744 & -2.6466333 & -3.0904793 \\
\hline & -2.5279024 & -0.6254795 & -2.4694110 \\
\hline & -2.6504762 & -0.2485786 & -3.4956260 \\
\hline & -2.7299844 & 0.2481141 & -1.3911858 \\
\hline & -3.0048791 & 1.2931431 & -1.5862781 \\
\hline & -3.2342900 & -0.1027986 & 2.7441344 \\
\hline C & -2.5517235 & -0.0260369 & 3.9730495 \\
\hline & -1.6866205 & 0.6523974 & 4.0582722 \\
\hline$C$ & -2.9705033 & -0.7974792 & 5.0697800 \\
\hline & -2.4312009 & -0.7286678 & 6.0266918 \\
\hline C & -4.0750860 & -1.6542245 & 4.9409895 \\
\hline & -4.4043051 & -2.2623210 & 5.7972652 \\
\hline & -4.7637136 & -1.7329920 & 3.7166275 \\
\hline & -5.6325370 & -2.4011708 & 3.6151865 \\
\hline & -4.3492552 & -0.9582364 & 2.623931 \\
\hline
\end{tabular}




\begin{tabular}{lrrr} 
H & -4.8931186 & -1.0199379 & 1.6692923 \\
& & & \\
S2 & & & \\
75 & \multicolumn{4}{r}{} \\
SCF & Energy $=-4548.290721847$ & \\
C & -0.0538966 & 4.6729997 & -1.4737749 \\
C & -0.5308518 & 3.5335728 & -0.7806584 \\
C & -1.7560904 & 2.9548155 & -1.1823698 \\
C & -2.4802468 & 3.4878515 & -2.2546784 \\
C & -2.0040217 & 4.6253240 & -2.9251243 \\
C & -0.7886464 & 5.2158853 & -2.5321542 \\
C & 0.2959656 & 2.9556443 & 0.2849766 \\
C & 1.5340821 & 2.3811448 & 0.1244947 \\
C & 1.8163928 & 1.7861313 & -1.2146597 \\
C & 0.9090222 & 0.8905008 & -1.8228179 \\
C & 1.1807963 & 0.3419268 & -3.0842637 \\
C & 2.3674121 & 0.6755902 & -3.7576581 \\
C & 3.2824747 & 1.5574572 & -3.1563151 \\
C & 3.0166773 & 2.1065461 & -1.8941624 \\
Br & 0.6799931 & 4.9927339 & 1.9832249 \\
C & 1.4944093 & 1.0479035 & 2.8426524 \\
C & 1.6339189 & -0.2760801 & 2.1260243 \\
C & 0.5690807 & 1.2277823 & 3.8479248 \\
C & 2.5332068 & 2.1112579 & 2.5656510 \\
C & 2.7608193 & 2.4142230 & 1.0664508 \\
O & 3.4437429 & 3.6448749 & 0.8869916 \\
H & 3.4469596 & 1.6403852 & 0.6663797 \\
H & 2.2454931 & 3.0507272 & 3.0747768 \\
H & 3.5145826 & 1.7987101 & 2.9893252 \\
H & 0.5915560 & 2.1252282 & 4.4869207 \\
H & -0.1038000 & 0.4071509 & 4.1460612 \\
H & 2.5766007 & -0.7741963 & 2.4444478 \\
H & 1.6915945 & -0.1545266 & 1.0262638 \\
H & 0.7910941 & -0.9563512 & 2.3545902 \\
H & 2.7981992 & 4.3362413 & 1.1854679 \\
H & -0.0097808 & 0.6176278 & -1.2831071 \\
H & 0.4632276 & -0.3564425 & -3.5414567 \\
H & 2.5815841 & 0.2462724 & -4.7481448 \\
H & 4.2126101 & 1.8274709 & -3.6797769 \\
H & 3.7148301 & 2.8177302 & -1.4280190 \\
H & -2.1260306 & 2.0704847 & -0.6453095 \\
H & -3.4309427 & 3.0244904 & -2.5551379 \\
H & -2.5830007 & 5.0564531 & -3.7557224 \\
H & -0.4131616 & 6.1067337 & -3.0579119 \\
H & 0.8928771 & 5.1254278 & -1.1468452 \\
Pd & -0.5191576 & 2.2957172 & 1.9793169
\end{tabular}




$\begin{array}{lrrr}\text { P } & -2.7249887 & 2.9491768 & 2.1574202 \\ \text { C } & -3.4872372 & 4.1680544 & 1.0133147 \\ \text { C } & -2.7461885 & 5.3333985 & 0.7144403 \\ \text { H } & -1.7537221 & 5.4836514 & 1.1704677 \\ \text { C } & -3.2564544 & 6.2747629 & -0.1886806 \\ \text { H } & -2.6660782 & 7.1728608 & -0.4229605 \\ \text { C } & -4.4999544 & 6.0615959 & -0.8082870 \\ \text { H } & -4.8926076 & 6.7975769 & -1.5263075 \\ \text { C } & -5.2353549 & 4.9018323 & -0.5166240 \\ \text { H } & -6.2076558 & 4.7270158 & -1.0023022 \\ \text { C } & -4.7339286 & 3.9560643 & 0.3921762 \\ \text { H } & -5.3103694 & 3.0451171 & 0.6074061 \\ \text { C } & -3.9065816 & 1.5354841 & 2.1431783 \\ \text { C } & -5.1533437 & 1.5938420 & 2.8013393 \\ \text { H } & -5.4310766 & 2.4984538 & 3.3634154 \\ \text { C } & -6.0299088 & 0.4995379 & 2.7446385 \\ \text { H } & -6.9995924 & 0.5484814 & 3.2632282 \\ \text { C } & -5.6711666 & -0.6574256 & 2.0304382 \\ \text { H } & -6.3603074 & -1.5146792 & 1.9901959 \\ \text { C } & -4.4279673 & -0.7231123 & 1.3792603 \\ \text { H } & -4.1384377 & -1.6318784 & 0.8301204 \\ \text { C } & -3.5456535 & 0.3669506 & 1.4428366 \\ \text { H } & -2.5503994 & 0.3191420 & 0.9696939 \\ \text { C } & -3.0181836 & 3.6819218 & 3.8233118 \\ \text { C } & -2.6892960 & 2.8906620 & 4.9466504 \\ \text { H } & -2.3052343 & 1.8693006 & 4.7976357 \\ \text { C } & -2.8506394 & 3.3976689 & 6.2421928 \\ \text { H } & -2.5961049 & 2.7706390 & 7.1102475 \\ \text { C } & -3.3271217 & 4.7078080 & 6.4320257 \\ \text { H } & -3.4440278 & 5.1106257 & 7.4494360 \\ \text { C } & -3.6494009 & 5.4992449 & 5.3188980 \\ \text { H } & -4.0227299 & 6.5249532 & 5.4603351 \\ \text { C } & -3.5012017 & 4.9898200 & 4.0174815 \\ \text { H } & -3.7603520 & 5.6133480 & 3.1500410 \\ & & & \\ \text { S3 } & & & \\ 75 & & & \\ \text { SCF } & \text { Energy }=-4548.294596929 & \\ \text { C } & 0.1379066 & 5.2386099 & -1.4819728 \\ \text { C } & -0.5950684 & 4.3489817 & -0.6602248 \\ \text { C } & -1.8788951 & 3.9305676 & -1.0866065 \\ \text { C } & -2.4085258 & 4.3685222 & -2.3056860 \\ \text { C } & -1.6706009 & 5.2497317 & -3.1117875 \\ \text { C } & -0.3966751 & 5.6810179 & -2.6963599 \\ \text { C } & -0.0125574 & 3.8131480 & 0.5739167 \\ & 1.0547834 & 2.9459484 & 0.7103415\end{array}$




$\begin{array}{lrrr}\text { C } & 1.5783682 & 2.2177917 & -0.4792561 \\ \mathrm{C} & 0.7122386 & 1.6633417 & -1.4535422 \\ \mathrm{C} & 1.2164239 & 0.9871110 & -2.5716899 \\ \mathrm{C} & 2.6025339 & 0.8359404 & -2.7456741 \\ \mathrm{C} & 3.4762263 & 1.3733157 & -1.7867850 \\ \mathrm{C} & 2.9731639 & 2.0533351 & -0.6678747 \\ \mathrm{Br} & 0.7047013 & 6.0891020 & 1.7423273 \\ \mathrm{C} & 0.1328127 & 0.7678628 & 2.5375215 \\ \mathrm{C} & -0.3583576 & -0.2062708 & 1.4974533 \\ \mathrm{C} & -0.6706184 & 1.1925676 & 3.5653122 \\ \mathrm{C} & 1.6012367 & 1.1763242 & 2.4481294 \\ \mathrm{C} & 1.7821748 & 2.6586472 & 2.0471461 \\ \mathrm{O} & 1.3358944 & 3.4322394 & 3.1337440 \\ \mathrm{H} & 2.8738608 & 2.8332912 & 1.8773011 \\ \mathrm{H} & 2.1032219 & 1.0371120 & 3.4278427 \\ \mathrm{H} & 2.1230740 & 0.5372483 & 1.7094895 \\ \mathrm{H} & -0.2642617 & 1.7878067 & 4.3957172 \\ \mathrm{H} & -1.7025493 & 0.8138329 & 3.6625608 \\ \mathrm{H} & -0.1168271 & 0.1422038 & 0.4730041 \\ \mathrm{H} & -1.4489442 & -0.3824056 & 1.5723639 \\ \mathrm{H} & 0.1628592 & -1.1812995 & 1.6226975 \\ \mathrm{H} & 1.2663270 & 4.3863081 & 2.8313945 \\ \mathrm{H} & -0.3732879 & 1.7658665 & -1.3236732 \\ \mathrm{H} & 0.5172089 & 0.5684535 & -3.3115151 \\ \mathrm{H} & 2.9990904 & 0.3021587 & -3.6223841 \\ \mathrm{H} & 4.5650412 & 1.2711317 & -1.9129783 \\ \mathrm{H} & 3.6773089 & 2.4870848 & 0.0562756 \\ \mathrm{H} & -2.4447617 & 3.2459235 & -0.4373799 \\ \mathrm{H} & -3.4092986 & 4.0360211 & -2.6168509 \\ \mathrm{H} & -2.0908908 & 5.6095690 & -4.0632681 \\ \mathrm{H} & 0.1822274 & 6.3734371 & -3.3262060 \\ \mathrm{H} & 1.1195817 & 5.5864242 & -1.1318168 \\ \mathrm{Pd} & -1.1512315 & 3.0566343 & 2.0493853 \\ \mathrm{P} & -2.8207686 & 4.5138957 & 2.4232773 \\ \mathrm{C} & -3.4892938 & 5.5178952 & 1.0426226 \\ \mathrm{C} & -2.6726578 & 6.5426336 & 0.5119814 \\ \mathrm{H} & -1.6891934 & 6.7476910 & 0.9656567 \\ \mathrm{C} & -3.1023075 & 7.2693178 & -0.6052419 \\ \mathrm{H} & -2.4545177 & 8.0568552 & -1.0178010 \\ \mathrm{C} & -4.3388152 & 6.9805133 & -1.2074085 \\ \mathrm{H} & -4.6690948 & 7.5484057 & -2.0904633 \\ \mathrm{C} & -5.1515500 & 5.9628673 & -0.6816930 \\ \mathrm{C} & -6.1239556 & 5.7367589 & -1.1455402 \\ & -4.7310504 & 5.2309601 & 0.4403969 \\ \mathrm{H} & -2.3720347 & 4.4362301 & 0.8497472 \\ & & 5.7478508 & 3.7206687\end{array}$




$\begin{array}{llll}\text { C } & -1.3817141 & 5.4690427 & 4.6118431 \\ \text { H } & -0.7881594 & 4.5528286 & 4.4687706 \\ \text { C } & -1.0707525 & 6.3754976 & 5.6351206 \\ \text { H } & -0.2378401 & 6.1591902 & 6.3206316 \\ \text { C } & -1.8030694 & 7.5674997 & 5.7657295 \\ \text { H } & -1.5503073 & 8.2843558 & 6.5618341 \\ \text { C } & -2.8477136 & 7.8541435 & 4.8703542 \\ \text { H } & -3.4139268 & 8.7933040 & 4.9642398 \\ \text { C } & -3.1657704 & 6.9479137 & 3.8467905 \\ \text { H } & -3.9717165 & 7.1783072 & 3.1337719 \\ \text { C } & -4.2884981 & 3.5958835 & 3.0553141 \\ \text { C } & -5.1042801 & 4.0729650 & 4.1005150 \\ \text { H } & -4.8768904 & 5.0390046 & 4.5748305 \\ \text { C } & -6.1987987 & 3.3115211 & 4.5413527 \\ \text { H } & -6.8275543 & 3.6876293 & 5.3627970 \\ \text { C } & -6.4917937 & 2.0757723 & 3.9406967 \\ \text { H } & -7.3502046 & 1.4824045 & 4.2903771 \\ \text { C } & -5.6800795 & 1.5938616 & 2.8989344 \\ \text { H } & -5.9003906 & 0.6229284 & 2.4296894 \\ \text { C } & -4.5778481 & 2.3451930 & 2.4657644 \\ \text { H } & -3.9173524 & 1.9643770 & 1.6687005\end{array}$

S4

75

SCF Energy $=-4548.294788801$

C $\quad-0.8373856 \quad 3.9707246$

C $\quad-1.0481673 \quad 3.0520576$

$-2.1511630$

$\begin{array}{llll}-1.9195216 & 1.9580862 & -1.3110249\end{array}$

$\begin{array}{llll}\text { C } & -2.5506885 & 1.7746926 & -2.5467783\end{array}$

$\begin{array}{llll}\text { C } & -2.3339773 & 2.6936421 & -3.5855340\end{array}$

$\begin{array}{llll}\text { C } & -1.4737669 & 3.7893180 & -3.3838322\end{array}$

$\begin{array}{llll}\text { C } & -0.3378019 & 3.1977911 & 0.1820492\end{array}$

$\begin{array}{llll}\text { C } & 1.0075390 & 3.0215259 & 0.4368051\end{array}$

$\begin{array}{llll}\text { C } & 1.8628717 & 2.3586458 & -0.5881143\end{array}$

$\begin{array}{llll}\text { C } & 1.4616898 & 1.1532337 & -1.2111250\end{array}$

$\begin{array}{llll}\text { C } & 2.2691362 & 0.5344413 & -2.1747773\end{array}$

$\begin{array}{llll}\text { C } & 3.5048972 & 1.0991942 & -2.5324509\end{array}$

$\begin{array}{llll}\text { C } & 3.9213009 & 2.2913747 & -1.9175098\end{array}$

$\begin{array}{llll}\text { C } & 3.1116627 & 2.9146561 & -0.9562896\end{array}$

$\begin{array}{llll}\mathrm{Br} & -0.9654707 & 5.6196974 & 0.7912710\end{array}$

$\begin{array}{llll}\text { C } & 1.1493865 & 1.3549757 & 3.1255082\end{array}$

$\begin{array}{llll}\text { C } & 0.8413541 & 1.6617727 & 4.5703198\end{array}$

$\begin{array}{llll}\text { C } & 0.4902355 & 0.3704365 & 2.4318629\end{array}$

$\begin{array}{llll}\text { C } & 2.2628870 & 2.1621559 & 2.4817247\end{array}$

$\begin{array}{llll}\text { C } & 1.7281722 & 3.4227010 & 1.7371055\end{array}$

$\begin{array}{llll}0 & 0.9430025 & 4.1349363 & 2.6622723\end{array}$ 


\begin{tabular}{|c|c|c|c|}
\hline & 2.6235862 & 4.0235580 & 1.4363925 \\
\hline $\mathrm{H}$ & 2.9675237 & 2.5105390 & 3.2636303 \\
\hline & 2.8331607 & 1.5449030 & 1.7604590 \\
\hline & 0.8244242 & 0.0633237 & 1.4284861 \\
\hline & -0.2530499 & -0.2703035 & 2.9355787 \\
\hline & 0.6930212 & 2.7516226 & 4.7024152 \\
\hline & 1.7043779 & 1.3739947 & 5.2104761 \\
\hline & -0.0558023 & 1.1223407 & 4.9314885 \\
\hline & 0.4030673 & 4.8167766 & 2.1669742 \\
\hline & 0.4993865 & 0.7036299 & -0.9292071 \\
\hline & 1.9316293 & -0.4011345 & -2.6463617 \\
\hline & 4.1409662 & 0.6125082 & -3.287 \\
\hline & 4.8831047 & 2.7490808 & -2.1957258 \\
\hline & 3.4404878 & 3.8618270 & -0.50 \\
\hline & -2.0857002 & 1.2543274 & -0.4814595 \\
\hline & -3.2295179 & 0.9217396 & -2.690 \\
\hline & -2.8410734 & 2.5620866 & -4.5535636 \\
\hline & -1.3022069 & 4.5112314 & -4.1966990 \\
\hline & -0.1833627 & 4.8354564 & -1.9724298 \\
\hline & -0.9796641 & 2.2262153 & 1.8376260 \\
\hline & -3.1668074 & 2.6954613 & 2.1243612 \\
\hline & -3.4819442 & 4.2091562 & 3.1028042 \\
\hline & -2.4463012 & 4.6849434 & 3.9315463 \\
\hline & -1.4709186 & 4.1733667 & 3.9176883 \\
\hline & -2.6490956 & 5.8250307 & 4.7215278 \\
\hline & -1.8336108 & 6.1998258 & 5.3579210 \\
\hline & -3.8803348 & 6.5008909 & 4.6779848 \\
\hline & -4.0346794 & 7.4032051 & 5.2891615 \\
\hline & -4.9101911 & 6.0360034 & 3.8422567 \\
\hline & -5.8706909 & 6.5717449 & 3.7990357 \\
\hline & -4.7141706 & 4.8915061 & 3.0534826 \\
\hline & -5.5133255 & 4.5332740 & 2.3871327 \\
\hline & -3.9713047 & 1.3399657 & 3.0794297 \\
\hline & -4.9089437 & 1.5721899 & 4.1053697 \\
\hline & -5.1958225 & 2.6027602 & 4.3610047 \\
\hline & -5.4684564 & 0.4903230 & 4.8045039 \\
\hline & -6.1953814 & 0.6801099 & 5.6089600 \\
\hline & -5.1050812 & -0.8278298 & 4.4818440 \\
\hline & -5.5462991 & -1.6721910 & 5.0328225 \\
\hline & -4.1693986 & -1.0655705 & 3.4597955 \\
\hline & -3.8759567 & -2.0957263 & 3.2066453 \\
\hline & -3.5973364 & 0.0131825 & 2.7697996 \\
\hline & -2.8431960 & -0.1610250 & 1.9843897 \\
\hline & -4.2258112 & 2.8760133 & 0.6372253 \\
\hline & -5.1096561 & 1.8596417 & 0.2209237 \\
\hline & -5.2556395 & 0.9656820 & 0.844823 \\
\hline
\end{tabular}




$\begin{array}{llll}\text { C } & -5.8068799 & 1.9889391 & -0.9910968 \\ \text { H } & -6.4973383 & 1.1924413 & -1.3084739 \\ \text { C } & -5.6289708 & 3.1288894 & -1.7920144 \\ \text { H } & -6.1724098 & 3.2238031 & -2.7443296 \\ \text { C } & -4.7528014 & 4.1452172 & -1.3752445 \\ \text { H } & -4.5978314 & 5.0361233 & -2.0016006 \\ \text { C } & -4.0490059 & 4.0224815 & -0.1706560 \\ \text { H } & -3.3370027 & 4.8040036 & 0.1386735\end{array}$

\section{Optimized Geometries(MXn)(TS_ab)}

\begin{tabular}{lrrr} 
R1 & \multicolumn{3}{l}{} \\
75 & \multicolumn{4}{l}{} \\
SCF & Energy $=-4548.276110082$ & \\
C & 0.9072926 & 3.2294051 & -3.0262411 \\
C & 0.1280717 & 3.5860169 & -1.8985236 \\
C & -1.2764796 & 3.6254894 & -2.0304096 \\
C & -1.8868206 & 3.3046327 & -3.2526328 \\
C & -1.1063916 & 2.9427854 & -4.3623156 \\
C & 0.2942459 & 2.9082193 & -4.2412385 \\
C & 0.8199189 & 3.8757248 & -0.6177513 \\
C & 1.7354147 & 3.0822528 & 0.0343219 \\
C & 2.2104897 & 1.8531176 & -0.6635692 \\
C & 1.3267229 & 0.8107787 & -1.0243108 \\
C & 1.7803906 & -0.2940105 & -1.7555907 \\
C & 3.1263356 & -0.3777671 & -2.1513371 \\
C & 4.0115862 & 0.6583110 & -1.8133123 \\
C & 3.5613623 & 1.7640218 & -1.0778348 \\
Br & -0.1236283 & 5.3714741 & 0.4746762 \\
C & 1.2536556 & 1.4213432 & 2.5606476 \\
C & -0.0958065 & 0.9667647 & 2.0692992 \\
C & 2.2253897 & 0.5555274 & 2.9283821 \\
C & 1.4736052 & 2.9170004 & 2.5971234 \\
C & 2.3335015 & 3.4332477 & 1.4077194 \\
O & 3.6746697 & 2.9918413 & 1.5343401 \\
H & 2.3777830 & 4.5381181 & 1.4931828 \\
H & 0.5059138 & 3.4573957 & 2.5878265 \\
H & 2.0175445 & 3.2200056 & 3.5151120 \\
H & 3.1767337 & 0.9097419 & 3.3567132 \\
H & 2.0857132 & -0.5339207 & 2.8459981 \\
H & -0.3957111 & 1.5231440 & 1.1573922 \\
H & -0.8705070 & 1.1954622 & 2.8341779 \\
H & -0.1277379 & -0.1188508 & 1.8560682 \\
H & 3.6567508 & 2.0130255 & 1.4606033
\end{tabular}




$\begin{array}{lrrr}\text { H } & 0.2668754 & 0.8842096 & -0.7476027 \\ \mathrm{H} & 1.0734088 & -1.0924062 & -2.0277878 \\ \mathrm{H} & 3.4800816 & -1.2421178 & -2.7335537 \\ \mathrm{H} & 5.0596732 & 0.6232194 & -2.1450192 \\ \mathrm{H} & 4.2434703 & 2.5954260 & -0.8603950 \\ \mathrm{H} & -1.8901215 & 3.9153720 & -1.1657637 \\ \mathrm{H} & -2.9836569 & 3.3398616 & -3.3364111 \\ \mathrm{H} & -1.5873852 & 2.6940087 & -5.3204011 \\ \mathrm{H} & 0.9211762 & 2.6309304 & -5.1021061 \\ \mathrm{H} & 2.0001045 & 3.2014196 & -2.9377265 \\ \mathrm{Pd} & 1.9931006 & 5.6847815 & -0.9778479 \\ \mathrm{P} & 3.9370926 & 6.1687301 & -1.9982435 \\ \mathrm{C} & 4.0524341 & 7.6418188 & -3.0985804 \\ \mathrm{C} & 4.9724120 & 7.7190385 & -4.1653569 \\ \mathrm{H} & 5.6339255 & 6.8659993 & -4.3798642 \\ \mathrm{C} & 5.0383383 & 8.8782435 & -4.9540889 \\ \mathrm{H} & 5.7548346 & 8.9320015 & -5.7881166 \\ \mathrm{C} & 4.1917115 & 9.9667537 & -4.6813362 \\ \mathrm{H} & 4.2434751 & 10.8733217 & -5.3034711 \\ \mathrm{C} & 3.2729236 & 9.8926654 & -3.6206001 \\ \mathrm{H} & 2.6024683 & 10.7397337 & -3.4100968 \\ \mathrm{C} & 3.1983850 & 8.7315745 & -2.8357484 \\ \mathrm{H} & 2.4676381 & 8.6465456 & -2.0145287 \\ \mathrm{C} & 5.3600754 & 6.3725283 & -0.8435265 \\ \mathrm{C} & 5.4351039 & 5.4907267 & 0.2575166 \\ \mathrm{H} & 4.6578687 & 4.7272030 & 0.4193498 \\ \mathrm{C} & 6.5069103 & 5.5765942 & 1.1574473 \\ \mathrm{H} & 6.5514967 & 4.8772579 & 2.0059931 \\ \mathrm{C} & 7.5004267 & 6.5562080 & 0.9831667 \\ \mathrm{H} & 8.3342297 & 6.6322210 & 1.6978053 \\ \mathrm{C} & 7.4203114 & 7.4441995 & -0.1019373 \\ \mathrm{H} & 8.1909460 & 8.2181261 & -0.2401548 \\ \mathrm{C} & 6.3583014 & 7.3517235 & -1.0172506 \\ \mathrm{H} & 6.3038588 & 8.0483920 & -1.8665915 \\ \mathrm{C} & 4.5113615 & 4.8120512 & -3.1119831 \\ \mathrm{C} & 3.7245374 & 4.5151287 & -4.2470885 \\ \mathrm{H} & 2.8242282 & 5.1138158 & -4.4554933 \\ \mathrm{C} & 4.0806188 & 3.4616491 & -5.0999289 \\ \mathrm{H} & 3.4619249 & 3.2422164 & -5.9834888 \\ \mathrm{C} & 5.2206320 & 2.6845839 & -4.8264306 \\ \mathrm{H} & 5.4933227 & 1.8509728 & -5.4908403 \\ \mathrm{C} & 6.0100884 & 2.9813044 & -3.7046916 \\ & 6.9108237 & 2.3857979 & -3.4901767 \\ \mathrm{H} & 6.6611611 & 4.0413829 & -2.8506425 \\ \mathrm{H} & & & \end{array}$


SCF Energy $=-4548.277431785$

\begin{tabular}{|c|c|c|c|}
\hline $\mathrm{C}$ & 1.6518852 & 3.4290272 & -2.8378573 \\
\hline C & 0.4997957 & 3.7361724 & -2.0666059 \\
\hline C & -0.7438312 & 3.8272698 & -2.7279173 \\
\hline C & -0.8364997 & 3.6156819 & -4.1131126 \\
\hline C & 0.3078269 & 3.3048204 & -4.8633489 \\
\hline C & 1.5521420 & 3.2122429 & -4.2141974 \\
\hline C & 0.6603103 & 3.9911584 & -0.6105699 \\
\hline C & 1.4292465 & 3.2786301 & 0.2867339 \\
\hline C & 2.1906401 & 2.0990443 & -0.2089821 \\
\hline C & 1.5193369 & 0.9850091 & -0.7622369 \\
\hline & 2.2406906 & -0.1211024 & -1.2267831 \\
\hline C & 3.6454559 & -0.1356946 & -1.1506842 \\
\hline C & 4.3227696 & 0.9720095 & -0.6177847 \\
\hline $\mathrm{C}$ & 3.6037669 & 2.0842577 & -0.1515868 \\
\hline $\mathrm{Br}$ & -0.7614881 & 5.2570613 & 0.1680825 \\
\hline$C$ & 0.3207227 & 1.2936886 & 2.3541775 \\
\hline C & 1.5058711 & 0.4582284 & 2.7750399 \\
\hline & -0.7763469 & 0.7355010 & 1.7993979 \\
\hline $\mathrm{C}$ & 0.3947969 & 2.7873896 & 2.5762318 \\
\hline C & 1.4860821 & 3.5712844 & 1.7935244 \\
\hline U & 2.7857128 & 3.3948048 & 2.3385840 \\
\hline & 1.2610321 & 4.6457109 & 1.9445757 \\
\hline $\mathrm{H}$ & -0.5835918 & 3.2416320 & 2.3206937 \\
\hline $\mathrm{H}$ & 0.5880865 & 3.0021159 & 3.6509994 \\
\hline & -1.6334837 & 1.3517531 & 1.4847790 \\
\hline & -0.8501941 & -0.3521338 & 1.6391872 \\
\hline $\mathrm{H}$ & 1.8515093 & 0.7279771 & 3.7948079 \\
\hline $\mathrm{H}$ & 2.3673666 & 0.6083030 & 2.0865936 \\
\hline & 1.2718030 & -0.6236550 & 2.7543143 \\
\hline $\mathrm{H}$ & 3.0726461 & 2.4836740 & 2.1305090 \\
\hline $\mathrm{H}$ & 0.4224122 & 0.9981660 & -0.8140985 \\
\hline $\mathrm{H}$ & 1.7027283 & -0.9816574 & -1.6528586 \\
\hline $\mathrm{H}$ & 4.2098057 & -1.0063894 & -1.5177533 \\
\hline $\mathrm{H}$ & 5.4218634 & 0.9870213 & -0.5804285 \\
\hline $\mathrm{H}$ & 4.1337610 & 2.9750655 & 0.2117005 \\
\hline $\mathrm{H}$ & -1.6428520 & 4.0804105 & -2.1492255 \\
\hline $\mathrm{H}$ & -1.8166918 & 3.6967079 & -4.6070586 \\
\hline $\mathrm{H}$ & 0.2345468 & 3.1458751 & -5.9496485 \\
\hline & 2.4625988 & 2.9878069 & -4.7897373 \\
\hline $\mathrm{H}$ & 2.6337065 & 3.3894697 & -2.3509614 \\
\hline $\mathrm{Pd}$ & 1.7246171 & 5.8627794 & -0.5536918 \\
\hline $\mathrm{P}$ & 3.4530867 & 6.6954510 & -1.7046898 \\
\hline & 4.3971107 & 8.1891750 & -1.1742147 \\
\hline
\end{tabular}




\begin{tabular}{|c|c|c|c|}
\hline & 5.7817270 & 8.3498129 & -1.3914593 \\
\hline & 6.3614770 & 7.5407996 & -1.8608818 \\
\hline & 6.4240666 & 9.5342294 & -0.9962588 \\
\hline & 7.5055273 & 9.6511657 & -1.1656297 \\
\hline & 5.6911039 & 10.5660016 & -0.3860890 \\
\hline & 6.1978544 & 11.4922016 & -0.0749256 \\
\hline & 4.3121263 & 10.4086971 & -0.1642461 \\
\hline & 3.7359459 & 11.2103725 & 0.3223110 \\
\hline & 3.6692061 & 9.2228562 & -0.5495288 \\
\hline & 2.5925999 & 9.0777852 & -0.3625338 \\
\hline & 4.7685393 & 5.4086218 & -1.8374474 \\
\hline & 4.9432037 & 4.6338948 & -3.0019309 \\
\hline & 4.3406155 & 4.8519235 & -3.8956934 \\
\hline & 5.8843193 & 3.5907511 & -3.0234522 \\
\hline & 6.0067983 & 2.9876216 & -3.9359408 \\
\hline & 6.6730364 & 3.3248130 & -1.8934707 \\
\hline & 7.4191511 & 2.5162376 & -1.9180498 \\
\hline & 6.503 & 4.05 & -0.7284626 \\
\hline & 7.1129055 & 3.8890207 & 0.16 \\
\hline & 5.5475374 & 5.11 & -0.69 \\
\hline & 5.3976360 & 5.7 & 2325 \\
\hline & 3.0531788 & 7.0573748 & -3.4 \\
\hline & 1.7998090 & 6.6497038 & -3.9690160 \\
\hline & 1.0830914 & 6.1707182 & -3.2833515 \\
\hline & 1.4764771 & 6.8514166 & -5.3200794 \\
\hline & 0.4981950 & 6.5188617 & -5.6976235 \\
\hline & 2.3977444 & 7.4733387 & -6.1779223 \\
\hline & 2.1436185 & 7.6367571 & -7.2365100 \\
\hline & 3.6444699 & 7.8958046 & -5.6817653 \\
\hline & 4.3650560 & 8.3918497 & -6.3499637 \\
\hline & 3.9730965 & 7.6886352 & -4.3338145 \\
\hline & 4.9483568 & 8.0230944 & -3.9494213 \\
\hline \\
\hline \\
\hline \multicolumn{4}{|c|}{.274603} \\
\hline & 1.7371565 & 4.5683459 & -3.1201044 \\
\hline & 1.2846232 & 4.6912355 & -1.7875066 \\
\hline & -0.0515252 & 5.0847724 & -1.5580093 \\
\hline & -0.9124622 & 5.3386735 & -2.6366341 \\
\hline & -0.4520261 & 5.2120364 & -3.9576539 \\
\hline & 0.8789623 & 4.8266036 & -4.1938502 \\
\hline & 2.2104352 & 4.3628071 & -0.6719776 \\
\hline & 2.8193030 & 3.1491391 & -0.4436510 \\
\hline & 2.8035901 & 2.1364958 & -1.5378086 \\
\hline & 1.6143785 & 1.5046925 & -1.963897 \\
\hline
\end{tabular}




\begin{tabular}{lrrr} 
C & 1.6335362 & 0.5896624 & -3.0256404 \\
$\mathrm{C}$ & 2.8406567 & 0.2816423 & -3.6772773 \\
$\mathrm{C}$ & 4.0307627 & 0.8997327 & -3.2607303 \\
$\mathrm{C}$ & 4.0146951 & 1.8236281 & -2.2032724 \\
$\mathrm{Br}$ & 1.9929059 & 5.6585332 & 0.9272483 \\
$\mathrm{C}$ & 1.4033019 & 2.2748013 & 2.2743817 \\
$\mathrm{C}$ & 0.1645911 & 2.2855280 & 1.4152265 \\
$\mathrm{C}$ & 1.3960053 & 2.7449372 & 3.5394044 \\
$\mathrm{C}$ & 2.6784412 & 1.7270316 & 1.6641872 \\
$\mathrm{C}$ & 3.5211342 & 2.7506162 & 0.8510242 \\
$\mathrm{O}$ & 4.8301436 & 2.2281614 & 0.6365196 \\
$\mathrm{H}$ & 3.6623519 & 3.6546976 & 1.4728928 \\
$\mathrm{H}$ & 3.3540842 & 1.3646364 & 2.4648550 \\
$\mathrm{H}$ & 2.4529351 & 0.8593746 & 1.0038388 \\
$\mathrm{H}$ & 2.3042385 & 2.7289470 & 4.1630142 \\
$\mathrm{H}$ & 0.4848257 & 3.1694011 & 3.9898951 \\
$\mathrm{H}$ & -0.0744996 & 1.2619625 & 1.0537846 \\
$\mathrm{H}$ & 0.3111388 & 2.9114896 & 0.5105676 \\
$\mathrm{H}$ & -0.7137899 & 2.6754897 & 1.9642674 \\
$\mathrm{H}$ & 4.7449785 & 1.4732982 & 0.0190138 \\
$\mathrm{H}$ & 0.6671813 & 1.7532894 & -1.4658470 \\
$\mathrm{H}$ & 0.6953639 & 0.1144374 & -3.3502511 \\
$\mathrm{H}$ & 2.8510001 & -0.4355824 & -4.5119182 \\
$\mathrm{H}$ & 4.9805531 & 0.6789632 & -3.7715665 \\
$\mathrm{H}$ & 4.9343876 & 2.3542321 & -1.9171950 \\
$\mathrm{H}$ & -0.4107355 & 5.1993527 & -0.5253588 \\
$\mathrm{H}$ & -1.9515611 & 5.6438096 & -2.4402896 \\
$\mathrm{H}$ & -1.1270945 & 5.4221996 & -4.8011398 \\
$\mathrm{H}$ & 1.2662907 & 4.7376364 & -5.2197134 \\
$\mathrm{C}$ & 2.7759202 & 4.2778475 & -3.3077752 \\
$\mathrm{H}$ & 6.3081762 & 4.1175760 & -0.1899534 \\
$\mathrm{Pd}$ & 3.9084790 & 5.7148942 & -0.8088537 \\
$\mathrm{P}$ & 5.5490316 & 5.8524125 & -2.3474250 \\
$\mathrm{C}$ & 6.5468802 & 7.4084040 & -2.3513367 \\
$\mathrm{C}$ & 5.9783138 & 8.5441003 & -1.7394500 \\
$\mathrm{H}$ & 4.9954844 & 8.4411987 & -1.2482931 \\
$\mathrm{C}$ & 6.6610386 & 9.7711726 & -1.7496638 \\
$\mathrm{H}$ & 6.2081088 & 10.6532835 & -1.2720446 \\
$\mathrm{C}$ & 7.9244231 & 9.8661983 & -2.3556785 \\
$\mathrm{H}$ & 8.4659134 & 10.8245120 & -2.3556526 \\
$\mathrm{C}$ & 8.5021052 & 8.7322844 & -2.9536756 \\
$\mathrm{H}$ & 9.4962016 & 8.8016622 & -3.4213074 \\
$\mathrm{H}$ & 8.8165119 & 7.5082044 & -2.9558231 \\
\hline
\end{tabular}




$\begin{array}{lllr}\text { C } & 7.8698188 & 2.7815436 & -0.9070603 \\ \text { H } & 7.9603210 & 2.2617889 & 0.0582790 \\ \text { C } & 8.6429360 & 2.3825358 & -2.0094077 \\ \text { H } & 9.3571939 & 1.5505722 & -1.9118146 \\ \text { C } & 8.4946400 & 3.0395348 & -3.2441427 \\ \text { H } & 9.0938727 & 2.7248928 & -4.1123323 \\ \text { C } & 7.5768901 & 4.0929214 & -3.3769514 \\ \text { H } & 7.4469591 & 4.5848216 & -4.3525856 \\ \text { C } & 4.9583455 & 5.7636345 & -4.0927907 \\ \text { C } & 4.6331866 & 6.9405000 & -4.7978375 \\ \text { H } & 4.8314757 & 7.9220577 & -4.3417143 \\ \text { C } & 4.0575651 & 6.8650828 & -6.0755718 \\ \text { H } & 3.8088819 & 7.7918998 & -6.6146310 \\ \text { C } & 3.8011072 & 5.6163854 & -6.6666518 \\ \text { H } & 3.3513510 & 5.5606399 & -7.6695249 \\ \text { C } & 4.1222642 & 4.4393323 & -5.9688988 \\ \text { H } & 3.9210217 & 3.4544810 & -6.4173151 \\ \text { C } & 4.6928549 & 4.5108245 & -4.6895510 \\ \text { H } & 4.9244362 & 3.5829485 & -4.1473538\end{array}$

R4

75

SCF Energy $=-4548.277808098$

$\begin{array}{lrrr}\text { C } & 1.0713160 & 5.3728791 & -2.6082947 \\ \text { C } & 0.6323202 & 4.6092286 & -1.5081177 \\ \text { C } & -0.6684570 & 4.0550822 & -1.5278606 \\ \text { C } & -1.4887815 & 4.2182264 & -2.6523110 \\ \text { C } & -1.0244366 & 4.9439928 & -3.7642140 \\ \text { C } & 0.2541956 & 5.5254240 & -3.7367664 \\ \text { C } & 1.4900637 & 4.3893623 & -0.3208022 \\ \text { C } & 2.0175894 & 3.1938381 & 0.1155574 \\ \text { C } & 2.1827137 & 2.0777233 & -0.8666692 \\ \text { C } & 1.1048794 & 1.3437941 & -1.4094184 \\ \text { C } & 1.3332289 & 0.2991540 & -2.3176541 \\ \text { C } & 2.6422459 & -0.0435129 & -2.6979558 \\ \text { C } & 3.7244661 & 0.6733578 & -2.1647046 \\ \text { C } & 3.4974469 & 1.7241624 & -1.2617001 \\ \text { Br } & 1.1374854 & 5.9121030 & 1.0870552 \\ \text { C } & 0.1688354 & 2.3427187 & 2.4658114 \\ \text { C } & -0.0066522 & 3.3354279 & 3.5873549 \\ \text { C } & -0.8725345 & 1.9021001 & 1.7277045 \\ \text { C } & 1.5802279 & 1.8602969 & 2.2148336 \\ \text { C } & 2.5036523 & 2.9114029 & 1.5364353 \\ \text { O } & 3.8616339 & 2.4892771 & 1.6077348 \\ \text { H } & 2.4628901 & 3.8566392 & 2.1100946 \\ \text { H } & 2.0739385 & 1.5994078 & 3.1757848\end{array}$




\begin{tabular}{|c|c|c|c|}
\hline & 1.5709008 & 0.9443041 & 1.5867239 \\
\hline $\mathrm{H}$ & -0.7335367 & 1.1493511 & 0.9363033 \\
\hline & -1.8973597 & 2.2646072 & 1.9066048 \\
\hline & 0.6411329 & 4.2254505 & 3.4451504 \\
\hline & 0.2862421 & 2.8796100 & 4.5582627 \\
\hline & -1.0533780 & 3.6860816 & 3.6692809 \\
\hline & 3.9577310 & 1.7144195 & 1.0165591 \\
\hline & 0.0789003 & 1.5981771 & -1.1184425 \\
\hline & 0.4767616 & -0.2550053 & -2.7312003 \\
\hline & 2.8154435 & -0.8632131 & -3.4115990 \\
\hline & 4.7571965 & 0.4385108 & -2.4628852 \\
\hline & 4.3439148 & 2.3190846 & -0.8946095 \\
\hline & -1.0239965 & 3.5094686 & -0.6404446 \\
\hline & -2.4976220 & 3.77 & 25950 \\
\hline & -1.6673414 & 5.06 & 93007 \\
\hline & 0.62414 & 6.1 & 8684 \\
\hline & 2.0672416 & 5.8 & 2092 \\
\hline & 3.3247948 & 5.5 & -0 \\
\hline & 5.1244032 & 5.4 & -1 \\
\hline & 6.3406778 & 6.86 & 4724 \\
\hline & 7.6842603 & 6.69 & 0606 \\
\hline & 8.0462647 & 5.700 & -2.2 \\
\hline & 8.5585190 & 7.7943272 & -1.9 \\
\hline & 9.6087188 & 7.6559371 & -2.2 \\
\hline & 8.0966716 & 9.0668283 & -1.6 \\
\hline & 8.7859620 & 9.9249541 & -1.6 \\
\hline & 6.7581899 & 9.2398155 & 228659 \\
\hline & 6.3961201 & 10.2330725 & 68058 \\
\hline & 5.8846202 & 8.1 & 55502 \\
\hline & 4.8376545 & 8.2508916 & -0.8 \\
\hline & 6.1820637 & 3.9927971 & -1.34 \\
\hline & 6.4114588 & 3.5896029 & -0.0 \\
\hline & 5.9577781 & 4.1630449 & 29108 \\
\hline & 7.1551764 & 2.4318442 & 0.2599313 \\
\hline & 7.3145089 & 2.1192516 & 1.3028115 \\
\hline & 7.6668136 & 1.6601734 & -0.7982248 \\
\hline & 8.2351761 & 0.7415851 & -0.5868386 \\
\hline & 7.4445416 & 2.0597127 & -2.1270391 \\
\hline & 7.8439090 & 1.4584966 & -2.9582610 \\
\hline & 6.7091790 & 3.2248736 & -2.4012650 \\
\hline & 6.5244715 & 3.5275049 & -3.4425122 \\
\hline & 4.6455328 & 5.3482251 & -3.3739507 \\
\hline & 3.8539014 & 4.2432490 & -3.7628374 \\
\hline & 3.5846901 & 3.4745933 & -3.0238501 \\
\hline & 3.3952992 & 4.1303085 & -5.0809612 \\
\hline & 2.7789481 & 3.2647098 & -5.365843 \\
\hline
\end{tabular}




$\begin{array}{llll}\mathrm{C} & 3.7005602 & 5.1286156 & -6.0235585 \\ \mathrm{H} & 3.3296841 & 5.0457162 & -7.0564960 \\ \mathrm{C} & 4.4769382 & 6.2341194 & -5.6396022 \\ \mathrm{H} & 4.7178622 & 7.0198326 & -6.3718955 \\ \mathrm{C} & 4.9545412 & 6.3437004 & -4.3220957 \\ \mathrm{H} & 5.5675900 & 7.2088293 & -4.0291957\end{array}$

S1

75

SCF Energy $=-4548.277294829$

$\begin{array}{lrrr}\text { C } & 2.6939437 & 4.7918292 & -2.5016794 \\ \mathrm{C} & 1.6021984 & 4.4331782 & -1.6854918 \\ \mathrm{C} & 0.3949669 & 4.0224424 & -2.2954517 \\ \mathrm{C} & 0.2996623 & 3.9357227 & -3.6909593 \\ \mathrm{C} & 1.4072218 & 4.2590729 & -4.4960992 \\ \mathrm{C} & 2.6016912 & 4.6904605 & -3.8970423 \\ \mathrm{C} & 1.7261370 & 4.4327067 & -0.2040378 \\ \mathrm{C} & 2.1899852 & 3.4227623 & 0.6252658 \\ \mathrm{C} & 3.0387219 & 2.3386812 & 0.0509432 \\ \mathrm{C} & 2.7858750 & 1.7157965 & -1.1952795 \\ \mathrm{C} & 3.6652374 & 0.7563116 & -1.7149354 \\ \mathrm{C} & 4.8244735 & 0.3892684 & -1.0111955 \\ \mathrm{C} & 5.0858962 & 0.9893218 & 0.2319822 \\ \mathrm{C} & 4.2063651 & 1.9447478 & 0.7556759 \\ \mathrm{Br} & 0.3409634 & 5.8190994 & 0.5673159 \\ \mathrm{C} & 0.4319514 & 1.2397522 & 1.7020343 \\ \mathrm{C} & -0.3324407 & 1.1876892 & 0.4058623 \\ \mathrm{C} & 1.0300846 & 0.1545892 & 2.2344144 \\ \mathrm{C} & 0.5356202 & 2.5974773 & 2.3718538 \\ \mathrm{C} & 1.9003731 & 3.3048230 & 2.1343174 \\ \mathrm{O} & 1.9925043 & 4.4869974 & 2.9107758 \\ \mathrm{H} & 2.6792104 & 2.6368837 & 2.5478610 \\ \mathrm{H} & -0.2717975 & 3.2704090 & 2.0140863 \\ \mathrm{H} & 0.4255620 & 2.5061444 & 3.4715934 \\ \mathrm{H} & 1.5808817 & 0.2115088 & 3.1873404 \\ \mathrm{H} & 1.0000126 & -0.8243663 & 1.7313064 \\ \mathrm{H} & 0.0686532 & 1.9302513 & -0.3149293 \\ \mathrm{H} & -1.3992842 & 1.4563535 & 0.5680161 \\ \mathrm{H} & -0.2896343 & 0.1876913 & -0.0664336 \\ \mathrm{H} & 1.3139978 & 5.1139249 & 2.5786397 \\ \mathrm{H} & 1.8882802 & 1.9770947 & -1.7671770 \\ \mathrm{H} & 3.4365651 & 0.2890602 & -2.6849648 \\ \mathrm{H} & 5.5156270 & -0.3596609 & -1.4263275 \\ \mathrm{H} & 5.9923893 & 0.7300048 & 0.7989865 \\ \mathrm{H} & 4.4588269 & 2.4289048 & 1.7078286 \\ \mathrm{H} & -0.4698197 & 3.7719700 & -1.6632435\end{array}$




\begin{tabular}{|c|c|c|c|}
\hline & -0.6441586 & 3.6103906 & -4.1542053 \\
\hline $\mathrm{H}$ & 1.3321870 & 4.1839883 & -5.5916377 \\
\hline & 3.4745946 & 4.9543811 & -4.5125670 \\
\hline & 3.6208734 & 5.1331358 & -2.0261616 \\
\hline & 5.5257813 & 8.8632025 & 0.6145703 \\
\hline & 6.1995386 & 7.6461350 & 0.8399840 \\
\hline & 7.5364419 & 7.6617639 & 1.2874575 \\
\hline & 8.1916225 & 8.8844501 & 1.4981763 \\
\hline & 7.5209676 & 10.0967343 & 1.2577964 \\
\hline & 6.1882107 & 0.0851189 & 0.8138955 \\
\hline & 5.2812480 & 6.0840768 & 0.4985231 \\
\hline & 5.9823534 & 5.5449692 & -1.1175259 \\
\hline & 6.5392017 & 6.465 & -2.0284000 \\
\hline & 6.9290003 & 6.041 & -3.3097346 \\
\hline & 6.7744378 & 4.6979572 & -3.6915218 \\
\hline & 6.2252744 & 3.7752830 & -2.7835403 \\
\hline & 5.8250316 & 4.1949038 & -1.5079557 \\
\hline$d$ & 3.0225954 & 5.9613655 & 0.5135375 \\
\hline & 5.9931354 & 4.8664653 & 1.6873787 \\
\hline & 7.1777405 & 4.1438201 & 1.4356939 \\
\hline & 7.6306597 & 3.1884250 & 2.3598627 \\
\hline & 6.9117584 & 2.9547053 & 3.5446587 \\
\hline & 5.7357116 & 3.6802627 & 3.8035540 \\
\hline & 5.2729512 & 4.6275936 & 2.8773005 \\
\hline & 7.7331241 & 4.3077265 & 0.5003783 \\
\hline & 8.5492973 & 2.6193257 & 2.1500544 \\
\hline & 7.2649267 & 2.1994611 & 4.2633048 \\
\hline & 5.1589877 & 3.4935750 & 4.7220523 \\
\hline & 4.3124721 & 5.1411468 & 3.0435631 \\
\hline & 6.6651482 & 7.5184079 & -1.7349824 \\
\hline & 7.3608263 & 6.7690749 & -4.0140330 \\
\hline & 7.0822451 & 4.3702648 & -4.6961472 \\
\hline & 6.0903743 & 2.7206630 & -3.0664666 \\
\hline & 5.3748589 & 3.4697372 & -0.8154135 \\
\hline & 8.0632665 & 6.7149317 & 1.4787080 \\
\hline & 9.2333496 & 8.8918579 & 1.8539215 \\
\hline & 8.0378618 & 11.0539472 & 1.4255206 \\
\hline & 5.6571775 & 11.0320605 & 0.6329556 \\
\hline & 4.4723109 & 8.8331941 & 0.2898029 \\
\hline \\
\hline \multicolumn{4}{|l|}{5} \\
\hline \multicolumn{4}{|l|}{$\mathrm{SCF}$} \\
\hline & 0.3437317 & 4.5145154 & -3.0225382 \\
\hline & -0.0098208 & 3.9110200 & -1.799315 \\
\hline & -0.9943911 & 2.8958428 & -1.791187 \\
\hline
\end{tabular}




\begin{tabular}{|c|c|c|c|}
\hline & -1.5833420 & 2.4712422 & -2.9891068 \\
\hline & -1.1961425 & 3.0538381 & -4.2102830 \\
\hline & -0.2336054 & 4.0758342 & -4.2231971 \\
\hline & 0.6310963 & 4.3119660 & -0.5223690 \\
\hline & 1.6190092 & 3.6628360 & 0.1983447 \\
\hline & 2.5113083 & 2.6919113 & -0.5000559 \\
\hline & 2.0512541 & 1.6737399 & -1.3661774 \\
\hline & 2.9555757 & 0.8371920 & -2.0360492 \\
\hline & 4.3410414 & 0.9877549 & -1.8566436 \\
\hline & 4.8137486 & 1.9813227 & -0.9827169 \\
\hline & 3.9124736 & 2.8176125 & -0.3114831 \\
\hline & -0.7326088 & 5.5446429 & 0.5320736 \\
\hline & 0.7923698 & 1.5239067 & 2.0748280 \\
\hline & 2.0256481 & 0.67 & 2.25 \\
\hline & -0.3442468 & 1.03 & 1.5 \\
\hline & 0.88 & 2.96 & 2.5 \\
\hline & 1.896 & 3.8 & 1.7 \\
\hline & 1.954 & 5.14 & 2.2 \\
\hline & 2.910 & 3.42 & 1.8 \\
\hline & -0.1133843 & $3.44^{\circ}$ & 2.4 \\
\hline & 1.1968788 & 3.03 & $3.5^{\circ}$ \\
\hline & -1.2391631 & 1.6655266 & 1.4237243 \\
\hline & -0.4204864 & -0.0152317 & 1.2063030 \\
\hline & 2.3604698 & 0.6931382 & 3.31 \\
\hline & 2.8726889 & 1.0590276 & 1.6517184 \\
\hline & 1.8497408 & -0.3751794 & 1.9641050 \\
\hline & 1.0669060 & 5.549 & 2.0938655 \\
\hline & 0.9752211 & 1.5253781 & -1.5063546 \\
\hline & 2.5677527 & 0.0522597 & -2.7033106 \\
\hline & 5.0463958 & 0.3325015 & -2.38 \\
\hline & 5.8935047 & 2.1270991 & -0.83 \\
\hline & 4.3036249 & 3.6161989 & 0.33 \\
\hline & -1.2783421 & 2.4377190 & -0.8324443 \\
\hline & -2.3446077 & 1.6765635 & -2.9726180 \\
\hline & -1.6538168 & 2.7133651 & -5.1517435 \\
\hline & 0.0766182 & 4.5400885 & -5.1709959 \\
\hline & 1.0849565 & 5.3248199 & -3.0141923 \\
\hline & 1.6149445 & 6.1964496 & -0.5617893 \\
\hline & 3.4958004 & 6.8363886 & -1.6308356 \\
\hline & 4.9735382 & 6.1544688 & -0.7641524 \\
\hline & 6.0976681 & 5.6415328 & -1.4427158 \\
\hline & 6.1284938 & 5.6573323 & -2.5423708 \\
\hline & 7.1612964 & 5.0782138 & $-0.718910 s$ \\
\hline & 8.0302963 & 4.6683627 & -1.2561915 \\
\hline & 7.1145022 & 5.0314020 & 0.684735 \\
\hline & 7.9463395 & 4.5827990 & 1.248960 \\
\hline
\end{tabular}




\begin{tabular}{|c|c|c|c|}
\hline & 5.9979205 & 5.5481749 & 1.3656759 \\
\hline & 5.9476404 & 5.5011543 & 2.4640050 \\
\hline & 4.9275927 & 6.1002821 & 0.6458470 \\
\hline & 4.0199289 & 6.4393789 & 1.1709682 \\
\hline & 3.9416907 & 8.5904466 & -1.9802476 \\
\hline & 5.2698634 & 9.0128537 & -2.1927931 \\
\hline & 6.0950772 & 8.2887341 & -2.1192305 \\
\hline & 5.5394853 & 10.3576778 & -2.4893675 \\
\hline & 6.5786189 & 10.6844545 & -2.6482356 \\
\hline & 4.4875171 & 11.2859052 & -2.5824464 \\
\hline & 4.7030283 & 12.3401495 & -2.8141337 \\
\hline & 3.1628991 & 10.8689653 & -2.3689910 \\
\hline & 2.3376921 & 11.5945488 & -2.4322311 \\
\hline & 2.8912744 & 9.52 & -2.05 \\
\hline & 1.8610060 & $9.1 \varepsilon$ & -1.86 \\
\hline & 3.5820764 & 6.0 & -3.2 \\
\hline & 3.6197998 & 4.6 & -3.3 \\
\hline & 3.6889177 & 4.0 & -2.4 \\
\hline & 3.549 & 3.9 & -4.6 \\
\hline & 3.5761265 & $2.8 \mathrm{~s}$ & -4.63 \\
\hline & 3.4242396 & 4.741 & -5.78 \\
\hline & 3.3583337 & 4.2318701 & -6.7626534 \\
\hline & 3.3811660 & 6.1439668 & -5.7233395 \\
\hline & 3.2855885 & 6.7372648 & -6.6457393 \\
\hline & 3.4638377 & 6.8000941 & -4.4830748 \\
\hline & 3.4315279 & 7.8988647 & -4.438017 \\
\hline \\
\hline \\
\hline \\
\hline & 0.4844363 & 3.8250301 & -2.9037465 \\
\hline & -0.1523029 & 3.9341996 & -1.6403151 \\
\hline & -1.5598315 & 3.8343659 & -1.5792302 \\
\hline & -2.3080579 & 3.6211381 & -2.7456489 \\
\hline & -1.6682172 & 3.5083765 & -3.9914648 \\
\hline & -0.2683190 & 3.6118833 & -4.0641451 \\
\hline & 0.7002099 & 4.1317765 & -0.4450730 \\
\hline & 1.7355919 & 3. 3327791 & -0.0218829 \\
\hline & 2.0883973 & 2.1543209 & -0.8672643 \\
\hline & 1.1639281 & 1.1183282 & -1.1233586 \\
\hline & 1.5087307 & 0.0473701 & -1.9592615 \\
\hline & 2.7803253 & -0.0040313 & -2.5560613 \\
\hline & 3.7055612 & 1.0240546 & -2.308927 \\
\hline & 3.3648127 & 2.0942413 & -1.4699214 \\
\hline & -0.3668868 & 5.4110202 & 1.056828 \\
\hline & 0.9896311 & 2.5209595 & 2.962205 \\
\hline
\end{tabular}




\begin{tabular}{|c|c|c|c|}
\hline & -0.2111826 & 1.8916029 & 2.3036645 \\
\hline & 0.8898321 & 3.2417889 & 4.1006417 \\
\hline & 2.3248201 & 2.3657003 & 2.2666596 \\
\hline & 2.6149544 & 3.4898698 & 1.2211763 \\
\hline & 2.6313740 & 4.7575289 & 1.8341674 \\
\hline & 3.6560982 & 3.3217816 & 0.8698606 \\
\hline & 3.1459903 & 2.4151509 & 3.0090262 \\
\hline & 2.3927763 & 1.3831576 & 1.7545165 \\
\hline & 1.7769764 & 3.6954998 & 4.5697048 \\
\hline & -0.0803700 & 3.3977665 & 4.5983976 \\
\hline & -0.0481873 & 0.8052806 & 2.1371584 \\
\hline & -0.3920377 & 2.3446301 & 1.3064477 \\
\hline & -1.1287474 & 2.0231405 & 2.9083453 \\
\hline & 1.7129353 & 4.963 & 2.1254941 \\
\hline & 0.1640222 & 1.166 & -0.66 \\
\hline & $0.77 €$ & -0.7 & -2.1 \\
\hline & 3.04 & $-0 . \varepsilon$ & -3.2 \\
\hline & 4.697 & 3094 & -2.78 \\
\hline & 4.07 & 9725 & -1.31 \\
\hline & -2.0609609 & 3.9339004 & -0.606 \\
\hline & -3.4039829 & 3.5441286 & -2.6798731 \\
\hline & -2.2601279 & 3.3441760 & -4.9046126 \\
\hline & 0.2441574 & 3.5276271 & -5.0344455 \\
\hline & 1.5795231 & 3.8921616 & -2.9547303 \\
\hline & 1.5467011 & 5.9768582 & -0.6588055 \\
\hline & 3.2443664 & 7.2168649 & -1.5320372 \\
\hline & 3.0209825 & 8.5144853 & -2.8152381 \\
\hline & 3.9522622 & 8.7321485 & -3.8527832 \\
\hline & 4.8433998 & 8.0909747 & -3.9317494 \\
\hline & 3.7361438 & 9.7564748 & -4.7878173 \\
\hline & 4.4636909 & 9.9201720 & -5.5975551 \\
\hline & 2.5940198 & 10.5701114 & -4.6918595 \\
\hline & 2.4258768 & 11.3710584 & -5.4278718 \\
\hline & 1.6623832 & 10.3539314 & -3.6623313 \\
\hline & 0.7623336 & 10.9832228 & -3.5906139 \\
\hline & 1.8709625 & 9.3254161 & -2.7306524 \\
\hline & 1.1378078 & 9.1309190 & -1.9313536 \\
\hline & 4.4447003 & 7.9456655 & -0.3418191 \\
\hline & 4.5237289 & 7.3477737 & 0.9352816 \\
\hline & 3.8541938 & 6.5076660 & 1.1901166 \\
\hline & 5.4517672 & 7.8275547 & 1.8729721 \\
\hline & 5.5124523 & 7.3547805 & 2.8651336 \\
\hline & 6.2859449 & 8.9129297 & 1.5540642 \\
\hline & 7.0040882 & 9.2943566 & 2.2960824 \\
\hline & 6.1946383 & 9.5180339 & 0.2891029 \\
\hline & 6.8399032 & 10.3739359 & 0.0383552 \\
\hline
\end{tabular}




$\begin{array}{llll}\mathrm{C} & 5.2799606 & 9.0350575 & -0.6604384 \\ \mathrm{H} & 5.2113245 & 9.5117861 & -1.6494508 \\ \mathrm{C} & 4.2553533 & 5.9383084 & -2.4089861 \\ \mathrm{C} & 3.7324592 & 5.3671978 & -3.5919841 \\ \mathrm{H} & 2.7861316 & 5.7484868 & -4.0061964 \\ \mathrm{C} & 4.4114780 & 4.3250784 & -4.2373306 \\ \mathrm{H} & 3.9936829 & 3.8879349 & -5.1567719 \\ \mathrm{C} & 5.6192736 & 3.8343964 & -3.7086662 \\ \mathrm{H} & 6.1517890 & 3.0158637 & -4.2159012 \\ \mathrm{C} & 6.1408394 & 4.3944866 & -2.5317027 \\ \mathrm{H} & 7.0861146 & 4.0169331 & -2.1126872 \\ \mathrm{C} & 5.4654696 & 5.4438486 & -1.8836080 \\ \mathrm{H} & 5.8807784 & 5.8814894 & -0.9640550\end{array}$

S4

75

SCF Energy $=-4548.276842071$

$\begin{array}{lrrr}\text { C } & 0.3639818 & 3.9519859 & -2.8787243 \\ \text { C } & -0.1213569 & 4.0365486 & -1.5473428 \\ \text { C } & -1.5173935 & 4.0434456 & -1.3315125 \\ \text { C } & -2.4025196 & 3.9603342 & -2.4151131 \\ \text { C } & -1.9131672 & 3.8730248 & -3.7298383 \\ \text { C } & -0.5260977 & 3.8688584 & -3.9558800 \\ \text { C } & 0.8661009 & 4.1042686 & -0.4482598 \\ \text { C } & 1.8648577 & 3.2112802 & -0.1513259 \\ \text { C } & 2.0239794 & 2.0398159 & -1.0625033 \\ \text { C } & 0.9641869 & 1.1394806 & -1.3101312 \\ \text { C } & 1.1282413 & 0.0741429 & -2.2054262 \\ \text { C } & 2.3513478 & -0.1067671 & -2.8745260 \\ \text { C } & 3.4086155 & 0.7882538 & -2.6405890 \\ \text { C } & 3.2485750 & 1.8523436 & -1.7416733 \\ \text { Br } & 0.0854266 & 5.4533398 & 1.2013438 \\ \text { C } & 1.2249764 & 2.2884419 & 2.7679795 \\ \text { C } & 1.2685304 & 3.1440581 & 4.0093043 \\ \text { C } & 0.0856288 & 1.7152394 & 2.3270390 \\ \text { C } & 2.5322040 & 2.1052201 & 2.0258295 \\ \text { C } & 2.8622778 & 3.2428647 & 1.0078760 \\ \text { O } & 3.0680452 & 4.4863458 & 1.6481345 \\ \text { H } & 3.8469209 & 2.9778772 & 0.5646342 \\ \text { H } & 3.3805601 & 2.0675987 & 2.7411077 \\ \text { H } & 2.5255024 & 1.1475334 & 1.4667572 \\ \text { H } & 0.0801410 & 1.0667927 & 1.4377971 \\ \text { H } & -0.8746833 & 1.8745958 & 2.8422594 \\ \text { H } & 1.7496484 & 4.1219600 & 3.8062172 \\ \text { H } & 1.8902061 & 2.6558403 & 4.7919806 \\ \text { H } & 0.2595390 & 3.3226655 & 4.4278763\end{array}$




$\begin{array}{lrrr}\text { H } & 2.1887113 & 4.9042559 & 1.8038557 \\ \mathrm{H} & 0.0012811 & 1.2948668 & -0.8035887 \\ \mathrm{H} & 0.2922613 & -0.6186650 & -2.3857469 \\ \mathrm{H} & 2.4771975 & -0.9405993 & -3.5816829 \\ \mathrm{H} & 4.3644992 & 0.6705630 & -3.1727788 \\ \mathrm{H} & 4.0660299 & 2.5752362 & -1.5996258 \\ \mathrm{H} & -1.8974422 & 4.1192187 & -0.3034560 \\ \mathrm{H} & -3.4874817 & 3.9645897 & -2.2303266 \\ \mathrm{H} & -2.6125902 & 3.8110182 & -4.5772708 \\ \mathrm{H} & -0.1315911 & 3.8010124 & -4.9810372 \\ \mathrm{H} & 1.4493542 & 3.9278047 & -3.0475691 \\ \mathrm{Pd} & 1.8168938 & 5.8872053 & -0.7325369 \\ \mathrm{P} & 3.5549157 & 7.0041918 & -1.6935570 \\ \mathrm{C} & 4.8730281 & 7.6422724 & -0.5791868 \\ \mathrm{C} & 5.0195314 & 7.0043156 & 0.6725769 \\ \mathrm{H} & 4.3393885 & 6.1810482 & 0.9530513 \\ \mathrm{C} & 6.0315715 & 7.4195123 & 1.5520871 \\ \mathrm{H} & 6.1443154 & 6.9151025 & 2.5238852 \\ \mathrm{C} & 6.8845485 & 8.4805695 & 1.2020886 \\ \mathrm{H} & 7.6691536 & 8.8117240 & 1.8995568 \\ \mathrm{C} & 6.7276547 & 9.1251183 & -0.0364571 \\ \mathrm{H} & 7.3879894 & 9.9618841 & -0.3113394 \\ \mathrm{C} & 5.7279756 & 8.7063074 & -0.9293189 \\ \mathrm{H} & 5.6088074 & 9.2136500 & -1.8980225 \\ \mathrm{C} & 4.4124759 & 5.6556606 & -2.6275429 \\ \mathrm{C} & 3.7769059 & 5.1300864 & -3.7760531 \\ \mathrm{H} & 2.8386446 & 5.5833555 & -4.1321646 \\ \mathrm{C} & 4.3360190 & 4.0439750 & -4.4623784 \\ \mathrm{H} & 3.8308313 & 3.6427611 & -5.3538667 \\ \mathrm{C} & 5.5345330 & 3.4633615 & -4.0093990 \\ \mathrm{H} & 5.9727004 & 2.6099757 & -4.5484425 \\ \mathrm{C} & 6.1668273 & 3.9771772 & -2.8662386 \\ \mathrm{H} & 7.1053954 & 3.5287923 & -2.5060831 \\ \mathrm{C} & 5.6121354 & 5.0703613 & -2.1773781 \\ \mathrm{H} & 1.5611024 & 9.0566297 & -1.9804032 \\ \mathrm{H} & 6.1144385 & 5.4714178 & -1.2850524 \\ \mathrm{C} & 3.3481642 & 8.3165559 & -2.9637695 \\ \mathrm{C} & 4.2347977 & 8.4729383 & -4.0502767 \\ \mathrm{H} & 5.0764866 & 7.7746370 & -4.1744443 \\ \mathrm{C} & 4.0369654 & 9.5101038 & -4.9749877 \\ \mathrm{H} & 4.7292797 & 9.6261814 & -5.8229311 \\ \mathrm{H} & 2.9578217 & 10.3973976 & -4.8199940 \\ & 2.8036846 & 11.2083107 & -5.5481198 \\ \mathrm{H} & 1.0708756 & 10.2428461 & -3.7411559 \\ \mathrm{H} & 2.2609238 & 10.9305054 & -3.6227387 \\ & & & -2.8190483 \\ \mathrm{H} & & \end{array}$




\section{Optimized Geometries(BXnb)}

$\begin{array}{lrrr}\text { R1 } & & & \\ \text { 75 } & & & \\ \text { SCF } & \text { Energy }=-4548.320737654 & \\ \text { C } & 1.5447813 & -0.3125753 & -2.3753259 \\ \text { C } & 1.3985209 & 0.3697932 & -1.1423771 \\ \text { C } & 0.9723511 & 1.7196579 & -1.1519853 \\ \text { C } & 0.7044316 & 2.3672917 & -2.3636460 \\ \text { C } & 0.8630063 & 1.6830655 & -3.5820386 \\ \text { C } & 1.2903979 & 0.3446150 & -3.5841706 \\ \text { C } & 1.6931816 & -0.3485422 & 0.1046717 \\ \text { C } & 2.8744852 & -0.9185349 & 0.4442537 \\ \text { C } & 4.0766693 & -0.6119260 & -0.3977299 \\ \text { C } & 4.9273353 & -1.6483819 & -0.8464536 \\ \text { C } & 6.0496293 & -1.3542034 & -1.6362808 \\ \text { C } & 6.3473678 & -0.0266157 & -1.9845086 \\ \text { C } & 5.5189631 & 1.0111721 & -1.5246050 \\ \text { C } & 4.3970457 & 0.7220162 & -0.7364293 \\ \text { Br } & 0.2888609 & 1.7485225 & 2.4778255 \\ \text { C } & 0.6980376 & -1.9246172 & 2.4859155 \\ \text { C } & -0.1913528 & -1.8688303 & 3.7142710 \\ \text { C } & 0.1675420 & -2.5178554 & 1.2907925 \\ \text { C } & 2.1699540 & -1.7525734 & 2.7865844 \\ \text { C } & 3.1421909 & -1.8667642 & 1.6159799 \\ \text { O } & 3.2784214 & -3.2286706 & 1.1692924 \\ \text { H } & 4.1532695 & -1.6175707 & 1.9977002 \\ \text { H } & 2.3002687 & -0.7660616 & 3.2809433 \\ \text { H } & 2.4771037 & -2.5221366 & 3.5311696 \\ \text { H } & 0.8164492 & -2.8877600 & 0.4809488 \\ \text { H } & -0.8396473 & -2.9680945 & 1.3141424 \\ \text { H } & 0.0046891 & -0.9507959 & 4.3026752 \\ \text { H } & 0.0175559 & -2.7444823 & 4.3697747 \\ \text { H } & -1.2671322 & -1.8939527 & 3.4511516 \\ \text { H } & 2.3837165 & -3.5335081 & 0.9262409 \\ \text { H } & 4.6893881 & -2.6843297 & -0.5674294 \\ \text { H } & 6.6963974 & -2.1741251 & -1.9852933 \\ \text { H } & 7.2268775 & 0.2001340 & -2.6062944 \\ \text { H } & 5.7511771 & 2.0572281 & -1.7765749 \\ \text { H } & 3.7581611 & 1.5364774 & -0.3670858 \\ \text { H } & 0.8640398 & 2.2384020 & -0.1878147 \\ \text { H } & 0.3669717 & 3.4144534 & -2.3566768 \\ \text { H } & 0.6455143 & 2.1927094 & -4.5328683 \\ \text { H } & 1.4095773 & -0.1967130 & -4.5338537\end{array}$




$\begin{array}{lrrr}\text { H } & 1.8806748 & -1.3586226 & -2.3666090 \\ \text { Pd } & -0.0041368 & -0.4098009 & 1.1706244 \\ \text { P } & -1.8359791 & 0.0962971 & -0.3732356 \\ \text { C } & -2.7253619 & 1.6900229 & -0.5768114 \\ \text { C } & -2.1338679 & 2.8703515 & -0.0866949 \\ \text { H } & -1.1657104 & 2.8166349 & 0.4336529 \\ \text { C } & -2.8113044 & 4.0951385 & -0.2103424 \\ \text { H } & -2.3484885 & 5.0130771 & 0.1823606 \\ \text { C } & -4.0757257 & 4.1455720 & -0.8177821 \\ \text { H } & -4.6035647 & 5.1067522 & -0.9140543 \\ \text { C } & -4.6787661 & 2.9641162 & -1.2870917 \\ \text { H } & -5.6789333 & 2.9984394 & -1.7454580 \\ \text { C } & -4.0106152 & 1.7381749 & -1.1609092 \\ \text { H } & -4.4898292 & 0.8102768 & -1.5095584 \\ \text { C } & -1.7535174 & -0.6413111 & -2.0457440 \\ \text { C } & -1.2067892 & -1.9404906 & -2.1582915 \\ \text { H } & -0.8932150 & -2.4735514 & -1.2486592 \\ \text { C } & -1.0445449 & -2.5377560 & -3.4150719 \\ \text { H } & -0.6259821 & -3.5530301 & -3.4872364 \\ \text { C } & -1.3939978 & -1.8324397 & -4.5798937 \\ \text { H } & -1.2566795 & -2.2959460 & -5.5684973 \\ \text { C } & -1.9067297 & -0.5300993 & -4.4762523 \\ \text { H } & -2.1651919 & 0.0348538 & -5.3846881 \\ \text { C } & -2.0890431 & 0.0645177 & -3.2174155 \\ \text { H } & -2.4788888 & 1.0893579 & -3.1474140 \\ \text { C } & -3.0851600 & -0.9006314 & 0.5489470 \\ \text { C } & -3.3189097 & -0.4993055 & 1.8865305 \\ \text { H } & -2.8278365 & 0.4075510 & 2.2782849 \\ \text { C } & -4.1602713 & -1.2564154 & 2.7135003 \\ \text { H } & -4.3349544 & -0.9356945 & 3.7516643 \\ \text { C } & -4.7729307 & -2.4224274 & 2.2197037 \\ \text { H } & -5.4287813 & -3.0198545 & 2.8707317 \\ \text { C } & -4.5478261 & -2.8192250 & 0.8913904 \\ \text { H } & -5.0330069 & -3.7255062 & 0.4975377 \\ \text { C } & -3.7085237 & -2.0627407 & 0.0552801 \\ \text { H } & -3.5389030 & -2.3771488 & -0.9850391 \\ & & & \\ \text { R2 } & & & \\ 75 & & & \\ \text { SCF } & \text { Energy }=-4548.309398392 & \\ \text { C } & 1.7333764 & 1.9158247 & -2.1015977 \\ \text { C } & 0.9644016 & 1.8557860 & -0.9060919 \\ \text { C } & -0.0860597 & 2.8032859 & -0.7728300 \\ \text { C } & -0.4171911 & 3.6927055 & -1.8026910 \\ \text { C } & 0.3190044 & 3.6942637 & -2.9978555 \\ \text { C } & 1.4092167 & 2.8153284 & -3.1240993\end{array}$




\begin{tabular}{|c|c|c|c|}
\hline & 1.2006340 & 0.8759860 & \\
\hline & 2.2282741 & 0.0109522 & 0.3840676 \\
\hline & 3.1374494 & -0.3963544 & -0.7278663 \\
\hline & 2.6348622 & -1.2593068 & -1.7279277 \\
\hline & 3.4657204 & -1.7106288 & -2.7610839 \\
\hline & 4.8134866 & -1.3112615 & -2.8097173 \\
\hline & 5.3229446 & -0.4570955 & -1.8176032 \\
\hline & 4.4933223 & -0.0018805 & -0.7780602 \\
\hline & 0.6587303 & 2.0685956 & 3.3259521 \\
\hline & 1.9459258 & -3.1202428 & 1.0654886 \\
\hline & 3.3444110 & -3.5987478 & 0.7535703 \\
\hline & 0.8611557 & -3.74 & 0.5617308 \\
\hline & 1.7786591 & -1.96 & 2.026 \\
\hline & 2.530 & -0.6 & 1.7 \\
\hline & 3.930 & $-0.7 !$ & $1.9^{\circ}$ \\
\hline & 2.17 & 0.0 & 2.5 \\
\hline & 0.70 & -1.7 & 2.1 \\
\hline & 2.114 & -2.2 & 3.0 \\
\hline & -0.154 & -3.4 & 0.8 \\
\hline & 0.953 & -4.6 & -0.1 \\
\hline & 3.92 & -3.75 & 1.6 \\
\hline & 3.9000804 & -2.863 & 0.1 \\
\hline & 3. 3315872 & -4.5478419 & 0.183 \\
\hline & 4.2976428 & -1.3467818 & 1.28 \\
\hline & 1.5850086 & -1.5735575 & -1.6752984 \\
\hline & 3.0555916 & -2.3806366 & -3.5317737 \\
\hline & 5.467 & -1.6652130 & -3.6215677 \\
\hline & 6.3752654 & -0.13 & -1.85 \\
\hline & .880 & 0.67 & -0.00 \\
\hline & -0.6486165 & 2.83 & 0.1 \\
\hline & -1.2567540 & 4.385 & -1.66 \\
\hline & 0.0603899 & 4.385 & -3.8 \\
\hline & 2.0163665 & $2.81^{\circ}$ & -4.0424732 \\
\hline & 2.5730951 & 1.2312106 & -2.2496139 \\
\hline & -0.2966049 & 0.8048829 & 1.5079180 \\
\hline & -1.6858375 & -0.1849375 & -0.0337970 \\
\hline & -2.6138798 & -1.5681223 & 0.7520725 \\
\hline & -3.0216911 & 0.9909385 & -0.5173011 \\
\hline & -1.1148392 & -0.8766844 & -1.6353637 \\
\hline & -3.6919795 & -2.2010341 & 0.0980260 \\
\hline & -4.3755552 & -3.2502104 & 0.7279614 \\
\hline & -3.9957390 & -3.6689246 & 2.0165520 \\
\hline & -2.9328949 & -3.0334063 & 2.6769441 \\
\hline & -2.2436501 & -1.9847747 & 2.0459732 \\
\hline & -3.9958961 & -1.8717850 & -0.9068213 \\
\hline & -5.2132765 & -3.7437080 & 0.212452 \\
\hline
\end{tabular}




$\begin{array}{rrrr}\text { H } & -4.5356494 & -4.4924612 & 2.5078326 \\ \text { H } & -2.6346406 & -3.3528635 & 3.6864789 \\ \text { H } & -1.4069232 & -1.4789982 & 2.5564238 \\ \text { C } & -3.7231593 & 0.8905333 & -1.7357472 \\ \text { C } & -4.7434756 & 1.8066539 & -2.0374479 \\ \text { C } & -5.0746739 & 2.8226167 & -1.1259877 \\ \text { C } & -4.3836426 & 2.9212363 & 0.0939908 \\ \text { C } & -3.3603593 & 2.0116767 & 0.3956467 \\ \text { H } & -3.4604229 & 0.1063133 & -2.4606475 \\ \text { H } & -5.2801662 & 1.7274185 & -2.9950126 \\ \text { H } & -5.8709181 & 3.5425317 & -1.3684206 \\ \text { H } & -4.6351019 & 3.7164577 & 0.8116008 \\ \text { H } & -2.8010710 & 2.1017035 & 1.3421250 \\ \text { C } & -0.9490399 & -2.2666152 & -1.8063878 \\ \text { C } & -0.4359205 & -2.7762165 & -3.0094547 \\ \text { C } & -0.0822436 & -1.9037516 & -4.0515080 \\ \text { C } & -0.2561107 & -0.5192685 & -3.8900178 \\ \text { C } & -0.7719075 & -0.0045137 & -2.6924640 \\ \text { H } & -1.2267459 & -2.9552490 & -0.9992807 \\ \text { H } & -0.3081267 & -3.8626201 & -3.1266018 \\ \text { H } & 0.3254751 & -2.3030573 & -4.9924078 \\ \text { H } & 0.0176645 & 0.1751759 & -4.6976812 \\ \text { H } & -0.9165517 & 1.0785423 & -2.5859975\end{array}$

R3

75

SCF Energy $=-4548.318865396$

$\begin{array}{lrrr}\text { C } & 1.5445026 & 2.1603760 & -2.0883332 \\ \text { C } & 1.4017818 & 0.8117257 & -1.6787224 \\ \text { C } & 1.2740795 & -0.1868111 & -2.6730932 \\ \text { C } & 1.2962916 & 0.1503693 & -4.0320920 \\ \text { C } & 1.4501496 & 1.4896050 & -4.4267090 \\ \text { C } & 1.5779804 & 2.4916262 & -3.4490168 \\ \text { C } & 1.3859127 & 0.4912912 & -0.2406307 \\ \text { C } & 2.2448330 & -0.2759971 & 0.4739087 \\ \text { C } & 3.3205636 & -1.0421012 & -0.2153971 \\ \text { C } & 3.4790567 & -2.4236730 & 0.0589354 \\ \text { C } & 4.4760613 & -3.1755229 & -0.5846690 \\ \text { C } & 5.3320765 & -2.5628774 & -1.5131937 \\ \text { C } & 5.1893867 & -1.1905896 & -1.7857762 \\ \text { C } & 4.1996159 & -0.4371021 & -1.1413587 \\ \text { Br } & 1.0706566 & 3.2582565 & 1.5791502 \\ \text { C } & 0.0572497 & -1.0457796 & 3.4177625 \\ \text { C } & -0.8799706 & -2.1717324 & 3.7811883 \\ \text { C } & -0.1379991 & 0.2091518 & 3.8791209 \\ \text { C } & 1.2032032 & -1.4764531 & 2.5246614\end{array}$




$\begin{array}{lrrr}\text { C } & 2.1867284 & -0.3923548 & 2.0061783 \\ \mathrm{O} & 3.4887632 & -0.5410111 & 2.5587748 \\ \mathrm{H} & 1.8459919 & 0.6007263 & 2.3561770 \\ \mathrm{H} & 1.8112790 & -2.2164423 & 3.0929151 \\ \mathrm{H} & 0.7614896 & -2.0456114 & 1.6817426 \\ \mathrm{H} & 0.5227671 & 1.0536192 & 3.6363413 \\ \mathrm{H} & -0.9840325 & 0.4277762 & 4.5503068 \\ \mathrm{H} & -1.3301795 & -2.6189579 & 2.8695979 \\ \mathrm{H} & -1.6990123 & -1.8356540 & 4.4462693 \\ \mathrm{H} & -0.3321519 & -2.9929275 & 4.2926469 \\ \mathrm{H} & 3.9381300 & -1.2540835 & 2.0616630 \\ \mathrm{H} & 2.7959719 & -2.9202010 & 0.7651565 \\ \mathrm{H} & 4.5789345 & -4.2485805 & -0.3611455 \\ \mathrm{H} & 6.1130762 & -3.1505318 & -2.0187369 \\ \mathrm{H} & 5.8641259 & -0.6993205 & -2.5033501 \\ \mathrm{H} & 4.1005372 & 0.6380224 & -1.3448930 \\ \mathrm{H} & 1.1652122 & -1.2390056 & -2.3780623 \\ \mathrm{H} & 1.1870011 & -0.6404305 & -4.7891293 \\ \mathrm{H} & 1.4631129 & 1.7523401 & -5.4952947 \\ \mathrm{H} & 1.6989065 & 3.5438308 & -3.7486457 \\ \mathrm{H} & 1.6253057 & 2.9365269 & -1.3117595 \\ \mathrm{Pd} & -0.1409254 & 1.3481366 & 0.6926974 \\ \mathrm{P} & -1.6342613 & -0.1181509 & -0.1806034 \\ \mathrm{C} & -1.8793030 & 0.0870381 & -1.9840900 \\ \mathrm{C} & -1.7294971 & 1.3852225 & -2.5159325 \\ \mathrm{H} & -1.4576001 & 2.2161754 & -1.8458714 \\ \mathrm{C} & -1.8869039 & 1.6001275 & -3.8903542 \\ \mathrm{H} & -1.7520314 & 2.6107934 & -4.3021440 \\ \mathrm{C} & -2.1877356 & 0.5227268 & -4.7418820 \\ \mathrm{H} & -2.2959328 & 0.6914014 & -5.8239700 \\ \mathrm{C} & -2.3387416 & -0.7696351 & -4.2145777 \\ \mathrm{H} & -2.5716560 & -1.6145390 & -4.8802178 \\ \mathrm{C} & -2.1861765 & -0.9911585 & -2.8365567 \\ \mathrm{H} & -2.2949757 & -2.0048168 & -2.4239953 \\ \mathrm{C} & -3.2451040 & 0.3326385 & 0.5718166 \\ \mathrm{C} & -3.2832466 & 0.4999206 & 1.9744251 \\ \mathrm{H} & -2.3635755 & 0.3527250 & 2.5639567 \\ \mathrm{C} & -4.4857017 & 0.8533720 & 2.6031678 \\ \mathrm{H} & -4.5113028 & 0.9845954 & 3.6953530 \\ \mathrm{C} & -5.6491194 & 1.0507340 & 1.8391445 \\ \mathrm{H} & -6.5902860 & 1.3357001 & 2.3332535 \\ \mathrm{C} & -5.6095146 & 0.8919241 & 0.4434126 \\ \mathrm{C} & -6.5190222 & 1.0501964 & -0.1557049 \\ & -4.4110033 & 0.5334322 & -0.1939667 \\ & -1.5366598 & 0.4104048 & -1.2865092 \\ & & -1.9382118 & 0.0343144\end{array}$




$\begin{array}{lrrr}\text { C } & -2.6195345 & -2.6901978 & 0.5182623 \\ \text { H } & -3.5550450 & -2.1830906 & 0.7953875 \\ \text { C } & -2.5084586 & -4.0842004 & 0.6524821 \\ \text { H } & -3.3619824 & -4.6627105 & 1.0368183 \\ \text { C } & -1.3159747 & -4.7369978 & 0.2994059 \\ \text { H } & -1.2327660 & -5.8290414 & 0.4060261 \\ \text { C } & -0.2289088 & -3.9910487 & -0.1877323 \\ \text { H } & 0.7123638 & -4.4906629 & -0.4618784 \\ \text { C } & -0.3316719 & -2.5984988 & -0.3139038 \\ \text { H } & 0.5297142 & -2.0144496 & -0.6647833\end{array}$

R4

75

SCF Energy $=-4548.324435477$

$\begin{array}{lll}\text { C } & 5.5763538 & 0.7332095\end{array}$

$\begin{array}{lll}\text { C } & 4.5793736 & -0.1898601\end{array}$

0.3098261

$-0.0949790$

C $\quad 4.8566405-1.0386169$

$-1.1937247$

$\begin{array}{lll}\text { C } & 6.0787867 & -0.9602413\end{array}$

$-1.8746467$

7.0575901

$-0.0379028$

$-1.4667032$

6.7999806

0.8050126

$-0.3726295$

Pd $\quad 0.2151601$

$-0.2388009$

0.5797835

P $\quad-2.1139392$

$-0.3719367$

0.4807162

C 2.1189989

$-0.3153619$

$-0.1020525$

$-0.3357046-0.1322580$

$\mathrm{Br} \quad 0.3805043$

2.1316975

0.8675160

C $\quad 1.7771750$

$-0.1994107$

$-1.5478409$

C $\quad 2.0400719$

1.0067084

$-2.2526224$

C $\quad 1.5321945$

1.1955393

$-3.5412535$

C $\quad 0.7277231$

0.2077374

$-4.1475682$

0.4569322

$-0.9893194$

$-3.4681751$

C $\quad 0.9897960$

$-1.2008339$

$-2.1866747$

C $\quad 3.1506091$

$-0.2489829$

2.1062645

4.3956017

$-0.1995861$

2.7787086

$-1.5172379$

2.5295593

$-1.4696857$

2.3178711

$-2.3502240$

1.4133086

$-0.7920190$

3.4196452

2.4308238

0.6527672

3.6167510

2.5868463

$-1.6408180$

2.0091913

2.7882072

$-2.3917787$

0.8494313

0.8120963

$-3.0792010$

1.5988914

$-0.8417082$

$-2.6302717$

4.3718564

$-1.0181759$

$-1.3520959$

3. 1928101

$-0.7506058$

3.5849140

$\mathrm{H}$

0.4206448

0.2439960

$-0.8322663$

2.3350123 


\begin{tabular}{lrrr} 
H & 4.0958995 & -1.7686174 & -1.5063885 \\
$\mathrm{H}$ & 6.2716746 & -1.6300828 & -2.7266655 \\
$\mathrm{H}$ & 8.0192797 & 0.0219988 & -1.9985617 \\
$\mathrm{H}$ & 7.5577510 & 1.5353295 & -0.0494925 \\
$\mathrm{H}$ & 5.3767711 & 1.3995845 & 1.1594133 \\
$\mathrm{H}$ & 0.7994049 & -2.1466600 & -1.6604393 \\
$\mathrm{H}$ & -0.1774747 & -1.7626763 & -3.9262538 \\
$\mathrm{H}$ & 0.3081852 & 0.3800199 & -5.1501786 \\
$\mathrm{H}$ & 1.7428753 & 2.1342879 & -4.0758314 \\
$\mathrm{H}$ & 2.6163195 & 1.7944053 & -1.7482050 \\
$\mathrm{C}$ & -2.6395756 & 1.2448850 & -0.9042209 \\
$\mathrm{C}$ & -3.3164431 & -0.5733645 & 1.2510133 \\
$\mathrm{C}$ & -2.5212049 & -1.5714379 & -1.3910930 \\
$\mathrm{C}$ & -3.2430579 & -1.2283149 & -2.5528844 \\
$\mathrm{C}$ & -3.4517393 & -2.1798403 & -3.5642721 \\
$\mathrm{C}$ & -2.9490579 & -3.4835931 & -3.4265598 \\
$\mathrm{C}$ & -2.2366412 & -3.8351936 & -2.2667112 \\
$\mathrm{C}$ & -2.0180300 & -2.8841183 & -1.2598069 \\
$\mathrm{H}$ & -3.6305904 & -0.2068870 & -2.6728161 \\
$\mathrm{H}$ & -4.0100051 & -1.8959130 & -4.4693179 \\
$\mathrm{H}$ & -3.1095985 & -4.2259441 & -4.2230025 \\
$\mathrm{H}$ & -1.8372582 & -4.8541206 & -2.1502755 \\
$\mathrm{H}$ & -1.4347780 & -3.1556869 & -0.3702682 \\
$\mathrm{C}$ & -3.9082897 & 1.8182977 & -0.6976149 \\
$\mathrm{C}$ & -4.2682548 & 2.9867627 & -1.3899067 \\
$\mathrm{C}$ & -3.3692319 & 3.5754237 & -2.2943847 \\
$\mathrm{C}$ & -2.1038611 & 2.9992570 & -2.5028670 \\
$\mathrm{C}$ & -1.7322289 & 1.8444092 & -1.8025931 \\
$\mathrm{H}$ & -4.6154045 & 1.3522619 & 0.0046998 \\
$\mathrm{H}$ & -5.2580861 & 3.4375976 & -1.2213985 \\
$\mathrm{H}$ & -3.6534405 & 4.4915105 & -2.8343859 \\
$\mathrm{H}$ & -1.3904454 & 3.4616068 & -3.2012570 \\
$\mathrm{H}$ & -0.7342457 & 1.4081016 & -1.9484480 \\
$\mathrm{C}$ & -4.2255706 & -1.6477224 & 1.2896601 \\
$\mathrm{C}$ & -5.0654650 & -1.8135162 & 2.4045436 \\
$\mathrm{C}$ & -5.0077927 & -0.9063447 & 3.4745534 \\
$\mathrm{C}$ & -4.1028693 & 0.1707970 & 3.4343859 \\
$\mathrm{C}$ & -3.2516294 & 0.3349524 & 2.3334443 \\
$\mathrm{H}$ & -4.2787864 & -2.3510882 & 0.4451793 \\
$\mathrm{H}$ & -5.7738246 & -2.6555117 & 2.4324286 \\
$\mathrm{H}$ & -5.6688805 & -1.0378427 & 4.3444678 \\
$\mathrm{H}$ & -4.0542454 & 0.8834509 & 4.2715430 \\
$\mathrm{H}$ & -2.5270642 & 1.1656034 & 2.3009965 \\
& & & \\
$\mathrm{H}$ & & & \\
\hline
\end{tabular}




$\begin{array}{lrrr}\text { SCF } & \text { Energy }=-4548.326624207 & \\ \text { C } & 0.8898678 & 0.9904195 & -2.5390340 \\ \text { C } & 1.2526621 & 1.4050645 & -1.2351622 \\ \text { C } & 1.4869964 & 2.7878131 & -1.0082767 \\ \text { C } & 1.3397167 & 3.7178565 & -2.0440572 \\ \text { C } & 0.9492497 & 3.2942422 & -3.3282886 \\ \text { C } & 0.7353417 & 1.9281407 & -3.5702981 \\ \text { C } & 1.3526856 & 0.4741415 & -0.0941360 \\ \text { C } & 2.3128395 & -0.4234116 & 0.2194454 \\ \text { C } & 3.4362594 & -0.6319983 & -0.7468779 \\ \text { C } & 3.2254067 & -1.2418005 & -2.0008204 \\ \text { C } & 4.2980142 & -1.4513246 & -2.8821485 \\ \text { C } & 5.5958392 & -1.0578229 & -2.5190632 \\ \text { C } & 5.8169451 & -0.4655295 & -1.2630920 \\ \text { C } & 4.7484578 & -0.2563200 & -0.3793281 \\ \text { Br } & 1.2159312 & 2.3573957 & 2.7821161 \\ \text { C } & 0.7779592 & -3.0063356 & 1.7171385 \\ \text { C } & -0.4286738 & -3.5556508 & 2.4357234 \\ \text { C } & 1.3378814 & -3.6560871 & 0.6717176 \\ \text { C } & 1.2933831 & -1.6974616 & 2.2602688 \\ \text { C } & 2.5366724 & -1.1026341 & 1.5886577 \\ \text { O } & 3.2416933 & -0.2213079 & 2.4517713 \\ \text { H } & 3.2481500 & -1.9343112 & 1.4081045 \\ \text { H } & 0.4607382 & -0.9451571 & 2.2450807 \\ \text { H } & 1.5413922 & -1.8230591 & 3.3370597 \\ \text { H } & 2.2098607 & -3.2634093 & 0.1272864 \\ \text { H } & 0.9277102 & -4.6173729 & 0.3230824 \\ \text { H } & -1.2720143 & -2.8356740 & 2.3910878 \\ \text { H } & -0.2093522 & -3.7096464 & 3.5142277 \\ \text { H } & -0.7692865 & -4.5174208 & 2.0067391 \\ \text { H } & 2.6685563 & 0.5714325 & 2.6058391 \\ \text { H } & 2.2126076 & -1.5692931 & -2.2730766 \\ \text { H } & 4.1169346 & -1.9281659 & -3.8577897 \\ \text { H } & 6.4365493 & -1.2171009 & -3.2114266 \\ \text { H } & 6.8329782 & -0.1597609 & -0.9694317 \\ \text { H } & 4.9049725 & 0.1960733 & 0.6110576 \\ \text { H } & 1.7640993 & 3.1154126 & 0.0048962 \\ \text { H } & 1.5258031 & 4.7841622 & -1.8453981 \\ \text { H } & 0.8201146 & 4.0276614 & -4.1386772 \\ \text { H } & 0.4438501 & 1.5813355 & -4.5730953 \\ \text { H } & 0.7247055 & -0.0721252 & -2.7424007 \\ \text { Pd } & -0.0744337 & 1.0164222 & 1.1945064 \\ \text { C } & -1.7557550 & 0.0976030 & -0.0167299 \\ & -2.8566793 & 1.4730375 & -0.5296289 \\ \text {-1.1620224 } & 2.6163727 & -1.1033637 \\ & & & -1.2016278\end{array}$




$\begin{array}{lrrr}\text { C } & -3.0596043 & 3.6768151 & -1.5447760 \\ \text { H } & -2.5861812 & 4.5628872 & -1.9931295 \\ \text { C } & -4.4570788 & 3.6112338 & -1.4043331 \\ \text { H } & -5.0845202 & 4.4495117 & -1.7431994 \\ \text { C } & -5.0531654 & 2.4788689 & -0.8243438 \\ \text { H } & -6.1464520 & 2.4282285 & -0.7087743 \\ \text { C } & -4.2571025 & 1.4073256 & -0.3883379 \\ \text { H } & -4.7225135 & 0.5190421 & 0.0641297 \\ \text { C } & -1.4917895 & -0.8597498 & -1.5496255 \\ \text { C } & -0.6164962 & -1.9632900 & -1.4926421 \\ \text { H } & -0.1396034 & -2.2503146 & -0.5435631 \\ \text { C } & -0.3278358 & -2.6869735 & -2.6579510 \\ \text { H } & 0.3591093 & -3.5450892 & -2.6039565 \\ \text { C } & -0.8990312 & -2.3068681 & -3.8857772 \\ \text { H } & -0.6591984 & -2.8661288 & -4.8027483 \\ \text { C } & -1.7765591 & -1.2107484 & -3.9402687 \\ \text { H } & -2.2273756 & -0.9112811 & -4.8983710 \\ \text { C } & -2.0777574 & -0.4871371 & -2.7752508 \\ \text { H } & -2.7519358 & 0.3797187 & -2.8224694 \\ \text { C } & -2.8002290 & -0.9851954 & 1.0316875 \\ \text { C } & -2.9346409 & -0.6485709 & 2.3965115 \\ \text { H } & -2.4171620 & 0.2394218 & 2.7960465 \\ \text { C } & -3.7052496 & -1.4522875 & 3.2478275 \\ \text { H } & -3.8010747 & -1.1865293 & 4.3110459 \\ \text { C } & -4.3372160 & -2.6037801 & 2.7455163 \\ \text { H } & -4.9309095 & -3.2422681 & 3.4167949 \\ \text { C } & -4.2032480 & -2.9431307 & 1.3892811 \\ \text { H } & -4.6934401 & -3.8461606 & 0.9954107 \\ \text { C } & -3.4402440 & -2.1357194 & 0.5300069 \\ \text { H } & -3.3297222 & -2.4047028 & -0.5305628\end{array}$

S2

75

SCF Energy $=-4548.323676785$

$\begin{array}{lrrr}\text { C } & 2.0413866 & 2.0597133 & -1.7571702 \\ \text { C } & 1.4483376 & 0.8102762 & -1.4525116 \\ \text { C } & 0.8465255 & 0.0732792 & -2.4985518 \\ \text { C } & 0.8599744 & 0.5564043 & -3.8130501 \\ \text { C } & 1.4597219 & 1.7917448 & -4.1052670 \\ \text { C } & 2.0494719 & 2.5413902 & -3.0720798 \\ \text { C } & 1.4866465 & 0.3432588 & -0.0523746 \\ \text { C } & 2.4558601 & -0.4037093 & 0.5301351 \\ \text { C } & 3.5255869 & -0.9828450 & -0.3447661 \\ \text { C } & 3.2202572 & -1.8899118 & -1.3819265 \\ \text { C } & 4.2367634 & -2.4403042 & -2.1775848 \\ \text { C } & 5.5778467 & -2.0953202 & -1.9457932\end{array}$




$\begin{array}{lrrr}\text { C } & 5.8942051 & -1.2045989 & -0.9053709 \\ \mathrm{C} & 4.8805890 & -0.6543353 & -0.1075439 \\ \mathrm{Br} & 1.4987641 & 3.0082940 & 2.0106178 \\ \mathrm{C} & 0.6682294 & -1.9840997 & 2.7027307 \\ \mathrm{C} & 1.3533791 & -3.2579167 & 2.2797117 \\ \mathrm{C} & -0.6680420 & -1.9140048 & 2.8730166 \\ \mathrm{C} & 1.5403029 & -0.7805642 & 2.9757878 \\ \mathrm{C} & 2.7394278 & -0.5784734 & 2.0354221 \\ \mathrm{O} & 3.5668609 & 0.4841035 & 2.4916693 \\ \mathrm{H} & 3.3806923 & -1.4806079 & 2.1130570 \\ \mathrm{H} & 0.9156232 & 0.1393445 & 2.9982565 \\ \mathrm{H} & 1.9903667 & -0.8723894 & 3.9900746 \\ \mathrm{H} & -1.1434767 & -0.9716922 & 3.1867540 \\ \mathrm{H} & -1.3193217 & -2.7876643 & 2.7114128 \\ \mathrm{H} & 2.1355134 & -3.5550424 & 3.0116803 \\ \mathrm{H} & 1.8775972 & -3.1297264 & 1.3070416 \\ \mathrm{H} & 0.6360984 & -4.0943581 & 2.1769306 \\ \mathrm{H} & 3.0360537 & 1.3163036 & 2.4056112 \\ \mathrm{H} & 2.1737204 & -2.1733084 & -1.5601333 \\ \mathrm{H} & 3.9776162 & -3.1452318 & -2.9824489 \\ \mathrm{H} & 6.3758095 & -2.5226477 & -2.5719860 \\ \mathrm{H} & 6.9433744 & -0.9305466 & -0.7148461 \\ \mathrm{H} & 5.1133216 & 0.0416011 & 0.7117529 \\ \mathrm{H} & 0.3587945 & -0.8858274 & -2.2783928 \\ \mathrm{H} & 0.3863396 & -0.0320454 & -4.6125499 \\ \mathrm{H} & 1.4617795 & 2.1738922 & -5.1372116 \\ \mathrm{H} & 2.5204328 & 3.5113994 & -3.2927048 \\ \mathrm{H} & 2.4834847 & 2.6442969 & -0.9366249 \\ \mathrm{Pd} & 0.0781662 & 1.2882222 & 0.9913823 \\ \mathrm{P} & -1.6752994 & 0.2717403 & -0.0201003 \\ \mathrm{C} & -1.9527968 & 1.0198754 & -1.6777205 \\ \mathrm{C} & -1.4422823 & 2.3171069 & -1.8997731 \\ \mathrm{H} & -0.8733968 & 2.8179100 & -1.0987997 \\ \mathrm{C} & -1.6078583 & 2.9339452 & -3.1463450 \\ \mathrm{H} & -4.2115603 & -3.9766899 & -0.8012511 \\ \mathrm{H} & -1.1909433 & 3.9374204 & -3.3152454 \\ \mathrm{C} & -2.2756877 & 2.2587349 & -4.1817444 \\ \mathrm{H} & -2.3920819 & 2.7372843 & -5.1658461 \\ \mathrm{C} & -2.7799682 & 0.9659928 & -3.9652438 \\ \mathrm{H} & -3.2951935 & 0.4311516 & -4.7774964 \\ \mathrm{C} & -2.6209390 & 0.3447732 & -2.7168344 \\ \mathrm{H} & -2.9984067 & -0.6759003 & -2.5605576 \\ & -1.8617935 & -1.5299125 & -0.2731203 \\ \mathrm{H} & -4.0413818 & -1.5219963 & -0.3608065 \\ & -3.2221290 & -3.5104559 & -0.6801349 \\ & & & \\ \mathrm{H} & & \end{array}$




$\begin{array}{lrrr}\text { C } & -2.0575824 & -4.2918483 & -0.7731460 \\ \text { H } & -2.1349344 & -5.3721469 & -0.9683891 \\ \text { C } & -0.7963677 & -3.6950035 & -0.6077728 \\ \text { H } & 0.1179645 & -4.3041130 & -0.6649737 \\ \text { C } & -0.6979383 & -2.3209472 & -0.3460782 \\ \text { H } & 0.2803167 & -1.8515670 & -0.1702638 \\ \text { C } & -3.1464978 & 0.7863966 & 0.9560507 \\ \text { C } & -3.6157423 & -0.0186029 & 2.0176420 \\ \text { H } & -3.1611285 & -1.0015721 & 2.2017450 \\ \text { C } & -4.6671746 & 0.4302823 & 2.8294710 \\ \text { H } & -5.0296805 & -0.2069328 & 3.6501138 \\ \text { C } & -5.2523789 & 1.6870985 & 2.5993341 \\ \text { H } & -6.0744203 & 2.0385857 & 3.2407445 \\ \text { C } & -4.7856429 & 2.4924744 & 1.5474224 \\ \text { H } & -5.2418269 & 3.4759882 & 1.3595105 \\ \text { C } & -3.7383047 & 2.0474000 & 0.7270678 \\ \text { H } & -3.3831649 & 2.6785901 & -0.1006870\end{array}$

S3

75

SCF Energy $=-4548.302784434$

$\begin{array}{lrrr}\text { C } & 1.5486570 & -0.6713482 & -2.0521954 \\ \text { C } & 1.3211888 & -1.3584914 & -0.8344892 \\ \text { C } & 1.0363107 & -2.7453075 & -0.8648773 \\ \text { C } & 1.0105080 & -3.4376311 & -2.0810241 \\ \text { C } & 1.2636819 & -2.7523821 & -3.2839053 \\ \text { C } & 1.5306334 & -1.3733809 & -3.2636327 \\ \text { C } & 1.4172816 & -0.6246890 & 0.4216175 \\ \text { C } & 2.4343250 & 0.1516690 & 0.8653049 \\ \text { C } & 3.7424426 & 0.1745275 & 0.1564933 \\ \text { C } & 4.4271992 & -1.0117949 & -0.1845374 \\ \text { C } & 5.6511649 & -0.9591140 & -0.8665965 \\ \text { C } & 6.2070648 & 0.2817672 & -1.2209320 \\ \text { C } & 5.5271651 & 1.4677433 & -0.8939014 \\ \text { C } & 4.3045303 & 1.4201154 & -0.2085976 \\ \text { Br } & 0.7934913 & 2.8221558 & -0.4232654 \\ \text { C } & 1.6981970 & -1.2387661 & 3.3870161 \\ \text { C } & 2.3362436 & -2.5368038 & 2.9799379 \\ \text { C } & 0.3727896 & -1.1686597 & 3.7616251 \\ \text { C } & 2.5856310 & -0.0195247 & 3.3800815 \\ \text { C } & 2.3304098 & 0.9367688 & 2.1684740 \\ \text { D } & 1.0833697 & 1.5542683 & 2.3637617 \\ \text { H } & 3.1552215 & 1.6856347 & 2.1872932 \\ \text { H } & 2.4168346 & 0.5896274 & 4.2909364 \\ \text { H } & 3.6496273 & -0.3267181 & 3.3578010 \\ \text { H } & -0.0257281 & -0.2480047 & 4.2189818\end{array}$




\begin{tabular}{|c|c|c|c|}
\hline & -0.2151830 & -2.0948352 & 3.8841430 \\
\hline & 2.8272082 & -2.4249385 & 1.9911592 \\
\hline & 1.6095823 & -3.3699954 & 2.9289933 \\
\hline & 3.1375971 & -2.8025348 & 3.7029167 \\
\hline & 0.8956586 & 2.0638127 & 1.4906604 \\
\hline & 3.9860287 & -1.9838894 & 0.0820818 \\
\hline & 6.1737731 & -1.8932385 & -1.1235314 \\
\hline & 7.1669471 & 0.3240920 & -1.7580564 \\
\hline & 5.9454360 & 2.4425239 & -1.1883042 \\
\hline & 3.7308592 & 2.3388566 & -0.0088576 \\
\hline & 0.8548303 & -3.2702130 & 0.0859265 \\
\hline & 0.7967756 & -4.5172774 & -2.0949248 \\
\hline & 1.2402336 & -3.2946855 & -4.2411585 \\
\hline & 1.7082985 & -0.8334858 & -4.2051986 \\
\hline & 1.7282390 & 0.4141147 & -2.0095841 \\
\hline d & -0.1826198 & -0.6561195 & 1.6150316 \\
\hline & -1.7313559 & -0.1668898 & 0.0149495 \\
\hline & -1.6178302 & -0.3088271 & -1.8024497 \\
\hline & -1.1779829 & 0.8185045 & -2.5324579 \\
\hline & -0.851 & 2785 & -1.994 \\
\hline & -1.1121308 & 2824 & -3.9310318 \\
\hline $\mathrm{H}$ & -0.7706420 & 7749 & -4.4943418 \\
\hline & -1.4588561 & -0.43 & -4.6038627 \\
\hline $\mathrm{H}$ & -1.4010189 & -0.4805681 & -5.7020731 \\
\hline $\mathrm{C}$ & -1.8748837 & -1.5571030 & -3.8730824 \\
\hline $\mathrm{H}$ & -2.1 & -2.48 & -4.394 \\
\hline $\mathrm{C}$ & -1.9 & -1.49 & -2.4748091 \\
\hline & -2.30 & -2.3 & -1.91 \\
\hline & -2.5 & 027 & 0.31 \\
\hline C & -2.1 & 2.1 & 1.4 \\
\hline & -1.3 & & 2.0 \\
\hline & -2.7 & & 528 \\
\hline & -2.4 & & 2 . \\
\hline & -3.7 & & 740 \\
\hline & -4.2 & & \\
\hline C & -4.1 & & -0.2 \\
\hline $\mathrm{H}$ & -4.8 & & -0.9625 \\
\hline $\mathrm{C}$ & -3.51107 & 7856 & -0.556440 \\
\hline $\mathrm{H}$ & -3.7943515 & 9208 & -1.453676 \\
\hline $\mathrm{C}$ & -3.0007764 & -1.4063178 & 0.526774 \\
\hline $\mathrm{C}$ & -4.3124328 & -1.0426560 & 0.893911 \\
\hline $\mathrm{H}$ & -4.6193351 & 0.0122847 & 0.850818 \\
\hline & -5.2211613 & -2.0253673 & 1.319741 \\
\hline $\mathrm{H}$ & -6.2418925 & -1.7320016 & 1.608296 \\
\hline C & -4.8330479 & -3.3735930 & 1.379308 \\
\hline & -5.5491013 & -4.1394539 & 1.713682 \\
\hline
\end{tabular}




$\begin{array}{llll}\text { C } & -3.5241984 & -3.7418705 & 1.0203955 \\ \text { H } & -3.2117851 & -4.7958212 & 1.0724327 \\ \text { C } & -2.6080665 & -2.7641473 & 0.6082974 \\ \text { H } & -1.5742985 & -3.0431220 & 0.3522518\end{array}$

S4

75

$\mathrm{SCF}$ Energy $=-4548.308208183$

C $\quad 1.6674408 \quad-0.4441330$

C $\quad 1.4222394 \quad-1.3239031$

$\begin{array}{lll}\text { C } & 1.1177369 & -2.6815662\end{array}$

$\begin{array}{lll}\text { C } & 1.0915486 & -3.1585487\end{array}$

$\begin{array}{lll}\text { C } & 1.3628137 & -2.2843591\end{array}$

C $\quad 1.6482925 \quad-0.9324126$

$\begin{array}{lll}\text { C } & 1.4917387 & -0.7993273\end{array}$

$\begin{array}{lll}\text { C } & 2.4643037 & -0.0707980\end{array}$

$\begin{array}{lll}\text { C } & 3.7984354 & 0.0503745\end{array}$

$\begin{array}{lll}\text { C } & 4.5130783 & -1.0935361\end{array}$

$-2.1255723$

$-1.0420155$

$-1.3068822$

$-2.6225639$

$-3.6911382$

$-3.4375465$

0.3171237

0.9087343

0.2592287

$-0.1574741$

$\begin{array}{lll}\text { C } & 5.7638943 & -0.9649909\end{array}$

$-0.7784701$

6.3152669

0.3095682

$-0.9924561$

5.6067863

1.4533337

$-0.5845161$

4.3574746

1.3305241

0.0404858

$\mathrm{Br} \quad 0.9570940$

2.8194049

$-0.1674751$

$\begin{array}{ll}-1.3612664 & 3.4716586 \\ -0.8790916 & 4.4786339\end{array}$

$\begin{array}{lll}\text { C } & 0.0784001 & -0.8790916\end{array}$

C $\quad 0.9117482-2.5446566$

2.7625620

$-0.5582521 \quad 3.3767078$

$\begin{array}{rr}2.3710699 & -0.5582521 \\ 2.2993847 & 0.5636263\end{array}$

$\begin{array}{lll}0 & 1.0970620 & 1.2649407\end{array}$

2.2900891

2.4740287

$3.1837370 \quad 1.2246280$

2.4478941

4.3527910

$-0.0636160$

3.1550362

$\begin{array}{ll}2.5495686 & -0.0636160 \\ 3.2422390 & -1.2043531\end{array}$

2.1864471

$\begin{array}{ll}1.7407016 & -2.9823517 \\ 0.0670217 & -3.2147626\end{array}$

2.9995339

$-0.0680687$

0.2131996

4.3657002

$-1.0544175 \quad 5.5077445$

$-0.8947663 \quad-1.4018779$

4.3877612

$1.8819816 \quad 1.6590788$

1.0003685

0.0088455

4.0737404

$-2.0885530$

$-1.8653058$

$-1.0969925$

6.3114453

0.4118978

$-1.4825119$

7.2956619

2.4552460

$-0.7676979$

3. 7626603

2.2203980

0.3017479

0.9173370

$-3.3572803$

$-0.4607965$

$\mathrm{H} \quad 0.8617354$

$-4.2168013$

$-2.8193913$

$\mathrm{H} \quad 1.3380934$

$-2.6572261$

$-4.7262466$ 


$\begin{array}{lrrr}\text { H } & 1.8383634 & -0.2439783 & -4.2738730 \\ \mathrm{H} & 1.8516590 & 0.6187561 & -1.9010861 \\ \mathrm{Pd} & -0.1395958 & -1.0878610 & 1.4110347 \\ \mathrm{P} & -1.6843392 & -0.2065981 & -0.0732881 \\ \mathrm{C} & -2.4083417 & 1.3803744 & 0.4570519 \\ \mathrm{C} & -1.9852776 & 1.9462007 & 1.6746670 \\ \mathrm{H} & -1.1795906 & 1.4609285 & 2.2457088 \\ \mathrm{C} & -2.5560720 & 3.1467738 & 2.1202856 \\ \mathrm{H} & -2.2089794 & 3.5958153 & 3.0624672 \\ \mathrm{C} & -3.5379759 & 3.7889950 & 1.3488906 \\ \mathrm{H} & -3.9736693 & 4.7393283 & 1.6929323 \\ \mathrm{C} & -3.9525438 & 3.2305287 & 0.1273981 \\ \mathrm{H} & -4.7127700 & 3.7397425 & -0.4840011 \\ \mathrm{C} & -3.3900194 & 2.0269030 & -0.3229987 \\ \mathrm{H} & -3.7026433 & 1.5963798 & -1.2859117 \\ \mathrm{C} & -3.0027523 & -1.4634144 & 0.2348736 \\ \mathrm{C} & -4.3090156 & -1.1157113 & 0.6345814 \\ \mathrm{H} & -4.5845449 & -0.0562641 & 0.7379101 \\ \mathrm{C} & -5.2520536 & -2.1202392 & 0.9065286 \\ \mathrm{H} & -6.2688055 & -1.8392909 & 1.2204464 \\ \mathrm{C} & -4.9037831 & -3.4750748 & 0.7808622 \\ \mathrm{H} & -5.6469034 & -4.2578649 & 0.9954167 \\ \mathrm{C} & -3.6009864 & -3.8292333 & 0.3889868 \\ \mathrm{H} & -3.3196203 & -4.8889784 & 0.2947845 \\ \mathrm{C} & -2.6513386 & -2.8310531 & 0.1274447 \\ \mathrm{H} & -1.6230869 & -3.1050104 & -0.1577865 \\ \mathrm{C} & -1.5475144 & -0.0877365 & -1.8928073 \\ \mathrm{C} & -1.8882130 & -1.1672590 & -2.7319618 \\ \mathrm{H} & -2.2531906 & -2.1124343 & -2.3071602 \\ \mathrm{C} & -1.7757282 & -1.0302342 & -4.1229720 \\ \mathrm{H} & -2.0404169 & -1.8766779 & -4.7741782 \\ \mathrm{C} & -1.3338923 & 0.1809795 & -4.6808761 \\ \mathrm{H} & -1.2540009 & 0.2856059 & -5.7738104 \\ \mathrm{C} & -0.9887500 & 1.2529557 & -3.8420848 \\ \mathrm{H} & -0.6257981 & 2.2002080 & -4.2686147 \\ \mathrm{C} & -1.0829399 & 1.1247786 & -2.4492239 \\ \mathrm{H} & -0.7548274 & 1.9459916 & -1.7855997\end{array}$

\section{Optimized Geometries(MXnb)}

R1

75

SCF Energy $=-4548.327011132$ 


$\begin{array}{lrrr}\text { C } & -0.4729729 & -0.9931025 & -2.9168287 \\ \text { C } & -1.0686939 & -1.2521951 & -1.6572436 \\ \text { C } & -2.4381620 & -1.6108454 & -1.6193472 \\ \text { C } & -3.1783622 & -1.7167626 & -2.8046615 \\ \text { C } & -2.5753273 & -1.4531475 & -4.0466172 \\ \text { C } & -1.2178760 & -1.0916973 & -4.0965657 \\ \text { C } & -0.2717465 & -1.1033781 & -0.4278202 \\ \text { C } & 0.9360861 & -1.6511397 & -0.1353850 \\ \text { C } & 1.5089050 & -2.6677062 & -1.0690422 \\ \text { C } & 0.7241458 & -3.7558317 & -1.5177287 \\ \text { C } & 1.2334848 & -4.6871280 & -2.4309707 \\ \text { C } & 2.5430987 & -4.5535166 & -2.9226426 \\ \text { C } & 3.3325519 & -3.4750670 & -2.4929115 \\ \text { C } & 2.8243430 & -2.5400312 & -1.5777083 \\ \text { Br } & -2.5857423 & -1.4728610 & 2.1624791 \\ \text { C } & 1.3997690 & -3.5652252 & 2.2303651 \\ \text { C } & 0.1734991 & -4.3378688 & 1.8259596 \\ \text { C } & 2.6085233 & -4.1502396 & 2.4065356 \\ \text { C } & 1.2182911 & -2.0749097 & 2.3995578 \\ \text { C } & 1.7035693 & -1.2833018 & 1.1510939 \\ \text { O } & 3.1065211 & -1.4034542 & 1.0115236 \\ \text { H } & 1.5025482 & -0.2106276 & 1.3545515 \\ \text { H } & 0.1544191 & -1.8282973 & 2.5896019 \\ \text { H } & 1.8185469 & -1.6944968 & 3.2514549 \\ \text { H } & 3.4711198 & -3.5812666 & 2.7908766 \\ \text { H } & 2.7592634 & -5.2260475 & 2.2232900 \\ \text { H } & -0.3796788 & -3.8155699 & 1.0209869 \\ \text { H } & -0.5347847 & -4.3826489 & 2.6815134 \\ \text { H } & 0.4089569 & -5.3699302 & 1.5027141 \\ \text { H } & 3.2926758 & -2.3693346 & 1.0317473 \\ \text { H } & -0.3033160 & -3.8630592 & -1.1449447 \\ \text { H } & 0.6010573 & -5.5251108 & -2.7615870 \\ \text { H } & 2.9445128 & -5.2852747 & -3.6401509 \\ \text { H } & 4.3567326 & -3.3536235 & -2.8786769 \\ \text { H } & 3.4392504 & -1.6937090 & -1.2492650 \\ \text { H } & -2.9075271 & -1.8006654 & -0.6416947 \\ \text { H } & -4.2396090 & -2.0048351 & -2.7563527 \\ \text { H } & -3.1626278 & -1.5255980 & -4.9746164 \\ \text { H } & -0.7362397 & -0.8792005 & -5.0626611 \\ \text { H } & 0.5915427 & -0.7244157 & -2.9622600 \\ \text { Pd } & -1.1962816 & 0.0712840 & 0.8842850 \\ \text { P } & -0.2373501 & 1.8447653 & -0.1191869 \\ \text { C } & -0.7813520 & 3.3221552 & 0.8220194 \\ \text { C } & -1.0576441 & 4.5498208 & 0.1875287 \\ \text { C } & -0.9547873 & 4.6369353 & -0.9043759 \\ & -1.4697963 & 5.6520579 & 0.9517863\end{array}$




$\begin{array}{rrrr}\text { H } & -1.6875621 & 6.6094334 & 0.4547076 \\ \mathrm{C} & -1.6085104 & 5.5348240 & 2.3457997 \\ \mathrm{H} & -1.9367118 & 6.4010092 & 2.9399705 \\ \mathrm{C} & -1.3367808 & 4.3111255 & 2.9806181 \\ \mathrm{H} & -1.4518168 & 4.2136321 & 4.0703934 \\ \mathrm{C} & -0.9275620 & 3.2043707 & 2.2214445 \\ \mathrm{H} & -0.7211258 & 2.2379890 & 2.7120313 \\ \mathrm{C} & 1.5857143 & 1.9763037 & -0.1940668 \\ \mathrm{C} & 2.3061058 & 1.1533700 & -1.0877301 \\ \mathrm{H} & 1.7700620 & 0.4875606 & -1.7763110 \\ \mathrm{C} & 3.7066710 & 1.1730697 & -1.0844918 \\ \mathrm{H} & 4.2583208 & 0.5259640 & -1.7820718 \\ \mathrm{C} & 4.4017648 & 2.0014493 & -0.1889315 \\ \mathrm{H} & 5.5019120 & 2.0049895 & -0.1821902 \\ \mathrm{C} & 3.6893114 & 2.8197085 & 0.7020079 \\ \mathrm{H} & 4.2281617 & 3.4716575 & 1.4058428 \\ \mathrm{C} & 2.2861021 & 2.8111742 & 0.7013433 \\ \mathrm{H} & 1.7324845 & 3.4546030 & 1.4004030 \\ \mathrm{C} & -0.8408387 & 2.1116792 & -1.8244079 \\ \mathrm{C} & -2.1798241 & 1.7524908 & -2.0900806 \\ \mathrm{H} & -2.7947517 & 1.3205202 & -1.2850676 \\ \mathrm{C} & -2.7048482 & 1.9091637 & -3.3780534 \\ \mathrm{H} & -3.7427156 & 1.6096476 & -3.5826162 \\ \mathrm{C} & -1.8968658 & 2.4183308 & -4.4095317 \\ \mathrm{H} & -2.3054213 & 2.5249699 & -5.4257813 \\ \mathrm{C} & -0.5669160 & 2.7832077 & -4.1466153 \\ \mathrm{H} & 0.0659015 & 3.1843386 & -4.9526756 \\ \mathrm{C} & -0.0358414 & 2.6344358 & -2.8548413 \\ \mathrm{H} & 1.0074090 & 2.9157358 & -2.6505473\end{array}$

R2

75

SCF Energy $=-4548.325584991$

C $\quad 0.9488272 \quad-0.7999505$

C $\quad-0.2309964-1.0310193$

C $\quad-1.4650803 \quad-1.1504501$

C $\quad-1.5139338-1.0306366$

$\begin{array}{lll}\text { C } & -0.3391722 & -0.7814017\end{array}$

$\begin{array}{lll}\text { C } & 0.8905401 & -0.6745064\end{array}$

C $\quad-0.1570888-1.0711916$

C $\quad 0.6904835-1.7713876$

$\begin{array}{lll}\text { C } & 1.5505417 & -2.8242612\end{array}$

$-2.7601126$

$-2.0090645$

$-2.6973094$

$-4.0921536$

$-4.8257996$

$-4.1540801$

$-0.5420106$

$\begin{array}{lll}1.5505417 & -2.8242612 \\ \text { C } & 0.9647108 & -3.8662146\end{array}$

0.2467422

$-0.3678945$

$-1.1219006$

$\begin{array}{llll}\text { C } & 1.7582016 & -4.8609413 & -1.7062908\end{array}$

$\begin{array}{llll}\text { C } & 3.1554936 & -4.8389894 & -1.5475384\end{array}$

$\begin{array}{llll}\text { C } & 3.7514122 & -3.8051616 & -0.8090397\end{array}$ 


\begin{tabular}{lrrr} 
C & 2.9577139 & -2.8035081 & -0.2254348 \\
$\mathrm{Br}$ & -3.3733708 & -1.3397922 & 0.6163432 \\
$\mathrm{C}$ & -0.1218739 & -4.0190974 & 2.1307266 \\
$\mathrm{C}$ & 1.1535731 & -4.7388322 & 2.4979864 \\
$\mathrm{C}$ & -1.1300770 & -4.6399100 & 1.4828991 \\
$\mathrm{C}$ & -0.2378286 & -2.5561927 & 2.4982665 \\
$\mathrm{C}$ & 0.7164491 & -1.5801617 & 1.7667016 \\
$\mathrm{O}$ & 2.0393279 & -1.5777201 & 2.3073790 \\
$\mathrm{H}$ & 0.3266274 & -0.5577842 & 1.9664981 \\
$\mathrm{H}$ & -1.2714819 & -2.2157201 & 2.2879040 \\
$\mathrm{H}$ & -0.0496271 & -2.4131638 & 3.5860851 \\
$\mathrm{H}$ & -2.0437597 & -4.0924213 & 1.2011368 \\
$\mathrm{H}$ & -1.0613701 & -5.7046550 & 1.2065065 \\
$\mathrm{H}$ & 1.4342108 & -4.5574253 & 3.5574191 \\
$\mathrm{H}$ & 2.0051161 & -4.3950341 & 1.8669533 \\
$\mathrm{H}$ & 1.0706698 & -5.8314694 & 2.3386671 \\
$\mathrm{H}$ & 2.4042644 & -2.4763759 & 2.1885901 \\
$\mathrm{H}$ & -0.1285677 & -3.8890200 & -1.2263663 \\
$\mathrm{H}$ & 1.2818236 & -5.6657454 & -2.2866723 \\
$\mathrm{H}$ & 3.7774937 & -5.6240697 & -2.0037573 \\
$\mathrm{H}$ & 4.8456140 & -3.7688653 & -0.6925625 \\
$\mathrm{H}$ & 3.4271051 & -1.9765708 & 0.3215987 \\
$\mathrm{H}$ & -2.3804780 & -1.3299039 & -2.1127781 \\
$\mathrm{H}$ & -2.4792291 & -1.1288847 & -4.6116955 \\
$\mathrm{H}$ & -0.3826656 & -0.6745541 & -5.9204369 \\
$\mathrm{H}$ & 1.8160310 & -0.4863377 & -4.7192362 \\
$\mathrm{H}$ & 1.9093296 & -0.7211652 & -2.2343290 \\
$\mathrm{Pd}$ & -1.4947877 & 0.1611862 & 0.2502883 \\
$\mathrm{P}$ & -0.0564674 & 1.8907595 & 0.0054620 \\
$\mathrm{C}$ & 0.2316296 & 2.8049445 & 1.5680142 \\
$\mathrm{C}$ & 1.2992078 & 3.7178422 & 1.7009123 \\
$\mathrm{H}$ & 1.9969692 & 3.8700939 & 0.8637294 \\
$\mathrm{C}$ & 1.4741837 & 4.4182143 & 2.9039871 \\
$\mathrm{H}$ & 2.3097475 & 5.1269872 & 3.0066814 \\
$\mathrm{C}$ & 0.5898431 & 4.2105910 & 3.9769374 \\
$\mathrm{H}$ & 0.7337315 & 4.7575201 & 4.9210106 \\
$\mathrm{C}$ & -1.2074975 & 1.1933221 & -1.7002906 \\
$\mathrm{C}$ & -0.4705160 & 3.2978258 & 3.8492390 \\
$\mathrm{H}$ & -1.1575034 & 3.1248382 & 4.6909602 \\
$\mathrm{C}$ & -0.6485186 & 2.5935088 & 2.6487947 \\
$\mathrm{H}$ & -1.4645660 & 1.8583562 & 2.5479147 \\
$\mathrm{C}$ & 1.6301253 & 1.6653684 & -0.6742130 \\
$\mathrm{H}$ & 1.9919511 & 2.1702699 & -1.9380168 \\
\hline
\end{tabular}




$\begin{array}{lrrr}\text { H } & 5.2145921 & 1.0040292 & -2.1013913 \\ \mathrm{C} & 3.8489761 & 0.6964175 & -0.4343790 \\ \mathrm{H} & 4.5767766 & 0.1246610 & 0.1602922 \\ \mathrm{C} & 2.5652706 & 0.9235737 & 0.0808621 \\ \mathrm{H} & 2.3039027 & 0.5111566 & 1.0663906 \\ \mathrm{C} & -0.8549217 & 3.0832436 & -1.1395794 \\ \mathrm{C} & -1.3761418 & 2.5687473 & -2.3492853 \\ \mathrm{H} & -1.3072654 & 1.4911278 & -2.5651301 \\ \mathrm{C} & -1.9828938 & 3.4330688 & -3.2708911 \\ \mathrm{H} & -2.3818479 & 3.0268269 & -4.2122920 \\ \mathrm{C} & -2.0920269 & 4.8058558 & -2.9863782 \\ \mathrm{H} & -2.5783136 & 5.4807368 & -3.7069558 \\ \mathrm{C} & -1.5889339 & 5.3142393 & -1.7776795 \\ \mathrm{H} & -1.6810582 & 6.3867519 & -1.5487350 \\ \mathrm{C} & -0.9678221 & 4.4576733 & -0.8537911 \\ \mathrm{H} & -0.5748332 & 4.8573040 & 0.0925000\end{array}$

R3

75

SCF Energy $=-4548.324308437$$$
\text { c }
$$

$$
-0.4879848
$$

2.4669046

$-1.0717938$

1.9361655

$-1.5398595$

$-1.4324894$

2.8350325

4.2185135

$-0.8470680$

4.7333322

$-0.3773921$

$-1.1445539$

3.8511875

0.4738774

$-1.6213036$

$-0.4228666$

$-2.4034437$

0.0634831

$-3.5861271$

0.8134672

1.2805498

1.0042452

$-3.9056785$

$-2.7297581$

$-1.9827513$

0.2662874

$-0.1976468$

0.4370570

Br $\quad-1.8690313$

$-3.1846968$

$-4.1575867$

$-2.6260695$

$-2.2769250$

$-1.1454207$

$-2.9355793$

$-2.7882553$

$-2.6254167$

$-1.4808254$

$-1.9329298$

$-1.7802026$

$-0.6025750$

0.3886273

0.1985299

$-0.9724436$

$-1.9600640$

$-0.4355312$

$-1.3367723$

$-2.5138737$

$-2.3235749$

$-3.4172114$

$-4.7290520$

$-4.9353394$

$-3.8401471$

3.2713283

0.7036980

0.8950206

1.7416585

$-0.7177531$

$-0.9631725$

$-2.5641901$

$-1.1674929$

$-0.7236338$

$-2.1161924$

$-2.3447449$

$-2.5981824$

$-3.7144383$

$-0.3811003$

$-3.5992493$

$-2.3658479$

$-0.8082829$

$-1.9188528$

$-3.7646609$

$-1.4337823$

1.5741089

$-2.8427308$

$-2.6542641$

2.7827306 


\begin{tabular}{|c|c|c|c|}
\hline & -5.0972588 & -1.3318447 & 0.3317488 \\
\hline H & -3.7278306 & -0.1987402 & 0.5063803 \\
\hline & -4.3951318 & -0.9828718 & 1.9619528 \\
\hline & -1.6331413 & -2.4478631 & -3.0486147 \\
\hline & -3.9155612 & 1.0318915 & -1.2979539 \\
\hline & -5.2431922 & 1.8658663 & -3.2434252 \\
\hline & -4.4880759 & 1.3709598 & -5.5877725 \\
\hline & -2.3783567 & 0.0623179 & -5.9585847 \\
\hline & -1.0325295 & -0.7213950 & -4.0096044 \\
\hline & -1.9787809 & 2.4299454 & 1.3134978 \\
\hline & -1.8068124 & 4.9022372 & 0.9755911 \\
\hline & -0.7562057 & 5.8212994 & -1.1133624 \\
\hline & 0.0827592 & 4.2419751 & -2.88 \\
\hline & -0.1270664 & 1.7774572 & -2.55 \\
\hline & -0.4407909 & -0.0404945 & 1.3 \\
\hline & 1.5332931 & -0.4710071 & 0.3672150 \\
\hline & 2.7868963 & 0.2518895 & 1.5011058 \\
\hline & 2.4672820 & 1.4608890 & 2.1572402 \\
\hline & 1.4725782 & 1.9121175 & 2.007209 \\
\hline & 3.4064679 & 2.0662073 & 3.0055263 \\
\hline & 3.1517588 & 3.0069009 & 3.5160147 \\
\hline & 4.6585944 & 1.4633824 & 3.2132419 \\
\hline & 5.3904248 & 1.9332327 & 3.8876781 \\
\hline & 4.9728132 & 0.2540743 & 2.5698071 \\
\hline & 5.9495890 & -0.2238297 & 2.7391269 \\
\hline C & 4.0415318 & -0.3533545 & 1.7134580 \\
\hline & 4.2863961 & -1.3036133 & 1.2165303 \\
\hline C & 1.9399660 & -2.2554792 & 0.2720456 \\
\hline C & 1.2587645 & -3.1294322 & 1.1452512 \\
\hline $\mathrm{H}$ & 0.4791491 & -2.7261828 & 1.8126821 \\
\hline C & 1.5568167 & -4.4994064 & 1.1417055 \\
\hline $\mathrm{H}$ & 1.0182239 & -5.1763388 & 1.8215848 \\
\hline C & 2.5316433 & -5.0045628 & 0.2644721 \\
\hline & 2.7589040 & -6.0812773 & 0.2553925 \\
\hline C & 3.2109039 & -4.1368226 & -0.6070108 \\
\hline & 3.9711252 & -4.5317775 & -1.2977536 \\
\hline C & 2.9195865 & -2.7635737 & -0.6055261 \\
\hline$\Pi$ & 3.4458710 & -2.0868171 & -1.2946525 \\
\hline C & 1.9642070 & 0.2094656 & -1.2775951 \\
\hline C & 2.6521039 & 1.4345602 & -1.3817055 \\
\hline $\mathrm{H}$ & 2.9916933 & 1.9541575 & -0.4743518 \\
\hline C & 2.9016036 & 1.9967912 & -2.6438103 \\
\hline $\mathrm{H}$ & 3.4358633 & 2.9558567 & -2.7147858 \\
\hline C & 2.4663801 & 1.3414306 & -3.8057478 \\
\hline $\mathrm{H}$ & 2.6617979 & 1.7821470 & -4.7949621 \\
\hline & 1.7757330 & 0.1206560 & -3.701698 \\
\hline
\end{tabular}




$\begin{array}{llll}\mathrm{H} & 1.4313957 & -0.3957243 & -4.6105288 \\ \mathrm{C} & 1.5155645 & -0.4465052 & -2.4455440 \\ \mathrm{H} & 0.9468859 & -1.3875181 & -2.3832826\end{array}$

R4

75

SCF Energy $=-4548.324727949$

C

C

C

C

$\mathrm{C}$

C

C

$\mathrm{H}$

$\mathrm{H}$

$\mathrm{H}$

$\mathrm{H}$

$\mathrm{H}$

$\mathrm{H}$

$\mathrm{H} \quad-0.2989760$
$-0.7660744$

0.1393449

1.3823914

1.7002406

0.7958373

$-0.4384940$

$-0.2266739$

$-1.3941257$

$-2.3539020$

$-1.9278146$

$-2.8127783$

$-4.1470066$

$-4.5833486$

$-3.6955273$

2.9616304

$-0.4666859$

0.4440385

$-0.1027348$

$-1.8143378$

$-1.7428348$

$-2.9587972$

$-0.9503786$

$-2.2527035$

$-2.5211956$

$-0.7922452$

0.8989595

0.6418815

$-0.0235622$

1. 4267511

$-3.6931765$

$-0.8834658$

$-2.4583178$

$-4.8422269$

$-5.6207638$

$-4.0300854$

2.0927944

2.6669140

1.0539521

$-1.1556638$
-2.8260815
-1.8683751
-2.3184776
-3.6826589
-4.6227180
-4.1872426
-0.4412964
0.1158060
-0.7034997
-1.3242264
-2.0954832
-2.2603119
-1.6550826
-0.8868382
1.0210384
2.5649087
3.5815593
1.8293911
2.3838711
1.5834857
1.7503220
2.0146170
3.3722775
1.8709054
1.0965504
1.9254829
3.3229728
4.5905502
3.6296749
1.5258491
-1.1926771
-2.5716115
-2.8651709
-1.7939674
-0.4678151
-1.5785128
-4.0147783
-5.6927949
-9124512 


$\begin{array}{lrrr}\text { H } & -0.3299954 & -1.7334444 & -2.4879987 \\ \text { Pd } & -0.4367819 & 1.2548011 & 0.6214591 \\ \text { P } & 1.5716087 & 0.2400746 & 0.4816611 \\ \text { C } & 2.7624385 & 1.5941751 & 0.1257700 \\ \text { C } & 4.0554781 & 1.6278159 & 0.6842433 \\ \text { H } & 4.3757395 & 0.8373018 & 1.3787854 \\ \text { C } & 4.9286360 & 2.6775422 & 0.3592212 \\ \text { H } & 5.9362169 & 2.7044984 & 0.8010449 \\ \text { C } & 4.5181243 & 3.6925688 & -0.5218233 \\ \text { H } & 5.2050536 & 4.5157406 & -0.7701430 \\ \text { C } & 3.2274469 & 3.6630323 & -1.0768315 \\ \text { H } & 2.8982646 & 4.4610239 & -1.7589369 \\ \text { C } & 2.3463355 & 2.6213807 & -0.7502721 \\ \text { H } & 1.3244280 & 2.6007203 & -1.1639536 \\ \text { C } & 2.1485017 & -0.5129908 & 2.0500555 \\ \text { C } & 1.5686035 & -0.0609154 & 3.2533206 \\ \text { H } & 0.7691858 & 0.6972372 & 3.2154941 \\ \text { C } & 1.9984911 & -0.5810844 & 4.4823321 \\ \text { H } & 1.5365014 & -0.2274755 & 5.4160778 \\ \text { C } & 3.0074442 & -1.5583425 & 4.5165453 \\ \text { H } & 3.3387340 & -1.9738441 & 5.4802140 \\ \text { C } & 3.5889157 & -2.0105322 & 3.3200767 \\ \text { H } & 4.3770618 & -2.7781619 & 3.3450092 \\ \text { C } & 3.1639854 & -1.4907313 & 2.0875487 \\ \text { H } & 3.6135927 & -1.8527508 & 1.1513221 \\ \text { C } & 1.9177516 & -1.0219491 & -0.7996827 \\ \text { C } & 1.4993357 & -2.3542067 & -0.5862472 \\ \text { H } & 1.0053748 & -2.6414347 & 0.3560473 \\ \text { C } & 1.6915133 & -3.3099277 & -1.5946921 \\ \text { H } & 1.3672388 & -4.3480490 & -1.4265530 \\ \text { C } & 2.2853691 & -2.9486774 & -2.8173355 \\ \text { H } & 2.4275821 & -3.7031036 & -3.6057752 \\ \text { C } & 2.6911337 & -1.6222515 & -3.0303417 \\ \text { H } & 3.1495235 & -1.3292656 & -3.9865541 \\ \text { C } & 2.5085286 & -0.6586590 & -2.0255540 \\ \text { H } & 2.8243246 & 0.3799098 & -2.1992739 \\ & & & \\ \text { S1 } & & & \\ 75 & & & \\ \text { SCF } & \text { Energy }=-4548.309690220 & \\ \text { C } & -0.4469101 & 1.8876687 & -2.2690489 \\ \text { C } & -1.3790947 & 1.3636012 & -1.3394668 \\ \text { C } & -2.7447527 & 1.7252419 & -1.4930708 \\ \text { C } & -3.1680649 & 2.5229360 & -2.5632219 \\ \text { C } & -2.2342502 & 2.9931900 & -3.5015833 \\ & -0.8729105 & 2.6768414 & -3.3424559\end{array}$




\begin{tabular}{|c|c|c|c|}
\hline & -1.0361485 & 0.5559051 & -0.1619781 \\
\hline & -1.5801477 & -0.6106097 & 0.2886926 \\
\hline & -1.9294539 & -1.6885277 & -0.6913873 \\
\hline & -2.6312669 & -1.4975981 & -1.9036239 \\
\hline & -2.7793602 & -2.5428241 & -2.8278432 \\
\hline & -2.2237554 & -3.8073071 & -2.5761918 \\
\hline & -1.5126224 & -4.0123766 & -1.3819900 \\
\hline & -1.3700617 & -2.9725420 & -0.4554111 \\
\hline & -1.2892510 & 2.9801808 & 1.9746096 \\
\hline & -4.2245462 & -1.7808933 & 1.3761217 \\
\hline & -5.1666984 & -1.1208969 & 0.4053754 \\
\hline & -4.2120401 & -3.1148920 & 1.5739314 \\
\hline & -3.2789814 & -0.8666305 & 2.1336444 \\
\hline & -1.7658375 & -0.9764024 & 1.7736892 \\
\hline & -1.0302713 & -0.2290497 & 2.7177714 \\
\hline & -1.45 & -2.0 & 5395 \\
\hline & -3.56 & 0.1 & 1.9 \\
\hline & -3.3 & -1.0 & 3.2 \\
\hline & -3.5 & -3.5 & 2.2 \\
\hline & -4 & -3.7 & $1 . C$ \\
\hline & -4 . & -0.5 & -0.3 \\
\hline & -5.8 & -0.4 & 0.5 \\
\hline & -5.7 & -1.8 & -0.1 \\
\hline & -1.302 & 0.7 & 2.6 \\
\hline & -3.0624698 & -0.5173075 & -2.13 \\
\hline & -3.3398468 & -2.3608708 & -3.7577353 \\
\hline & -2.3410248 & -4.6240945 & -3.3042304 \\
\hline & -1.0549373 & -4.9900862 & -1.1675476 \\
\hline & -0.7788642 & -3.1500726 & 0.4533176 \\
\hline & -3.4623190 & 1.3880256 & -0.7315155 \\
\hline & -4.2318568 & 2.7885359 & -2.6 \\
\hline & -2.5626324 & 3.62 & -4.34 \\
\hline & -0.1297624 & 3.0 & -4.06 \\
\hline & 0.6198835 & 1.67 & -2.14 \\
\hline & 3.8062773 & 2.0 & 0.70 \\
\hline & 3.6137257 & 0.6233540 & 0.6214135 \\
\hline & 4.6875472 & -0.2483559 & 0.8912561 \\
\hline & 5.9437292 & 0.2756775 & 1.2324064 \\
\hline & 6.1351727 & 1.6665726 & 1.3053720 \\
\hline & 5.0659465 & 2.5388395 & 1.0412004 \\
\hline & 1.9426086 & 0.0238237 & 0.1527289 \\
\hline & 2.0310532 & -0.2011293 & -1.6659075 \\
\hline & 2.9561127 & 0.5194838 & -2.4483234 \\
\hline & 2.8756931 & 0.4711428 & $-3.849666 \theta$ \\
\hline & 1.8780577 & -0.2948825 & -4.4761640 \\
\hline & 0.9672300 & -1.0287127 & -3.697072 \\
\hline
\end{tabular}




$\begin{array}{lrrr}\text { C } & 1.0407570 & -0.9850421 & -2.2989709 \\ \text { Pd } & 0.3481649 & 1.4927509 & 0.9159328 \\ \text { C } & 1.8541740 & -1.6988852 & 0.7822373 \\ \text { C } & 2.2985927 & -2.7970591 & 0.0145677 \\ \text { C } & 2.2117391 & -4.0975250 & 0.5340377 \\ \text { C } & 1.6883752 & -4.3101724 & 1.8209727 \\ \text { C } & 1.2616708 & -3.2159520 & 2.5921267 \\ \text { C } & 1.3444811 & -1.9121021 & 2.0783061 \\ \text { H } & 2.6976786 & -2.6377327 & -0.9972212 \\ \text { H } & 2.5498143 & -4.9502029 & -0.0739574 \\ \text { H } & 1.6110634 & -5.3322905 & 2.2218476 \\ \text { H } & 0.8455170 & -3.3749749 & 3.5983247 \\ \text { H } & 0.9499129 & -1.0630540 & 2.6556738 \\ \text { H } & 3.7318311 & 1.1291593 & -1.9617046 \\ \text { H } & 3.5986348 & 1.0384492 & -4.4553152 \\ \text { H } & 1.8125366 & -0.3215376 & -5.5743424 \\ \text { H } & 0.1790061 & -1.6310137 & -4.1721622 \\ \text { H } & 0.3107550 & -1.5488404 & -1.7030794 \\ \text { H } & 4.5359105 & -1.3368176 & 0.8397717 \\ \text { H } & 6.7797565 & -0.4074234 & 1.4460741 \\ \text { H } & 7.1214117 & 2.0722216 & 1.5771789 \\ \text { H } & 5.2094982 & 3.6278491 & 1.1049870 \\ \text { H } & 2.9584132 & 2.6970469 & 0.5045058 \\ & & & \\ \text { S2 } & & & \\ \text { 75 } & & & \\ \text { SCF } & \text { Energy }=-4548.312853955 & \\ \text { C } & -1.9904918 & -0.4536652 & -2.1241148 \\ \text { C } & -1.3435821 & -1.3946903 & -1.2835712 \\ \text { C } & -1.6099252 & -2.7728458 & -1.5042941 \\ \text { C } & -2.4273325 & -3.1930837 & -2.5614406 \\ \text { C } & -3.0175646 & -2.2460644 & -3.4138985 \\ \text { C } & -2.8010224 & -0.8750167 & -3.1820950 \\ \text { C } & -0.5098284 & -1.0531775 & -0.1260795 \\ \text { C } & 0.6711012 & -1.5899923 & 0.2898468 \\ \text { C } & 1.7286292 & -1.9363046 & -0.7132847 \\ \text { C } & 1.5680802 & -2.7716911 & -1.8399966 \\ \text { C } & 2.5966799 & -2.9158859 & -2.7830542 \\ \text { C } & 3.8156457 & -2.2351868 & -2.6274119 \\ \text { C } & 3.9963394 & -1.4061892 & -1.5084393 \\ \text { C } & 2.9701218 & -1.2623705 & -0.5644004 \\ \text { Br } & -2.7929822 & -1.3075934 & 2.1712162 \\ \text { C } & 1.4247318 & -4.3637856 & 1.2440896 \\ \text { C } & 2.9307170 & -4.4283694 & 1.2303981 \\ \text { C } & 0.6629280 & -5.2323157 & 0.5450539 \\ & 0.7922550 & -3.3014129 & 2.1117445\end{array}$




$\begin{array}{lrrr}\text { C } & 1.0818175 & -1.8089236 & 1.7593355 \\ \mathrm{O} & 0.4717423 & -1.0047347 & 2.7464327 \\ \mathrm{H} & 2.1705838 & -1.6175776 & 1.8533039 \\ \mathrm{H} & -0.3122726 & -3.4145928 & 2.1043825 \\ \mathrm{H} & 1.1322248 & -3.4243728 & 3.1642534 \\ \mathrm{H} & -0.4370526 & -5.1908052 & 0.5913043 \\ \mathrm{H} & 1.1133454 & -6.0189329 & -0.0809659 \\ \mathrm{H} & 3.3274704 & -4.5462369 & 2.2621627 \\ \mathrm{H} & 3.3712000 & -3.4939467 & 0.8238714 \\ \mathrm{H} & 3.3007587 & -5.2701898 & 0.6145575 \\ \mathrm{H} & -0.4953696 & -1.2184491 & 2.7698225 \\ \mathrm{H} & 0.6325760 & -3.3239336 & -1.9771749 \\ \mathrm{H} & 2.4421524 & -3.5782513 & -3.6486728 \\ \mathrm{H} & 4.6187292 & -2.3528302 & -3.3705529 \\ \mathrm{H} & 4.9388550 & -0.8557013 & -1.3673585 \\ \mathrm{H} & 3.1170360 & -0.5820817 & 0.2864323 \\ \mathrm{H} & -1.1808552 & -3.5037910 & -0.8052427 \\ \mathrm{H} & -2.6156841 & -4.2671878 & -2.7101668 \\ \mathrm{H} & -3.6642698 & -2.5726188 & -4.2424416 \\ \mathrm{H} & -3.2760424 & -0.1228291 & -3.8295517 \\ \mathrm{H} & -1.8562181 & 0.6178746 & -1.9412427 \\ \mathrm{Pd} & -1.4002731 & 0.3283615 & 0.9991110 \\ \mathrm{P} & -0.0165694 & 1.9463460 & 0.1337531 \\ \mathrm{C} & 1.7428417 & 1.9136560 & 0.6557887 \\ \mathrm{C} & 2.7720418 & 2.4065635 & -0.1752974 \\ \mathrm{H} & 2.5322008 & 2.8107984 & -1.1691717 \\ \mathrm{C} & 4.1059060 & 2.3596569 & 0.2575025 \\ \mathrm{H} & 4.9048508 & 2.7368884 & -0.3986174 \\ \mathrm{C} & 4.4202538 & 1.8266441 & 1.5196303 \\ \mathrm{H} & 5.4684981 & 1.7810550 & 1.8521224 \\ \mathrm{C} & 3.3946652 & 1.3477457 & 2.3519696 \\ \mathrm{H} & 3.6337665 & 0.9208790 & 3.3376629 \\ \mathrm{C} & 2.0576928 & 1.3905599 & 1.9253432 \\ \mathrm{H} & 1.2638394 & 0.9512470 & 2.5477436 \\ \mathrm{C} & -0.6370142 & 3.6040535 & 0.6224623 \\ \mathrm{C} & 0.2162953 & 4.7023883 & 0.8486668 \\ \mathrm{H} & 1.3050174 & 4.5803826 & 0.7482234 \\ \mathrm{C} & -0.3258487 & 5.9453926 & 1.2096573 \\ \mathrm{H} & 0.3432709 & 6.8004908 & 1.3894179 \\ \mathrm{C} & -1.7165737 & 6.0993514 & 1.3457129 \\ \mathrm{H} & -2.1362295 & 7.0755205 & 1.6322079 \\ \mathrm{C} & -2.5703850 & 5.0054791 & 1.1255200 \\ \mathrm{C} & -3.6588187 & 5.1193929 & 1.2393113 \\ & -2.0324814 & 3.7589960 & 0.7704038 \\ & 0.0997889 & 2.0203160 & -1.6957328\end{array}$




$\begin{array}{lrrr}\mathrm{C} & 0.8526199 & 1.0273383 & -2.3614551 \\ \mathrm{H} & 1.4530403 & 0.3069679 & -1.7896898 \\ \mathrm{C} & 0.8195632 & 0.9409382 & -3.7589115 \\ \mathrm{H} & 1.3987010 & 0.1519320 & -4.2608205 \\ \mathrm{C} & 0.0388690 & 1.8407146 & -4.5047025 \\ \mathrm{H} & 0.0049384 & 1.7643483 & -5.6019700 \\ \mathrm{C} & -0.6962077 & 2.8408892 & -3.8464249 \\ \mathrm{H} & -1.2995505 & 3.5554810 & -4.4266416 \\ \mathrm{C} & -0.6673219 & 2.9346354 & -2.4453293 \\ \mathrm{H} & -1.2523473 & 3.7127500 & -1.9329213\end{array}$

S3

75

SCF Energy $=-4548.330436499$

$\begin{array}{lll}\text { C } & -1.1080454 & -0.5678198\end{array}$

C $\quad-1.3253599 \quad-0.9793852$

$-2.4444733$

$-1.1061965$

$\begin{array}{lll}-1.325359 & -0.952081 & -1.2528168\end{array}$

$-0.6958182$

$-3.7212137-1.1087189$

$-1.5925090$

$-3.4911784$

$-0.6798110$

$-2.9119752$

$-2.1775662$

$-0.4155327$

$-3.3343360$

$-0.1983868$

$-1.0614847$

$-0.1567203$

0.9858622

$-1.7053693$

$-0.3276978$

C $\quad 1.1957197$

$-2.5599431$

$-1.5339031$

C $\quad 0.2171091$

$-3.4898666$

$-1.9549469$

C $\quad 0.4172840$

$-4.2813139$

$-3.0931586$

C $\quad 1.6051923$

$-4.1695967$

$-3.8362437$

C 2.5934544

$-3.2621190$

$-3.4217600$

C $\quad 2.3935137$

$-2.4691129$

$-2.2814782$

$\mathrm{Br} \quad-1.6387659$

$-1.6573014$

2.9721845

$-3.6827725$

2.0759309

C $\quad 0.4409832$

$-4.3772221$

1.5109245

$-3.5146571$

3.4073050

1.8266398

$-3.0752607$

1.0958731

2.1825829

$-1.6529857$

0.6364641

$-0.8221851$

1.7685698

$-1.2087700$

0.0654721

3.0280806

$-2.9547491$

1.5761433

2.7606843

$-3.7233835$

0.2062543

2.7162274

$-3.0021327$

3.8080075

1.0914604

$-3.8938036$

4.1336942

0.7296885

$-5.1105057$

0.7293186

$-0.2352363$

$-3.6378909$

1.0337849

$-0.1396653$

$-4.8918805$

2. 2992587

1.4894976

$-1.3428654$

2.4172112

$-0.7101205$

$-3.5885899$

$-1.3742389$

$\mathrm{H}$

$-0.3606792$

$-4.9969530$

$-3.3999216$ 


$\begin{array}{lrrr}\text { H } & 1.7625418 & -4.7926038 & -4.7296605 \\ \mathrm{H} & 3.5308420 & -3.1680882 & -3.9916401 \\ \mathrm{H} & 3.1707921 & -1.7551405 & -1.9755712 \\ \mathrm{H} & -2.8309897 & -1.5671444 & 0.3445078 \\ \mathrm{H} & -4.7453259 & -1.3285503 & -1.2541617 \\ \mathrm{H} & -4.3332792 & -0.5534249 & -3.6092228 \\ \mathrm{H} & -1.9834228 & -0.0832470 & -4.3652437 \\ \mathrm{H} & -0.0808059 & -0.3834704 & -2.7854583 \\ \mathrm{Pd} & -0.6164003 & -0.0241092 & 1.4790303 \\ \mathrm{P} & 0.2673816 & 1.7743836 & 0.4473204 \\ \mathrm{C} & -0.7887682 & 2.4888907 & -0.8633024 \\ \mathrm{C} & -0.2917130 & 3.3158011 & -1.8921455 \\ \mathrm{H} & 0.7784365 & 3.5679962 & -1.9294061 \\ \mathrm{C} & -1.1640790 & 3.8040588 & -2.8777669 \\ \mathrm{H} & -0.7733524 & 4.4433797 & -3.6839157 \\ \mathrm{C} & -2.5297295 & 3.4747286 & -2.8381117 \\ \mathrm{H} & -3.2073549 & 3.8495954 & -3.6201692 \\ \mathrm{C} & -3.0291613 & 2.6644027 & -1.8048817 \\ \mathrm{H} & -4.0947915 & 2.3952683 & -1.7744436 \\ \mathrm{C} & -2.1624001 & 2.1718782 & -0.8205615 \\ \mathrm{H} & -2.5348671 & 1.5082648 & -0.0242556 \\ \mathrm{C} & 0.4293113 & 3.0689573 & 1.7373292 \\ \mathrm{C} & 0.8322619 & 2.6586255 & 3.0283382 \\ \mathrm{H} & 1.0367890 & 1.5922587 & 3.2191755 \\ \mathrm{C} & 0.9651722 & 3.6050639 & 4.0554105 \\ \mathrm{H} & 1.2788550 & 3.2805584 & 5.0588240 \\ \mathrm{C} & 0.6855280 & 4.9589264 & 3.8038673 \\ \mathrm{H} & 0.7814582 & 5.6997475 & 4.6119958 \\ \mathrm{C} & 0.2732947 & 5.3663000 & 2.5234370 \\ \mathrm{H} & 0.0467719 & 6.4254292 & 2.3281448 \\ \mathrm{C} & 0.1438707 & 4.4262364 & 1.4893981 \\ \mathrm{H} & -0.1871929 & 4.7439294 & 0.4896176 \\ \mathrm{C} & 1.9409915 & 1.6184683 & -0.2842831 \\ \mathrm{C} & 2.0905708 & 1.1606701 & -1.6098789 \\ \mathrm{H} & 1.2032263 & 0.9906647 & -2.2330496 \\ \mathrm{C} & 3.3687763 & 0.9261218 & -2.1376947 \\ \mathrm{H} & 3.4704211 & 0.5783281 & -3.1764519 \\ \mathrm{C} & 4.5083272 & 1.1270772 & -1.3407941 \\ \mathrm{H} & 5.5109862 & 0.9363209 & -1.7522404 \\ \mathrm{C} & 4.3625663 & 1.5757220 & -0.0170841 \\ \mathrm{H} & 5.2501935 & 1.7339068 & 0.6135147 \\ & 3.0868506 & 1.8238375 & 0.5100967 \\ & 2.9822934 & 2.1670337 & 1.5483708 \\ & & & \\ \mathrm{H} & & & \\ \mathrm{H} & & & \\ \mathrm{H} & & & \end{array}$




$\begin{array}{lrrr}\text { SCF } & \text { Energy }=-4548.326599873 & \\ \text { C } & -1.0609715 & -0.4950362 & -2.4397624 \\ \text { C } & -1.2447221 & -0.9808505 & -1.1214537 \\ \text { C } & -2.5614627 & -1.2850190 & -0.6948902 \\ \text { C } & -3.6493205 & -1.1100808 & -1.5608272 \\ \text { C } & -3.4510637 & -0.6205585 & -2.8634240 \\ \text { C } & -2.1500380 & -0.3167006 & -3.2994576 \\ \text { C } & -0.0937901 & -1.1184343 & -0.2076488 \\ \text { C } & 1.0717237 & -1.7767732 & -0.4405901 \\ \text { C } & 1.1971051 & -2.6150427 & -1.6722072 \\ \text { C } & 0.1669131 & -3.4987158 & -2.0692131 \\ \text { C } & 0.2842233 & -4.2642250 & -3.2362034 \\ \text { C } & 1.4367598 & -4.1690923 & -4.0351550 \\ \text { C } & 2.4737038 & -3.3053625 & -3.6478757 \\ \text { C } & 2.3575866 & -2.5406210 & -2.4768513 \\ \text { Br } & -1.8157986 & -1.4204252 & 3.0843503 \\ \text { C } & 1.2890868 & -3.6487359 & 2.0348274 \\ \text { C } & 1.4680118 & -3.2496780 & 3.4779864 \\ \text { C } & 0.1739106 & -4.2645035 & 1.5898327 \\ \text { C } & 2.4133474 & -3.2890037 & 1.0876340 \\ \text { C } & 2.2846330 & -1.8486988 & 0.4927048 \\ \text { O } & 2.3637244 & -0.8590790 & 1.4955170 \\ \text { H } & 3.1850971 & -1.6868240 & -0.1396561 \\ \text { H } & 3.3919425 & -3.3276678 & 1.6105301 \\ \text { H } & 2.4567915 & -4.0053519 & 0.2425274 \\ \text { H } & 0.0726353 & -4.5686698 & 0.5367795 \\ \text { H } & -0.6745497 & -4.4663417 & 2.2610246 \\ \text { H } & 1.7660020 & -2.1847597 & 3.5600196 \\ \text { H } & 2.2919693 & -3.8364252 & 3.9418437 \\ \text { H } & 0.5440085 & -3.4014460 & 4.0664179 \\ \text { H } & 1.4498738 & -0.7514376 & 1.8755976 \\ \text { H } & -0.7347829 & -3.5785816 & -1.4470379 \\ \text { H } & -0.5311897 & -4.9459557 & -3.5220601 \\ \text { H } & 1.5287135 & -4.7710056 & -4.9518355 \\ \text { H } & 3.3832681 & -3.2235328 & -4.2627927 \\ \text { H } & 3.1734638 & -1.8615595 & -2.1904627 \\ \text { H } & -2.7152619 & -1.6525814 & 0.3314611 \\ \text { H } & -4.6635062 & -1.3565461 & -1.2114887 \\ \text { H } & -4.3087492 & -0.4736676 & -3.5372179 \\ \text { H } & -1.9819786 & 0.0693344 & -4.3158676 \\ \text { H } & -0.0460752 & -0.2681476 & -2.7934179 \\ \text { Pd } & -0.5194247 & -0.1427862 & 1.4799524 \\ \text { P } & 0.4103040 & 1.6593051 & 0.4913565 \\ \text { C } & 0.4272386 & 2.9459807 & 1.7974358 \\ \text { C } & 0.7374867 & 2.5591450 & 3.1194567 \\ \text { H } & 0.9498969 & 1.5014190 & 3.3444924\end{array}$




$\begin{array}{lrrr}\text { C } & 0.7653484 & 3.5189929 & 4.1421932 \\ \text { H } & 1.0042187 & 3.2120825 & 5.1712393 \\ \text { C } & 0.4772594 & 4.8631903 & 3.8519139 \\ \text { H } & 0.4915342 & 5.6142814 & 4.6560943 \\ \text { C } & 0.1621653 & 5.2485724 & 2.5373675 \\ \text { H } & -0.0686724 & 6.3005022 & 2.3111202 \\ \text { C } & 0.1355516 & 4.2944607 & 1.5089615 \\ \text { H } & -0.1177582 & 4.5924335 & 0.4807379 \\ \text { C } & 2.1049377 & 1.6585703 & -0.1980705 \\ \text { C } & 2.3650680 & 1.0029821 & -1.4203767 \\ \text { H } & 1.5460324 & 0.5330869 & -1.9784344 \\ \text { C } & 3.6718423 & 0.9492960 & -1.9253267 \\ \text { H } & 3.8606393 & 0.4471487 & -2.8860435 \\ \text { C } & 4.7310373 & 1.5332871 & -1.2103928 \\ \text { H } & 5.7570469 & 1.4836367 & -1.6049640 \\ \text { C } & 4.4746221 & 2.1839866 & 0.0072686 \\ \text { H } & 5.2989313 & 2.6441634 & 0.5723959 \\ \text { C } & 3.1677688 & 2.2512017 & 0.5118052 \\ \text { H } & 2.9749029 & 2.7583665 & 1.4676327 \\ \text { C } & -0.6766058 & 2.3195509 & -0.8230491 \\ \text { C } & -0.1900390 & 2.9770898 & -1.9695781 \\ \text { H } & 0.8907808 & 3.1318232 & -2.0994544 \\ \text { C } & -1.0895657 & 3.4232813 & -2.9517240 \\ \text { H } & -0.7068526 & 3.9301505 & -3.8504454 \\ \text { C } & -2.4693243 & 3.2195723 & -2.7906399 \\ \text { H } & -3.1695335 & 3.5588549 & -3.5688420 \\ \text { C } & -2.9567969 & 2.5767274 & -1.6394247 \\ \text { H } & -4.0354338 & 2.4046886 & -1.5140450 \\ \text { C } & -2.0655469 & 2.1261282 & -0.6589489 \\ \text { H } & -2.4380577 & 1.5912447 & 0.2292613\end{array}$

\section{Optimized Geometries (BXnc)}

$\begin{array}{lccc}\text { R1 } & & & \\ 75 & & & \\ \text { SCF } & \text { Energy }=-4548.338894608 & \\ \text { C } & -1.1655986 & 2.4014617 & -0.4483549 \\ \text { C } & -1.0215485 & 1.1681360 & -1.1357378 \\ \text { C } & -2.1947491 & 0.5725807 & -1.6627296 \\ \text { C } & -3.4513404 & 1.1643854 & -1.4821957 \\ \text { C } & -3.5721527 & 2.3850030 & -0.7976166 \\ \text { C } & -2.4186495 & 3.0065465 & -0.2923733 \\ \text { C } & 0.3152153 & 0.5472003 & -1.2266883\end{array}$




$\begin{array}{lrrr}\text { C } & 0.8971215 & -0.0283725 & -2.3140060 \\ \mathrm{C} & 0.2737066 & -0.0886752 & -3.6618711 \\ \mathrm{C} & 0.3318581 & -1.2858684 & -4.4152226 \\ \mathrm{C} & -0.2741945 & -1.3756874 & -5.6774898 \\ \mathrm{C} & -0.9411243 & -0.2653086 & -6.2214438 \\ \mathrm{C} & -0.9902077 & 0.9363994 & -5.4933363 \\ \mathrm{C} & -0.3886983 & 1.0241047 & -4.2308266 \\ \mathrm{Br} & 1.6266467 & 1.5428172 & 2.9439405 \\ \mathrm{C} & 2.8125198 & 1.8685744 & -1.1362276 \\ \mathrm{C} & 2.1354130 & 3.0798021 & -1.7287435 \\ \mathrm{C} & 3.2222788 & 1.8630481 & 0.1859478 \\ \mathrm{C} & 3.2504356 & 0.8021657 & -2.1239276 \\ \mathrm{C} & 2.3561808 & -0.4648968 & -2.2177841 \\ \mathrm{O} & 2.6782240 & -1.2825539 & -1.1007943 \\ \mathrm{H} & 2.6402374 & -0.9894632 & -3.1592815 \\ \mathrm{H} & 3.2935046 & 1.2535566 & -3.1358995 \\ \mathrm{H} & 4.2703852 & 0.4474206 & -1.8702941 \\ \mathrm{H} & 3.9142632 & 1.0913887 & 0.5564719 \\ \mathrm{H} & 3.0986632 & 2.7538177 & 0.8187392 \\ \mathrm{H} & 1.2728519 & 2.7918266 & -2.3615779 \\ \mathrm{H} & 2.8555258 & 3.6097904 & -2.3901363 \\ \mathrm{H} & 1.7914895 & 3.7893789 & -0.9528487 \\ \mathrm{H} & 1.9261606 & -1.8880746 & -0.9460876 \\ \mathrm{H} & 0.8418850 & -2.1634258 & -3.9899926 \\ \mathrm{H} & -0.2245821 & -2.3203905 & -6.2407500 \\ \mathrm{H} & -1.4142426 & -0.3330490 & -7.2127316 \\ \mathrm{H} & -1.4987202 & 1.8165576 & -5.9157135 \\ \mathrm{H} & -0.4335069 & 1.9668156 & -3.6667461 \\ \mathrm{H} & -2.1187107 & -0.3566827 & -2.2430481 \\ \mathrm{H} & -4.3462969 & 0.6669610 & -1.8856571 \\ \mathrm{H} & -4.5609746 & 2.8464214 & -0.6565497 \\ \mathrm{H} & -2.4949895 & 3.9629826 & 0.2466526 \\ \mathrm{H} & -0.2715980 & 2.8741799 & -0.0145061 \\ \mathrm{Pd} & 1.2739011 & 0.7419454 & 0.5563832 \\ \mathrm{P} & 0.0400467 & -1.0422607 & 1.2985181 \\ \mathrm{C} & -1.7364039 & -0.7118993 & 1.5755500 \\ \mathrm{C} & -2.0749717 & 0.5584706 & 2.0864471 \\ \mathrm{H} & -1.2740924 & 1.2849982 & 2.2967430 \\ \mathrm{C} & -3.4190758 & 0.8771292 & 2.3218800 \\ \mathrm{H} & -3.6800185 & 1.8725703 & 2.7102374 \\ \mathrm{C} & -4.4279520 & -0.0614933 & 2.0486519 \\ \mathrm{C} & -5.4833119 & 0.1980228 & 2.2224088 \\ \mathrm{H} & -4.0901865 & -1.3318433 & 1.5538492 \\ \mathrm{H} & -2.7464341 & -1.6616264 & 1.3197399 \\ & & -2.6574867 & 0.9347708\end{array}$




$\begin{array}{lrrr}\text { C } & 0.0917678 & -2.5239138 & 0.1962296 \\ \text { C } & 0.9538479 & -3.6088599 & 0.4718680 \\ \text { H } & 1.5476832 & -3.6166173 & 1.3964481 \\ \text { C } & 1.0649065 & -4.6746630 & -0.4364142 \\ \text { H } & 1.7480823 & -5.5071942 & -0.2111435 \\ \text { C } & 0.3070940 & -4.6828698 & -1.6185231 \\ \text { H } & 0.3944802 & -5.5213213 & -2.3258067 \\ \text { C } & -0.5682111 & -3.6171042 & -1.8896347 \\ \text { H } & -1.1732756 & -3.6122658 & -2.8082877 \\ \text { C } & -0.6686611 & -2.5429440 & -0.9948400 \\ \text { H } & -1.3497934 & -1.7139925 & -1.2137130 \\ \text { C } & 0.6653406 & -1.7358636 & 2.8769591 \\ \text { C } & 2.0583458 & -1.7830163 & 3.0938594 \\ \text { H } & 2.7381232 & -1.3280213 & 2.3585469 \\ \text { C } & 2.5639168 & -2.3851901 & 4.2537743 \\ \text { H } & 3.6508816 & -2.4167937 & 4.4211826 \\ \text { C } & 1.6846979 & -2.9219990 & 5.2108896 \\ \text { H } & 2.0835668 & -3.3803194 & 6.1285985 \\ \text { C } & 0.2980127 & -2.8625828 & 5.0010212 \\ \text { H } & -0.3935623 & -3.2722296 & 5.7527356 \\ \text { C } & -0.2147475 & -2.2757736 & 3.8321173 \\ \text { H } & -1.3012465 & -2.2280058 & 3.6683782 \\ & & & \\ \text { R2 } & & & \\ \text { 75 } & & & \\ \text { SCF } & \text { Energy = } & -4548.318797620 & \\ \text { C } & 2.4678109 & 0.7548753 & -2.1626642 \\ \text { C } & 2.0554557 & -0.3112123 & -1.3247944 \\ \text { C } & 1.5421071 & -1.4873986 & -1.9300645 \\ \text { C } & 1.4462251 & -1.5954193 & -3.3245469 \\ \text { C } & 1.8634319 & -0.5330627 & -4.1429939 \\ \text { C } & 2.3751332 & 0.6389537 & -3.5547328 \\ \text { C } & 2.0920221 & -0.2031879 & 0.1428062 \\ \text { C } & 3.1714397 & -0.0854862 & 0.9452359 \\ \text { C } & 4.5556223 & 0.0059525 & 0.3933551 \\ \text { C } & 5.0066477 & -0.8557240 & -0.6338876 \\ \text { C } & 6.3094685 & -0.7529806 & -1.1395938 \\ \text { C } & 7.1984287 & 0.2063558 & -0.6245823 \\ \text { C } & 6.7717783 & 1.0598322 & 0.4056095 \\ \text { C } & 5.4659075 & 0.9603836 & 0.9106054 \\ \text { Br } & 0.2024597 & 2.2748779 & -0.0627371 \\ \text { C } & 1.0044567 & -1.6334468 & 2.1177117 \\ \text { C } & 1.7690614 & -2.8515962 & 1.6527123 \\ \text { C } & -0.3993880 & -1.6553332 & 2.0910760 \\ \text { C } & 1.7345140 & -0.6436528 & 3.0057426 \\ \text { C } & 3.0927818 & -0.1524145 & 2.4690707\end{array}$




\begin{tabular}{lrrr} 
O & 4.0782026 & -1.0688412 & 2.9657380 \\
$\mathrm{H}$ & 3.2732405 & 0.8646016 & 2.8998719 \\
$\mathrm{H}$ & 1.0723879 & 0.2186797 & 3.2153134 \\
$\mathrm{H}$ & 1.9653305 & -1.1306286 & 3.9794485 \\
$\mathrm{H}$ & -0.9847670 & -1.0484383 & 2.8012831 \\
$\mathrm{H}$ & -0.9219144 & -2.5337070 & 1.6824353 \\
$\mathrm{H}$ & 1.9449851 & -3.5042739 & 2.5354580 \\
$\mathrm{H}$ & 2.7617323 & -2.5961663 & 1.2440418 \\
$\mathrm{H}$ & 1.1939299 & -3.4321048 & 0.9057097 \\
$\mathrm{H}$ & 4.8962527 & -0.9066108 & 2.4534815 \\
$\mathrm{H}$ & 4.3206494 & -1.6160173 & -1.0323285 \\
$\mathrm{H}$ & 6.6364893 & -1.4342321 & -1.9399878 \\
$\mathrm{H}$ & 8.2218731 & 0.2846439 & -1.0214309 \\
$\mathrm{H}$ & 7.4580635 & 1.8162690 & 0.8162721 \\
$\mathrm{H}$ & 5.1350895 & 1.6495988 & 1.7025874 \\
$\mathrm{H}$ & 1.2193336 & -2.3186076 & -1.2860151 \\
$\mathrm{H}$ & 1.0386972 & -2.5132363 & -3.7744792 \\
$\mathrm{H}$ & 1.7883872 & -0.6157966 & -5.2379054 \\
$\mathrm{H}$ & 2.7012875 & 1.4760174 & -4.1909192 \\
$\mathrm{H}$ & 2.8432287 & 1.6759859 & -1.6978059 \\
$\mathrm{Pd}$ & 0.0874541 & -0.1687925 & 0.5778020 \\
$\mathrm{P}$ & -2.2056252 & -0.0303910 & 0.0726197 \\
$\mathrm{C}$ & -3.1256564 & 1.4096793 & 0.7349701 \\
$\mathrm{C}$ & -2.4545728 & -0.0330859 & -1.7515710 \\
$\mathrm{C}$ & -3.2322060 & -1.4638645 & 0.6017411 \\
$\mathrm{C}$ & -4.2486679 & 1.9532090 & 0.0832236 \\
$\mathrm{C}$ & -4.9510392 & 3.0172426 & 0.6710233 \\
$\mathrm{C}$ & -4.5386069 & 3.5374699 & 1.9091817 \\
$\mathrm{C}$ & -3.4130661 & 3.0004124 & 2.5571951 \\
$\mathrm{C}$ & -2.7016515 & 1.9453775 & 1.9680710 \\
$\mathrm{H}$ & -4.5671218 & 1.5537876 & -0.8907863 \\
$\mathrm{H}$ & -5.8235786 & 3.4457010 & 0.1549346 \\
$\mathrm{H}$ & -5.0900463 & 4.3732956 & 2.3659808 \\
$\mathrm{H}$ & -3.0759240 & 3.4176932 & 3.5178913 \\
$\mathrm{H}$ & -1.7946452 & 1.5455543 & 2.4461858 \\
$\mathrm{C}$ & -3.6666407 & -0.5035573 & -2.3065907 \\
$\mathrm{C}$ & -3.8525779 & -0.5016900 & -3.6970185 \\
$\mathrm{C}$ & -2.8308584 & -0.0381475 & -4.5438098 \\
$\mathrm{C}$ & -1.6238450 & 0.4229247 & -3.9950107 \\
$\mathrm{C}$ & -1.4310138 & 0.4271566 & -2.6051049 \\
$\mathrm{H}$ & -4.4616883 & -0.8863106 & -1.6495136 \\
$\mathrm{H}$ & -4.7997900 & -0.8688900 & -4.1206125 \\
$\mathrm{H}$ & -2.9765390 & -0.0422678 & -5.6349365 \\
& -0.8125165 & 0.7776875 & -4.6469730 \\
$\mathrm{H}$ & -4.1858498 & -1.3654574 & -2.1792369 \\
& & & \\
\hline
\end{tabular}




$\begin{array}{llll}\text { C } & -4.8667509 & -2.5130916 & 2.0756422 \\ \text { C } & -4.6038535 & -3.7621708 & 1.4928640 \\ \text { C } & -3.6514092 & -3.8651006 & 0.4619937 \\ \text { C } & -2.9651439 & -2.7253709 & 0.0227404 \\ \text { H } & -4.4012260 & -0.3870554 & 2.0868863 \\ \text { H } & -5.6123326 & -2.4254833 & 2.8805276 \\ \text { H } & -5.1404643 & -4.6581175 & 1.8396098 \\ \text { H } & -3.4419294 & -4.8414234 & -0.0009394 \\ \text { H } & -2.2167355 & -2.8070843 & -0.7817191\end{array}$

R3

75

SCF Energy $=-4548.319139529$

C $\quad 0.2082874 \quad 2.6414696$

$\begin{array}{lll}\text { C } & -0.2993797 & 1.6093136\end{array}$

$\begin{array}{lll}\text { C } & -0.9179756 & 1.9966357\end{array}$

$-1.7001936$

$\begin{array}{lll}-1.0009180 & 3.3415877 & -4.1287334\end{array}$

$\begin{array}{lll}0.1196404 & 3.9880362 & -2.0775135\end{array}$

$\begin{array}{lll}-0.2867695 & 0.1993576 & -2.1277724\end{array}$

$\begin{array}{lll}-0.2676397 & -0.9095222 & -2.9119391\end{array}$

$\begin{array}{lll}0.1818006 & -0.9024988 & -4.3220267\end{array}$

$\begin{array}{lll}-0.5222019 & -1.5851193 & -5.3456357\end{array}$

$\begin{array}{lll}-0.0736435 & -1.5343757 & -6.6750422\end{array}$

$\begin{array}{lll}1.0836779 & -0.8114424 & -7.0079028\end{array}$

$\begin{array}{llll}\text { C } & 1.7998056 & -0.1444573 & -5.9973042\end{array}$

$\begin{array}{llll}\text { C } & 1.3595933 & -0.1950224 & -4.6693380\end{array}$

$\begin{array}{llll}\mathrm{Br} & 2.0987197 & -0.0323226 & -0.3198772\end{array}$

$\begin{array}{llll}\text { C } & -2.4419863 & -0.8216522 & -0.9932605\end{array}$

$\begin{array}{llll}\text { C } & -3.2123520 & 0.1793177 & -1.8214556\end{array}$

$\begin{array}{llll}\text { C } & -2.4232714 & -0.6904451 & 0.4027277\end{array}$

$\begin{array}{llll}\text { C } & -2.1012610 & -2.1412175 & -1.6653991\end{array}$

$\begin{array}{llll}\text { C } & -0.6572988 & -2.2253244 & -2.2279432\end{array}$

$\begin{array}{llll}0 & -0.6027984 & -3.3795628 & -3.0449539\end{array}$

$\mathrm{H} \quad 0.0327803 \quad-2.3300226 \quad-1.3497610$

$\mathrm{H} \quad-2.2443948 \quad-2.9832792 \quad-0.9580262$

H $\quad-2.7888026 \quad-2.3026189 \quad-2.5211315$

$\mathrm{H} \quad-2.2846863 \quad-1.5768582 \quad 1.0423580$

$\begin{array}{llll}\mathrm{H} & -2.9558269 & 0.1483844 & 0.8775149\end{array}$

$\mathrm{H} \quad-2.8166472 \quad 0.2617315 \quad-2.8494396$

$\mathrm{H} \quad-3.2160031 \quad 1.1874753 \quad-1.3665147$

$\mathrm{H} \quad-4.2641191 \quad-0.1761395 \quad-1.8969038$

$\begin{array}{llll}\mathrm{H} & 0.2403494 & -3.3463270 & -3.5365879\end{array}$

$\mathrm{H} \quad-1.4314006 \quad-2.1471168 \quad-5.0923802$

$\mathrm{H} \quad-0.6390375 \quad-2.0609008 \quad-7.4595464$

$\begin{array}{llll}\mathrm{H} & 1.4315968 & -0.7732935 & -8.0514508\end{array}$ 


$\begin{array}{lrrr}\text { H } & 2.7154885 & 0.4134126 & -6.2466629 \\ \text { H } & 1.9223182 & 0.3080669 & -3.8690124 \\ \text { H } & -1.3284155 & 1.2205558 & -4.4136786 \\ \text { H } & -1.4807959 & 3.6082526 & -5.0830592 \\ \text { H } & -0.5536127 & 5.4061294 & -3.5892987 \\ \text { H } & 0.5274414 & 4.7636177 & -1.4111530 \\ \text { H } & 0.6889869 & 2.3651842 & -0.7532417 \\ \text { Pd } & -0.4032177 & -0.0536846 & -0.0720273 \\ \text { P } & -0.0251779 & 0.0177263 & 2.2804350 \\ \text { C } & 0.6767137 & 1.5795109 & 2.9652939 \\ \text { C } & 1.5171274 & 2.3818926 & 2.1662624 \\ \text { H } & 1.7638229 & 2.0539353 & 1.1450037 \\ \text { C } & 2.0693444 & 3.5613078 & 2.6887996 \\ \text { H } & 2.7244538 & 4.1778576 & 2.0549666 \\ \text { C } & 1.7917212 & 3.9514183 & 4.0090704 \\ \text { H } & 2.2242124 & 4.8786278 & 4.4149258 \\ \text { C } & 0.9609181 & 3.1521415 & 4.8118444 \\ \text { H } & 0.7420136 & 3.4478549 & 5.8491817 \\ \text { C } & 0.4036894 & 1.9723248 & 4.2950803 \\ \text { H } & -0.2521433 & 1.3570128 & 4.9277818 \\ \text { C } & 1.1049476 & -1.3173496 & 2.8332769 \\ \text { C } & 1.1144867 & -2.5184440 & 2.0947584 \\ \text { H } & 0.5089817 & -2.5924778 & 1.1781837 \\ \text { C } & 1.9120832 & -3.5934661 & 2.5120017 \\ \text { H } & 1.9186350 & -4.5268812 & 1.9294282 \\ \text { C } & 2.7165083 & -3.4684791 & 3.6575991 \\ \text { H } & 3.3519220 & -4.3079738 & 3.9782337 \\ \text { C } & 2.7196084 & -2.2671109 & 4.3859953 \\ \text { H } & 3.3579892 & -2.1628922 & 5.2764345 \\ \text { C } & 1.9141005 & -1.1920648 & 3.9781696 \\ \text { H } & 1.9231335 & -0.2493725 & 4.5450040 \\ \text { C } & -1.5051446 & -0.2290175 & 3.3504418 \\ \text { C } & -1.7328646 & -1.4311671 & 4.0476154 \\ \text { H } & -0.9731409 & -2.2262131 & 4.0290045 \\ \text { C } & -2.9236707 & -1.6129339 & 4.7724505 \\ \text { H } & -3.0900755 & -2.5555390 & 5.3156970 \\ \text { C } & -3.8920607 & -0.5980607 & 4.8102046 \\ \text { H } & -4.8218337 & -0.7417086 & 5.3809410 \\ \text { C } & -3.6690738 & 0.6050846 & 4.1162311 \\ \text { H } & -4.4228977 & 1.4065086 & 4.1430669 \\ \text { C } & -2.4873686 & 0.7865540 & 3.3852693 \\ \text { H } & -2.3162617 & 1.7301060 & 2.8429963 \\ \text { R4 } & & & \\ \text { SCF } & \text { Energy }=-4548.331369847 & \\ & & & \end{array}$




\begin{tabular}{|c|c|c|c|}
\hline & 1.1637123 & -5.5508535 & \\
\hline & 0.0951317 & -4.6672686 & 0.1657364 \\
\hline & -0.9762561 & -5.1497181 & -0.6234802 \\
\hline & -0.9765623 & -6.4626793 & -1.1128291 \\
\hline & 0.0902947 & -7.3282255 & -0.8160203 \\
\hline & 1.1573623 & -6.8666637 & -0.0276147 \\
\hline & 0.1070858 & -3.2572244 & 0.6361852 \\
\hline & -0.2143375 & -0.2939553 & 0.3522801 \\
\hline & -0.3017088 & 2.1025651 & 0.1356232 \\
\hline & -0.1697094 & -2.2291108 & -0.1929562 \\
\hline & 2.2200228 & -0.2274126 & -0.2556841 \\
\hline & -0.3301764 & -2.2060972 & -1.6517160 \\
\hline & 0.7058588 & -2.6857295 & -2.4 \\
\hline & 0.60 & -2.56 & -3.8 \\
\hline & -0.52 & -1.9 & -4.4 \\
\hline & -1.5 & -1.4 & -3.6 \\
\hline & -1.4 & -1.6 & -2.2 \\
\hline & 0.3 & -2.9 & 2.1 \\
\hline & 0.69 & -4.0 & 2.9 \\
\hline & -0.97 & -2.2 & 2.6 \\
\hline & -1.2512756 & -0.86 & 2.2 \\
\hline & -2.1780692 & -0.5436841 & 1.2 \\
\hline & -0.8126917 & 27462 & 3.26 \\
\hline & 1.1569789 & -2.21 & 2.21 \\
\hline & -0.8806260 & -2.32 & 3.78 \\
\hline & -1.8269567 & -2.94 & 2.39 \\
\hline & -2.7238589 & -1.36 & 0.75 \\
\hline & -2.670 & 0.44 & 1.23 \\
\hline & -1.403 & 0.06 & 4.1 \\
\hline & -0.9663629 & 1.2 & 2.8 \\
\hline & 0.253 & 0.0 & 3.5 \\
\hline & 0.1424925 & -4.81 & 2.6 \\
\hline & -1.8126532 & -4.47 & -0.8 \\
\hline & -1.8190559 & -6.8151474 & -1.72 \\
\hline & 0.0896299 & -8.3603039 & -1.1983255 \\
\hline & 2.0014457 & -7.5347235 & 0.2030463 \\
\hline & 2.0068287 & -5.1887142 & 1.0645906 \\
\hline & -2.2696211 & -1.2187801 & -1.6129866 \\
\hline & -2.4457589 & -1.0107154 & -4.0962929 \\
\hline & -0.5984218 & -1.8720291 & -5.5626785 \\
\hline & 1.4194871 & -2.9431866 & -4.5215458 \\
\hline & 1.5982887 & -3.1267418 & -2.0286873 \\
\hline & -0.4746418 & 2.4765301 & -1.6608216 \\
\hline & 1.1253064 & 3.0985431 & 0.7172542 \\
\hline & -1.7405701 & 2.9739450 & 0.879565 \\
\hline & -1.6005999 & 3.7929678 & 2.018969 \\
\hline
\end{tabular}




$\begin{array}{lrrr}\text { C } & -2.7350337 & 4.3368667 & 2.6437771 \\ \text { C } & -4.0180583 & 4.0733653 & 2.1381515 \\ \text { C } & -4.1649083 & 3.2602924 & 1.0004139 \\ \text { C } & -3.0360228 & 2.7081675 & 0.3783531 \\ \text { H } & -0.5970365 & 4.0109756 & 2.4134382 \\ \text { H } & -2.6119771 & 4.9776724 & 3.5301169 \\ \text { H } & -4.9047483 & 4.5035548 & 2.6275417 \\ \text { H } & -5.1668559 & 3.0533188 & 0.5946928 \\ \text { H } & -3.1555297 & 2.0761184 & -0.5155596 \\ \text { C } & -1.1490769 & 3.6309769 & -2.1170764 \\ \text { C } & -1.2672722 & 3.8810089 & -3.4928993 \\ \text { C } & -0.7170650 & 2.9826098 & -4.4233622 \\ \text { C } & -0.0439338 & 1.8354339 & -3.9732751 \\ \text { C } & 0.0798853 & 1.5802495 & -2.5993143 \\ \text { H } & -1.5954856 & 4.3299582 & -1.3942689 \\ \text { H } & -1.7956349 & 4.7823425 & -3.8393771 \\ \text { H } & -0.8166763 & 3.1780578 & -5.5020663 \\ \text { H } & 0.3841879 & 1.1203355 & -4.6909750 \\ \text { H } & 0.6190018 & 0.6858900 & -2.2513117 \\ \text { C } & 1.8822138 & 2.6066221 & 1.7993040 \\ \text { C } & 2.9399913 & 3.3674963 & 2.3195638 \\ \text { C } & 3.2576953 & 4.6120920 & 1.7508837 \\ \text { C } & 2.5155283 & 5.0961542 & 0.6600066 \\ \text { C } & 1.4502456 & 4.3429885 & 0.1431692 \\ \text { H } & 1.6519535 & 1.6142264 & 2.2122362 \\ \text { H } & 3.5304362 & 2.9766842 & 3.1618262 \\ \text { H } & 4.0948857 & 5.2032595 & 2.1522699 \\ \text { H } & 2.7706606 & 6.0650624 & 0.2045290 \\ \text { H } & 0.8761992 & 4.7192242 & -0.7165195\end{array}$

S1

75

SCF Energy $=-4548.341425160$

C $\quad-2.2996591 \quad 1.0062732$

C $\quad-1.6766063 \quad-0.2058107$

C $\quad-2.4777496 \quad-1.2183595$

$-2.5926963$

$-2.1971339$

$-1.6075269$

$\begin{array}{lll}-3.8545000 & -1.0276129 & -1.4229173\end{array}$

$\begin{array}{lll}-4.4570145 & 0.1760933 & -1.8216126\end{array}$

$\begin{array}{lll}-3.6740722 & 1.1891223 & -2.4086511\end{array}$

$\begin{array}{lll}-0.2211549 & -0.3617511 & -2.2860983\end{array}$

$\begin{array}{llll}\text { C } & 0.6267210 & -0.2661623 & -3.3314616\end{array}$

$\begin{array}{llll}\text { C } & 0.1627511 & -0.0658345 & -4.7267527\end{array}$

$\begin{array}{llll}\text { C } & -0.9701122 & -0.7368211 & -5.2417795\end{array}$

C $\quad-1.4066041-0.5012188-6.5528066$

$\begin{array}{llll}\text { C } & -0.7212650 & 0.4084240 & -7.3764435\end{array}$

$\begin{array}{llll}\text { C } & 0.4083291 & 1.0791206 & -6.8773179\end{array}$ 


$\begin{array}{lrrr}\text { C } & 0.8499062 & 0.8423880 & -5.5676621 \\ \mathrm{Br} & 0.2921491 & 2.1303539 & -0.4140058 \\ \mathrm{C} & 2.0306683 & -1.7440767 & -0.8575687 \\ \mathrm{C} & 3.0927320 & -1.3931742 & 0.1591590 \\ \mathrm{C} & 0.8857495 & -2.4897350 & -0.4855027 \\ \mathrm{C} & 2.4742932 & -1.6372840 & -2.3034858 \\ \mathrm{C} & 2.1304728 & -0.3268618 & -3.0768594 \\ \mathrm{O} & 2.6581552 & 0.8350750 & -2.4653120 \\ \mathrm{H} & 2.6431072 & -0.4225932 & -4.0577964 \\ \mathrm{H} & 3.5797146 & -1.7320735 & -2.3209104 \\ \mathrm{H} & 2.0583674 & -2.4788198 & -2.8928933 \\ \mathrm{H} & 0.3135966 & -3.0057823 & -1.2728602 \\ \mathrm{H} & 0.8167009 & -2.9209334 & 0.5247328 \\ \mathrm{H} & 3.5077249 & -0.3875617 & -0.0537119 \\ \mathrm{H} & 3.9351619 & -2.1148811 & 0.0697557 \\ \mathrm{H} & 2.7191429 & -1.4353573 & 1.1992337 \\ \mathrm{H} & 1.9614068 & 1.2176423 & -1.8743394 \\ \mathrm{H} & -1.5023443 & -1.4570158 & -4.6039182 \\ \mathrm{H} & -2.2873358 & -1.0379571 & -6.9378396 \\ \mathrm{H} & -1.0650690 & 0.5920089 & -8.4057198 \\ \mathrm{H} & 0.9481332 & 1.7986779 & -7.5120400 \\ \mathrm{H} & 1.7176692 & 1.3890714 & -5.1678428 \\ \mathrm{H} & -2.0024713 & -2.1582998 & -1.2915835 \\ \mathrm{H} & -4.4581234 & -1.8205567 & -0.9566641 \\ \mathrm{H} & -5.5367648 & 0.3290454 & -1.6728004 \\ \mathrm{H} & -4.1423344 & 2.1356971 & -2.7188995 \\ \mathrm{H} & -1.6769353 & 1.8021041 & -3.0231829 \\ \mathrm{Pd} & 0.3076229 & -0.4130915 & -0.3472777 \\ \mathrm{P} & 0.0758384 & -0.3275691 & 2.0433684 \\ \mathrm{C} & -1.7169061 & -0.0893893 & 2.3944444 \\ \mathrm{C} & -2.4954532 & 0.6484687 & 1.4770028 \\ \mathrm{H} & -2.0284486 & 1.0719130 & 0.5745357 \\ \mathrm{C} & -3.8623290 & 0.8503683 & 1.7192842 \\ \mathrm{H} & -4.4563423 & 1.4210346 & 0.9907984 \\ \mathrm{C} & -4.4645028 & 0.3189887 & 2.8711376 \\ \mathrm{H} & 1.2250619 & -4.2988429 & 4.1856106 \\ \mathrm{H} & -5.5383960 & 0.4749425 & 3.0556783 \\ \mathrm{C} & -3.6935426 & -0.4163007 & 3.7876881 \\ \mathrm{H} & -4.1597596 & -0.8356006 & 4.6923351 \\ \mathrm{C} & -2.3250544 & -0.6206606 & 3.5532578 \\ \mathrm{H} & -1.7287902 & -1.2062132 & 4.2686318 \\ \mathrm{C} & 0.4915780 & -1.8546502 & 2.9773517 \\ & 1.6171958 & -1.9215225 & 3.8236094 \\ \mathrm{H} & 1.2064006 & -1.0134690 & 4.0197907 \\ & 2.8808821 & -3.1395361 & 4.4216987 \\ & & & \\ \mathrm{H} & & \end{array}$




$\begin{array}{rrrr}\mathrm{H} & 1.5101597 & -5.2507328 & 4.6582706 \\ \mathrm{C} & 0.1001085 & -4.2384865 & 3.3438673 \\ \mathrm{H} & -0.4990709 & -5.1424744 & 3.1566391 \\ \mathrm{C} & -0.2609747 & -3.0274099 & 2.7367466 \\ \mathrm{H} & -1.1424715 & -2.9837718 & 2.0778300 \\ \mathrm{C} & 0.9385560 & 0.9863672 & 2.9898252 \\ \mathrm{C} & 2.1574376 & 1.4837306 & 2.4871927 \\ \mathrm{H} & 2.5258424 & 1.1307172 & 1.5143243 \\ \mathrm{C} & 2.8768214 & 2.4439464 & 3.2146741 \\ \mathrm{H} & 3.8257374 & 2.8317592 & 2.8150471 \\ \mathrm{C} & 2.3748874 & 2.9231912 & 4.4359690 \\ \mathrm{H} & 2.9345615 & 3.6844046 & 5.0005466 \\ \mathrm{C} & 1.1510096 & 2.4406271 & 4.9308296 \\ \mathrm{H} & 0.7498602 & 2.8232948 & 5.8815642 \\ \mathrm{C} & 0.4327094 & 1.4736562 & 4.2111418 \\ \mathrm{H} & -0.5295859 & 1.1037460 & 4.5950142\end{array}$

S2

75

SCF Energy $=-4548.330443648$

$\begin{array}{lrrr}\text { C } & -1.9024475 & 1.2311906 & -3.0886615 \\ \text { C } & -1.5813991 & 0.0713554 & -2.3383258 \\ \text { C } & -2.6391482 & -0.6441280 & -1.7235456 \\ \text { C } & -3.9698933 & -0.2225065 & -1.8587728 \\ \text { C } & -4.2720011 & 0.9256617 & -2.6095019 \\ \text { C } & -3.2313340 & 1.6475030 & -3.2233183 \\ \text { C } & -0.1850138 & -0.3166045 & -2.1123961 \\ \text { C } & 0.8449022 & -0.4044176 & -2.9904222 \\ \text { C } & 0.7037995 & -0.2680783 & -4.4602769 \\ \text { C } & -0.3896343 & -0.8402386 & -5.1521909 \\ \text { C } & -0.5186782 & -0.6935771 & -6.5396673 \\ \text { C } & 0.4432255 & 0.0262832 & -7.2695392 \\ \text { C } & 1.5378746 & 0.5952061 & -6.5970963 \\ \text { C } & 1.6696810 & 0.4463436 & -5.2086905 \\ \text { Br } & 0.0752188 & 2.0996809 & -0.2575980 \\ \text { C } & 0.9088986 & -2.4869307 & -0.9545615 \\ \text { C } & -0.1480485 & -3.3082868 & -1.6576367 \\ \text { C } & 0.8980458 & -2.3874458 & 0.4436387 \\ \text { C } & 2.1499784 & -2.1656618 & -1.7625270 \\ \text { C } & 2.2128282 & -0.7428713 & -2.4156158 \\ \text { O } & 2.6991748 & 0.2173746 & -1.4857255 \\ \text { H } & 2.9599875 & -0.8108330 & -3.2346859 \\ \text { H } & 3.0550306 & -2.2612588 & -1.1297184 \\ \text { H } & 2.2306773 & -2.9078400 & -2.5841245 \\ \text { H } & 1.8287860 & -2.1622943 & 0.9892576 \\ \text { H } & 0.1313902 & -2.9227582 & 1.0256832\end{array}$




\begin{tabular}{lrrr} 
H & 0.2649273 & -4.3243925 & -1.8443196 \\
$\mathrm{H}$ & -0.4166088 & -2.8778489 & -2.6409199 \\
$\mathrm{H}$ & -1.0661403 & -3.4196055 & -1.0503928 \\
$\mathrm{H}$ & 1.9565461 & 0.8204527 & -1.2393498 \\
$\mathrm{H}$ & -1.1436478 & -1.4067440 & -4.5864914 \\
$\mathrm{H}$ & -1.3760260 & -1.1501813 & -7.0577059 \\
$\mathrm{H}$ & 0.3414257 & 0.1406302 & -8.3593916 \\
$\mathrm{H}$ & 2.2945906 & 1.1650299 & -7.1580625 \\
$\mathrm{H}$ & 2.5174032 & 0.9135372 & -4.6843710 \\
$\mathrm{H}$ & -2.4018472 & -1.5342483 & -1.1226951 \\
$\mathrm{H}$ & -4.7746995 & -0.7923258 & -1.3696332 \\
$\mathrm{H}$ & -5.3148953 & 1.2619844 & -2.7128406 \\
$\mathrm{H}$ & -3.4593011 & 2.5549420 & -3.8031975 \\
$\mathrm{H}$ & -1.0864482 & 1.8097033 & -3.5425576 \\
$\mathrm{Pd}$ & 0.1235494 & -0.4241791 & -0.0756735 \\
$\mathrm{P}$ & -0.0348234 & -0.0430490 & 2.2471367 \\
$\mathrm{C}$ & -1.7028830 & 0.5663803 & 2.7187244 \\
$\mathrm{C}$ & -2.6015747 & 0.9885954 & 1.7173562 \\
$\mathrm{H}$ & -2.2897006 & 0.9825901 & 0.6628646 \\
$\mathrm{C}$ & -3.8897931 & 1.4214790 & 2.0674157 \\
$\mathrm{H}$ & -4.5815893 & 1.7437582 & 1.2749045 \\
$\mathrm{C}$ & -4.2892013 & 1.4402155 & 3.4134331 \\
$\mathrm{H}$ & -5.2995575 & 1.7820947 & 3.6853749 \\
$\mathrm{C}$ & -3.4003595 & 1.0111575 & 4.4145911 \\
$\mathrm{H}$ & -3.7124481 & 1.0134104 & 5.4700175 \\
$\mathrm{C}$ & -2.1147877 & 0.5676146 & 4.0704806 \\
$\mathrm{H}$ & -1.4356973 & 0.1983810 & 4.8537175 \\
$\mathrm{C}$ & 0.1749392 & -1.4802120 & 3.3794072 \\
$\mathrm{C}$ & 1.3810284 & -1.7086064 & 4.0705938 \\
$\mathrm{H}$ & 2.1908006 & -0.9659977 & 4.0171477 \\
$\mathrm{C}$ & 1.5467909 & -2.8765756 & 4.8343570 \\
$\mathrm{H}$ & 2.4910630 & -3.0423605 & 5.3749203 \\
$\mathrm{C}$ & 0.5141474 & -3.8239485 & 4.9138976 \\
$\mathrm{H}$ & 0.6457181 & -4.7362018 & 5.5150280 \\
$\mathrm{C}$ & -0.6911002 & -3.6014671 & 4.2238892 \\
$\mathrm{H}$ & -1.5063934 & -4.3383801 & 4.2841612 \\
$\mathrm{C}$ & -0.8593206 & -2.4397892 & 3.4576048 \\
$\mathrm{H}$ & -1.8055265 & -2.2668907 & 2.9209936 \\
$\mathrm{C}$ & 1.2271249 & 1.1425603 & 2.8477118 \\
$\mathrm{C}$ & 2.4722378 & 1.1376377 & 2.1832450 \\
$\mathrm{H}$ & 2.6137218 & 0.5058561 & 1.2916531 \\
$\mathrm{C}$ & 3.5071995 & 1.9709527 & 2.6309677 \\
$\mathrm{H}$ & 4.4734933 & 1.9673439 & 2.1048509 \\
& 3.2997177 & 2.8243532 & 3.7281802 \\
\hline & 2.1085084 & 3.4875085 & 4.0708870 \\
& & & \\
$\mathrm{H}$ & &
\end{tabular}




$\begin{array}{llll}\mathrm{H} & 1.8831885 & 3.5235973 & 5.2252619 \\ \mathrm{C} & 1.0176472 & 2.0020953 & 3.9415201 \\ \mathrm{H} & 0.0399501 & 2.0304748 & 4.4436323\end{array}$

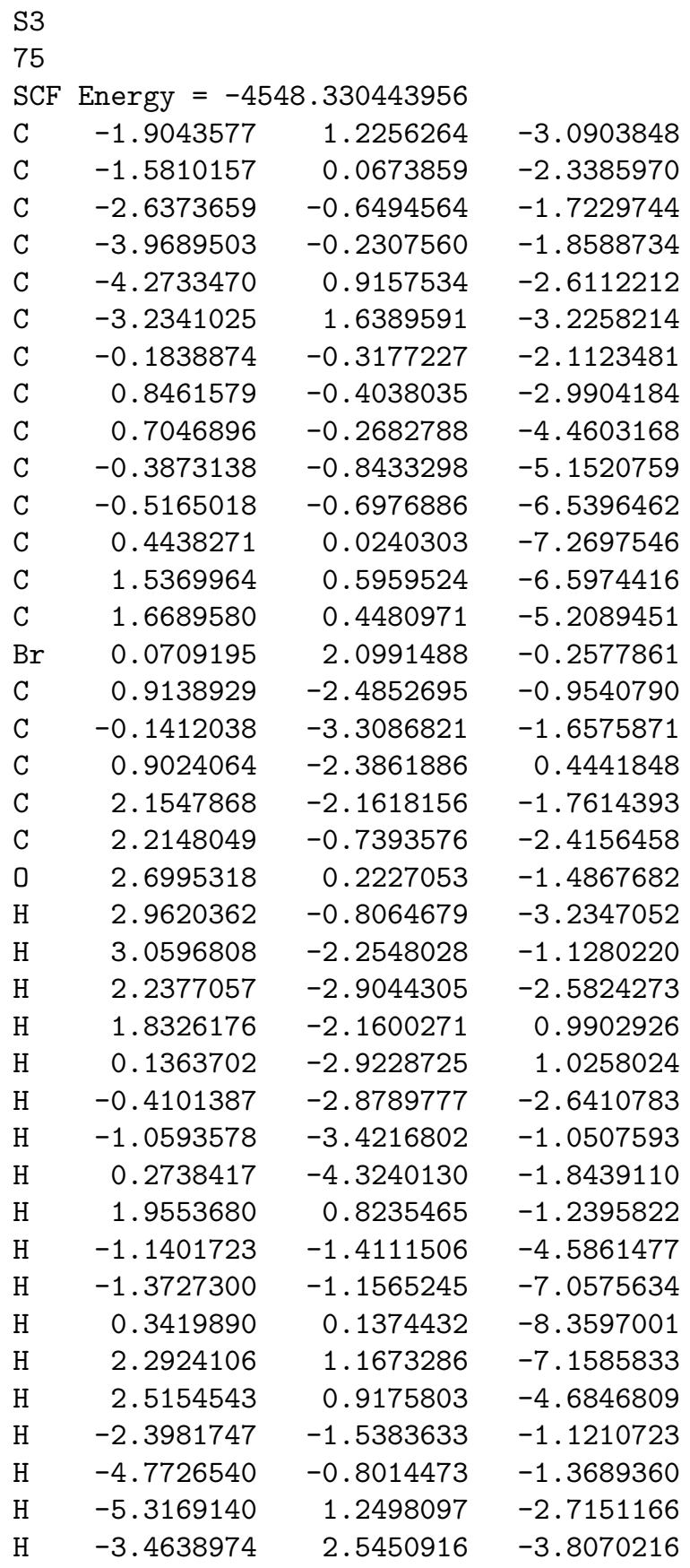




$\begin{array}{lrrr}\text { H } & -1.0895255 & 1.8053570 & -3.5448209 \\ \text { Pd } & 0.1254193 & -0.4245225 & -0.0756187 \\ \text { P } & -0.0320902 & -0.0428990 & 2.2474834 \\ \text { C } & -1.7011146 & 0.5627764 & 2.7203144 \\ \text { C } & -2.6017773 & 0.9822760 & 1.7195719 \\ \text { H } & -2.2909743 & 0.9762231 & 0.6647562 \\ \text { C } & -3.8906025 & 1.4124998 & 2.0706647 \\ \text { H } & -4.5839371 & 1.7327838 & 1.2786911 \\ \text { C } & -4.2887154 & 1.4311462 & 3.4170715 \\ \text { H } & -5.2995959 & 1.7708502 & 3.6897863 \\ \text { C } & -3.3978993 & 1.0047485 & 4.4176069 \\ \text { H } & -3.7089286 & 1.0069419 & 5.4733463 \\ \text { C } & -2.1116385 & 0.5639883 & 4.0724881 \\ \text { H } & -1.4308536 & 0.1969840 & 4.8553015 \\ \text { C } & 1.2276049 & 1.1459675 & 2.8462591 \\ \text { C } & 2.4724462 & 1.1429901 & 2.1812673 \\ \text { H } & 2.6151145 & 0.5102234 & 1.2905580 \\ \text { C } & 3.5056684 & 1.9793305 & 2.6273397 \\ \text { H } & 4.4717214 & 1.9771990 & 2.1007758 \\ \text { C } & 3.2967390 & 2.8337333 & 3.7235029 \\ \text { H } & 4.1040558 & 3.4994305 & 4.0647494 \\ \text { C } & 2.0511545 & 2.8508878 & 4.3728169 \\ \text { H } & 1.8794316 & 3.5313813 & 5.2205886 \\ \text { C } & 1.0167307 & 2.0063563 & 3.9391264 \\ \text { H } & 0.0392386 & 2.0330842 & 4.4417313 \\ \text { C } & 0.1821591 & -1.4790646 & 3.3801309 \\ \text { C } & 1.3896367 & -1.7045603 & 4.0698776 \\ \text { H } & 2.1980313 & -0.9605948 & 4.0144676 \\ \text { C } & 1.5586626 & -2.8714861 & 4.8345065 \\ \text { H } & 2.5038552 & -3.0347868 & 5.3742156 \\ \text { C } & 0.5280534 & -3.8209041 & 4.9160922 \\ \text { H } & 0.6620860 & -4.7322235 & 5.5180949 \\ \text { C } & -0.6783765 & -3.6015666 & 4.2271544 \\ \text { H } & -1.4919133 & -4.3403211 & 4.2886046 \\ \text { C } & -0.8499604 & -2.4407792 & 3.4602671 \\ \text { H } & -1.7971782 & -2.2702850 & 2.9246760 \\ & & & \\ \text { S4 } & & & \\ 75 & \text { C } & & \\ \text { SCF } & \text { Energy }=-4548.336642912 & \\ \text { C } & -2.2605826 & 0.8843552 & -1.2414207 \\ \text { C } & -1.6418605 & -0.0950277 & -2.0672399 \\ \text { C } & -2.4442814 & -1.1512119 & -2.5798871 \\ \text { C } & -3.8050416 & -1.2287984 & -2.2655958 \\ & -4.3961609 & -0.2692167 & -1.4203177 \\ & -3.6209909 & 0.7874500 & -0.9167845\end{array}$




\begin{tabular}{lrrr} 
C & -0.1909920 & -0.1433807 & -2.2579625 \\
$\mathrm{C}$ & 0.6011965 & -0.2275525 & -3.3451010 \\
$\mathrm{C}$ & 0.0662031 & 0.0042941 & -4.7108243 \\
$\mathrm{C}$ & 0.4841086 & -0.7825923 & -5.8102936 \\
$\mathrm{C}$ & -0.0585949 & -0.5817631 & -7.0882684 \\
$\mathrm{C}$ & -1.0248238 & 0.4155050 & -7.2989104 \\
$\mathrm{C}$ & -1.4404860 & 1.2128025 & -6.2183745 \\
$\mathrm{C}$ & -0.9011287 & 1.0116276 & -4.9414507 \\
$\mathrm{Br}$ & 1.0070536 & 2.1550528 & -0.4210765 \\
$\mathrm{C}$ & 1.7750309 & -1.9460888 & -0.9254791 \\
$\mathrm{C}$ & 2.9184551 & -1.7889685 & 0.0509717 \\
$\mathrm{C}$ & 0.5302716 & -2.4654273 & -0.4997938 \\
$\mathrm{C}$ & 2.1780120 & -1.9438564 & -2.3891127 \\
$\mathrm{C}$ & 2.0760543 & -0.5942674 & -3.1591844 \\
$\mathrm{O}$ & 2.8511307 & 0.4285001 & -2.5725370 \\
$\mathrm{H}$ & 2.5187390 & -0.7760926 & -4.1613802 \\
$\mathrm{H}$ & 3.2388720 & -2.2668784 & -2.4437572 \\
$\mathrm{H}$ & 1.5731646 & -2.6874906 & -2.9466581 \\
$\mathrm{H}$ & -0.1768364 & -2.8534219 & -1.2501375 \\
$\mathrm{H}$ & 0.4294157 & -2.8794569 & 0.5141675 \\
$\mathrm{H}$ & 3.5022045 & -0.8780395 & -0.1903384 \\
$\mathrm{H}$ & 3.6115376 & -2.6539380 & -0.0508974 \\
$\mathrm{H}$ & 2.5743146 & -1.7466176 & 1.1020180 \\
$\mathrm{H}$ & 2.2808292 & 0.9391091 & -1.9444356 \\
$\mathrm{H}$ & 1.2293851 & -1.5785444 & -5.6613266 \\
$\mathrm{H}$ & 0.2756850 & -1.2125173 & -7.9263845 \\
$\mathrm{H}$ & -1.4464972 & 0.5762148 & -8.3026781 \\
$\mathrm{H}$ & -2.1854638 & 2.0081181 & -6.3742772 \\
$\mathrm{H}$ & -1.2106697 & 1.6517642 & -4.1032664 \\
$\mathrm{H}$ & -1.9726898 & -1.9048832 & -3.2279355 \\
$\mathrm{H}$ & -4.4127912 & -2.0501225 & -2.6756531 \\
$\mathrm{H}$ & -5.4624840 & -0.3468035 & -1.1590525 \\
$\mathrm{H}$ & -4.0707577 & 1.5426454 & -0.2554604 \\
$\mathrm{H}$ & -1.6506068 & 1.7153074 & -0.8598665 \\
$\mathrm{Pd}$ & 0.3449514 & -0.3145046 & -0.3317066 \\
$\mathrm{P}$ & 0.0213375 & -0.2200691 & 2.0514955 \\
$\mathrm{C}$ & 1.2054689 & 0.6991615 & 3.1051696 \\
$\mathrm{C}$ & 2.4988999 & 0.9639296 & 2.6160616 \\
$\mathrm{H}$ & 2.7628125 & 0.6606550 & 1.5937707 \\
$\mathrm{C}$ & 3.4260773 & 1.6451166 & 3.4208976 \\
$\mathrm{H}$ & 4.4330521 & 1.8566660 & 3.0314042 \\
$\mathrm{C}$ & 3.0621655 & 2.0708678 & 4.7083703 \\
$\mathrm{C}$ & 3.7879964 & 2.6112401 & 5.3349780 \\
& 1.7661392 & 1.8197455 & 5.1940426 \\
\hline & 0.8370192 & 1.1386465 & 4.3946546
\end{tabular}




$\begin{array}{rrr}-0.1817562 & 0.9502857 & 4.7667294 \\ -0.1904884 & -1.8351479 & 2.8984195 \\ 0.6122939 & -2.2335338 & 3.9862785 \\ 1.3574207 & -1.5381721 & 4.3990171 \\ 0.4612279 & -3.5139730 & 4.5442982 \\ 1.0949614 & -3.8144556 & 5.3924554 \\ -0.4935474 & -4.4046993 & 4.0287955 \\ -0.6116975 & -5.4053679 & 4.4709873 \\ -1.2928422 & -4.0168373 & 2.9391004 \\ -2.0372779 & -4.7125054 & 2.5232541 \\ -1.1341003 & -2.7463651 & 2.3674993 \\ -1.7414208 & -2.4570165 & 1.4952057 \\ -1.5684598 & 0.6717823 & 2.3553482 \\ -2.7264272 & 0.0520654 & 2.8632095 \\ -2.7073260 & -1.0066122 & 3.1562287 \\ -3.9140155 & 0.7886440 & 3.0102208 \\ -4.8129559 & 0.2927123 & 3.4068680 \\ -3.9531859 & 2.1474908 & 2.6620547 \\ -4.8847717 & 2.7217609 & 2.7790748 \\ -2.7940449 & 2.7719840 & 2.1678836 \\ -2.8119441 & 3.8380127 & 1.8950286 \\ -1.6085467 & 2.0417910 & 2.0117779 \\ -0.7096181 & 2.5264912 & 1.5997594\end{array}$

\section{Optimized Geometries (MXnc)}

\begin{tabular}{lrrr} 
R3 & & & \\
75 & \multicolumn{4}{l}{} \\
SCF & Energy $=-4548.341224723$ & \\
C & 2.1698052 & 2.0847436 & 0.8564030 \\
C & 1.5467395 & 1.6300254 & -0.3318908 \\
C & 0.9931745 & 2.5993737 & -1.2068190 \\
C & 1.0707130 & 3.9679159 & -0.9140143 \\
C & 1.6875007 & 4.4011962 & 0.2730445 \\
C & 2.2326138 & 3.4519742 & 1.1546256 \\
C & 1.4192940 & 0.1961410 & -0.6397765 \\
C & 2.3797337 & -0.7544170 & -0.4980258 \\
C & 3.7799520 & -0.4722013 & -0.0884844 \\
C & 4.5301131 & 0.5590085 & -0.7027768 \\
C & 5.8389821 & 0.8427384 & -0.2934372 \\
C & 6.4371711 & 0.0957818 & 0.7369816 \\
C & 5.7122293 & -0.9371418 & 1.3516289 \\
C & 4.3980925 & -1.2200692 & 0.9450924
\end{tabular}




$\begin{array}{lrrr}\text { Br } & -2.8882652 & -0.4088734 & -1.9909818 \\ \mathrm{C} & 1.1671705 & -1.0388299 & -3.1817859 \\ \mathrm{C} & 1.9165700 & 0.1478836 & -3.7311453 \\ \mathrm{C} & -0.1857042 & -1.1992254 & -3.4241793 \\ \mathrm{C} & 1.9998318 & -2.1407124 & -2.5605276 \\ \mathrm{C} & 2.0255092 & -2.1568509 & -0.9924296 \\ \mathrm{O} & 2.8400676 & -3.2076001 & -0.5160548 \\ \mathrm{H} & 0.9899736 & -2.3895789 & -0.6599473 \\ \mathrm{H} & 1.6314347 & -3.1347386 & -2.8851258 \\ \mathrm{H} & 3.0516460 & -2.0420786 & -2.9024838 \\ \mathrm{H} & -0.6817262 & -2.1663242 & -3.2511404 \\ \mathrm{H} & -0.7368784 & -0.4798097 & -4.0474631 \\ \mathrm{H} & 2.5489077 & -0.1895273 & -4.5813639 \\ \mathrm{H} & 2.6000205 & 0.5803182 & -2.9747181 \\ \mathrm{H} & 1.2369273 & 0.9403904 & -4.0965872 \\ \mathrm{H} & 3.7713893 & -2.9235930 & -0.6181966 \\ \mathrm{H} & 4.0652169 & 1.1521518 & -1.5035694 \\ \mathrm{H} & 6.3992146 & 1.6533191 & -0.7839578 \\ \mathrm{H} & 7.4658481 & 0.3189011 & 1.0581260 \\ \mathrm{H} & 6.1688909 & -1.5249265 & 2.1628184 \\ \mathrm{H} & 3.8273460 & -2.0171473 & 1.4405479 \\ \mathrm{H} & 0.4791449 & 2.2593041 & -2.1188712 \\ \mathrm{H} & 0.6343849 & 4.6994376 & -1.6110189 \\ \mathrm{H} & 1.7380347 & 5.4742737 & 0.5121571 \\ \mathrm{H} & 2.7057354 & 3.7776979 & 2.0935320 \\ \mathrm{H} & 2.5907133 & 1.3496975 & 1.5549461 \\ \mathrm{Pd} & -0.4284821 & -0.3061896 & -1.3358947 \\ \mathrm{P} & -1.2133637 & -0.1141005 & 0.8017854 \\ \mathrm{C} & -2.4440565 & 1.2243761 & 0.9958060 \\ \mathrm{C} & -2.0383339 & 2.5360637 & 0.6703148 \\ \mathrm{H} & -1.0151915 & 2.7257395 & 0.3143554 \\ \mathrm{C} & -2.9479279 & 3.5964766 & 0.7836657 \\ \mathrm{H} & -2.6242575 & 4.6172294 & 0.5301418 \\ \mathrm{C} & -4.2668340 & 3.3534663 & 1.2045093 \\ \mathrm{H} & -4.9825130 & 4.1857471 & 1.2845471 \\ \mathrm{C} & -4.6738897 & 2.0445307 & 1.5097400 \\ \mathrm{H} & -5.7103649 & 1.8472037 & 1.8225397 \\ \mathrm{C} & -3.7670829 & 0.9780258 & 1.4061419 \\ \mathrm{H} & -4.0926373 & -0.0482455 & 1.6264138 \\ \mathrm{C} & -2.0043293 & -1.6705632 & 1.3810652 \\ \mathrm{C} & -2.2351042 & -2.7453697 & 0.5019008 \\ \mathrm{H} & -1.9875598 & -2.6306999 & -0.5631277 \\ \mathrm{C} & -2.8027108 & -3.9357034 & 0.9836408 \\ \mathrm{H} & -2.9781815 & -4.7721256 & 0.2906108 \\ & -3.1493962 & -4.0564635 & 2.3386690 \\ & -3.5963819 & -4.9901028 & 2.7128521\end{array}$




$\begin{array}{lrrr}\text { C } & -2.9207397 & -2.9847349 & 3.2193691 \\ \text { H } & -3.1874261 & -3.0754063 & 4.2832322 \\ \text { C } & -2.3422205 & -1.7982491 & 2.7466577 \\ \text { H } & -2.1437774 & -0.9686379 & 3.4417035 \\ \text { C } & -0.0207887 & 0.1599911 & 2.1795094 \\ \text { C } & -0.0485183 & 1.2978299 & 3.0060347 \\ \text { H } & -0.8050453 & 2.0777326 & 2.8434702 \\ \text { C } & 0.8945265 & 1.4427856 & 4.0380765 \\ \text { H } & 0.8655805 & 2.3382265 & 4.6769530 \\ \text { C } & 1.8713310 & 0.4582094 & 4.2452895 \\ \text { H } & 2.6144511 & 0.5757988 & 5.0483762 \\ \text { C } & 1.8953326 & -0.6861094 & 3.4264663 \\ \text { H } & 2.6529805 & -1.4658525 & 3.5914014 \\ \text { C } & 0.9546819 & -0.8374122 & 2.3999464 \\ \text { H } & 0.9712453 & -1.7383180 & 1.7697076 \\ & & & \\ \text { R4 } & & & \\ \text { 75 } & & & \\ \text { SCF } & \text { Energy }=-4548.350861461 & \\ \text { C } & -1.4247687 & -2.1041770 & -1.8337582 \\ \text { C } & -1.5777782 & -1.5984382 & -0.5190485 \\ \text { C } & -2.8440654 & -1.0764296 & -0.1458515 \\ \text { C } & -3.9119949 & -1.0557995 & -1.0539652 \\ \text { C } & -3.7385134 & -1.5503103 & -2.3584455 \\ \text { C } & -2.4919859 & -2.0763096 & -2.7399680 \\ \text { C } & -0.4358065 & -1.5299723 & 0.4081315 \\ \text { C } & 0.4777548 & -2.5019130 & 0.6538552 \\ \text { C } & 0.3115448 & -3.8888019 & 0.1435593 \\ \text { C } & -0.9158567 & -4.5725352 & 0.3163208 \\ \text { C } & -1.1028047 & -5.8601077 & -0.2024729 \\ \text { C } & -0.0628100 & -6.5006859 & -0.8987279 \\ \text { C } & 1.1657642 & -5.8420757 & -1.0673476 \\ \text { C } & 1.3546298 & -4.5502183 & -0.5512542 \\ \text { Br } & -0.9340286 & 2.6393441 & 2.0751221 \\ \text { C } & 0.3184787 & -0.7136784 & 3.2976155 \\ \text { C } & 1.2043306 & 0.3513122 & 3.9013067 \\ \text { C } & -1.0678212 & -0.5577726 & 3.2166791 \\ \text { C } & 0.9912347 & -2.0466394 & 3.0588586 \\ \text { C } & 1.6156189 & -2.2107665 & 1.6338271 \\ \text { O } & 2.6554391 & -3.1688857 & 1.6369325 \\ \text { H } & 2.0933458 & -1.2425853 & 1.3688751 \\ \text { H } & 1.8322463 & -2.1515453 & 3.7739879 \\ \text { H } & 0.2889663 & -2.8877613 & 3.2283050 \\ \text { H } & -1.6982613 & -1.4365217 & 3.0095165 \\ \text { H } & -1.5564045 & 0.3294536 & 3.6461946 \\ & 2.1469694 & 0.4587344 & 3.3258866\end{array}$




$\begin{array}{lrrr}\text { H } & 1.4910883 & 0.0385225 & 4.9299252 \\ \mathrm{H} & 0.6997029 & 1.3339764 & 3.9431777 \\ \mathrm{H} & 2.2364294 & -4.0535011 & 1.6356124 \\ \mathrm{H} & -1.7293404 & -4.0698089 & 0.8592339 \\ \mathrm{H} & -2.0676211 & -6.3703435 & -0.0600847 \\ \mathrm{H} & -0.2095830 & -7.5124542 & -1.3060721 \\ \mathrm{H} & 1.9850175 & -6.3342645 & -1.6137907 \\ \mathrm{H} & 2.3122327 & -4.0326665 & -0.6949567 \\ \mathrm{H} & -2.9790639 & -0.6752381 & 0.8703294 \\ \mathrm{H} & -4.8839657 & -0.6448780 & -0.7415515 \\ \mathrm{H} & -4.5730463 & -1.5262812 & -3.0755395 \\ \mathrm{H} & -2.3441065 & -2.4622904 & -3.7600304 \\ \mathrm{H} & -0.4506045 & -2.5084641 & -2.1391680 \\ \mathrm{Pd} & -0.3842282 & 0.2822611 & 1.2606419 \\ \mathrm{P} & 0.5635140 & 1.1843313 & -0.6436495 \\ \mathrm{C} & -0.4960646 & 2.4512163 & -1.4324835 \\ \mathrm{C} & 0.0083904 & 3.6925927 & -1.8600320 \\ \mathrm{H} & 1.0681887 & 3.9398745 & -1.7018915 \\ \mathrm{C} & -0.8500031 & 4.6184138 & -2.4749572 \\ \mathrm{H} & -0.4562880 & 5.5935656 & -2.7994029 \\ \mathrm{C} & -2.2050069 & 4.3052517 & -2.6696517 \\ \mathrm{H} & -2.8748943 & 5.0346559 & -3.1498436 \\ \mathrm{C} & -2.7074100 & 3.0640289 & -2.2410418 \\ \mathrm{H} & -3.7700858 & 2.8162053 & -2.3824917 \\ \mathrm{C} & -1.8598480 & 2.1404496 & -1.6149456 \\ \mathrm{H} & -2.2565211 & 1.1772122 & -1.2608094 \\ \mathrm{C} & 2.1786392 & 2.0023010 & -0.3118881 \\ \mathrm{C} & 2.4354552 & 2.5641517 & 0.9554101 \\ \mathrm{H} & 1.6534804 & 2.5241594 & 1.7296211 \\ \mathrm{C} & 3.6610971 & 3.2004192 & 1.2040190 \\ \mathrm{H} & 3.8520468 & 3.6375893 & 2.1957401 \\ \mathrm{C} & 4.6367544 & 3.2804905 & 0.1965030 \\ \mathrm{H} & 5.5992727 & 3.7753420 & 0.3970525 \\ \mathrm{C} & 4.3804588 & 2.7284016 & -1.0697833 \\ \mathrm{H} & 0.1820573 & -0.7069957 & -5.2770433 \\ \mathrm{H} & 5.1384416 & 2.7914968 & -1.8652663 \\ \mathrm{C} & 3.1568647 & 2.0911050 & -1.3266615 \\ \mathrm{H} & 2.9621999 & 1.6569885 & -2.3179687 \\ \mathrm{C} & 0.9827862 & 0.0471512 & -2.0266155 \\ \mathrm{C} & 1.9375059 & -0.9676668 & -1.7942378 \\ \mathrm{H} & 2.4430079 & -1.0356480 & -0.8206651 \\ \mathrm{C} & 2.2564261 & -1.8794829 & -2.8089035 \\ & 3.0040017 & -2.6640346 & -2.6215710 \\ \mathrm{H} & 1.6232822 & -1.7936342 & -4.0624938 \\ & 0.6713282 & -2.5158410 & -4.8548439 \\ & & & \\ \mathrm{H} & & \end{array}$




$\begin{array}{lrrr}\text { C } & 0.3577898 & 0.1348847 & -3.2845559 \\ \text { H } & -0.3898545 & 0.9183074 & -3.4711822 \\ & & & \\ \text { S3 } & & & \\ 75 & & & \\ \text { SCF } & \text { Energy }=-4548.350729778 & \\ \text { C } & 0.5517158 & -2.1907089 & -2.1012357 \\ \text { C } & -0.5861864 & -1.6025846 & -1.4954572 \\ \text { C } & -1.5954488 & -1.0755376 & -2.3417919 \\ \text { C } & -1.4770428 & -1.1401646 & -3.7371410 \\ \text { C } & -0.3358081 & -1.7170572 & -4.3214206 \\ \text { C } & 0.6751761 & -2.2389737 & -3.4955239 \\ \text { C } & -0.7175585 & -1.4878678 & -0.0330986 \\ \text { C } & -0.4955833 & -2.4614703 & 0.8883690 \\ \text { C } & -0.1420606 & -3.8632022 & 0.5513103 \\ \text { C } & -0.7912182 & -4.5685651 & -0.4889260 \\ \text { C } & -0.4224451 & -5.8821417 & -0.8074847 \\ \text { C } & 0.6031281 & -6.5247323 & -0.0921720 \\ \text { C } & 1.2489666 & -5.8429253 & 0.9524686 \\ \text { C } & 0.8760938 & -4.5295199 & 1.2742706 \\ \text { Br } & -1.9198683 & 2.8668389 & 0.4668309 \\ \text { C } & -3.0267303 & -1.1214279 & 1.5386408 \\ \text { C } & -3.7781766 & -1.8650212 & 0.4630139 \\ \text { C } & -3.1948611 & 0.2434474 & 1.7030852 \\ \text { C } & -2.3020379 & -1.9732358 & 2.5604246 \\ \text { C } & -0.7560719 & -2.1387548 & 2.3589526 \\ \text { O } & -0.0403191 & -1.0201069 & 2.8548979 \\ \text { H } & -0.4436270 & -2.9913917 & 2.9929686 \\ \text { H } & -2.4448389 & -1.5555855 & 3.5774969 \\ \text { H } & -2.7463831 & -2.9896492 & 2.5450628 \\ \text { H } & -2.8648222 & 0.7470620 & 2.6244274 \\ \text { H } & -3.9026146 & 0.8032460 & 1.0743269 \\ \text { H } & -4.6157612 & -2.4224402 & 0.9368387 \\ \text { H } & -3.1347783 & -2.6145822 & -0.0371379 \\ \text { H } & -4.2035853 & -1.1851794 & -0.2989130 \\ \text { H } & -0.2377045 & -0.2767951 & 2.2186970 \\ \text { H } & -1.5924482 & -4.0720119 & -1.0549051 \\ \text { H } & -0.9433357 & -6.4115151 & -1.6199112 \\ \text { H } & 0.8927023 & -7.5561973 & -0.3437833 \\ \text { H } & 2.0513995 & -6.3372924 & 1.5213874 \\ \text { H } & 1.3937407 & -3.9988732 & 2.0878038 \\ \text { H } & -2.4750140 & -0.5953809 & -1.8875166 \\ \text { H } & -2.2743696 & -0.7233443 & -4.3707930 \\ \text { H } & -0.2335745 & -1.7571891 & -5.4164319 \\ \text { H } & 1.5771811 & -2.6852132 & -3.9410134 \\ & 1.3437590 & -2.6036149 & -1.4633171\end{array}$




$\begin{array}{lrrr}\text { Pd } & -1.2718817 & 0.4076803 & 0.4846649 \\ \text { P } & 0.8494983 & 1.1471259 & -0.0123699 \\ \text { C } & 0.8934531 & 2.2987244 & -1.4304925 \\ \text { C } & 1.3288806 & 3.6298846 & -1.2957548 \\ \text { H } & 1.6607353 & 4.0029454 & -0.3166738 \\ \text { C } & 1.3191901 & 4.4811802 & -2.4117308 \\ \text { H } & 1.6532405 & 5.5239688 & -2.3020786 \\ \text { C } & 0.8762015 & 4.0102015 & -3.6584444 \\ \text { H } & 0.8678338 & 4.6825672 & -4.5297542 \\ \text { C } & 0.4334933 & 2.6825259 & -3.7890010 \\ \text { H } & 0.0752453 & 2.3080258 & -4.7597004 \\ \text { C } & 0.4337703 & 1.8278002 & -2.6783161 \\ \text { H } & 0.0653831 & 0.7974211 & -2.7797552 \\ \text { C } & 1.6182877 & 2.0288658 & 1.4089565 \\ \text { C } & 3.0135953 & 2.2508313 & 1.3939648 \\ \text { H } & 3.6170780 & 1.8996728 & 0.5439205 \\ \text { C } & 3.6319945 & 2.9108573 & 2.4650204 \\ \text { H } & 4.7186327 & 3.0837445 & 2.4438810 \\ \text { C } & 2.8680297 & 3.3403797 & 3.5641074 \\ \text { H } & 3.3564919 & 3.8505008 & 4.4082980 \\ \text { C } & 1.4830348 & 3.1136504 & 3.5842753 \\ \text { H } & 0.8793737 & 3.4461333 & 4.4419285 \\ \text { C } & 0.8557896 & 2.4635544 & 2.5098266 \\ \text { H } & -0.2333541 & 2.3139505 & 2.5069235 \\ \text { C } & 2.1556399 & -0.0952920 & -0.3810333 \\ \text { C } & 2.8938501 & -0.0919559 & -1.5791414 \\ \text { H } & 2.6974632 & 0.6701928 & -2.3457450 \\ \text { C } & 3.8770701 & -1.0709440 & -1.8009792 \\ \text { H } & 4.4466467 & -1.0646070 & -2.7424998 \\ \text { C } & 4.1224898 & -2.0562135 & -0.8327254 \\ \text { H } & 4.8841750 & -2.8300020 & -1.0123523 \\ \text { C } & 3.3950195 & -2.0505419 & 0.3716507 \\ \text { H } & 3.5882896 & -2.8178987 & 1.1354157 \\ \text { C } & 2.4164986 & -1.0746073 & 0.6027795 \\ \text { H } & 1.8421569 & -1.0756361 & 1.5423825 \\ & & & \\ \text { S4 } & & & \\ \text { 75 } & & & \\ \text { SCF } & \text { Energy }=-4548.359538668 & \\ \text { C } & -1.1476955 & -2.1636887 & -1.9407094 \\ \text { C } & -1.4922049 & -1.6117690 & -0.6818748 \\ \text { C } & -2.7829647 & -1.0388323 & -0.5338907 \\ \text { C } & -3.6893625 & -1.0133554 & -1.6033951 \\ \text { C } & -3.3249201 & -1.5529112 & -2.8489824 \\ \text { C } & -2.0525703 & -2.1293415 & -3.0083898 \\ & -0.5259961 & -1.5536943 & 0.4276317\end{array}$




\begin{tabular}{|c|c|c|c|}
\hline & 0.2878053 & -2.5407604 & \\
\hline & 0.2257409 & -3.9351968 & 0.3729999 \\
\hline & -1.0084041 & -4.5973097 & 0.1765999 \\
\hline & -1.0507664 & -5.8976100 & -0.3435471 \\
\hline & 0.1393465 & -6.5681464 & -0.6757219 \\
\hline & 1.3737804 & -5.9291544 & -0.4719825 \\
\hline & 1.4168307 & -4.6290782 & 0.0528859 \\
\hline & -1.0770956 & 2.7078758 & 1.8378953 \\
\hline & -0.2492883 & -0.6372110 & 3.3930588 \\
\hline & 0.5239904 & 0.4247343 & 4.1391601 \\
\hline & -1.5835070 & -0.4477526 & 3.0267038 \\
\hline & 0.4188312 & -1.9953952 & 3.3246218 \\
\hline & 1.2550100 & -2.2576890 & 2.0293598 \\
\hline & 2.189 & -1.21 & 44444 \\
\hline & 1.863 & -3.16 & 53785 \\
\hline & 1.111 & -2.0 & 4.1 \\
\hline & -0.327 & -2.8 & 3.4 \\
\hline & -2.187 & -1.31 & 2.7 \\
\hline & -2.117 & 0.4 & 3.3 \\
\hline & 1.572 & 0.46 & 3.7 \\
\hline & 0.5667649 & 0.14 & 42377 \\
\hline & 0.0594712 & 1.42 & 4.0 \\
\hline & 1.6379321 & -0.42 & 362768 \\
\hline & -1.9400080 & -4.0758172 & 0.4389621 \\
\hline & -2.0220788 & -6.3953200 & -0.4868250 \\
\hline & 0.1047789 & -7.5891725 & -1.0849121 \\
\hline & 2.3117515 & -6.44 & -0.7259162 \\
\hline & 2.3872640 & -4.13 & 0.1983937 \\
\hline & -3.0672434 & -0.6020541 & 0.4355374 \\
\hline & -4.6834318 & -0.5624605 & -1.4633114 \\
\hline & -4.0307570 & -1.52 & -3.6 \\
\hline & -1.7548424 & -2.5510525 & -3.9 \\
\hline & -0.1541162 & -2.6107460 & -2.0738013 \\
\hline & -0.5269573 & 0.3030371 & 1.1967839 \\
\hline & 0.7066257 & 1.1012253 & -0.5970263 \\
\hline & 2.2903602 & 1.8716749 & -0.0653086 \\
\hline & 2.4098092 & 2.4293929 & 1.2240592 \\
\hline & 1.5394329 & 2.4215276 & 1.8983451 \\
\hline & 3.6194388 & 3.0182145 & 1.6224924 \\
\hline & 3.7041475 & 3.4504193 & 2.6309051 \\
\hline & 4.7135522 & 3.0555576 & 0.7428730 \\
\hline & 5.6627362 & 3.5131313 & 1.0608952 \\
\hline & 4.5950717 & 2.5058297 & -0.5448641 \\
\hline & 5.4484494 & 2.5332247 & -1.2392808 \\
\hline & 3.3898083 & 1.9137239 & -0.950489 \\
\hline & 3.3034126 & 1.4763423 & -1.955676 \\
\hline
\end{tabular}




$\begin{array}{rrrr}\text { C } & 1.2639717 & -0.0909487 & -1.8812096 \\ \mathrm{C} & 0.8554478 & 0.0148315 & -3.2241165 \\ \mathrm{H} & 0.1967060 & 0.8373543 & -3.5354188 \\ \mathrm{C} & 1.2796492 & -0.9374068 & -4.1659476 \\ \mathrm{H} & 0.9512906 & -0.8490560 & -5.2124978 \\ \mathrm{C} & 2.1080676 & -1.9991927 & -3.7720890 \\ \mathrm{H} & 2.4318218 & -2.7503256 & -4.5084066 \\ \mathrm{C} & 2.5251792 & -2.1003365 & -2.4322966 \\ \mathrm{H} & 3.1750018 & -2.9304105 & -2.1183341 \\ \mathrm{C} & 2.1080036 & -1.1534205 & -1.4870051 \\ \mathrm{H} & 2.4303594 & -1.2409276 & -0.4379157 \\ \mathrm{C} & -0.1982813 & 2.3812334 & -1.5390243 \\ \mathrm{C} & 0.3789614 & 3.6237058 & -1.8566552 \\ \mathrm{H} & 1.3996732 & 3.8580178 & -1.5214156 \\ \mathrm{C} & -0.3601048 & 4.5671126 & -2.5885191 \\ \mathrm{H} & 0.0888607 & 5.5430785 & -2.8273678 \\ \mathrm{C} & -1.6671558 & 4.2709595 & -3.0074708 \\ \mathrm{H} & -2.2436820 & 5.0143941 & -3.5786157 \\ \mathrm{C} & -2.2424157 & 3.0284953 & -2.6877567 \\ \mathrm{H} & -3.2693726 & 2.7936722 & -3.0054864 \\ \mathrm{C} & -1.5159257 & 2.0864999 & -1.9475924 \\ \mathrm{H} & -1.9737759 & 1.1227101 & -1.6793661\end{array}$

\section{Optimized Geometries(BXn)(TS-cd)-exo}

\begin{tabular}{lrrr} 
R1 & & & \\
75 & \multicolumn{4}{l}{} \\
SCF & Energy $=-4548.297673059$ & \\
C & 1.1481402 & 4.3305207 & -0.6635396 \\
C & 0.4251666 & 3.3075167 & 0.0158307 \\
C & -0.9705697 & 3.5115920 & 0.2623081 \\
C & -1.5912418 & 4.7199701 & -0.1011796 \\
C & -0.8464117 & 5.7296601 & -0.7191947 \\
C & 0.5178916 & 5.5223619 & -1.0163055 \\
C & 1.0866648 & 2.1315231 & 0.5921584 \\
C & 1.0592310 & 0.8030365 & 0.2940590 \\
C & 0.2493802 & 0.1872407 & -0.7718832 \\
C & -0.1729231 & -1.1585691 & -0.6197152 \\
C & -0.9809289 & -1.7710850 & -1.5871882 \\
C & -1.3797682 & -1.0618857 & -2.7327524 \\
C & -0.9581909 & 0.2693906 & -2.9006433 \\
C & -0.1546994 & 0.8881275 & -1.9348612 \\
Br & 1.2837990 & 5.6995095 & 2.9501429
\end{tabular}




$\begin{array}{lrrr}\text { C } & 2.8578734 & 2.2147728 & 1.8325133 \\ \text { C } & 3.7825207 & 3.2571655 & 1.2414825 \\ \mathrm{C} & 2.3643782 & 2.4660521 & 3.1867079 \\ \mathrm{C} & 3.1690961 & 0.7650397 & 1.4862403 \\ \mathrm{C} & 1.9139529 & -0.0723534 & 1.1960095 \\ \mathrm{O} & 1.2304504 & -0.5125932 & 2.3681142 \\ \mathrm{H} & 2.2215442 & -0.9954380 & 0.6600660 \\ \mathrm{H} & 3.7988626 & 0.7526255 & 0.5728561 \\ \mathrm{H} & 3.7506373 & 0.2904071 & 2.3048499 \\ \mathrm{H} & 2.1185399 & 1.5847610 & 3.8023351 \\ \mathrm{H} & 2.8148832 & 3.3095223 & 3.7369904 \\ \mathrm{H} & 3.9000758 & 3.1204083 & 0.1487926 \\ \mathrm{H} & 4.7914630 & 3.1446974 & 1.6970874 \\ \mathrm{H} & 3.4242689 & 4.2847204 & 1.4536901 \\ \mathrm{H} & 0.5112915 & 0.1255770 & 2.5528859 \\ \mathrm{H} & 0.1015746 & -1.7034895 & 0.2949317 \\ \mathrm{H} & -1.3053141 & -2.8132287 & -1.4421593 \\ \mathrm{H} & -2.0110969 & -1.5445031 & -3.4939302 \\ \mathrm{H} & -1.2536454 & 0.8312322 & -3.7999617 \\ \mathrm{H} & 0.1792945 & 1.9237367 & -2.0874694 \\ \mathrm{H} & -1.5613607 & 2.6888656 & 0.6827234 \\ \mathrm{H} & -2.6586297 & 4.8629877 & 0.1186516 \\ \mathrm{H} & -1.3247950 & 6.6845837 & -0.9826648 \\ \mathrm{H} & 1.0963916 & 6.3161749 & -1.5114320 \\ \mathrm{H} & 2.2152279 & 4.1811103 & -0.8700753 \\ \mathrm{Pd} & 0.6802940 & 3.2752707 & 2.3357205 \\ \mathrm{P} & -1.0787754 & 2.5042965 & 3.6767579 \\ \mathrm{C} & -2.6320541 & 3.4343148 & 3.3456178 \\ \mathrm{C} & -2.4977113 & 4.8272504 & 3.1526454 \\ \mathrm{H} & -1.4941687 & 5.2882480 & 3.1687911 \\ \mathrm{C} & -3.6372829 & 5.6119529 & 2.9239888 \\ \mathrm{H} & -3.5256130 & 6.6969924 & 2.7772540 \\ \mathrm{C} & -4.9099867 & 5.0169003 & 2.8690946 \\ \mathrm{H} & -5.8011840 & 5.6345037 & 2.6795727 \\ \mathrm{C} & -5.0428116 & 3.6307956 & 3.0574440 \\ \mathrm{H} & -6.0373715 & 3.1605307 & 3.0192430 \\ \mathrm{C} & -3.9088458 & 2.8391692 & 3.3025360 \\ \mathrm{H} & -4.0190061 & 1.7556455 & 3.4576647 \\ \mathrm{C} & -1.5199440 & 0.7380912 & 3.4713783 \\ \mathrm{C} & -1.1278245 & -0.2452912 & 4.4086914 \\ \mathrm{H} & -0.6515932 & 0.0575690 & 5.3522070 \\ \mathrm{C} & -1.3280245 & -1.6069543 & 4.1330519 \\ & -1.0071952 & -2.3598375 & 4.8684524 \\ \mathrm{C} & -1.9256597 & -2.0092634 & 2.9286404 \\ & -2.0787390 & -3.0780438 & 2.7173722 \\ & & -1.0409457 & 1.9855034\end{array}$




$\begin{array}{lrrr}\text { H } & -2.7603132 & -1.3423996 & 1.0286463 \\ \text { C } & -2.0974377 & 0.3180207 & 2.2468586 \\ \text { H } & -2.3877189 & 1.0610281 & 1.4896095 \\ \text { C } & -0.8607966 & 2.7445070 & 5.4856803 \\ \text { C } & 0.2188036 & 3.5298908 & 5.9342011 \\ \text { H } & 0.9015882 & 3.9828640 & 5.1975027 \\ \text { C } & 0.3804281 & 3.7765595 & 7.3079147 \\ \text { H } & 1.2212308 & 4.3974128 & 7.6521414 \\ \text { C } & -0.5303743 & 3.2434758 & 8.2332674 \\ \text { H } & -0.4004792 & 3.4363246 & 9.3091844 \\ \text { C } & -1.6196056 & 2.4736401 & 7.7850888 \\ \text { H } & -2.3442581 & 2.0683271 & 8.5076917 \\ \text { C } & -1.7905892 & 2.2299873 & 6.4153348 \\ \text { H } & -2.6521475 & 1.6415190 & 6.0644621 \\ & & & \\ \text { R2 } & & & \\ 75 & & & \\ \text { SCF } & \text { Energy }=-4548.311633101 & \\ \text { C } & -0.7199096 & 3.7382082 & -1.4534648 \\ \text { C } & -1.1980273 & 2.5729915 & -0.7971641 \\ \text { C } & -2.4820872 & 2.0848556 & -1.1521503 \\ \text { C } & -3.2497687 & 2.7233080 & -2.1364524 \\ \text { C } & -2.7557129 & 3.8636899 & -2.7884689 \\ \text { C } & -1.4885250 & 4.3661928 & -2.4392204 \\ \text { C } & -0.4108740 & 1.9618269 & 0.2904126 \\ \text { C } & 0.9086949 & 1.6552168 & 0.3286162 \\ \text { C } & 1.8421702 & 1.8454602 & -0.8087054 \\ \text { C } & 1.4836122 & 1.4742189 & -2.1262395 \\ \text { C } & 2.3813602 & 1.6462906 & -3.1872515 \\ \text { C } & 3.6592067 & 2.1864183 & -2.9559980 \\ \text { C } & 4.0334240 & 2.5472516 & -1.6516200 \\ \text { C } & 3.1358847 & 2.3754938 & -0.5860803 \\ \text { Br } & -0.4322019 & 4.8797047 & 1.8128829 \\ \text { C } & -1.0258372 & 0.3680466 & 1.6339295 \\ \text { C } & -1.0792095 & -0.7083479 & 0.5645778 \\ \text { C } & -2.2521233 & 0.7227948 & 2.3032864 \\ \text { C } & 0.2754822 & 0.4494608 & 2.4202988 \\ \text { C } & 1.4510519 & 0.8932282 & 1.5319362 \\ \text { O } & 2.1319801 & -0.2891151 & 1.0909604 \\ \text { H } & 2.1457052 & 1.5343891 & 2.1280838 \\ \text { H } & 0.1495755 & 1.1500047 & 3.2681003 \\ \text { H } & 0.5330075 & -0.5499494 & 2.8320935 \\ \text { H } & -2.2542738 & 0.7516069 & 3.4070905 \\ \text { H } & -3.1983222 & 0.3592708 & 1.8679224 \\ & -1.2732980 & -1.6758750 & 1.0787250 \\ & -0.1185848 & -0.7935895 & 0.0262174\end{array}$




\begin{tabular}{lrrr} 
H & -1.8993863 & -0.5473527 & -0.1588640 \\
H & 2.7156396 & -0.0174210 & 0.3536664 \\
H & 0.4886675 & 1.0428097 & -2.3065267 \\
H & 2.0840010 & 1.3505667 & -4.2049266 \\
H & 4.3630591 & 2.3207616 & -3.7911585 \\
H & 5.0303107 & 2.9729301 & -1.4606355 \\
H & 3.4255542 & 2.6837392 & 0.4299606 \\
H & -2.8850110 & 1.2013014 & -0.6389028 \\
H & -4.2463235 & 2.3303347 & -2.3877045 \\
H & -3.3601897 & 4.3671360 & -3.5578476 \\
H & -1.0984578 & 5.2696864 & -2.9322786 \\
H & 0.2465728 & 4.1587286 & -1.1476818 \\
Pd & -1.7087166 & 2.6851725 & 1.8225767 \\
P & -3.4807168 & 3.6899902 & 2.8325284 \\
$\mathrm{C}$ & -3.1275862 & 4.8318577 & 4.2211404 \\
$\mathrm{C}$ & -4.4496876 & 4.6482072 & 1.5974799 \\
$\mathrm{C}$ & -4.7295912 & 2.5363915 & 3.5364040 \\
$\mathrm{C}$ & -3.9959943 & 5.8887373 & 4.5552161 \\
$\mathrm{C}$ & -3.7156056 & 6.6983861 & 5.6670382 \\
$\mathrm{C}$ & -2.5726949 & 6.4539456 & 6.4472782 \\
$\mathrm{C}$ & -1.7023918 & 5.4033702 & 6.1102501 \\
$\mathrm{C}$ & -1.9728124 & 4.5979246 & 4.9941618 \\
$\mathrm{H}$ & -4.8855087 & 6.0869137 & 3.9391234 \\
$\mathrm{H}$ & -4.3914434 & 7.5288036 & 5.9217752 \\
$\mathrm{H}$ & -2.3535080 & 7.0928795 & 7.3162993 \\
$\mathrm{H}$ & -0.7972196 & 5.2210829 & 6.7086811 \\
$\mathrm{H}$ & -1.2804187 & 3.7966352 & 4.6953097 \\
$\mathrm{C}$ & -5.8407599 & 4.8410192 & 1.7496787 \\
$\mathrm{C}$ & -6.5567872 & 5.5775834 & 0.7937999 \\
$\mathrm{C}$ & -5.8921228 & 6.1230858 & -0.3180128 \\
$\mathrm{C}$ & -4.5096792 & 5.9299915 & -0.4709105 \\
$\mathrm{C}$ & -3.7856915 & 5.1947373 & 0.4795650 \\
$\mathrm{H}$ & -6.3675849 & 4.4021595 & 2.6101060 \\
$\mathrm{H}$ & -7.6408102 & 5.7230635 & 0.9174754 \\
$\mathrm{H}$ & -6.4566789 & 6.6967454 & -1.0690383 \\
$\mathrm{H}$ & -3.9821126 & 6.3459030 & -1.3415777 \\
$\mathrm{H}$ & -2.7000790 & 5.0492648 & 0.3648285 \\
$\mathrm{C}$ & -4.9124533 & 2.3917941 & 4.9250998 \\
$\mathrm{C}$ & -5.8100257 & 1.4308625 & 5.4220572 \\
$\mathrm{C}$ & -6.5332270 & 0.6140738 & 4.5392818 \\
$\mathrm{C}$ & -6.3543004 & 0.7561140 & 3.1510188 \\
$\mathrm{C}$ & -5.4529596 & 1.7055459 & 2.6517300 \\
$\mathrm{H}$ & -4.3550517 & 3.0375424 & 5.6195994 \\
& -5.9473998 & 1.3264478 & 6.5090657 \\
\hline & -6.9205569 & 0.1203339 & 2.4534285
\end{tabular}




\begin{tabular}{lrrr} 
H & -5.3116360 & 1.8162397 & 1.5649901 \\
& & & \\
R3 & & & \\
75 & \multicolumn{4}{r}{} \\
SCF & Energy $=-4548.313087721$ & \\
C & -0.9987642 & 5.7904963 & 0.0635466 \\
C & -0.7042357 & 4.4046010 & 0.0561261 \\
C & -1.0433257 & 3.6621636 & -1.1056013 \\
C & -1.6219263 & 4.2861211 & -2.2167281 \\
C & -1.8958721 & 5.6656547 & -2.1949606 \\
C & -1.5856184 & 6.4126022 & -1.0467293 \\
C & -0.1428281 & 3.7242297 & 1.2303699 \\
C & 0.6533425 & 2.6205327 & 1.2604702 \\
C & 1.4632284 & 2.1513940 & 0.1184350 \\
C & 1.5197129 & 0.7845110 & -0.2545417 \\
C & 2.2867745 & 0.3819520 & -1.3592297 \\
C & 3.0141987 & 1.3275512 & -2.1004669 \\
C & 2.9728699 & 2.6838696 & -1.7298490 \\
C & 2.2070170 & 3.0954074 & -0.6328766 \\
Br & 2.1861486 & 5.8392148 & 1.9490610 \\
C & -1.5161564 & 3.1113584 & 2.7901967 \\
C & -2.6824886 & 2.9125206 & 1.8452466 \\
C & -1.6642330 & 4.1362754 & 3.7956298 \\
C & -0.7049107 & 1.8658788 & 3.1583860 \\
C & 0.7385097 & 1.9365377 & 2.6377708 \\
O & 1.2671985 & 0.6236889 & 2.6441605 \\
H & 1.3518355 & 2.6031886 & 3.2975224 \\
H & -0.7137202 & 1.7053246 & 4.2553915 \\
H & -1.1632963 & 0.9751065 & 2.6815218 \\
H & -1.3706842 & 3.8953556 & 4.8321607 \\
H & -2.5521480 & 4.7884137 & 3.7278683 \\
H & -2.4203730 & 2.2760813 & 0.9800740 \\
H & -3.0676901 & 3.8771181 & 1.4633339 \\
H & -3.5005825 & 2.4148095 & 2.4120832 \\
H & 2.1748854 & 0.6731557 & 2.2873223 \\
H & 0.9374908 & 0.0470464 & 0.3142098 \\
H & 2.3093393 & -0.6805076 & -1.6470665 \\
H & 3.6150400 & 1.0078186 & -2.9656423 \\
H & 3.5485195 & 3.4292821 & -2.2996436 \\
H & 2.1863417 & 4.1497297 & -0.3160938 \\
H & -0.8399905 & 2.5821589 & -1.1281824 \\
H & -1.8674928 & 3.6886902 & -3.1081476 \\
H & -2.3545501 & 6.1536261 & -3.0684459 \\
H & -1.7970810 & 7.4922594 & -1.0136709 \\
H & -0.7461468 & 6.3791420 & 0.9586078 \\
Pd & -0.0096678 & 5.0453209 & 2.9405002 \\
& & &
\end{tabular}




$\begin{array}{rrrr}\text { P } & 0.1342279 & 6.6806500 & 4.5373088 \\ \mathrm{C} & -0.1684021 & 8.3912103 & 3.9275234 \\ \mathrm{C} & 0.2224430 & 8.7306286 & 2.6150019 \\ \mathrm{H} & 0.7117606 & 7.9708409 & 1.9836124 \\ \mathrm{C} & 0.0222303 & 10.0358544 & 2.1403139 \\ \mathrm{H} & 0.3321448 & 10.2912593 & 1.1156881 \\ \mathrm{C} & -0.5666604 & 11.0092807 & 2.9643240 \\ \mathrm{H} & -0.7255223 & 12.0309608 & 2.5867773 \\ \mathrm{C} & -0.9516935 & 10.6760814 & 4.2736940 \\ \mathrm{H} & -1.4102534 & 11.4347882 & 4.9260501 \\ \mathrm{C} & -0.7547004 & 9.3728783 & 4.7565301 \\ \mathrm{H} & -1.0650128 & 9.1167928 & 5.7801394 \\ \mathrm{C} & 1.7457425 & 6.7455443 & 5.4104981 \\ \mathrm{C} & 2.5065900 & 5.5622487 & 5.4934804 \\ \mathrm{H} & 2.1539002 & 4.6600750 & 4.9709611 \\ \mathrm{C} & 3.7110690 & 5.5521032 & 6.2117693 \\ \mathrm{H} & 4.3041115 & 4.6271320 & 6.2703668 \\ \mathrm{C} & 4.1685078 & 6.7257807 & 6.8345572 \\ \mathrm{H} & 5.1199869 & 6.7203150 & 7.3878135 \\ \mathrm{C} & 3.4180296 & 7.9099517 & 6.7402258 \\ \mathrm{H} & 3.7803800 & 8.8331839 & 7.2172560 \\ \mathrm{C} & 2.2066293 & 7.9222324 & 6.0313456 \\ \mathrm{H} & 1.6238664 & 8.8518682 & 5.9512383 \\ \mathrm{C} & -1.0788329 & 6.5075295 & 5.9113393 \\ \mathrm{C} & -0.6913700 & 6.0452515 & 7.1844043 \\ \mathrm{H} & 0.3720096 & 5.8644740 & 7.3995904 \\ \mathrm{C} & -1.6584891 & 5.8213241 & 8.1790728 \\ \mathrm{H} & -1.3444126 & 5.4623579 & 9.1709550 \\ \mathrm{C} & -3.0159354 & 6.0603236 & 7.9137918 \\ \mathrm{H} & -3.7709804 & 5.8874289 & 8.6954232 \\ \mathrm{C} & -3.4077644 & 6.5226957 & 6.6447497 \\ \mathrm{H} & -4.4701098 & 6.7140053 & 6.4300258 \\ \mathrm{C} & -2.4474234 & 6.7383311 & 5.6470091 \\ \mathrm{H} & -2.7582286 & 7.1007640 & 4.6546953\end{array}$

R4

75

SCF Energy $=-4548.284203803$

$\begin{array}{lll}\text { C } & 2.6019619 & 0.3405809\end{array}$

$\begin{array}{lll}\text { C } & 1.3101758 & 0.9148291\end{array}$

$-0.3584184$

$-0.2866735$

$\begin{array}{llll}\text { C } & 0.3272207 & 0.4770310 & -1.2042113\end{array}$

$\begin{array}{llll}\text { C } & 0.6347871 & -0.4841385 & -2.1776795\end{array}$

$\begin{array}{llll}\text { C } & 1.9273965 & -1.0285059 & -2.2542717\end{array}$

$\begin{array}{llll}\text { C } & 2.9084366 & -0.6141464 & -1.3376779\end{array}$

$\begin{array}{llll}\text { C } & 1.0283693 & 1.9289890 & 0.7634953\end{array}$

$\begin{array}{llll}\mathrm{Pd} & -0.2915538 & 5.2743771 & 0.8422542\end{array}$ 


\begin{tabular}{lrrr} 
P & -1.6514391 & 7.0348029 & 0.6500834 \\
$\mathrm{C}$ & 0.3274467 & 3.0682886 & 0.5551348 \\
$\mathrm{Br}$ & 1.6937953 & 6.3400143 & -0.3800974 \\
$\mathrm{C}$ & -0.4145827 & 3.4011640 & -0.6840351 \\
$\mathrm{C}$ & 0.2545862 & 3.6728769 & -1.9058343 \\
$\mathrm{C}$ & -0.4773519 & 3.8959783 & -3.0747639 \\
$\mathrm{C}$ & -1.8847264 & 3.8757746 & -3.0567365 \\
$\mathrm{C}$ & -2.5623147 & 3.6011095 & -1.8594967 \\
$\mathrm{C}$ & -1.8374173 & 3.3492552 & -0.6849400 \\
$\mathrm{C}$ & 1.3844868 & 1.5779711 & 2.2088124 \\
$\mathrm{O}$ & 1.4975010 & 0.1915491 & 2.4592858 \\
$\mathrm{C}$ & 0.2464146 & 2.2550082 & 2.9913021 \\
$\mathrm{C}$ & 0.1715845 & 3.7103298 & 2.5587635 \\
$\mathrm{C}$ & -1.0906972 & 4.4460942 & 2.5810051 \\
$\mathrm{C}$ & 1.4168260 & 4.4828527 & 3.0131060 \\
$\mathrm{H}$ & 2.3625872 & 2.0099737 & 2.5143868 \\
$\mathrm{H}$ & 0.4272990 & 2.2146916 & 4.0877113 \\
$\mathrm{H}$ & -0.7003048 & 1.7206070 & 2.7718667 \\
$\mathrm{H}$ & -2.0178032 & 3.8539150 & 2.4847024 \\
$\mathrm{H}$ & -1.1813183 & 5.2828999 & 3.2962182 \\
$\mathrm{H}$ & 1.6927844 & 4.1715657 & 4.0449179 \\
$\mathrm{H}$ & 1.2469724 & 5.5758578 & 3.0038089 \\
$\mathrm{H}$ & 2.2860745 & 4.2958662 & 2.3549484 \\
$\mathrm{H}$ & 0.8226321 & -0.2595037 & 1.9149774 \\
$\mathrm{H}$ & -0.6880814 & 0.8924220 & -1.1502643 \\
$\mathrm{H}$ & -0.1452151 & -0.8103951 & -2.8823296 \\
$\mathrm{H}$ & 2.1683884 & -1.7794677 & -3.0218842 \\
$\mathrm{H}$ & 3.9226001 & -1.0392994 & -1.3842901 \\
$\mathrm{H}$ & 3.3728866 & 0.6549701 & 0.3594504 \\
$\mathrm{H}$ & -2.3597186 & 3.0827063 & 0.2452494 \\
$\mathrm{H}$ & -3.6620962 & 3.5795593 & -1.8384472 \\
$\mathrm{H}$ & -2.4525600 & 4.0817604 & -3.9755129 \\
$\mathrm{H}$ & -5.7338865 & 4.8052285 & 2.3777603 \\
$\mathrm{H}$ & 0.0571708 & 4.1090863 & -4.0126374 \\
$\mathrm{H}$ & 1.3504708 & 3.7294790 & -1.9058195 \\
$\mathrm{C}$ & -2.3145493 & 7.1310832 & -1.0624806 \\
$\mathrm{C}$ & -0.9434658 & 8.6921285 & 0.9624729 \\
$\mathrm{C}$ & -3.1416803 & 6.9640570 & 1.7218724 \\
$\mathrm{C}$ & -3.4447989 & 7.9656783 & 2.6638138 \\
$\mathrm{C}$ & -4.5641238 & 7.8270866 & 3.5030031 \\
$\mathrm{C}$ & -5.3902523 & 6.6974670 & 3.4011450 \\
-5.0908439 & 5.6946336 & 2.4605456 \\
\hline & -3.9675342 & 5.8207918 & 1.6334774 \\
$\mathrm{H}$ & -4.7939858 & 8.8561057 & 2.7413221 \\
& -6.2676279 & 6.6125728 & 4.2397131 \\
& & & \\
$\mathrm{H}$ & &
\end{tabular}




$\begin{array}{rrrr}\text { H } & -3.7188869 & 5.0313942 & 0.9078644 \\ \text { C } & -3.6935979 & 7.2235284 & -1.3400964 \\ \text { C } & -4.1361582 & 7.3007806 & -2.6709089 \\ \text { C } & -3.2084286 & 7.3004729 & -3.7249818 \\ \text { C } & -1.8342449 & 7.2132341 & -3.4454737 \\ \text { C } & -1.3828376 & 7.1182779 & -2.1221344 \\ \text { H } & -4.4247148 & 7.2405290 & -0.5193147 \\ \text { H } & -5.2142868 & 7.3698226 & -2.8818921 \\ \text { H } & -3.5582352 & 7.3651572 & -4.7667225 \\ \text { H } & -1.1017392 & 7.2026798 & -4.2665085 \\ \text { H } & -0.3066244 & 7.0168659 & -1.8991631 \\ \text { C } & 0.2108796 & 8.8035273 & 1.7604420 \\ \text { C } & 0.7399640 & 10.0706028 & 2.0493568 \\ \text { C } & 0.1264470 & 11.2240330 & 1.5329454 \\ \text { C } & -1.0181135 & 11.1125810 & 0.7239500 \\ \text { C } & -1.5541161 & 9.8484893 & 0.4357806 \\ \text { H } & 0.7026311 & 7.8883738 & 2.1211503 \\ \text { H } & 1.6471879 & 10.1552773 & 2.6660194 \\ \text { H } & 0.5489443 & 12.2165207 & 1.7520390 \\ \text { H } & -1.4919247 & 12.0152049 & 0.3092208 \\ \text { H } & -2.4439392 & 9.7556651 & -0.2054740\end{array}$

S1

75

SCF Energy $=-4548.284551216$

$\begin{array}{lll}\text { C } & -0.2754617 & 3.8087611\end{array}$

$\begin{array}{lll}\text { C } & -0.3895729 & 2.4223774\end{array}$

C $\quad-1.6889171 \quad 1.8391492$

$\begin{array}{lll}\text { C } & -2.8324708 & 2.6278290\end{array}$

C $\quad-2.7009979 \quad 3.9980673$

C $\quad-1.4203122 \quad 4.5781094$

C $\quad 0.8030429 \quad 1.5558610$

C $\quad 1.6187048 \quad 1.1905100$

C $\quad 1.5711164 \quad 1.7454333$

$\begin{array}{lll}\text { C } & 0.3639593 & 2.0198661\end{array}$

$\begin{array}{lll}\text { C } & 0.3848732 & 2.5304328\end{array}$

C $\quad 1.6060193 \quad 2.7808106$

C $\quad 2.8113352 \quad 2.4984856$

C $\quad 2.7959995 \quad 1.9782435$

$\mathrm{Br} \quad 1.2497819 \quad 4.2038124$

$\begin{array}{lll}\text { C } & 1.4069376 & 0.1609985\end{array}$

$\begin{array}{lll}\text { C } & 2.5651172 & 0.6552800\end{array}$

$\begin{array}{lll}\text { C } & 0.1488004 & -0.1094442\end{array}$

$\begin{array}{lll}\text { C } & 1.8964353 & -0.7529396\end{array}$

$\begin{array}{lll}\text { C } & 2.6101119 & 0.0759270\end{array}$

$\begin{array}{lll}0 & 3.9175121 & 0.5361431\end{array}$
0.2795252
0.5636926
0.6016548
0.4022081
0.1330018
0.0594747
0.7092639
$-0.3102612$
$-1.6888378$
$-2.3721318$
$-3.6774795$
$-4.3258703$
$-3.6622689$
$-2.3602556$
3. 2635496
2. 1674803
3. 0552783
2. 8681997
1.0532228
$-0.0276622$
0.3189109 


$\begin{array}{lrrr}\text { H } & 2.7589228 & -0.5402628 & -0.9381950 \\ \text { H } & 2.5804926 & -1.5139785 & 1.4864619 \\ \text { H } & 1.0432497 & -1.2803365 & 0.5821674 \\ \text { H } & -0.6281307 & -0.6565460 & 2.3048729 \\ \text { H } & 0.2116775 & -0.4156283 & 3.9274866 \\ \text { H } & 2.9436817 & 1.6522828 & 2.7528706 \\ \text { H } & 3.4112853 & -0.0618744 & 3.0268634 \\ \text { H } & 2.2321173 & 0.7767578 & 4.1043711 \\ \text { H } & 3.8008652 & 1.2991645 & 0.9171317 \\ \text { H } & -0.5985006 & 1.8180789 & -1.8842462 \\ \text { H } & -0.5657762 & 2.7321461 & -4.1944223 \\ \text { H } & 1.6175500 & 3.1882378 & -5.3482584 \\ \text { H } & 3.7744060 & 2.6841327 & -4.1621174 \\ \text { H } & 3.7375355 & 1.7562330 & -1.8358929 \\ \text { H } & -1.7797582 & 0.7542693 & 0.7548115 \\ \text { H } & -3.8301531 & 2.1673974 & 0.4553145 \\ \text { H } & -3.5973425 & 4.6174463 & -0.0149057 \\ \text { H } & -1.3147073 & 5.6520837 & -0.1548893 \\ \text { H } & 0.7228690 & 4.2636604 & 0.2607078 \\ \text { Pd } & 0.0358273 & 1.9697217 & 2.8749046 \\ \text { P } & -1.4826288 & 2.2233160 & 4.4851372 \\ \text { C } & -2.7794410 & 3.3996510 & 3.9255701 \\ \text { C } & -2.3524469 & 4.6581341 & 3.4505631 \\ \text { H } & -1.2734030 & 4.8843247 & 3.4016288 \\ \text { C } & -3.3006530 & 5.5980876 & 3.0248680 \\ \text { H } & -2.9605459 & 6.5773078 & 2.6557937 \\ \text { C } & -4.6710454 & 5.2883637 & 3.0488231 \\ \text { H } & -5.4112008 & 6.0267418 & 2.7043025 \\ \text { C } & -5.0946160 & 4.0314071 & 3.5096533 \\ \text { H } & -6.1665174 & 3.7821566 & 3.5321112 \\ \mathrm{C} & -4.1538096 & 3.0880313 & 3.9545839 \\ \text { H } & -4.4932091 & 2.1114974 & 4.3284384 \\ \mathrm{C} & -2.3961227 & 0.6915816 & 4.9232186 \\ \mathrm{C} & -2.4319007 & 0.1796498 & 6.2344093 \\ \text { H } & -1.9383861 & 0.7281804 & 7.0498138 \\ \mathrm{C} & -3.0968932 & -1.0302815 & 6.4993664 \\ \mathrm{H} & -3.1163466 & -1.4257908 & 7.5262054 \\ \mathrm{C} & -3.7354751 & -1.7290737 & 5.4635392 \\ \mathrm{H} & -4.2578099 & -2.6743100 & 5.6748309 \\ \mathrm{C} & -3.7015332 & -1.2206746 & 4.1518426 \\ \mathrm{H} & -4.1978926 & -1.7661597 & 3.3349153 \\ \mathrm{C} & -3.0267200 & -0.0237079 & 3.8803417 \\ \mathrm{H} & -2.9813104 & 0.3693996 & 2.8533582 \\ \mathrm{C} & -0.9099860 & 2.8918974 & 6.0876606 \\ \mathrm{H} & 0.4475694 & 2.7630308 & 6.4372346 \\ & 1.1487489 & 2.3290366 & 5.7095608\end{array}$




$\begin{array}{rrrr}\text { C } & 0.8937986 & 3.2272082 & 7.6839642 \\ \text { H } & 1.9567227 & 3.1338050 & 7.9521564 \\ \text { C } & -0.0094796 & 3.8298883 & 8.5751871 \\ \text { H } & 0.3444347 & 4.2022859 & 9.5486149 \\ \text { C } & -1.3625385 & 3.9725048 & 8.2202419 \\ \text { H } & -2.0678495 & 4.4558299 & 8.9131471 \\ \text { C } & -1.8152273 & 3.5064152 & 6.9768489 \\ \text { H } & -2.8713936 & 3.6248553 & 6.6902717\end{array}$

S2

75

SCF Energy $=-4548.322221800$

C $\quad-0.4125416 \quad 4.1757735$

$\begin{array}{lll}\text { C } & -0.7782921 & 3.2239259\end{array}$

$\begin{array}{lll}\text { C } & -2.1553910 & 3.0911576\end{array}$

$-1.0191032$

$-3.1271217$

3.8598016

0.2886602

$-2.7493153$

4.7842094

$-0.3675394$

$-1.3870809$

4.9372599

$-1.3535617$

$-1.6722351$

C $\quad 0.2705220$

2.5325390

0.7368223

1.4709826

2.0203727

0.3657281

C $\quad 1.9769655$

1.9271631

$-1.0173248$

1.1407643

1.5384937

$-2.0899717$

C $\quad 1.6473974$

1.4556727

$-3.3934047$

1.7613061

$-3.6507243$

C $\quad 3.8358254$

2.1443218

$-2.5912344$

$2.2228444-1.2838301$

3.3353307

5.4679371

1.6387899

$-0.0305366$

1.1912599

2.4187282

$-0.7987835$

0.1663745

1.6001842

$-0.7376620$

1.8707271

3.4781879

2.5966188

1.4496097

0.8880341

1.5090825

3.3639815

1.5507137

2.0149725

3.0814030

2.6099174

1.1354175

$1.2325967 \quad 3.5859913$

1.8062883

$-0.2135129$

2.5573375

$-0.2647666$

1.9141440

4.4744622

$-1.8367320$

1.7751012

3.5121049

$-0.9144733$

$-0.7431588$

2.2310310

$-0.2596196$

$-0.1154568$

0.6764421

$-1.8129997$

0.5079752

1.3250510

2.6399843

3.4540536

1.9401036

1.2949828

$-1.8892860$

0.0870047

1.1457652

$-4.2166205$

3. 3910498

1.6984881

$-4.6759756$

H $\quad 4.8913591$

2. 3892820

$-2.7852349$ 


$\begin{array}{lrrr}\text { H } & 3.9774171 & 2.5461088 & -0.4505776 \\ \text { H } & -2.4653311 & 2.3904170 & 1.0749266 \\ \text { H } & -4.1867084 & 3.7424222 & -0.0953916 \\ \text { H } & -3.5112407 & 5.3911443 & -1.8655401 \\ \text { H } & -1.0769892 & 5.6719715 & -2.4307174 \\ \text { H } & 0.6513867 & 4.3314122 & -1.2376180 \\ \text { Pd } & -0.0626311 & 3.6096206 & 2.5510336 \\ \text { P } & -0.8912125 & 5.0478187 & 4.1114268 \\ \text { C } & -2.1059480 & 6.1928854 & 3.3439381 \\ \text { C } & -2.0368882 & 6.4433730 & 1.9575408 \\ \text { H } & -1.2496692 & 5.9670383 & 1.3537663 \\ \text { C } & -2.9669947 & 7.3044880 & 1.3559784 \\ \text { H } & -2.9067922 & 7.4852890 & 0.2728238 \\ \text { C } & -3.9669470 & 7.9189633 & 2.1268681 \\ \text { H } & -4.6965508 & 8.5917576 & 1.6506727 \\ \text { C } & -4.0410238 & 7.6685246 & 3.5081974 \\ \text { H } & -4.8266075 & 8.1432948 & 4.1155750 \\ \text { C } & -3.1172054 & 6.8057158 & 4.1169269 \\ \text { H } & -3.1925839 & 6.5930904 & 5.1940075 \\ \text { C } & -1.8593046 & 4.2654842 & 5.4653121 \\ \text { C } & -1.3600922 & 4.1707488 & 6.7786799 \\ \text { H } & -0.4014524 & 4.6458032 & 7.0345246 \\ \text { C } & -2.0883459 & 3.4778882 & 7.7611123 \\ \text { H } & -1.6917320 & 3.4114778 & 8.7856129 \\ \text { C } & -3.3172249 & 2.8799630 & 7.4415000 \\ \text { H } & -3.8872140 & 2.3413434 & 8.2134787 \\ \text { C } & -3.8192794 & 2.9726830 & 6.1306297 \\ \text { H } & -4.7838481 & 2.5084344 & 5.8746448 \\ \text { C } & -3.0927425 & 3.6553592 & 5.1458878 \\ \text { H } & -3.4863919 & 3.7287764 & 4.1197670 \\ \text { C } & 0.3452072 & 6.0674211 & 4.9966048 \\ \text { C } & 1.6261831 & 5.5125944 & 5.1943244 \\ \text { H } & 1.8630729 & 4.5315939 & 4.7541923 \\ \text { C } & 2.5928800 & 6.2239560 & 5.9193930 \\ \text { H } & 3.5928763 & 5.7894707 & 6.0667689 \\ \text { C } & 2.2910324 & 7.4969448 & 6.4325029 \\ \text { H } & 3.0544115 & 8.0605087 & 6.9902063 \\ \text { C } & 1.0201586 & 8.0575434 & 6.2210582 \\ \text { H } & 0.7870005 & 9.0603254 & 6.6100458 \\ \text { C } & 0.0448944 & 7.3447728 & 5.5058779 \\ \text { H } & -0.9455667 & 7.7899506 & 5.3311355\end{array}$

S3

75

$\mathrm{SCF}$ Energy $=-4548.322188901$

$\begin{array}{lll}\text { C } & -0.0415537 & 5.2239124\end{array}$

$-0.5573673$ 


\begin{tabular}{|c|c|c|c|}
\hline & -0.6061273 & 4.4105619 & 0.4623278 \\
\hline & -1.9512970 & 4.6586819 & 0.8416232 \\
\hline & -2.7067807 & 5.6609072 & 0.2168230 \\
\hline & -2.1378025 & 6.4447690 & -0.7986806 \\
\hline & -0.8014565 & 6.2201739 & -1.1787481 \\
\hline & 0.2493719 & 3.4629526 & 1.1933264 \\
\hline & 1.2502806 & 2.6450076 & 0.7805102 \\
\hline & 1.6528273 & 2.4153688 & -0.6204690 \\
\hline & 0.6978948 & 2.2649502 & -1.6530535 \\
\hline & 1.1073344 & 2.0458864 & -2.9747373 \\
\hline & 2.4759308 & 1.9754698 & -3.2906628 \\
\hline & 3.4325470 & 2.1193786 & -2.2713130 \\
\hline & 3.0279406 & 2.3325668 & -0.9458416 \\
\hline & 2.2023803 & 5.97 & 2.0018049 \\
\hline & -0.3299173 & 2.25 & 2.9047595 \\
\hline & -1.384 & $1.4^{\circ}$ & 2.1 \\
\hline & $-0.775 s$ & 3.1 & 3.98 \\
\hline & $1.017 \mathrm{~s}$ & 1.5 & 3.0 \\
\hline & 2.031 & 1.95 & 1.8 \\
\hline & 3.100 & 2.763 & 2.35 \\
\hline & 2.5047948 & 1.030 & 1.50 \\
\hline & 1.4960394 & 1.7893283 & 3.9935848 \\
\hline & 0.8485936 & 0.4633675 & 2.9833740 \\
\hline & -0.2639197 & 3.0178023 & 4.9590287 \\
\hline & -1.8569011 & 3.3107061 & 4.0691071 \\
\hline & -0.9858535 & 1.0623389 & 1.1907956 \\
\hline & -2.2795660 & 2.0845467 & 1.9005868 \\
\hline & -1.7142177 & 0.6356166 & 2.7803844 \\
\hline & 2.8243773 & 3.7161236 & 2.2873243 \\
\hline & -0.3729468 & 2.3158206 & -1.4067041 \\
\hline & 0.3518755 & 1.9248868 & -3.7662297 \\
\hline & 2.7953998 & 1.8062359 & -4.3301793 \\
\hline & 4.5057714 & 2.0698427 & -2.5111078 \\
\hline & 3.7684285 & 2.4720350 & -0.1438072 \\
\hline & -2.4031296 & 4.0714286 & 1.6517300 \\
\hline & -3.7445505 & 5.8372244 & 0.5369705 \\
\hline & -2.7286229 & 7.2349247 & -1.2861247 \\
\hline & -0.3386515 & 6.8408572 & -1.9609984 \\
\hline & 1.0133642 & 5.0837769 & -0.8245877 \\
\hline & 0.3003340 & 4.5934172 & 3.0052678 \\
\hline & -0.0351480 & 6.2025485 & 4.5823252 \\
\hline & -0.9304299 & 7.6335137 & 3.8570211 \\
\hline & -0.8655482 & 7.8541757 & 2.4653566 \\
\hline & -0.2676032 & 7.1817813 & 1.8318775 \\
\hline & -1.5569701 & 8.9343508 & 1.8963550 \\
\hline & -1.5044863 & 9.0907756 & 0.8089647 \\
\hline
\end{tabular}




$\begin{array}{lrrr}\text { C } & -2.3134880 & 9.7977343 & 2.7050619 \\ \text { H } & -2.8566906 & 10.6424614 & 2.2544138 \\ \text { C } & -2.3834437 & 9.5783656 & 4.0918984 \\ \text { H } & -2.9801226 & 10.2487359 & 4.7290914 \\ \text { C } & -1.6983199 & 8.4982042 & 4.6683331 \\ \text { H } & -1.7748388 & 8.3147298 & 5.7506631 \\ \text { C } & 1.4715188 & 6.8481805 & 5.3974853 \\ \text { C } & 2.5605675 & 5.9651873 & 5.5465053 \\ \text { H } & 2.5007530 & 4.9560881 & 5.1102575 \\ \text { C } & 3.7170538 & 6.3871203 & 6.2179302 \\ \text { H } & 4.5665929 & 5.6965858 & 6.3273148 \\ \text { C } & 3.7976957 & 7.6951849 & 6.7253161 \\ \text { H } & 4.7107920 & 8.0300741 & 7.2405815 \\ \text { C } & 2.7193590 & 8.5809559 & 6.5618742 \\ \text { H } & 2.7865935 & 9.6100339 & 6.9461916 \\ \text { C } & 1.5547020 & 8.1599843 & 5.9007324 \\ \text { H } & 0.7160562 & 8.8582862 & 5.7634194 \\ \text { C } & -1.1136633 & 5.7107181 & 5.9887823 \\ \text { C } & -0.5975355 & 5.4861016 & 7.2797060 \\ \text { H } & 0.4643229 & 5.6858255 & 7.4861307 \\ \text { C } & -1.4385781 & 5.0153843 & 8.3027753 \\ \text { H } & -1.0270499 & 4.8454807 & 9.3093097 \\ \text { C } & -2.7964531 & 4.7697982 & 8.0464645 \\ \text { H } & -3.4533845 & 4.4047021 & 8.8502615 \\ \text { C } & -3.3158238 & 4.9933981 & 6.7583465 \\ \text { H } & -4.3804387 & 4.8053803 & 6.5518261 \\ \text { C } & -2.4793773 & 5.4549522 & 5.7331874 \\ \text { H } & -2.8863195 & 5.6309702 & 4.7248660 \\ & & & \\ \text { S4 } & & & \\ \text { 75 } & & & \\ \text { SCF } & \text { Energy }=-4548.281567865 & \\ \text { C } & -0.8428647 & 4.3691572 & -0.5995209 \\ \text { C } & -0.7310152 & 3.3730844 & 0.4043931 \\ \text { C } & -1.8828347 & 2.5922917 & 0.7046144 \\ \text { C } & -3.1055760 & 2.8388239 & 0.0686106 \\ \text { C } & -3.2055576 & 3.8511031 & -0.9021775 \\ \text { C } & -2.0659208 & 4.5946591 & -1.2449658 \\ \text { C } & 0.5281970 & 3.0292956 & 1.1293699 \\ \text { C } & 1.3943162 & 2.0636703 & 0.7277429 \\ \text { C } & 1.4744902 & 1.6128000 & -0.6809582 \\ \text { C } & 1.3712109 & 0.2451254 & -1.0238125 \\ \text { C } & 1.4216333 & -0.1620171 & -2.3641559 \\ \text { C } & 1.5906131 & 0.7888140 & -3.3855731 \\ & 1.7121789 & 2.1487712 & -3.0566160 \\ & & & -1.7167268\end{array}$




$\begin{array}{lrrr}\text { Br } & 1.2255291 & 6.5715024 & 1.0581365 \\ \mathrm{C} & 0.8947826 & 3.0040354 & 3.1764523 \\ \mathrm{C} & 2.0676515 & 3.9129636 & 3.5895788 \\ \mathrm{C} & -0.4099595 & 3.3784076 & 3.7313550 \\ \mathrm{C} & 1.3315201 & 1.5483517 & 3.0674316 \\ \mathrm{C} & 2.2003980 & 1.3635564 & 1.8064942 \\ \mathrm{O} & 3.5476899 & 1.8086236 & 1.9153177 \\ \mathrm{H} & 2.2810577 & 0.2818887 & 1.5684616 \\ \mathrm{H} & 1.8937809 & 1.2641888 & 3.9828035 \\ \mathrm{H} & 0.4459415 & 0.8867896 & 2.9833284 \\ \mathrm{H} & -1.2322560 & 2.6505059 & 3.6242629 \\ \mathrm{H} & -0.4190402 & 3.9019180 & 4.7037206 \\ \mathrm{H} & 2.6277217 & 4.3062804 & 2.7183263 \\ \mathrm{H} & 2.7771745 & 3.3559976 & 4.2364119 \\ \mathrm{H} & 1.7090910 & 4.8072014 & 4.1353651 \\ \mathrm{H} & 3.5394674 & 2.7779193 & 1.8060517 \\ \mathrm{H} & 1.2254485 & -0.5057983 & -0.2322557 \\ \mathrm{H} & 1.3259888 & -1.2296022 & -2.6143982 \\ \mathrm{H} & 1.6344639 & 0.4674932 & -4.4372969 \\ \mathrm{H} & 1.8591685 & 2.8981223 & -3.8491495 \\ \mathrm{H} & 1.7787346 & 3.6168776 & -1.4501437 \\ \mathrm{H} & -1.7987493 & 1.7897793 & 1.4521916 \\ \mathrm{H} & -3.9866528 & 2.2319453 & 0.3281941 \\ \mathrm{H} & -4.1678997 & 4.0560862 & -1.3935449 \\ \mathrm{H} & -2.1318508 & 5.3804781 & -2.0105198 \\ \mathrm{H} & 0.0287279 & 4.9986651 & -0.8210212 \\ \mathrm{Pd} & -0.2648956 & 4.8558194 & 2.2666860 \\ \mathrm{P} & -1.9026965 & 6.2004360 & 2.9594274 \\ \mathrm{C} & -1.4195389 & 7.7766698 & 3.7516825 \\ \mathrm{C} & -0.1018877 & 7.9307711 & 4.2219600 \\ \mathrm{H} & 0.6237839 & 7.1209033 & 4.0538645 \\ \mathrm{C} & 0.2792645 & 9.1260758 & 4.8516616 \\ \mathrm{H} & 1.3122477 & 9.2482714 & 5.2101139 \\ \mathrm{C} & -0.6493971 & 10.1685379 & 5.0036754 \\ \mathrm{H} & -0.3467357 & 11.1080066 & 5.4908251 \\ \mathrm{C} & -1.9620319 & 10.0214694 & 4.5200375 \\ \mathrm{H} & -2.6856016 & 10.8438574 & 4.6267907 \\ \mathrm{C} & -2.3485375 & 8.8293513 & 3.8906195 \\ \mathrm{H} & -3.3703392 & 8.7152547 & 3.4969145 \\ \mathrm{C} & -3.0834044 & 5.4100535 & 4.1208215 \\ \mathrm{C} & -3.3898433 & 5.9479390 & 5.3851714 \\ \mathrm{H} & -2.9436567 & 6.9031231 & 5.6980852 \\ \mathrm{C} & -4.2629246 & 5.2621010 & 6.2475731 \\ & -4.4933586 & 5.6858802 & 7.2368211 \\ \mathrm{C} & -5.5398397 & 4.0459230 & 5.8508627 \\ & & 3.5134946 & 6.5273421\end{array}$




$\begin{array}{llll}\text { C } & -4.5336144 & 3.5051257 & 4.5884595 \\ \text { H } & -4.9791288 & 2.5490295 & 4.2740881 \\ \text { C } & -3.6510507 & 4.1753882 & 3.7314436 \\ \text { H } & -3.3901202 & 3.7454838 & 2.7513889 \\ \text { C } & -2.9291944 & 6.7246836 & 1.5264363 \\ \text { C } & -4.3022464 & 6.4333528 & 1.4153050 \\ \text { H } & -4.8190405 & 5.8831856 & 2.2140472 \\ \text { C } & -5.0168437 & 6.8512564 & 0.2799963 \\ \text { H } & -6.0889474 & 6.6165464 & 0.1966661 \\ \text { C } & -4.3699180 & 7.5687001 & -0.7379403 \\ \text { H } & -4.9333564 & 7.8970230 & -1.6247031 \\ \text { C } & -2.9998236 & 7.8635595 & -0.6228031 \\ \text { H } & -2.4843272 & 8.4202756 & -1.4200885 \\ \text { C } & -2.2752436 & 7.4369112 & 0.4975356 \\ \text { H } & -1.1905235 & 7.6252801 & 0.5694014\end{array}$

\section{Optimized Geometries(MXn)(TS-cd)-exo}

\begin{tabular}{lcrr} 
R3 & & & \\
75 & \multicolumn{4}{l}{} \\
SCF & Energy $=-4548.313313657$ & \\
C & 1.9560263 & 4.8470913 & -3.0484575 \\
C & 1.9136423 & 4.6605127 & -1.6413528 \\
C & 1.2745904 & 5.6681780 & -0.8689127 \\
C & 0.6921652 & 6.7903754 & -1.4732969 \\
C & 0.7395526 & 6.9514054 & -2.8681773 \\
C & 1.3783449 & 5.9724302 & -3.6474440 \\
C & 2.6337123 & 3.5297997 & -1.0144175 \\
C & 2.8884782 & 2.2816726 & -1.5362747 \\
C & 2.2450989 & 1.6645614 & -2.7085351 \\
C & 0.8910538 & 1.9486020 & -3.0256408 \\
C & 0.2638986 & 1.3461426 & -4.1210574 \\
C & 0.9667109 & 0.4319999 & -4.9273255 \\
C & 2.2984947 & 0.1183532 & -4.6133968 \\
C & 2.9305520 & 0.7199907 & -3.5155853 \\
Br & 5.2648850 & 6.3006314 & 1.5694388 \\
C & 2.4321704 & 3.0425908 & 0.8544496 \\
C & 0.9164726 & 2.8447598 & 0.8715520 \\
C & 2.9610645 & 4.0674773 & 1.7411817 \\
C & 3.2174419 & 1.7205806 & 0.8212600 \\
C & 3.7644527 & 1.4247866 & -0.6035855 \\
D & 3.8278395 & 0.0427588 & -0.8936480 \\
H & 4.8161815 & 1.7758860 & -0.6754357
\end{tabular}




$\begin{array}{lrrr}\text { H } & 4.0380089 & 1.7288349 & 1.5629308 \\ \mathrm{H} & 2.5398939 & 0.8842793 & 1.0945492 \\ \mathrm{H} & 3.7461785 & 3.7836557 & 2.4598189 \\ \mathrm{H} & 2.2794848 & 4.8537115 & 2.1083015 \\ \mathrm{H} & 0.6477271 & 2.3887117 & 1.8491407 \\ \mathrm{H} & 0.5896953 & 2.1615501 & 0.0634447 \\ \mathrm{H} & 0.3554312 & 3.7914439 & 0.7774619 \\ \mathrm{H} & 2.9127486 & -0.2728474 & -1.0307788 \\ \mathrm{H} & 0.3299423 & 2.6535344 & -2.3967448 \\ \mathrm{H} & -0.7866450 & 1.5861417 & -4.3446367 \\ \mathrm{H} & 0.4727183 & -0.0415396 & -5.7891019 \\ \mathrm{H} & 2.8550314 & -0.6039909 & -5.2300458 \\ \mathrm{H} & 3.9663927 & 0.4566732 & -3.2680652 \\ \mathrm{H} & 1.2560898 & 5.5886694 & 0.2248894 \\ \mathrm{H} & 0.2114544 & 7.5528731 & -0.8423387 \\ \mathrm{H} & 0.2920788 & 7.8375644 & -3.3424393 \\ \mathrm{H} & 1.4444461 & 6.0882593 & -4.7395059 \\ \mathrm{H} & 2.4633878 & 4.1045290 & -3.6748052 \\ \mathrm{Pd} & 4.0727242 & 4.7007485 & 0.0923605 \\ \mathrm{P} & 5.4820956 & 5.3120458 & -1.6677866 \\ \mathrm{C} & 5.5426138 & 7.1382788 & -1.8233179 \\ \mathrm{C} & 4.3097561 & 7.8226182 & -1.7695844 \\ \mathrm{H} & 3.3785874 & 7.2612362 & -1.6022284 \\ \mathrm{C} & 4.2753465 & 9.2154460 & -1.9180441 \\ \mathrm{H} & 3.3098342 & 9.7418056 & -1.8778330 \\ \mathrm{C} & 5.4698772 & 9.9349992 & -2.0978126 \\ \mathrm{H} & 5.4436550 & 11.0305498 & -2.2008260 \\ \mathrm{C} & 6.6983259 & 9.2553672 & -2.1329874 \\ \mathrm{H} & 7.6363756 & 9.8169770 & -2.2601158 \\ \mathrm{C} & 6.7377799 & 7.8575045 & -2.0000578 \\ \mathrm{H} & 7.7007829 & 7.3268936 & -2.0212713 \\ \mathrm{C} & 7.2267324 & 4.7551910 & -1.4862422 \\ \mathrm{C} & 7.7070152 & 4.3717060 & -0.2174765 \\ \mathrm{H} & 7.0359076 & 4.4350424 & 0.6529759 \\ \mathrm{C} & 9.0362883 & 3.9451250 & -0.0703180 \\ \mathrm{H} & 4.4055621 & 5.2124674 & -5.6803332 \\ \mathrm{C} & 9.4036299 & 3.6485130 & 0.9237818 \\ \mathrm{H} & 9.8925871 & 3.8969359 & -1.1825705 \\ \mathrm{H} & 10.9334885 & 3.5590010 & -1.0640566 \\ \mathrm{C} & 9.4175387 & 4.2787178 & -2.4490566 \\ \mathrm{C} & 10.0840585 & 4.2419030 & -3.3242671 \\ \mathrm{H} & 7.0895996 & 4.7045240 & -2.6032574 \\ \mathrm{H} & 5.1091777 & 4.7747198 & -3.3867138 \\ & 4.7429049 & 5.6817305 & -4.4011196 \\ & & & \\ \mathrm{H} & & \end{array}$




$\begin{array}{llll}\mathrm{H} & 4.1165096 & 5.9293451 & -6.4639586 \\ \mathrm{C} & 4.4354206 & 3.8366090 & -5.9587277 \\ \mathrm{H} & 4.1666979 & 3.4673529 & -6.9598182 \\ \mathrm{C} & 4.8188913 & 2.9299870 & -4.9554249 \\ \mathrm{H} & 4.8546884 & 1.8541666 & -5.1741014 \\ \mathrm{C} & 5.1474921 & 3.3934551 & -3.6739541 \\ \mathrm{H} & 5.4499859 & 2.6817228 & -2.8908756\end{array}$

R4

75

SCF Energy $=-4548.282843287$

C $\quad 1.8205174 \quad 4.4978894$

C $\quad 1.4659191 \quad 4.2309770$

C $\quad 0.5799682 \quad 5.1370643$

$\begin{array}{lll}\text { C } & 0.1257583 & 6.2905561\end{array}$

C $\quad 0.5205670 \quad 6.5579744$

C $\quad 1.3596834 \quad 5.6478062$

C $\quad 1.9043989 \quad 2.9766310$

$-2.5680283$

$-1.2180802$

$-0.5679475$

$-1.2268347$

$-2.5477678$

C $\quad 1.6507279$

1.7368516

$-3.2155039$

$-0.5483933$

C $\quad 1.0399983$

1.4625330

$-1.0473712$

$-0.1198746$

2.1326514

$-2.3733374$

$-0.6630791$

1.8488910

$-2.8252627$

$-0.0626461$

0.8883559

$-4.0862513$

$-4.9173034$

$\begin{array}{ll}0.1952693 & -4.4676480 \\ 0.4719805 & -3.2039135\end{array}$

C $\quad 1.6132144$

0.4719805

2.1395823

$\mathrm{Br} \quad 3.5018858$

6.5442910

2.4745985

1.3463739

C $\quad 3.8838469$

2.0328791

1.4829407

C $\quad 2.0395375$

3.6254024

2.1669916

C $\quad 1.5114191$

1.2435252

1.2692414

1.7869882

0.5600953

$-0.0808042$

0.9298526

$-0.5264618$

$-0.3644669$

$\mathrm{H} \quad 2.8106073$

0.1249497

$-0.0842986$

1.7315668

0.5765304

2.1304709

1.5292090

1.3093398

2.2713120

3.8470623

3.0732453

3.8343965

0.5372179

4.2889107

1.6264820

2.2452298

$3.9532256 \quad 1.2256989$

$4.5463927 \quad 2.8635298$

1.7929534

$0.0762429-0.1557975$

$-0.6636707$

$-0.5949170$

2.8850014

$-2.1805008$

$-1.5646236$

2.3836213

$-4.4215622$

$-0.4877012$

0.6704577

$-5.9087462$

1.5404241

$-0.5721616$

$-5.1038632$

H $\quad 2.4919733$

$-0.0847223$

$-2.8482790$ 


\begin{tabular}{|c|c|c|c|}
\hline & 0.2331605 & 4.9228411 & 0.4512423 \\
\hline & -0.5436777 & 6.9838679 & -0.6962767 \\
\hline & 0.1685880 & 7.4663782 & -3.0590089 \\
\hline & 1.6616519 & 5.8331398 & -4.2571004 \\
\hline & 2.4467125 & 3.7787466 & -3.1063587 \\
\hline & 3.0956720 & 4.6209136 & 0.6531042 \\
\hline & 4.8445097 & 5.1995442 & -0.8101368 \\
\hline & 4.9194239 & 6.9605097 & -1.3017150 \\
\hline & 6.1258156 & 7.5968462 & -1.6540900 \\
\hline & 7.0761345 & 7.0450354 & -1.5923153 \\
\hline & 6.1109344 & 8.9353135 & -2.0767320 \\
\hline & 7.0541388 & 9.4339770 & -2.3473159 \\
\hline & 4.8953851 & 9.6379572 & -2.1490832 \\
\hline & 4.8873719 & 10.6883463 & -2.47 \\
\hline & 3.6937434 & 9.0045905 & -1.78 \\
\hline & 2.74 & 9.5548051 & -1.83 \\
\hline & 3.70 & 7.670 & -1.35 \\
\hline & 2.7714629 & 7.1748434 & -1.04 \\
\hline & 6.501 & 4.8298687 & $-0.0 s$ \\
\hline & 6.7156628 & 5.0778636 & 1.28 \\
\hline & 5.8937822 & 5.4891079 & 1.8897712 \\
\hline & 7.9683453 & 4.8131417 & 1.8534165 \\
\hline & 8.1265114 & 5.0116070 & 2.9244305 \\
\hline & 9.0136452 & 4.2952312 & 1.0694150 \\
\hline & 9.9934171 & 4.0835744 & 1.5242767 \\
\hline & 8.8040868 & 4.0474899 & -0.2970452 \\
\hline & 9.6187775 & 3.6436637 & -0.9174863 \\
\hline & 7.5542121 & 4.3139785 & -0.8791295 \\
\hline & 7.3940969 & 4.1163077 & -1.9492156 \\
\hline & 4.8967061 & 4.2963107 & -2.4085009 \\
\hline & 4.8336384 & 2.8859890 & -2.3656296 \\
\hline & 4.8479798 & 2.3713881 & -1.3935807 \\
\hline & 4.7252073 & 2.1457956 & -3.5496240 \\
\hline & 4.6704875 & 1.0485803 & -3.5066547 \\
\hline & 4.6659055 & 2.8062414 & -4.7902402 \\
\hline & 4.5558045 & 2.2252662 & -5.7179892 \\
\hline & 4.7463867 & 4.2073856 & -4.8393548 \\
\hline & 4.7069473 & 4.7280567 & -5.8082177 \\
\hline & 4.8665081 & 4.9522949 & -3.6542590 \\
\hline & 4.9086541 & 6.0502230 & -3.6925430 \\
\hline \\
\hline \\
\hline \multicolumn{4}{|c|}{11} \\
\hline & 1.2452779 & 3.7580659 & -2.784076 \\
\hline & 0.6138933 & 4.0113530 & -1.537515 \\
\hline
\end{tabular}




\begin{tabular}{lrrr} 
C & -0.6669736 & 4.6278830 & -1.5686304 \\
$\mathrm{C}$ & -1.2929841 & 4.9480909 & -2.7806860 \\
$\mathrm{C}$ & -0.6538702 & 4.6798004 & -4.0025083 \\
$\mathrm{C}$ & 0.6201213 & 4.0878002 & -3.9920960 \\
$\mathrm{C}$ & 1.3309315 & 3.7718988 & -0.2672873 \\
$\mathrm{C}$ & 2.3183298 & 2.8593197 & 0.0309915 \\
$\mathrm{C}$ & 2.7488931 & 1.6807920 & -0.7348061 \\
$\mathrm{C}$ & 1.8575710 & 0.9714223 & -1.5788169 \\
$\mathrm{C}$ & 2.2746230 & -0.1761753 & -2.2624618 \\
$\mathrm{C}$ & 3.5907852 & -0.6514176 & -2.1196998 \\
$\mathrm{C}$ & 4.4805839 & 0.0259247 & -1.2698537 \\
$\mathrm{C}$ & 4.0636567 & 1.1724227 & -0.5804564 \\
$\mathrm{Br}$ & 0.9456622 & 8.1694988 & 1.0778074 \\
$\mathrm{C}$ & 0.4505346 & 3.8249008 & 1.4885042 \\
$\mathrm{C}$ & -0.6592248 & 2.8123055 & 1.2126152 \\
$\mathrm{C}$ & 0.0421147 & 5.1813023 & 1.8148844 \\
$\mathrm{C}$ & 1.6086617 & 3.2528676 & 2.3214677 \\
$\mathrm{C}$ & 2.8696580 & 3.0526371 & 1.4470453 \\
$\mathrm{O}$ & 3.8051657 & 4.1211396 & 1.5250709 \\
$\mathrm{H}$ & 3.4269938 & 2.1566122 & 1.7794405 \\
$\mathrm{H}$ & 1.8534494 & 3.9098406 & 3.1781808 \\
$\mathrm{H}$ & 1.2989420 & 2.2709818 & 2.7346305 \\
$\mathrm{H}$ & 0.4298502 & 5.6305106 & 2.7428872 \\
$\mathrm{H}$ & -0.9780875 & 5.5078944 & 1.5500369 \\
$\mathrm{H}$ & -1.1569939 & 2.5858423 & 2.1804241 \\
$\mathrm{H}$ & -0.2514452 & 1.8695049 & 0.7983173 \\
$\mathrm{H}$ & -1.4312899 & 3.1943898 & 0.5217889 \\
$\mathrm{H}$ & 3.3289182 & 4.9234004 & 1.1764542 \\
$\mathrm{H}$ & 0.8222130 & 1.3232123 & -1.6850621 \\
$\mathrm{H}$ & 1.5621159 & -0.7117558 & -2.9080704 \\
$\mathrm{H}$ & 3.9166189 & -1.5529527 & -2.6599382 \\
$\mathrm{H}$ & 5.5102471 & -0.3416338 & -1.1422054 \\
$\mathrm{H}$ & 4.7725615 & 1.7036037 & 0.0716597 \\
$\mathrm{H}$ & -1.1717275 & 4.8867052 & -0.6299020 \\
$\mathrm{H}$ & -2.2827518 & 5.4283290 & -2.7651815 \\
$\mathrm{H}$ & -1.1395238 & 4.9411071 & -4.9544820 \\
$\mathrm{H}$ & 1.1481674 & 3.8896369 & -4.9365703 \\
$\mathrm{H}$ & 2.2464483 & 3.3123122 & -2.8010854 \\
$\mathrm{Pd}$ & 1.4313408 & 5.8347917 & 0.3938697 \\
$\mathrm{P}$ & 3.0936065 & 6.6202522 & -1.0663273 \\
$\mathrm{C}$ & 2.4226704 & 7.9706966 & -2.1080072 \\
$\mathrm{H}$ & 3.1175761 & 9.1700179 & -2.3449684 \\
$\mathrm{H}$ & 2.0963206 & 9.3400787 & -1.8731220 \\
\hline & 1.29839898 & 9.9379153 & -3.7709829
\end{tabular}




$\begin{array}{lrrr}\text { H } & 0.8572577 & 10.7112118 & -4.4182218 \\ \mathrm{C} & 0.6034226 & 8.7392955 & -3.5315263 \\ \mathrm{H} & -0.3834153 & 8.5683038 & -3.9874796 \\ \mathrm{C} & 1.1568129 & 7.7616530 & -2.6943270 \\ \mathrm{H} & 0.6028687 & 6.8344851 & -2.4869489 \\ \mathrm{C} & 4.5853641 & 7.3127876 & -0.2406860 \\ \mathrm{C} & 5.8128726 & 7.4057424 & -0.9327373 \\ \mathrm{H} & 5.8846243 & 7.0524466 & -1.9720110 \\ \mathrm{C} & 6.9425339 & 7.9389168 & -0.2943656 \\ \mathrm{H} & 7.8958249 & 8.0076100 & -0.8402862 \\ \mathrm{C} & 6.8577965 & 8.3783047 & 1.0378977 \\ \mathrm{H} & 7.7467171 & 8.7909520 & 1.5391236 \\ \mathrm{C} & 5.6384218 & 8.2863915 & 1.7283956 \\ \mathrm{H} & 5.5658970 & 8.6284926 & 2.7717703 \\ \mathrm{C} & 4.5036953 & 7.7560703 & 1.0952135 \\ \mathrm{H} & 3.5398308 & 7.7028368 & 1.6245095 \\ \mathrm{C} & 3.8456271 & 5.4824593 & -2.2973072 \\ \mathrm{C} & 3.6869330 & 5.6616890 & -3.6859380 \\ \mathrm{H} & 3.1248699 & 6.5265904 & -4.0649888 \\ \mathrm{C} & 4.2304598 & 4.7288237 & -4.5829193 \\ \mathrm{H} & 4.0973820 & 4.8735463 & -5.6659518 \\ \mathrm{C} & 4.9373446 & 3.6153530 & -4.1005082 \\ \mathrm{H} & 5.3575984 & 2.8796194 & -4.8027386 \\ \mathrm{C} & 5.1129474 & 3.4450796 & -2.7162596 \\ \mathrm{H} & 5.6680729 & 2.5761520 & -2.3369437 \\ \mathrm{C} & 4.5671768 & 4.3692546 & -1.8138584 \\ \mathrm{H} & 4.6893123 & 4.2295489 & -0.7281674 \\ & & & \end{array}$

\begin{tabular}{lrrr} 
S4 & & & \\
75 & \multicolumn{4}{l}{} \\
SCF & Energy $=-4548.281344605$ & \\
C & 1.4654681 & 3.9525342 & -2.7314229 \\
C & 0.9351189 & 3.8759723 & -1.4144440 \\
C & -0.4299358 & 4.2379850 & -1.2213582 \\
C & -1.1989543 & 4.7247009 & -2.2910453 \\
C & -0.6417414 & 4.8379071 & -3.5744261 \\
C & 0.6892301 & 4.4348600 & -3.7883092 \\
C & 1.7376351 & 3.3076829 & -0.2972830 \\
C & 2.2646704 & 2.0534984 & -0.3258281 \\
C & 2.2920565 & 1.1591766 & -1.5112863 \\
C & 1.1634608 & 0.9354448 & -2.3321139 \\
C & 1.2497254 & 0.0837857 & -3.4419299 \\
C & 2.4596398 & -0.5608802 & -3.7521900 \\
C & 3.5794487 & -0.3674152 & -2.9269026 \\
C & 3.4951110 & 0.4775819 & -1.8103532 \\
Br & 0.4502482 & 7.7052456 & 0.9964287
\end{tabular}




\begin{tabular}{|c|c|c|c|}
\hline & 1.9154921 & 3.7418562 & 1.6767719 \\
\hline $\mathrm{C}$ & 3.2636277 & 4.3811703 & 2.0861355 \\
\hline & 0.7521570 & 4.5879785 & 1.9563500 \\
\hline & 1.8988699 & 2.2511885 & 2.0186011 \\
\hline & 2.7552466 & 1.4865145 & 0.9958348 \\
\hline & 4.1702098 & 1.5577049 & 1.1922095 \\
\hline & 2.5300636 & 0.4015161 & 1.0403423 \\
\hline & 2.2758312 & 2.1082324 & 3.0535667 \\
\hline & 0.8630874 & 1.8614506 & 1.9691006 \\
\hline & -0.2436473 & 4.1184358 & 1.8750103 \\
\hline & 0.8300127 & 5.3343341 & 2.7637925 \\
\hline & 4.0549860 & 4.2603074 & 1.3198058 \\
\hline & 3.6358889 & 3.9155163 & 3.0235833 \\
\hline & 3.1719592 & 5.4734613 & 2.2437102 \\
\hline & 4.4337016 & 2.48 & 1.03 \\
\hline & 0.2116 & 1.42 & -2.0 \\
\hline & 0.3607 & -0.08 & -4.0 \\
\hline & 2.5247265 & -1.22 & -4.6 \\
\hline & 4.5264395 & -0.88 & -3.1 \\
\hline & 4.3608720 & 0.62 & -1.1 \\
\hline & -0.8932103 & 4.1122504 & -0.23 \\
\hline & -2.2445789 & 5.0156983 & -2.1115979 \\
\hline & -1.2448070 & 5.2258656 & -4.4088377 \\
\hline & 1.1320937 & 4.4943727 & -4.7936214 \\
\hline & 2.4881613 & 3.6079425 & -2.9150769 \\
\hline & 1.5017794 & 5.5969322 & 0.2762772 \\
\hline & 3.0718172 & 6.6787348 & -1.1122655 \\
\hline & 4.4089060 & 7.4837138 & -0.1323434 \\
\hline & 4.0599168 & 8.1593260 & 1.0575943 \\
\hline & 3.0020788 & 8.1975684 & 1.3672822 \\
\hline & 5.0533916 & 8.7822634 & 1.8272063 \\
\hline & 4.7715138 & 9.3080893 & 2.7521652 \\
\hline & 6.3993924 & 8.7331980 & 1.4249554 \\
\hline & 7.1768548 & 9.2178193 & 2.0351027 \\
\hline & 6.7496823 & 8.0633212 & 0.2413966 \\
\hline & 7.8013046 & 8.0235569 & -0.0814403 \\
\hline & 5.7601007 & 7.4415019 & -0.5373806 \\
\hline & 6.0394956 & 6.9177240 & -1.4632305 \\
\hline & 4.0160996 & 5.5990098 & -2.2600868 \\
\hline & 4.0174871 & 5.7927104 & -3.6550037 \\
\hline & 3.5268790 & 6.6792162 & -4.0818668 \\
\hline & 4.6107485 & 4.8382063 & -4.4982109 \\
\hline & 4.5930963 & 4.9895182 & -5.5882387 \\
\hline & 5.2117683 & 3.6898373 & -3.9577142 \\
\hline & 5.6576523 & 2.9337900 & -4.6208387 \\
\hline & 5.2386783 & 3.5054655 & -2.5634217 \\
\hline
\end{tabular}




$\begin{array}{lrrr}\text { H } & 5.7065775 & 2.6083919 & -2.1337356 \\ \mathrm{C} & 4.6418542 & 4.4526477 & -1.7224445 \\ \mathrm{H} & 4.6294242 & 4.2889899 & -0.6353227 \\ \mathrm{C} & 2.4111243 & 7.9970661 & -2.1944126 \\ \mathrm{C} & 3.1888326 & 9.0955404 & -2.6100378 \\ \mathrm{H} & 4.2214552 & 9.2060129 & -2.2456203 \\ \mathrm{C} & 2.6398887 & 10.0478787 & -3.4830106 \\ \mathrm{H} & 3.2455503 & 10.9090345 & -3.8040572 \\ \mathrm{C} & 1.3186924 & 9.9045103 & -3.9416392 \\ \mathrm{H} & 0.8901520 & 10.6545794 & -4.6238687 \\ \mathrm{C} & 0.5413497 & 8.8124724 & -3.5197747 \\ \mathrm{H} & -0.4976102 & 8.7043138 & -3.8656003 \\ \mathrm{C} & 1.0807819 & 7.8632768 & -2.6395695 \\ \mathrm{H} & 0.4724814 & 7.0186929 & -2.2847752\end{array}$

\section{Optimized Geometries(BXnd/d')-exo}

\begin{tabular}{lrrr} 
R1 & \multicolumn{3}{l}{} \\
75 & \multicolumn{4}{l}{} \\
SCF & Energy $=-4548.347409027$ & \\
C & -0.3922850 & 3.3506488 & -1.6713277 \\
C & -0.2743158 & 1.9449217 & -1.6431824 \\
C & -1.3922264 & 1.1877227 & -1.2150279 \\
C & -2.5774336 & 1.8121701 & -0.8044314 \\
C & -2.6662871 & 3.2136709 & -0.8076562 \\
C & -1.5748010 & 3.9770782 & -1.2497725 \\
C & 0.9826574 & 1.2456085 & -2.0027587 \\
C & 1.1373363 & 0.2969677 & -2.9757863 \\
C & 0.1436565 & -0.1778475 & -3.9567894 \\
C & 0.2637181 & -1.4804512 & -4.5034424 \\
C & -0.6673922 & -1.9583952 & -5.4369997 \\
C & -1.7367589 & -1.1481950 & -5.8524913 \\
C & -1.8551931 & 0.1541540 & -5.3355359 \\
C & -0.9268130 & 0.6361651 & -4.4040895 \\
Br & 0.4737417 & 3.4985057 & 1.9219334 \\
C & 2.2613475 & 1.4188479 & -1.1533444 \\
C & 2.6849450 & 2.8771592 & -0.9230570 \\
C & 2.1187242 & 0.6505278 & 0.1773677 \\
C & 3.3170405 & 0.6769913 & -2.0354668 \\
C & 2.5330421 & -0.3143521 & -2.9135163 \\
O & 2.5219415 & -1.6565821 & -2.4067215 \\
H & 2.9861117 & -0.4039382 & -3.9234922 \\
H & 3.8169048 & 1.4286768 & -2.6803099
\end{tabular}




$\begin{array}{lrrr}\text { H } & 4.1027901 & 0.1608409 & -1.4493605 \\ \mathrm{H} & 2.1460990 & -0.4410564 & -0.0000678 \\ \mathrm{H} & 2.9089027 & 0.9257766 & 0.9068841 \\ \mathrm{H} & 2.7797830 & 3.4145132 & -1.8880942 \\ \mathrm{H} & 3.6756143 & 2.8980020 & -0.4240569 \\ \mathrm{H} & 1.9747914 & 3.4209189 & -0.2714455 \\ \mathrm{H} & 1.8679341 & -1.6896535 & -1.6802899 \\ \mathrm{H} & 1.0870270 & -2.1211624 & -4.1577408 \\ \mathrm{H} & -0.5551346 & -2.9754092 & -5.8443128 \\ \mathrm{H} & -2.4679576 & -1.5234765 & -6.5846509 \\ \mathrm{H} & -2.6764244 & 0.8070112 & -5.6688954 \\ \mathrm{H} & -1.0206666 & 1.6628330 & -4.0262176 \\ \mathrm{H} & -1.3185746 & 0.0945534 & -1.2428703 \\ \mathrm{H} & -3.4256297 & 1.1992834 & -0.4657708 \\ \mathrm{H} & -3.5865667 & 3.7110733 & -0.4665601 \\ \mathrm{H} & -1.6379924 & 5.0751645 & -1.2582654 \\ \mathrm{H} & 0.4518632 & 3.9606041 & -2.0170917 \\ \mathrm{Pd} & 0.4047070 & 1.1773659 & 1.1646730 \\ \mathrm{P} & -0.1852227 & -0.9681160 & 1.4024347 \\ \mathrm{C} & -1.9966907 & -0.9922250 & 1.7224764 \\ \mathrm{C} & -2.5790639 & 0.1968832 & 2.2120905 \\ \mathrm{H} & -1.9582594 & 1.0980006 & 2.3583250 \\ \mathrm{C} & -3.9501961 & 0.2434300 & 2.5030425 \\ \mathrm{H} & -4.3937667 & 1.1770384 & 2.8796447 \\ \mathrm{C} & -4.7502093 & -0.8944563 & 2.3057932 \\ \mathrm{H} & -5.8271686 & -0.8565617 & 2.5289405 \\ \mathrm{C} & -4.1743055 & -2.0816801 & 1.8221256 \\ \mathrm{H} & -4.7980723 & -2.9750748 & 1.6676178 \\ \mathrm{C} & -2.8019025 & -2.1342457 & 1.5327759 \\ \mathrm{H} & -2.3602988 & -3.0649370 & 1.1474408 \\ \mathrm{C} & 0.0950705 & -2.2848134 & 0.1527784 \\ \mathrm{C} & 1.2326855 & -3.1186757 & 0.2508661 \\ \mathrm{H} & 1.9206922 & -3.0083930 & 1.1016974 \\ \mathrm{C} & 1.4805683 & -4.0961167 & -0.7262390 \\ \mathrm{H} & 2.3740353 & -4.7318920 & -0.6430260 \\ \mathrm{C} & 0.6039394 & -4.2485588 & -1.8117595 \\ \mathrm{H} & 0.8065438 & -5.0082105 & -2.5808684 \\ \mathrm{C} & -0.5209403 & -3.4150294 & -1.9244239 \\ \mathrm{H} & -1.2025083 & -3.5124776 & -2.7810366 \\ \mathrm{C} & -0.7723031 & -2.4358525 & -0.9530777 \\ \mathrm{H} & -1.6640331 & -1.8011552 & -1.0497183 \\ \mathrm{C} & 0.5530981 & -1.6796894 & 2.9318674 \\ & 1.4632256 & -0.9019889 & 3.6735473 \\ \mathrm{H} & 2.7135385 & 0.1102453 & 3.3138627 \\ & 2.7308784 & -0.7998818 & 5.4333373\end{array}$




$\begin{array}{rrrr}\text { C } & 1.6685949 & -2.6954733 & 5.3046191 \\ \text { H } & 2.1043966 & -3.0946145 & 6.2330899 \\ \text { C } & 0.7543508 & -3.4716519 & 4.5686565 \\ \text { H } & 0.4736073 & -4.4758271 & 4.9207514 \\ \text { C } & 0.1957247 & -2.9670216 & 3.3864150 \\ \text { H } & -0.5186069 & -3.5772559 & 2.8136816\end{array}$

$\begin{array}{lrrr}\text { R2 (d') } & & & \\ 75 & & & \\ \text { SCF } & \text { Energy }=-4548.350329009 & \\ \text { C } & 1.3167660 & -0.8129386 & -1.5888877 \\ \text { C } & 1.6039153 & -1.8354948 & -0.6506412 \\ \text { C } & 1.3451066 & -3.1732670 & -1.0226760 \\ \text { C } & 0.8102155 & -3.4776393 & -2.2863246 \\ \text { C } & 0.5144685 & -2.4509768 & -3.1974204 \\ \text { C } & 0.7732243 & -1.1145454 & -2.8426183 \\ \text { C } & 2.1136243 & -1.4577183 & 0.6922732 \\ \text { C } & 3.1422914 & -0.5841548 & 0.9306617 \\ \text { C } & 4.0398467 & 0.0316191 & -0.0626441 \\ \text { C } & 4.5687648 & -0.7217142 & -1.1382889 \\ \text { C } & 5.4171494 & -0.1243222 & -2.0789793 \\ \text { C } & 5.7517770 & 1.2368529 & -1.9646456 \\ \text { C } & 5.2368815 & 1.9932011 & -0.8987304 \\ \text { C } & 4.3941277 & 1.3967488 & 0.0504026 \\ \text { Br } & 1.0859947 & 2.6529975 & 1.1804330 \\ \text { C } & 1.4431670 & -1.9258302 & 2.0144144 \\ \text { C } & 1.7410863 & -3.3945023 & 2.3775252 \\ \text { C } & -0.0475274 & -1.6319519 & 1.8573745 \\ \text { C } & 2.0950920 & -0.9546584 & 3.0560489 \\ \text { C } & 3.3764207 & -0.4041356 & 2.4235127 \\ \text { O } & 4.4910457 & -1.1981470 & 2.8527221 \\ \text { H } & 3.5344660 & 0.6674054 & 2.6881618 \\ \text { H } & 1.4048995 & -0.1017249 & 3.2677141 \\ \text { H } & 2.3056251 & -1.4411684 & 4.0282043 \\ \text { H } & -0.6185902 & -1.6387956 & 2.8096405 \\ \text { H } & -0.5339079 & -2.2859647 & 1.1125458 \\ \text { H } & 1.3146873 & -3.6289554 & 3.3741917 \\ \text { H } & 2.8376449 & -3.5538579 & 2.4193368 \\ \text { H } & 1.2947035 & -4.1023882 & 1.6529493 \\ \text { H } & 5.2365927 & -0.9724011 & 2.2633923 \\ \text { H } & 4.3074023 & -1.7863092 & -1.2251933 \\ \text { H } & 5.8229616 & -0.7249365 & -2.9073543 \\ \text { H } & 6.4160486 & 1.7064223 & -2.7062097 \\ \text { H } & 5.4866383 & 3.0612590 & -0.8079212 \\ \text { H } & 3.9545976 & 2.0010336 & 0.8573448 \\ \text { H } & 1.5752027 & -3.9875592 & -0.3231075\end{array}$




$\begin{array}{lrrr}\text { H } & 0.6201136 & -4.5277942 & -2.5561531 \\ \text { H } & 0.0793732 & -2.6885555 & -4.1798391 \\ \text { H } & 0.5294915 & -0.2970091 & -3.5368104 \\ \text { H } & 1.5011229 & 0.2330920 & -1.3039186 \\ \text { Pd } & 0.1693463 & 0.3256208 & 1.2567600 \\ \text { P } & -1.7949934 & 0.3592082 & 0.2520227 \\ \text { C } & -2.9618162 & 1.5504483 & 1.0156122 \\ \text { C } & -1.6817780 & 0.9060975 & -1.5045420 \\ \text { C } & -2.7112890 & -1.2230828 & 0.1657518 \\ \text { C } & -4.1937982 & 1.8391934 & 0.3909208 \\ \text { C } & -5.0799766 & 2.7486660 & 0.9847656 \\ \text { C } & -4.7378135 & 3.3776356 & 2.1957184 \\ \text { C } & -3.5069503 & 3.0989583 & 2.8107121 \\ \text { C } & -2.6158071 & 2.1872141 & 2.2220307 \\ \text { H } & -4.4557347 & 1.3566726 & -0.5630547 \\ \text { H } & -6.0402134 & 2.9742850 & 0.4966598 \\ \text { H } & -5.4332626 & 4.0957978 & 2.6561922 \\ \text { H } & -3.2308354 & 3.6001675 & 3.7504200 \\ \text { H } & -1.6336610 & 1.9776024 & 2.6745389 \\ \text { C } & -2.5037599 & 0.3483639 & -2.5061153 \\ \text { C } & -2.3970671 & 0.7962448 & -3.8324124 \\ \text { C } & -1.4778016 & 1.8039684 & -4.1663751 \\ \text { C } & -0.6701741 & 2.3709142 & -3.1650479 \\ \text { C } & -0.7678652 & 1.9268849 & -1.8385895 \\ \text { H } & -3.2212327 & -0.4459987 & -2.2568931 \\ \text { H } & -3.0364428 & 0.3499316 & -4.6091224 \\ \text { H } & -1.3904898 & 2.1476839 & -5.2083489 \\ \text { H } & 0.0521472 & 3.1619845 & -3.4166869 \\ \text { H } & -0.1203377 & 2.3594964 & -1.0566342 \\ \text { C } & -3.8198017 & -1.4862758 & 0.9928735 \\ \text { C } & -4.4217353 & -2.7559807 & 0.9752685 \\ \text { C } & -3.9198951 & -3.7659165 & 0.1387748 \\ \text { C } & -2.8053475 & -3.5076296 & -0.6794676 \\ \text { C } & -2.1966829 & -2.2463010 & -0.6625502 \\ \text { H } & -4.2138266 & -0.6974896 & 1.6504902 \\ \text { H } & -5.2901022 & -2.9548100 & 1.6214364 \\ \text { H } & -4.3942834 & -4.7588726 & 0.1280219 \\ \text { H } & -2.3965693 & -4.2946441 & -1.3308558 \\ \text { H } & -1.3152487 & -2.0562638 & -1.2923903 \\ & & & \\ \text { R3(d' }) & & & \\ 75 & & & \\ \text { SCF } & \text { Energy }=-4548.348159889 & \\ \text { C } & -1.0172138 & 2.3499909 & -1.1522557 \\ \text { C } & -1.3207521 & 1.5002420 & -2.2374903 \\ & -1.4870476 & 2.0699671 & -3.5185992\end{array}$




\begin{tabular}{|c|c|c|c|}
\hline & -1.3495124 & 3.4527056 & -3.7077050 \\
\hline & -1.0410935 & 4.2888012 & -2.6214059 \\
\hline & -0.8747803 & 3.7321208 & -1.3425574 \\
\hline & -1.4874037 & 0.0293263 & -2.0857386 \\
\hline & -0.7196616 & -0.9473302 & -2.7212104 \\
\hline & 0.4110163 & -0.6460278 & -3.6269442 \\
\hline & 0.5181851 & -1.2847687 & -4.8857337 \\
\hline & 1.5452335 & -0.9428249 & -5.7801331 \\
\hline & 2.4795991 & 0.0474347 & -5.4425340 \\
\hline & 2.3739782 & 0.7014550 & -4.2027296 \\
\hline & 1.3570673 & 0.3588366 & -3.3064975 \\
\hline & 2.1473735 & -1.4504168 & -0.6 \\
\hline & -2.7556538 & -0.5823580 & -1.42 \\
\hline & -4.0 & 0.2267913 & -1.65 \\
\hline & -2.3 & $-0.62 c$ & 0.02 \\
\hline & -2. & -1.98 & -2.0 \\
\hline & -1. & -2.33 & -2.5 \\
\hline & -1. & -3.16 & -3.6 \\
\hline & $-0 . \varepsilon$ & -2.886 & -1.7 \\
\hline & 19905 & -2.752 & -1.4 \\
\hline & 71908 & -1.9474816 & -2.9 \\
\hline & -2.5993180 & -1.5192298 & 0.6 \\
\hline & -2.5791258 & 0.2942654 & 0.58 \\
\hline & -4.2462928 & 0.3386601 & -2.74 \\
\hline & -3.9462898 & 1.2415616 & -1.22 \\
\hline & -4.8987116 & -0.2799098 & -1.18 \\
\hline & -0.5836425 & -3.593 & $-3.7 \mathrm{~s}$ \\
\hline & -0.24 & -2.0168527 & -5.1 \\
\hline & 1.6 & -1.44 & -6.7 \\
\hline & 3.28 & 0.3 & -6.1 \\
\hline & 3.1 & 1.47 & -3.9 \\
\hline & 1.29 & 0.860 & -2.3 \\
\hline & -1.7097462 & 1.4122909 & -4.37 \\
\hline & -1.4753616 & 3.8793251 & -4.7144313 \\
\hline & -0.9229901 & 5.3723824 & -2.7737697 \\
\hline & -0.6167982 & 4.3730297 & -0.4859027 \\
\hline & -0.8485420 & 1.9047492 & -0.1614235 \\
\hline & -0.2621466 & -0.7566016 & -0.2813745 \\
\hline & 0.1863576 & -0.3917055 & 1.8990865 \\
\hline & 1.0804496 & 1.2011668 & 2.1152390 \\
\hline & 1.8895460 & 1.6840227 & 1.0652077 \\
\hline & 1.9974462 & 1.0815484 & 0.1495897 \\
\hline & 2.5674103 & 2.9040717 & 1.2072001 \\
\hline & 3.1925140 & 3.2738969 & 0.3807198 \\
\hline & & 3.6481468 & 2.3926226 \\
\hline & 2.9777915 & 4.6073468 & 2.498600 \\
\hline
\end{tabular}




$\begin{array}{lrrr}\text { C } & 1.6522465 & 3.1652158 & 3.4449979 \\ \text { H } & 1.5587005 & 3.7412808 & 4.3781077 \\ \text { C } & 0.9682605 & 1.9473037 & 3.3095486 \\ \text { H } & 0.3342056 & 1.5826161 & 4.1308273 \\ \text { C } & 1.1779488 & -1.6796382 & 2.7455221 \\ \text { C } & 1.0770122 & -3.0098390 & 2.2919356 \\ \text { H } & 0.4814847 & -3.2286150 & 1.3929571 \\ \text { C } & 1.7581797 & -4.0327158 & 2.9674965 \\ \text { H } & 1.6803489 & -5.0697153 & 2.6081275 \\ \text { C } & 2.5561285 & -3.7293145 & 4.0837602 \\ \text { H } & 3.1007631 & -4.5310799 & 4.6051745 \\ \text { C } & 2.6698672 & -2.4008060 & 4.5262864 \\ \text { H } & 3.3044534 & -2.1588902 & 5.3922430 \\ \text { C } & 1.9796117 & -1.3748850 & 3.8618832 \\ \text { H } & 2.0768554 & -0.3344498 & 4.2050843 \\ \text { C } & -1.2780388 & -0.2088448 & 2.9948236 \\ \text { C } & -1.6998720 & -1.2693418 & 3.8210841 \\ \text { H } & -1.0963159 & -2.1867690 & 3.8838072 \\ \text { C } & -2.8816863 & -1.1536094 & 4.5712269 \\ \text { H } & -3.1985684 & -1.9862698 & 5.2172774 \\ \text { C } & -3.6511370 & 0.0186848 & 4.5040566 \\ \text { H } & -4.5749180 & 0.1083356 & 5.0952284 \\ \text { C } & -3.2369521 & 1.0786774 & 3.6787640 \\ \text { H } & -3.8351163 & 2.0005029 & 3.6198868 \\ \text { C } & -2.0620220 & 0.9645713 & 2.9228307 \\ \text { H } & -1.7427594 & 1.7993558 & 2.2803259 \\ & & & \\ \text { R4 } & & & \\ \text { 75 } & & & \\ \text { SCF } & \text { Energy }=-4548.344829393 & \\ \text { C } & -0.2109747 & -6.3224073 & 1.4091955 \\ \text { C } & -0.3187460 & -5.2067480 & 0.5404480 \\ \text { C } & -0.1659142 & -5.4328635 & -0.8512050 \\ \text { C } & 0.1135179 & -6.7123074 & -1.3472096 \\ \text { C } & 0.2423835 & -7.8032532 & -0.4701905 \\ \text { C } & 0.0732312 & -7.6008845 & 0.9091229 \\ \text { C } & -0.5881962 & -3.8673349 & 1.0912181 \\ \text { Pd } & 0.1226289 & 0.2873189 & -0.6674065 \\ \text { P } & -0.4511429 & 2.4299228 & -0.4718091 \\ \text { C } & -0.3034584 & -2.6499088 & 0.5457595 \\ \text { Br } & 2.2243808 & 0.9754939 & -1.9079701 \\ \text { C } & 0.3749353 & -2.3613369 & -0.7410968 \\ \text { C } & 1.7517216 & -2.6539891 & -0.9239812 \\ \text { C } & 2.3471919 & -2.5142321 & -2.1795671 \\ \text { C } & 1.5875452 & -2.0879511 & -3.2869392 \\ & 0.2323056 & -1.7840802 & -3.1308441\end{array}$




\begin{tabular}{|c|c|c|c|}
\hline & -0.3798421 & -1.8979822 & -1.8625349 \\
\hline & -1.2257270 & -3.6741538 & 2.4728805 \\
\hline & -2.2673081 & -4.5858151 & 2.7923783 \\
\hline & -1.6637939 & -2.1918079 & 2.4501426 \\
\hline & -0.6857825 & -1.4929471 & 1.4684716 \\
\hline & -1.3110871 & -0.3292858 & 0.6925174 \\
\hline & 0.5803893 & -1.0044217 & 2.2194257 \\
\hline & -0.4593581 & -3.8425885 & 3.2640539 \\
\hline & -1.6912004 & -1.7240148 & 3.4538389 \\
\hline & -2.6918221 & -2.1559071 & 2.0299908 \\
\hline & -2.2267979 & -0.6390135 & 0.1434191 \\
\hline & -1.5916890 & 0.4818989 & 1.3908132 \\
\hline & 0.3301619 & -0.1357127 & 2.8624925 \\
\hline & 1.365 & -0.6 & 37684 \\
\hline & 1.008 & -1.8 & 2786 \\
\hline & -2.851 & -4 . & 340 \\
\hline & -0.280 & -4 . & -1 . \\
\hline & 0.227 & -6 . & -2 . \\
\hline & 0.462 & $-8 . \varepsilon$ & $-0 . \varepsilon$ \\
\hline & 0.1585171 & -8.4 & 1.6 \\
\hline & -0.3696076 & -6.1848149 & 2.4 \\
\hline & -1.4681179 & -1.7776314 & -1.7 \\
\hline & -0.3657041 & -1.4460145 & -3.9896260 \\
\hline & 2.0668486 & -1.9765527 & -4.2 \\
\hline & 3.4176376 & -2.7335917 & -2.3025615 \\
\hline & 2.3345833 & -3.0090446 & -0.0622168 \\
\hline & -0.9668642 & 3.2097919 & -2.0573367 \\
\hline & 0.8872292 & 3.47 & 30235 \\
\hline & -1.8830187 & 2.8015794 & 0.6214937 \\
\hline & -1.7261034 & 3.445 & 1.8635779 \\
\hline & -2.8394146 & 3.6427719 & 2.6986969 \\
\hline & -4.1105668 & 3.2024785 & 90756 \\
\hline & -4.2697510 & 2.557 & 1.0589845 \\
\hline & -3.1622437 & 2.3512465 & 0.2269574 \\
\hline & -0.7318731 & 3.7952579 & 2.1771927 \\
\hline & -2.7091245 & 4.1486482 & 3.6673441 \\
\hline & -4.9805857 & 3.3592681 & 2.9543823 \\
\hline & -5.2638687 & 2.2073583 & 0.7420749 \\
\hline & -3.2837607 & 1.8357297 & -0.7382659 \\
\hline & -1.8390656 & 4.3218356 & -2.0478818 \\
\hline & -2.2074250 & 4.9368686 & -3.2541390 \\
\hline & -1.7150998 & 4.4457784 & -4.4750294 \\
\hline & -0.8504244 & 3.3390173 & -4.4853708 \\
\hline & -0.4747679 & 2.7189626 & -3.2836639 \\
\hline & -2.2369093 & 4.7036764 & -1.0963519 \\
\hline & -2.8857328 & 5.8034510 & -3.238206 \\
\hline
\end{tabular}




$\begin{array}{lrrr}\text { H } & -2.0088152 & 4.9269805 & -5.4204420 \\ \text { H } & -0.4603609 & 2.9509009 & -5.4384516 \\ \text { H } & 0.2232727 & 1.8665388 & -3.2799240 \\ \text { C } & 1.7669133 & 2.8976686 & 1.1524326 \\ \text { C } & 2.7615092 & 3.6797712 & 1.7555980 \\ \text { C } & 2.8938788 & 5.0367004 & 1.4134670 \\ \text { C } & 2.0269215 & 5.6102894 & 0.4689057 \\ \text { C } & 1.0207470 & 4.8347672 & -0.1289857 \\ \text { H } & 1.6745801 & 1.8262578 & 1.3854048 \\ \text { H } & 3.4487010 & 3.2225735 & 2.4831994 \\ \text { H } & 3.6836872 & 5.6468913 & 1.8773324 \\ \text { H } & 2.1369931 & 6.6688951 & 0.1889290 \\ \text { H } & 0.3489450 & 5.2837593 & -0.8750482\end{array}$

S1

75

SCF Energy $=-4548.346217859$

$\begin{array}{lrrr}\text { C } & -0.4947448 & 1.8316805 & -2.7552077 \\ \text { C } & -0.6085071 & 0.4667821 & -2.3861290 \\ \text { C } & -1.8562902 & 0.0001873 & -1.8687824 \\ \text { C } & -2.9565841 & 0.8813107 & -1.7704782 \\ \text { C } & -2.8196631 & 2.2209952 & -2.1438835 \\ \text { C } & -1.5851323 & 2.6956313 & -2.6289300 \\ \text { C } & 0.4917308 & -0.4938613 & -2.6450838 \\ \text { C } & 0.9004176 & -0.9855404 & -3.8508618 \\ \text { C } & 0.3579241 & -0.7035827 & -5.1927474 \\ \text { C } & -0.9899523 & -0.3158740 & -5.3966976 \\ \text { C } & -1.4779741 & -0.0674585 & -6.6861284 \\ \mathrm{C} & -0.6366119 & -0.1994946 & -7.8042930 \\ \text { C } & 0.6996174 & -0.5904395 & -7.6188876 \\ \text { C } & 1.1921753 & -0.8425895 & -6.3306861 \\ \mathrm{Br} & -1.2269402 & 2.6729231 & 0.8720285 \\ \text { C } & 1.3305104 & -1.0135787 & -1.4770794 \\ \mathrm{C} & 2.3281672 & 0.0832684 & -1.0217207 \\ \mathrm{C} & 0.4443823 & -1.4197759 & -0.2925979 \\ \mathrm{C} & 2.0743937 & -2.2094015 & -2.1363905 \\ \mathrm{C} & 2.1135953 & -1.8932470 & -3.6498309 \\ \mathrm{O} & 3.3319263 & -1.2709341 & -4.0901376 \\ \mathrm{H} & 2.0407946 & -2.8179012 & -4.2597715 \\ \mathrm{H} & 3.0912530 & -2.3775127 & -1.7305870 \\ \mathrm{H} & 1.4841014 & -3.1350591 & -1.9760431 \\ \mathrm{H} & -0.2954582 & -2.2002974 & -0.5722865 \\ \mathrm{H} & 1.0711425 & -1.8218001 & 0.5263527 \\ \mathrm{H} & 1.8019258 & 1.0241754 & -0.7540952 \\ \mathrm{H} & 3.0655966 & 0.3140144 & -1.8168723 \\ \mathrm{H} & 2.8870713 & -0.2594310 & -0.1269516\end{array}$




$\begin{array}{lrrr}\text { H } & 3.2788465 & -0.3336366 & -3.8210129 \\ \mathrm{H} & -1.6693091 & -0.2275560 & -4.5387703 \\ \mathrm{H} & -2.5302702 & 0.2270841 & -6.8191367 \\ \mathrm{H} & -1.0230154 & -0.0021456 & -8.8158174 \\ \mathrm{H} & 1.3685643 & -0.6978999 & -8.4866155 \\ \mathrm{H} & 2.2449870 & -1.1234639 & -6.1822014 \\ \mathrm{H} & -1.9993053 & -1.0753941 & -1.6947097 \\ \mathrm{H} & -3.9166379 & 0.5037483 & -1.3894845 \\ \mathrm{H} & -3.6705241 & 2.9100205 & -2.0441100 \\ \mathrm{H} & -1.4769407 & 3.7540733 & -2.9065541 \\ \mathrm{H} & 0.4636391 & 2.1930358 & -3.1546063 \\ \mathrm{Pd} & -0.5461478 & 0.3060846 & 0.2795508 \\ \mathrm{P} & -0.5385946 & -0.2712593 & 2.4287729 \\ \mathrm{C} & -2.2230491 & -0.3679496 & 3.1651207 \\ \mathrm{C} & -3.2629897 & 0.4384127 & 2.6590510 \\ \mathrm{H} & -3.0538729 & 1.1227982 & 1.8208897 \\ \mathrm{C} & -4.5369753 & 0.3840674 & 3.2450445 \\ \mathrm{H} & -5.3427621 & 1.0182546 & 2.8454485 \\ \mathrm{C} & -4.7845718 & -0.4704010 & 4.3320207 \\ \mathrm{H} & -5.7865665 & -0.5120930 & 4.7857692 \\ \mathrm{C} & -3.7499824 & -1.2747952 & 4.8384148 \\ \mathrm{H} & -3.9363971 & -1.9465854 & 5.6900494 \\ \mathrm{C} & -2.4730284 & -1.2280109 & 4.2583082 \\ \mathrm{H} & -1.6703755 & -1.8678509 & 4.6530300 \\ \mathrm{C} & 0.1606811 & -1.9232003 & 2.8311799 \\ \mathrm{C} & 1.3861104 & -2.0684645 & 3.5088124 \\ \mathrm{H} & 1.9218782 & -1.1778708 & 3.8682005 \\ \mathrm{C} & 1.9206544 & -3.3499165 & 3.7275394 \\ \mathrm{H} & 2.8780728 & -3.4558661 & 4.2597509 \\ \mathrm{C} & 1.2365764 & -4.4885363 & 3.2746013 \\ \mathrm{H} & 1.6568994 & -5.4906189 & 3.4481174 \\ \mathrm{C} & 0.0128999 & -4.3459159 & 2.5950800 \\ \mathrm{H} & -0.5258274 & -5.2353842 & 2.2349831 \\ \mathrm{C} & -0.5198093 & -3.0708313 & 2.3677341 \\ \mathrm{H} & -1.4714287 & -2.9574945 & 1.8257397 \\ \mathrm{C} & 0.4272211 & 0.8687223 & 3.4904240 \\ \mathrm{C} & 1.5329749 & 1.5325615 & 2.9230600 \\ \mathrm{H} & -0.7556173 & 0.5491213 & 5.2919495 \\ \mathrm{H} & 1.7388328 & 1.4107763 & 1.8490933 \\ \mathrm{C} & 2.3387601 & 2.3574168 & 3.7204057 \\ \mathrm{H} & 3.1987820 & 2.8773842 & 3.2725664 \\ \mathrm{C} & 2.0336877 & 2.5366432 & 5.0806190 \\ \mathrm{H} & 2.6589033 & 3.1947843 & 5.7029153 \\ & 0.9224028 & 1.8867743 & 5.6430045 \\ \mathrm{H} & 0.6737748 & 2.0359237 & 6.7046508 \\ & 0.1190482 & 1.0502178 & 4.8520608 \\ & & & \end{array}$




$\begin{array}{lrrr}\text { S2 }\left(d^{\prime}\right) & & & \\ 75 & & & \\ \text { SCF } & \text { Energy }=-4548.360809179 & \\ \text { C } & -2.5213938 & -1.1567697 & -1.0791237 \\ \text { C } & -1.6883068 & -1.6875477 & -2.0867518 \\ \text { C } & -2.2865737 & -2.2523133 & -3.2347883 \\ \text { C } & -3.6822110 & -2.2777596 & -3.3719105 \\ \text { C } & -4.5027479 & -1.7424303 & -2.3643865 \\ \text { C } & -3.9171499 & -1.1814304 & -1.2175636 \\ \text { C } & -0.2046471 & -1.7163658 & -1.9790614 \\ \text { C } & 0.7239974 & -0.9442681 & -2.6900140 \\ \text { C } & 0.4545440 & 0.1700120 & -3.6197037 \\ \text { C } & -0.8021333 & 0.8214481 & -3.6832553 \\ \text { C } & -1.0226500 & 1.8723390 & -4.5787767 \\ \text { C } & 0.0059777 & 2.3132598 & -5.4293921 \\ \text { C } & 1.2642588 & 1.6970927 & -5.3611384 \\ \text { C } & 1.4862915 & 0.6420453 & -4.4655340 \\ \text { Br } & 1.2488056 & 2.0705987 & -1.0356225 \\ \text { C } & 0.4945347 & -2.8928155 & -1.2331117 \\ \text { C } & -0.2369376 & -4.2334353 & -1.3173586 \\ \text { C } & 0.5110437 & -2.2920859 & 0.1665255 \\ \text { C } & 1.8892881 & -2.9223911 & -1.9081165 \\ \text { C } & 2.1273133 & -1.5474310 & -2.5652151 \\ \text { O } & 3.0224979 & -0.7408468 & -1.7930589 \\ \text { H } & 2.5620333 & -1.6886776 & -3.5824969 \\ \text { H } & 2.7036133 & -3.1644877 & -1.1980793 \\ \text { H } & 1.8855894 & -3.7022434 & -2.6968107 \\ \text { H } & 1.4293524 & -2.4676104 & 0.7622353 \\ \text { H } & -0.3920522 & -2.5386373 & 0.7531574 \\ \text { H } & 0.3266983 & -5.0082404 & -0.7579826 \\ \text { H } & -0.3404314 & -4.5728297 & -2.3691864 \\ \text { H } & -1.2527908 & -4.1640923 & -0.8800701 \\ \text { H } & 2.6789463 & 0.1874115 & -1.7797775 \\ \text { H } & -1.6072896 & 0.5196288 & -3.0036675 \\ \text { H } & -2.0073120 & 2.3633756 & -4.6031729 \\ \text { H } & -0.1688326 & 3.1447061 & -6.1288940 \\ \text { H } & 2.0870161 & 2.0452848 & -6.0037079 \\ \text { H } & 2.4872836 & 0.1924994 & -4.4104960 \\ \text { H } & -1.6434963 & -2.6568514 & -4.0306516 \\ \text { H } & -4.1325239 & -2.7142192 & -4.2763881 \\ \text { H } & -5.5973757 & -1.7573860 & -2.4770905 \\ \text { H } & -4.5476042 & -0.7467762 & -0.4271186 \\ \text { H } & -2.0486215 & -0.6892854 & -0.2025897 \\ \text { Pd } & 0.5584114 & -0.2934775 & -0.3608854 \\ \text { P } & 0.2237131 & 0.3704092 & 1.7732967\end{array}$




$\begin{array}{lrrr}\text { C } & -1.2887908 & 1.3951308 & 1.9656345 \\ \text { C } & -1.8022822 & 2.0891712 & 0.8501460 \\ \text { H } & -1.2746858 & 2.0267530 & -0.1146101 \\ \text { C } & -2.9635797 & 2.8656540 & 0.9834916 \\ \text { H } & -3.3577347 & 3.4029922 & 0.1079161 \\ \text { C } & -3.6178438 & 2.9565764 & 2.2230629 \\ \text { H } & -4.5301399 & 3.5642926 & 2.3228904 \\ \text { C } & -3.1078087 & 2.2677269 & 3.3368728 \\ \text { H } & -3.6172831 & 2.3346725 & 4.3101636 \\ \text { C } & -1.9492150 & 1.4863957 & 3.2111923 \\ \text { H } & -1.5650951 & 0.9324762 & 4.0806153 \\ \text { C } & -0.0334396 & -0.9772810 & 2.9947410 \\ \text { C } & 0.9996255 & -1.3889688 & 3.8590902 \\ \text { H } & 1.9530353 & -0.8405474 & 3.8716537 \\ \text { C } & 0.8107822 & -2.4919875 & 4.7085554 \\ \text { H } & 1.6222946 & -2.8025161 & 5.3839555 \\ \text { C } & -0.4061974 & -3.1914367 & 4.7010385 \\ \text { H } & -0.5523084 & -4.0535668 & 5.3691100 \\ \text { C } & -1.4384319 & -2.7877068 & 3.8356504 \\ \text { H } & -2.3942179 & -3.3330324 & 3.8223080 \\ \text { C } & -1.2524591 & -1.6916764 & 2.9822304 \\ \text { H } & -2.0629585 & -1.3812116 & 2.3047838 \\ \text { C } & 1.6296198 & 1.3162904 & 2.4675292 \\ \text { C } & 2.9180949 & 1.0289297 & 1.9718644 \\ \text { H } & 3.0324494 & 0.3145893 & 1.1414951 \\ \text { C } & 4.0357878 & 1.6763342 & 2.5178803 \\ \text { H } & 5.0393697 & 1.4528729 & 2.1262906 \\ \text { C } & 3.8698708 & 2.6232079 & 3.5430770 \\ \text { H } & 4.7466721 & 3.1405503 & 3.9613748 \\ \text { C } & 2.5840361 & 2.9210259 & 4.0248839 \\ \text { H } & 2.4514517 & 3.6723827 & 4.8180430 \\ \text { C } & 1.4622366 & 2.2668131 & 3.4918468 \\ \text { H } & 0.4554795 & 2.5076608 & 3.8636728 \\ & & & \\ \text { S3(d') } & & & \\ \text { 75 } & & & \\ \text { SCF } & \text { Energy }=-4548.360809395 & \\ \text { C } & -2.5180354 & -1.1636736 & -1.0771955 \\ \text { C } & -1.6844830 & -1.6915355 & -2.0859785 \\ \text { C } & -2.2822909 & -2.2569373 & -3.2339424 \\ \text { C } & -3.6779739 & -2.2858173 & -3.3698907 \\ \text { C } & -4.4989948 & -1.7533357 & -2.3612504 \\ \text { C } & -3.9138411 & -1.1917616 & -1.2144843 \\ \text { C } & -0.2006685 & -1.7166124 & -1.9795663 \\ & 0.7252424 & -0.9423101 & -2.6914721 \\ & 0.4520427 & 0.1711733 & -3.6209927\end{array}$




$\begin{array}{lrrr}\text { C } & -0.8060332 & 0.8201285 & -3.6824553 \\ \mathrm{C} & -1.0302725 & 1.8702329 & -4.5779726 \\ \mathrm{C} & -0.0040621 & 2.3128667 & -5.4306191 \\ \mathrm{C} & 1.2555644 & 1.6992282 & -5.3644328 \\ \mathrm{C} & 1.4813114 & 0.6449374 & -4.4688535 \\ \mathrm{Br} & 1.2415285 & 2.0744621 & -1.0370428 \\ \mathrm{C} & 0.5021469 & -2.8909168 & -1.2336740 \\ \mathrm{C} & -0.2256385 & -4.2335901 & -1.3176144 \\ \mathrm{C} & 0.5177781 & -2.2904457 & 0.1661080 \\ \mathrm{C} & 1.8966745 & -2.9166378 & -1.9093689 \\ \mathrm{C} & 2.1303920 & -1.5411513 & -2.5668576 \\ \mathrm{O} & 3.0230874 & -0.7316469 & -1.7948140 \\ \mathrm{H} & 2.5655571 & -1.6812770 & -3.5840953 \\ \mathrm{H} & 2.7120608 & -3.1562705 & -1.1997207 \\ \mathrm{H} & 1.8947808 & -3.6965901 & -2.6979702 \\ \mathrm{H} & 1.4366255 & -2.4643358 & 0.7614674 \\ \mathrm{H} & -0.3845318 & -2.5393053 & 0.7530103 \\ \mathrm{H} & -0.3283102 & -4.5735028 & -2.3693611 \\ \mathrm{H} & -1.2416194 & -4.1669895 & -0.8801978 \\ \mathrm{H} & 0.3402005 & -5.0067087 & -0.7581216 \\ \mathrm{H} & 2.6771728 & 0.1957447 & -1.7824673 \\ \mathrm{H} & -1.6092954 & 0.5169878 & -3.0012114 \\ \mathrm{H} & -2.0159306 & 2.3593488 & -4.6007034 \\ \mathrm{H} & -0.1817628 & 3.1437266 & -6.1300905 \\ \mathrm{H} & 2.0764531 & 2.0488239 & -6.0086229 \\ \mathrm{H} & 2.4832578 & 0.1973083 & -4.4156371 \\ \mathrm{H} & -1.6388726 & -2.6592468 & -4.0306598 \\ \mathrm{H} & -4.1279441 & -2.7227335 & -4.2743203 \\ \mathrm{H} & -5.5936804 & -1.7709754 & -2.4730018 \\ \mathrm{H} & -4.5447446 & -0.7593657 & -0.4231585 \\ \mathrm{H} & -2.0456209 & -0.6957239 & -0.2007077 \\ \mathrm{Pd} & 0.5598918 & -0.2916501 & -0.3608513 \\ \mathrm{P} & 0.2264154 & 0.3706514 & 1.7736099 \\ \mathrm{C} & -1.2883687 & 1.3913796 & 1.9689010 \\ \mathrm{C} & -1.8061494 & 2.0836021 & 0.8542708 \\ \mathrm{H} & -1.2805310 & 2.0221226 & -0.1116155 \\ \mathrm{C} & -2.9691395 & 2.8571601 & 0.9898747 \\ \mathrm{H} & -3.3666590 & 3.3930701 & 0.1149447 \\ \mathrm{C} & -3.6208625 & 2.9469392 & 2.2308639 \\ \mathrm{H} & -4.5344839 & 3.5523649 & 2.3324715 \\ \mathrm{C} & -3.1065959 & 2.2598537 & 3.3438181 \\ \mathrm{H} & -3.6140441 & 2.3259422 & 4.3182255 \\ \mathrm{C} & -1.9462795 & 1.4814516 & 3.2158808 \\ & -1.5588474 & 0.9288460 & 4.0846703 \\ \mathrm{C} & 1.6311680 & 1.3197040 & 2.4659918 \\ & & 1.0370995 & 1.9667069\end{array}$




$\begin{array}{rrrr}\mathrm{H} & 3.0337509 & 0.3244333 & 1.1349130 \\ \mathrm{C} & 4.0363418 & 1.6870430 & 2.5110578 \\ \mathrm{H} & 5.0396371 & 1.4672901 & 2.1166441 \\ \mathrm{C} & 3.8700499 & 2.6316929 & 3.5382326 \\ \mathrm{H} & 4.7462834 & 3.1510386 & 3.9552391 \\ \mathrm{C} & 2.5844970 & 2.9247430 & 4.0237142 \\ \mathrm{H} & 2.4515562 & 3.6743696 & 4.8184493 \\ \mathrm{C} & 1.4634071 & 2.2679809 & 3.4923295 \\ \mathrm{H} & 0.4568485 & 2.5051825 & 3.8670127 \\ \mathrm{C} & -0.0251554 & -0.9784163 & 2.9946677 \\ \mathrm{C} & 1.0115372 & -1.3897229 & 3.8548588 \\ \mathrm{H} & 1.9641553 & -0.8398569 & 3.8648860 \\ \mathrm{C} & 0.8273182 & -2.4941602 & 4.7034735 \\ \mathrm{H} & 1.6416168 & -2.8043377 & 5.3756764 \\ \mathrm{C} & -0.3886211 & -3.1954573 & 4.6992206 \\ \mathrm{H} & -0.5311029 & -4.0587138 & 5.3666225 \\ \mathrm{C} & -1.4244415 & -2.7921443 & 3.8379427 \\ \mathrm{H} & -2.3794019 & -3.3389700 & 3.8270944 \\ \mathrm{C} & -1.2430976 & -1.6946715 & 2.9853701 \\ \mathrm{H} & -2.0563213 & -1.3846446 & 2.3110020\end{array}$

S4

75

SCF Energy $=-4548.346204019$

$\begin{array}{lll}\text { C } & -0.1069724 & 2.7009164\end{array}$

C $\quad 0.4617814 \quad 2.3805340$

C $1.8036324 \quad 1.8904010$

C $\quad 2.5499910 \quad 1.7718942$

C $\quad 1.9680713 \quad 2.0977145$

$\begin{array}{lll}\text { C } & 0.6365352 & 2.5550096\end{array}$

C $\quad-0.2596743 \quad 2.6665910$

$-1.9112145$

$-0.6518258$

$-0.6124170$

$-1.8063367$

$-3.0343783$

$-3.0846864$

2. 6665910

0.6123094

$-0.5024795$

3.8863219

1.1745692

$-0.1116245$

5.2214559

0.6857275

$-0.8713153$

6.3573050

1.0638832

$-0.5166791$

7.6396202

0.6217542

C $\quad 0.6058264$

7.8213351

$-0.2026337$

C $\quad 1.3741229$

6.7055516

$-0.5773549$

C $\quad 1.0225435$

5.4218932

$-0.1401988$

$\mathrm{Br} \quad 0.4165496$

$-0.9509506$

$-2.8409649$

$-0.8540833$

1. 5122117

1. 4190225

$-2.1537604$

1.0131360

0.7357296

0.1384302

0.3486306

1.5389563

$-1.1654876$

2. 2078329

2.7742900

$-1.3370881$

3.7087066

2.4427770

$-2.7017009$

4.1177509

2. 2524825

$\mathrm{H} \quad-0.9699166$

4.3518141

3. 2697705 


\begin{tabular}{|c|c|c|c|}
\hline & -2.0589459 & 1.8009537 & 3.2872298 \\
\hline$H$ & -0.2955007 & 2.0850626 & 3.4518857 \\
\hline & 1.0927164 & 0.6616348 & \\
\hline & -0.3021682 & -0.4571313 & 2.1559612 \\
\hline & -2.9419165 & 1.7928643 & 0.7479443 \\
\hline & -2.5458200 & 0.1231190 & 1.2690850 \\
\hline & -1.9693078 & 0.7230300 & -0.3204326 \\
\hline & -2.9614297 & 3.8148952 & 1.3610656 \\
\hline & -1.7648913 & 6.2107014 & 1.6878011 \\
\hline & -1.1262252 & 8.505 & 0.9227290 \\
\hline & 0.8841412 & 8.8283126 & -0.5490127 \\
\hline & 2.2628307 & 6.83 & -1.2136679 \\
\hline & 1.6482797 & 4.56 & -0.4270478 \\
\hline & 2.2999385 & 1.75 & 0.3586677 \\
\hline & 3.5883225 & 1.41 & -1.7592022 \\
\hline & 2.5432250 & 1.98 & -3.9640772 \\
\hline & 0.1770863 & 2.795 & $-4.054389 s$ \\
\hline & -1.1384152 & 3.0797914 & -1.9441212 \\
\hline & 0.5115764 & -0.2783489 & -0.3991631 \\
\hline & 0.6404155 & -2.4201742 & 0.1965129 \\
\hline & -0.6552541 & -3.4669375 & -0.5691352 \\
\hline & -1.9092371 & -2.8831793 & -0.8384078 \\
\hline & -2.0515370 & -1.8077160 & -0.6550543 \\
\hline & -2.9489715 & -3.6663013 & -1.3586750 \\
\hline & -3.9251918 & -3.20 & -1.5712740 \\
\hline & -2.7360810 & -5.029 & -1.6291540 \\
\hline & -3.5486810 & -5.640 & -2.0503527 \\
\hline & -1.4816327 & -5.6076768 & -1.3750669 \\
\hline & -1.3080000 & -6.67 & -1.5986778 \\
\hline & -0.4404241 & -4.8309749 & -0.8423374 \\
\hline & 0.5436634 & -5.2839772 & -0.6528807 \\
\hline C & 0.4753512 & -2.7867431 & 1.9909398 \\
\hline & -0.6642622 & -3.4220500 & 2.5196223 \\
\hline & -1.4600789 & -3.7691850 & 1.8447784 \\
\hline & -0.7816816 & -3.6143670 & 3.9070588 \\
\hline & -1.6745948 & -4.1135027 & 4.3129009 \\
\hline C & 0.2347556 & -3.1773246 & 4.7704101 \\
\hline & 0.1406412 & -3.3301581 & 5.8561073 \\
\hline C & 1.3737752 & -2.5402987 & 4.2447518 \\
\hline & 2.1722028 & -2.1927656 & 4.9176425 \\
\hline C & 1.4916232 & -2.3392338 & $2.863807 \varepsilon$ \\
\hline $\mathrm{H}$ & 2.3768494 & -1.829 & 2.4526684 \\
\hline C & 2.2544219 & -3.2038037 & -0.2139224 \\
\hline C & 2.7203083 & -4.3051045 & $0.539217 \varepsilon$ \\
\hline 11 & 2.1365948 & -4.6766189 & 1.3940925 \\
\hline & 3.9346366 & -4.9230494 & 0.203735 \\
\hline
\end{tabular}




$\begin{array}{lllr}\text { H } & 4.2899022 & -5.7810995 & 0.7944096 \\ \text { C } & 4.6931930 & -4.4461821 & -0.8784042 \\ \text { H } & 5.6475448 & -4.9297949 & -1.1371569 \\ \text { C } & 4.2315821 & -3.3506626 & -1.6263075 \\ \text { H } & 4.8204191 & -2.9739089 & -2.4763059 \\ \text { C } & 3.0175804 & -2.7272872 & -1.2991862 \\ \text { H } & 2.6360199 & -1.8827924 & -1.8955857\end{array}$

\section{Optimized Geometries(MXnd)-exo}

\begin{tabular}{lrrr} 
R3 & \multicolumn{3}{l}{} \\
75 & \multicolumn{4}{l}{} \\
SCF & Energy $=-4548.350665449$ & \\
C & 2.8075176 & 2.0689683 & -0.1974133 \\
C & 1.8210211 & 1.6517857 & -1.1168033 \\
C & 0.8152004 & 2.5849947 & -1.4753772 \\
C & 0.8037283 & 3.8799724 & -0.9454049 \\
C & 1.8050011 & 4.2826815 & -0.0463753 \\
C & 2.8013351 & 3.3694795 & 0.3268120 \\
C & 1.8259323 & 0.3080160 & -1.7452211 \\
C & 2.0961792 & -0.9775268 & -1.2058948 \\
C & 2.5265539 & -1.4505501 & 0.1366792 \\
C & 3.7635723 & -1.0838540 & 0.7074794 \\
C & 4.1942807 & -1.6562282 & 1.9152912 \\
C & 3.4004097 & -2.6152428 & 2.5656882 \\
C & 2.1905076 & -3.0272076 & 1.9797592 \\
C & 1.7736770 & -2.4647772 & 0.7669841 \\
Br & -2.4384395 & -1.2387751 & -1.8364115 \\
C & 1.6741458 & 0.1138047 & -3.3093155 \\
C & 2.0168341 & 1.3231682 & -4.1756034 \\
C & 0.2237643 & -0.3687544 & -3.3968782 \\
C & 2.6155300 & -1.0752254 & -3.5571012 \\
C & 2.4326555 & -1.9956294 & -2.3444295 \\
O & 3.5488613 & -2.8150970 & -2.0606247 \\
H & 1.5926588 & -2.7064442 & -2.4825985 \\
H & 2.4231347 & -1.5771370 & -4.5265562 \\
H & 3.6698107 & -0.7167811 & -3.5549385 \\
H & 0.0553809 & -1.3193270 & -3.9355053 \\
H & -0.5082322 & 0.3976417 & -3.7146858 \\
H & 1.9810707 & 1.0377661 & -5.2466667 \\
H & 3.0358776 & 1.7004393 & -3.9491611 \\
H & 1.3092594 & 2.1605709 & -4.0277667 \\
H & 4.3029034 & -2.2280946 & -1.8565748
\end{tabular}




$\begin{array}{lrrr}\text { H } & 4.4063348 & -0.3535027 & 0.1951514 \\ \mathrm{H} & 5.1563569 & -1.3472490 & 2.3512282 \\ \mathrm{H} & 3.7296441 & -3.0501077 & 3.5212270 \\ \mathrm{H} & 1.5586621 & -3.7825697 & 2.4698938 \\ \mathrm{H} & 0.8382722 & -2.7990542 & 0.2981639 \\ \mathrm{H} & 0.0207311 & 2.2743895 & -2.1687840 \\ \mathrm{H} & 0.0033932 & 4.5778563 & -1.2338426 \\ \mathrm{H} & 1.7995001 & 5.3001318 & 0.3720301 \\ \mathrm{H} & 3.5810390 & 3.6645898 & 1.0437857 \\ \mathrm{H} & 3.5945484 & 1.3719565 & 0.1068498 \\ \mathrm{Pd} & -0.1099843 & -0.5792549 & -1.3606024 \\ \mathrm{P} & -0.6868387 & -0.1123689 & 0.9801855 \\ \mathrm{C} & -2.2803814 & 0.8144454 & 1.0020434 \\ \mathrm{C} & -2.5391486 & 1.7377326 & -0.0311251 \\ \mathrm{H} & -1.8233766 & 1.8364600 & -0.8589239 \\ \mathrm{C} & -3.7066209 & 2.5134461 & -0.0105781 \\ \mathrm{H} & -3.9001441 & 3.2296790 & -0.8233268 \\ \mathrm{C} & -4.6355395 & 2.3593179 & 1.0324696 \\ \mathrm{H} & -5.5586542 & 2.9586606 & 1.0422946 \\ \mathrm{C} & -4.3881751 & 1.4305964 & 2.0563463 \\ \mathrm{H} & -5.1167203 & 1.2992348 & 2.8708895 \\ \mathrm{C} & -3.2126946 & 0.6625936 & 2.0453429 \\ \mathrm{H} & -3.0234021 & -0.0638530 & 2.8490581 \\ \mathrm{C} & -1.0235678 & -1.5476415 & 2.0857342 \\ \mathrm{C} & -1.5461820 & -2.7142551 & 1.4864413 \\ \mathrm{H} & -1.7620544 & -2.7151291 & 0.4050894 \\ \mathrm{C} & -1.7916260 & -3.8553345 & 2.2637292 \\ \mathrm{H} & -2.1955954 & -4.7607787 & 1.7860414 \\ \mathrm{C} & -1.5198907 & -3.8446741 & 3.6433511 \\ \mathrm{H} & -1.7052156 & -4.7441166 & 4.2501688 \\ \mathrm{C} & -1.0177969 & -2.6795310 & 4.2455986 \\ \mathrm{H} & -0.8132521 & -2.6601503 & 5.3270183 \\ \mathrm{C} & -0.7738173 & -1.5326328 & 3.4725705 \\ \mathrm{H} & -0.3779406 & -0.6255561 & 3.9507003 \\ \mathrm{C} & 0.3713105 & 0.9970775 & 2.0120579 \\ \mathrm{C} & 0.0407315 & 2.3562474 & 2.1910073 \\ \mathrm{H} & -0.8634974 & 2.7686375 & 1.7234704 \\ \mathrm{C} & 0.8569453 & 3.1873529 & 2.9732608 \\ \mathrm{H} & 0.5819118 & 4.2448157 & 3.1037357 \\ \mathrm{C} & 2.0133517 & 2.6790167 & 3.5842572 \\ \mathrm{H} & 2.6480170 & 3.3334999 & 4.2009856 \\ \mathrm{C} & 2.3521183 & 1.3279596 & 3.4037595 \\ \mathrm{C} & 1.5537628 & 0.9093442 & 3.8760917 \\ \mathrm{H} & 1.8026197 & -0.5640406 & 2.5190166\end{array}$


75

SCF Energy $=-4548.334216959$

$\begin{array}{lll}\text { C } & 0.3647759 & -2.2475880\end{array}$

$-0.9838870$

$-0.4108382-1.9754068$

0.1847310

C $\quad-1.7116136 \quad-1.3818699$

0.0162889

C $\quad-2.2063326-1.1331562$

$-1.2977976$

$-1.4431511-1.4590643$

$-2.4146386$

$-0.1519489-2.0177930$

$-2.2542560$

$-0.0201503$

$-2.5359085$

1.5009041

0.2023245

$-3.8387606$

1.8353639

0.0679316

$-5.0393644$

0.9950690

$-0.8515408$

$-5.0954856$

$-0.0823892$

$-0.9530614$

$-6.2399474$

$-0.8830675$

$-0.1466932$

$-7.3618556$

$-0.6232199$

0.7507609

$-7.3316448$

0.4569526

C $\quad 0.8523902$

$-6.1887353$

1. 2624617

$-0.7639529$

2.5913906

1.8750611

C $\quad 0.2498230$

$-1.5850835$

2.6629798

$1.6860485-1.0126701$

2.5282403

$-0.7681884-0.4459569$

2.6518031

$-2.5538055$

3.8740321

3. 3088021

$-3.9224128$

$-5.0484730$

4.0129763

0.1292900

$-4.0220233$

3.3764003

0.7637692

$-2.2199008$

$-0.8860625-2.6589336$

$-1.8132541-0.7978721$

4.7492350

4.1947336

$-0.5585844$

0.3266037

2.7694585

$2.4506182-1.8126254$

3.4141855

2.5957228

1.8779267

$-0.2686609$

3.3276591

1.8168465

$-0.4970868$

1.5533996

$-0.8274059$

$-5.1025529$

3.8227596

$-1.5066818$

$-4.2365302$

$-0.2821604$

$-1.6752776$

$-6.2590895$

$-1.7135185$

$-0.2280774$

$-8.2612979$

$-1.2522389$

$-8.2102140 \quad 0.6790385$

1. 3756583

$-6.1852178$

2.1178809

$-1.4051750$

0.8459807

$-3.2255634-0.7355359$

$-1.4141442$

$-1.8391919$

$-1.2897027$

$-3.4255628$

H $\quad 0.4427789$

$-2.2677957$

$-3.1429473$

$\mathrm{H} \quad 1.3558244$

$-2.7061128$

$-0.8555068$

$-0.5149897$

0.4046237

0.7601701

P 0.2628729

1. 6773306

$-1.1456197$

$-0.9240470$

3. 0232704

$-1.5482962$ 


\begin{tabular}{lrrr} 
C & -0.5465924 & 4.3628974 & -1.7534499 \\
H & 0.5072555 & 4.6592162 & -1.6561505 \\
C & -1.5202450 & 5.3282158 & -2.0561552 \\
H & -1.2185771 & 6.3763261 & -2.2040195 \\
C & -2.8723399 & 4.9637859 & -2.1606085 \\
H & -3.6324406 & 5.7242966 & -2.3953305 \\
C & -3.2543723 & 3.6285854 & -1.9457096 \\
H & -4.3146662 & 3.3398165 & -2.0054717 \\
C & -2.2863492 & 2.6651923 & -1.6289916 \\
H & -2.5821856 & 1.6266469 & -1.4179250 \\
C & 1.8730591 & 2.5090604 & -0.7861317 \\
C & 2.4310776 & 2.4540815 & 0.5062149 \\
H & 1.8770406 & 1.9550305 & 1.3134113 \\
C & 3.6720028 & 3.0572010 & 0.7676933 \\
H & 4.0968430 & 3.0045177 & 1.7814202 \\
C & 4.3632378 & 3.7261931 & -0.2543340 \\
H & 5.3344486 & 4.2007152 & -0.0465710 \\
C & 3.8135882 & 3.7843262 & -1.5469280 \\
H & 4.3518536 & 4.3034678 & -2.3545466 \\
C & 2.5798775 & 3.1735288 & -1.8144317 \\
H & 2.1682545 & 3.1996497 & -2.8343296 \\
C & 0.6656942 & 0.9834190 & -2.8092948 \\
C & 1.8596887 & 0.2430872 & -2.9534737 \\
H & 2.5358260 & 0.1272685 & -2.0926667 \\
C & 2.1935011 & -0.3355185 & -4.1852900 \\
H & 3.1322872 & -0.9012306 & -4.2862168 \\
C & 1.3285878 & -0.2003261 & -5.2862262 \\
H & 1.5885298 & -0.6568313 & -6.2532079 \\
C & 0.1364762 & 0.5287414 & -5.1469251 \\
H & -0.5405965 & 0.6483360 & -6.0065721 \\
C & -0.1924937 & 1.1228670 & -3.9166867 \\
H & -1.1201144 & 1.7055691 & -3.8191967 \\
& & & \\
S3 & & & \\
75 & & & \\
SCF & Energy $=-4548.357596040$ & \\
C & -0.4302162 & -2.8888102 & -1.9654395 \\
C & -1.3280007 & -1.9070489 & -1.4918475 \\
C & -1.7980712 & -0.9417453 & -2.4200299 \\
C & -1.3920745 & -0.9630617 & -3.7591707 \\
C & -0.5135036 & -1.9595699 & -4.2145031 \\
C & -0.0350140 & -2.9181312 & -3.3101258 \\
C & -1.8108727 & -1.8689581 & -0.0890982 \\
C & -1.1271513 & -2.1092980 & 1.1309798 \\
& 0.2495756 & -2.5618266 & 1.4629380 \\
\hline & 0.7471463 & -3.8216529 & 1.0637167
\end{tabular}




\begin{tabular}{|c|c|c|c|}
\hline & 2.0029366 & -4.2677676 & 1.5044245 \\
\hline & 2.7762717 & -3.4700062 & 2.3651465 \\
\hline & 2.2645675 & -2.2414676 & 2.8170306 \\
\hline & 1.0052946 & -1.8042093 & 2.3851278 \\
\hline & -1.6490119 & 2.4396231 & 1.3649457 \\
\hline & -3.3400666 & -1.6590348 & 0.2669256 \\
\hline & -4.3406696 & -2.1031175 & -0.7985324 \\
\hline & -3.3488209 & -0.1659207 & 0.5951891 \\
\hline & -3.4708631 & -2.4684929 & 1.5672971 \\
\hline & -2.1255139 & -2.3069729 & \\
\hline & -2.0575924 & -1.2928129 & 3.2888037 \\
\hline & -1.8532081 & -3.2402395 & 2.8357092 \\
\hline & -4.3245470 & -2.139 & 4338 \\
\hline & -3.6162 & -3.54 & 5747 \\
\hline & -3.8715 & 0.12 & 6853 \\
\hline & -3.648 & 0.50 & 576 \\
\hline & -5.37 & $-1.9 s$ & -0 . \\
\hline & -4.18 & -3.1 & -1 \\
\hline & -4.266 & -1.50 & -1 \\
\hline & -2.0462770 & -0.43 & $2 . \varepsilon$ \\
\hline & 0.1327574 & -4.47 & 0.4 \\
\hline & 2.3783172 & -5.247 & 1.1 \\
\hline & 3.7685664 & -3.812 & 2.6 \\
\hline & 2.8488614 & $-1.607 !$ & 3.5 \\
\hline & 0.5925071 & -0.8589956 & 2.7 \\
\hline & -2.4786620 & -0.1509411 & -2.0 \\
\hline & -1.7642635 & -0.19 & -4.4 \\
\hline & -0.1943791 & -1.9805928 & -5.2 \\
\hline & 0.666 & -3.694 & -3.6 \\
\hline & -0.0400733 & -3.644 & -1.2 \\
\hline & $-1.288^{\circ}$ & 0.16 & 0.6 \\
\hline & 1.0070592 & 0.6174 & -0. \\
\hline & 0.9466973 & 2.198 & -0.9 \\
\hline & 1.9778991 & 3.1524722 & -0.9037457 \\
\hline & 2.8344417 & 2.9836325 & -0.2349478 \\
\hline & 1.9078075 & 4.3245276 & -1.6741679 \\
\hline & 2.7131733 & 5.0713869 & -1.6034132 \\
\hline & 0.8149372 & 4.5456594 & -2.5277139 \\
\hline & 0.7614876 & 5.4664571 & -3.1282242 \\
\hline & -0.2160086 & 3.5936367 & -2.6050655 \\
\hline & -1.0813158 & 3.7664458 & -3.2625618 \\
\hline & -0.1554236 & 2.4296680 & -1.8266032 \\
\hline & -0.9717431 & 1.6943085 & -1.8656745 \\
\hline & 2.2517039 & 0.9516767 & 1.2810079 \\
\hline & 3.6284532 & 0.6976967 & 1.1196514 \\
\hline & 4.0068938 & 0.2995323 & 0.167540 \\
\hline
\end{tabular}




$\begin{array}{lrrr}\text { C } & 4.5181923 & 0.9394168 & 2.1789650 \\ \text { H } & 5.5909192 & 0.7312666 & 2.0466153 \\ \text { C } & 4.0425292 & 1.4426665 & 3.4008518 \\ \text { H } & 4.7407817 & 1.6255222 & 4.2318355 \\ \text { C } & 2.6723630 & 1.7174767 & 3.5569940 \\ \text { H } & 2.2931407 & 2.1204374 & 4.5082057 \\ \text { C } & 1.7789910 & 1.4745861 & 2.5039050 \\ \text { H } & 0.7044503 & 1.6913006 & 2.6196762 \\ \text { C } & 1.9033427 & -0.4756716 & -1.2226957 \\ \text { C } & 1.9573700 & -0.1668796 & -2.5975116 \\ \text { H } & 1.4705859 & 0.7414639 & -2.9774713 \\ \text { C } & 2.6412544 & -1.0101143 & -3.4865549 \\ \text { H } & 2.6759219 & -0.7521348 & -4.5557437 \\ \text { C } & 3.2762600 & -2.1714073 & -3.0203019 \\ \text { H } & 3.8159316 & -2.8271896 & -3.7204309 \\ \text { C } & 3.2193073 & -2.4881659 & -1.6530789 \\ \text { H } & 3.7122207 & -3.3934346 & -1.2673875 \\ \text { C } & 2.5379133 & -1.6496426 & -0.7610050 \\ \text { H } & 2.5292752 & -1.8956387 & 0.3061943 \\ & & & \\ \text { S4 } & & & \\ \text { 75 } & & & \\ \text { SCF } & \text { Energy }=-4548.335835518 & \\ \text { C } & 0.7714860 & -2.3270620 & -0.6252851 \\ \text { C } & -0.3133379 & -1.9865731 & 0.2406005 \\ \text { C } & -1.5101121 & -1.4427953 & -0.3460470 \\ \text { C } & -1.6030862 & -1.3190950 & -1.7644313 \\ \text { C } & -0.5517627 & -1.7214304 & -2.5806000 \\ \text { C } & 0.6421522 & -2.2218295 & -2.0050641 \\ \text { C } & -0.3362302 & -2.4865920 & 1.6386767 \\ \text { C } & -0.3780231 & -3.7832378 & 2.0597237 \\ \text { C } & -0.3894662 & -5.0153923 & 1.2513269 \\ \text { C } & -0.9630874 & -5.0588029 & -0.0437773 \\ \text { C } & -0.9674784 & -6.2468216 & -0.7862440 \\ \text { C } & -0.4036960 & -7.4199334 & -0.2554322 \\ \text { C } & 0.1570137 & -7.3949609 & 1.0323203 \\ \text { C } & 0.1620552 & -6.2090420 & 1.7802108 \\ \text { Br } & -0.7949562 & 2.6219448 & 1.6445290 \\ \text { C } & -0.3543192 & -1.4839360 & 2.7828113 \\ \text { C } & 1.0698256 & -0.9177961 & 3.0139312 \\ \text { C } & -1.3110035 & -0.3490763 & 2.4137987 \\ \text { C } & -0.8041994 & -2.3756087 & 3.9748729 \\ \text { C } & -0.3855074 & -3.8174971 & 3.5897339 \\ \text { O } & 0.8800883 & -4.2336531 & 4.1260702 \\ \text { H } & -1.1063215 & -4.5670469 & 3.9774488 \\ \text { H } & -0.3721026 & -2.0732868 & 4.9487087\end{array}$




$\begin{array}{lrrr}\text { H } & -1.9094442 & -2.3345645 & 4.0628919 \\ \mathrm{H} & -2.3489035 & -0.7037873 & 2.2457937 \\ \mathrm{H} & -1.3227639 & 0.4613196 & 3.1650348 \\ \mathrm{H} & 1.7604257 & -1.7014277 & 3.3867931 \\ \mathrm{H} & 1.0404906 & -0.0994232 & 3.7610018 \\ \mathrm{H} & 1.4916315 & -0.5011057 & 2.0755308 \\ \mathrm{H} & 1.5668350 & -3.7640334 & 3.6143182 \\ \mathrm{H} & -1.4328806 & -4.1596038 & -0.4649055 \\ \mathrm{H} & -1.4241497 & -6.2581746 & -1.7877838 \\ \mathrm{H} & -0.4082597 & -8.3515903 & -0.8414434 \\ \mathrm{H} & 0.5972575 & -8.3088738 & 1.4599714 \\ \mathrm{H} & 0.6169828 & -6.1795805 & 2.7812064 \\ \mathrm{H} & -2.4391690 & -1.4216324 & 0.2398894 \\ \mathrm{H} & -2.5424074 & -0.9545110 & -2.2062934 \\ \mathrm{H} & -0.6381140 & -1.6496123 & -3.6735185 \\ \mathrm{H} & 1.4643245 & -2.5403752 & -2.6595055 \\ \mathrm{H} & 1.6806676 & -2.7532568 & -0.1772075 \\ \mathrm{Pd} & -0.5718995 & 0.3959539 & 0.6051786 \\ \mathrm{P} & 0.5161327 & 1.6100624 & -1.1987000 \\ \mathrm{C} & 2.0910443 & 2.4066532 & -0.6595985 \\ \mathrm{C} & 2.5186482 & 2.2914870 & 0.6777277 \\ \mathrm{H} & 1.8813431 & 1.7691689 & 1.4047546 \\ \mathrm{C} & 3.7348242 & 2.8639106 & 1.0835018 \\ \mathrm{H} & 4.0575743 & 2.7658854 & 2.1309483 \\ \mathrm{C} & 4.5309159 & 3.5612932 & 0.1612390 \\ \mathrm{H} & 5.4822147 & 4.0125236 & 0.4821722 \\ \mathrm{C} & 4.1126670 & 3.6774267 & -1.1757453 \\ \mathrm{H} & 4.7346454 & 4.2179556 & -1.9054011 \\ \mathrm{C} & 2.9037689 & 3.0972982 & -1.5870390 \\ \mathrm{H} & 2.5958935 & 3.1668422 & -2.6409353 \\ \mathrm{C} & 1.0703110 & 0.9417334 & -2.8316590 \\ \mathrm{C} & 0.3044986 & 1.0907068 & -4.0046137 \\ \mathrm{H} & -0.6334700 & 1.6638594 & -3.9763856 \\ \mathrm{C} & 0.7379710 & 0.5200185 & -5.2128800 \\ \mathrm{H} & 0.1323404 & 0.6494522 & -6.1229017 \\ \mathrm{C} & 1.9422194 & -0.2007666 & -5.2646967 \\ \mathrm{H} & 2.2838152 & -0.6416332 & -6.2133514 \\ \mathrm{C} & 2.7154575 & -0.3446995 & -4.0992984 \\ \mathrm{H} & 3.6651068 & -0.9005209 & -4.1318176 \\ \mathrm{C} & 2.2803163 & 0.2164526 & -2.8906254 \\ \mathrm{H} & 2.8881936 & 0.0963634 & -1.9812435 \\ \mathrm{C} & -0.6087684 & 2.9735448 & -1.7122158 \\ & -0.1996097 & 4.3099579 & -1.8687895 \\ \mathrm{H} & 0.8400625 & 4.5960370 & -1.6568348 \\ & -0.8021419 & 6.3322627 & -2.3741147\end{array}$




$\begin{array}{llll}\text { C } & -2.4640530 & 4.9358848 & -2.5148002 \\ \text { H } & -3.1881221 & 5.7050807 & -2.8234113 \\ \text { C } & -2.8788727 & 3.6033940 & -2.3499123 \\ \text { H } & -3.9294949 & 3.3251575 & -2.5228050 \\ \text { C } & -1.9582741 & 2.6289794 & -1.9393205 \\ \text { H } & -2.2869417 & 1.5928887 & -1.7648113\end{array}$

\section{Optimized Geometries(BXnd(CH-coord))}

$\begin{array}{lrrr}\text { R3 } & & & \\ \text { 75 } & & & \\ \text { SCF } & \text { Energy }=-4548.345774304 & \\ \text { C } & -2.8987673 & -1.7193821 & -0.8959902 \\ \text { C } & -1.9338827 & -2.6047919 & -0.3234747 \\ \text { C } & -1.4590397 & -3.6656672 & -1.1394558 \\ \text { C } & -1.8616519 & -3.7831378 & -2.4700868 \\ \text { C } & -2.7663254 & -2.8624187 & -3.0386043 \\ \text { C } & -3.2941039 & -1.8446667 & -2.2432110 \\ \text { C } & -1.4051802 & -2.3697537 & 1.0365676 \\ \text { C } & -0.1640583 & -2.7014112 & 1.5115048 \\ \text { C } & 0.9804988 & -3.3302790 & 0.8173397 \\ \text { C } & 1.6797014 & -4.4047613 & 1.4211502 \\ \text { C } & 2.8213850 & -4.9500288 & 0.8095454 \\ \text { C } & 3.2848270 & -4.4324823 & -0.4110554 \\ \text { C } & 2.5928354 & -3.3711034 & -1.0215471 \\ \text { C } & 1.4537316 & -2.8252080 & -0.4177066 \\ \text { Br } & -0.0617353 & -0.2087948 & -2.6281030 \\ \text { C } & -2.1607962 & -1.4644248 & 2.0417836 \\ \text { C } & -3.6339699 & -1.8525687 & 2.2618645 \\ \text { C } & -2.0058665 & 0.0073719 & 1.5582838 \\ \text { C } & -1.3508151 & -1.7110643 & 3.3423360 \\ \text { C } & 0.0606607 & -2.0703843 & 2.8885956 \\ \text { O } & 0.6983703 & -2.8909040 & 3.8597759 \\ \text { H } & 0.6663964 & -1.1389589 & 2.7316790 \\ \text { H } & -1.3871157 & -0.8509979 & 4.0387014 \\ \text { H } & -1.7488498 & -2.6029418 & 3.8704258 \\ \text { H } & -1.3916713 & 0.5878730 & 2.2669179 \\ \text { H } & -2.9818047 & 0.5295080 & 1.4474289 \\ \text { H } & -3.7233111 & -2.9317657 & 2.5031827 \\ \text { H } & -4.2741430 & -1.6518591 & 1.3821508 \\ \text { H } & -4.0491341 & -1.2742306 & 3.1127967 \\ \text { H } & 1.6160018 & -3.0301843 & 3.5561424 \\ \text { H } & 1.2969279 & -4.8293048 & 2.3607760\end{array}$




\begin{tabular}{lrrr} 
H & 3.3460880 & -5.7914380 & 1.2879314 \\
$\mathrm{H}$ & 4.1798382 & -4.8597104 & -0.8888183 \\
$\mathrm{H}$ & 2.9403704 & -2.9509750 & -1.9775330 \\
$\mathrm{H}$ & 0.9281571 & -1.9944589 & -0.9154663 \\
$\mathrm{H}$ & -0.7560104 & -4.3932713 & -0.7156378 \\
$\mathrm{H}$ & -1.4607612 & -4.6045266 & -3.0831509 \\
$\mathrm{H}$ & -3.0596740 & -2.9537161 & -4.0944521 \\
$\mathrm{H}$ & -4.0171471 & -1.1273184 & -2.6577674 \\
$\mathrm{H}$ & -3.4014236 & -0.9621313 & -0.2771299 \\
$\mathrm{Pd}$ & -1.1315482 & 0.0137715 & -0.3368563 \\
$\mathrm{P}$ & 0.1310399 & 1.7893956 & 0.0935788 \\
$\mathrm{C}$ & -0.2047588 & 3.2752751 & -0.9298466 \\
$\mathrm{C}$ & -0.9305536 & 3.1657915 & -2.1318594 \\
$\mathrm{H}$ & -1.2844642 & 2.1752251 & -2.4569629 \\
$\mathrm{C}$ & -1.1624415 & 4.3093528 & -2.9125648 \\
$\mathrm{H}$ & -1.7290776 & 4.2180488 & -3.8513892 \\
$\mathrm{C}$ & -0.6738095 & 5.5601016 & -2.5014716 \\
$\mathrm{H}$ & -0.8590026 & 6.4542325 & -3.1161543 \\
$\mathrm{C}$ & 0.0508753 & 5.6710735 & -1.3019978 \\
$\mathrm{H}$ & 0.4352203 & 6.6492294 & -0.9752764 \\
$\mathrm{C}$ & 0.2837360 & 4.5342055 & -0.5141278 \\
$\mathrm{H}$ & 0.8426552 & 4.6243785 & 0.4292966 \\
$\mathrm{C}$ & 1.8993556 & 1.3751646 & -0.1478042 \\
$\mathrm{C}$ & 2.3719328 & 0.1789444 & 0.4292336 \\
$\mathrm{H}$ & 1.6746540 & -0.4711609 & 0.9767517 \\
$\mathrm{C}$ & 3.7105411 & -0.2013175 & 0.2699452 \\
$\mathrm{H}$ & 4.0615004 & -1.1473048 & 0.7077405 \\
$\mathrm{C}$ & 4.5828171 & 0.6072385 & -0.4788240 \\
$\mathrm{H}$ & 5.6315433 & 0.3039831 & -0.6180878 \\
$\mathrm{C}$ & 4.1106404 & 1.7934078 & -1.0648023 \\
$\mathrm{H}$ & 4.7877884 & 2.4193792 & -1.6654253 \\
$\mathrm{C}$ & 2.7711730 & 2.1818266 & -0.9019806 \\
$\mathrm{H}$ & 2.3998100 & 3.1004163 & -1.3780383 \\
$\mathrm{C}$ & 0.0489128 & 2.4707581 & 1.8001935 \\
$\mathrm{C}$ & 1.0602991 & 2.2469116 & 2.7538855 \\
$\mathrm{H}$ & 1.9791239 & 1.7161587 & 2.4662086 \\
$\mathrm{C}$ & 0.8994002 & 2.7088094 & 4.0714078 \\
$\mathrm{H}$ & 1.6957938 & 2.5312298 & 4.8098261 \\
$\mathrm{C}$ & -0.2658238 & 3.3971011 & 4.4432804 \\
$\mathrm{H}$ & -0.3886201 & 3.7574044 & 5.4757373 \\
$\mathrm{H}$ & -1.2769509 & 3.6244244 & 3.4923404 \\
& -2.1935273 & 4.1622792 & 3.7779941 \\
\hline & -1.1247741 & 3.1592289 & 2.1798613 \\
& & & \\
$\mathrm{H}$ & & & \\
$\mathrm{H}$ & & &
\end{tabular}


75

SCF Energy $=-4548.344978397$

$\begin{array}{lll}\text { C } & 0.6208841 & -6.5735041\end{array}$

C $\quad-0.0351404 \quad-5.4113952$

C $\quad-0.8956761 \quad-5.5495565$

C $\quad-1.0663349-6.7887116$

C $\quad-0.3886612 \quad-7.9261414$

C $\quad 0.4508008 \quad-7.8114479$

$\begin{array}{lll}\text { C } & 0.1804188 & -4.1153359\end{array}$

$\mathrm{Pd} \quad-0.3984707$

$\mathrm{P} \quad-0.1493317$

C $\quad 0.0427673$

$\mathrm{Br} \quad 0.4087863$

C $\quad-0.3346813$

C $\quad 0.5336803$

C 0.1196054

C $\quad-1.1708047$

C $\quad-2.0416248$

C $\quad-1.6307127$

C $\quad 0.6696726$

0.1135940

C $\quad 0.3667967$

C 0.4237333

C $\quad-0.5558061$

C $\quad 1.8721434$

$\mathrm{H} \quad 1.7663573$

$\mathrm{H} \quad 1.0450151$

$\mathrm{H} \quad-0.6690087$

$\mathrm{H} \quad-1.5981951$

$\mathrm{H} \quad-0.3055145$

$\mathrm{H} \quad 2.1652392$

$\mathrm{H} \quad 1.9617195$

$\mathrm{H} \quad 2.5918988$

$\mathrm{H} \quad-0.8484643$

$\mathrm{H} \quad-1.4486336$

$\mathrm{H} \quad-1.7405865$

$\mathrm{H} \quad-0.5237250$

H $\quad 0.9769110$

$\mathrm{H} \quad 1.2578368$

H $\quad-2.3581859$

$\mathrm{H} \quad-3.0490909$

$\mathrm{H} \quad-1.4842072$

H 0.8094359

$\mathrm{H} \quad 1.5325787$

C $\quad-0.2452629$

C 1.5150106
0.1602304

2. 1761903

$-2.8601668$

0.9424962

$-2.4812829$

$-2.7448262$

$-2.5047919$

$-2.0047563$

$-1.7277352$

$-1.9441453$

$-4.0234482$

$-4.9817926$

$-2.5552397$

$-1.7713709$

$-0.5918404$

$-1.2993124$

$-4.2165447$

$-2.1493670$

$-2.5233464$

$-0.9290810$

0.1485132

$-0.4994125$

$-0.8831687$

$-2.1370118$

$-4.9979199$

$-4.6775801$

$-6.8676220$

$-8.8991720$

$-8.6972108$

$-6.5047059$

$-1.8492377$

$-1.3334253$

$-1.8138914$

$-2.7027126$

$-3.1532051$

3. 6351280

2. 2383630
0.9091586

0.4296741

$-0.6889221$

$-1.3185577$

$-0.8463223$

0.2735980

1.0957386

$-0.4176745$

0.4691023

0.5794890

$-2.6781218$

$-0.8037631$

$-1.8939858$

$-3.2062304$

$-3.4698116$

$-2.4128093$

$-1.0774639$

2.5462878

3. 4353911

2.9219390

1.5838398

1. 5196140

1. 2970312

2.5825520

3. 6980899

3. 3236097

1. 7048071

2. 3074803

2.0053618

0.2724771

1. 3934727

3. 2669944

$-1.0620520$

$-2.1850812$

$-1.3426459$

0.6614867

1.8007873

$-0.2591755$

$-2.6100963$

$-4.5060354$

$-4.0393962$

$-1.6854347$

$-0.6389234$

1. 2234325 


$\begin{array}{lrrr}\text { C } & -1.3552854 & 2.6713773 & 1.7663513 \\ \text { C } & -1.1489647 & 3.8427268 & 2.5262832 \\ \text { C } & -2.1161307 & 4.2565917 & 3.4534322 \\ \text { C } & -3.3017370 & 3.5176868 & 3.6140635 \\ \text { C } & -3.5204063 & 2.3660693 & 2.8411587 \\ \text { C } & -2.5498558 & 1.9430581 & 1.9193006 \\ \text { H } & -0.2348496 & 4.4387867 & 2.3845715 \\ \text { H } & -1.9478136 & 5.1671639 & 4.0480680 \\ \text { H } & -4.0610692 & 3.8476050 & 4.3391542 \\ \text { H } & -4.4524905 & 1.7923515 & 2.9551250 \\ \text { H } & -2.7070251 & 1.0472431 & 1.3004363 \\ \text { C } & 0.6472877 & 4.7160503 & -0.5169197 \\ \text { C } & 0.4764955 & 5.8558110 & -1.3210677 \\ \text { C } & -0.5856545 & 5.9204223 & -2.2358398 \\ \text { C } & -1.4794700 & 4.8404964 & -2.3523841 \\ \text { C } & -1.3085543 & 3.6973946 & -1.5619729 \\ \text { H } & 1.4838115 & 4.6651052 & 0.1954052 \\ \text { H } & 1.1822522 & 6.6955479 & -1.2316694 \\ \text { H } & -0.7154248 & 6.8128750 & -2.8666610 \\ \text { H } & -2.3058162 & 4.8830264 & -3.0775606 \\ \text { H } & -1.9833767 & 2.8357621 & -1.6715102 \\ \text { C } & 1.7254989 & 2.2319038 & 2.6158666 \\ \text { C } & 3.0310586 & 2.1249567 & 3.1248915 \\ \text { C } & 4.1240602 & 2.0251805 & 2.2499634 \\ \text { C } & 3.9125135 & 2.0277191 & 0.8587485 \\ \text { C } & 2.6145059 & 2.1251807 & 0.3419900 \\ \text { H } & 0.8714309 & 2.2988273 & 3.3051158 \\ \text { H } & 3.1913205 & 2.1154929 & 4.2135972 \\ \text { H } & 5.1448159 & 1.9379877 & 2.6518904 \\ \text { H } & 4.7658245 & 1.9372957 & 0.1699282 \\ \text { H } & 2.4347422 & 2.0782989 & -0.7452863\end{array}$

S3

75

SCF Energy $=-4548.350703175$

$\begin{array}{lrrr}\text { C } & -2.6152754 & -1.5115865 & -2.1943885 \\ \text { C } & -1.3697556 & -1.0480122 & -2.7309144 \\ \text { C } & -1.4411933 & -0.0075796 & -3.6986017 \\ \text { C } & -2.6494573 & 0.6153744 & -4.0052686 \\ \text { C } & -3.8530321 & 0.2105469 & -3.3905609 \\ \text { C } & -3.8329373 & -0.8737844 & -2.5140134 \\ \text { C } & -0.0757493 & -1.6028639 & -2.2760932 \\ \text { C } & 1.1750528 & -1.0785358 & -2.4842968 \\ \text { C } & 1.5931348 & 0.2595172 & -2.9386432 \\ \text { C } & 0.9639681 & 1.4221632 & -2.4295871 \\ \text { C } & 1.4416468 & 2.6943013 & -2.7601606\end{array}$




$\begin{array}{lrrr}\text { C } & 2.5675656 & 2.8419118 & -3.5903617 \\ \mathrm{C} & 3.2120233 & 1.6995470 & -4.0926411 \\ \mathrm{C} & 2.7319976 & 0.4193247 & -3.7678496 \\ \mathrm{Br} & -2.1816252 & 1.7180861 & -0.3357692 \\ \mathrm{C} & -0.0090273 & -2.8846086 & -1.4030524 \\ \mathrm{C} & -0.4330955 & -4.1374496 & -2.1994775 \\ \mathrm{C} & -0.8584250 & -2.6936963 & -0.1178605 \\ \mathrm{C} & 1.4995875 & -2.9493630 & -1.0252549 \\ \mathrm{C} & 2.2566740 & -2.0418455 & -1.9982159 \\ \mathrm{O} & 3.3446841 & -1.4163847 & -1.3187573 \\ \mathrm{H} & 2.6520115 & -2.6216329 & -2.8725416 \\ \mathrm{H} & 1.6528387 & -2.5196184 & -0.0151756 \\ \mathrm{H} & 1.8837204 & -3.9877073 & -0.9999547 \\ \mathrm{H} & -0.2888184 & -3.0053051 & 0.7746678 \\ \mathrm{H} & -1.8077883 & -3.2727957 & -0.1214546 \\ \mathrm{H} & 0.2275806 & -4.2888721 & -3.0785048 \\ \mathrm{H} & -1.4728759 & -4.0549469 & -2.5732216 \\ \mathrm{H} & -0.3716260 & -5.0423974 & -1.5608999 \\ \mathrm{H} & 3.6513158 & -0.6843991 & -1.8931099 \\ \mathrm{H} & 0.0980492 & 1.3307067 & -1.7541782 \\ \mathrm{H} & 0.9355741 & 3.5766223 & -2.3407343 \\ \mathrm{H} & 2.9449795 & 3.8446821 & -3.8421810 \\ \mathrm{H} & 4.0908203 & 1.8028283 & -4.7475334 \\ \mathrm{H} & 3.2275692 & -0.4698696 & -4.1904296 \\ \mathrm{H} & -0.5239634 & 0.3234929 & -4.1983648 \\ \mathrm{H} & -2.6585148 & 1.4380394 & -4.7361382 \\ \mathrm{H} & -4.7958533 & 0.7276283 & -3.6202773 \\ \mathrm{H} & -4.7638355 & -1.2456873 & -2.0613820 \\ \mathrm{H} & -2.6595741 & -2.4295468 & -1.5986439 \\ \mathrm{Pd} & -1.4350171 & -0.6879395 & -0.0775692 \\ \mathrm{P} & -0.3344428 & -0.2086526 & 1.7896104 \\ \mathrm{C} & -1.3820655 & 0.3893883 & 3.1742117 \\ \mathrm{C} & -2.7445492 & 0.6802750 & 2.9760785 \\ \mathrm{H} & -3.1749155 & 0.5744740 & 1.9688594 \\ \mathrm{C} & -3.5248013 & 1.1306431 & 4.0536054 \\ \mathrm{H} & -4.5894950 & 1.3581872 & 3.8941091 \\ \mathrm{C} & -2.9499519 & 1.2943636 & 5.3239176 \\ \mathrm{H} & -3.5643835 & 1.6483557 & 6.1658384 \\ \mathrm{C} & -1.5881636 & 1.0043236 & 5.5225691 \\ \mathrm{H} & -1.1343047 & 1.1310274 & 6.5171552 \\ \mathrm{C} & -0.8047480 & 0.5479987 & 4.4532910 \\ \mathrm{H} & 0.2586190 & 0.3114817 & 4.6103694 \\ \mathrm{C} & 0.8763507 & 1.1225884 & 1.4532026 \\ & 1.9398308 & 0.8709676 & 0.5617476 \\ \mathrm{H} & 2.0779449 & -0.1120511 & 0.0871287 \\ & & 1.8966407 & 0.2427920\end{array}$




$\begin{array}{rrrr}\text { H } & 3.6600107 & 1.6923464 & -0.4619917 \\ \mathrm{C} & 2.6733767 & 3.1803551 & 0.7852110 \\ \mathrm{H} & 3.3726529 & 3.9872913 & 0.5187505 \\ \mathrm{C} & 1.5987152 & 3.4368605 & 1.6532652 \\ \mathrm{H} & 1.4511376 & 4.4455531 & 2.0677634 \\ \mathrm{C} & 0.6994336 & 2.4138523 & 1.9891262 \\ \mathrm{H} & -0.1511600 & 2.6219881 & 2.6528147 \\ \mathrm{C} & 0.6089732 & -1.5537208 & 2.6204152 \\ \mathrm{C} & 2.0144071 & -1.6124193 & 2.6652618 \\ \mathrm{H} & 2.6167036 & -0.8076385 & 2.2226582 \\ \mathrm{C} & 2.6533775 & -2.7013938 & 3.2832111 \\ \mathrm{H} & 3.7527800 & -2.7373734 & 3.3115800 \\ \mathrm{C} & 1.8981719 & -3.7327212 & 3.8611648 \\ \mathrm{H} & 2.4024509 & -4.5839620 & 4.3429341 \\ \mathrm{C} & 0.4931681 & -3.6747994 & 3.8233308 \\ \mathrm{H} & -0.1063270 & -4.4788746 & 4.2762015 \\ \mathrm{C} & -0.1480339 & -2.5952139 & 3.2030196 \\ \mathrm{H} & -1.2481005 & -2.5528718 & 3.1675897\end{array}$

S4

75

SCF Energy $=-4548.346538392$

$\begin{array}{lrrr}\text { C } & 2.7914445 & 0.5719182 & -1.8438432 \\ \text { C } & 2.4994738 & -0.3264515 & -0.7867639 \\ \text { C } & 1.9507915 & -1.6063146 & -1.1097202 \\ \text { C } & 1.7456207 & -1.9697179 & -2.4605132 \\ \text { C } & 2.0455291 & -1.0677361 & -3.4845069 \\ \text { C } & 2.5612740 & 0.2052989 & -3.1721012 \\ \text { C } & 2.8625481 & -0.0038652 & 0.6148237 \\ \text { C } & 4.1145657 & 0.0492565 & 1.1553137 \\ \text { C } & 5.4113298 & -0.2072856 & 0.5020099 \\ \text { C } & 6.5854497 & 0.4067260 & 1.0064698 \\ \text { C } & 7.8325386 & 0.1722097 & 0.4102870 \\ \text { C } & 7.9401532 & -0.6834073 & -0.6983343 \\ \text { C } & 6.7859650 & -1.3073743 & -1.2025474 \\ \text { C } & 5.5372745 & -1.0745443 & -0.6117277 \\ \text { Br } & -0.9072841 & 0.4914505 & -2.6577459 \\ \text { C } & 1.7667596 & 0.3559065 & 1.6193671 \\ \text { C } & 1.2806492 & 1.8054346 & 1.3654192 \\ \text { C } & 0.5906504 & -0.6272931 & 1.5137673 \\ \text { C } & 2.5366382 & 0.2539313 & 2.9662496 \\ \text { C } & 4.0248526 & 0.4883801 & 2.6171182 \\ \text { O } & 4.4682921 & 1.8420021 & 2.8037207 \\ \text { H } & 4.6954888 & -0.1113382 & 3.2672556 \\ \text { H } & 2.1823112 & 0.9703390 & 3.7332419 \\ \text { H } & 2.4197518 & -0.7714521 & 3.3741310\end{array}$




$\begin{array}{lrrr}\text { H } & 0.9311923 & -1.6739916 & 1.6647987 \\ \mathrm{H} & -0.1544559 & -0.4061009 & 2.3057041 \\ \mathrm{H} & 2.1011770 & 2.5347961 & 1.5214529 \\ \mathrm{H} & 0.4584457 & 2.0641495 & 2.0610306 \\ \mathrm{H} & 0.8955391 & 1.9308125 & 0.3324463 \\ \mathrm{H} & 4.0994849 & 2.3602515 & 2.0624274 \\ \mathrm{H} & 6.4953304 & 1.0961916 & 1.8584335 \\ \mathrm{H} & 8.7293176 & 0.6661461 & 0.8150678 \\ \mathrm{H} & 8.9194769 & -0.8673694 & -1.1658111 \\ \mathrm{H} & 6.8589425 & -1.9894729 & -2.0634860 \\ \mathrm{H} & 4.6512399 & -1.5885197 & -1.0071140 \\ \mathrm{H} & 1.8402225 & -2.3605098 & -0.3180325 \\ \mathrm{H} & 1.3416803 & -2.9651153 & -2.6957909 \\ \mathrm{H} & 1.8625935 & -1.3435879 & -4.5327398 \\ \mathrm{H} & 2.7805128 & 0.9190233 & -3.9794450 \\ \mathrm{H} & 3.2119566 & 1.5573664 & -1.5984006 \\ \mathrm{Pd} & -0.1519743 & -0.4011509 & -0.4214500 \\ \mathrm{P} & -2.1753627 & -0.1839864 & 0.4577490 \\ \mathrm{C} & -2.2471291 & 1.4541059 & 1.2666432 \\ \mathrm{C} & -2.2481384 & 1.6175923 & 2.6654310 \\ \mathrm{H} & -2.3170883 & 0.7406924 & 3.3251495 \\ \mathrm{C} & -2.1455783 & 2.9054078 & 3.2186870 \\ \mathrm{H} & -2.1412330 & 3.0288999 & 4.3121903 \\ \mathrm{C} & -2.0434009 & 4.0274426 & 2.3815585 \\ \mathrm{H} & -1.9599910 & 5.0341775 & 2.8181223 \\ \mathrm{C} & -2.0390225 & 3.8628703 & 0.9839594 \\ \mathrm{H} & -1.9472067 & 4.7390998 & 0.3247133 \\ \mathrm{C} & -2.1315509 & 2.5828550 & 0.4232496 \\ \mathrm{H} & -2.0783787 & 2.4398547 & -0.6691577 \\ \mathrm{C} & -2.6785359 & -1.4327267 & 1.7104015 \\ \mathrm{C} & -3.8555200 & -1.2527466 & 2.4683153 \\ \mathrm{H} & -4.4519935 & -0.3352671 & 2.3524070 \\ \mathrm{C} & -4.2744170 & -2.2502070 & 3.3604115 \\ \mathrm{H} & -5.1893295 & -2.1025128 & 3.9538594 \\ \mathrm{C} & -3.5347982 & -3.4394262 & 3.4875308 \\ \mathrm{H} & -3.8683958 & -4.2222570 & 4.1854279 \\ \mathrm{C} & -2.3775323 & -3.6313853 & 2.7160126 \\ \mathrm{H} & -1.8032091 & -4.5659399 & 2.8034984 \\ \mathrm{C} & -1.9495239 & -2.6307431 & 1.8293283 \\ \mathrm{H} & -1.0491195 & -2.7666022 & 1.2121188 \\ \mathrm{C} & -3.6231442 & -0.2442695 & -0.6670397 \\ \mathrm{C} & -4.7096032 & 0.6379328 & -0.5205836 \\ \mathrm{H} & -4.6695560 & 1.4461461 & 0.2243922 \\ & -5.8408365 & 0.4935897 & -1.3416849 \\ \mathrm{H} & -5.6848735 & 1.1914349 & -1.2328313 \\ & & -0.5321382 & -2.2980159\end{array}$




$\begin{array}{llll}\text { H } & -6.7772363 & -0.6410130 & -2.9421008 \\ \mathrm{C} & -4.8061700 & -1.4157303 & -2.4390958 \\ \mathrm{H} & -4.8377527 & -2.2131982 & -3.1964376 \\ \mathrm{C} & -3.6714227 & -1.2707715 & -1.6315561 \\ \mathrm{H} & -2.8054916 & -1.9368478 & -1.7591043\end{array}$

\section{Optimized Geometries(BXn(TS-de))}

\begin{tabular}{lrrr} 
R3 & \multicolumn{4}{l}{} \\
75 & \multicolumn{4}{l}{} \\
SCF & Energy $=-4548.298413933$ & \\
C & -1.1288940 & 5.0810525 & 0.2205093 \\
C & -0.9161748 & 3.6956040 & 0.0483167 \\
C & -0.9567290 & 3.1482218 & -1.2535840 \\
C & -1.2471107 & 3.9637749 & -2.3553136 \\
C & -1.4958705 & 5.3353987 & -2.1700683 \\
C & -1.4287482 & 5.8973878 & -0.8836968 \\
C & -0.6374732 & 2.8722162 & 1.2346631 \\
C & 0.4521454 & 2.0988834 & 1.4770008 \\
C & 1.6513632 & 1.9924269 & 0.6252610 \\
C & 2.2359545 & 0.7384522 & 0.3278968 \\
C & 3.4012046 & 0.6625844 & -0.4529358 \\
C & 3.9973231 & 1.8349340 & -0.9460991 \\
C & 3.4210792 & 3.0848654 & -0.6582766 \\
C & 2.2598820 & 3.1672562 & 0.1210827 \\
Br & 1.3406936 & 7.0523764 & 1.0151264 \\
C & -1.5659276 & 2.9621323 & 2.4525239 \\
C & -3.0543791 & 2.8814417 & 2.0948998 \\
C & -1.2493256 & 4.2454708 & 3.2200888 \\
C & -1.0777977 & 1.7398785 & 3.2923555 \\
C & 0.3852313 & 1.5088582 & 2.8839516 \\
D & 0.7006495 & 0.1228840 & 2.9912717 \\
H & 1.0816029 & 2.0968117 & 3.5363146 \\
H & -1.2169542 & 1.8722443 & 4.3841191 \\
H & -1.6443593 & 0.8377794 & 2.9807663 \\
H & -0.2845429 & 4.1528370 & 3.7594067 \\
H & -2.0466573 & 4.5259119 & 3.9328682 \\
H & -3.2665238 & 1.9830748 & 1.4821418 \\
H & -3.3751914 & 3.7689747 & 1.5118342 \\
H & -3.6718777 & 2.8325056 & 3.0150980 \\
H & 1.6622373 & 0.0402075 & 2.8456842 \\
H & 1.7430112 & -0.1782880 & 0.6842497 \\
H & 3.8403840 & -0.3195471 & -0.6856388
\end{tabular}




\begin{tabular}{lrrr} 
H & 4.9121421 & 1.7752119 & -1.5557223 \\
$\mathrm{H}$ & 3.8799533 & 4.0100816 & -1.0378318 \\
$\mathrm{H}$ & 1.8259539 & 4.1513701 & 0.3574227 \\
$\mathrm{H}$ & -0.7548409 & 2.0742699 & -1.3830942 \\
$\mathrm{H}$ & -1.2809545 & 3.5284372 & -3.3658777 \\
$\mathrm{H}$ & -1.7307346 & 5.9776520 & -3.0331396 \\
$\mathrm{H}$ & -1.5799844 & 6.9778129 & -0.7468830 \\
$\mathrm{H}$ & -2.1221693 & 5.5590083 & 1.7447602 \\
$\mathrm{Pd}$ & -0.6411613 & 5.8797607 & 2.0533853 \\
$\mathrm{P}$ & -0.1699858 & 7.0474093 & 4.0920250 \\
$\mathrm{C}$ & -0.4068871 & 8.8716360 & 4.0467420 \\
$\mathrm{C}$ & -0.3638085 & 9.5717500 & 2.8242603 \\
$\mathrm{H}$ & -0.1702255 & 9.0204951 & 1.8911412 \\
$\mathrm{C}$ & -0.5303295 & 10.9663543 & 2.8120551 \\
$\mathrm{H}$ & -0.4906482 & 11.5078756 & 1.8549989 \\
$\mathrm{C}$ & -0.7462934 & 11.6663776 & 4.0102382 \\
$\mathrm{H}$ & -0.8787601 & 12.7589372 & 3.9959881 \\
$\mathrm{C}$ & -0.7946740 & 10.9682002 & 5.2294601 \\
$\mathrm{H}$ & -0.9691964 & 11.5094931 & 6.1716821 \\
$\mathrm{C}$ & -0.6269334 & 9.5765484 & 5.2508090 \\
$\mathrm{H}$ & -0.6723471 & 9.0342406 & 6.2066753 \\
$\mathrm{C}$ & 1.5607768 & 6.7939196 & 4.6299284 \\
$\mathrm{C}$ & 1.9815287 & 5.4958042 & 4.9939271 \\
$\mathrm{H}$ & 1.2540913 & 4.6707224 & 5.0424430 \\
$\mathrm{C}$ & 3.3279458 & 5.2500618 & 5.2969857 \\
$\mathrm{H}$ & 3.6474384 & 4.2366846 & 5.5820876 \\
$\mathrm{C}$ & 4.2668827 & 6.2936990 & 5.2260738 \\
$\mathrm{H}$ & 5.3243264 & 6.0978951 & 5.4584200 \\
$\mathrm{C}$ & 3.8533691 & 7.5822373 & 4.8532316 \\
$\mathrm{H}$ & 4.5872361 & 8.3992675 & 4.7870503 \\
$\mathrm{C}$ & 2.5056309 & 7.8351060 & 4.5531240 \\
$\mathrm{H}$ & 2.1875181 & 8.8412990 & 4.2460939 \\
$\mathrm{C}$ & -1.2054477 & 6.5806621 & 5.5378570 \\
$\mathrm{C}$ & -0.6860538 & 6.2948276 & 6.8150254 \\
$\mathrm{H}$ & 0.4005377 & 6.3174569 & 6.9826794 \\
$\mathrm{C}$ & -1.5569057 & 5.9949814 & 7.8768944 \\
$\mathrm{H}$ & -1.1453193 & 5.7786649 & 8.8743354 \\
$\mathrm{C}$ & -2.9453090 & 5.9808532 & 7.6718186 \\
$\mathrm{H}$ & -3.6240458 & 5.7467399 & 8.5056677 \\
$\mathrm{C}$ & -3.4680560 & 6.2678338 & 6.3979817 \\
$\mathrm{H}$ & -4.5556111 & 6.2592378 & 6.2314312 \\
$\mathrm{C}$ & -2.6033170 & 6.5625474 & 5.3357488 \\
$\mathrm{H}$ & -3.0093863 & 6.7858024 & 4.3359525 \\
& & & \\
\hline & & & \\
\hline
\end{tabular}




\begin{tabular}{|c|c|c|c|}
\hline $\mathrm{CF}$ & inergy $=-45$ & 3.295973134 & \\
\hline C & 2.9035519 & -0.1395570 & 0.8163669 \\
\hline & 1.7050964 & 0.4935919 & 0.4088194 \\
\hline & 0.7489788 & -0.2752795 & -0.3000344 \\
\hline & 0.9992583 & -1.6137634 & -0.6234196 \\
\hline & 2.2076830 & -2.2218367 & -0.2368331 \\
\hline & 3.1546170 & -1.4800350 & 0.4876731 \\
\hline & 1.4218574 & 1.8895475 & \\
\hline & -1.3923043 & 5.2865331 & -0.9245534 \\
\hline & -2.4643239 & 7.0862755 & 0.2374659 \\
\hline & 0.8081500 & 2.8841695 & 0.0685210 \\
\hline & -0.8167677 & 6.9710114 & -2.72 \\
\hline & 0.5043408 & 2.9025855 & -1.37 \\
\hline & 1.2737193 & & -2 . \\
\hline & 0.9888474 & & -3 . \\
\hline & -0.0782803 & & -4 . \\
\hline & -0.8054967 & & -3 . \\
\hline & -0.4960914 & & -1 \\
\hline & 971 & & \\
\hline & 1.7483484 & & \\
\hline & 0.8444324 & & \\
\hline & 0.5057564 & & 477 \\
\hline & -0.9875536 & 547 & 949 \\
\hline & 1.3944756 & 415 & 956 \\
\hline & 2.8594301 & 51 & 7704 \\
\hline & 1.2508647 & 900 & 5279 \\
\hline & -0.0689042 & & 2.8 \\
\hline & -1.5863501 & & 050 \\
\hline & -1.2432837 & & 039 \\
\hline & 1.1538409 & & 379 \\
\hline & 1.2602 & & -0 . \\
\hline & 2.4643885 & & 354 \\
\hline & 0.9428653 & 22 & 63973 \\
\hline & -0.1982824 & 0.1978434 & -0.5980193 \\
\hline & 0.2432680 & -2.1911491 & -1.1772899 \\
\hline & 2.4043627 & -3.2745251 & -0.4906270 \\
\hline & 4.0978276 & -1.9505551 & 0.8049048 \\
\hline & 3.6455907 & 0.4281657 & 1.3951088 \\
\hline & -2.1248014 & 3.9396653 & -0.8903744 \\
\hline & -1.5959428 & 4.2642293 & -3.7447843 \\
\hline & -0.3234280 & 2.7207989 & -5.2282209 \\
\hline & 1.6049082 & 1.3938390 & -4.2805108 \\
\hline & 2.1175338 & 1.52705 & -1.8549835 \\
\hline & -3.3100947 & $8.3969^{\circ}$ & -0.7208957 \\
\hline & -1.1657212 & 7.9349679 & 1.2115027 \\
\hline & -3.7515972 & 6.6077240 & 1.463046 \\
\hline
\end{tabular}




$\begin{array}{lrrr}\text { C } & -4.3096245 & 7.5622071 & 2.3408947 \\ \text { C } & -5.3202348 & 7.1874293 & 3.2370741 \\ \text { C } & -5.7896753 & 5.8615370 & 3.2573045 \\ \text { C } & -5.2488936 & 4.9128470 & 2.3754498 \\ \text { C } & -4.2328441 & 5.2847290 & 1.4799090 \\ \text { H } & -3.9539441 & 8.6033312 & 2.3189159 \\ \text { H } & -5.7493941 & 7.9355002 & 3.9206791 \\ \text { H } & -6.5849038 & 5.5705570 & 3.9602040 \\ \text { H } & -5.6197871 & 3.8768631 & 2.3814352 \\ \text { H } & -3.8082713 & 4.5509460 & 0.7787404 \\ \text { C } & -3.2595057 & 9.7531148 & -0.3455582 \\ \text { C } & -3.9840533 & 10.7081070 & -1.0772532 \\ \text { C } & -4.7615565 & 10.3122639 & -2.1773299 \\ \text { C } & -4.8117307 & 8.9580567 & -2.5518012 \\ \text { C } & -4.0838362 & 8.0020472 & -1.8310538 \\ \text { H } & -2.6418348 & 10.0662063 & 0.5093254 \\ \text { H } & -3.9365776 & 11.7684667 & -0.7863894 \\ \text { H } & -5.3254676 & 11.0631182 & -2.7512383 \\ \text { H } & -5.4097803 & 8.6467876 & -3.4212698 \\ \text { H } & -4.0955802 & 6.9457079 & -2.1384489 \\ \text { C } & -1.0104792 & 7.7170591 & 2.5963152 \\ \text { C } & 0.1059676 & 8.2404055 & 3.2699523 \\ \text { C } & 1.0675316 & 8.9855140 & 2.5695254 \\ \text { C } & 0.9141449 & 9.2043003 & 1.1886393 \\ \text { C } & -0.1900064 & 8.6760961 & 0.5063055 \\ \text { H } & -1.7618587 & 7.1372289 & 3.1519345 \\ \text { H } & 0.2218737 & 8.0625423 & 4.3497003 \\ \text { H } & 1.9419444 & 9.3931585 & 3.0988658 \\ \text { H } & 1.6700126 & 9.7790215 & 0.6327428 \\ \text { H } & -0.2864142 & 8.8014284 & -0.5837034\end{array}$

S3

75

SCF Energy $=-4548.301392910$

$\begin{array}{lrrr}\text { C } & -1.2638786 & 4.7790970 & 0.2680791 \\ \text { C } & -1.3040587 & 3.3704567 & 0.0807666 \\ \text { C } & -1.6916152 & 2.8317204 & -1.1574710 \\ \text { C } & -2.0429185 & 3.6876460 & -2.2152524 \\ \text { C } & -1.9767470 & 5.0790401 & -2.0428088 \\ \text { C } & -1.5858518 & 5.6262453 & -0.8054532 \\ \text { C } & -0.6912567 & 2.6212546 & 1.1992169 \\ \text { C } & 0.6301772 & 2.2924131 & 1.2629940 \\ \text { C } & 1.6539365 & 2.3063001 & 0.1946858 \\ \text { C } & 1.7037874 & 3.3104827 & -0.8015606 \\ \text { C } & 2.7169510 & 3.2985885 & -1.7708142 \\ \text { C } & 3.6936330 & 2.2894611 & -1.7716583\end{array}$




$\begin{array}{lrrr}\text { C } & 3.6617567 & 1.2919203 & -0.7821838 \\ \mathrm{C} & 2.6569890 & 1.3066728 & 0.1949929 \\ \mathrm{Br} & 1.4790486 & 6.5588676 & 1.0456168 \\ \mathrm{C} & -1.3768018 & 2.5921213 & 2.5787862 \\ \mathrm{C} & -2.5838644 & 1.6310035 & 2.5639066 \\ \mathrm{C} & -1.8557162 & 4.0027658 & 2.9557045 \\ \mathrm{C} & -0.2307816 & 2.0760204 & 3.4995963 \\ \mathrm{C} & 1.0544781 & 2.3303687 & 2.7192220 \\ \mathrm{O} & 1.4501437 & 3.7136278 & 2.9750519 \\ \mathrm{H} & 1.8991012 & 1.6653083 & 2.9992668 \\ \mathrm{H} & -0.2130597 & 2.5781808 & 4.4869127 \\ \mathrm{H} & -0.3386372 & 0.9862129 & 3.6705162 \\ \mathrm{H} & -1.9015021 & 4.1394678 & 4.0502109 \\ \mathrm{H} & -2.8491227 & 4.2180304 & 2.5192157 \\ \mathrm{H} & -2.2659712 & 0.6015279 & 2.2988479 \\ \mathrm{H} & -3.3373254 & 1.9626373 & 1.8211428 \\ \mathrm{H} & -3.0719136 & 1.6011259 & 3.5601753 \\ \mathrm{H} & 1.9830594 & 4.0503417 & 2.2159651 \\ \mathrm{H} & 0.9732388 & 4.1280611 & -0.7922420 \\ \mathrm{H} & 2.7473707 & 4.0994295 & -2.5247774 \\ \mathrm{H} & 4.4855800 & 2.2848770 & -2.5361013 \\ \mathrm{H} & 4.4252263 & 0.4990311 & -0.7706264 \\ \mathrm{H} & 2.6333228 & 0.5205025 & 0.9657692 \\ \mathrm{H} & -1.6730556 & 1.7405019 & -1.2993748 \\ \mathrm{H} & -2.3443063 & 3.2651654 & -3.1856750 \\ \mathrm{H} & -2.2273979 & 5.7512721 & -2.8778555 \\ \mathrm{H} & -1.5095286 & 6.7172796 & -0.6875845 \\ \mathrm{H} & -1.7400504 & 5.8587915 & 1.5264784 \\ \mathrm{Pd} & -0.4539068 & 5.3177034 & 2.1475423 \\ \mathrm{P} & 0.0073303 & 6.4634036 & 4.2016433 \\ \mathrm{C} & -0.9041635 & 8.0610220 & 4.1753063 \\ \mathrm{C} & -1.0438450 & 8.7261774 & 2.9388467 \\ \mathrm{H} & -0.6277832 & 8.2653494 & 2.0288698 \\ \mathrm{H} & 4.8756929 & 5.6153541 & 4.8374506 \\ \mathrm{C} & -1.6818162 & 9.9747000 & 2.8816004 \\ \mathrm{H} & -1.7836178 & 10.4884514 & 1.9137466 \\ \mathrm{C} & -2.1880572 & 10.5653250 & 4.0515658 \\ \mathrm{H} & -2.6941629 & 11.5415169 & 4.0028252 \\ \mathrm{C} & -2.0428999 & 9.9095366 & 5.2857915 \\ \mathrm{H} & -2.4302131 & 10.3717047 & 6.2065698 \\ \mathrm{C} & -1.3979680 & 8.6645030 & 5.3511419 \\ \mathrm{H} & -1.2754242 & 8.1601256 & 6.3208587 \\ \mathrm{H} & 1.7299596 & 6.9688529 & 4.6075996 \\ & 2.5617900 & 6.0065099 & 4.5603841 \\ \mathrm{H} & 4.0779178 & 6.3756243 & 4.2575524 \\ & & & \\ \mathrm{H} & & \end{array}$




$\begin{array}{lrrr}\text { C } & 4.3818830 & 7.6996447 & 5.2236807 \\ \text { H } & 5.4178382 & 7.9863546 & 5.4602296 \\ \text { C } & 3.3600839 & 8.6614977 & 5.2543115 \\ \text { H } & 3.5908700 & 9.7059398 & 5.5136472 \\ \text { C } & 2.0381946 & 8.3007049 & 4.9494628 \\ \text { H } & 1.2472872 & 9.0632415 & 4.9694769 \\ \text { C } & -0.5976498 & 5.6259546 & 5.7220048 \\ \text { C } & 0.2793968 & 4.9442955 & 6.5895911 \\ \text { H } & 1.3653786 & 5.0104761 & 6.4328508 \\ \text { C } & -0.2276443 & 4.1897478 & 7.6598908 \\ \text { H } & 0.4692366 & 3.6661353 & 8.3318143 \\ \text { C } & -1.6124178 & 4.1087501 & 7.8789987 \\ \text { H } & -2.0062560 & 3.5186916 & 8.7201855 \\ \text { C } & -2.4928382 & 4.7879919 & 7.0199579 \\ \text { H } & -3.5796059 & 4.7330693 & 7.1845811 \\ \mathrm{C} & -1.9916068 & 5.5345937 & 5.9434751 \\ \text { H } & -2.6878582 & 6.0606569 & 5.2726188\end{array}$

S4

75

SCF Energy $=-4548.299601431$

$\begin{array}{lrrr}\text { C } & 1.3763977 & 3.8864895 & -1.7935601 \\ \text { C } & 0.5437194 & 3.9853846 & -0.6460929 \\ \text { C } & -0.6784753 & 4.7000402 & -0.7733934 \\ \text { C } & -1.0983128 & 5.1551904 & -2.0400844 \\ \text { C } & -0.2865041 & 4.9866905 & -3.1709043 \\ \text { C } & 0.9716233 & 4.3740746 & -3.0379181 \\ \text { C } & 0.9922637 & 3.3895395 & 0.6255530 \\ \text { C } & 1.8282084 & 2.3132431 & 0.7949425 \\ \text { C } & 2.3188268 & 1.3392835 & -0.1983990 \\ \text { C } & 3.6460091 & 0.8512224 & -0.1331456 \\ \text { C } & 4.1000708 & -0.1101570 & -1.0489831 \\ \text { C } & 3.2379759 & -0.6103430 & -2.0382088 \\ \text { C } & 1.9105819 & -0.1480230 & -2.0994895 \\ \text { C } & 1.4546222 & 0.8129566 & -1.1893985 \\ \text { Br } & -1.8708252 & 7.7083225 & 0.0428173 \\ \text { C } & 0.5441301 & 3.9686522 & 1.9706381 \\ \text { C } & 1.0581121 & 5.4072280 & 2.1987800 \\ \text { C } & -0.9774973 & 3.8617026 & 2.0907327 \\ \text { C } & 1.1699788 & 2.9773623 & 3.0049357 \\ \text { C } & 2.2689702 & 2.2093639 & 2.2475918 \\ \text { O } & 3.5885757 & 2.7526370 & 2.4230849 \\ \text { H } & 2.3530427 & 1.1588262 & 2.5937474 \\ \text { H } & 1.5722908 & 3.4967600 & 3.8964170 \\ \text { H } & 0.4041688 & 2.2549044 & 3.3561707 \\ \text { H } & -1.3126582 & 2.8323086 & 1.8651667\end{array}$




$\begin{array}{lrrr}\text { H } & -1.3153432 & 4.1265169 & 3.1113728 \\ \mathrm{H} & 2.1661304 & 5.4178333 & 2.2360281 \\ \mathrm{H} & 0.6829041 & 5.8081984 & 3.1612514 \\ \mathrm{H} & 0.7388623 & 6.0968844 & 1.3918382 \\ \mathrm{H} & 3.6168627 & 3.5695476 & 1.8867401 \\ \mathrm{H} & 4.3152216 & 1.2508736 & 0.6431131 \\ \mathrm{H} & 5.1383336 & -0.4711837 & -0.9884325 \\ \mathrm{H} & 3.5952000 & -1.3643071 & -2.7560631 \\ \mathrm{H} & 1.2228213 & -0.5460026 & -2.8613499 \\ \mathrm{H} & 0.4143139 & 1.1670507 & -1.2343759 \\ \mathrm{H} & -2.1831085 & 3.9444924 & 0.2513381 \\ \mathrm{H} & -2.0542721 & 5.6887936 & -2.1353031 \\ \mathrm{H} & -0.6250750 & 5.3645875 & -4.1478530 \\ \mathrm{H} & 1.6405328 & 4.2783979 & -3.9064760 \\ \mathrm{H} & 2.3635604 & 3.4158323 & -1.6894920 \\ \mathrm{Pd} & -1.8027552 & 5.3076956 & 0.8434493 \\ \mathrm{P} & -3.1464321 & 6.0223266 & 2.6950168 \\ \mathrm{C} & -1.9901776 & 6.6613631 & 3.9645128 \\ \mathrm{C} & -1.6240408 & 5.8965766 & 5.0915383 \\ \mathrm{H} & -2.1401958 & 4.9498397 & 5.3076185 \\ \mathrm{C} & -0.5984627 & 6.3432499 & 5.9418576 \\ \mathrm{H} & -0.3159325 & 5.7386954 & 6.8168369 \\ \mathrm{C} & 0.0610409 & 7.5537168 & 5.6769142 \\ \mathrm{H} & 0.8646425 & 7.9009804 & 6.3436645 \\ \mathrm{C} & -0.3040332 & 8.3187050 & 4.5545360 \\ \mathrm{H} & 0.2157047 & 9.2638135 & 4.3369508 \\ \mathrm{C} & -1.3171788 & 7.8748267 & 3.6941097 \\ \mathrm{H} & -1.5703541 & 8.4453948 & 2.7867541 \\ \mathrm{C} & -4.1185723 & 4.7125106 & 3.5476799 \\ \mathrm{C} & -4.7929607 & 4.9816667 & 4.7584707 \\ \mathrm{H} & -4.7219916 & 5.9793817 & 5.2172519 \\ \mathrm{C} & -5.5558029 & 3.9807729 & 5.3758672 \\ \mathrm{H} & -6.0762162 & 4.1954550 & 6.3214668 \\ \mathrm{C} & -5.6607092 & 2.7081086 & 4.7858525 \\ \mathrm{H} & -6.2615500 & 1.9246786 & 5.2720346 \\ \mathrm{C} & -5.0058900 & 2.4411923 & 3.5733779 \\ \mathrm{H} & -5.0933372 & 1.4501625 & 3.1032675 \\ \mathrm{C} & -4.2371513 & 3.4409010 & 2.9551611 \\ \mathrm{H} & -3.7269669 & 3.2444540 & 2.0005614 \\ \mathrm{C} & -4.3945087 & 7.3382299 & 2.4453046 \\ \mathrm{C} & -4.6323670 & 8.3403963 & 3.4053945 \\ \mathrm{H} & -4.0152053 & 8.3912629 & 4.3147554 \\ & -5.6464205 & 9.2877503 & 3.1898448 \\ \mathrm{H} & -5.8248514 & 10.0746991 & 3.9382425 \\ & -7.2159867 & 9.9806117 & 1.8541852\end{array}$




$\begin{array}{llll}\text { C } & -6.1868129 & 8.2337552 & 1.0641729 \\ \text { H } & -6.7864254 & 8.1974501 & 0.1423886 \\ \text { C } & -5.1710321 & 7.2908859 & 1.2698132 \\ \text { H } & -4.9605892 & 6.5248974 & 0.5085248\end{array}$

\section{Optimized Geometries(BXne)}

\begin{tabular}{lrrr} 
R3 & & & \\
75 & \multicolumn{4}{l}{} \\
SCF & Energy $=-4548.305156062$ & \\
C & -1.8169781 & 0.1299533 & -0.4239995 \\
C & -1.5902119 & 0.3858594 & -1.7961403 \\
C & -2.0420486 & 1.6306276 & -2.3066073 \\
C & -2.7207475 & 2.5477618 & -1.4983419 \\
C & -2.9898558 & 2.2346128 & -0.1562425 \\
C & -2.5437562 & 1.0170358 & 0.3824322 \\
C & -0.9877532 & -0.6167852 & -2.6837770 \\
C & -0.1231040 & -0.4353638 & -3.7223059 \\
C & 0.5854724 & 0.7954885 & -4.1092287 \\
C & 0.7383047 & 1.1515492 & -5.4702689 \\
C & 1.4385212 & 2.3139993 & -5.8272147 \\
C & 2.0124406 & 3.1296140 & -4.8376585 \\
C & 1.8912755 & 2.7670756 & -3.4840343 \\
C & 1.1842753 & 1.6134592 & -3.1227994 \\
Br & 1.6587599 & -1.3867512 & -0.6133451 \\
C & -1.4569449 & -2.0776777 & -2.5597247 \\
C & -2.9089223 & -2.1738653 & -3.0876810 \\
C & -1.4196809 & -2.6058434 & -1.1161220 \\
C & -0.4428181 & -2.7977613 & -3.4936389 \\
C & 0.0544797 & -1.7355748 & -4.4950303 \\
O & -0.6565505 & -1.7038383 & -5.7462244 \\
H & 1.1114613 & -1.9049108 & -4.7841462 \\
H & 0.4224480 & -3.1243773 & -2.8832833 \\
H & -0.8684475 & -3.6817243 & -4.0089709 \\
H & -0.6725649 & -3.4136534 & -0.9821739 \\
H & -2.4120855 & -2.9515897 & -0.7754131 \\
H & -2.9848557 & -1.8278700 & -4.1390728 \\
H & -3.5902124 & -1.5466806 & -2.4775559 \\
H & -3.2708356 & -3.2216092 & -3.0495731 \\
H & -1.5517610 & -1.3689761 & -5.5436314 \\
H & 0.2950862 & 0.4992127 & -6.2370668 \\
H & 1.5413997 & 2.5825340 & -6.8899520 \\
H & 2.5677006 & 4.0369575 & -5.1205051
\end{tabular}




$\begin{array}{lrrr}\text { H } & 2.3732518 & 3.3790521 & -2.7055437 \\ \text { H } & 1.1151330 & 1.2979957 & -2.0738376 \\ \text { H } & -1.8553572 & 1.8529730 & -3.3672085 \\ \text { H } & -3.0577766 & 3.5051661 & -1.9226230 \\ \text { H } & -3.5353487 & 2.9403147 & 0.4876145 \\ \text { H } & -2.7285313 & 0.7960447 & 1.4415553 \\ \text { H } & -2.0211002 & -1.5660241 & 1.0833747 \\ \text { Pd } & -0.6790619 & -1.3254496 & 0.3614448 \\ \text { P } & 0.2026479 & -0.0423375 & 2.2482297 \\ \text { C } & 0.0985934 & 1.7378462 & 1.8206620 \\ \text { C } & 0.5917296 & 2.1221576 & 0.5545252 \\ \text { H } & 1.1012422 & 1.3714544 & -0.0704390 \\ \text { C } & 0.3995714 & 3.4301532 & 0.0953394 \\ \text { H } & 0.7555099 & 3.7120294 & -0.9060644 \\ \text { C } & -0.2756333 & 4.3668935 & 0.8981940 \\ \text { H } & -0.4382953 & 5.3908303 & 0.5293519 \\ \text { C } & -0.7492674 & 3.9925033 & 2.1650252 \\ \text { H } & -1.2757183 & 4.7239370 & 2.7969053 \\ \text { C } & -0.5691144 & 2.6780514 & 2.6275449 \\ \text { H } & -0.9653772 & 2.3803718 & 3.6091875 \\ \text { C } & 1.9507301 & -0.3252668 & 2.7112652 \\ \text { C } & 2.3629646 & -1.6547380 & 2.9387920 \\ \text { H } & 1.6473238 & -2.4808041 & 2.8064191 \\ \text { C } & 3.6849702 & -1.9246043 & 3.3140867 \\ \text { H } & 4.0027536 & -2.9638161 & 3.4863242 \\ \text { C } & 4.6076559 & -0.8719128 & 3.4494152 \\ \text { H } & 5.6491816 & -1.0864748 & 3.7327360 \\ \text { C } & 4.2021305 & 0.4508031 & 3.2126478 \\ \text { H } & 4.9234192 & 1.2761738 & 3.3109393 \\ \text { C } & 2.8746344 & 0.7278324 & 2.8455342 \\ \text { H } & 2.5566414 & 1.7633588 & 2.6550505 \\ \text { C } & -0.6800201 & -0.1705214 & 3.8570266 \\ \text { C } & -0.0543173 & 0.2182019 & 5.0613417 \\ \text { H } & 0.9900044 & 0.5645872 & 5.0462937 \\ \text { C } & -0.7604091 & 0.1583563 & 6.2721302 \\ \text { H } & -0.2656983 & 0.4613866 & 7.2073668 \\ \text { C } & -2.0925756 & -0.2903043 & 6.2926682 \\ \text { H } & -2.6427882 & -0.3388045 & 7.2445437 \\ \text { C } & -2.7164119 & -0.6875116 & 5.0986973 \\ \text { H } & -3.7547966 & -1.0514329 & 5.1106536 \\ \text { C } & -2.0106410 & -0.6329934 & 3.8864910 \\ \text { H } & -2.4857714 & -0.9675673 & 2.9498906 \\ \text { R3(TS-eX) } & & & \\ \text { Energy }=-4548.293869515 & \\ & & & \end{array}$




\begin{tabular}{|c|c|c|c|}
\hline & -1.3469473 & -1.1019616 & -0.6612764 \\
\hline & -1.2669476 & -2.4939673 & -0.4735918 \\
\hline & -1.7042685 & -3.3242797 & -1.5381873 \\
\hline & -2.1952841 & -2.7794152 & -2.7287663 \\
\hline & -2.2792563 & -1.3847333 & -2.8814048 \\
\hline & -1.8586987 & -0.5394093 & -1.8394624 \\
\hline & -0.8378605 & -3.0613446 & 0.8069598 \\
\hline & -0.1501031 & -4.2141185 & \\
\hline & 0.5605384 & -5.0584986 & 0.0803807 \\
\hline & 0.5636063 & -6.4686253 & 0.1984542 \\
\hline & 1.2663184 & -7.2568926 & -0.7252703 \\
\hline & 1.9825955 & -6.65 & -1.77 \\
\hline & 2.0030897 & -5.24 & $-1.8 \varepsilon$ \\
\hline & 1.302 & -4. & $-0 . \varsigma$ \\
\hline & 1.864 & -0. & -0.2 \\
\hline & -1.23 & -2 . & 2.0 \\
\hline & -2.76 & -2 . & 2.2 \\
\hline & -0.524 & -0 . & 2.1 \\
\hline & -0.63 & -3 & 3.2 \\
\hline & -0.2237012 & -4.5 & 2.5 \\
\hline & -1.1544771 & -5 & 2.7 \\
\hline & 0.7387729 & -4.9 & 2.9 \\
\hline & 0.2496624 & -2.7 & 3.6 \\
\hline & -1.3671084 & -3.4 & 4.0 \\
\hline & 0.5709153 & -1.1 & $2.1 \varepsilon$ \\
\hline & -0.9238935 & -0.2 & $2.8 \varepsilon$ \\
\hline & -3.2659878 & -3 & 2.21 \\
\hline & -3.1689315 & -1.5 & 1.34 \\
\hline & -3.0276924 & -1.6 & 3.1 \\
\hline & -1.9364458 & -5.4 & 2.2 \\
\hline & -0.0048670 & -6 & 1.0 \\
\hline & 1.2540114 & -8.3 & -0.6 \\
\hline & 2.534 & -7.27222 & -2.4 \\
\hline & 2.5814480 & -4.7677549 & -2.6855185 \\
\hline & 1.3459865 & -3.3574803 & -1.0295987 \\
\hline & -1.6516443 & -4.4148174 & -1.4060475 \\
\hline & -2.5267899 & -3.4462244 & -3.5387044 \\
\hline & -2.6774065 & -0.9415786 & -3.8068275 \\
\hline & -1.9218114 & 0.5514633 & -1.9654196 \\
\hline & -1.8994736 & 0.7272085 & 0.9051969 \\
\hline & -0.4666717 & 0.3002606 & 0.4696958 \\
\hline & 0.0952430 & 2.6489965 & -0.0421157 \\
\hline & -0.2855598 & 2.7572942 & -1.8384928 \\
\hline & 0.4454276 & 1.9291837 & -2.7208301 \\
\hline & 1.2470044 & & -2.3252833 \\
\hline & 0.1140972 & 1.8993240 & -4.081622 \\
\hline
\end{tabular}




$\begin{array}{lrrr}\text { H } & 0.6818755 & 1.2466803 & -4.7615718 \\ \mathrm{C} & -0.9491042 & 2.6789938 & -4.5715007 \\ \mathrm{H} & -1.2133151 & 2.6436765 & -5.6392894 \\ \mathrm{C} & -1.6752254 & 3.5011018 & -3.6945473 \\ \mathrm{H} & -2.5056807 & 4.1163054 & -4.0730101 \\ \mathrm{C} & -1.3453292 & 3.5455909 & -2.3295078 \\ \mathrm{H} & -1.9159540 & 4.1906420 & -1.6455753 \\ \mathrm{C} & 1.8300669 & 3.2082175 & 0.1000661 \\ \mathrm{C} & 2.5722813 & 2.7924029 & 1.2237490 \\ \mathrm{H} & 2.1168162 & 2.1068734 & 1.9540327 \\ \mathrm{C} & 3.8930766 & 3.2308242 & 1.3921436 \\ \mathrm{H} & 4.4712790 & 2.8988340 & 2.2673012 \\ \mathrm{C} & 4.4832710 & 4.0721276 & 0.4334622 \\ \mathrm{H} & 5.5242409 & 4.4057324 & 0.5611260 \\ \mathrm{C} & 3.7503209 & 4.4762582 & -0.6948067 \\ \mathrm{H} & 4.2140881 & 5.1273783 & -1.4512155 \\ \mathrm{C} & 2.4247361 & 4.0461537 & -0.8644853 \\ \mathrm{H} & 1.8519282 & 4.3525460 & -1.7525925 \\ \mathrm{C} & -0.8986106 & 3.9990003 & 0.7092362 \\ \mathrm{C} & -0.3910570 & 5.3076936 & 0.8485298 \\ \mathrm{H} & 0.6377873 & 5.5334248 & 0.5317762 \\ \mathrm{C} & -1.1973263 & 6.3185335 & 1.3945444 \\ \mathrm{H} & -0.7934835 & 7.3363602 & 1.5050640 \\ \mathrm{C} & -2.5128277 & 6.0345413 & 1.7983553 \\ \mathrm{H} & -3.1417252 & 6.8297451 & 2.2264921 \\ \mathrm{C} & -3.0207151 & 4.7312671 & 1.6638108 \\ \mathrm{H} & -4.0474160 & 4.5011575 & 1.9862308 \\ \mathrm{C} & -2.2141475 & 3.7146646 & 1.1305101 \\ \mathrm{H} & -2.5964069 & 2.6847319 & 1.0411614\end{array}$

$\begin{array}{lrrr}\text { R3X } & & & \\ \text { 75 } & & & \\ \text { SCF } & \text { Energy }=-4548.305137061 & \\ \text { C } & -1.2244911 & -0.4910931 & 1.4333816 \\ \text { C } & -1.2440629 & -1.8453310 & 1.0269840 \\ \text { C } & -2.4494141 & -2.3341932 & 0.4593230 \\ \text { C } & -3.5836040 & -1.5244240 & 0.3457499 \\ \text { C } & -3.5523692 & -0.2035042 & 0.8211137 \\ \text { C } & -2.3705412 & 0.3142086 & 1.3749939 \\ \text { C } & -0.1019675 & -2.7402539 & 1.2518826 \\ \text { C } & 0.3727116 & -3.7422640 & 0.4583545 \\ \text { C } & -0.0161295 & -4.0667252 & -0.9238662 \\ \text { C } & -0.1681883 & -5.4099040 & -1.3424843 \\ \text { C } & -0.5143831 & -5.7065313 & -2.6695722 \\ \text { C } & -0.6967272 & -4.6733953 & -3.6040695 \\ \text { C } & -0.5178549 & -3.3368649 & -3.2039597\end{array}$




\begin{tabular}{lrrr} 
C & -0.1826355 & -3.0358737 & -1.8779866 \\
$\mathrm{Br}$ & 2.2866267 & -0.5971334 & -0.0102504 \\
$\mathrm{C}$ & 0.6181793 & -2.6774083 & 2.6109911 \\
$\mathrm{C}$ & -0.3244056 & -3.2569821 & 3.6934814 \\
$\mathrm{C}$ & 1.0209748 & -1.2505368 & 3.0187194 \\
$\mathrm{C}$ & 1.8427411 & -3.5983243 & 2.3433618 \\
$\mathrm{C}$ & 1.4296089 & -4.5486443 & 1.2012150 \\
$\mathrm{O}$ & 0.9121586 & -5.8214081 & 1.6307047 \\
$\mathrm{H}$ & 2.2883941 & -4.8072495 & 0.5492271 \\
$\mathrm{H}$ & 2.6792465 & -2.9694058 & 1.9795559 \\
$\mathrm{H}$ & 2.1803716 & -4.1552504 & 3.2400461 \\
$\mathrm{H}$ & 2.1207302 & -1.1152209 & 3.0471281 \\
$\mathrm{H}$ & 0.5807586 & -0.9565248 & 3.9883694 \\
$\mathrm{H}$ & -0.6247699 & -4.2988002 & 3.4584100 \\
$\mathrm{H}$ & -1.2494919 & -2.6506112 & 3.7731984 \\
$\mathrm{H}$ & 0.1747782 & -3.2643497 & 4.6838538 \\
$\mathrm{H}$ & 0.0477114 & -5.6383972 & 2.0476403 \\
$\mathrm{H}$ & -0.0111764 & -6.2110505 & -0.6052916 \\
$\mathrm{H}$ & -0.6377914 & -6.7561469 & -2.9781048 \\
$\mathrm{H}$ & -0.9608774 & -4.9089991 & -4.6463232 \\
$\mathrm{H}$ & -0.6186081 & -2.5241460 & -3.9403082 \\
$\mathrm{H}$ & -0.0015499 & -2.0006467 & -1.5614930 \\
$\mathrm{H}$ & -2.4744529 & -3.3798363 & 0.1198849 \\
$\mathrm{H}$ & -4.5032521 & -1.9316623 & -0.0999313 \\
$\mathrm{H}$ & -4.4413541 & 0.4406683 & 0.7513032 \\
$\mathrm{H}$ & -2.3451675 & 1.3585943 & 1.7115954 \\
$\mathrm{H}$ & -0.1559064 & 0.9532203 & 2.8334964 \\
$\mathrm{Pd}$ & 0.6092074 & 0.2913757 & 1.6685057 \\
$\mathrm{P}$ & 0.2934343 & 2.2453315 & 0.2317361 \\
$\mathrm{C}$ & -1.0537362 & 1.8653376 & -0.9531385 \\
$\mathrm{C}$ & -0.9812498 & 0.6281418 & -1.6299685 \\
$\mathrm{H}$ & -0.0865645 & -0.0008865 & -1.4981450 \\
$\mathrm{C}$ & -2.0505213 & 0.2007676 & -2.4253774 \\
$\mathrm{H}$ & -2.0009036 & -0.7795435 & -2.9207578 \\
$\mathrm{C}$ & -3.1946427 & 1.0073827 & -2.5603774 \\
$\mathrm{H}$ & -4.0403538 & 0.6633456 & -3.1746906 \\
$\mathrm{C}$ & -3.2607252 & 2.2461298 & -1.9041935 \\
$\mathrm{H}$ & -4.1537384 & 2.8808175 & -2.0098770 \\
$\mathrm{C}$ & -2.1952309 & 2.6761810 & -1.0955758 \\
$\mathrm{H}$ & -2.2617706 & 3.6348220 & -0.5608958 \\
$\mathrm{C}$ & 1.7118471 & 2.7648302 & -0.8018740 \\
$\mathrm{C}$ & 2.9488294 & 2.9794364 & -0.1592744 \\
$\mathrm{H}$ & 3.0414716 & 2.8045828 & 0.9237518 \\
& 4.0612970 & 3.3965253 & -0.9007245 \\
$\mathrm{H}$ & 3.0251069 & 3.5588346 & -0.3954071 \\
\hline & & 3.5869043 & -2.2897606
\end{tabular}




$\begin{array}{rrrr}\text { H } & 4.8286826 & 3.9034230 & -2.8730999 \\ \mathrm{C} & 2.7234812 & 3.3629512 & -2.9327721 \\ \mathrm{H} & 2.6354398 & 3.5043746 & -4.0206319 \\ \mathrm{C} & 1.6019694 & 2.9539680 & -2.1920590 \\ \mathrm{H} & 0.6407795 & 2.7737290 & -2.6953546 \\ \mathrm{C} & -0.2380969 & 3.8164003 & 1.0274878 \\ \mathrm{C} & -0.0854767 & 5.0534597 & 0.3646242 \\ \mathrm{H} & 0.3959030 & 5.0864314 & -0.6242770 \\ \mathrm{C} & -0.5421437 & 6.2353461 & 0.9668966 \\ \mathrm{H} & -0.4185179 & 7.1963164 & 0.4448712 \\ \mathrm{C} & -1.1516456 & 6.1942404 & 2.2330563 \\ \mathrm{H} & -1.5065768 & 7.1234646 & 2.7039203 \\ \mathrm{C} & -1.2965510 & 4.9673665 & 2.9011337 \\ \mathrm{H} & -1.7613660 & 4.9308851 & 3.8978589 \\ \mathrm{C} & -0.8363335 & 3.7838889 & 2.3027134 \\ \mathrm{H} & -0.9239220 & 2.8215109 & 2.8329019\end{array}$

R4

75

SCF Energy $=-4548.298276561$

C $\quad-0.3263803 \quad-6.5809788$

$\begin{array}{lll}\text { C } & -0.7665213 & -5.3170139\end{array}$

C $\quad-1.9906128 \quad-5.2530006$

C $\quad-2.7257049 \quad-6.4124539$

C $\quad-2.2619310 \quad-7.6654521$

$\begin{array}{lll}\text { C } & -1.0626686 & -7.7424359\end{array}$

C $\quad-0.0154644 \quad-4.0914565$

$\begin{array}{lll}\mathrm{Pd} & -0.0333349 & 0.3108517\end{array}$

$\begin{array}{lll}\mathrm{P} & -0.0056722 & 2.5514624\end{array}$

$\begin{array}{lll}\text { C } & 0.2016656 & -2.9736521\end{array}$

$\begin{array}{lll}\text { Br } & 2.0711258 & 0.9099301\end{array}$

$\begin{array}{lll}\text { C } & -0.0215654 & -2.7804811\end{array}$

$\begin{array}{lll}\text { C } & -0.0260040 & -3.9006427\end{array}$

$\begin{array}{lll}\text { C } & -0.2580915 & -3.7595188\end{array}$

$\begin{array}{lll}\text { C } & -0.4828277 & -2.4817644\end{array}$

C $\quad-0.4259824 \quad-1.3566934$

$\begin{array}{lll}\text { C } & -0.1735089 & -1.4926617\end{array}$

$\begin{array}{lll}\text { C } & 0.6310087 & -3.9397045\end{array}$

$\begin{array}{lll}0 & -0.0578170 & -4.5642610\end{array}$

$\begin{array}{lll}\text { C } & 0.7735455 & -2.4082359\end{array}$

$\begin{array}{lll}\text { C } & 0.8503585 & -1.8546946\end{array}$

$\begin{array}{lll}\text { C } & 0.0016218 & -0.5928028\end{array}$

1.1589195

0.6977858

$-0.0133887$

$-0.2857256$

0.1552708

0.8817211

1.0207993

$-0.8185620$

0.1105219

0.2580190

$-2.0992836$

$-1.1844927$

$-2.0605446$

$-3.4299920$

$-3.9714189$

$-3.1342721$

$-1.7553850$

2.3966780

3.4676790

2.5384232

1.0776907

1.0502457

0.6602623

$2.3148186-1.6186155$

2.3926308

3.1537770

$1.6379517-2.0928870$

3.0478845 


\begin{tabular}{|c|c|c|c|}
\hline & -1.0243186 & -0.8139339 & 1.3949784 \\
\hline & 0.4431398 & 0.2187927 & 1.6622222 \\
\hline & 2.7560747 & -0.7879477 & 1.2481252 \\
\hline & 2.3951849 & -1.3523282 & -0.4108832 \\
\hline & 2.9152142 & -2.5328455 & 0.8388931 \\
\hline & -1.0113102 & -4.4168905 & 3.3142335 \\
\hline & -2.3570910 & -4.2733266 & -0.3535565 \\
\hline & -3.6726465 & -6.3389587 & -0.8421406 \\
\hline & -2.8404665 & -8.5770709 & -0.0580290 \\
\hline & -0.6972455 & -8.7167540 & 1.2406977 \\
\hline & 0.6039036 & -6.6512467 & 1.7397799 \\
\hline & -1.5132232 & 0.0769397 & -0.4789370 \\
\hline & -0.5435357 & -0.3553962 & -3.5773040 \\
\hline & -0.6662898 & -2.3535425 & -5.0493725 \\
\hline & -0.2524651 & -4.6471977 & -4.0803331 \\
\hline & 0.1684707 & -4.8980957 & -1.6435926 \\
\hline & 0.5033041 & 3.8962308 & -1.0196011 \\
\hline & 1.1758020 & 2.6264398 & 1.515556 \\
\hline & -1.5800696 & 3.1684088 & 0.8361126 \\
\hline & -1.8718275 & 4.5471286 & 0.8994521 \\
\hline & -3.0626449 & 4.9877078 & 1.4973367 \\
\hline & -3.9678449 & 4.0595171 & 2.0391403 \\
\hline & -3.6830133 & 2.6852394 & 1.9759047 \\
\hline & -2.4974729 & 2.2407246 & 1.3710636 \\
\hline & -1.1650938 & 5.2753893 & 0.4749126 \\
\hline & -3.2855562 & 6.0646720 & 1.5382429 \\
\hline & -4.9015527 & 4.4079771 & 2.5059024 \\
\hline & -4.3920113 & 1.9533004 & 2.3911627 \\
\hline & -2.2807791 & 1.1642676 & 1.3060976 \\
\hline & 1.3734141 & 4.9278844 & -0.6185692 \\
\hline & 1.7057197 & 5.9521147 & -1.5204951 \\
\hline & 1.1693457 & 5.9511769 & -2.8180228 \\
\hline & 0.3007192 & 4.9207771 & -3.2190178 \\
\hline & -0.0269847 & 3.8922147 & -2.3261178 \\
\hline & 1.7974016 & 4.9243610 & 0.3963325 \\
\hline & 2.3912327 & 6.7537013 & -1.2063418 \\
\hline & 1.4351962 & 6.7522811 & -3.5241553 \\
\hline & -0.1110901 & 4.9104237 & -4.2392222 \\
\hline & -0.6850632 & 3.0699898 & -2.646489 \\
\hline & 0.7639791 & 2.9483423 & 2.8245779 \\
\hline & 1.6878816 & 2.9005250 & 3.8820557 \\
\hline & 3.0215790 & 2.5356584 & 3.6392578 \\
\hline & 3.4335936 & 2.2164229 & 2.332897 \\
\hline & 2.5176889 & 2.2536407 & 1.2724988 \\
\hline & -0.2798890 & 3.2346090 & 3.0187615 \\
\hline & 1.3599354 & 3.1511157 & 4.902283 \\
\hline
\end{tabular}




\begin{tabular}{lrrr} 
H & 3.7422280 & 2.4970540 & 4.4701584 \\
H & 4.4770237 & 1.9267080 & 2.1368958 \\
H & 2.8264763 & 1.9780114 & 0.2502628 \\
& & & \\
S3 & & & \\
75 & \multicolumn{3}{c}{} \\
SCF & Energy $=-4548.305956452$ & \\
C & -1.5632412 & -1.7695660 & -1.3238376 \\
C & -1.8702445 & -2.8655888 & -0.4786208 \\
C & -2.4429384 & -4.0373307 & -0.9994339 \\
C & -2.7164222 & -4.1219174 & -2.3753212 \\
C & -2.4022815 & -3.0445572 & -3.2192364 \\
C & -1.8267241 & -1.8680328 & -2.6982313 \\
C & -1.3763590 & -2.6344911 & 0.8948526 \\
C & -0.1164351 & -2.9383754 & 1.3165000 \\
C & 0.8921777 & -3.8334587 & 0.7070912 \\
C & 1.0689957 & -3.9441069 & -0.6929608 \\
C & 2.0699058 & -4.7743432 & -1.2175740 \\
C & 2.9076818 & -5.5110969 & -0.3646605 \\
C & 2.7488142 & -5.4033924 & 1.0275329 \\
C & 1.7566995 & -4.5663252 & 1.5561744 \\
Br & 1.4114961 & -0.3314527 & -1.8681285 \\
C & -2.0377154 & -1.5314565 & 1.7447253 \\
C & -3.3706262 & -2.0290236 & 2.3412211 \\
C & -2.3112243 & -0.2915899 & 0.8749581 \\
C & -0.9619200 & -1.2771219 & 2.8422225 \\
C & 0.3275817 & -1.8348889 & 2.2536626 \\
O & 0.8710493 & -0.7969779 & 1.3678542 \\
H & 1.1082778 & -2.0941472 & 2.9986050 \\
H & -0.8612196 & -0.2067736 & 3.1100837 \\
H & -1.2088291 & -1.8401761 & 3.7642837 \\
H & -2.3846435 & 0.6235984 & 1.4897827 \\
H & -3.2360873 & -0.4153757 & 0.2825993 \\
H & -3.2074369 & -2.9183107 & 2.9843683 \\
H & -4.0767119 & -2.3103213 & 1.5341113 \\
H & -3.8457252 & -1.2354077 & 2.9545839 \\
H & 1.4786050 & -1.2028047 & 0.7012030 \\
H & 0.4461954 & -3.3489132 & -1.3716661 \\
H & 2.2017394 & -4.8323738 & -2.3084321 \\
H & 3.6905972 & -6.1619745 & -0.7829928 \\
H & 3.4026692 & -5.9736141 & 1.7050855 \\
H & 1.6323455 & -4.4884715 & 2.6477676 \\
H & -2.6423144 & -4.8880339 & -0.3301348 \\
H & -3.1617053 & -5.0381798 & -2.7917042 \\
H & -2.6018923 & -3.1161454 & -4.2998998 \\
H & -1.5587814 & -1.0440287 & -3.3754270
\end{tabular}




\begin{tabular}{|c|c|c|c|}
\hline & -1.7034775 & 0.5187433 & -1.3429188 \\
\hline $\mathrm{Pd}$ & -0.6936955 & -0.1142474 & -0.4221539 \\
\hline P & 0.0523508 & 2.1027836 & 0.2463914 \\
\hline & -0.5642174 & 3.3231897 & -0.9824588 \\
\hline & -0.5463003 & 2.9512797 & -2.3442711 \\
\hline & -0.1892078 & 1.9479098 & -2.6272818 \\
\hline & -0.9533337 & 3.8667005 & -3.3267036 \\
\hline & -0.9336059 & 3.5699861 & -4.3861766 \\
\hline & -1.3837977 & 5.1531843 & -2.9607643 \\
\hline & -1.7095324 & 5.8667694 & -3.7328229 \\
\hline & -1.3926798 & 5.5294727 & -1.6071267 \\
\hline & -1.7205657 & 6.5390182 & -1.3160131 \\
\hline & -0.9777850 & 4.6218003 & -0.6197099 \\
\hline & -0.9730139 & 4.9 & 0.4374755 \\
\hline & 1.8436424 & 2.5 & 0.36 \\
\hline & 2.702 & 1.6 & 1.1 \\
\hline & 2.294 & 0.7 & 1.5 \\
\hline & 4.066 & $1.9^{\circ}$ & 1.2 \\
\hline & 4.727 & 1.3 & 1.7 \\
\hline & 4.5913 & 3.1 & 0.5 \\
\hline & 5.6644217 & 3.340 & 0.6541916 \\
\hline & 3.7424176 & 3.9421828 & -0.1683935 \\
\hline & 4.1463201 & 4.8305167 & -0.6773400 \\
\hline & 2.3740605 & 3.6476712 & -0.2739363 \\
\hline & 1.7204312 & 4.3040643 & -0.8649775 \\
\hline & -0.6628686 & 2.6488154 & 1.8467224 \\
\hline & 0.0993121 & 2.6506929 & 3.0327278 \\
\hline & 1.1800282 & 2.4542296 & 2.9889114 \\
\hline & -0.5144177 & 2.91 & 4.2685281 \\
\hline & 0.0935154 & 2.91 & 5.1859597 \\
\hline & -1.8920490 & 3.17 & 4.3359765 \\
\hline & -2.3694927 & 3.3809038 & 5.3058444 \\
\hline & -2.6573143 & 3.1803128 & 3.1574753 \\
\hline & -3.7368384 & 3.3900695 & 3.1991365 \\
\hline & -2.0509740 & 2.9102975 & 1.9217601 \\
\hline & -2.6585837 & 2.9116601 & 1.0039407 \\
\hline \\
\hline \multicolumn{4}{|l|}{5} \\
\hline \multicolumn{4}{|l|}{ SCF } \\
\hline & 3.9918152 & 0.3766472 & -1.8903994 \\
\hline & 2.8334422 & 0.2313909 & -1.079807 \\
\hline & 1.5673339 & 0.2448154 & -1.7145087 \\
\hline & 1.4841654 & 0.3180468 & -3.118569 \\
\hline & 2.6436368 & 0.4117292 & -3.904345 \\
\hline & 3.9030868 & 0.4597557 & -3.281622 \\
\hline
\end{tabular}




$\begin{array}{lrrr}\text { C } & 2.9667063 & 0.1357014 & 0.3824488 \\ \text { C } & 4.0183049 & -0.3509961 & 1.1170863 \\ \text { C } & 5.1957381 & -1.1170429 & 0.6681930 \\ \text { C } & 6.4646334 & -0.8999196 & 1.2573870 \\ \text { C } & 7.5759547 & -1.6600859 & 0.8623150 \\ \text { C } & 7.4417750 & -2.6559021 & -0.1186830 \\ \text { C } & 6.1816152 & -2.8938279 & -0.6973359 \\ \text { C } & 5.0707938 & -2.1364415 & -0.3072750 \\ \text { Br } & -0.8777635 & 2.4779271 & -1.6501381 \\ \mathrm{C} & 1.8479869 & 0.6732372 & 1.2762888 \\ \mathrm{C} & 1.6418363 & 2.1909394 & 1.1127605 \\ \mathrm{C} & 0.5736950 & -0.1286716 & 1.0602143 \\ \mathrm{C} & 2.3530469 & 0.3188384 & 2.7183756 \\ \mathrm{C} & 3.8626152 & 0.0344594 & 2.5803301 \\ \mathrm{O} & 4.6947647 & 1.1588139 & 2.9089072 \\ \mathrm{H} & 4.1946021 & -0.7671781 & 3.2717694 \\ \mathrm{H} & 2.1565360 & 1.1338095 & 3.4414748 \\ \mathrm{H} & 1.8473512 & -0.5925138 & 3.0994310 \\ \mathrm{H} & 0.7584734 & -1.2030686 & 1.2376474 \\ \mathrm{H} & -0.2586203 & 0.2290521 & 1.6978435 \\ \mathrm{H} & 2.5520359 & 2.7370370 & 1.4321646 \\ \mathrm{H} & 0.7962339 & 2.5357224 & 1.7423763 \\ \mathrm{H} & 1.4147536 & 2.4652990 & 0.0651242 \\ \mathrm{H} & 4.6173091 & 1.7805969 & 2.1582254 \\ \mathrm{H} & 6.5621979 & -0.1139533 & 2.0207985 \\ \mathrm{H} & 8.5565845 & -1.4709041 & 1.3255441 \\ \mathrm{H} & 8.3142862 & -3.2512255 & -0.4281176 \\ \mathrm{H} & 6.0634993 & -3.6826497 & -1.4560595 \\ \mathrm{H} & 4.0850026 & -2.3303924 & -0.7549218 \\ \mathrm{H} & 0.0196282 & -1.3477458 & -0.7709208 \\ \mathrm{H} & 0.5002402 & 0.3362464 & -3.6123289 \\ \mathrm{H} & 2.5576815 & 0.4797482 & -4.9998249 \\ \mathrm{H} & 4.8175612 & 0.5744735 & -3.8828608 \\ \mathrm{H} & 4.9749274 & 0.4326411 & -1.4027165 \\ \mathrm{Pd} & -0.2602943 & 0.1627392 & -0.8217188 \\ \mathrm{P} & -2.5265826 & -0.0572432 & 0.0162144 \\ \mathrm{C} & -2.6934962 & 0.8098613 & 1.6274319 \\ \mathrm{C} & -3.0585753 & 0.1323972 & 2.8083251 \\ \mathrm{H} & -3.3226374 & -0.9344189 & 2.7718504 \\ \mathrm{C} & -3.0817033 & 0.8206507 & 4.0330603 \\ \mathrm{H} & -3.3658920 & 0.2857345 & 4.9519332 \\ \mathrm{H} & -2.7445480 & 2.1824178 & 4.0850379 \\ & -2.7614628 & 2.7179397 & 5.0463506 \\ \mathrm{C} & -2.3816487 & 2.8594372 & 2.9067958 \\ & -2.3484235 & 2.1799515 & 1.6812562\end{array}$




$\begin{array}{rrr}-2.0389499 & 2.6970110 & 0.7575350 \\ -3.1160368 & -1.7616967 & 0.3798861 \\ -4.4886727 & -2.0865537 & 0.3515854 \\ -5.2258951 & -1.3207957 & 0.0678968 \\ -4.9114947 & -3.3839411 & 0.6791531 \\ -5.9835500 & -3.6310287 & 0.6505559 \\ -3.9714500 & -4.3644725 & 1.0394056 \\ -4.3059046 & -5.3814837 & 1.2942105 \\ -2.6032193 & -4.0468833 & 1.0648414 \\ -1.8619233 & -4.8130692 & 1.3373938 \\ -2.1763623 & -2.7526313 & 0.7303565 \\ -1.1041039 & -2.5067777 & 0.7328687 \\ -3.8454563 & 0.6358208 & -1.0448882 \\ -4.9103476 & 1.3961627 & -0.5251688 \\ -4.9524793 & 1.6173826 & 0.5514685 \\ -5.9097287 & 1.8791840 & -1.3859861 \\ -6.7373674 & 2.4787290 & -0.9777394 \\ -5.8510283 & 1.6020621 & -2.7610552 \\ -6.6328847 & 1.9855932 & -3.4338506 \\ -4.7874964 & 0.8429582 & -3.2805408 \\ -4.7323251 & 0.6345378 & -4.3594528 \\ -3.7836125 & 0.3662504 & -2.4276248 \\ -2.9364375 & -0.2059979 & -2.8360046\end{array}$

\section{Optimized Geometries(BXn(TS-df))}

$\begin{array}{lrrr}\text { R3 } & & & \\ 75 & & & \\ \text { SCF } & \text { Energy }=-4548.291180302 & \\ \text { C } & -1.6782502 & 4.6480355 & 1.8948365 \\ \text { C } & -0.8650336 & 4.2193285 & 0.8130627 \\ \text { C } & -0.7782049 & 5.0280407 & -0.3420525 \\ \text { C } & -1.5282744 & 6.2056211 & -0.4416908 \\ \text { C } & -2.4017942 & 6.5711684 & 0.5971424 \\ \text { C } & -2.4942262 & 5.7881773 & 1.7599722 \\ \text { C } & -0.3128997 & 2.8766497 & 0.9405318 \\ \text { C } & 0.8615912 & 2.2786615 & 0.6021886 \\ \text { C } & 2.0407765 & 2.8776226 & -0.0387624 \\ \text { C } & 2.8795574 & 2.0981336 & -0.8710029 \\ \text { C } & 4.0193491 & 2.6617182 & -1.4640747 \\ \text { C } & 4.3500448 & 4.0067193 & -1.2298275 \\ \text { C } & 3.5352386 & 4.7839942 & -0.3863065 \\ \text { C } & 2.3951184 & 4.2265328 & 0.2040645\end{array}$




$\begin{array}{lrrr}\text { Br } & 1.3919320 & 3.5731792 & 3.7588012 \\ \mathrm{C} & -1.2814048 & 1.9305437 & 1.6588236 \\ \mathrm{C} & -2.3579829 & 1.4413351 & 0.6606429 \\ \mathrm{C} & -1.9877746 & 2.6998561 & 2.7817321 \\ \mathrm{C} & -0.3253327 & 0.7881296 & 2.0847534 \\ \mathrm{C} & 0.8393251 & 0.8227818 & 1.0597130 \\ \mathrm{O} & 0.7027379 & -0.0934886 & -0.0416107 \\ \mathrm{H} & 1.7994256 & 0.5412525 & 1.5383042 \\ \mathrm{H} & 0.0926069 & 1.0239922 & 3.0835576 \\ \mathrm{H} & -0.8057213 & -0.2096915 & 2.1186545 \\ \mathrm{H} & -1.7628448 & 2.2797322 & 3.7954961 \\ \mathrm{H} & -3.0806013 & 2.7775491 & 2.6690382 \\ \mathrm{H} & -1.9009255 & 0.8661342 & -0.1689512 \\ \mathrm{H} & -2.9093412 & 2.2976085 & 0.2218810 \\ \mathrm{H} & -3.0868527 & 0.7765976 & 1.1684739 \\ \mathrm{H} & 0.0354537 & 0.2931399 & -0.6405345 \\ \mathrm{H} & 2.6118218 & 1.0461155 & -1.0500086 \\ \mathrm{H} & 4.6573545 & 2.0426263 & -2.1135630 \\ \mathrm{H} & 5.2475295 & 4.4457389 & -1.6917739 \\ \mathrm{H} & 3.8048861 & 5.8299340 & -0.1712607 \\ \mathrm{H} & 1.7796068 & 4.8162863 & 0.8950989 \\ \mathrm{H} & -0.1336265 & 4.6986517 & -1.1701211 \\ \mathrm{H} & -1.4542842 & 6.8289188 & -1.3452141 \\ \mathrm{H} & -3.0089726 & 7.4852116 & 0.5171909 \\ \mathrm{H} & -3.1621218 & 6.1033584 & 2.5721157 \\ \mathrm{H} & -2.4598218 & 4.7866521 & 4.2801511 \\ \mathrm{Pd} & -1.0294234 & 4.3510625 & 3.8683603 \\ \mathrm{P} & -0.3445988 & 6.2914547 & 4.9239600 \\ \mathrm{C} & -0.0837702 & 7.4280484 & 3.5050987 \\ \mathrm{C} & 0.7963794 & 7.0089538 & 2.4821617 \\ \mathrm{H} & 1.3413273 & 6.0573743 & 2.5976180 \\ \mathrm{C} & 0.9465742 & 7.7887319 & 1.3296428 \\ \mathrm{H} & 1.6200748 & 7.4487407 & 0.5289683 \\ \mathrm{C} & 0.2212903 & 8.9848656 & 1.1846507 \\ \mathrm{H} & 0.3321887 & 9.5886291 & 0.2712581 \\ \mathrm{C} & -0.6515737 & 9.4020363 & 2.2008850 \\ \mathrm{H} & -1.2221896 & 10.3367380 & 2.0909268 \\ \mathrm{C} & -0.8086624 & 8.6260010 & 3.3619054 \\ \mathrm{H} & -1.5003505 & 8.9512476 & 4.1524615 \\ \mathrm{C} & 1.2240706 & 6.3014613 & 5.8625498 \\ \mathrm{C} & 1.4114701 & 5.3119521 & 6.8489088 \\ \mathrm{H} & 0.6477127 & 4.5327853 & 6.9923102 \\ \mathrm{C} & 2.5787453 & 5.3097818 & 7.6229228 \\ & 2.7251387 & 4.5327275 & 8.3879268 \\ \mathrm{C} & 3.5703184 & 6.2831285 & 7.4060384 \\ & & 6.2718668 & 8.0066361\end{array}$




$\begin{array}{lrrr}\text { C } & 3.3898175 & 7.2614117 & 6.4156667 \\ \text { H } & 4.1679291 & 8.0191458 & 6.2381924 \\ \text { C } & 2.2168354 & 7.2749078 & 5.6428166 \\ \text { H } & 2.0762428 & 8.0365756 & 4.8618167 \\ \text { C } & -1.5064892 & 7.2064588 & 6.0196988 \\ \text { C } & -1.0186497 & 8.1794558 & 6.9188033 \\ \text { H } & 0.0644008 & 8.3496301 & 7.0071166 \\ \text { C } & -1.9130884 & 8.9266049 & 7.7001146 \\ \text { H } & -1.5241145 & 9.6804477 & 8.4012176 \\ \text { C } & -3.2975625 & 8.7128310 & 7.5908858 \\ \text { H } & -3.9963529 & 9.2987557 & 8.2069491 \\ \text { C } & -3.7869985 & 7.7421054 & 6.7012720 \\ \text { H } & -4.8694047 & 7.5623711 & 6.6181820 \\ \text { C } & -2.8942485 & 6.9885452 & 5.9242149 \\ \text { H } & -3.2682472 & 6.2103344 & 5.2387588\end{array}$

R4

75

SCF Energy $=-4548.270695756$

$\begin{array}{lrrr}\text { C } & 4.2163593 & 0.9612720 & -0.2210436 \\ \text { C } & 2.8037558 & 0.9031780 & -0.1981911 \\ \text { C } & 2.1662624 & -0.1908686 & -0.8294999 \\ \text { C } & 2.9148839 & -1.1742739 & -1.4915711 \\ \text { C } & 4.3171392 & -1.0887873 & -1.5298797 \\ \text { C } & 4.9640972 & -0.0192819 & -0.8883504 \\ \text { C } & 2.0270257 & 1.9081491 & 0.5546616 \\ \text { Pd } & -1.9535198 & 4.9838007 & -0.1272497 \\ \text { P } & -2.7755323 & 7.0640996 & 0.4195671 \\ \text { C } & 0.9367646 & 2.6492516 & 0.1929109 \\ \text { Br } & -0.2890865 & 6.1064739 & -1.7276074 \\ \text { C } & 0.0738662 & 2.6387925 & -0.9851535 \\ \text { C } & 0.3926832 & 2.0409929 & -2.2256294 \\ \text { C } & -0.5986712 & 1.8151612 & -3.1888485 \\ \text { C } & -1.9404427 & 2.1219506 & -2.9060736 \\ \text { C } & -2.2775554 & 2.7492530 & -1.6912772 \\ \text { C } & -1.2527023 & 3.1146265 & -0.7970408 \\ \text { C } & 2.3374710 & 2.1332235 & 2.0412773 \\ \text { O } & 2.8433399 & 0.9972733 & 2.7159255 \\ \text { C } & 0.9805497 & 2.6585283 & 2.5749773 \\ \text { C } & 0.4086340 & 3.4638024 & 1.3738465 \\ \text { C } & -1.1104069 & 3.4679286 & 1.3211276 \\ \text { C } & 0.9951691 & 4.8931266 & 1.4109168 \\ \text { H } & 3.1246438 & 2.9113259 & 2.1705156 \\ \text { H } & 1.0700342 & 3.2550454 & 3.5045271 \\ \text { H } & 0.3439915 & 1.7720393 & 2.7860059 \\ \text { H } & -1.5628961 & 2.4692941 & 1.4491400\end{array}$




\begin{tabular}{|c|c|c|c|}
\hline & -1.5511395 & 4.1597372 & 2.0655776 \\
\hline & 0.5479935 & 5.4722111 & 2.2448145 \\
\hline & 0.8035110 & 5.4347457 & 0.4662673 \\
\hline & 2.0915757 & 4.8600472 & 1.5650518 \\
\hline & 2.3183916 & 0.2301952 & 2.4144951 \\
\hline & 1.0692690 & -0.2648380 & -0.7913808 \\
\hline & 2.3993985 & -2.0175613 & -1.9763231 \\
\hline & 4.9050789 & -1.8598348 & -2.0504729 \\
\hline & 6.0625350 & 0.0495344 & -0.9039349 \\
\hline & 4.7285592 & 1.7910652 & 0.2877159 \\
\hline & -3.2274072 & 4.4385495 & 0.5746534 \\
\hline & -3.3277546 & 3.0059563 & -1.4856712 \\
\hline & -2.7323419 & 1.8895105 & -3.6344441 \\
\hline & -0.3285563 & 1.3560620 & -4.1515732 \\
\hline & 1.4290786 & 1.73 & -2.4193945 \\
\hline & -2.9199925 & 8.2918986 & -0.9269448 \\
\hline & -1.5699271 & 7.7609581 & 1.618306 \\
\hline & -4.3832413 & 7.1938102 & 1.3177683 \\
\hline & -5.0872192 & 8.4159779 & 1.3615528 \\
\hline & -6.2714860 & 8.5216857 & 2.1062472 \\
\hline & -6.7618768 & 7.4142369 & 2.8186026 \\
\hline & -6.0667899 & 6.1947938 & 2.7766887 \\
\hline & -4.8867318 & 6.0846709 & 2.0245944 \\
\hline & -4.7129588 & 9.2863670 & 0.8038130 \\
\hline & -6.8166030 & 9.4774922 & 2.127317 \\
\hline & -7.6918379 & 7.5001258 & 3.4008192 \\
\hline & -6.4484617 & 5.3201204 & 3.3246513 \\
\hline & -4.3473211 & 5.1252237 & 1.9707605 \\
\hline & -2.6487153 & 9.6595342 & -0.7227380 \\
\hline & -2.8204124 & 10.5709708 & -1.7772628 \\
\hline & -3.2640571 & 10.1215871 & -3.0315414 \\
\hline & -3.5294922 & 8.7564405 & -3.2364247 \\
\hline & -3.3507677 & 7.8408538 & -2.1907453 \\
\hline & -2.2880699 & 10.0095449 & 0.2559810 \\
\hline & -2.6006560 & 11.6374407 & -1.6175935 \\
\hline & -3.3927671 & 10.8370527 & -3.8578481 \\
\hline & -3.8593229 & 8.3994397 & -4.2234196 \\
\hline & -3.5196408 & 6.7659691 & -2.3544431 \\
\hline & -1.8362253 & 7.7354633 & 3.0025924 \\
\hline & -0.8512464 & 8.1498262 & 3.9137589 \\
\hline & 0.3967870 & 8.5961629 & 3.4497071 \\
\hline & 0.6604357 & 8.6242467 & 2.068975 \\
\hline & -0.3119370 & 8.2019393 & 1.1522964 \\
\hline & -2.8148598 & 7.3933689 & 3.370027 \\
\hline & -1.0645266 & 8.1264026 & 4.9932131 \\
\hline & 1.1673233 & 8.9201229 & 4.165666 \\
\hline
\end{tabular}




\begin{tabular}{|c|c|c|c|}
\hline H & 1.6397443 & 8.9640561 & 1.6996499 \\
\hline 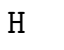 & -0.0897618 & 8.1674877 & 0.0741698 \\
\hline \multicolumn{4}{|l|}{3} \\
\hline \multicolumn{4}{|l|}{5} \\
\hline \multicolumn{4}{|c|}{-4548.267382916} \\
\hline & -1.7776425 & 4.3857301 & 0.7303083 \\
\hline & -1.4182078 & 3.0915475 & 0.2437730 \\
\hline & -1.7564745 & 2.6860694 & -1.0535469 \\
\hline & -2.4918574 & 3.5555401 & -1.8792792 \\
\hline & -2.9050437 & 4.8079082 & -1.3896227 \\
\hline & -2.5728471 & 5.2190377 & -0.0878237 \\
\hline & -0.7285216 & 2.3893070 & 1.3314190 \\
\hline & 0.5923727 & 2.1100943 & 1.5205021 \\
\hline & 1.6830263 & 1.9806857 & 0.5352451 \\
\hline & 1.6706002 & 2.6693265 & -0.7009318 \\
\hline & 2.7382607 & 2.5389892 & $-1.5 s$ \\
\hline & 3.837 & 1.72 & -1.2 \\
\hline & 3.868 & 1.03 & -0.0 \\
\hline $\mathrm{C}$ & 2.8042531 & 1.17 & 7989 \\
\hline $\mathrm{Br}$ & 1.616 & 5.95 & 3747 \\
\hline $\mathrm{C}$ & -1.5126285 & 2.5528021 & 1543 \\
\hline $\mathrm{C}$ & -2.6588776 & 1.527 & 2.7272947 \\
\hline $\mathrm{C}$ & -2.1074220 & 3.9806835 & 2.6253780 \\
\hline$C$ & -0.4168196 & 2.2961610 & 3.7220003 \\
\hline & 0.9029089 & 2.4430074 & 2.9698008 \\
\hline & 1.2191584 & 3.8764476 & 2.9639991 \\
\hline & 1.7608007 & 1.9128134 & 3.4317861 \\
\hline & -0.4693963 & 2.9909428 & 4.5824996 \\
\hline & -0.5025037 & 1.2642584 & 4.1160976 \\
\hline & -1.9723568 & 4.4909683 & 3.6030980 \\
\hline & -3.1902196 & 4.0116579 & 2.4177344 \\
\hline & -2.2670980 & 0.4912865 & 2.6846077 \\
\hline & -3.3632640 & 1.6634011 & 1.8812736 \\
\hline & -3.2242149 & 1.6508704 & 3.6746159 \\
\hline & 1.7671449 & 4.1170844 & 2.1624750 \\
\hline & 0.8391847 & 3.3448789 & -0.9361759 \\
\hline & 2.7188533 & 3.1008126 & -2.5436200 \\
\hline & 4.6755212 & 1.6248969 & -1.9921590 \\
\hline & 4.7269389 & 0.4005060 & 0.1999821 \\
\hline & 2.8315935 & 0.6312182 & 1.8032670 \\
\hline & -1.4520934 & 1.6907652 & -1.4098708 \\
\hline & -2.7652014 & 3.2447710 & -2.8985885 \\
\hline & -3.5012746 & 5.4767116 & -2.0293403 \\
\hline & -2.9195576 & 6.1966765 & 0.2793584 \\
\hline & -1.2641929 & 6.5540244 & 1.708456 \\
\hline
\end{tabular}




$\begin{array}{lrrr}\text { Pd } & -0.4249465 & 5.3719631 & 2.1028396 \\ \text { P } & 0.1601192 & 6.7383612 & 3.9789208 \\ \text { C } & -0.7209512 & 8.3466822 & 4.0093506 \\ \text { C } & -0.7936008 & 9.0690255 & 2.7984677 \\ \text { H } & -0.3555463 & 8.6385078 & 1.8832748 \\ \text { C } & -1.4085962 & 10.3291618 & 2.7672333 \\ \text { H } & -1.4585364 & 10.8876588 & 1.8203974 \\ \text { C } & -1.9639532 & 10.8725657 & 3.9388856 \\ \text { H } & -2.4547429 & 11.8572345 & 3.9109154 \\ \text { C } & -1.8903704 & 10.1577043 & 5.1460312 \\ \text { H } & -2.3196677 & 10.5822527 & 6.0663244 \\ \text { C } & -1.2630298 & 8.9018099 & 5.1861643 \\ \text { H } & -1.1934744 & 8.3495795 & 6.1350871 \\ \text { C } & 1.9029964 & 7.2112681 & 4.3122171 \\ \text { C } & 2.9180196 & 6.2405243 & 4.1658526 \\ \text { H } & 2.6488244 & 5.2188051 & 3.8668064 \\ \text { C } & 4.2570503 & 6.5834574 & 4.4023057 \\ \text { H } & 5.0408600 & 5.8200382 & 4.2844300 \\ \text { C } & 4.5991401 & 7.8962399 & 4.7662546 \\ \text { H } & 5.6522645 & 8.1653217 & 4.9389865 \\ \text { C } & 3.5933902 & 8.8673506 & 4.8985925 \\ \text { H } & 3.8542968 & 9.8996795 & 5.1771929 \\ \text { C } & 2.2499944 & 8.5297469 & 4.6731838 \\ \text { H } & 1.4700445 & 9.2977797 & 4.7739684 \\ \text { C } & -0.4069532 & 5.8370591 & 5.4675351 \\ \text { C } & 0.4927412 & 5.1456022 & 6.3058298 \\ \text { H } & 1.5756445 & 5.2801333 & 6.1724021 \\ \text { C } & 0.0097578 & 4.2867663 & 7.3051692 \\ \text { H } & 0.7226404 & 3.7543033 & 7.9529952 \\ \text { C } & -1.3726898 & 4.1068901 & 7.4833647 \\ \text { H } & -1.7466852 & 3.4304303 & 8.2663796 \\ \text { C } & -2.2752935 & 4.7986605 & 6.6577598 \\ \text { H } & -3.3598782 & 4.6705756 & 6.7939479 \\ \text { C } & -1.7989705 & 5.6508701 & 5.6503741 \\ \text { H } & -2.5119951 & 6.1912878 & 5.0082568 \\ & & & \\ \text { S4 } & & & \\ \text { 75 } & & & \\ \text { SCF } & \text { Energy }=-4548.271411494 & \\ \text { C } & 0.3609562 & 3.5261089 & -1.8800841 \\ \text { C } & 0.1833923 & 3.3964797 & -0.4846033 \\ \text { C } & -1.1425016 & 3.4198774 & 0.0259345 \\ \text { C } & -2.2428258 & 3.2832114 & -0.8427016 \\ \text { C } & -2.0386684 & 3.3770775 & -2.2331999 \\ \text { C } & -0.7416894 & 3.5508769 & -2.7435739 \\ & 1.2354453 & 3.0561493 & 0.4712860\end{array}$




\begin{tabular}{lrrr} 
C & 2.4279133 & 2.3982339 & 0.3279780 \\
$\mathrm{C}$ & 3.1322863 & 1.9296152 & -0.8824612 \\
$\mathrm{C}$ & 4.5281730 & 2.1255993 & -1.0097439 \\
$\mathrm{C}$ & 5.2199901 & 1.6398329 & -2.1294782 \\
$\mathrm{C}$ & 4.5368199 & 0.9354569 & -3.1341630 \\
$\mathrm{C}$ & 3.1555184 & 0.7072837 & -3.0033743 \\
$\mathrm{C}$ & 2.4604944 & 1.1957447 & -1.8889879 \\
$\mathrm{Br}$ & -0.9909832 & 6.6613006 & 0.5293082 \\
$\mathrm{C}$ & 0.8303806 & 3.1956797 & 1.9373312 \\
$\mathrm{C}$ & 1.1311619 & 4.5969317 & 2.5148263 \\
$\mathrm{C}$ & -0.6482473 & 2.8486500 & 2.0374946 \\
$\mathrm{C}$ & 1.7550142 & 2.1440579 & 2.6175854 \\
$\mathrm{C}$ & 3.0099977 & 2.0923192 & 1.7075143 \\
$\mathrm{O}$ & 4.0624830 & 2.9983934 & 2.0706414 \\
$\mathrm{H}$ & 3.4874521 & 1.0910423 & 1.7342902 \\
$\mathrm{H}$ & 2.0094630 & 2.3927746 & 3.6665100 \\
$\mathrm{H}$ & 1.2740843 & 1.1439033 & 2.6030731 \\
$\mathrm{H}$ & -0.8824112 & 1.8118480 & 1.7409792 \\
$\mathrm{H}$ & -1.0518859 & 3.0345662 & 3.0521746 \\
$\mathrm{H}$ & 2.2229928 & 4.7425556 & 2.6295505 \\
$\mathrm{H}$ & 0.6811483 & 4.7014545 & 3.5234682 \\
$\mathrm{H}$ & 0.7318107 & 5.4030259 & 1.8713463 \\
$\mathrm{H}$ & 3.7675817 & 3.8879356 & 1.7941592 \\
$\mathrm{H}$ & 5.0603312 & 2.6606860 & -0.2096245 \\
$\mathrm{H}$ & 6.3040757 & 1.8108001 & -2.2153136 \\
$\mathrm{H}$ & 5.0801854 & 0.5546274 & -4.0122845 \\
$\mathrm{H}$ & 2.6154435 & 0.1356720 & -3.7737965 \\
$\mathrm{H}$ & 1.3837187 & 0.9997185 & -1.7832297 \\
$\mathrm{H}$ & -3.0218445 & 3.4411553 & 2.0390301 \\
$\mathrm{H}$ & -3.2650878 & 3.1662397 & -0.4521981 \\
$\mathrm{H}$ & -2.9042490 & 3.3286867 & -2.9114064 \\
$\mathrm{H}$ & -0.5842412 & 3.6613182 & -3.8268097 \\
$\mathrm{H}$ & 1.3804044 & 3.5824435 & -2.2848940 \\
$\mathrm{Pd}$ & -2.0570783 & 4.5584862 & 1.5551373 \\
$\mathrm{P}$ & -3.2665891 & 5.8998900 & 2.9841157 \\
$\mathrm{C}$ & -2.1710363 & 6.3521376 & 4.3896618 \\
$\mathrm{C}$ & -2.4511713 & 5.9414164 & 5.7086306 \\
$\mathrm{H}$ & -3.3692753 & 5.3779677 & 5.9289036 \\
$\mathrm{C}$ & -1.5559559 & 6.2561215 & 6.7445843 \\
$\mathrm{H}$ & -1.7787110 & 5.9317612 & 7.7724250 \\
$\mathrm{C}$ & -0.3865087 & 6.9833104 & 6.4714389 \\
$\mathrm{H}$ & 0.3127216 & 7.2283014 & 7.2853480 \\
$\mathrm{H}$ & -0.1097641 & 7.3954945 & 5.1555797 \\
\hline
\end{tabular}




$\begin{array}{lrrr}\text { C } & -4.7575046 & 5.2010123 & 3.8143462 \\ \text { C } & -5.7974651 & 6.0446203 & 4.2583340 \\ \text { H } & -5.7513546 & 7.1245497 & 4.0566200 \\ \text { C } & -6.8940145 & 5.5071743 & 4.9504168 \\ \text { H } & -7.7043708 & 6.1723991 & 5.2849240 \\ \text { C } & -6.9595672 & 4.1287195 & 5.2123290 \\ \text { H } & -7.8213624 & 3.7099477 & 5.7537104 \\ \text { C } & -5.9261860 & 3.2845427 & 4.7723876 \\ \text { H } & -5.9741397 & 2.2025333 & 4.9672281 \\ \text { C } & -4.8343488 & 3.8179533 & 4.0710795 \\ \text { H } & -4.0315480 & 3.1579846 & 3.7053468 \\ \text { C } & -3.8634938 & 7.4871553 & 2.2990332 \\ \text { C } & -3.8298310 & 8.6789494 & 3.0480403 \\ \text { H } & -3.3916393 & 8.6822035 & 4.0570557 \\ \text { C } & -4.3443571 & 9.8640461 & 2.4968436 \\ \text { H } & -4.3101769 & 10.7964617 & 3.0804427 \\ \text { C } & -4.8938494 & 9.8597628 & 1.2048003 \\ \text { H } & -5.2912480 & 10.7908667 & 0.7731388 \\ \text { C } & -4.9241977 & 8.6695834 & 0.4567415 \\ \text { H } & -5.3383805 & 8.6677623 & -0.5625211 \\ \text { C } & -4.4038706 & 7.4865507 & 0.9973694 \\ \text { H } & -4.3904109 & 6.5603005 & 0.4033112\end{array}$

\section{Optimized Geometries(BXnf)}

\begin{tabular}{lrrr} 
R3 & & & \\
75 & \multicolumn{4}{l}{} \\
SCF & Energy $=-4548.353461584$ & \\
C & -2.3363214 & 4.2485956 & 0.9700810 \\
C & -1.1627403 & 3.6435489 & 0.4202291 \\
C & -0.7160170 & 3.9896183 & -0.8659519 \\
C & -1.4096925 & 4.9695552 & -1.5952336 \\
C & -2.5319039 & 5.6113057 & -1.0461080 \\
C & -3.0099621 & 5.2611501 & 0.2427119 \\
C & -0.6618460 & 2.7048548 & 1.4233862 \\
C & 0.5438787 & 2.3023645 & 1.9190518 \\
C & 1.8986365 & 2.6359615 & 1.4598216 \\
C & 2.9796693 & 1.7611633 & 1.7263951 \\
C & 4.2886540 & 2.1187307 & 1.3693812 \\
C & 4.5415912 & 3.3532778 & 0.7466094 \\
C & 3.4739737 & 4.2314431 & 0.4873631 \\
C & 2.1673195 & 3.8786393 & 0.8393065 \\
Br & 0.5688911 & 7.0695680 & 0.1483110
\end{tabular}




$\begin{array}{lrrr}\text { C } & -1.8428511 & 2.2910484 & 2.3084891 \\ \mathrm{C} & -2.6324227 & 1.1262507 & 1.6740565 \\ \mathrm{C} & -2.7124440 & 3.5857736 & 2.2943587 \\ \mathrm{C} & -1.1330164 & 1.8655094 & 3.6128222 \\ \mathrm{C} & 0.3023659 & 1.4579756 & 3.1721179 \\ \mathrm{O} & 0.4745307 & 0.0537922 & 2.9238652 \\ \mathrm{H} & 1.0421223 & 1.6897457 & 3.9672730 \\ \mathrm{H} & -1.0626174 & 2.7335909 & 4.3013207 \\ \mathrm{H} & -1.6317639 & 1.0407527 & 4.1589767 \\ \mathrm{H} & -2.4379751 & 4.2419417 & 3.1447653 \\ \mathrm{H} & -3.8023648 & 3.3909378 & 2.3679662 \\ \mathrm{H} & -2.0067130 & 0.2144010 & 1.5989826 \\ \mathrm{H} & -2.9833821 & 1.3879166 & 0.6550100 \\ \mathrm{H} & -3.5182756 & 0.8756913 & 2.2931544 \\ \mathrm{H} & 0.1336193 & -0.1097491 & 2.0235950 \\ \mathrm{H} & 2.7732069 & 0.7938609 & 2.2089763 \\ \mathrm{H} & 5.1186864 & 1.4262617 & 1.5789643 \\ \mathrm{H} & 5.5705159 & 3.6337199 & 0.4731762 \\ \mathrm{H} & 3.6516343 & 5.2169922 & 0.0313881 \\ \mathrm{H} & 1.3648816 & 4.6153396 & 0.6856822 \\ \mathrm{H} & 0.1781776 & 3.5102793 & -1.2878120 \\ \mathrm{H} & -1.0540053 & 5.2580841 & -2.5941130 \\ \mathrm{H} & -3.0522178 & 6.3926524 & -1.6185618 \\ \mathrm{H} & -3.9565390 & 5.6815754 & 0.6148454 \\ \mathrm{H} & -2.6433078 & 6.5823452 & 2.6074870 \\ \mathrm{Pd} & -1.4009000 & 6.5934751 & 1.6709308 \\ \mathrm{P} & -0.3711104 & 7.1937703 & 3.5321007 \\ \mathrm{C} & -0.1002543 & 9.0033797 & 3.7151578 \\ \mathrm{C} & 0.2544973 & 9.7474557 & 2.5685908 \\ \mathrm{H} & 0.3454540 & 9.2335065 & 1.5960498 \\ \mathrm{C} & 0.4946895 & 11.1249428 & 2.6786838 \\ \mathrm{H} & 0.7775592 & 11.6972162 & 1.7822269 \\ \mathrm{C} & 0.3638752 & 11.7721753 & 3.9191707 \\ \mathrm{H} & 0.5440228 & 12.8550551 & 3.9992682 \\ \mathrm{C} & -0.0076837 & 11.0355253 & 5.0554751 \\ \mathrm{H} & -0.1221021 & 11.5380183 & 6.0279543 \\ \mathrm{C} & -0.2387334 & 9.6541333 & 4.9586926 \\ \mathrm{H} & -0.5335869 & 9.0870339 & 5.8527838 \\ \mathrm{C} & 1.2890366 & 6.4142052 & 3.6066682 \\ \mathrm{C} & 1.3752551 & 5.0534090 & 3.9681966 \\ \mathrm{H} & 0.4615905 & 4.4875697 & 4.2023856 \\ \mathrm{C} & 2.6247290 & 4.4227586 & 4.0325621 \\ & 2.6892146 & 3.3625405 & 4.3142008 \\ \mathrm{C} & 3.7903236 & 5.1341117 & 3.7074113 \\ & 3.7661258 & 4.6284906 & 3.7370512 \\ & & 6.4811890 & 3.3211373\end{array}$




$\begin{array}{lrrr}\mathrm{H} & 4.6113473 & 7.0393506 & 3.0525137 \\ \mathrm{C} & 2.4567266 & 7.1256349 & 3.2715919 \\ \mathrm{H} & 2.3924064 & 8.1807694 & 2.9724930 \\ \mathrm{C} & -1.1121214 & 6.6724443 & 5.1387912 \\ \mathrm{C} & -0.2814843 & 6.5501441 & 6.2764495 \\ \mathrm{H} & 0.8002652 & 6.7267948 & 6.1870281 \\ \mathrm{C} & -0.8307644 & 6.1931042 & 7.5166925 \\ \mathrm{H} & -0.1737435 & 6.0949218 & 8.3940095 \\ \mathrm{C} & -2.2108293 & 5.9566318 & 7.6375249 \\ \mathrm{H} & -2.6382041 & 5.6692611 & 8.6100842 \\ \mathrm{C} & -3.0408490 & 6.0811551 & 6.5119903 \\ \mathrm{H} & -4.1217905 & 5.8939062 & 6.5972486 \\ \mathrm{C} & -2.4924796 & 6.4353723 & 5.2692958 \\ \mathrm{H} & -3.1341311 & 6.5220485 & 4.3773898\end{array}$

R4

75

SCF Energy $=-4548.346461001$

$\begin{array}{lrrr}\text { C } & 4.4288726 & 1.4136278 & -0.0700892 \\ \text { C } & 3.0329714 & 1.2300438 & -0.2327702 \\ \text { C } & 2.5887586 & 0.4021147 & -1.2929760 \\ \text { C } & 3.4996714 & -0.1869363 & -2.1790278 \\ \text { C } & 4.8801905 & 0.0302587 & -2.0234756 \\ \text { C } & 5.3382875 & 0.8278428 & -0.9615771 \\ \text { C } & 2.0859766 & 1.8495326 & 0.7036840 \\ \text { Pd } & -2.4660388 & 4.8848251 & -0.4111033 \\ \text { P } & -2.8208041 & 6.9749987 & 0.2454564 \\ \text { C } & 0.8080061 & 2.2837215 & 0.4986851 \\ \text { Br } & -0.5890874 & 5.5778106 & -1.9624172 \\ \text { C } & -0.2139274 & 2.2994633 & -0.5454364 \\ \text { C } & -0.1389074 & 2.2464515 & -1.9490010 \\ \text { C } & -1.3206467 & 2.2950814 & -2.7093081 \\ \text { C } & -2.5724141 & 2.3922638 & -2.0864920 \\ \text { C } & -2.6734257 & 2.4509749 & -0.6680485 \\ \text { C } & -1.4842972 & 2.4170887 & 0.0998028 \\ \text { C } & 2.4408810 & 2.0472077 & 2.1900826 \\ \text { O } & 3.2889543 & 1.0520836 & 2.7365560 \\ \text { C } & 1.0469777 & 2.1541816 & 2.8677965 \\ \text { C } & 0.1697217 & 2.8167029 & 1.7888286 \\ \text { C } & -1.3179246 & 2.4033741 & 1.6124867 \\ \text { C } & 0.2775217 & 4.3559343 & 1.8418536 \\ \text { H } & 3.0038978 & 2.9992131 & 2.3275645 \\ \text { H } & 1.0777026 & 2.6993490 & 3.8320244 \\ \text { H } & 0.6942649 & 1.1184231 & 3.0655842 \\ \text { H } & -1.4803285 & 1.3681199 & 1.9891114 \\ \text { H } & -2.0345101 & 3.0695787 & 2.1311823\end{array}$




$\begin{array}{rrr}-0.2195577 & 4.7573236 & 2.7482568 \\ -0.2060165 & 4.8118891 & 0.9539985 \\ 1.3333670 & 4.6900650 & 1.8467088 \\ 2.9384852 & 0.1890717 & 2.4417687 \\ 1.5132422 & 0.2025607 & -1.4016406 \\ 3.1301578 & -0.8287190 & -2.9936223 \\ 5.5963208 & -0.4306017 & -2.7205784 \\ 6.4177659 & 0.9933089 & -0.8233218 \\ 4.7995891 & 2.0196776 & 0.7676120 \\ -3.6396251 & 4.5566605 & 0.5593110 \\ -3.6560933 & 2.3691107 & -0.1821422 \\ -3.4899249 & 2.4224004 & -2.6921187 \\ -1.2589273 & 2.2869950 & -3.8068544 \\ 0.8371162 & 2.1973816 & -2.4498238 \\ -2.7717996 & 8.2078564 & -1.1082865 \\ -1.5283513 & 7.5094602 & 1.4378122 \\ -4.3953216 & 7.3679635 & 1.1250961 \\ -4.9529872 & 8.6610942 & 1.0382752 \\ -6.1049749 & 8.9827144 & 1.7730477 \\ -6.7045402 & 8.0240499 & 2.6059708 \\ -6.1517167 & 6.7355378 & 2.6964425 \\ -5.0076959 & 6.4077884 & 1.9536226 \\ -4.4885909 & 9.4179665 & 0.3901370 \\ -6.5374268 & 9.9912910 & 1.6902013 \\ -7.6087530 & 8.2785447 & 3.1794056 \\ -6.6195897 & 5.9762138 & 3.3410967 \\ -4.5814836 & 5.3928778 & 2.0006238 \\ -2.2045955 & 9.4843288 & -0.9287895 \\ -2.2351641 & 10.4151912 & -1.9802650 \\ -2.8341785 & 10.0771092 & -3.2042104 \\ -3.4002970 & 8.8022913 & -3.3817982 \\ -3.3646819 & 7.8662276 & -2.3401711 \\ -1.7296501 & 9.7463536 & 0.0280236 \\ -1.7828384 & 11.4088213 & -1.8414136 \\ -2.8517543 & 10.8066302 & -4.0280656 \\ -3.8567781 & 8.5296517 & -4.3450393 \\ -3.7728467 & 6.8538256 & -2.4807100 \\ -1.8382571 & 7.7870761 & 2.7841812 \\ -0.8138926 & 8.1327158 & 3.6811916 \\ 0.5160527 & 8.2110376 & 3.2384183 \\ 0.8229573 & 7.9388720 & 1.8933461 \\ -0.1895182 & 7.5806475 & 0.9937708 \\ -2.8791408 & 7.7347546 & 3.1343628 \\ -1.0609598 & 8.3448479 & 4.7324732 \\ 1.3166037 & 8.4817180 & 3.9435769 \\ 1.8644648 & 7.9914586 & 1.5418109\end{array}$


3.7358131

$-0.6433042$

$$
-2.7235968
$$

4.5328429

$-0.5692432$

$$
-3.5020850
$$

4.5680351

0.5983823

$$
-3.1444388
$$

3.7746842

2.3878990

1.7246645

0.8247085

C $\quad 1.4058736$

2.6883958

0.4738455

C $\quad 1.8700511$

3.1245535

$-0.8450209$

1.2287805

2. 6150935

$-2.0041582$

C $\quad 1.6297848$

3.0061040

$-3.2881270$

2.6982896

3.9049911

$-3.4486626$

C $\quad 3.3648635$

4.3944838

$-2.3120059$

C 2.9616336

4.0120111

$-1.0260919$

$\mathrm{Br} \quad 0.3108388$

5.9078497

1. 2791702

C $\quad 0.0307921$

1. 7140491

2. 1946869

0.1036687

0.1815767

2.0394130

$-1.3482531$

2.1541934

2. 2711122

2. 7705372

2.8797594

2. 2928986

2. 6199386

1. 7294593

2.9510465

3.8636010

1. 9579432

3.1100290

1.8712485

1. 0505185

3. 2233827

1. 6312168

1.7362064

1. 5919836

3. 3871396

$-1.2489699$

2.7439715

$-1.9894035$

1. 2801035

3. 6397057

3.7049957

1.0564934

$-0.1221903$

3. 0153794

$-0.7260919$

$-0.1954358$

1. 5604658

0.0375034

$-0.3164334$

1. 4065939

2.2361860

4.5501290

3. 0297512

1.9161016

H $\quad 0.4253936$

1.8733410

$-1.8791593$

$2.5947635-4.1704723$

1.1154877

4. 2132144

$-4.4560716$

3.0172798

5.0927490

$-2.4285983$

4. 2080982

4.3987275

$-0.1347503$

$-0.9536335$

3. 7448744

$-1.5512473$

$-3.0100135$

5.1522983

$-1.4317854$

$-4.4137195$

5. 1821133

0.6397815

$-3.8419270$

3. 6693747

2.5677250

$-2.8383027$

5.2679867

3. 8214148

Pd $\quad-1.6824359$

5.4347351

2. 7950776 


\begin{tabular}{|c|c|c|c|}
\hline & -0.7261557 & 6.5660676 & 4.4404285 \\
\hline & -0.0652662 & 8.2234134 & 4.0126506 \\
\hline & -0.5480040 & 8.8802985 & 2.8649404 \\
\hline & -1.2480100 & 8.3550231 & 2.1987791 \\
\hline & -0.1102376 & 10.1795394 & 2.5675162 \\
\hline & -0.4854796 & 10.6867751 & 1.6662259 \\
\hline & 0.8157634 & 10.8213414 & 3.4060203 \\
\hline & 1.1655629 & 11.8367706 & 3.1654589 \\
\hline & 1.3008590 & 10.1651690 & 4.5503347 \\
\hline & 2.0299820 & 10.6633455 & 5.2070194 \\
\hline & 0.8576587 & 8.8707131 & 4.8594352 \\
\hline & 1.2412599 & 8.3617381 & 5.7559115 \\
\hline & 0.6838164 & 5.5697476 & 5.0472100 \\
\hline & 0.4354435 & 4.5024423 & 5.9379611 \\
\hline & -0.5810080 & 4.3353093 & 6.3258132 \\
\hline & 1.4852173 & 3.6586415 & 6.3269560 \\
\hline & 1.2890244 & 2.8303583 & 7.0243855 \\
\hline & 2.7793397 & 3.8622414 & 5.8171677 \\
\hline & 3.5989285 & 3.1902836 & 6.1126362 \\
\hline & 3.0211681 & 4.9066754 & 4.9112193 \\
\hline & 4.0217193 & 5.0436891 & 4.4787002 \\
\hline & 1.9782627 & 5.7629488 & 4.5260058 \\
\hline & 2.1582658 & 6.5635917 & 3.7945151 \\
\hline & -1.7160732 & 6.9357962 & 5.9510550 \\
\hline & -1.0859213 & 7.1146924 & 7.2008198 \\
\hline & -0.0048346 & 6.9403461 & 7.3042873 \\
\hline & -1.8403685 & 7.4938515 & 8.3214076 \\
\hline & -1.3432322 & 7.6191010 & 9.2952166 \\
\hline & -3.2236909 & 7.7089957 & 8.2021449 \\
\hline & -3.8132038 & 8.0059357 & 9.0827251 \\
\hline & -3.8529883 & 7.5374702 & 6.9581776 \\
\hline & -4.9368014 & 7.7003940 & 6.8594073 \\
\hline & -3.1029718 & 7.1483282 & 5.8377904 \\
\hline & -3.5911006 & 6.9948734 & 4.862750 \\
\hline
\end{tabular}

S4

75

SCF Energy $=-4548.348016586$

$\begin{array}{lrrr}\text { C } & -0.1896339 & 3.3416333 & -1.5541851 \\ \text { C } & -0.0498103 & 2.7802718 & -0.2724482 \\ \text { C } & -1.1971764 & 2.3027390 & 0.4317383 \\ \text { C } & -2.4789088 & 2.3688929 & -0.1663003 \\ \text { C } & -2.5964820 & 2.9405268 & -1.4646870 \\ \text { C } & -1.4652377 & 3.4185615 & -2.1406043 \\ \text { C } & 1.1099265 & 2.5734547 & 0.5937045 \\ \text { C } & 2.4545293 & 2.3582218 & 0.4849439\end{array}$




$\begin{array}{lrrr}\text { C } & 3.3247295 & 2.3933634 & -0.7001681 \\ \mathrm{C} & 4.6791471 & 2.7900276 & -0.5720003 \\ \mathrm{C} & 5.5273665 & 2.8108579 & -1.6882888 \\ \mathrm{C} & 5.0489894 & 2.4252208 & -2.9516026 \\ \mathrm{C} & 3.7133624 & 2.0067386 & -3.0875076 \\ \mathrm{C} & 2.8612959 & 1.9892917 & -1.9756028 \\ \mathrm{Br} & -1.2757558 & 6.1537750 & -0.0554065 \\ \mathrm{C} & 0.5955927 & 2.3643499 & 2.0255630 \\ \mathrm{C} & 0.3750676 & 3.7178148 & 2.7345854 \\ \mathrm{C} & -0.7861025 & 1.6955037 & 1.7647073 \\ \mathrm{C} & 1.7506277 & 1.5670631 & 2.6645272 \\ \mathrm{C} & 3.0123749 & 2.0151934 & 1.8711391 \\ \mathrm{O} & 3.7299124 & 3.1126450 & 2.4585379 \\ \mathrm{H} & 3.7614583 & 1.1981177 & 1.8127087 \\ \mathrm{H} & 1.8787666 & 1.7388297 & 3.7513767 \\ \mathrm{H} & 1.5990512 & 0.4798164 & 2.5002584 \\ \mathrm{H} & -0.6649826 & 0.5955780 & 1.6440995 \\ \mathrm{H} & -1.5292969 & 1.8701022 & 2.5672170 \\ \mathrm{H} & 1.3317247 & 4.2514866 & 2.8966577 \\ \mathrm{H} & -0.0944827 & 3.5704667 & 3.7283578 \\ \mathrm{H} & -0.2811252 & 4.3815739 & 2.1361090 \\ \mathrm{H} & 3.2128284 & 3.9176946 & 2.2610533 \\ \mathrm{H} & 5.0449381 & 3.0992760 & 0.4183567 \\ \mathrm{H} & 6.5735690 & 3.1326180 & -1.5701882 \\ \mathrm{H} & 5.7158993 & 2.4426418 & -3.8269594 \\ \mathrm{H} & 3.3348064 & 1.6814187 & -4.0688152 \\ \mathrm{H} & 1.8282300 & 1.6290975 & -2.0834101 \\ \mathrm{H} & -3.6557465 & 3.4637954 & 1.8970300 \\ \mathrm{H} & -3.3357509 & 1.8504574 & 0.2868919 \\ \mathrm{H} & -3.5877386 & 2.9996922 & -1.9373194 \\ \mathrm{H} & -1.5800288 & 3.8860291 & -3.1288765 \\ \mathrm{H} & 0.6861718 & 3.7427158 & -2.0817673 \\ \mathrm{Pd} & -2.7254940 & 4.4410049 & 1.1193248 \\ \mathrm{P} & -3.3637951 & 5.8946224 & 2.6719200 \\ \mathrm{C} & -2.0194946 & 6.1762587 & 3.8939906 \\ \mathrm{C} & -2.1452822 & 5.7664141 & 5.2362901 \\ \mathrm{H} & -3.0812187 & 5.3110978 & 5.5902536 \\ \mathrm{C} & -1.0711067 & 5.9403990 & 6.1247669 \\ \mathrm{H} & -1.1730754 & 5.6143646 & 7.1709510 \\ \mathrm{C} & 0.1235807 & 6.5300571 & 5.6821796 \\ \mathrm{H} & 0.9640830 & 6.6632546 & 6.3801273 \\ \mathrm{C} & 0.2444646 & 6.9463528 & 4.3444098 \\ \mathrm{C} & 1.1797848 & 7.4058916 & 3.9905829 \\ & -0.8159053 & 6.7648330 & 3.4470464 \\ \mathrm{H} & -4.8135547 & 5.4660576 & 3.7306085\end{array}$




$\begin{array}{rrrr}\text { C } & -5.6444290 & 6.4819315 & 4.2477685 \\ \text { H } & -5.4730567 & 7.5303172 & 3.9647038 \\ \text { C } & -6.6961738 & 6.1567827 & 5.1190336 \\ \text { H } & -7.3449280 & 6.9559465 & 5.5083354 \\ \text { C } & -6.9219711 & 4.8213176 & 5.4895719 \\ \text { H } & -7.7479198 & 4.5693989 & 6.1718655 \\ \text { C } & -6.0958339 & 3.8064044 & 4.9777426 \\ \text { H } & -6.2707595 & 2.7564648 & 5.2570900 \\ \text { C } & -5.0527680 & 4.1269525 & 4.0964435 \\ \text { H } & -4.4165884 & 3.3342582 & 3.6714637 \\ \text { C } & -3.7801001 & 7.5553287 & 2.0219818 \\ \text { C } & -3.4737352 & 8.7281183 & 2.7394571 \\ \text { H } & -2.9289343 & 8.6639431 & 3.6928546 \\ \text { C } & -3.8539121 & 9.9790059 & 2.2261377 \\ \text { H } & -3.6050548 & 10.8950091 & 2.7830492 \\ \text { C } & -4.5421779 & 10.0602518 & 1.0049667 \\ \text { H } & -4.8339621 & 11.0421379 & 0.6024508 \\ \text { C } & -4.8473315 & 8.8886688 & 0.2899802 \\ \text { H } & -5.3727622 & 8.9510337 & -0.6746615 \\ \text { C } & -4.4624981 & 7.6385747 & 0.7920247 \\ \text { H } & -4.6639918 & 6.7182915 & 0.2230032\end{array}$

\section{Optimized Geometries (BXni)}

\begin{tabular}{lrrr} 
R3 & & & \\
79 & \multicolumn{4}{l}{} \\
SCF & Energy $=-2238.417427572$ & \\
C & -1.3225238 & 2.7342787 & 2.4227937 \\
C & -0.1033371 & 2.2554442 & 1.8924999 \\
C & 0.9602344 & 1.9462334 & 2.7631374 \\
C & 0.7964421 & 2.0854315 & 4.1501749 \\
C & -0.4198057 & 2.5510666 & 4.6747096 \\
C & -1.4748555 & 2.8876338 & 3.8079905 \\
P & 0.0264890 & 1.9473095 & 0.0926023 \\
C & 1.8062497 & 1.6073390 & -0.1848025 \\
C & 2.2333151 & 0.2684425 & -0.2753874 \\
C & 3.5952420 & -0.0278155 & -0.4278964 \\
C & 4.5331633 & 1.0148470 & -0.4949941 \\
C & 4.1078523 & 2.3540119 & -0.4168553 \\
C & 2.7463415 & 2.6554023 & -0.2635701 \\
Pd & -1.0996581 & 0.2577127 & -0.8101327 \\
O & -0.2540005 & 0.6910990 & -2.7232740 \\
C & 0.7220867 & -0.0050318 & -3.1962781
\end{tabular}




\begin{tabular}{rrrr} 
O & 1.1683636 & -1.0856343 & -2.7730782 \\
$\mathrm{C}$ & -1.4891442 & -2.3299335 & -1.5616782 \\
$\mathrm{C}$ & -1.7572747 & -2.9319805 & -0.3093774 \\
$\mathrm{C}$ & -2.7310356 & -3.9499471 & -0.2492958 \\
$\mathrm{C}$ & -3.4280333 & -4.3366963 & -1.4062530 \\
$\mathrm{C}$ & -3.1787394 & -3.7045328 & -2.6372125 \\
$\mathrm{C}$ & -2.1997241 & -2.7018081 & -2.7150598 \\
$\mathrm{C}$ & -1.1249346 & -2.3525353 & 0.8992545 \\
$\mathrm{C}$ & 0.1583507 & -2.4556335 & 1.3296740 \\
$\mathrm{C}$ & 0.4320966 & -1.4289527 & 2.4315207 \\
$\mathrm{C}$ & -0.9628305 & -0.9870752 & 2.8724235 \\
$\mathrm{C}$ & -1.9299913 & -1.3159342 & 1.7039640 \\
$\mathrm{C}$ & 1.2523028 & -3.3079366 & 0.8154420 \\
$\mathrm{C}$ & 1.9083409 & -4.1922505 & 1.7087606 \\
$\mathrm{C}$ & 2.9873194 & -4.9789753 & 1.2770467 \\
$\mathrm{C}$ & 3.4354531 & -4.8941182 & -0.0517531 \\
$\mathrm{C}$ & 2.7918712 & -4.0205882 & -0.9439677 \\
$\mathrm{C}$ & 1.7091607 & -3.2335336 & -0.5206278 \\
$\mathrm{C}$ & -3.2598931 & -1.8827504 & 2.2483400 \\
$\mathrm{C}$ & -2.2749686 & -0.1079896 & 0.8049032 \\
$\mathrm{O}$ & 1.1797820 & -1.8860590 & 3.5539615 \\
$\mathrm{C}$ & -0.2668020 & 3.5915914 & -0.6712572 \\
$\mathrm{C}$ & -0.4737788 & 3.6551552 & -2.0669621 \\
$\mathrm{C}$ & -0.6354200 & 4.9040389 & -2.6847706 \\
$\mathrm{C}$ & -0.5946769 & 6.0865912 & -1.9260671 \\
$\mathrm{C}$ & -0.3829907 & 6.0217147 & -0.5392356 \\
$\mathrm{C}$ & -0.2166162 & 4.7782523 & 0.0914235 \\
$\mathrm{O}$ & 1.2829602 & 0.5722238 & -4.3080889 \\
$\mathrm{H}$ & 1.9888938 & -0.0511517 & -4.5728585 \\
$\mathrm{H}$ & 0.9783555 & -0.5814110 & 1.9486694 \\
$\mathrm{H}$ & -1.0084201 & 0.0700328 & 3.1876772 \\
$\mathrm{H}$ & -1.2196567 & -1.6120818 & 3.7520673 \\
$\mathrm{H}$ & -2.4849729 & 0.7818875 & 1.4280382 \\
$\mathrm{H}$ & -3.1864843 & -0.3300011 & 0.1967209 \\
$\mathrm{H}$ & -3.0781781 & -2.7779751 & 2.8784351 \\
$\mathrm{H}$ & -3.9511182 & -2.1655816 & 1.4297364 \\
$\mathrm{H}$ & -3.7679718 & -1.1196352 & 2.8737728 \\
$\mathrm{H}$ & 2.0650965 & -2.1304460 & 3.2208203 \\
$\mathrm{H}$ & 1.5412825 & -4.2717114 & 2.7426341 \\
$\mathrm{H}$ & 3.4764358 & -5.6685330 & 1.9824507 \\
$\mathrm{H}$ & 4.2829305 & -5.5097600 & -0.3904510 \\
& 3.1328874 & -3.9390684 & -1.9873382 \\
$\mathrm{H}$ & 1.2560219 & -2.5335297 & -1.2365043 \\
\hline
\end{tabular}




$\begin{array}{rrrr}\text { H } & -1.9618544 & -2.2158702 & -3.6728364 \\ \text { H } & -0.6095259 & -1.6541844 & -1.6773920 \\ \text { H } & -0.4851090 & 2.7207174 & -2.6536994 \\ \text { H } & -0.7952627 & 4.9506012 & -3.7725839 \\ \text { H } & -0.7267000 & 7.0628673 & -2.4170400 \\ \text { H } & -0.3446907 & 6.9444664 & 0.0594934 \\ \text { H } & -0.0448297 & 4.7337050 & 1.1764062 \\ \text { H } & 1.4901779 & -0.5394738 & -0.2661294 \\ \text { H } & 3.9070641 & -1.0795482 & -0.5108678 \\ \text { H } & 5.6024688 & 0.7865303 & -0.6217521 \\ \text { H } & 4.8421750 & 3.1710660 & -0.4830369 \\ \text { H } & 2.4115876 & 3.7022403 & -0.2116530 \\ \text { H } & 1.9145207 & 1.5845691 & 2.3552755 \\ \text { H } & 1.6242401 & 1.8191699 & 4.8235147 \\ \text { H } & -0.5474813 & 2.6536303 & 5.7627064 \\ \text { H } & -2.4268158 & 3.2609370 & 4.2142617 \\ \text { H } & -2.1503598 & 2.9958357 & 1.7461067\end{array}$

$\mathrm{R} 4$

79

SCF Energy $=-2238.427040432$

$\begin{array}{lrrr}\text { C } & 1.7810942 & 3.7592127 & 1.8588095 \\ \text { C } & 0.8656017 & 2.6873038 & 1.9307594 \\ \text { C } & 0.4866750 & 2.1936405 & 3.2004867 \\ \text { C } & 1.0181494 & 2.7617081 & 4.3681409 \\ \text { C } & 1.9324619 & 3.8242483 & 4.2877873 \\ \text { C } & 2.3093884 & 4.3224552 & 3.0295297 \\ \text { P } & 0.2888408 & 1.8788861 & 0.3790140 \\ \text { C } & -1.4304542 & 1.3576644 & 0.7544456 \\ \text { C } & -2.2673744 & 2.1028852 & 1.6108261 \\ \text { C } & -3.5666741 & 1.6478372 & 1.8891669 \\ \text { C } & -4.0356094 & 0.4543645 & 1.3158961 \\ \text { C } & -3.2099049 & -0.2789797 & 0.4466829 \\ \text { C } & -1.9119688 & 0.1707758 & 0.1630757 \\ \text { Pd } & 1.4610568 & 0.1103981 & -0.3331615 \\ \text { C } & 1.4504508 & -0.4563669 & 1.6441281 \\ \text { C } & 1.6384086 & -1.9786481 & 1.8134576 \\ \text { C } & 0.8026993 & -2.6535065 & 0.7095791 \\ \text { C } & -0.3250349 & -3.2453490 & 1.2245046 \\ \text { C } & -0.4813526 & -2.8867311 & 2.6974969 \\ \text { C } & 0.9437932 & -2.4982433 & 3.1070993 \\ \text { C } & -1.3532560 & -4.0500711 & 0.5419140 \\ \text { C } & -2.7180986 & -3.9301839 & 0.9022058 \\ \text { C } & -3.6964734 & -4.7085383 & 0.2650079 \\ \text { C } & -3.3336468 & -5.6297584 & -0.7312131 \\ \text { C } & -1.9779655 & -5.7790941 & -1.0761323\end{array}$




$\begin{array}{rrrr}\text { C } & -0.9989297 & -5.0034642 & -0.4439080 \\ \text { C } & 1.0885254 & -2.3909246 & -0.7230761 \\ \text { C } & 2.4195631 & -2.1860449 & -1.2108884 \\ \text { C } & 2.6690284 & -1.9983008 & -2.5857516 \\ \text { C } & 1.6153807 & -1.9778725 & -3.5023323 \\ \text { C } & 0.2947403 & -2.1439646 & -3.0398091 \\ \text { C } & 0.0345886 & -2.3395093 & -1.6845381 \\ \text { O } & -1.4486885 & -1.8324616 & 2.8633155 \\ \text { C } & 3.1388104 & -2.3379335 & 1.8770564 \\ \text { O } & -0.6281071 & 0.8767222 & -2.5092119 \\ \text { C } & 0.1902589 & 3.2505692 & -0.8287690 \\ \text { C } & -1.0197574 & 3.9244753 & -1.0820153 \\ \text { C } & -1.0536114 & 4.9676544 & -2.0185774 \\ \text { C } & 0.1123871 & 5.3335993 & -2.7124737 \\ \text { C } & 1.3157060 & 4.6522161 & -2.4694143 \\ \text { C } & 1.3597263 & 3.6118231 & -1.5294656 \\ \text { H } & -0.8746577 & -3.7290673 & 3.3036828 \\ \text { H } & 1.4709065 & -3.4055680 & 3.4702417 \\ \text { H } & 0.9516324 & -1.7499603 & 3.9239523 \\ \text { H } & 0.4792819 & -0.1827277 & 2.0989292 \\ \text { H } & 2.2372073 & 0.1267238 & 2.1710112 \\ \text { H } & 3.5186712 & -2.0958243 & 2.8909398 \\ \text { H } & 3.7525024 & -1.7494070 & 1.1690284 \\ \text { H } & 3.3156318 & -3.4178395 & 1.6972246 \\ \text { H } & -1.3718502 & -1.2324446 & 2.0916405 \\ \text { H } & 0.0614939 & -5.1310417 & -0.7066968 \\ \text { H } & -1.6813235 & -6.5135431 & -1.8404462 \\ \text { H } & -4.1022731 & -6.2391797 & -1.2302104 \\ \text { H } & -4.7533390 & -4.5949285 & 0.5518072 \\ \text { H } & -2.9938404 & -3.2046932 & 1.6814140 \\ \text { H } & -1.0022862 & -2.4453836 & -1.3452919 \\ \text { H } & -0.5478613 & -2.0804647 & -3.7429982 \\ \text { H } & 1.8101345 & -1.7964318 & -4.5686850 \\ \text { H } & 3.7032067 & -1.8455297 & -2.9261132 \\ \text { H } & 3.2711593 & -2.2829509 & -0.5307622 \\ \text { H } & -1.9006947 & 3.0313485 & 2.0728797 \\ \text { H } & -4.2129783 & 2.2271453 & 2.5656654 \\ \text { H } & -5.0492677 & 0.0944552 & 1.5479312 \\ \text { H } & -3.5716253 & -1.2118920 & -0.0103315 \\ \text { H } & -1.2641422 & -0.3622336 & -0.5465999 \\ \text { H } & -1.9399068 & 3.6189262 & -0.5644016 \\ \text { H } & -2.0022078 & 5.4890509 & -2.2173465 \\ \text { H } & 0.0789707 & 6.1440367 & -3.4564203 \\ & 2.2257246 & 4.9175244 & -3.0275337 \\ \text { H } & -0.2360553 & 1.3703391 & 3.2844436\end{array}$




$\begin{array}{rrrr}\mathrm{H} & 0.7101932 & 2.3686592 & 5.3488237 \\ \mathrm{H} & 2.3469339 & 4.2679958 & 5.2055628 \\ \mathrm{H} & 3.0178976 & 5.1612943 & 2.9559530 \\ \mathrm{H} & 2.0760446 & 4.1634778 & 0.8799090 \\ \mathrm{C} & 0.5310097 & 1.0084320 & -2.9420977 \\ \mathrm{O} & 1.6388847 & 0.7818307 & -2.3284754 \\ \mathrm{O} & 0.7283365 & 1.4543750 & -4.2301738 \\ \mathrm{H} & -0.1779923 & 1.6168469 & -4.5601094\end{array}$

S3

79

SCF Energy $=-2238.447495460$

C $\quad-1.1684261 \quad-1.5839154$

$\begin{array}{lll}\text { C } & 0.0987336 & -1.0763137\end{array}$

$\begin{array}{lll}\text { C } & 0.9893830 & -1.8777563\end{array}$

C $\quad 0.6111917 \quad-3.1737224$

C $\quad-0.6563280 \quad-3.6719195$

$\begin{array}{lll}\text { C } & -1.5449118 & -2.8728318\end{array}$

$\begin{array}{lll}\mathrm{P} & 0.6010052 & 0.5774594\end{array}$

C $\quad 1.8807972 \quad 1.1082825$

C $\quad 3.2144073 \quad 1.2434648$

$\begin{array}{lll}\text { C } & 4.2172458 & 1.6145087\end{array}$

C $\quad 3.8909125 \quad 1.8584273$

$\begin{array}{lll}\text { C } & 2.5592156 & 1.7322073\end{array}$

$\begin{array}{lll}\text { C } & 1.5554544 & 1.3582171\end{array}$

$\begin{array}{lll}\mathrm{Pd} & 1.3503506 & 0.7999292\end{array}$

$\begin{array}{lll}\text { C } & 2.1852236 & -1.0584319\end{array}$

2.0392392

2.3949030

3.1371762

3.5234073

3.1800111

2.4400823

1.7843416

2.9950865

2.5606455

3.4710666

4.8146591

5.2492768

4.3445437

$-0.2921795$

$-0.2772557$

$\begin{array}{llll}\text { C } & 1.4392502 & -2.1031105 & -1.1726611\end{array}$

$\begin{array}{llll}\text { C } & 0.3311445 & -1.4167568 & -2.0202399\end{array}$

$\begin{array}{llll}\text { C } & 0.7841322 & -1.1181122 & -3.2776855\end{array}$

$\begin{array}{llll}\text { C } & 2.2090311 & -1.6448443 & -3.4836432\end{array}$

C $\quad 2.3827845 \quad-2.6125113 \quad-2.2957764$

$\begin{array}{llll}\text { C } & -1.0279524 & -1.1514700 & -1.4713959\end{array}$

$\begin{array}{llll}\text { C } & -1.5011423 & 0.1518795 & -1.1856159\end{array}$

$\begin{array}{llll}\text { C } & -2.7836482 & 0.3564516 & -0.6541417\end{array}$

$\begin{array}{llll}\text { C } & -3.6392503 & -0.7338970 & -0.4242682\end{array}$

$\begin{array}{llll}\text { C } & -3.1998325 & -2.0312694 & -0.7368890\end{array}$

$\begin{array}{llll}\text { C } & -1.9075044 & -2.2363514 & -1.2452127\end{array}$

$\begin{array}{llll}\text { C } & 0.1183406 & -0.3327255 & -4.3328749\end{array}$

$\begin{array}{llll}\text { C } & 0.9183328 & 0.3425115 & -5.2965036\end{array}$

$\begin{array}{llll}\text { C } & 0.3327067 & 1.1456167 & -6.2874069\end{array}$

$\begin{array}{llll}\text { C } & -1.0636956 & 1.2914113 & -6.3508696\end{array}$

$\begin{array}{llll}\text { C } & -1.8668063 & 0.6013989 & -5.4279805\end{array}$

$\begin{array}{llll}\text { C } & -1.2879518 & -0.2028530 & -4.4364565\end{array}$

$\begin{array}{llll}\text { C } & 0.9400321 & -3.2665654 & -0.3057176\end{array}$

$\begin{array}{llll}0 & 3.2087265 & -0.6323731 & -3.5553845\end{array}$ 


$\begin{array}{rrrr}\text { O } & 2.0235237 & 1.3935548 & -2.2826144 \\ \text { C } & -0.8675080 & 1.6283272 & 2.1380111 \\ \text { C } & -1.0782755 & 2.7812718 & 1.3534293 \\ \text { C } & -2.1853851 & 3.6063168 & 1.6075470 \\ \text { C } & -3.0861999 & 3.2901076 & 2.6375774 \\ \text { C } & -2.8760095 & 2.1433527 & 3.4219039 \\ \text { C } & -1.7716178 & 1.3138309 & 3.1758445 \\ \text { H } & 2.2664374 & -2.2004384 & -4.4473984 \\ \text { H } & 3.4437012 & -2.6840698 & -1.9862852 \\ \text { H } & 2.0463144 & -3.6265106 & -2.5993733 \\ \text { H } & 3.1574388 & -0.7734810 & -0.7314750 \\ \text { H } & 2.3623026 & -1.4617544 & 0.7384492 \\ \text { H } & 0.4623157 & -4.0527967 & -0.9248145 \\ \text { H } & 0.2108763 & -2.9336751 & 0.4526238 \\ \text { H } & 1.7947635 & -3.7319115 & 0.2258552 \\ \text { H } & 2.8540713 & 0.1687098 & -3.0956173 \\ \text { H } & 2.0120238 & 0.2309739 & -5.2385004 \\ \text { H } & 0.9749041 & 1.6591951 & -7.0200606 \\ \text { H } & -1.5228737 & 1.9272817 & -7.1226114 \\ \text { H } & -2.9629651 & 0.6872968 & -5.4807147 \\ \text { H } & -1.9370011 & -0.7470904 & -3.7392776 \\ \text { H } & -1.5774441 & -3.2540110 & -1.4936017 \\ \text { H } & -3.8659129 & -2.8938179 & -0.5816004 \\ \text { H } & -4.6461527 & -0.5715644 & -0.0110579 \\ \text { H } & -3.1097821 & 1.3798874 & -0.4179802 \\ \text { H } & -0.8690390 & 1.0263583 & -1.3910520 \\ \text { H } & -0.3974389 & 3.0197755 & 0.5237154 \\ \text { H } & -2.3457199 & 4.4991268 & 0.9847118 \\ \text { H } & -3.9569192 & 3.9357244 & 2.8285393 \\ \text { H } & -3.5792815 & 1.8879762 & 4.2290658 \\ \text { H } & -1.6261277 & 0.4077695 & 3.7816449 \\ \text { H } & 3.4495925 & 1.0628412 & 1.4991018 \\ \text { H } & 5.2565343 & 1.7194600 & 3.1252382 \\ \text { H } & 4.6756471 & 2.1546605 & 5.5272086 \\ \text { H } & 2.3004512 & 1.9308923 & 6.3003179 \\ \text { H } & 0.5141076 & 1.2678310 & 4.6874084 \\ \text { H } & 1.9776846 & -1.4852630 & 3.4186678 \\ \text { H } & 1.3122442 & -3.7962587 & 4.0994456 \\ \text { H } & -0.9507648 & -4.6869477 & 3.4865036 \\ \text { H } & -2.5350706 & -3.2572139 & 2.1539344 \\ \text { H } & -1.8597809 & -0.9734710 & 1.4422108 \\ \text { C } & 1.2258573 & 2.4061385 & -2.2981201 \\ \text { O } & 0.6788975 & 2.8233217 & -1.2392871 \\ \text { H } & 0.9387760 & 3.0033880 & -3.4619566 \\ & 1.2360960 & 2.4034922 & -4.1862377\end{array}$


SCF Energy $=-2238.429904093$

\begin{tabular}{|c|c|c|c|}
\hline $\mathrm{C}$ & -1.4763787 & 4.0962215 & -1.3861767 \\
\hline C & -0.9848367 & 3.8914474 & -0.0786155 \\
\hline C & -1.0011303 & 4.9539972 & 0.8504206 \\
\hline $\mathrm{C}$ & -1.5020662 & 6.2102850 & 0.4730321 \\
\hline C & -1.9879064 & 6.4141972 & -0.8285761 \\
\hline C & -1.9730871 & 5.3561001 & -1.7531615 \\
\hline$P$ & -0.2593377 & 2.2488002 & 0.3525149 \\
\hline C & -0.1775440 & 2.3375701 & 2.1828320 \\
\hline C & 1.0418029 & 2.3815918 & 2.8846826 \\
\hline C & 1.0438346 & 2.3924024 & 4.2889166 \\
\hline $\mathrm{C}$ & -0.1676917 & 2.3616696 & 4.9984933 \\
\hline C & -1.3879930 & 2.3319982 & 4.3001785 \\
\hline $\mathrm{C}$ & -1.3945090 & 2.3190379 & 2.8982976 \\
\hline $\mathrm{Pd}$ & -1.3623660 & 0.6240782 & -0.6803819 \\
\hline $\mathrm{C}$ & -1.7339892 & -0.5195788 & 0.9558756 \\
\hline C & -0.7356149 & -1.5504449 & 1.5127975 \\
\hline C & -0.2135956 & -2.6124877 & 0.5281100 \\
\hline C & -0.3548020 & -3.8847185 & 1.0092788 \\
\hline C & -0.9326296 & -3.8600153 & 2.4192509 \\
\hline C & -1.5111719 & -2.4387752 & 2.5401011 \\
\hline C & 0.5739178 & -2.2474991 & -0.6734558 \\
\hline $\mathrm{C}$ & 1.9776566 & -2.4077511 & -0.6552463 \\
\hline C & 2.7504210 & -2.0806131 & -1.7795368 \\
\hline $\mathrm{C}$ & 2.1348414 & -1.5711521 & -2.9362172 \\
\hline C & 0.7407954 & -1.4110922 & -2.9676277 \\
\hline C & -0.0360558 & -1.7533364 & -1.8471718 \\
\hline C & 0.0631555 & -5.1476352 & 0.3697946 \\
\hline $\mathrm{C}$ & 0.4177501 & -6.2651294 & 1.1669440 \\
\hline $\mathrm{C}$ & 0.8022920 & -7.4770189 & 0.5755606 \\
\hline $\mathrm{C}$ & 0.8368827 & -7.6061469 & -0.8225431 \\
\hline $\mathrm{C}$ & 0.4750848 & -6.5103182 & -1.6251464 \\
\hline $\mathrm{C}$ & 0.0918206 & -5.2973453 & -1.0388580 \\
\hline C & 0.4752007 & -0.8701842 & 2.1830989 \\
\hline 0 & 0.0721528 & -4.1593654 & 3.4046894 \\
\hline 0 & -1.5164506 & 1.3265809 & -2.8285803 \\
\hline $\mathrm{C}$ & 1.4847401 & 2.3968313 & -0.2009219 \\
\hline C & 2.1359460 & 1.2592674 & -0.7159631 \\
\hline C & 3.4796697 & 1.3438112 & -1.1131843 \\
\hline $\mathrm{C}$ & 4.1717907 & 2.5605648 & -1.0080565 \\
\hline $\mathrm{C}$ & 3.5178558 & 3.7015093 & -0.5093234 \\
\hline $\mathrm{C}$ & 2.1761173 & 3.6238481 & -0.1091909 \\
\hline $\mathrm{H}$ & -1.7093330 & -4.6372227 & 2.5745309 \\
\hline $\mathrm{H}$ & -1.4370245 & -2.0585331 & 3.5778537 \\
\hline
\end{tabular}




$\begin{array}{rrrr}\text { H } & -2.5852059 & -2.4626313 & 2.2644170 \\ \text { H } & -2.6177880 & -1.0445365 & 0.5289448 \\ \text { H } & -2.0732886 & 0.1199208 & 1.7955720 \\ \text { H } & 1.1467572 & -1.6215107 & 2.6463823 \\ \text { H } & 0.1436983 & -0.1786672 & 2.9793738 \\ \text { H } & 1.0738139 & -0.2963042 & 1.4494816 \\ \text { H } & 0.8758943 & -3.6741775 & 3.1342744 \\ \text { H } & 0.4116576 & -6.1555994 & 2.2609525 \\ \text { H } & 1.0801276 & -8.3289053 & 1.2153138 \\ \text { H } & 1.1384293 & -8.5579961 & -1.2858444 \\ \text { H } & 0.4844131 & -6.6028115 & -2.7220758 \\ \text { H } & -0.2069520 & -4.4568693 & -1.6788686 \\ \text { H } & -1.1321701 & -1.6957594 & -1.9040981 \\ \text { H } & 0.2481735 & -1.0178034 & -3.8687493 \\ \text { H } & 2.7413671 & -1.3036253 & -3.8144499 \\ \text { H } & 3.8412900 & -2.2264169 & -1.7516817 \\ \text { H } & 2.4562799 & -2.8089111 & 0.2503281 \\ \text { H } & 1.5830202 & 0.3140066 & -0.8180242 \\ \text { H } & 3.9767628 & 0.4502183 & -1.5161566 \\ \text { H } & 5.2235550 & 2.6254047 & -1.3260088 \\ \text { H } & 4.0542119 & 4.6599143 & -0.4385414 \\ \text { H } & 1.6597643 & 4.5192374 & 0.2680914 \\ \text { H } & 1.9918337 & 2.3917657 & 2.3315253 \\ \text { H } & 2.0008086 & 2.4203877 & 4.8314787 \\ \text { H } & -0.1628544 & 2.3632098 & 6.0988546 \\ \text { H } & -2.3402853 & 2.3144979 & 4.8511483 \\ \text { H } & -2.3491216 & 2.3021981 & 2.3499382 \\ \text { H } & -0.6242202 & 4.8009068 & 1.8719330 \\ \text { H } & -1.5128075 & 7.0332898 & 1.2038535 \\ \text { H } & -2.3827172 & 7.3990232 & -1.1214394 \\ \text { H } & -2.3573169 & 5.5077818 & -2.7732318 \\ \text { H } & -1.4813898 & 3.2657731 & -2.1094302 \\ \text { C } & -2.4173534 & 0.4546445 & -3.0139673 \\ \text { O } & -2.9677695 & 0.3679612 & -4.2459539 \\ \text { O } & -2.8127064 & -0.3495591 & -2.1001202 \\ \text { H } & -3.6282422 & -0.3534007 & -4.1934056\end{array}$

\title{
Optimized Geometries (TS-BXni-e')
}

\author{
R3 \\ 79 \\ SCF Energy $=-2238.384370682$ \\ $\begin{array}{lll}\text { C } & -2.3079442 & 6.2475034\end{array}$ \\ 5.6941869
}




$\begin{array}{lrrr}\text { C } & -0.9459473 & 6.1309523 & 5.3361505 \\ \text { C } & -0.1044509 & 5.2750147 & 6.0739059 \\ \text { C } & -0.6224891 & 4.5315313 & 7.1461170 \\ \text { C } & -1.9777956 & 4.6452891 & 7.4954891 \\ \text { C } & -2.8172247 & 5.5126273 & 6.7745664 \\ \text { P } & -0.3597225 & 6.9705105 & 3.8197076 \\ \text { C } & 1.4681629 & 6.7687544 & 3.8616508 \\ \text { C } & 2.0410514 & 5.7424942 & 3.0842739 \\ \text { C } & 3.4313325 & 5.5590731 & 3.0620126 \\ \text { C } & 4.2555124 & 6.4065967 & 3.8201589 \\ \text { C } & 3.6879885 & 7.4346522 & 4.5967993 \\ \text { C } & 2.2971467 & 7.6205699 & 4.6187612 \\ \text { Pd } & -1.0306370 & 6.2170727 & 1.7943657 \\ \text { O } & 0.3026812 & 7.3386059 & 0.2810205 \\ \text { C } & 1.6189414 & 7.3533404 & 0.4244343 \\ \text { O } & 2.4215781 & 6.5521170 & -0.0320247 \\ \text { C } & -1.1604147 & 5.1740447 & -0.2449753 \\ \text { C } & -1.0700364 & 3.7690624 & 0.0016707 \\ \text { C } & -1.3721460 & 2.8499707 & -1.0198561 \\ \text { C } & -1.8010605 & 3.3026513 & -2.2803072 \\ \text { C } & -1.9539066 & 4.6766436 & -2.5210009 \\ \text { C } & -1.6313440 & 5.5983958 & -1.5094155 \\ \text { C } & -0.8459692 & 3.3103150 & 1.3974958 \\ \text { C } & 0.2703418 & 2.8129052 & 1.9924193 \\ \text { C } & 0.0670422 & 2.7331321 & 3.5087135 \\ \text { C } & -1.4494943 & 2.8636898 & 3.6905437 \\ \text { C } & -2.0266159 & 3.4618077 & 2.3767758 \\ \text { C } & 1.5857941 & 2.5760609 & 1.3676063 \\ \text { C } & 2.3569928 & 1.4376514 & 1.7135951 \\ \text { C } & 3.6357898 & 1.2455072 & 1.1685647 \\ \text { C } & 4.1728246 & 2.1843629 & 0.2710188 \\ \text { C } & 3.4118307 & 3.3094697 & -0.0901741 \\ \text { C } & 2.1298741 & 3.4977091 & 0.4435631 \\ \text { C } & -3.2486402 & 2.6531860 & 1.8872622 \\ \text { C } & -2.4474217 & 4.9438613 & 2.5021551 \\ \text { O } & 0.5313016 & 1.5516034 & 4.1634240 \\ \text { C } & -0.6224093 & 8.7580914 & 4.1285182 \\ \text { C } & -0.5623845 & 9.6209277 & 3.0126881 \\ \text { C } & -0.7070769 & 11.0037481 & 3.1948007 \\ \text { C } & -0.9196710 & 11.5306346 & 4.4808465 \\ \text { C } & -0.9824132 & 10.6717841 & 5.5907140 \\ \text { C } & -0.8313764 & 9.2861370 & 5.4188970 \\ \text { O } & 1.9989060 & 8.4315950 & 1.1718569 \\ & 2.9668906 & 8.3382015 & 1.2862601 \\ \text { H } & 0.5897674 & 3.6163396 & 3.9520289 \\ & & 3.4524035 & 4.5869999\end{array}$




\begin{tabular}{|c|c|c|c|}
\hline & -1.8352246 & 1.8346918 & 3.8386129 \\
\hline & -2.7494446 & 5.1874525 & 3.5370225 \\
\hline & -3.2797501 & 5.1929967 & 1.8103621 \\
\hline & -2.9883736 & 1.5811214 & 1.7685175 \\
\hline & -3.6137697 & 3.0300772 & 0.9108295 \\
\hline & -4.0785063 & 2.7293589 & 2.6202985 \\
\hline & 1.5021359 & 1.6247820 & 4.2193933 \\
\hline & 1.9228624 & 0.6860110 & 2.3881036 \\
\hline & 4.2152877 & 0.3502569 & 1.4427770 \\
\hline & 5.1799156 & 2.0362491 & -0.1482638 \\
\hline & 3.8128258 & 4.0672135 & -0.7792317 \\
\hline & 1.5747745 & 4.4023626 & 0.1670773 \\
\hline & -1.2892397 & 1.7719734 & -0.8138778 \\
\hline & -2.0328714 & 2.5737947 & -3.0722777 \\
\hline & -2.3092580 & 5.0319247 & -3.5005767 \\
\hline & -1.6997458 & $6.67 \varepsilon$ & -1.7192152 \\
\hline & -0.2213797 & 6.215 & -0.0162548 \\
\hline & -0.3897369 & 9.203 & 2.0088236 \\
\hline & -0.6566708 & 11.6742569 & 2.3238025 \\
\hline & -1.0390304 & 12.6160992 & 4.6184422 \\
\hline & -1.1488303 & 11.0817297 & 6.5985138 \\
\hline & -0.8784366 & 8.6131477 & 6.2880464 \\
\hline & 1.3920676 & 5.1136187 & 2.4577073 \\
\hline & 3.8566024 & 4.7677040 & 2.4275378 \\
\hline & 5.3480718 & 6.2741480 & 3.7999518 \\
\hline & 4.3360542 & 8.1032874 & 5.1838259 \\
\hline & 1.8556948 & 8.4368355 & 5.2098519 \\
\hline & 0.9575077 & 5.1870500 & 5.8030339 \\
\hline & 0.0382501 & 3.8548997 & 7.7080804 \\
\hline & -2.3825173 & 4.0570246 & 8.3326065 \\
\hline & -3.8786386 & 5.6090009 & 7.0482317 \\
\hline & -2.9681455 & 6.9215124 & 5.1262417 \\
\hline \\
\hline \\
\hline \multicolumn{4}{|c|}{ Energy $=-22$} \\
\hline & -0.1128410 & 8.5967984 & 1.3540464 \\
\hline & -1.0677323 & 7.5942731 & 1.6343705 \\
\hline & -1.1794993 & 7.0870000 & 2.9433058 \\
\hline & -0.3497881 & 7.5825991 & 3.9628691 \\
\hline & 0.5883722 & 8.5881474 & 3.6835930 \\
\hline & 0.7034062 & 9.0969986 & 2.3777357 \\
\hline & -2.0078736 & 6.8778099 & 0.2341621 \\
\hline & -3.2887289 & 5.8235376 & 1.0316233 \\
\hline & -4.5563123 & 6.3262878 & 1.3845774 \\
\hline & -5.5052015 & 5.4766765 & 1.974612 \\
\hline
\end{tabular}




\begin{tabular}{|c|c|c|c|}
\hline & -5.1956675 & 4.1273902 & 2.2178236 \\
\hline & -3.9309921 & 3.6232170 & 1.8697205 \\
\hline & -2.9816598 & 4.4675043 & 1.2763894 \\
\hline & -0.8954483 & 5.4468625 & -1.1683523 \\
\hline & 0.7917862 & 5.6804486 & -0.0314320 \\
\hline & 1.9500465 & 4.6583473 & -0.1234210 \\
\hline & 1.3690254 & 3.2691809 & -0.4109831 \\
\hline & 1.2231013 & 2.5144117 & 0.7164912 \\
\hline & 1.7260945 & 3.3046709 & 1.9247385 \\
\hline & 2.5644017 & 4.4332422 & 1.2846034 \\
\hline & 0.5426162 & 1.2170893 & 0.8706442 \\
\hline & 0.7224152 & 0.4565031 & 2.05 \\
\hline & 0.0943770 & -0.7850912 & 2.2206426 \\
\hline & -0.7432832 & -1.2 & 61123 \\
\hline & -0.9520633 & -0.5 & 67150 \\
\hline & -0.322 & 0.6 & -0.1 \\
\hline & 0.99 & $3 . c$ & -1.8 \\
\hline & 1.7841255 & 2.2 & -2.6 \\
\hline & 1.5232494 & 2.1 & -4.0 \\
\hline & 0.4783411 & 2.9 & -4.5 \\
\hline & -0.3325741 & 3.72 & -3.7 \\
\hline & -0.0998180 & 3.772 & -2.3410757 \\
\hline & 0.6367872 & 3.7544431 & 2.7434258 \\
\hline & 2.9827848 & 5.1029284 & -1.1676993 \\
\hline & -2.5199547 & 2.9737158 & -1.54 \\
\hline & -2.9448588 & 8.2923463 & -0.4645111 \\
\hline & -3.1332359 & 9.4 & 0.2538731 \\
\hline & -3.8871531 & 10.5340480 & -0.3090261 \\
\hline & -4.4583933 & 10.3821729 & -1.5838124 \\
\hline & -4.2776012 & 9.1837806 & -2.29 \\
\hline & -3.5227820 & 8.1382163 & -1.7442545 \\
\hline & 2.3491157 & 2.692 & 95197 \\
\hline & 3.6121100 & 4.0836021 & 91873 \\
\hline & 2.5733687 & 5.3432765 & 1.9170325 \\
\hline & 0.4232681 & 5.6925846 & 1.0131593 \\
\hline & 1.1520693 & 6.7089099 & -0.2547251 \\
\hline & 3.4657930 & 6.0482567 & -0.8459392 \\
\hline & 2.5001937 & 5.2812095 & -2.1495996 \\
\hline & 3.7748481 & 4.3385622 & -1.3057534 \\
\hline & -0.0549140 & 4.0785651 & 2.1344696 \\
\hline & -0.5219091 & 1.2737004 & -1.0337693 \\
\hline & -1.6214035 & -0.9228343 & -0.7412792 \\
\hline & -1.2397603 & -2.2702636 & 1.3482124 \\
\hline & 0.2590941 & -1.3571314 & 3.146765 \\
\hline & 1.3616874 & 0.8477622 & 2.8561948 \\
\hline & -1.2781744 & 3.4742800 & -1.725705 \\
\hline
\end{tabular}




$\begin{array}{rrrr}\text { H } & -1.1929073 & 4.2824302 & -4.1488412 \\ \text { H } & 0.2850261 & 2.9393258 & -5.6712226 \\ \text { H } & 2.1485676 & 1.5686204 & -4.7007913 \\ \text { H } & 2.6142357 & 1.6427835 & -2.2368636 \\ \text { H } & -4.8033993 & 7.3794831 & 1.1841613 \\ \text { H } & -6.4982898 & 5.8706516 & 2.2385166 \\ \mathrm{H} & -5.9473527 & 3.4641350 & 2.6725758 \\ \mathrm{H} & -3.6807310 & 2.5662067 & 2.0445906 \\ \mathrm{H} & -2.0077806 & 4.0644891 & 0.9547293 \\ \mathrm{H} & -2.6903518 & 9.6115907 & 1.2537344 \\ \mathrm{H} & -4.0299611 & 11.4690942 & 0.2539181 \\ \mathrm{H} & -5.0477874 & 11.2008681 & -2.0232276 \\ \mathrm{H} & -4.7272993 & 9.0595765 & -3.2926559 \\ \mathrm{H} & -3.3961820 & 7.1873960 & -2.2841696 \\ \mathrm{H} & -1.9121035 & 6.2979198 & 3.1664659 \\ \mathrm{H} & -0.4386411 & 7.1750410 & 4.9808134 \\ \mathrm{H} & 1.2363233 & 8.9755415 & 4.4845153 \\ \mathrm{H} & 1.4408074 & 9.8824368 & 2.1532266 \\ \mathrm{H} & -0.0117430 & 8.9858145 & 0.3287821 \\ \mathrm{C} & -3.2757979 & 3.9295311 & -1.9433495 \\ \mathrm{O} & -2.9047958 & 5.1054174 & -2.2084956 \\ \mathrm{O} & -4.5901743 & 3.6502140 & -2.0999836 \\ \mathrm{H} & -4.6929793 & 2.7066788 & -1.8597325\end{array}$

S3

79

SCF Energy $=-2238.387563153$

5.3336687

C -1.3721945

6.0124823

$-1.3721945$

6.0788551

6.0910190

C $\quad-0.8599683$

6.4601895

7.3490027

C $\quad-1.5489796$

6.1050977

8.5185622

$-2.7444481$

5.3691931

8.4374402

$-3.2526367$

4.9832548

7.1854832

$-0.5447356$

6.5387122

4.5298681

C $\quad 1.2445273$

6.5665385

4.9185907

5.3989395

5.4553244

C $\quad 3.2238064$

5.3103009

5.5905385

C 4.0383251

6.3934091

5.2183584

C $\quad 3.4542096$

7.5685819

4.7193578

C $\quad 2.0615015$

7.6557432

4.5582936

$\mathrm{Pd} \quad-0.9894452$

5.4462725

2.6028184

C $\quad-0.1258369$

3. 7580524

3.3691026

C $\quad-0.3400463$

2.4818794

2.5583643

C $\quad 0.0331624$

2.6978596

1.0879347

C $\quad 1.2875112$

2.2604192

0.7951749

C 1.9649754

1.7347965

2.0583332 


\begin{tabular}{rrrr} 
C & 0.7639221 & 1.4671485 & 2.9959306 \\
$\mathrm{C}$ & -0.9245533 & 3.4098169 & 0.2218572 \\
$\mathrm{C}$ & -1.2378851 & 4.7573796 & 0.5533144 \\
$\mathrm{C}$ & -2.2209071 & 5.4339637 & -0.2123189 \\
$\mathrm{C}$ & -2.8832853 & 4.7936631 & -1.2696237 \\
$\mathrm{C}$ & -2.5433878 & 3.4685692 & -1.5978057 \\
$\mathrm{C}$ & -1.5664122 & 2.7798469 & -0.8608218 \\
$\mathrm{C}$ & 2.0226152 & 2.4439852 & -0.4688536 \\
$\mathrm{C}$ & 1.8715021 & 3.6207744 & -1.2409404 \\
$\mathrm{C}$ & 2.5731958 & 3.7810500 & -2.4430580 \\
$\mathrm{C}$ & 3.4433779 & 2.7756189 & -2.8985223 \\
$\mathrm{C}$ & 3.6171362 & 1.6107337 & -2.1322607 \\
$\mathrm{C}$ & 2.9195436 & 1.4508960 & -0.9266821 \\
$\mathrm{C}$ & -1.7646010 & 1.9386419 & 2.7361581 \\
$\mathrm{O}$ & 2.8918192 & 2.6823721 & 2.5705877 \\
$\mathrm{O}$ & 2.2244123 & 5.2155510 & 1.7719684 \\
$\mathrm{C}$ & -1.0198225 & 8.2662261 & 4.1551608 \\
$\mathrm{C}$ & -0.6981421 & 8.7534615 & 2.8647302 \\
$\mathrm{C}$ & -1.0706358 & 10.0572299 & 2.5035053 \\
$\mathrm{C}$ & -1.7694709 & 10.8705457 & 3.4118641 \\
$\mathrm{C}$ & -2.0970468 & 10.3808979 & 4.6880757 \\
$\mathrm{C}$ & -1.7251295 & 9.0805053 & 5.0633018 \\
$\mathrm{H}$ & 2.5341751 & 0.7937036 & 1.8842039 \\
$\mathrm{H}$ & 1.0578538 & 1.5562342 & 4.0606254 \\
$\mathrm{H}$ & 0.3757008 & 0.4398615 & 2.8283312 \\
$\mathrm{H}$ & 0.9363617 & 4.0643595 & 3.3695485 \\
$\mathrm{H}$ & -0.5214546 & 3.6393616 & 4.4000037 \\
$\mathrm{H}$ & -1.9154961 & 1.0137102 & 2.1437526 \\
$\mathrm{H}$ & -2.5134017 & 2.6860936 & 2.4032768 \\
$\mathrm{H}$ & -1.9621843 & 1.7050929 & 3.8028531 \\
$\mathrm{H}$ & 2.6600901 & 3.5785923 & 2.2164507 \\
$\mathrm{H}$ & 1.2290304 & 4.4311680 & -0.8729126 \\
$\mathrm{H}$ & -1.9858123 & 8.6948915 & 6.0601261 \\
$\mathrm{H}$ & 2.4486462 & 4.7086848 & -3.0224189 \\
$\mathrm{H}$ & 3.9950403 & 2.9048457 & -3.8423539 \\
$\mathrm{H}$ & 4.3043399 & 0.8217542 & -2.4747774 \\
$\mathrm{H}$ & 3.0615959 & 0.5362812 & -0.3315125 \\
$\mathrm{H}$ & -1.3007015 & 1.7427493 & -1.1149515 \\
$\mathrm{H}$ & -3.0461345 & 2.9656849 & -2.4384241 \\
$\mathrm{H}$ & -3.6493724 & 5.3287385 & -1.8512330 \\
$\mathrm{H}$ & -2.4425215 & 6.4924308 & 0.0053926 \\
\hline & -0.1875298 & 5.5296778 & 0.7547869 \\
$\mathrm{H}$ & -0.8125197 & 10.4339815 & 1.5026145 \\
$\mathrm{H}$ & -2.6479611 & 11.0161414 & 3.1229928 \\
& & & \\
$\mathrm{H}$ & &
\end{tabular}




\begin{tabular}{|c|c|c|c|}
\hline H & 1.2033783 & 4.5503061 & 5.7572532 \\
\hline $\mathrm{H}$ & 3.6741842 & 4.3850168 & 5.9780128 \\
\hline $\mathrm{H}$ & 5.1313203 & 6.3208134 & 5.3219901 \\
\hline & 4.0854948 & 8.4274347 & 4.4441480 \\
\hline & 1.6152990 & 8.5665729 & 4.1363097 \\
\hline & 0.0815690 & 7.0271287 & 7.4075173 \\
\hline & -1.1491425 & 6.4011517 & 9.5001734 \\
\hline & -3.2790055 & 5.0884923 & 9.3575619 \\
\hline & -4.1830272 & 4.3993010 & 7.1223142 \\
\hline & -2.9406081 & 5.0261264 & 5.0221817 \\
\hline & 1.8404560 & 6.2915201 & 1.2825941 \\
\hline & 0.7724201 & 6.5269049 & 0.5990588 \\
\hline & 2.5937844 & 7.4243112 & 1.4834109 \\
\hline & 3.3359659 & 7.1195772 & 2.0465164 \\
\hline \\
\hline \\
\hline \multicolumn{4}{|c|}{85} \\
\hline & -5.0098986 & 7.2424207 & 1.0717648 \\
\hline & -4.7284723 & 6.7356164 & 2.3 \\
\hline & -5.7493630 & 6.6778467 & 3.3313985 \\
\hline & -7.0445229 & 7.1171545 & 3.0133102 \\
\hline & -7.3241797 & 7.6204073 & 1.7317769 \\
\hline & -6.3053407 & 7.6853966 & 0.7658101 \\
\hline & -2.9957648 & 6.2400245 & 2.7100829 \\
\hline & -3.1010171 & 5.1913415 & 4.2092508 \\
\hline & -2.2366564 & 5.4024067 & 5.3016915 \\
\hline & -2.2257790 & 4.4993019 & 6.3765723 \\
\hline & -3.0743944 & 3.3801028 & 6.3700690 \\
\hline & -3.9391810 & 3.1653581 & 5.2832394 \\
\hline C & -3.9498277 & 4.0613548 & 4.2041081 \\
\hline $\mathrm{Pd}$ & -1.6784163 & 5.4937382 & 1.0105372 \\
\hline $\mathrm{C}$ & -1.7055594 & 3.5597701 & 1.6392742 \\
\hline 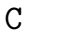 & -0.3920004 & 2.8317258 & 1.9157319 \\
\hline 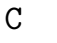 & 0.5423330 & 2.8484263 & 0.7054267 \\
\hline & 0.9077666 & 1.5929757 & 0.3036580 \\
\hline & 0.4174773 & 0.5562147 & 1.3048366 \\
\hline & -0.6814292 & 1.3044436 & 2.0871343 \\
\hline & 1.0827559 & 4.1370670 & 0.2342719 \\
\hline & 2.4910664 & 4.2740322 & 0.1672238 \\
\hline & 3.0877365 & 5.5102025 & -0.1033725 \\
\hline & 2.2802601 & 6.6428502 & -0.3098733 \\
\hline & 0.8864037 & 6.5139795 & -0.2676741 \\
\hline & 0.2498318 & 5.2686673 & -0.0040144 \\
\hline & 1.7234214 & 1.2242225 & -0.8658907 \\
\hline & 2.5604783 & 0.0817984 & -0.829525 \\
\hline
\end{tabular}




\begin{tabular}{|c|c|c|c|}
\hline & 3.3241362 & -0.2813705 & -1.9485378 \\
\hline & 3.2654352 & 0.4816525 & -3.1264633 \\
\hline & 2.4274519 & 1.6100047 & -3.1794765 \\
\hline & 1.6628944 & 1.9764074 & -2.0650678 \\
\hline & 0.3002606 & 3.4359650 & 3.1550822 \\
\hline & 1.4998191 & 0.0891569 & 2.1305778 \\
\hline & -2.1386355 & 7.2515738 & -0.4207085 \\
\hline & -2.1955949 & 7.7878535 & 3.3015271 \\
\hline & -0.8108232 & 7.9553580 & 3.0895529 \\
\hline & -0.1640665 & 9.1102722 & 3.5554448 \\
\hline & -0.8955770 & 10.1032864 & 4.2277393 \\
\hline & -2.2769767 & 9.9415344 & 4.4327035 \\
\hline & -2.9288300 & 8.7883368 & 3.9703985 \\
\hline & 0.0285983 & -0.36153 & 0.8173381 \\
\hline & -0.7030273 & 0.988 & 34697 \\
\hline & -1.6699 & 1.06 & 33422 \\
\hline & -2.2122729 & 3.1 & 5008 \\
\hline & -2.3648613 & 3.44 & 2641 \\
\hline & 1.2106460 & 2.86 & 3.4 \\
\hline & -0.3887021 & 3.4279825 & 4.0 \\
\hline & 0.5941444 & 4.4865496 & 2.9610471 \\
\hline & 2.0135897 & 0.8836821 & 2.375 \\
\hline & 2.6170210 & -0.4989306 & 0.1028466 \\
\hline & 3.9741713 & -1.1686190 & -1.8983175 \\
\hline & 3.8650704 & 0.1950873 & -4.0039176 \\
\hline & 2.3624848 & 2.2058538 & -4.1028576 \\
\hline & 0.9946465 & 2.8478668 & 97447 \\
\hline & -0.8633761 & 5.2513451 & -0.8946889 \\
\hline & 0.2577727 & 7.3994847 & -0.4469443 \\
\hline & 2.7373564 & 7.6244756 & -0.5091089 \\
\hline & 4.1848752 & 5.5934181 & -0.1386505 \\
\hline & 3.1163927 & 3.3894041 & 0.3592226 \\
\hline & -0.2517083 & 7.1777800 & 2.5431481 \\
\hline & 0.9151804 & 9.2364884 & 3.3823081 \\
\hline & -0.3895076 & 11.0122130 & 4.5871410 \\
\hline & -2.8530635 & 10.7227365 & 4.9514545 \\
\hline & -4.0120735 & 8.6683649 & 4.1209662 \\
\hline & -1.5657879 & 6.2738500 & 5.3068683 \\
\hline & -1.5477136 & 4.6731501 & 7.2256649 \\
\hline & -3.0620687 & 2.6727172 & 7.2128050 \\
\hline & -4.6054740 & 2.2896998 & 5.2712264 \\
\hline & -4.6250576 & 3.8856508 & 3.3524796 \\
\hline & -5.5325895 & 6.2878039 & 4.3368074 \\
\hline & -7.8389720 & 7.0673755 & 3.7734892 \\
\hline & -8.3408147 & 7.9627676 & 1.4854012 \\
\hline & -6.5189005 & 8.0817689 & -0.238290 \\
\hline
\end{tabular}




$\begin{array}{llll}\mathrm{H} & -4.2031643 & 7.2990303 & 0.3235752 \\ \mathrm{C} & -2.1313861 & 6.7472317 & -1.5758830 \\ \mathrm{O} & -2.7470164 & 7.4533606 & -2.5553734 \\ \mathrm{O} & -1.6027331 & 5.6272598 & -1.9250826 \\ \mathrm{H} & -2.6258509 & 6.9325420 & -3.3758587\end{array}$

\section{Optimized Geometries (BXne')}

\begin{tabular}{lrrr} 
R3 & \multicolumn{3}{l}{} \\
79 & \multicolumn{4}{l}{} \\
SCF & Energy $=-2238.403176950$ & \\
C & -1.3299944 & 2.9218906 & 2.3524434 \\
C & -0.1375799 & 2.3531814 & 1.8507678 \\
C & 0.8888156 & 1.9977082 & 2.7477157 \\
C & 0.7207557 & 2.1965405 & 4.1278712 \\
C & -0.4664195 & 2.7603493 & 4.6216529 \\
C & -1.4877426 & 3.1321849 & 3.7298695 \\
P & -0.0566067 & 1.9450245 & 0.0633459 \\
C & 1.7085071 & 1.4782895 & -0.1839354 \\
C & 2.0319531 & 0.1097585 & -0.0739955 \\
C & 3.3421076 & -0.3338125 & -0.3094203 \\
C & 4.3360241 & 0.5919946 & -0.6694703 \\
C & 4.0189362 & 1.9594891 & -0.7792943 \\
C & 2.7100724 & 2.4058144 & -0.5327632 \\
Pd & -1.3703725 & 0.1335633 & -0.6422242 \\
O & -0.4944093 & -0.0644058 & -2.9193989 \\
C & 0.8523372 & -0.2031207 & -3.0565548 \\
O & 1.4477346 & -1.2577797 & -3.0920445 \\
C & -2.1062821 & -1.7026497 & -1.1388067 \\
C & -1.7236378 & -2.7575024 & -0.2625694 \\
C & -1.8767504 & -4.1026399 & -0.6440412 \\
C & -2.4339327 & -4.4221087 & -1.8954126 \\
C & -2.8442140 & -3.3980732 & -2.7607746 \\
C & -2.6788411 & -2.0458909 & -2.3846153 \\
C & -1.1695671 & -2.3267556 & 1.0394503 \\
C & 0.0785522 & -2.5126929 & 1.5477254 \\
C & 0.2928642 & -1.6000661 & 2.7569170 \\
C & -1.1188954 & -1.1165736 & 3.1198904 \\
C & -2.0393609 & -1.3963761 & 1.8965634 \\
C & 1.1894815 & -3.2532703 & 0.9176114 \\
C & 2.1044413 & -3.9979022 & 1.7028367 \\
C & 3.1985333 & -4.6484866 & 1.1107720 \\
C & 3.4035903 & -4.5719733 & -0.2771448
\end{tabular}




\begin{tabular}{rrrr} 
C & 2.4971374 & -3.8458578 & -1.0701619 \\
$\mathrm{C}$ & 1.4023623 & -3.2008550 & -0.4813170 \\
$\mathrm{C}$ & -3.3319515 & -2.1272008 & 2.3204636 \\
$\mathrm{C}$ & -2.4192430 & -0.1224472 & 1.1003562 \\
$\mathrm{O}$ & 0.9168164 & -2.1806313 & 3.9065033 \\
$\mathrm{C}$ & -0.1981128 & 3.5770646 & -0.7664277 \\
$\mathrm{C}$ & -0.6300277 & 3.5860500 & -2.1093466 \\
$\mathrm{C}$ & -0.7195664 & 4.7981277 & -2.8100672 \\
$\mathrm{C}$ & -0.3908199 & 6.0070450 & -2.1730747 \\
$\mathrm{C}$ & 0.0303708 & 6.0027340 & -0.8323503 \\
$\mathrm{C}$ & 0.1292339 & 4.7918262 & -0.1287010 \\
$\mathrm{O}$ & 1.3882439 & 1.0242292 & -3.1529083 \\
$\mathrm{H}$ & 2.3596438 & 0.9147794 & -3.0753877 \\
$\mathrm{H}$ & 0.9178374 & -0.7402168 & 2.4089996 \\
$\mathrm{H}$ & -1.1297559 & -0.0556263 & 3.4342990 \\
$\mathrm{H}$ & -1.4445917 & -1.7245618 & 3.9882384 \\
$\mathrm{H}$ & -2.2585051 & 0.7864030 & 1.7079084 \\
$\mathrm{H}$ & -3.4756271 & -0.1511307 & 0.7688042 \\
$\mathrm{H}$ & -3.0976227 & -3.0598606 & 2.8738620 \\
$\mathrm{H}$ & -3.9408188 & -2.3950657 & 1.4333238 \\
$\mathrm{H}$ & -3.9465373 & -1.4775007 & 2.9778188 \\
$\mathrm{H}$ & 1.8742134 & -2.2017515 & 3.7255339 \\
$\mathrm{H}$ & 1.9234669 & -4.0878126 & 2.7834215 \\
$\mathrm{H}$ & 3.8923971 & -5.2278725 & 1.7395749 \\
$\mathrm{H}$ & 4.2641110 & -5.0794704 & -0.7394631 \\
$\mathrm{H}$ & 2.6439153 & -3.7576895 & -2.1567785 \\
$\mathrm{H}$ & 0.7229287 & -2.6177719 & -1.1152195 \\
$\mathrm{H}$ & -1.5520665 & -4.8993926 & 0.0427892 \\
$\mathrm{H}$ & -2.5548591 & -5.4758850 & -2.1891912 \\
$\mathrm{H}$ & -3.3034364 & -3.6446645 & -3.7311265 \\
$\mathrm{H}$ & -3.0325444 & -1.2569853 & -3.0715788 \\
$\mathrm{H}$ & -0.9021824 & -0.9751675 & -2.8922401 \\
$\mathrm{H}$ & -0.8936697 & 2.6361725 & -2.5985432 \\
$\mathrm{H}$ & -1.0561848 & 4.7993866 & -3.8577887 \\
$\mathrm{H}$ & -0.4690500 & 6.9579007 & -2.7218569 \\
$\mathrm{H}$ & 0.2835752 & 6.9489172 & -0.3301322 \\
$\mathrm{H}$ & 0.4563969 & 4.7870221 & 0.9220154 \\
$\mathrm{H}$ & 1.2350192 & -0.6135228 & 0.1576091 \\
$\mathrm{H}$ & 3.5669564 & -1.4079491 & -0.2386591 \\
$\mathrm{H}$ & 5.3619599 & 0.2477068 & -0.8705570 \\
$\mathrm{H}$ & 4.7976643 & 2.6845032 & -1.0621850 \\
& 2.4600022 & 3.4719166 & -0.6367424 \\
$\mathrm{H}$ & 1.8224159 & 1.5615178 & 2.3642878 \\
\hline
\end{tabular}




\begin{tabular}{lrrr} 
H & -2.1358810 & 3.2040530 & 1.6567498 \\
& & & \\
R4 & & & \\
79 & \multicolumn{3}{r}{} \\
SCF & Energy $=-2238.419766294$ & \\
C & 1.4122567 & 2.5902602 & 2.6046786 \\
C & 0.1535446 & 2.2134880 & 2.0818836 \\
C & -0.9248817 & 1.9987569 & 2.9631705 \\
C & -0.7425112 & 2.1472802 & 4.3481844 \\
C & 0.5080565 & 2.5240960 & 4.8611107 \\
C & 1.5846813 & 2.7525922 & 3.9857640 \\
P & 0.0542601 & 1.9705128 & 0.2649856 \\
C & -1.6415032 & 1.3283658 & -0.0461005 \\
C & -2.5021203 & 1.9496957 & -0.9721482 \\
C & -3.7048543 & 1.3236203 & -1.3427321 \\
C & -4.0613269 & 0.0872442 & -0.7808192 \\
C & -3.2120615 & -0.5287327 & 0.1550777 \\
C & -2.0020391 & 0.0792033 & 0.5128647 \\
Pd & 1.3290421 & 0.2542975 & -0.7359889 \\
C & 2.3421218 & -0.2565210 & 0.9723072 \\
C & 2.5198380 & -1.7534502 & 1.3356123 \\
C & 1.4131858 & -2.5335304 & 0.6116352 \\
C & 0.3463781 & -2.7997477 & 1.4249362 \\
C & 0.6456033 & -2.3009930 & 2.8418585 \\
C & 2.1636681 & -2.0402247 & 2.8184338 \\
C & -0.9928331 & -3.3038926 & 1.0753717 \\
C & -1.9910248 & -3.4096795 & 2.0796509 \\
C & -3.2808723 & -3.8730430 & 1.7819367 \\
C & -3.6225209 & -4.2353402 & 0.4683220 \\
C & -2.6540124 & -4.1177098 & -0.5437734 \\
C & -1.3633691 & -3.6637379 & -0.2465919 \\
C & 1.6432935 & -2.7040722 & -0.8370461 \\
C & 1.7794366 & -3.9688044 & -1.4488124 \\
C & 2.1382912 & -4.0602636 & -2.8024916 \\
C & 2.3610341 & -2.8893555 & -3.5479468 \\
C & 2.2121030 & -1.6278930 & -2.9431190 \\
C & 1.8659029 & -1.5120342 & -1.5787054 \\
O & -0.1410760 & -1.1511612 & 3.2039144 \\
C & 3.9293169 & -2.2388696 & 0.9680123 \\
O & -1.0544101 & -1.0244919 & -2.3647959 \\
C & 0.0157178 & 3.6885858 & -0.3855014 \\
C & -0.4992239 & 4.7589279 & 0.3754417 \\
C & -0.5305520 & 6.0528925 & -0.1658633 \\
C & -0.0535181 & 6.2847521 & -1.4678323 \\
C & 0.4576790 & 5.2198502 & -2.2282377 \\
& 0.4991021 & 3.9238207 & -1.6897335
\end{tabular}




$\begin{array}{lrrr}\text { H } & 0.3769535 & -3.0597011 & 3.6063421 \\ \text { H } & 2.6947510 & -2.9587572 & 3.1436534 \\ \text { H } & 2.4496751 & -1.2254124 & 3.5139746 \\ \text { H } & 1.8038895 & 0.2576578 & 1.7919382 \\ \text { H } & 3.3222282 & 0.2554239 & 0.8489776 \\ \text { H } & 4.6846504 & -1.7394286 & 1.6090908 \\ \text { H } & 4.1641278 & -2.0031160 & -0.0885884 \\ \text { H } & 4.0276878 & -3.3355981 & 1.1045145 \\ \text { H } & 0.2150516 & -0.3809221 & 2.7183619 \\ \text { H } & -0.6270805 & -3.5886162 & -1.0557500 \\ \text { H } & -2.9046539 & -4.3840444 & -1.5820698 \\ \text { H } & -4.6348421 & -4.5982468 & 0.2344307 \\ \text { H } & -4.0293694 & -3.9430396 & 2.5862238 \\ \text { H } & -1.7627950 & -3.0908611 & 3.1047863 \\ \text { H } & -0.3406405 & -1.1998009 & -1.6791231 \\ \text { H } & 2.3658063 & -0.7185661 & -3.5457744 \\ \text { H } & 2.6530753 & -2.9589450 & -4.6078630 \\ \text { H } & 2.2514476 & -5.0480262 & -3.2749769 \\ \text { H } & 1.6069247 & -4.8792372 & -0.8536949 \\ \text { H } & -2.2215618 & 2.9136299 & -1.4209035 \\ \text { H } & -4.3665979 & 1.8129274 & -2.0740357 \\ \text { H } & -5.0031298 & -0.4025484 & -1.0721116 \\ \text { H } & -3.4744497 & -1.5016157 & 0.5939068 \\ \text { H } & -1.3404487 & -0.4337061 & 1.2273156 \\ \text { H } & -0.8725185 & 4.5761998 & 1.3943430 \\ \text { H } & -0.9300872 & 6.8859587 & 0.4324101 \\ \text { H } & -0.0784225 & 7.3010875 & -1.8897077 \\ \text { H } & 0.8325273 & 5.3982887 & -3.2474461 \\ \text { H } & 0.8891299 & 3.0830834 & -2.2818500 \\ \text { H } & -1.9063450 & 1.7010378 & 2.5679998 \\ \text { H } & -1.5865847 & 1.9621712 & 5.0289630 \\ \text { H } & 0.6482651 & 2.6339462 & 5.9469687 \\ \text { H } & 2.5680905 & 3.0458797 & 4.3829735 \\ \text { H } & 2.2586755 & 2.7519344 & 1.9186584 \\ \text { C } & -0.7454246 & 0.1244189 & -2.9521647 \\ \text { O } & 0.2193453 & 0.8402467 & -2.6734096 \\ \text { O } & -1.5934596 & 0.4656891 & -3.9265666 \\ \text { H } & -2.3095069 & -0.2028614 & -3.9453276 \\ & & & \\ \text { S3 } & & & \\ \text { 79 } & & & \\ \text { SCF } & \text { Energy }=-2238.411308685 & \\ \text { C } & -0.3658341 & -2.4395423 & 3.6915636 \\ \text { C } & 0.3791844 & -1.2428446 & 3.6560674 \\ \text { C } & 1.4157869 & -1.0348627 & 4.5905208 \\ & 1.6943304 & -2.0139172 & 5.5559767\end{array}$




\begin{tabular}{|c|c|c|c|}
\hline & 0.9435373 & -3.2023504 & \\
\hline & -0.0859356 & -3.4149236 & 4.6615003 \\
\hline & -0.0579837 & -0.0019246 & 2.3825266 \\
\hline & 1.5365536 & 0.8113490 & 1.9748742 \\
\hline & 2.6182502 & -0.0098756 & 1.5866673 \\
\hline & 3.7902282 & 0.5620667 & 1.0761267 \\
\hline & 3.8996445 & 1.9576009 & 0.9575260 \\
\hline & 2.8350945 & 2.7787043 & 1.3610511 \\
\hline & 1.6545482 & 2.2107731 & 1.8667109 \\
\hline & -1.2005961 & -0.7037452 & 0.4431769 \\
\hline & 0.3597568 & -1.8527318 & -0.2237451 \\
\hline & 0.1716298 & -2.6267074 & -1.52 \\
\hline & -0.2781187 & -1.6332170 & -2.60 \\
\hline & 0.761 & -1.21 & -3.38 \\
\hline & 2.053 & -1.9 & -2.9 \\
\hline & 1.56 & -3.06 & $-2 . c$ \\
\hline & -1.67 & -1.1 & -2. \\
\hline & -2.23 & -0.8 & -1.2 \\
\hline & -3.5744227 & -0.4 & -1.1 \\
\hline & -4.3657893 & -0.34 & -2.3 \\
\hline & -3.8118295 & -0.6434855 & -3.6 \\
\hline & -2.4757636 & -1.0576548 & -3.7 \\
\hline & 0.8106520 & -0.0717256 & -4.3 \\
\hline & -0.0456111 & 1.0488515 & -4.1 \\
\hline & 0.0751527 & 2.16 & -5.0 \\
\hline & 1.0500455 & 2.19 & -6.0 \\
\hline & 1.9096931 & $1.0 s$ & -6.1 \\
\hline & 1.7965913 & -0.02 & -5.3 \\
\hline & -0.7803023 & -3.8 & -1.3 \\
\hline & 2.9911 & -1.048 & -2.3 \\
\hline & 1.6240 & $1.0 \varsigma$ & -1.3 \\
\hline & -1.0477 & 1.2734948 & 3.2 \\
\hline & -1.6997569 & $2.24 €$ & 2.46 \\
\hline & -2.4956494 & 3.2300091 & 3.0701099 \\
\hline & -2.6618522 & 3.2409792 & 4.4655614 \\
\hline & -2.0256022 & 2.2681193 & 5.2543768 \\
\hline & -1.2187926 & 1.2872502 & 4.6557736 \\
\hline & 2.5731048 & -2.3642090 & -3.8769636 \\
\hline & 2.3074365 & -3.2787615 & -1.2769757 \\
\hline & 1.4251901 & -3.9983444 & -2.6615525 \\
\hline & 1.1702967 & -1.1055069 & -0.319358 \\
\hline & 0.5345536 & -2.5302196 & 0.6415339 \\
\hline & -0.9477377 & -4.3423239 & -2.3053510 \\
\hline & -1.7629574 & -3.4809170 & -0.9578037 \\
\hline & -0.3536456 & -4.5435123 & -0.620950 \\
\hline & 2.4961924 & -0.2824432 & -2.012623 \\
\hline
\end{tabular}




$\begin{array}{rrrr}\mathrm{H} & -0.8004592 & 1.0529110 & -3.3784470 \\ \mathrm{H} & -0.5933028 & 3.0257459 & -4.8634655 \\ \mathrm{H} & 1.1439588 & 3.0663798 & -6.6853864 \\ \mathrm{H} & 2.6829901 & 1.1001501 & -6.9636260 \\ \mathrm{H} & 2.4907900 & -0.8646918 & -5.4518372 \\ \mathrm{H} & -2.0339039 & -1.2895825 & -4.6886029 \\ \mathrm{H} & -4.4267491 & -0.5601781 & -4.5204482 \\ \mathrm{H} & -5.4169286 & -0.0230354 & -2.2741695 \\ \mathrm{H} & -4.0265598 & -0.2189461 & -0.2176673 \\ \mathrm{H} & -0.6223104 & 1.0106445 & -0.5874061 \\ \mathrm{H} & -1.5718557 & 2.2425141 & 1.3693688 \\ \mathrm{H} & -2.9931314 & 3.9873579 & 2.4458677 \\ \mathrm{H} & -3.2932863 & 4.0077588 & 4.9392433 \\ \mathrm{H} & -2.1577770 & 2.2711742 & 6.3470155 \\ \mathrm{H} & -0.7241544 & 0.5258411 & 5.2765645 \\ \mathrm{H} & 2.5383486 & -1.1035923 & 1.6700239 \\ \mathrm{H} & 4.6135142 & -0.0892699 & 0.7484775 \\ \mathrm{H} & 4.8177296 & 2.4040907 & 0.5468125 \\ \mathrm{H} & 2.9157540 & 3.8730434 & 1.2774720 \\ \mathrm{H} & 0.8207580 & 2.8609296 & 2.1676142 \\ \mathrm{H} & 2.0078539 & -0.1078797 & 4.5521080 \\ \mathrm{H} & 2.5042484 & -1.8502997 & 6.2830025 \\ \mathrm{H} & 1.1679659 & -3.9698951 & 6.3495569 \\ \mathrm{H} & -0.6683147 & -4.3482418 & 4.6837202 \\ \mathrm{H} & -1.1601059 & -2.6006836 & 2.9444018 \\ \mathrm{C} & 0.8292849 & 2.0279456 & -1.2917195 \\ \mathrm{O} & -0.4296172 & 1.9744821 & -0.8685099 \\ \mathrm{O} & 1.1259076 & 3.2795006 & -1.6914092 \\ \mathrm{H} & 2.0442609 & 3.2281303 & -2.0271394\end{array}$

S4

79

SCF Energy $=-2238.409022733$

$\begin{array}{lrrr}\text { C } & -0.4812136 & 3.9118792 & -2.0798671 \\ \text { C } & -0.9533640 & 3.5021043 & -0.8125591 \\ \text { C } & -2.2377890 & 3.9000463 & -0.3910353 \\ \text { C } & -3.0416060 & 4.6910350 & -1.2293153 \\ \text { C } & -2.5710101 & 5.0896110 & -2.4903442 \\ \text { C } & -1.2867853 & 4.7010174 & -2.9109837 \\ \text { P } & 0.1238374 & 2.3773013 & 0.1690381 \\ \text { C } & -0.6753187 & 2.3242377 & 1.8218109 \\ \text { C } & -0.1439918 & 2.9669210 & 2.9557976 \\ \text { C } & -0.7722101 & 2.8252181 & 4.2046749 \\ \text { C } & -1.9344208 & 2.0480057 & 4.3290023 \\ \text { C } & -2.4680218 & 1.4015760 & 3.1999780 \\ \text { C } & -1.8357272 & 1.5288609 & 1.9555945\end{array}$




\begin{tabular}{|c|c|c|c|}
\hline $\mathrm{Pd}$ & 0.5670354 & 0.2239421 & -0.7237280 \\
\hline C & 0.8388641 & -0.4654915 & 1.1763552 \\
\hline C & 1.5461328 & -1.7986538 & 1.4310178 \\
\hline C & 0.8499656 & -2.9006645 & 0.6390888 \\
\hline c & 0.2888282 & -3.8534037 & 1.4480993 \\
\hline C & 0.7683039 & -3.6782695 & 2.8807018 \\
\hline C & 1.3071335 & -2.2320336 & 2.9162037 \\
\hline C & 0.9260115 & -2.8595636 & -0.8296233 \\
\hline c & 1.1246347 & -4.0691610 & -1.5422852 \\
\hline C & 1.3220441 & -4.0704653 & -2.9265311 \\
\hline c & 1.3336851 & -2.8499389 & -3.6219098 \\
\hline C & 1.1339525 & -1.6455920 & -2.9259032 \\
\hline C & 0.9238085 & -1.6179949 & -1.5256554 \\
\hline C & -0.6157550 & -4.9548696 & 1.0738983 \\
\hline C & -0.5800723 & -6.1892280 & 1.7678617 \\
\hline C & -1.4696983 & -7.2228302 & 1.4379172 \\
\hline C & -2.4154390 & -7.0467601 & 0.4147127 \\
\hline $\mathrm{C}$ & -2.4703190 & -5.8203267 & -0.2726550 \\
\hline C & -1.5851128 & -4.7862549 & 0.0541766 \\
\hline C & 3.0452193 & -1.7014778 & 1.0873452 \\
\hline 0 & 1.7618902 & -4.6685290 & 3.2107541 \\
\hline 0 & -0.1308808 & 1.0722490 & -2.8149385 \\
\hline C & 1.6451655 & 3.3739116 & 0.4166776 \\
\hline C & 2.8859744 & 2.7040748 & 0.4321129 \\
\hline $\mathrm{C}$ & 4.0719871 & 3.4259732 & 0.6407643 \\
\hline $\mathrm{C}$ & 4.0245779 & 4.8173652 & 0.8266922 \\
\hline C & 2.7897427 & 5.4901278 & 0.8008939 \\
\hline C & 1.6018218 & 4.7729775 & 0.5954719 \\
\hline $\mathrm{H}$ & -0.0377042 & -3.8433566 & 3.6257035 \\
\hline $\mathrm{H}$ & 2.2291660 & -2.1816145 & 3.5284745 \\
\hline $\mathrm{H}$ & 0.5608532 & -1.5648073 & 3.3934240 \\
\hline $\mathrm{H}$ & -0.2004016 & -0.5203542 & 1.5636831 \\
\hline $\mathrm{H}$ & 1.3613569 & 0.3413160 & 1.7379580 \\
\hline $\mathrm{H}$ & 3.5701315 & -2.6544927 & 1.3043841 \\
\hline $\mathrm{H}$ & 3.5270478 & -0.9023842 & 1.6883108 \\
\hline $\mathrm{H}$ & 3.1866707 & -1.4647363 & 0.0143807 \\
\hline $\mathrm{H}$ & 2.3545736 & -4.7159819 & 2.4345576 \\
\hline $\mathrm{H}$ & 0.1740972 & -6.3240866 & 2.5571693 \\
\hline $\mathrm{H}$ & -1.4207928 & -8.1772438 & 1.9848741 \\
\hline $\mathrm{H}$ & -3.1120622 & -7.8588837 & 0.1560844 \\
\hline $\mathrm{H}$ & -3.2166575 & -5.6672474 & -1.0675714 \\
\hline $\mathrm{H}$ & -1.6383474 & -3.8248962 & -0.4772615 \\
\hline $\mathrm{H}$ & -1.4027034 & -0.1455643 & -1.3376361 \\
\hline $\mathrm{H}$ & 1.1478054 & -0.7026847 & -3.4923343 \\
\hline $\mathrm{H}$ & 1.5078975 & -2.8309426 & -4.7095948 \\
\hline $\mathrm{H}$ & 1.4841542 & -5.0201979 & -3.4590364 \\
\hline
\end{tabular}




$\begin{array}{lrrr}\text { H } & 1.1426601 & -5.0171131 & -0.9844372 \\ \text { H } & 2.9116003 & 1.6148542 & 0.2672091 \\ \text { H } & 5.0377710 & 2.8988275 & 0.6492639 \\ \text { H } & 4.9550446 & 5.3835383 & 0.9849584 \\ \text { H } & 2.7532669 & 6.5815311 & 0.9383426 \\ \text { H } & 0.6348047 & 5.2979803 & 0.5658074 \\ \text { H } & 0.7687814 & 3.5733250 & 2.8647905 \\ \text { H } & -0.3457803 & 3.3252013 & 5.0874854 \\ \text { H } & -2.4223562 & 1.9372231 & 5.3090723 \\ \text { H } & -3.3726016 & 0.7818694 & 3.2925373 \\ \text { H } & -2.2361820 & 0.9979224 & 1.0775258 \\ \text { H } & -2.6105469 & 3.5980996 & 0.5982132 \\ \text { H } & -4.0416097 & 5.0001094 & -0.8887058 \\ \text { H } & -3.2034482 & 5.7065461 & -3.1466121 \\ \text { H } & -0.9116518 & 5.0074949 & -3.8987852 \\ \text { H } & 0.5156044 & 3.5969328 & -2.4203165 \\ \text { C } & -1.3387624 & 0.8599981 & -2.9213541 \\ \text { O } & -2.0369746 & 1.3307492 & -3.9586826 \\ \text { O } & -2.0729406 & 0.1659457 & -2.0510351 \\ \text { H } & -2.9713049 & 1.0599234 & -3.8453408\end{array}$

\section{Optimized Geometries (BXnj)}

\begin{tabular}{lrrr} 
R3 & & & \\
73 & \multicolumn{3}{l}{} \\
SCF & Energy $=-1973.528249972$ & \\
C & -0.3054954 & -1.4253055 & -1.7866323 \\
C & -0.3352563 & -2.7089766 & -1.1786563 \\
C & -0.7453731 & -3.8191581 & -1.9633185 \\
C & -1.0632127 & -3.6753859 & -3.3188511 \\
C & -0.9799352 & -2.4137624 & -3.9330581 \\
C & -0.6014875 & -1.3016893 & -3.1612689 \\
C & 0.0358547 & -2.8621786 & 0.2383185 \\
C & 0.4283547 & -3.9936271 & 0.8970822 \\
C & 0.6651318 & -5.3487278 & 0.3563099 \\
C & -0.1167933 & -6.4477397 & 0.7838475 \\
C & 0.1372304 & -7.7423479 & 0.3030512 \\
C & 1.1763259 & -7.9626570 & -0.6152706 \\
C & 1.9599379 & -6.8786672 & -1.0493187 \\
C & 1.7100159 & -5.5869355 & -0.5665899 \\
C & -0.0508862 & -1.6660868 & 1.1921108 \\
C & -1.5271015 & -1.3474744 & 1.5135241 \\
C & 0.6919336 & -0.4443924 & 0.6643151
\end{tabular}




$\begin{array}{lrrr}\text { C } & 0.6780366 & -2.2033356 & 2.4636774 \\ \mathrm{C} & 0.5993754 & -3.7334241 & 2.3902986 \\ \mathrm{O} & -0.5242360 & -4.1983329 & 3.1622580 \\ \mathrm{H} & 1.5304334 & -4.2115944 & 2.7764425 \\ \mathrm{H} & 1.7447092 & -1.9010339 & 2.4386757 \\ \mathrm{H} & 0.2368886 & -1.8198120 & 3.4041674 \\ \mathrm{H} & 1.7348967 & -0.7026122 & 0.3830772 \\ \mathrm{H} & 0.6990768 & 0.3590353 & 1.4302689 \\ \mathrm{H} & -2.0020420 & -2.2167816 & 2.0087195 \\ \mathrm{H} & -2.0836822 & -1.1050174 & 0.5863564 \\ \mathrm{H} & -1.5940394 & -0.4713000 & 2.1898832 \\ \mathrm{H} & -0.4452652 & -5.1689035 & 3.2146852 \\ \mathrm{H} & -0.9618992 & -6.2685182 & 1.4660265 \\ \mathrm{H} & -0.4895253 & -8.5826675 & 0.6395495 \\ \mathrm{H} & 1.3734320 & -8.9763303 & -0.9959061 \\ \mathrm{H} & 2.7750907 & -7.0430775 & -1.7708099 \\ \mathrm{H} & 2.3187505 & -4.7358104 & -0.9065751 \\ \mathrm{H} & -0.8322362 & -4.8083791 & -1.4949755 \\ \mathrm{H} & -1.3799557 & -4.5546904 & -3.9003514 \\ \mathrm{H} & -1.2164994 & -2.2944168 & -5.0021388 \\ \mathrm{H} & -0.5522668 & -0.3096235 & -3.6509336 \\ \mathrm{Pd} & -0.1309467 & 0.4076336 & -1.0006948 \\ \mathrm{P} & -0.2063240 & 2.5790984 & -0.1412538 \\ \mathrm{C} & -1.1022904 & 3.8595067 & -1.1119352 \\ \mathrm{C} & -0.9159156 & 3.8524329 & -2.5107604 \\ \mathrm{H} & -0.2734243 & 3.0821685 & -2.9682593 \\ \mathrm{C} & -1.5410691 & 4.8178340 & -3.3135102 \\ \mathrm{H} & -1.3897573 & 4.8052792 & -4.4034385 \\ \mathrm{C} & -2.3663078 & 5.7922726 & -2.7262136 \\ \mathrm{H} & -2.8631816 & 6.5455698 & -3.3560933 \\ \mathrm{C} & -2.5621360 & 5.7989158 & -1.3350529 \\ \mathrm{H} & -3.2114455 & 6.5582493 & -0.8731843 \\ \mathrm{C} & -1.9328041 & 4.8377863 & -0.5277458 \\ \mathrm{H} & -2.0949845 & 4.8395392 & 0.5605813 \\ \mathrm{C} & 1.3972404 & 3.3949647 & 0.2472730 \\ \mathrm{C} & 2.5807506 & 2.6433754 & 0.1072474 \\ \mathrm{H} & 2.5137314 & 1.6034196 & -0.2488729 \\ \mathrm{C} & 3.8233489 & 3.2230941 & 0.4124400 \\ \mathrm{H} & 4.7445470 & 2.6313581 & 0.3016176 \\ \mathrm{C} & 3.8883924 & 4.5551601 & 0.8514102 \\ \mathrm{H} & 4.8622202 & 5.0105057 & 1.0875267 \\ \mathrm{C} & 2.7094841 & 5.3114175 & 0.9831782 \\ \mathrm{H} & 2.7602092 & 6.3580261 & 1.3200487 \\ & 1.4671698 & 4.7353997 & 0.6815428 \\ & -1.58625117 & 5.3303479 & 0.7782986 \\ 2.5345208 & 1.4715395\end{array}$




$\begin{array}{lrll}\mathrm{C} & -0.3984202 & 2.6527617 & 2.6972420 \\ \mathrm{H} & 0.6721216 & 2.9050473 & 2.7056865 \\ \mathrm{C} & -1.0812253 & 2.4445933 & 3.9082059 \\ \mathrm{H} & -0.5373887 & 2.5320462 & 4.8609642 \\ \mathrm{C} & -2.4479884 & 2.1272765 & 3.9040293 \\ \mathrm{H} & -2.9799523 & 1.9637218 & 4.8532375 \\ \mathrm{C} & -3.1345952 & 2.0087079 & 2.6818735 \\ \mathrm{H} & -4.2036996 & 1.7486685 & 2.6717734 \\ \mathrm{C} & -2.4544269 & 2.1973378 & 1.4719983 \\ \mathrm{H} & -2.9857546 & 2.0677401 & 0.5163143\end{array}$

R4

73

SCF Energy $=-1973.530119062$

$-0.1631768$

$\begin{array}{llll}\text { C } & -0.5496771 & -5.3444599 & 0.3630169\end{array}$

$\begin{array}{llll}\text { C } & -1.3820764 & -5.4530452 & -0.7786598\end{array}$

$\begin{array}{llll}\text { C } & -1.7783873 & -6.7046252 & -1.2646881\end{array}$

$\begin{array}{llll}\text { C } & -1.3630804 & -7.8815034 & -0.6152388\end{array}$

$\begin{array}{llll}\text { C } & -0.5586063 & -7.7898335 & 0.5322709\end{array}$

$\begin{array}{llll}\text { C } & -0.1638528 & -4.0273747 & 0.8987417\end{array}$

$\begin{array}{llll}\mathrm{Pd} & 0.1514143 & 0.3970481 & -0.9936941\end{array}$

$\begin{array}{llll}P & 0.1591759 & 2.5736515 & -0.1415254\end{array}$

$\begin{array}{llll}\text { C } & 0.1914327 & -2.8834409 & 0.2318088\end{array}$

$\begin{array}{llll}\text { C } & 0.5141258 & -2.7027571 & -1.1930015\end{array}$

$\begin{array}{llll}\text { C } & 0.9921851 & -3.7811820 & -1.9830025\end{array}$

$\begin{array}{llll}\text { C } & 1.2801900 & -3.6154780 & -3.3416930\end{array}$

$\begin{array}{llll}\text { C } & 1.0967478 & -2.3618545 & -3.9531837\end{array}$

$\begin{array}{llll}\text { C } & 0.6577694 & -1.2780486 & -3.1740036\end{array}$

$\begin{array}{llll}\text { C } & 0.3993507 & -1.4213032 & -1.7928674\end{array}$

$\begin{array}{llll}\text { C } & -0.1606777 & -3.7916464 & 2.4087958\end{array}$

$\begin{array}{llll}0 & -1.1329789 & -4.5217317 & 3.1418879\end{array}$

$\begin{array}{llll}\text { C } & -0.2990939 & -2.2588565 & 2.5054515\end{array}$

$\begin{array}{llll}\text { C } & 0.3217259 & -1.6939046 & 1.1919470\end{array}$

$\begin{array}{llll}\text { C } & -0.4969553 & -0.4935644 & 0.7269396\end{array}$

$\begin{array}{llll}\text { C } & 1.8028417 & -1.3170563 & 1.4059493\end{array}$

$\mathrm{H} \quad \begin{array}{llll}\mathrm{H} & 0.8149649 & -4.1197103 & 2.8386039\end{array}$

$\mathrm{H} \quad 0.1567476 \quad-1.8255425 \quad 3.4173549$

$\mathrm{H} \quad-1.3869793 \quad-2.0351002 \quad 2.5356905$

$\mathrm{H} \quad-1.5486470 \quad-0.7885427 \quad 0.5262676$

$\begin{array}{llll}\mathrm{H} & -0.4733493 & 0.3046947 & 1.4982809\end{array}$

$\begin{array}{llll}\mathrm{H} & 1.8891279 & -0.4495957 & 2.0910494\end{array}$

$\begin{array}{llll}\mathrm{H} & 2.2708064 & -1.0364186 & 0.4414458\end{array}$

H $\quad 2.3721548 \quad-2.1650343 \quad 1.8383781$

$\mathrm{H} \quad-1.9598847 \quad-4.4840330 \quad 2.6230353$

$\mathrm{H} \quad-1.7144959 \quad-4.5338007 \quad-1.2828688$ 


\begin{tabular}{|c|c|c|c|}
\hline & -2.4232212 & -6.7637966 & -2.1550082 \\
\hline & -1.6757134 & -8.8655171 & -0.9966110 \\
\hline & -0.2383301 & -8.7041589 & 1.0551699 \\
\hline & 0.4546319 & -6.4757007 & 1.9292269 \\
\hline & 0.5369436 & -0.2908388 & -3.6609655 \\
\hline & 1.3083216 & -2.2260468 & -5.0254850 \\
\hline & 1.6515926 & -4.4689978 & -3.9291844 \\
\hline & 1.1480323 & -4.7620444 & -1.5126743 \\
\hline & 0.9413399 & 3.8859289 & -1.1658883 \\
\hline & 1.1239022 & 2.5820984 & 1.4212314 \\
\hline & -1.4574338 & 3.3147686 & 0.3315205 \\
\hline & -1.5724500 & 4.6614049 & 0.7358489 \\
\hline & -2.8218192 & 5.1777713 & 1.1083360 \\
\hline & -3.9627648 & 4.3554293 & 1.0777280 \\
\hline & -3.8533746 & 3.0166853 & 0.6686818 \\
\hline & -2.6039908 & 2.4966305 & 0.2923621 \\
\hline & -0.6817455 & 5.3077542 & 0.7534914 \\
\hline & -2.9080597 & 6.2293944 & 1.4214583 \\
\hline & -4.9422674 & 4.7640809 & 1.3691847 \\
\hline & -4.7454619 & 2.3731481 & 0.6366621 \\
\hline & -2.5040752 & 1.4522396 & -0.0422811 \\
\hline & 1.7599949 & 4.9026157 & -0.6326738 \\
\hline & 2.3019856 & 5.8843443 & -1.4777411 \\
\hline & 2.0301044 & 5.860 & -2.85 \\
\hline & 1.2161369 & 4.8475532 & -3.3923631 \\
\hline & 0.6782013 & 3.8617255 & -2.5521731 \\
\hline & 1.9812753 & 4.9188248 & 0.4450669 \\
\hline & 2.9425372 & 6.6737063 & -1.0557812 \\
\hline & 2.4586734 & 6.6295855 & -3.5155297 \\
\hline & 1.0055210 & 4.8214959 & -4.4721461 \\
\hline & 0.0448999 & 3.0620626 & -2.9703591 \\
\hline & 0.5069734 & 2.6996381 & 2.6820433 \\
\hline & 1.2646491 & 2.5376800 & 3.8550570 \\
\hline & 2.6393262 & 2.2672420 & 3.7773182 \\
\hline & 3.2589227 & 2.1491480 & 2.5196295 \\
\hline & 2.5045968 & 2.2919123 & 1.3479446 \\
\hline & -0.5695831 & 2.9152396 & 2.7477656 \\
\hline & 0.7733833 & 2.6248559 & 4.8359670 \\
\hline & 3.2303105 & 2.1412110 & 4.6969318 \\
\hline & 4.3345690 & 1.9271798 & 2.4522927 \\
\hline & 2.9845953 & 2.1640303 & 0.3652277 \\
\hline \\
\hline \\
\hline \\
\hline & -2.4039375 & -1.5754531 & -0.897816 \\
\hline
\end{tabular}




\begin{tabular}{|c|c|c|c|}
\hline & -1.7977654 & -2.7055801 & -0.2856861 \\
\hline & -2.1179838 & -4.0096014 & -0.7133856 \\
\hline & -3.0590257 & -4.1967073 & -1.7393627 \\
\hline & -3.6736532 & -3.0855193 & -2.3417208 \\
\hline & -3.3479054 & -1.7794502 & -1.9233288 \\
\hline & -0.8366261 & -2.4069452 & 0.7954743 \\
\hline & 0.4756829 & -2.7507109 & 0.9292828 \\
\hline & 1.3345355 & -3.4676271 & -0.0256490 \\
\hline & 1.1025569 & -3.4130678 & -1.4236552 \\
\hline & 1.9861832 & -4.0189721 & -2.3242312 \\
\hline & 3.1324810 & -4.6910061 & -1.8603887 \\
\hline & 3.3803276 & -4.7544898 & -0.4 \\
\hline & 2.4934381 & -4.1509001 & 0.4256291 \\
\hline & -1.32 & -1.53 & $1 . s$ \\
\hline & -2.24 & -2.37 & $2 . \varepsilon$ \\
\hline & -2.1 & -0.3 & 1. \\
\hline & -0.0 & -1.1 & 2. \\
\hline & 1.0 & -2.1 & 2.2 \\
\hline & 2.2 & -1.4 & 2.0 \\
\hline & 1.22 & -2.9 & $2 . c$ \\
\hline & 0.336 & -0.1 & 2.3 \\
\hline & -0.1103932 & -1.092 & 3.7 \\
\hline & -1.8698277 & 0.610 & 2.0 \\
\hline & -3.1954908 & -0.46 & 1.4 \\
\hline & -1.6973678 & -3.2347506 & 3.3 \\
\hline & -3.1148976 & -2.7642214 & 2.3 \\
\hline & -2.6317795 & -1.75 & 3.7 \\
\hline & 2.8530409 & -2.0082869 & 1.4 \\
\hline & 0.227 & -2.866 & -1.8 \\
\hline & 1.785 & -3.95 & -3.4 \\
\hline & 3.8283540 & -5.1 & -2. \\
\hline & 4.2697846 & -5.281 & -0.1 \\
\hline & 2.693 & -4.228 & 1.5 \\
\hline & -1.6240683 & -4.8728839 & -0.2409542 \\
\hline & -3.3195189 & -5.2152099 & -2.0657645 \\
\hline & -4.4196140 & -3.2349162 & -3.1385307 \\
\hline & -3.8394066 & -0.9198358 & -2.4078471 \\
\hline & -1.5364515 & 0.1888054 & -0.4843084 \\
\hline & 0.0039739 & 1.9439389 & -0.1429828 \\
\hline & -0.2315350 & 3.5879993 & -0.9344204 \\
\hline & -0.9271313 & 3.6272808 & -2.1608507 \\
\hline & -1.3364722 & 2.6941200 & -2.5814358 \\
\hline & -1.1046435 & 4.8438327 & -2.8361825 \\
\hline & -1.6480991 & 4.8653 & -3.7928966 \\
\hline & -0.5993296 & 6.0336925 & -2.2843245 \\
\hline & -0.7448392 & 6.9896883 & -2.809816 \\
\hline
\end{tabular}




$\begin{array}{lrrr}\text { C } & 0.0838724 & 6.0026762 & -1.0569979 \\ \text { H } & 0.4758218 & 6.9339127 & -0.6202461 \\ \text { C } & 0.2699268 & 4.7851363 & -0.3826982 \\ \text { H } & 0.8012634 & 4.7615822 & 0.5806859 \\ \text { C } & 1.6345334 & 1.3798964 & -0.7867555 \\ \text { C } & 1.8594092 & -0.0110636 & -0.8522472 \\ \text { H } & 1.0553932 & -0.6987451 & -0.5470024 \\ \text { C } & 3.0951986 & -0.5070691 & -1.2955995 \\ \text { H } & 3.2548994 & -1.5944658 & -1.3475371 \\ \text { C } & 4.1096675 & 0.3850167 & -1.6782966 \\ \text { H } & 5.0784751 & -0.0018962 & -2.0296816 \\ \text { C } & 3.8869997 & 1.7731336 & -1.6226174 \\ \text { H } & 4.6798822 & 2.4718851 & -1.9303710 \\ \text { C } & 2.6530164 & 2.2726538 & -1.1794922 \\ \text { H } & 2.4778843 & 3.3584268 & -1.1429892 \\ \text { C } & 0.3516577 & 2.3641848 & 1.6088602 \\ \text { C } & 1.5605743 & 2.0110396 & 2.2396119 \\ \text { H } & 2.3526608 & 1.5072913 & 1.6686424 \\ \text { C } & 1.7332750 & 2.2586339 & 3.6113005 \\ \text { H } & 2.6760131 & 1.9676461 & 4.0981964 \\ \text { C } & 0.7069999 & 2.8576454 & 4.3584446 \\ \text { H } & 0.8449223 & 3.0467141 & 5.4338045 \\ \text { C } & -0.5034518 & 3.2063918 & 3.7326135 \\ \text { H } & -1.3138469 & 3.6700021 & 4.3153866 \\ \text { C } & -0.6852903 & 2.9539393 & 2.3656439 \\ \text { H } & -1.6370342 & 3.2175388 & 1.8771571 \\ & & & \\ \text { S4 } & & & \\ \text { 73 } & & & \\ \text { SCF } & \text { Energy }=-1973.533273633 & \\ \text { C } & 3.8348029 & 1.0954248 & -1.9169227 \\ \text { C } & 2.7627198 & 0.6563887 & -1.0975146 \\ \text { C } & 1.4464874 & 0.6727738 & -1.6252521 \\ \text { C } & 1.2475043 & 1.0551612 & -2.9696373 \\ \text { C } & 2.3199009 & 1.4628852 & -3.7813251 \\ \text { C } & 3.6183036 & 1.4932497 & -3.2406238 \\ \text { C } & 2.9889795 & 0.2416002 & 0.2955506 \\ \text { C } & 4.1124141 & -0.3091762 & 0.8585152 \\ \text { C } & 5.3231160 & -0.8351298 & 0.2015926 \\ \text { C } & 6.5974464 & -0.6567966 & 0.7930636 \\ \text { C } & 7.7467216 & -1.1940946 & 0.1935939 \\ \text { C } & 7.6479023 & -1.9268144 & -1.0004071 \\ \text { C } & 6.3849663 & -2.1271869 & -1.5871883 \\ & 5.2359588 & -1.5916128 & -0.9929047 \\ \text { C. } & 1.6821002 & 0.4685201 & 1.3295174 \\ & & 1.9717523 & 1.5460750\end{array}$




\begin{tabular}{|c|c|c|c|}
\hline & 0.6016058 & -0.2702901 & 0.9397960 \\
\hline & 2.4801415 & -0.1936991 & 2.6137381 \\
\hline & 3.9987446 & -0.2990766 & 2.3758237 \\
\hline & 4.7540302 & 0.7886769 & 2.9422743 \\
\hline & 4.4310513 & -1.2075421 & 2.8440014 \\
\hline & 2.2533776 & 0.3760119 & 3.5363486 \\
\hline & 2.0649491 & -1.2148450 & 2.7396879 \\
\hline & 0.8109179 & -1.3492179 & 0.7741331 \\
\hline & -0.1654450 & -0.1504407 & 1.7334741 \\
\hline & 2.5139057 & 2.4869602 & 1.9346459 \\
\hline & 0.7940807 & 2.1143136 & 2.2818052 \\
\hline & 1.3117090 & 2.454 & 1633 \\
\hline & 4.5975656 & 1.5551119 & 0217 \\
\hline & 6.666 & -0.07 & 3166 \\
\hline & 8.729 & -1.03 & 7012 \\
\hline & 8.55 & -2.3 & -1 \\
\hline & 6.29 & -2.7 & -2 . \\
\hline & 4.24 & -1.7 & -1.4 \\
\hline & 0.227 & 1.0 & -3.4 \\
\hline & 2.1417811 & $1.7 €$ & -4.8 \\
\hline & 4.465 & 1.83 & -3.8 \\
\hline & 4.8504098 & 1.1309992 & -1.4 \\
\hline & -0.3310424 & 0.3133333 & -0.7802959 \\
\hline & -2.5294905 & 0.1114647 & 0.0066607 \\
\hline & -2.7604520 & 0.8944587 & 1.6531282 \\
\hline & -3.4869774 & 0.2960683 & 2.7006390 \\
\hline & -3.9659682 & -0.6821142 & 2.5468269 \\
\hline & -3.5934586 & 0.94 & 15167 \\
\hline & -4.1576 & 0.47 & 4.7 \\
\hline & -2.9816801 & 2.19 & 3900 \\
\hline & -3.0649156 & 2.70 & 5.1 \\
\hline & -2.2538818 & 2.794 & 3.0 \\
\hline & -1.7648039 & 3.7679913 & 3.2507420 \\
\hline & -2.1349632 & 2.1434931 & 1.8609100 \\
\hline & -1.5411757 & 2.5903688 & 1.0476522 \\
\hline & -3.2454819 & -1.5693480 & 0.2157167 \\
\hline & -4.6262781 & -1.8233846 & 0.0832037 \\
\hline & -5.3107834 & -1.0032165 & -0.1814264 \\
\hline & -5.1235728 & -3.1208866 & 0.2824636 \\
\hline & -6.2015125 & -3.3153562 & 0.1744144 \\
\hline & -4.2484763 & -4.1687595 & 0.6161210 \\
\hline & -4.6408744 & -5.1854921 & 0.7695634 \\
\hline & -2.8713301 & -3.9198665 & 0.7442442 \\
\hline & -2.1822641 & -4.7400874 & 0.9959596 \\
\hline & -2.3687391 & -2.6260318 & 0.5385727 \\
\hline & -1.2893703 & -2.4270845 & 0.619391 \\
\hline
\end{tabular}




$\begin{array}{lllr}\text { C } & -3.7623585 & 0.9606730 & -1.0615244 \\ \text { C } & -4.7402686 & 1.8430311 & -0.5616187 \\ \text { H } & -4.7917774 & 2.0467544 & 0.5182449 \\ \text { C } & -5.6432189 & 2.4620074 & -1.4420735 \\ \text { H } & -6.4035854 & 3.1523106 & -1.0461650 \\ \text { C } & -5.5781548 & 2.2024652 & -2.8207295 \\ \text { H } & -6.2860572 & 2.6905530 & -3.5075008 \\ \text { C } & -4.6034682 & 1.3224282 & -3.3236722 \\ \text { H } & -4.5464763 & 1.1192699 & -4.4037211 \\ \text { C } & -3.6957445 & 0.7083302 & -2.4494521 \\ \text { H } & -2.9276027 & 0.0217535 & -2.8424444\end{array}$

\section{Optimized Geometries (TS-BXnj/k-f')}

\begin{tabular}{lrrr} 
R3 & \multicolumn{3}{l}{} \\
73 & \multicolumn{4}{l}{} \\
SCF & Energy $=-1973.505963658$ & \\
C & 1.7104604 & 5.2455665 & 1.0463054 \\
C & 1.1751045 & 4.1423223 & 0.3112805 \\
C & 1.1788440 & 4.2156447 & -1.1033723 \\
C & 1.7349499 & 5.3181418 & -1.7657742 \\
C & 2.3414010 & 6.3562209 & -1.0364745 \\
C & 2.3596329 & 6.3052151 & 0.3697906 \\
C & 0.9075071 & 2.9388779 & 1.0987185 \\
C & 0.7115500 & 1.6234426 & 0.7652167 \\
C & 0.2306535 & 1.0391020 & -0.4964604 \\
C & -0.8707072 & 1.6111495 & -1.1822639 \\
C & -1.3589343 & 1.0430221 & -2.3663285 \\
C & -0.7667647 & -0.1199994 & -2.8897131 \\
C & 0.3124871 & -0.7123478 & -2.2122779 \\
C & 0.8013485 & -0.1443271 & -1.0271090 \\
C & 1.1405666 & 3.0936490 & 2.6054397 \\
C & -0.2182265 & 3.3414945 & 3.3059121 \\
C & 2.1072054 & 4.2485797 & 2.8357881 \\
C & 1.6642667 & 1.6804569 & 2.9779568 \\
C & 0.9743677 & 0.7317712 & 1.9772253 \\
O & -0.2368714 & 0.2070570 & 2.5434992 \\
H & 1.6453664 & -0.1198701 & 1.7044573 \\
H & 2.7607816 & 1.6326621 & 2.8175781 \\
H & 1.4511511 & 1.3895051 & 4.0251299 \\
H & 3.1244376 & 4.0516067 & 2.4507562 \\
H & 2.1882544 & 4.5547115 & 3.9015008 \\
H & -0.8682902 & 2.4545594 & 3.1851693
\end{tabular}




$\begin{array}{lrrr}\text { H } & -0.7227454 & 4.2345057 & 2.8802680 \\ \mathrm{H} & -0.0694729 & 3.5284358 & 4.3889652 \\ \mathrm{H} & -0.7925999 & -0.0750094 & 1.7916947 \\ \mathrm{H} & -1.3465893 & 2.5084528 & -0.7588061 \\ \mathrm{H} & -2.2154656 & 1.5052114 & -2.8806468 \\ \mathrm{H} & -1.1509892 & -0.5678690 & -3.8185985 \\ \mathrm{H} & 0.7807914 & -1.6242305 & -2.6133811 \\ \mathrm{H} & 1.6544055 & -0.6098034 & -0.5107959 \\ \mathrm{H} & 0.7753884 & 3.3808654 & -1.6916390 \\ \mathrm{H} & 1.7283302 & 5.3490556 & -2.8659625 \\ \mathrm{H} & 2.8128048 & 7.2025961 & -1.5599004 \\ \mathrm{H} & 2.8686499 & 7.1000853 & 0.9376291 \\ \mathrm{Pd} & 0.9696346 & 6.1464869 & 2.6981212 \\ \mathrm{P} & -0.0415154 & 7.3117795 & 4.3886940 \\ \mathrm{C} & -1.2238129 & 8.6450587 & 3.9163347 \\ \mathrm{C} & -0.9551403 & 9.3560922 & 2.7282318 \\ \mathrm{H} & -0.0878658 & 9.0638082 & 2.1135238 \\ \mathrm{C} & -1.7902560 & 10.4105834 & 2.3292403 \\ \mathrm{H} & -1.5732411 & 10.9592562 & 1.4002209 \\ \mathrm{C} & -2.9088191 & 10.7545220 & 3.1074235 \\ \mathrm{H} & -3.5691720 & 11.5761054 & 2.7906749 \\ \mathrm{C} & -3.1904176 & 10.0399741 & 4.2842240 \\ \mathrm{H} & -4.0715858 & 10.3006025 & 4.8903124 \\ \mathrm{C} & -2.3522528 & 8.9890756 & 4.6895903 \\ \mathrm{H} & -2.5840787 & 8.4205686 & 5.6029745 \\ \mathrm{C} & 1.0782628 & 8.1516114 & 5.5924096 \\ \mathrm{C} & 2.4530668 & 7.8481851 & 5.5331802 \\ \mathrm{H} & 2.8049394 & 7.1520369 & 4.7535531 \\ \mathrm{C} & 3.3472466 & 8.4322003 & 6.4454225 \\ \mathrm{H} & 4.4196037 & 8.1898987 & 6.3930898 \\ \mathrm{C} & 2.8726382 & 9.3287530 & 7.4164787 \\ \mathrm{H} & 3.5724873 & 9.7912116 & 8.1291839 \\ \mathrm{C} & 1.5025989 & 9.6418155 & 7.4736166 \\ \mathrm{H} & 1.1299550 & 10.3498719 & 8.2295431 \\ \mathrm{C} & 0.6074675 & 9.0565303 & 6.5660415 \\ \mathrm{H} & -0.4623389 & 9.3096894 & 6.6104731 \\ \mathrm{C} & -1.0507772 & 6.2058392 & 5.4575169 \\ \mathrm{C} & -0.5934805 & 5.7720728 & 6.7177824 \\ \mathrm{H} & 0.3341070 & 6.1897239 & 7.1365199 \\ \mathrm{C} & -1.3161687 & 4.8050119 & 7.4365558 \\ \mathrm{H} & -0.9489552 & 4.4705982 & 8.4188681 \\ \mathrm{C} & -2.5004682 & 4.2678053 & 6.9076356 \\ & -3.0636431 & 3.5089401 & 7.4713786 \\ \mathrm{C} & -2.9618266 & 4.6999498 & 5.6511668 \\ \mathrm{H} & -3.8864983 & 4.2792152 & 5.2279199 \\ & & & \\ \mathrm{H} & & \end{array}$




\begin{tabular}{|c|c|c|c|}
\hline H & -2.5911527 & 5.9757302 & 3.9332311 \\
\hline \multicolumn{4}{|l|}{4} \\
\hline \multicolumn{4}{|l|}{73} \\
\hline SCF & Energy $=-197$ & 3.504814435 & \\
\hline $\mathrm{C}$ & 4.1058588 & 0.9459690 & 0.0030686 \\
\hline C & 2.6950536 & 0.8994279 & -0.0959248 \\
\hline C & 2.1119739 & -0.1419531 & -0.8568485 \\
\hline C & 2.9113831 & -1.0782279 & -1.5271818 \\
\hline $\mathrm{C}$ & 4.3124213 & -1.0005138 & -1.4445880 \\
\hline $\mathrm{C}$ & 4.9048731 & 0.0126580 & -0.6721986 \\
\hline C & 1.8632107 & 1.8576020 & 0.6555806 \\
\hline $\mathrm{Pd}$ & -2.0443071 & 4.8816221 & -0.0498971 \\
\hline $\mathrm{P}$ & -2.6796254 & 7.0111992 & 0.4702556 \\
\hline & 0.7605614 & 2.5713513 & 0.2752777 \\
\hline & -0.0437358 & 2.6266476 & -0.9436089 \\
\hline & 0.3353883 & 2.1345434 & 40465 \\
\hline & -0.606 & 2.0 & 8374 \\
\hline & -1.955 & 2.3 & 1002 \\
\hline & $-2.351 \varepsilon$ & 2.8 & 3215 \\
\hline & -1.377 & 3.1 & 7368 \\
\hline & 2.13 & 2.0 & 0705 \\
\hline 0 & 2.67 & 0.9 & 3130 \\
\hline $\mathrm{C}$ & 0.7580435 & 2.5 & 2476 \\
\hline $\mathrm{C}$ & 0.1608666 & 3.341 & 1.4555615 \\
\hline C & -1.3570761 & 3.2786579 & 1.3172829 \\
\hline & 0.6707134 & 4.8010856 & 1.4677518 \\
\hline & 2.8988485 & 2.8875528 & 2.2904857 \\
\hline & 0.8177824 & 3238 & 3.5890996 \\
\hline & 0.1466990 & 1.6666049 & 2.8729439 \\
\hline & -1.7519347 & 2.2489873 & 1.3912701 \\
\hline $1+$ & -1.8979467 & 3.91 & 2.0497845 \\
\hline & 0.2129647 & 5.37 & 2.2987447 \\
\hline & 0.3999379 & 5.31 & 0.5220557 \\
\hline 11 & 1.7726505 & 4.8393448 & 1.5826759 \\
\hline $\mathrm{H}$ & 2.1536199 & 0.1881903 & 2.5455957 \\
\hline $\mathrm{H}$ & 1.0157312 & -0.2155742 & -0.9092206 \\
\hline & 2.4362627 & -1.8803625 & -2.1126676 \\
\hline & 4.9404280 & -1.7350978 & -1.9711514 \\
\hline & 6.0009306 & 0.0733744 & -0.5903865 \\
\hline & 4.5768195 & 1.7284275 & 0.6156053 \\
\hline & -3.4124377 & 3.1000150 & -1.5897429 \\
\hline & -2.7057833 & 2.1930493 & -3.8081589 \\
\hline & -0.2921461 & 1.6335892 & -4.2285604 \\
\hline & 1.3750926 & 1.8219410 & -2.3822498 \\
\hline & -2.8676658 & 8.2139579 & -0.9141043 \\
\hline
\end{tabular}




$\begin{array}{lrrr}\text { C } & -1.4336102 & 7.8120377 & 1.5627151 \\ \text { C } & -4.2525110 & 7.2078331 & 1.4166455 \\ \text { C } & -4.8605914 & 8.4618665 & 1.6308062 \\ \text { C } & -6.0385682 & 8.5524459 & 2.3869843 \\ \text { C } & -6.6178526 & 7.3937894 & 2.9347184 \\ \text { C } & -6.0193132 & 6.1417961 & 2.7194911 \\ \text { C } & -4.8423110 & 6.0485431 & 1.9587961 \\ \text { H } & -4.4151981 & 9.3703249 & 1.1988474 \\ \text { H } & -6.5104483 & 9.5337496 & 2.5482796 \\ \text { H } & -7.5430948 & 7.4685276 & 3.5262489 \\ \text { H } & -6.4734853 & 5.2316994 & 3.1399705 \\ \text { H } & -4.3637622 & 5.0742966 & 1.7645563 \\ \text { C } & -2.5991184 & 9.5926189 & -0.7830778 \\ \text { C } & -2.7964702 & 10.4556292 & -1.8727376 \\ \text { C } & -3.2635174 & 9.9507742 & -3.0981423 \\ \text { C } & -3.5249795 & 8.5769271 & -3.2362449 \\ \text { C } & -3.3213973 & 7.7110990 & -2.1511490 \\ \text { H } & -2.2178800 & 9.9885392 & 0.1704945 \\ \text { H } & -2.5807737 & 11.5296649 & -1.7648502 \\ \text { H } & -3.4146231 & 10.6292537 & -3.9514980 \\ \text { H } & -3.8783917 & 8.1755090 & -4.1981133 \\ \text { H } & -3.5009143 & 6.6284489 & -2.2554442 \\ \text { C } & -1.6402928 & 7.9465846 & 2.9501187 \\ \text { C } & -0.6054113 & 8.4168533 & 3.7760222 \\ \text { C } & 0.6393199 & 8.7613756 & 3.2254479 \\ \text { C } & 0.8487580 & 8.6318502 & 1.8405320 \\ \text { C } & -0.1768619 & 8.1529947 & 1.0147591 \\ \text { H } & -2.6140661 & 7.6788550 & 3.3864660 \\ \text { H } & -0.7765060 & 8.5162064 & 4.8588576 \\ \text { H } & 1.4483993 & 9.1291911 & 3.8743190 \\ \text { H } & 1.8228295 & 8.8970407 & 1.4020900 \\ \text { H } & -0.0021427 & 8.0328221 & -0.0658584\end{array}$

S3

73

SCF Energy $=-1973.521566115$

$\begin{array}{lrrr}\text { C } & -2.1964700 & 4.3705534 & 0.5641955 \\ \text { C } & -1.2156984 & 3.3987756 & 0.2045706 \\ \text { C } & -1.0357028 & 3.0505759 & -1.1476195 \\ \text { C } & -1.8467356 & 3.6441602 & -2.1302520 \\ \text { C } & -2.8402320 & 4.5743399 & -1.7714714 \\ \text { C } & -3.0301028 & 4.9337890 & -0.4230187 \\ \text { C } & -0.5904203 & 2.8053221 & 1.3935250 \\ \text { C } & 0.6759703 & 2.4973839 & 1.7929595 \\ \text { C } & 1.9356714 & 2.6358684 & 1.0506766 \\ \text { C } & 2.0814813 & 3.5657169 & -0.0105721\end{array}$




\begin{tabular}{|c|c|c|c|}
\hline & 3.3087913 & 3.7302168 & -0.6621182 \\
\hline$C$ & 4.4327078 & 2.9777179 & -0.2720653 \\
\hline & 4.3097345 & 2.0550991 & 0.7787372 \\
\hline & 3.0789353 & 1.8858658 & 1.4323228 \\
\hline & -1.6291699 & 2.5527802 & 2.4930087 \\
\hline & -2.3404845 & 1.2083079 & 2.2205192 \\
\hline & -2.6489875 & 3.7064635 & 2.4556269 \\
\hline & -0.7391242 & 2.4865630 & 3.7557042 \\
\hline & 0.6699538 & 2.0830578 & 3.2688769 \\
\hline & 1.6507814 & 2.7639130 & 4.0562242 \\
\hline & 0.8271147 & 0.9772461 & 3.3637281 \\
\hline & -0.6382227 & 3.4994286 & 4.1887893 \\
\hline & -1.1418157 & 1.8179447 & 412082 \\
\hline & -2.7872659 & $4.1 \mathrm{~s}$ & 70424 \\
\hline & -3.6467351 & 3.41 & 51011 \\
\hline & -1.6194588 & 0.3 & 2.2 \\
\hline & -2.842 & 1.2 & 1.2 \\
\hline & -3.1084730 & 1.0 & 2.9 \\
\hline & 2.4373188 & 79771 & 3.4 \\
\hline & 1.2225397 & 4.1844221 & -0.3 \\
\hline & 3.3950217 & 4.4673320 & -1.4 \\
\hline & 5.3977668 & 3.1140127 & -0.7826433 \\
\hline & 5.1788400 & 1.4575048 & 1.0936389 \\
\hline & 2.9992346 & 1.1467536 & 2.2441117 \\
\hline & -0.2771246 & 2.3030667 & -1.4245455 \\
\hline & -1.7167554 & 3. 3629892 & -3.1863523 \\
\hline & -3.4786852 & 5.0213318 & -2.5494442 \\
\hline & -3.8139292 & 5.6562865 & -0.1480344 \\
\hline & -1.5893347 & 5.5928729 & 2.0620372 \\
\hline & -0.4837331 & 6.7257824 & 3.7340213 \\
\hline & -0.8337755 & 8.4864392 & 4.1 \\
\hline & -1.1055613 & 9.3630666 & 3.0730291 \\
\hline & -1.1393217 & 8.9635036 & 2.0463315 \\
\hline & -1.3389032 & 0.7249479 & 3.3146496 \\
\hline & -1.5488639 & 1.4026299 & 2.4732047 \\
\hline & -1.3174026 & 11.2194799 & 4.6304262 \\
\hline & -1.5091036 & 12.2863383 & 4.8213921 \\
\hline & -1.0606475 & 10.3482716 & 5.7022695 \\
\hline & -1.0497150 & 10.7315958 & 6.7341493 \\
\hline & -0.8175411 & 8.9859876 & 5.4625963 \\
\hline & -0.6219117 & 8.3025727 & 6.3027433 \\
\hline & 1.3494425 & 6.6841785 & 3.5660498 \\
\hline & 1.8968780 & 5.7258990 & 2.6904176 \\
\hline & 1.2120697 & 5.0881624 & 2.1105146 \\
\hline & 3.2886715 & 5.5921309 & 2.5667190 \\
\hline & 3.7053058 & 4.8453687 & 1.874665 \\
\hline
\end{tabular}




$\begin{array}{lrrr}\text { C } & 4.1388770 & 6.4211863 & 3.3171234 \\ \mathrm{H} & 5.2307092 & 6.3204609 & 3.2196258 \\ \mathrm{C} & 3.5973453 & 7.3878150 & 4.1833750 \\ \mathrm{H} & 4.2638288 & 8.0442328 & 4.7636076 \\ \mathrm{C} & 2.2059525 & 7.5214043 & 4.3093238 \\ \mathrm{H} & 1.7833447 & 8.2800033 & 4.9853604 \\ \mathrm{C} & -0.7685374 & 5.8566331 & 5.3327968 \\ \mathrm{C} & 0.2481572 & 5.1424378 & 5.9952221 \\ \mathrm{H} & 1.2700305 & 5.1392326 & 5.5936134 \\ \mathrm{C} & -0.0566646 & 4.3853379 & 7.1390050 \\ \mathrm{H} & 0.7418163 & 3.8146873 & 7.6364264 \\ \mathrm{C} & -1.3692758 & 4.3421343 & 7.6326367 \\ \mathrm{H} & -1.6045726 & 3.7452338 & 8.5268542 \\ \mathrm{C} & -2.3882050 & 5.0546769 & 6.9729470 \\ \mathrm{H} & -3.4212170 & 5.0200569 & 7.3515579 \\ \mathrm{C} & -2.0926620 & 5.7996356 & 5.8237379 \\ \mathrm{H} & -2.8933766 & 6.3392077 & 5.2928240\end{array}$

S4

73

SCF Energy $=-1973.505801767$

$\begin{array}{lrrr}\text { C } & 0.2975208 & 3.6848534 & -1.8461033 \\ \text { C } & 0.0277904 & 3.4116733 & -0.4860497 \\ \text { C } & -1.3315031 & 3.3688601 & -0.0531736 \\ \text { C } & -2.3756205 & 3.3582004 & -1.0098844 \\ \text { C } & -2.0800201 & 3.6011370 & -2.3667121 \\ \text { C } & -0.7493931 & 3.8045429 & -2.7718781 \\ \text { C } & 1.0099090 & 3.0162505 & 0.5236112 \\ \text { C } & 2.2507923 & 2.4420933 & 0.4470348 \\ \text { C } & 3.0846492 & 2.1104152 & -0.7248690 \\ \text { C } & 4.4776585 & 2.3623346 & -0.6977233 \\ \text { C } & 5.2909739 & 2.0073896 & -1.7842784 \\ \text { C } & 4.7350865 & 1.3808220 & -2.9117290 \\ \text { C } & 3.3579163 & 1.0979920 & -2.9378163 \\ \text { C } & 2.5418476 & 1.4551516 & -1.8561150 \\ \text { C } & 0.4656541 & 3.0228908 & 1.9548555 \\ \text { C } & 0.5915007 & 4.4201757 & 2.6042649 \\ \text { C } & -0.9977186 & 2.5912381 & 1.8571911 \\ \text { C } & 1.4150816 & 2.0157718 & 2.6588217 \\ \text { C } & 2.7357255 & 2.0845018 & 1.8524047 \\ \text { O } & 3.6996631 & 3.0272295 & 2.3507325 \\ \text { H } & 3.2732391 & 1.1138719 & 1.8634068 \\ \text { H } & 1.5758677 & 2.2326884 & 3.7333144 \\ \text { H } & 1.0030378 & 0.9892463 & 2.5717190 \\ \text { H } & -1.1152858 & 1.5704984 & 1.4505144 \\ \text { H } & -1.5456139 & 2.6528986 & 2.8239593\end{array}$




$\begin{array}{lrrr}\text { H } & 1.6545608 & 4.7049202 & 2.7387661 \\ \mathrm{H} & 0.1154611 & 4.4340158 & 3.6049847 \\ \mathrm{H} & 0.0918712 & 5.1950105 & 1.9865285 \\ \mathrm{H} & 3.3561746 & 3.9138203 & 2.1258413 \\ \mathrm{H} & 4.9081594 & 2.8381685 & 0.1957344 \\ \mathrm{H} & 6.3706124 & 2.2197950 & -1.7477213 \\ \mathrm{H} & 5.3740236 & 1.1024086 & -3.7636018 \\ \mathrm{H} & 2.9164309 & 0.5852576 & -3.8063027 \\ \mathrm{H} & 1.4693245 & 1.2131338 & -1.8731692 \\ \mathrm{H} & -3.4198978 & 3.1862167 & -0.7036103 \\ \mathrm{H} & -2.8970561 & 3.6368006 & -3.1040851 \\ \mathrm{H} & -0.5213400 & 4.0200338 & -3.8267076 \\ \mathrm{H} & 1.3399636 & 3.7791674 & -2.1803055 \\ \mathrm{Pd} & -2.2726728 & 4.3609784 & 1.4503031 \\ \mathrm{P} & -3.2729623 & 5.7816147 & 2.9288097 \\ \mathrm{C} & -2.0527432 & 6.3720301 & 4.1733435 \\ \mathrm{C} & -2.0035624 & 5.8349952 & 5.4752251 \\ \mathrm{H} & -2.7835633 & 5.1338232 & 5.8072435 \\ \mathrm{C} & -0.9591582 & 6.1885424 & 6.3461492 \\ \mathrm{H} & -0.9285495 & 5.7613241 & 7.3601300 \\ \mathrm{C} & 0.0392993 & 7.0830023 & 5.9287753 \\ \mathrm{H} & 0.8564943 & 7.3579684 & 6.6124628 \\ \mathrm{C} & -0.0082438 & 7.6245315 & 4.6314070 \\ \mathrm{H} & 0.7720558 & 8.3248261 & 4.2964963 \\ \mathrm{C} & -1.0418204 & 7.2662093 & 3.7558425 \\ \mathrm{H} & -1.0639505 & 7.6756740 & 2.7337809 \\ \mathrm{C} & -4.6330941 & 5.0939020 & 3.9702802 \\ \mathrm{C} & -5.4526892 & 5.9037290 & 4.7831798 \\ \mathrm{H} & -5.3158112 & 6.9953029 & 4.7885842 \\ \mathrm{C} & -6.4491657 & 5.3203665 & 5.5795974 \\ \mathrm{H} & -7.0877725 & 5.9577143 & 6.2101254 \\ \mathrm{C} & -6.6351752 & 3.9262862 & 5.5707234 \\ \mathrm{H} & -7.4191896 & 3.4721768 & 6.1957479 \\ \mathrm{C} & -5.8254991 & 3.1159944 & 4.7584383 \\ \mathrm{H} & -5.9727723 & 2.0253982 & 4.7429039 \\ \mathrm{C} & -4.8299790 & 3.6986575 & 3.9570868 \\ \mathrm{H} & -4.1927114 & 3.0829595 & 3.3008672 \\ \mathrm{C} & -3.9954201 & 7.3426526 & 2.2662399 \\ \mathrm{C} & -4.0290296 & 8.5498015 & 2.9954942 \\ \mathrm{H} & -3.5712576 & 8.5991872 & 3.9951357 \\ \mathrm{C} & -4.6252121 & 9.6926798 & 2.4392363 \\ \mathrm{H} & -4.6440381 & 10.6325048 & 3.0120086 \\ & -5.1922119 & 9.6394441 & 1.1545777 \\ \mathrm{H} & -5.6562839 & 10.5378174 & 0.7199336 \\ & -5.5859295 & 8.3991018 & -0.5907146\end{array}$




$\begin{array}{llll}\mathrm{C} & -4.5526175 & 7.2998863 & 0.9713406 \\ \mathrm{H} & -4.4967624 & 6.3616913 & 0.3952797\end{array}$

\section{Optimized Geometries (BXnf')}

\begin{tabular}{lrrr} 
R3 & \multicolumn{3}{l}{} \\
73 & \multicolumn{4}{l}{} \\
SCF & Energy $=-1973.572264771$ & \\
C & 2.2534145 & -1.4973505 & -1.3856451 \\
C & 0.9945981 & -2.1678731 & -1.1445793 \\
C & 0.1034437 & -2.3823948 & -2.2177615 \\
C & 0.3863066 & -1.8260701 & -3.4792723 \\
C & 1.5531135 & -1.0675788 & -3.6932481 \\
C & 2.5345645 & -0.9355463 & -2.6624457 \\
C & 0.9985236 & -2.6070575 & 0.2464854 \\
C & 0.3410814 & -3.5185585 & 1.0346495 \\
C & -0.9480008 & -4.1863641 & 0.8253433 \\
C & -1.9870373 & -3.5753952 & 0.0785532 \\
C & -3.2264747 & -4.2035398 & -0.0989712 \\
C & -3.4681060 & -5.4644051 & 0.4728073 \\
C & -2.4588856 & -6.0785410 & 1.2339850 \\
C & -1.2217186 & -5.4465456 & 1.4144786 \\
C & 2.2479430 & -2.0356133 & 0.9504073 \\
C & 1.9765137 & -0.6900957 & 1.6589956 \\
C & 3.2120253 & -1.7685558 & -0.2337211 \\
C & 2.5350687 & -3.1371944 & 1.9884713 \\
C & 1.1300449 & -3.7108048 & 2.3335977 \\
O & 0.4757193 & -3.0980070 & 3.4483856 \\
H & 1.2141755 & -4.7815894 & 2.6149855 \\
H & 3.1404224 & -3.9448264 & 1.5256472 \\
H & 3.0584844 & -2.7853325 & 2.8991456 \\
H & 3.7981183 & -2.6825955 & -0.4794123 \\
H & 3.9306856 & -0.9465882 & -0.0497842 \\
H & 1.1609255 & -0.7618325 & 2.4017094 \\
H & 1.7081743 & 0.0890764 & 0.9174296 \\
H & 2.8924435 & -0.3643530 & 2.1928887 \\
H & 0.0972809 & -2.2538236 & 3.1297903 \\
H & -1.8206445 & -2.5748780 & -0.3408740 \\
H & -4.0141015 & -3.6986764 & -0.6791070 \\
H & -4.4408737 & -5.9601679 & 0.3356732 \\
H & -2.6391038 & -7.0613857 & 1.6957440 \\
H & -0.4469919 & -5.9407343 & 2.0176647 \\
H & -0.8145588 & -2.9651243 & -2.0735987
\end{tabular}




\begin{tabular}{|c|c|c|c|}
\hline & -0.3261157 & -1.9659948 & -4.3056117 \\
\hline & 1.7510378 & -0.6301187 & -4.6828941 \\
\hline & 3.5348669 & -0.5371466 & -2.8952248 \\
\hline & 1.3909952 & 0.6162356 & -1.5695581 \\
\hline & 0.0312302 & 2.0353334 & -0.4820210 \\
\hline & -1.4858650 & 2.6088351 & -1.3569344 \\
\hline & -1.3929966 & 2.8467249 & -2.7439086 \\
\hline & -0.4378790 & 2.6475297 & -3.2567290 \\
\hline & -2.5072579 & 3.3121650 & -3.4575652 \\
\hline & -2.4268725 & 3.4958491 & -4.5398172 \\
\hline & -3.7276704 & 3.5290527 & -2.7941639 \\
\hline & -4.6048634 & 3.8845179 & -3.3559911 \\
\hline & -3.8293666 & 3.2808419 & -1.4150780 \\
\hline & -4.7857593 & 3.4420309 & -0.8943869 \\
\hline & -2.7128296 & 2.8240813 & -0.6958642 \\
\hline & -2.7946193 & 2.6238562 & 0.3832017 \\
\hline & 0.7646443 & 3.5957616 & 0.1694097 \\
\hline & 2.1400705 & 3.5793253 & 0.4831450 \\
\hline & 2.7182441 & 2.6620616 & 0.282 \\
\hline & 2.7545245 & 4.7156011 & 1.03 \\
\hline & 3.8273192 & 4.6941664 & 1.27 \\
\hline & 2.0031819 & 5.882 & 1.25 \\
\hline & 2.4863953 & 6.77 & 1.6 \\
\hline & 0.6364439 & 5.9 & 0.9 \\
\hline & 0.0478 & 6.8 & 1.0 \\
\hline & 0.0159 & 4.7 & 0.3 \\
\hline & -1.0543 & 4.7 & 0.1 \\
\hline & -0.6634228 & 1.2511215 & 1.04 \\
\hline & -0.4698680 & 1.7834272 & 2.3321563 \\
\hline & 0.0407775 & 2.7494822 & 2.4551115 \\
\hline & -0.9068807 & 1.0714807 & 3.4626062 \\
\hline & -0.7380658 & 1.4886111 & 4.4670513 \\
\hline & -1.5556382 & -0.1652381 & 3.3143583 \\
\hline & -1.8976871 & -0.7213801 & 4.2003401 \\
\hline & -1.7629184 & -0.6953524 & 2.0263031 \\
\hline & -2.2633307 & -1.6671733 & 1.9019289 \\
\hline & -1.3084323 & 0.0038645 & 0.8999293 \\
\hline & -1.4237357 & -0.4273596 & -0.1074725 \\
\hline \\
\hline \\
\hline \multicolumn{4}{|c|}{3.572492206} \\
\hline & 1.8513723 & -4.3126501 & 2.1127389 \\
\hline & 0.9567141 & -4.1464560 & 1.0245913 \\
\hline & 1.3053208 & -4.7327426 & -0.2162790 \\
\hline & 2.5219701 & -5.4092436 & -0.380163 \\
\hline
\end{tabular}




$\begin{array}{lrrr}\text { C } & 3.4181140 & -5.5267403 & 0.6965691 \\ \text { C } & 3.0701912 & -4.9833145 & 1.9454543 \\ \text { C } & -0.2877896 & -3.3932067 & 1.2085274 \\ \text { Pd } & -1.4718943 & 0.7526084 & -1.7126496 \\ \text { P } & -0.0878019 & 2.1516857 & -0.6251619 \\ \text { C } & -0.9597383 & -2.5690903 & 0.3465944 \\ \text { C } & -0.9332276 & -2.1349336 & -1.0431215 \\ \text { C } & 0.0402741 & -2.2428407 & -2.0594288 \\ \text { C } & -0.2015006 & -1.6562559 & -3.3133531 \\ \text { C } & -1.4231560 & -1.0012488 & -3.5882443 \\ \text { C } & -2.4714206 & -0.9677092 & -2.6143373 \\ \text { C } & -2.1947838 & -1.4964042 & -1.3202458 \\ \text { C } & -1.0536654 & -3.4437300 & 2.5415708 \\ \text { O } & -1.0075452 & -4.6970765 & 3.2011196 \\ \text { C } & -2.4751761 & -2.9666492 & 2.1375907 \\ \text { C } & -2.1844951 & -1.9367193 & 1.0302721 \\ \text { C } & -3.1551926 & -1.7084011 & -0.1556387 \\ \text { C } & -1.8276644 & -0.5702833 & 1.6573097 \\ \text { H } & -0.6102945 & -2.7262373 & 3.2707083 \\ \text { H } & -3.0560184 & -2.5700961 & 2.9940357 \\ \text { H } & -3.0188620 & -3.8412443 & 1.7179991 \\ \text { H } & -3.7591417 & -2.6235476 & -0.3502239 \\ \text { H } & -3.8577559 & -0.8660481 & -0.0048858 \\ \text { H } & -2.6992002 & -0.1639087 & 2.2100740 \\ \text { H } & -1.5552168 & 0.1491690 & 0.8597354 \\ \text { H } & -0.9689445 & -0.6411057 & 2.3515202 \\ \text { H } & -1.1886915 & -5.3775068 & 2.5237692 \\ \text { H } & 0.5938280 & -4.6751628 & -1.0516235 \\ \text { H } & 2.7689663 & -5.8573665 & -1.3549760 \\ \text { H } & 4.3754638 & -6.0537891 & 0.5674272 \\ \text { H } & 3.7555094 & -5.0869294 & 2.8007178 \\ \text { H } & 1.5823537 & -3.9051242 & 3.0965921 \\ \text { H } & -3.4902081 & -0.6552995 & -2.8952436 \\ \text { H } & -1.6058309 & -0.5866633 & -4.5907131 \\ \text { H } & 0.5730708 & -1.6961857 & -4.0933341 \\ \text { H } & 1.0002648 & -2.7366947 & -1.8607798 \\ \text { C } & 1.1812878 & 3.0675970 & -1.5982148 \\ \text { C } & 0.9301100 & 1.1608526 & 0.5575267 \\ \text { C } & -0.8041789 & 3.4685913 & 0.4483322 \\ \text { C } & -0.0868829 & 4.6206541 & 0.8297412 \\ \text { C } & -0.6759780 & 5.5655464 & 1.6849616 \\ \text { C } & -1.9812209 & 5.3662042 & 2.1665056 \\ \text { C } & -2.7024515 & 4.2222235 & 1.7837790 \\ \text { H } & -2.1191266 & 3.2809642 & 0.9221014 \\ \text { H } & 0.9343407 & 4.7795122 & 0.4517204 \\ & -0.1123090 & 6.4652156 & 1.9759501\end{array}$




$\begin{array}{rrrr}\text { H } & -2.4407230 & 6.1093449 & 2.8359460 \\ \text { H } & -3.7284844 & 4.0673526 & 2.1508400 \\ \text { H } & -2.6771754 & 2.3879386 & 0.5966920 \\ \text { C } & 2.5167903 & 3.2073156 & -1.1690370 \\ \text { C } & 3.4337784 & 3.9340666 & -1.9465914 \\ \mathrm{C} & 3.0235627 & 4.5285210 & -3.1512498 \\ \text { C } & 1.6929432 & 4.3887027 & -3.5841997 \\ \mathrm{C} & 0.7778911 & 3.6547639 & -2.8157275 \\ \mathrm{H} & 2.8386302 & 2.7385389 & -0.2267973 \\ \mathrm{H} & 4.4764580 & 4.0351980 & -1.6082741 \\ \mathrm{H} & 3.7443597 & 5.0960544 & -3.7593793 \\ \mathrm{H} & 1.3700351 & 4.8454591 & -4.5321992 \\ \mathrm{H} & -0.2610105 & 3.5193858 & -3.1577285 \\ \mathrm{C} & 1.0235229 & 1.4486069 & 1.9322895 \\ \mathrm{C} & 1.6856296 & 0.5550820 & 2.7937854 \\ \mathrm{C} & 2.2599285 & -0.6222264 & 2.2889958 \\ \mathrm{C} & 2.1875766 & -0.9016340 & 0.9120250 \\ \mathrm{C} & 1.5258355 & -0.0159634 & 0.0535172 \\ \mathrm{H} & 0.5559875 & 2.3582368 & 2.3366325 \\ \mathrm{H} & 1.7412061 & 0.7789158 & 3.8702533 \\ \mathrm{H} & 2.7604369 & -1.3314777 & 2.9650818 \\ \mathrm{H} & 2.6270538 & -1.8289092 & 0.5156727 \\ \mathrm{H} & 1.4279094 & -0.2512518 & -1.0171428\end{array}$

S3

73

SCF Energy $=-1973.578348420$

C $\quad-2.9217349-1.3809345$

C $\quad-1.9116775 \quad-1.9853870$

0.9514153

$\begin{array}{ll}-1.9116775 & -1.9853870 \\ -2.1764439 & -2.1742892\end{array}$

0.1150032

$-3.4203817-1.7118396$

$-1.2826319$

$-4.3919993$

$-1.1368270$

$-1.8031075$

$-4.1500679$

$-0.9865243$

$-0.9664575$

$-0.8138476$

$-2.4297269$

0.4185290

0.9884495

C $\quad 0.5063485$

$-2.7798895$

0.9432279

C $\quad 1.4358374$

$-2.9815424$

$-0.1760848$

C $\quad 1.3243492$

$-2.2757745$

$-1.3971186$

C 2.2957638

$-2.3935964$

$-2.3959170$

C $\quad 3.4087675$

$-3.2319051$

$-2.2118819$

C $\quad 3.5326735$

$-3.9563763$

$-1.0154642$

C $\quad 2.5618470$

$-3.8318366$

$-0.0099813$

$-1.3276873$

$-2.3745743$

2.4345291

$-1.9699456$

$-3.7317453$

2.8057247

$-2.4274618-1.2849972$

2.3816712

$-0.0278529-2.1319242$

3. 2205892

1.0468573

$-2.8581691$

2.3866710 


\begin{tabular}{|c|c|c|c|}
\hline & 2.2980764 & -2.1978679 & 2.5597188 \\
\hline & 1.1392108 & -3.9268566 & 2.7125582 \\
\hline & 0.2284768 & -1.0521399 & 3.2066960 \\
\hline & -0.0617357 & -2.4709678 & 4.2748776 \\
\hline & -1.9812094 & -0.2814064 & 2.5543694 \\
\hline & -3.2338205 & -1.4263631 & 3.1293625 \\
\hline & -1.2408046 & -4.5617925 & 2.719501 \\
\hline & -2.8229990 & -3.9600055 & 2.134836 \\
\hline & -2.3488917 & -3.7071913 & 3.8483749 \\
\hline & 2.9069416 & -2.5722324 & 1.8911637 \\
\hline & 0.4847228 & -1.5599810 & -1.5311571 \\
\hline & 2.1930 & -1.8025966 & -3.31 \\
\hline & 4.1769 & -3.3178305 & -2.99 \\
\hline & 4.3919 & 72576 & -0.86 \\
\hline & 2.6638468 & -4.4320042 & 0.90 \\
\hline & -1.51713 & -2.7996754 & -1.90 \\
\hline & -3.6361129 & -1.8584651 & -2.8719222 \\
\hline & -5.3508880 & -0.8092250 & -1.3944546 \\
\hline & -4.9203635 & -0.5472670 & 1.070805 \\
\hline & -1.1772563 & -0.2060739 & -1.106198 \\
\hline & 0.1842702 & 1.4979662 & -0.607817 \\
\hline & 0.1229852 & 3.0700046 & -1.5727439 \\
\hline & -0.5730164 & 3.0673289 & -2.7970814 \\
\hline & -1.0798541 & 2.1408787 & -3.1152638 \\
\hline & -0.6208482 & 4.2281148 & -3.5868479 \\
\hline & -1.1652873 & 4.2189591 & -4.5434161 \\
\hline & 0.0187106 & 5.3996531 & -3.1501614 \\
\hline & -0.0229577 & 6.3120832 & -3.7645077 \\
\hline & 0.7075503 & 5.4093015 & -1.9236925 \\
\hline & 1.2045982 & 6.3286525 & -1.5780193 \\
\hline & 0.7617164 & 4.2492409 & -1.1373355 \\
\hline & 1.2985812 & 4.2597908 & -0.1766259 \\
\hline & 1.9577801 & 1.0200866 & -0.7263888 \\
\hline & 2.5105625 & 0.1417362 & 0.2313994 \\
\hline & 1.9266188 & -0.1959568 & 1.1004330 \\
\hline & 3.8194319 & -0.3339284 & 0.0750515 \\
\hline & 4.2418479 & -1.0087347 & 0.8336439 \\
\hline & 4.5799741 & 0.0283962 & -1.048047 \\
\hline & 5.6001792 & -0.3637623 & -1.174800 \\
\hline & 4.0282628 & 0.8883222 & -2.011537 \\
\hline & 4.6161727 & 1.1772851 & -2.8962336 \\
\hline & 2.7269317 & 1.3894750 & -1.849265 \\
\hline & 2.3025312 & 2.0688915 & -2.6035943 \\
\hline & -0.0199302 & 2.0726869 & 1.133625 \\
\hline & 1.0499572 & 2.4711191 & 1.960382 \\
\hline & 2.0812103 & 2.4360049 & 1.579241 \\
\hline
\end{tabular}




$\begin{array}{lrrr}\text { C } & 0.8039715 & 2.8912276 & 3.2781214 \\ \text { H } & 1.6464702 & 3.1884746 & 3.9210796 \\ \text { C } & -0.5089942 & 2.9295046 & 3.7756881 \\ \text { H } & -0.6974996 & 3.2560138 & 4.8097131 \\ \text { C } & -1.5804173 & 2.5420053 & 2.9519501 \\ \text { H } & -2.6109667 & 2.5649501 & 3.3379585 \\ \text { C } & -1.3363067 & 2.1068343 & 1.6410423 \\ \text { H } & -2.1626633 & 1.7670893 & 0.9946782\end{array}$

S4

73

SCF Energy $=-1973.576018477$

$\begin{array}{lrrr}\text { C } & 2.2251782 & 0.1760437 & -2.0551306 \\ \text { C } & 2.1557383 & -0.8726729 & -1.1129428 \\ \text { C } & 1.5537616 & -2.1282933 & -1.4725831 \\ \text { C } & 0.9918305 & -2.3184907 & -2.7673209 \\ \text { C } & 0.9824680 & -1.2008515 & -3.6619902 \\ \text { C } & 1.6208657 & 0.0119758 & -3.3127112 \\ \text { C } & 2.5911961 & -0.9824926 & 0.2736407 \\ \text { C } & 3.4529068 & -0.3696737 & 1.1427069 \\ \text { C } & 4.2391179 & 0.8605442 & 0.9829640 \\ \text { C } & 4.4541510 & 1.7083472 & 2.0998856 \\ \text { C } & 5.1846903 & 2.8976185 & 1.9675059 \\ \text { C } & 5.7318927 & 3.2619784 & 0.7254421 \\ \text { C } & 5.5567905 & 2.4128658 & -0.3815616 \\ \text { C } & 4.8226640 & 1.2256723 & -0.2543242 \\ \text { C } & 1.9704987 & -2.2555404 & 0.8790527 \\ \text { C } & 0.5637976 & -2.0134278 & 1.4678099 \\ \text { C } & 1.8098365 & -3.1564971 & -0.3769057 \\ \text { C } & 2.9737288 & -2.5741012 & 2.0054326 \\ \text { C } & 3.4971058 & -1.1748163 & 2.4447742 \\ \text { O } & 2.7479460 & -0.5564390 & 3.5039651 \\ \text { H } & 4.5320559 & -1.2423293 & 2.8414230 \\ \text { H } & 2.5419563 & -3.1296722 & 2.8611251 \\ \text { H } & 3.8283712 & -3.1573085 & 1.6028151 \\ \text { H } & 2.7613788 & -3.6890754 & -0.6005581 \\ \text { H } & 1.0084026 & -3.9151485 & -0.2863992 \\ \text { H } & 0.5622195 & -1.2296765 & 2.2461903 \\ \text { H } & 0.1854355 & -2.9492186 & 1.9269977 \\ \text { H } & -0.1411062 & -1.7059842 & 0.6692120 \\ \text { H } & 1.9469704 & -0.1636593 & 3.0999376 \\ \text { H } & 4.0198349 & 1.4182574 & 3.0680625 \\ \text { H } & 5.3289494 & 3.5472349 & 2.8446336 \\ \text { H } & 6.3038340 & 4.1965700 & 0.6226025 \\ \text { H } & 6.0066792 & 2.6731195 & -1.3521731 \\ \text { H } & 4.7218267 & 0.5492537 & -1.1145584\end{array}$




\begin{tabular}{|c|c|c|c|}
\hline & 0.6887842 & -3.3194238 & -3.1145109 \\
\hline H & 0.5523901 & -1.3193412 & -4.6675633 \\
\hline & 1.6299673 & 0.8404582 & -4.0362147 \\
\hline & 2.7013896 & 1.1300482 & -1.7930744 \\
\hline d & -0.7407652 & -1.3835852 & -1.7999288 \\
\hline & -2.1227373 & -0.0740647 & -0.6068290 \\
\hline & -1.1300304 & 0.8134178 & 0.6785051 \\
\hline & -1.4146480 & 0.7569692 & 2.0558657 \\
\hline & -2.3234183 & 0.2480193 & 2.4087870 \\
\hline & -0.5209535 & 1.3237357 & 2.9834553 \\
\hline & -0.7439452 & 1.2641279 & 4.0597908 \\
\hline & 0.6557527 & 1.9520232 & 2.5421319 \\
\hline & 1.3593730 & 2.3876842 & 3.2672986 \\
\hline & 0.9323255 & 2.02 & 1.1634929 \\
\hline & 1.8582355 & 2.50 & 0.8154444 \\
\hline & 0.0460807 & 1.46 & 6337 \\
\hline & 0.2794981 & 1.48 & -0.8345808 \\
\hline & -3.4695514 & -0.8506261 & 0.3843977 \\
\hline & -4.6125073 & -0.1450927 & 0.8121932 \\
\hline & -4.7471960 & 0.9075618 & 0.5211209 \\
\hline & -5.5798843 & -0.7856935 & 1.6027622 \\
\hline & -6.4725170 & -0.2308 & 1.9300069 \\
\hline & -5.4118529 & -2.1308 & 1.9738988 \\
\hline & -6.17 & -2.63 & 2.5932571 \\
\hline & -4.276 & -2.8 & 1.54 \\
\hline & -4.14 & -3.8 & 1.8251905 \\
\hline & -3.31 & -2.2 & 0.7 \\
\hline & -2.4 & -2.7 & 0.3 \\
\hline & -2.98 & 1.3 & -1.4 \\
\hline & -3.1 & 2.5 & -0.5 \\
\hline & -2.7 & & \\
\hline & -3.82 & & -1.611 \\
\hline & -3.9285664 & $4.5 \varsigma$ & -1.16 \\
\hline & -4.3701136 & 3.3178415 & $-2.876257 €$ \\
\hline & -4.9066519 & 4.1062605 & -3.425767 \\
\hline & -4.2232137 & 2.0395608 & -3.4433266 \\
\hline & -4.6431990 & 1.8253780 & -4.4379544 \\
\hline & -3.5283327 & 1.0387555 & -2.7487936 \\
\hline & -3.3857014 & 0.0407587 & -3.194040 \\
\hline
\end{tabular}

\section{Optimized Geometries (TS-BXne'-f")}

R3 
79

SCF Energy $=-2238.392395776$

$\begin{array}{lll}\text { C } & -0.7971417 & 7.5657802\end{array}$

$\begin{array}{lll}\text { C } & -0.7881010 & 7.2213357\end{array}$

$\begin{array}{lll}\text { C } & 0.1416588 & 7.8126538\end{array}$

$\begin{array}{lll}\text { C } & 1.0596419 & 8.7595495\end{array}$

$\begin{array}{lll}\text { C } & 1.0484672 & 9.1159701\end{array}$

$-1.8544049$

$-2.8122644$

$-3.9268072$

$-4.5839720$

$-4.1329139$

$-3.0273033$

$-2.3734045$

$-0.5314630$

1.6603321

2.0423786

1.1891330

0.7276655

1. 2182603

1. 6459243

$-0.2072231$

$-0.3422131$

$-1.2840608$

$-2.1241711$

$-2.0045727$

$-1.0619707$

0.9996060

1. 1272918

1.0266603

0.8123457

0.7084817

0.7748183

0.2754850

3. 5397761

$-2.0008424$

$-3.1060591$

$-3.3248806$

$-4.3169553$

$-5.0951243$

$-4.8753695$

$-3.8799317$

2.0881986

2. 4341792

0.7499840
6. 7211059

5.5281623

5.9003825

4. 9407606

3.6097454

3. 2294496

4. 1881483

5.6627788

5.6456312

4. 5536171

3. 3190202

2. 6870885

3. 4485233

4.8221584

1.5567826

0.8116335

$-0.5381386$

0.1947062

1. 2246434

3. 2046419

2. 0600638

2. 1797515

3. 4438339

4.6011513

4.4891829

3.5449777

4.2006290

2.8653848

7.9978223

9. 1520504

10.0734619

9. 8449676

8.6955250

7.7748855

2.9215830

5. 3212967

5.4744969
$-0.2238510$
0.9223545

1.4008893

2. 7667477

3. 6386052

3. 1588309

1.7977899

0.1488755

1. 1749685

1. 9507288

2.7384992

2.7566775

1.9764965

1.1855605

$-1.4296271$

$-1.1096694$

$-0.0904761$

$-0.4121248$

0.7059618

1. 9532002

1. 3761571

0.7895639

1. 9878491

2.0915926

1.0107769

$-0.1851171$

$-0.2944638$

$-1.8610563$

$-2.6708275$

$-4.0667210$

$-4.6570945$

$-3.8585563$

$-2.4492427$

3. 0108164

$-0.2255616$

$-3.0724517$

$-0.2813358$

0.4983699

0.1246633

$-1.0225218$

$-1.8019644$

$-1.4411492$

2.4082813

1. 9727005

1. 3918993 


$\begin{array}{lrrr}\text { H } & 1.3929949 & 6.6071794 & -0.6148157 \\ \text { H } & 2.4595365 & 5.8772001 & -1.8367576 \\ \text { H } & 4.1676620 & 5.0911009 & -0.0162463 \\ \text { H } & 3.7676916 & 3.8486394 & -1.2525378 \\ \text { H } & 3.8306649 & 3.3973954 & 0.4813139 \\ \text { H } & -0.5584345 & 3.8925809 & 2.6303358 \\ \text { H } & -0.9939190 & 1.7932914 & -1.2306065 \\ \text { H } & -2.6485221 & -0.0457133 & -1.0465725 \\ \text { H } & -2.8652303 & -1.3469475 & 1.0972804 \\ \text { H } & -1.3678104 & -0.7861800 & 3.0342499 \\ \text { H } & 0.2753286 & 1.0741730 & 2.8564134 \\ \text { H } & -1.2895349 & 3.2281461 & -3.6588516 \\ \text { H } & 0.5469946 & 5.5819232 & -4.3312325 \\ \text { H } & 0.7389975 & 3.5312421 & -5.7524507 \\ \text { H } & 1.1239463 & 1.2874003 & -4.7027621 \\ \text { H } & 1.3059663 & 1.0795690 & -2.2051073 \\ \text { H } & -4.2843857 & 6.9411347 & 1.9319597 \\ \text { H } & -5.4570016 & 5.2349141 & 3.3410294 \\ \text { H } & -4.6513034 & 2.8607948 & 3.3744058 \\ \text { H } & -2.6757272 & 2.1878320 & 1.9763455 \\ \text { H } & -1.5248916 & 3.9070599 & 0.5396914 \\ \text { H } & -2.7141967 & 9.3304191 & 1.3963696 \\ \text { H } & -4.4828293 & 10.9749773 & 0.7343240 \\ \text { H } & -5.8717110 & 10.5687644 & -1.3136728 \\ \text { H } & -5.4785948 & 8.5181258 & -2.7054290 \\ \text { H } & -3.6890931 & 6.8794231 & -2.0544892 \\ \text { H } & -1.4978207 & 6.4739295 & 3.1492091 \\ \text { H } & 0.1497910 & 7.5231059 & 4.7002478 \\ \text { H } & 1.7895207 & 9.2178282 & 3.8431554 \\ \text { H } & 1.7659928 & 9.8576446 & 1.4148854 \\ \text { H } & 0.1371543 & 8.7772282 & -0.1484170 \\ \text { C } & -2.7202244 & 3.8969285 & -2.5871301 \\ \text { O } & -2.6532568 & 5.0513136 & -2.9775930 \\ \text { O } & -3.5587102 & 3.5062538 & -1.6160809 \\ \text { H } & -3.3250642 & 2.5968965 & -1.3291701 \\ & & & \\ \text { R4 } & & & \\ 79 & & & \\ \text { SCF } & \text { Energy }=-2238.381672208 & \\ \text { C } & -2.3711864 & 6.3405158 & 5.3366655 \\ \text { C } & -0.9661016 & 6.2766146 & 5.2039513 \\ \text { C } & -0.2159350 & 5.5447594 & 6.1451606 \\ \text { C } & -0.8648237 & 4.8798829 & 7.1997522 \\ \text { C } & -2.2611076 & 4.9472240 & 7.3278283 \\ \text { P } & -3.0132518 & 5.6868437 & 6.3967874 \\ & -0.2153530 & 7.0478032 & 3.7057655\end{array}$




\begin{tabular}{|c|c|c|c|}
\hline & 1.5715503 & 6.6674737 & 3.9118234 \\
\hline & 2.0338620 & 5.4788968 & 3.3101676 \\
\hline & 3.3663484 & 5.0733461 & 3.4719798 \\
\hline & 4.2491579 & 5.8679667 & 4.2235591 \\
\hline & 3.7980308 & 7.0639677 & 4.8094164 \\
\hline & 2.4605928 & 7.4655818 & 4.6584973 \\
\hline & -1.1027346 & 6.0445600 & 1.8221389 \\
\hline & 1.0025337 & 6.6800467 & 0.2383826 \\
\hline & 0.5542707 & 7.6089806 & -0.4070122 \\
\hline & -0.6003958 & 8.2604914 & -0.1479972 \\
\hline & -1.7881780 & 4.9390522 & 0.2491820 \\
\hline & -0.9635656 & 3.7904152 & 0.0759210 \\
\hline & -0.6016719 & 3.3726491 & -1.2176400 \\
\hline & -1.1029921 & 4.06 & -2.3 \\
\hline & -1.9747587 & 5.159 & -2.1 \\
\hline & -2.333 & $5.5 \mathrm{~s}$ & -0.8 \\
\hline & -0.692 & 3.13 & 2020 \\
\hline & 0.400 & 2.6 & 3090 \\
\hline & 0.021 & 302465 & 3.3 \\
\hline & -1.2875971 & 2.9621910 & 3.6553051 \\
\hline & -1.9366939 & 3.1527276 & 2.2638646 \\
\hline & 1.7814465 & 2.5154596 & 1.4821367 \\
\hline & 2.6581442 & 1.5346257 & 2.0076064 \\
\hline & 4.0020873 & 1.4869054 & 1.6080094 \\
\hline & 4.4991912 & 2.4189979 & 0.6809793 \\
\hline & 3.6385959 & 3.4019438 & 0.1584722 \\
\hline & 2.2955581 & 3.45 & 0.5511715 \\
\hline & -2.8544960 & 1.9655470 & 1.8978610 \\
\hline & -2.6732778 & 4.4908477 & 2.0380272 \\
\hline & -0.1352902 & 0.7485200 & 3.4157061 \\
\hline & -0.3249506 & 8.8466698 & 4.0819708 \\
\hline & -0.2136364 & 9.7621933 & 3.0147225 \\
\hline & -0.2439033 & 1.1429045 & 3.2616978 \\
\hline & -0.3963915 & 11.6219710 & 4.5740489 \\
\hline & -0.5178265 & 10.7145861 & 5.6396607 \\
\hline & -0.4806847 & 9.3320666 & 5.3972827 \\
\hline & 1.1836057 & 8.1214039 & -1.4798645 \\
\hline & 0.6187782 & 8.8297437 & -1.8509834 \\
\hline & 0.8218110 & 2.4794429 & 4.1088346 \\
\hline & -1.0267588 & 3.9532118 & 4.0749474 \\
\hline & -1.9450249 & 2.4504217 & 4.3877501 \\
\hline & -2.8746526 & 5.0329173 & 2.9874589 \\
\hline & -3.6355307 & 4.3880778 & 1.5078083 \\
\hline & -2.3238606 & 1.0107898 & 2.0800943 \\
\hline & -3.1504419 & 2.0130757 & 0.8299115 \\
\hline & -3.7764065 & 1.9850826 & 2.515895 \\
\hline
\end{tabular}




$\begin{array}{lrrr}\text { H } & -0.2851494 & 0.4988272 & 4.3469086 \\ \mathrm{H} & 2.2556736 & 0.7994342 & 2.7200321 \\ \mathrm{H} & 4.6673383 & 0.7142714 & 2.0237913 \\ \mathrm{H} & 5.5551994 & 2.3851397 & 0.3722041 \\ \mathrm{H} & 4.0213999 & 4.1546819 & -0.5473406 \\ \mathrm{H} & 1.6541329 & 4.2633886 & 0.1772349 \\ \mathrm{H} & 0.0548169 & 2.4993980 & -1.3462424 \\ \mathrm{H} & -0.8316239 & 3.7375812 & -3.3464509 \\ \mathrm{H} & -2.3835191 & 5.6764403 & -3.0433405 \\ \mathrm{H} & -3.0147870 & 6.4510331 & -0.7469121 \\ \mathrm{H} & -1.0591259 & 7.7228247 & 0.5880232 \\ \mathrm{H} & -0.1135000 & 9.3897654 & 1.9844650 \\ \mathrm{H} & -0.1564183 & 11.8493462 & 2.4222793 \\ \mathrm{H} & -0.4268053 & 12.7052773 & 4.7660774 \\ \mathrm{H} & -0.6421265 & 11.0850001 & 6.6687631 \\ \mathrm{H} & -0.5766663 & 8.6225222 & 6.2327762 \\ \mathrm{H} & 1.3393041 & 4.8795341 & 2.7022915 \\ \mathrm{H} & 3.7121452 & 4.1451478 & 2.9937899 \\ \mathrm{H} & 5.2987591 & 5.5589073 & 4.3441268 \\ \mathrm{H} & 4.4935596 & 7.6913108 & 5.3879205 \\ \mathrm{H} & 2.1080133 & 8.4009772 & 5.1186106 \\ \mathrm{H} & 0.8774309 & 5.4850770 & 6.0436794 \\ \mathrm{H} & -0.2712477 & 4.3040874 & 7.9263137 \\ \mathrm{H} & -2.7666804 & 4.4219433 & 8.1522010 \\ \mathrm{H} & -4.1082487 & 5.7443052 & 6.4921329 \\ \mathrm{H} & -2.9591509 & 6.9039872 & 4.5943436 \\ & & & \\ \mathrm{~S} 3 & & & \\ 79 & \mathrm{C} & & \\ \mathrm{SCF} & \mathrm{Energy}=-2238.392793926 & \\ \mathrm{C} & -2.6741508 & 5.8949928 & 6.1749519 \\ \mathrm{C} & -1.3435203 & 6.3498857 & 6.0698608 \\ \mathrm{C} & -0.6322267 & 6.7011208 & 7.2356412 \\ \mathrm{C} & -1.2514371 & 6.6015803 & 8.4917664 \\ \mathrm{C} & -2.5808926 & 6.1568608 & 8.5911227 \\ \mathrm{C} & -3.2924542 & 5.8051680 & 7.4310938 \\ \mathrm{P} & -0.6107765 & 6.4869541 & 4.3894552 \\ \mathrm{C} & 1.1968115 & 6.3771362 & 4.7038829 \\ \mathrm{C} & 1.6930488 & 5.1643555 & 5.2287258 \\ \mathrm{C} & 3.0695163 & 4.9820756 & 5.4032146 \\ \mathrm{C} & 3.9659560 & 6.0014499 & 5.0364981 \\ \mathrm{C} & 3.4771346 & 7.2065009 & 4.5093915 \\ \mathrm{C} & 2.0943766 & 7.4007934 & 4.3481400 \\ \mathrm{C} & -1.4327560 & 4.9322009 & 2.8853269 \\ & -0.6718868 & 1.9286326 & 2.2601062 \\ & & & \\ \mathrm{H} & & \end{array}$




\begin{tabular}{|c|c|c|c|}
\hline & -0.1785525 & 2.6087095 & 0.9733239 \\
\hline & 1.1572158 & 2.4163798 & 0.7725368 \\
\hline & 1.7364401 & 1.6219529 & 1.9645068 \\
\hline & 0.6455741 & 1.8035932 & 3.0494864 \\
\hline & -1.2637269 & 3.3421876 & 0.3160204 \\
\hline & -2.1500113 & 3.9737751 & 1.2354419 \\
\hline & -3.3489103 & 4.5618213 & 0.7842969 \\
\hline & -3.6279319 & 4.5920963 & -0.5954763 \\
\hline & -2.7340563 & 4.0084428 & -1.5113761 \\
\hline & -1.5621274 & 3.3793404 & -1.0617671 \\
\hline & 2.0212380 & 2.9024770 & -0.3128880 \\
\hline & 1.6808453 & 4.0336843 & -1.0964842 \\
\hline & 2.5413147 & 4.5075641 & -2.0943603 \\
\hline & 3.7626611 & 3.85 & -2.3475549 \\
\hline & 4.1181692 & 2.73 & 71019 \\
\hline & 3.2661634 & 2.27 & -0.5 \\
\hline & -1.2573190 & 0.54 & 1.9 \\
\hline & 3.0269492 & 2.00 & 2.3 \\
\hline & 2.5321587 & 4.6 & 2.2 \\
\hline & -0.8807024 & 8.25 & 3.93 \\
\hline & -0.6760360 & 8.6248612 & 2.5855214 \\
\hline & -0.8526992 & 9.9602727 & 2.1937022 \\
\hline & -1.2509736 & 10.9264891 & 3.1335131 \\
\hline & -1.4654226 & 10.5541599 & 4.4706911 \\
\hline & -1.2780698 & 9.2217666 & 4.8726468 \\
\hline & 1.8236089 & 0.5448644 & 1.6857699 \\
\hline & 0.8572107 & 2.7698212 & 3.5544170 \\
\hline & 0.6437784 & 0.9951881 & 3.8077612 \\
\hline & -1.5849259 & 2.9699733 & 3.9915316 \\
\hline & -2.7851990 & 2.3626484 & 2.8034285 \\
\hline & -0.4950453 & -0.1092573 & 1.4310018 \\
\hline & -2.1008438 & 0.6475016 & 1.1921798 \\
\hline & -1.6331600 & 0.0296977 & 2.8149463 \\
\hline & 3.0045165 & 2.9929331 & 2.4336826 \\
\hline & 0.7472481 & 4.5723713 & -0.8930212 \\
\hline & 2.2573416 & 5.3991580 & -2.6739644 \\
\hline & 4.4369642 & 4.2305377 & -3.1345142 \\
\hline & 5.0761224 & 2.2278657 & -1.7581466 \\
\hline & 3.5757483 & 1.4240756 & 0.0573299 \\
\hline & -0.8836369 & 2.8894827 & -1.7755419 \\
\hline & -2.9659705 & 4.0215733 & -2.5871909 \\
\hline & -4.5542734 & 5.0669625 & -0.9538529 \\
\hline & -4.0578869 & 5.0086228 & 1.4982561 \\
\hline & 0.2993838 & 5.4225124 & 1.8055314 \\
\hline & -0.3616812 & 7.8701765 & 1.8488182 \\
\hline & -0.6845276 & 10.2444876 & 1.143971 \\
\hline
\end{tabular}




$\begin{array}{rrrr}\text { H } & -1.3988394 & 11.9716298 & 2.8217795 \\ \mathrm{H} & -1.7817430 & 11.3061321 & 5.2096411 \\ \mathrm{H} & -1.4469907 & 8.9353280 & 5.9209329 \\ \mathrm{H} & 0.9927672 & 4.3532478 & 5.4818716 \\ \mathrm{H} & 3.4475165 & 4.0273835 & 5.7979030 \\ \mathrm{H} & 5.0493839 & 5.8501392 & 5.1566638 \\ \mathrm{H} & 4.1742517 & 8.0079887 & 4.2207728 \\ \mathrm{H} & 1.7149993 & 8.3456205 & 3.9327671 \\ \mathrm{H} & 0.4096735 & 7.0462103 & 7.1567327 \\ \mathrm{H} & -0.6910338 & 6.8722961 & 9.3995860 \\ \mathrm{H} & -3.0621196 & 6.0771844 & 9.5777080 \\ \mathrm{H} & -4.3310651 & 5.4491662 & 7.5062641 \\ \mathrm{H} & -3.2128582 & 5.6028750 & 5.2582971 \\ \mathrm{C} & 2.2005934 & 5.6275469 & 1.5679044 \\ \mathrm{O} & 0.9576536 & 6.0063599 & 1.2883265 \\ \mathrm{O} & 3.0717619 & 6.4374727 & 0.9366711 \\ \mathrm{H} & 3.9569010 & 6.0769812 & 1.1478961\end{array}$

S4

79

SCF Energy $=-2238.386373030$

$\begin{array}{lrrr}\text { C } & -2.8212156 & 4.9405271 & 0.5537775 \\ \text { C } & -3.0642795 & 4.9789334 & 1.9438789 \\ \text { C } & -4.0794851 & 4.1696990 & 2.4905812 \\ \text { C } & -4.8399921 & 3.3350860 & 1.6529703 \\ \text { C } & -4.6066806 & 3.3173844 & 0.2679561 \\ \text { C } & -3.5985473 & 4.1286714 & -0.2814696 \\ \text { P } & -1.9845502 & 6.0839201 & 2.9510388 \\ \text { C } & -2.6637495 & 5.8674609 & 4.6546045 \\ \text { C } & -3.6272506 & 6.7369602 & 5.2050433 \\ \text { C } & -4.1242300 & 6.5131990 & 6.4994407 \\ \text { C } & -3.6719690 & 5.4177869 & 7.2531996 \\ \text { C } & -2.7115631 & 4.5469358 & 6.7102108 \\ \text { C } & -2.2041209 & 4.7765883 & 5.4231195 \\ \text { Pd } & 0.2353310 & 5.5534891 & 2.5520114 \\ \text { C } & 1.1337703 & 3.7274071 & 3.4206324 \\ \text { C } & 0.6472617 & 2.4935036 & 2.6342610 \\ \text { C } & 0.6772372 & 2.8780702 & 1.1455762 \\ \text { C } & -0.4003784 & 2.4084150 & 0.4538608 \\ \text { C } & -1.3071713 & 1.6249716 & 1.4008427 \\ \text { C } & -0.8353220 & 2.0896779 & 2.8037744 \\ \text { C } & 1.8056815 & 3.7699825 & 0.8688575 \\ \text { C } & 2.7233688 & 3.7064714 & -0.1970549 \\ \text { C } & 3.8271739 & 4.5749930 & -0.2169147 \\ \text { C } & 4.0200038 & 5.5001567 & 0.8324136 \\ \text { C } & 3.1154099 & 5.5631686 & 1.9119852\end{array}$




\begin{tabular}{rrrr} 
C & 1.9798497 & 4.7196220 & 1.9248755 \\
$\mathrm{C}$ & -0.7732429 & 2.6477077 & -0.9454624 \\
$\mathrm{C}$ & -1.6524846 & 1.7538949 & -1.6040371 \\
$\mathrm{C}$ & -2.0930945 & 2.0118069 & -2.9102489 \\
$\mathrm{C}$ & -1.6707321 & 3.1677781 & -3.5884052 \\
$\mathrm{C}$ & -0.7887575 & 4.0583522 & -2.9471298 \\
$\mathrm{C}$ & -0.3412840 & 3.8020782 & -1.6448311 \\
$\mathrm{C}$ & 1.6044013 & 1.3089813 & 2.8953052 \\
$\mathrm{O}$ & -1.2442111 & 0.2043109 & 1.1797430 \\
$\mathrm{O}$ & 0.6476968 & 7.7084354 & 1.1691062 \\
$\mathrm{C}$ & -2.5900919 & 7.7610348 & 2.4952084 \\
$\mathrm{C}$ & -1.7668188 & 8.8592210 & 2.8179426 \\
$\mathrm{C}$ & -2.1882525 & 10.1645958 & 2.5271193 \\
$\mathrm{C}$ & -3.4254336 & 10.3822085 & 1.8964295 \\
$\mathrm{C}$ & -4.2431014 & 9.2897408 & 1.5621379 \\
$\mathrm{C}$ & -3.8298482 & 7.9818219 & 1.8624375 \\
$\mathrm{H}$ & -2.3729456 & 1.8843248 & 1.2387893 \\
$\mathrm{H}$ & -1.0123464 & 1.3118347 & 3.5724503 \\
$\mathrm{H}$ & -1.4229991 & 2.9909315 & 3.0694164 \\
$\mathrm{H}$ & 0.4545159 & 4.0172962 & 4.2530027 \\
$\mathrm{H}$ & 2.1328245 & 3.5944834 & 3.8738492 \\
$\mathrm{H}$ & 1.2801463 & 0.3984609 & 2.3523969 \\
$\mathrm{H}$ & 1.6295783 & 1.0636072 & 3.9770545 \\
$\mathrm{H}$ & 2.6359071 & 1.5549703 & 2.5695276 \\
$\mathrm{H}$ & -0.3063501 & -0.0071155 & 1.0097380 \\
$\mathrm{H}$ & -1.9786207 & 0.8499727 & -1.0688045 \\
$\mathrm{H}$ & -2.7770731 & 1.3039979 & -3.4034390 \\
$\mathrm{H}$ & -2.0194881 & 3.3717708 & -4.6120407 \\
$\mathrm{H}$ & -0.4418684 & 4.9613021 & -3.4755757 \\
$\mathrm{H}$ & 0.3324031 & 4.5141240 & -1.1492985 \\
$\mathrm{H}$ & 2.2078371 & 6.4696415 & -0.1402433 \\
$\mathrm{H}$ & 3.2752785 & 6.2917559 & 2.7209461 \\
$\mathrm{H}$ & 4.8911422 & 6.1737612 & 0.8142582 \\
$\mathrm{H}$ & 4.5476235 & 4.5297775 & -1.0470773 \\
$\mathrm{H}$ & 2.5717298 & 2.9745296 & -1.0042216 \\
$\mathrm{H}$ & -0.7865289 & 8.6798971 & 3.2838186 \\
$\mathrm{H}$ & -1.5398698 & 11.0170426 & 2.7805622 \\
$\mathrm{H}$ & -3.7502803 & 11.4067171 & 1.6585311 \\
$\mathrm{H}$ & -5.2108151 & 9.4559015 & 1.0643630 \\
$\mathrm{H}$ & -4.4710952 & 7.1264820 & 1.6025779 \\
$\mathrm{H}$ & -3.9887977 & 7.5930568 & 4.6168593 \\
-4.8734832 & 7.2009028 & 6.9206913 \\
$\mathrm{H}$ & -4.0647455 & 5.2441392 & 8.2665599 \\
\hline
\end{tabular}




$\begin{array}{lrrr}\mathrm{H} & -5.6238058 & 2.6968181 & 2.0887219 \\ \mathrm{H} & -5.2017200 & 2.6614917 & -0.3849975 \\ \mathrm{H} & -3.3969351 & 4.1085780 & -1.3622572 \\ \mathrm{H} & -2.0169176 & 5.5587547 & 0.1293865 \\ \mathrm{C} & 0.4925570 & 7.2941333 & 0.0347835 \\ \mathrm{O} & -0.6678016 & 7.4240429 & -0.6370801 \\ \mathrm{O} & 1.4195492 & 6.6617514 & -0.7110900 \\ \mathrm{H} & -0.5858858 & 6.9555428 & -1.4940041\end{array}$

\section{Optimized Geometries (BXnf $\left.{ }^{66}\right)$}

\begin{tabular}{lrrr} 
R3 & \multicolumn{4}{l}{} \\
79 & \multicolumn{4}{l}{} \\
SCF & Energy $=-2238.439527857$ & \\
C & 2.1137780 & 2.4886544 & 0.5899693 \\
C & 0.9536382 & 1.9716986 & 1.2090855 \\
C & 0.9712460 & 1.7181401 & 2.5963353 \\
C & 2.1346241 & 1.9697323 & 3.3471836 \\
C & 3.2815596 & 2.4879008 & 2.7261680 \\
C & 3.2642513 & 2.7536212 & 1.3451326 \\
P & -0.4777610 & 1.5280615 & 0.1331710 \\
C & -1.8537931 & 1.3079331 & 1.3400909 \\
C & -2.7609191 & 2.3298472 & 1.6812351 \\
C & -3.8120742 & 2.0635640 & 2.5743505 \\
C & -3.9624867 & 0.7839918 & 3.1366623 \\
C & -3.0558228 & -0.2381484 & 2.8021774 \\
C & -2.0114079 & 0.0228027 & 1.9021043 \\
Pd & 0.1014303 & -0.2660091 & -1.0752212 \\
C & 2.9326072 & -0.7479858 & -1.7249100 \\
C & 3.1567067 & -1.6603565 & -0.4829154 \\
C & 1.7714590 & -2.2539501 & -0.1923615 \\
C & 1.5757870 & -2.4758055 & 1.1464198 \\
C & 2.7938024 & -1.9256715 & 1.9175337 \\
C & 3.5111347 & -1.0296535 & 0.8738328 \\
C & 0.4165100 & -3.0389830 & 1.8443443 \\
C & 0.5345528 & -3.5257575 & 3.1724520 \\
C & -0.5718723 & -4.0632986 & 3.8444771 \\
C & -1.8312790 & -4.1185984 & 3.2222900 \\
C & -1.9723990 & -3.6158485 & 1.9166878 \\
C & -0.8664180 & -3.0842091 & 1.2421319 \\
C & 1.0325787 & -2.2872336 & -1.4661865 \\
C & 0.0214556 & -3.1469384 & -1.9841683 \\
C & -0.4074978 & -2.9873068 & -3.3113680
\end{tabular}




\begin{tabular}{rrrr} 
C & 0.1222858 & -1.9594063 & -4.1254132 \\
$\mathrm{C}$ & 1.1370332 & -1.1182445 & -3.6452437 \\
$\mathrm{C}$ & 1.6425425 & -1.2973694 & -2.3327295 \\
$\mathrm{O}$ & 2.4680145 & -1.2436194 & 3.1169872 \\
$\mathrm{C}$ & 4.1302348 & -2.8021985 & -0.8532982 \\
$\mathrm{O}$ & -2.7003510 & -1.6497887 & -1.3704986 \\
$\mathrm{C}$ & -0.8867756 & 3.1087555 & -0.7083267 \\
$\mathrm{C}$ & -0.5887213 & 4.3663623 & -0.1416529 \\
$\mathrm{C}$ & -0.9577458 & 5.5433231 & -0.8116488 \\
$\mathrm{C}$ & -1.6305983 & 5.4705547 & -2.0436516 \\
$\mathrm{C}$ & -1.9299544 & 4.2186134 & -2.6071018 \\
$\mathrm{C}$ & -1.5552587 & 3.0368287 & -1.9487193 \\
$\mathrm{H}$ & 3.4561334 & -2.7601634 & 2.2444265 \\
$\mathrm{H}$ & 4.5976229 & -0.9274493 & 1.0657283 \\
$\mathrm{H}$ & 3.0537712 & -0.0207983 & 0.9366648 \\
$\mathrm{H}$ & 2.8183771 & 0.3155248 & -1.4297693 \\
$\mathrm{H}$ & 3.7725564 & -0.8027460 & -2.4492540 \\
$\mathrm{H}$ & 5.1395697 & -2.3962746 & -1.0716817 \\
$\mathrm{H}$ & 3.7758169 & -3.3464225 & -1.7527835 \\
$\mathrm{H}$ & 4.2218149 & -3.5378593 & -0.0294028 \\
$\mathrm{H}$ & 1.9189740 & -0.4706936 & 2.8673489 \\
$\mathrm{H}$ & -1.0105560 & -2.6445368 & 0.2480979 \\
$\mathrm{H}$ & -2.9533550 & -3.6224278 & 1.4170154 \\
$\mathrm{H}$ & -2.6967558 & -4.5427189 & 3.7538716 \\
$\mathrm{H}$ & -0.4497331 & -4.4400562 & 4.8716730 \\
$\mathrm{H}$ & 1.5019739 & -3.4622412 & 3.6867279 \\
$\mathrm{H}$ & -2.0368844 & -2.0669534 & -1.9758101 \\
$\mathrm{H}$ & 1.5747123 & -0.3469899 & -4.2967505 \\
$\mathrm{H}$ & -0.2601565 & -1.8230252 & -5.1468481 \\
$\mathrm{H}$ & -1.1630680 & -3.6727405 & -3.7251444 \\
$\mathrm{H}$ & -0.3817910 & -3.9549820 & -1.3578973 \\
$\mathrm{H}$ & -2.6573911 & 3.3285965 & 1.2326008 \\
$\mathrm{H}$ & -4.5251688 & 2.8628742 & 2.8276266 \\
$\mathrm{H}$ & -4.7913329 & 0.5818080 & 3.8319642 \\
$\mathrm{H}$ & -3.1557173 & -1.2472109 & 3.2299563 \\
$\mathrm{H}$ & -1.3159367 & -0.7819115 & 1.6191334 \\
$\mathrm{H}$ & -0.0619741 & 4.4230556 & 0.8230504 \\
$\mathrm{H}$ & -0.7193151 & 6.5225099 & -0.3686919 \\
$\mathrm{H}$ & -1.9186722 & 6.3946817 & -2.5678396 \\
$\mathrm{H}$ & -2.4550488 & 4.1567643 & -3.5722277 \\
$\mathrm{H}$ & -1.7850682 & 2.0554185 & -2.3945118 \\
& 0.0695295 & 1.3289733 & 3.0926993 \\
$\mathrm{H}$ & 2.1412423 & 1.7567823 & 4.4266475 \\
\hline
\end{tabular}




$\begin{array}{llrr}\mathrm{C} & -2.9703912 & -0.4157592 & -1.8716561 \\ \mathrm{O} & -2.6753241 & -0.0267660 & -2.9822609 \\ \mathrm{O} & -3.6554089 & 0.3302666 & -0.9869144 \\ \mathrm{H} & -3.6110614 & -0.0974957 & -0.1035187\end{array}$

\begin{tabular}{lrrr} 
R4 & & & \\
79 & \multicolumn{4}{l}{} \\
SCF & Energy $=-2238.436633861$ & \\
C & -2.3581003 & 2.4898521 & 1.1662024 \\
C & -0.9878477 & 2.1879870 & 1.3330428 \\
C & -0.4743737 & 2.0087191 & 2.6323144 \\
C & -1.3237104 & 2.1169893 & 3.7472744 \\
C & -2.6848118 & 2.4140043 & 3.5757960 \\
C & -3.1992280 & 2.6087859 & 2.2808575 \\
P & 0.0302072 & 1.9437633 & -0.1897257 \\
C & 1.6489035 & 1.4169547 & 0.4980224 \\
C & 1.7684038 & 0.0702885 & 0.8996964 \\
C & 2.9399470 & -0.3745609 & 1.5286663 \\
C & 4.0095785 & 0.5155837 & 1.7272611 \\
C & 3.9038873 & 1.8509602 & 1.3034483 \\
C & 2.7221868 & 2.3078565 & 0.6971609 \\
Pd & -1.1073159 & 0.4973140 & -1.4594858 \\
O & 2.0848955 & -1.3176182 & -2.3275180 \\
C & 2.1984876 & -0.3973718 & -3.1072069 \\
D & 1.3102072 & 0.5925831 & -3.3232337 \\
C & -2.3898608 & -1.3270639 & -1.1260558 \\
C & -1.3971984 & -2.3515415 & -0.9095663 \\
C & -0.7402256 & -2.9381398 & -2.0041265 \\
C & -0.9976320 & -2.4675630 & -3.3058036 \\
C & -1.9074609 & -1.4222245 & -3.5268178 \\
C & -2.6421596 & -0.8536828 & -2.4458388 \\
C & -1.3032775 & -2.5520499 & 0.5355621 \\
C & -0.3598649 & -2.8687030 & 1.4750582 \\
C & -0.9159388 & -2.5246517 & 2.8589942 \\
C & -2.1105257 & -1.5835410 & 2.5512103 \\
C & -2.6095207 & -2.0576667 & 1.1723375 \\
C & 1.0100799 & -3.3778807 & 1.3250119 \\
C & 1.6348482 & -4.0454667 & 2.4098295 \\
C & 2.9623398 & -4.4875305 & 2.3115763 \\
C & 3.6998866 & -4.2688447 & 1.1345934 \\
C & 3.0944664 & -3.5998548 & 0.0562843 \\
C & 1.7673126 & -3.1638836 & 0.1484486 \\
C & -3.5993967 & -3.2373990 & 1.2999516 \\
C & -3.1532230 & -1.0116496 & 0.1602511 \\
O & -1.2929812 & -3.7390626 & 3.5346431 \\
C & 0.3540588 & 3.6569546 & -0.7767594 \\
& & &
\end{tabular}




$\begin{array}{lrrr}\text { C } & 0.8889094 & 3.8224557 & -2.0728560 \\ \text { C } & 1.1858188 & 5.1071515 & -2.5508638 \\ \text { C } & 0.9391003 & 6.2353240 & -1.7485723 \\ \text { C } & 0.4011586 & 6.0738278 & -0.4613296 \\ \text { C } & 0.1126145 & 4.7890172 & 0.0273914 \\ \text { O } & 3.2845527 & -0.2519008 & -3.9033808 \\ \text { H } & 3.1540209 & 0.5593547 & -4.4347328 \\ \text { H } & -0.1326157 & -1.9979518 & 3.4553148 \\ \text { H } & -1.7354983 & -0.5421618 & 2.4747962 \\ \text { H } & -2.8805470 & -1.6105941 & 3.3499197 \\ \text { H } & -2.9563255 & 0.0228380 & 0.5071707 \\ \text { H } & -4.2492384 & -1.0953319 & 0.0051550 \\ \text { H } & -3.1800759 & -4.0136372 & 1.9681113 \\ \text { H } & -3.8028048 & -3.6915212 & 0.3083847 \\ \text { H } & -4.5637337 & -2.8847149 & 1.7224599 \\ \text { H } & -1.5418133 & -3.4919224 & 4.4450349 \\ \text { H } & 1.0481024 & -4.2367778 & 3.3198457 \\ \text { H } & 3.4251442 & -5.0103587 & 3.1630372 \\ \text { H } & 4.7436287 & -4.6112422 & 1.0624368 \\ \text { H } & 3.6570529 & -3.3804531 & -0.8631860 \\ \text { H } & 1.3567429 & -2.5845887 & -0.6860671 \\ \text { H } & -0.0197080 & -3.7507111 & -1.8466726 \\ \text { H } & -0.4636339 & -2.9140177 & -4.1570019 \\ \text { H } & -2.0943224 & -1.0602981 & -4.5487253 \\ \text { H } & -3.4825655 & -0.1711696 & -2.6500914 \\ \text { H } & 0.5252849 & 0.4844860 & -2.6618540 \\ \text { H } & 1.0759602 & 2.9371295 & -2.7003823 \\ \text { H } & 1.6052579 & 5.2301127 & -3.5610694 \\ \text { H } & 1.1640339 & 7.2430704 & -2.1296651 \\ \text { H } & 0.2061815 & 6.9541068 & 0.1701554 \\ \text { H } & -0.3022092 & 4.6632019 & 1.0385730 \\ \text { H } & 0.9334521 & -0.6241533 & 0.7193801 \\ \text { H } & 3.0217107 & -1.4238312 & 1.8452222 \\ \text { H } & 4.9347046 & 0.1615518 & 2.2070778 \\ \text { H } & 4.7449050 & 2.5459501 & 1.4502844 \\ \text { H } & 2.6323778 & 3.3577986 & 0.3814030 \\ \text { H } & 0.5900264 & 1.7710940 & 2.7715964 \\ \text { H } & -0.9150437 & 1.9646680 & 4.7578692 \\ \text { H } & -3.3482333 & 2.4918860 & 4.4503581 \\ \text { H } & -4.2653814 & 2.8423588 & 2.1391136 \\ \text { H } & -2.7604098 & 2.6162254 & 0.1478260\end{array}$

S3

79

SCF Energy $=-2238.442284859$

$\begin{array}{lll}\text { C } & -1.7996814 & -0.3876832\end{array}$

4.3629912 


$\begin{array}{lrrr}\text { C } & -0.4539588 & -0.5414774 & 3.9687231 \\ \text { C } & 0.4486956 & -1.2062305 & 4.8234444 \\ \text { C } & 0.0083272 & -1.7049782 & 6.0605494 \\ \text { C } & -1.3301994 & -1.5404542 & 6.4530919 \\ \text { C } & -2.2337849 & -0.8795747 & 5.6024008 \\ \text { P } & 0.0512395 & 0.1854818 & 2.3526369 \\ \text { C } & 1.6424265 & -0.6628819 & 1.9834237 \\ \text { C } & 1.6083585 & -2.0536910 & 1.7453699 \\ \text { C } & 2.7710761 & -2.7325095 & 1.3618748 \\ \text { C } & 3.9734964 & -2.0254650 & 1.1838931 \\ \text { C } & 4.0080058 & -0.6399247 & 1.4041425 \\ \text { C } & 2.8488931 & 0.0416842 & 1.8119124 \\ \text { Pd } & -1.5008018 & 0.0260200 & 0.7432027 \\ \text { C } & -2.4390807 & -2.1173083 & -1.2008961 \\ \text { C } & -1.8487784 & -2.2769722 & -2.6268531 \\ \text { C } & -1.3093436 & -0.8774814 & -2.9610708 \\ \text { C } & -0.1788480 & -0.9365063 & -3.7287797 \\ \text { C } & 0.2592671 & -2.4130340 & -3.8711373 \\ \text { C } & -0.6066499 & -3.1606536 & -2.8213062 \\ \text { C } & -2.1040990 & 0.0917489 & -2.2065954 \\ \text { C } & -2.7036594 & -0.6170713 & -1.0994221 \\ \text { C } & -3.5491016 & 0.0739812 & -0.1893720 \\ \text { C } & -3.7894152 & 1.4635626 & -0.3911755 \\ \text { C } & -3.2436400 & 2.1267984 & -1.5003794 \\ \text { C } & -2.4083890 & 1.4501334 & -2.4108683 \\ \text { C } & 0.6515428 & 0.1298845 & -4.3045156 \\ \text { C } & 0.6258094 & 1.4621665 & -3.8241590 \\ \text { C } & 1.4469372 & 2.4515421 & -4.3781308 \\ \text { C } & 2.3124344 & 2.1436759 & -5.4408207 \\ \text { C } & 2.3596824 & 0.8258977 & -5.9265510 \\ \text { C } & 1.5512337 & -0.1692287 & -5.3618925 \\ \text { C } & -2.9746215 & -2.6399695 & -3.6203909 \\ \text { O } & 1.6468298 & -2.6079523 & -3.6763548 \\ \text { O } & 1.6446801 & -1.1504360 & -1.3083434 \\ \text { C } & 0.5647339 & 1.8947567 & 2.8081982 \\ \text { C } & 0.3157029 & 2.9353057 & 1.8910597 \\ \text { C } & 0.7273294 & 4.2450090 & 2.1835702 \\ \text { C } & 1.3782084 & 4.5240186 & 3.3966184 \\ \text { C } & 1.6179323 & 3.4903505 & 4.3191884 \\ \text { C } & 1.2137507 & 2.1789275 & 4.0275743 \\ \text { H } & 0.0465453 & -2.7842235 & -4.9005917 \\ \text { H } & -0.0190167 & -3.1825356 & -1.8814576 \\ \text { H } & -0.8326959 & -4.2051993 & -3.1147372 \\ & -1.7012748 & -2.4366289 & -0.4337352 \\ \text { H } & -2.5622426 & -2.7097362 & -1.0341868 \\ & & -2.6434113 & -4.6635306\end{array}$




\begin{tabular}{|c|c|c|c|}
\hline & -3.8052113 & -1.9062771 & -3.5644612 \\
\hline & -3.3853938 & -3.6457109 & -3.3933901 \\
\hline & 1.8372705 & -2.1952719 & -2.7983198 \\
\hline & -0.0044037 & 1.7132656 & -2.9646152 \\
\hline & 1.4176981 & 3.4716245 & -3.9657769 \\
\hline & 2.9547317 & 2.9222793 & -5.8798361 \\
\hline & 3.0411728 & 0.5668210 & -6.7515830 \\
\hline & 1.6290680 & -1.2001183 & -5.7291927 \\
\hline & -2.0195517 & 1.9767777 & -3.2924357 \\
\hline & -3.4753617 & 3.1896682 & -1.6650785 \\
\hline & -4.4486290 & 2.0013211 & 0.3059347 \\
\hline & -4.1278042 & -0.4787013 & 0.5688588 \\
\hline & 0.2285129 & 0.5377349 & -0.3648320 \\
\hline & -0.2064571 & 2.7044011 & 0.9494570 \\
\hline & 0.5333929 & 5.0522368 & 1.4612015 \\
\hline & 1.6955934 & 5.5522434 & 3.6281098 \\
\hline & 2.1210558 & 3.7078400 & 5.2736930 \\
\hline & 1.3978460 & 1.3722848 & 4.7 \\
\hline & 0.6564661 & -2.5989012 & 5722 \\
\hline & 2.7346658 & -3.816 & 1.1752744 \\
\hline & 4.8823991 & -2.55 & 0.8636266 \\
\hline & 4.94 & -0.0 & 39089 \\
\hline & $2.87 \varepsilon$ & 1.1 & 1.9 \\
\hline & 1.496 & -1.3 & 4.5 \\
\hline & 0.717 & -2.2 & 6.7 \\
\hline & -1.67273 & -1.934 & 7.4 \\
\hline & -3.2851581 & -0.7551251 & 5.9032524 \\
\hline & -2.5021969 & 0.1164397 & 3.6792708 \\
\hline & 1.8682618 & 0.0559925 & -1.2538797 \\
\hline & 1.0647896 & 0.9829036 & -0.7509122 \\
\hline & 2.9953059 & 0.6341432 & -1.7099292 \\
\hline & 3.5175119 & -0.0850776 & -2.1190480 \\
\hline \\
\hline \\
\hline \\
\hline & -1.6264444 & 0.4107451 & -2.3129074 \\
\hline & -1.6573389 & 1.0482925 & -1.0543358 \\
\hline & -2.8739101 & 1.1125279 & -0.3434255 \\
\hline & -4.0417011 & 0.5571238 & -0.8898889 \\
\hline & -4.0068610 & -0.0638857 & -2.1494628 \\
\hline & -2.7976091 & -0.1309849 & -2.8598425 \\
\hline & -0.0946563 & 1.6261943 & -0.2786881 \\
\hline & -0.6516630 & 3.0126769 & 0.8078218 \\
\hline & -1.4451508 & 4.0679386 & 0.3115886 \\
\hline & -1.8470595 & 5.1068831 & 1.162912 \\
\hline
\end{tabular}




\begin{tabular}{lrrr} 
C & -1.4563406 & 5.1044407 & 2.5144408 \\
C & -0.6601874 & 4.0607616 & 3.0119122 \\
C & -0.2587526 & 3.0184001 & 2.1600655 \\
Pd & 0.8969717 & 0.0026773 & 0.8894698 \\
C & -0.0686909 & -1.1152806 & 3.3544874 \\
C & -1.1412045 & -2.0838591 & 2.7720617 \\
C & -0.6071491 & -2.4058789 & 1.3692380 \\
C & -1.6087002 & -2.5591942 & 0.4492740 \\
C & -2.9524980 & -2.3088886 & 1.1357022 \\
C & -2.5577317 & -1.5706391 & 2.4470612 \\
C & 0.8590854 & -2.3023530 & 1.4171283 \\
C & 1.8958484 & -2.9479525 & 0.6962946 \\
C & 3.2284690 & -2.7276088 & 1.0665967 \\
C & 3.5549419 & -1.8485410 & 2.1203696 \\
C & 2.5457640 & -1.2170143 & 2.8663042 \\
C & 1.1878715 & -1.4662404 & 2.5520341 \\
C & -1.5249838 & -2.8192086 & -0.9906120 \\
C & -2.6334561 & -3.3737588 & -1.6778916 \\
C & -2.5794990 & -3.5989064 & -3.0604178 \\
C & -1.4256440 & -3.2672224 & -3.7919147 \\
C & -0.3267095 & -2.6984171 & -3.1239894 \\
C & -0.3753140 & -2.4781089 & -1.7425573 \\
C & -1.1718252 & -3.3643268 & 3.6367434 \\
O & -3.7206589 & -3.5098187 & 1.3289497 \\
O & 4.1878196 & 0.2908320 & -0.2535561 \\
C & 0.7994454 & 2.5127744 & -1.6223151 \\
C & 2.1983686 & 2.6278224 & -1.4882452 \\
C & 2.9409386 & 3.3353207 & -2.4459272 \\
C & 2.2940540 & 3.9266508 & -3.5439050 \\
C & 0.8998159 & 3.8118697 & -3.6814213 \\
C & 0.1516709 & 3.1092963 & -2.7240235 \\
H & -3.5962972 & -1.6643301 & 0.5032102 \\
H & -3.3078026 & -1.7353553 & 3.2455603 \\
H & -2.5084048 & -0.4836155 & 2.2277800 \\
H & -0.3691361 & -0.0556009 & 3.1955314 \\
H & 0.0929415 & -1.2472799 & 4.4445773 \\
H & -1.8660071 & -4.1175393 & 3.2153538 \\
H & -1.5163947 & -3.1281256 & 4.6643879 \\
H & -0.1651164 & -3.8253923 & 3.7052143 \\
H & -3.0827458 & -4.2169801 & 1.5430548 \\
H & -3.5304198 & -3.6435151 & -1.1012832 \\
H & -3.4487551 & -4.0386935 & -3.5734451 \\
H & -1.3853085 & -3.4465144 & -4.8772258 \\
H & 0.5759248 & -2.4071877 & -3.6829780 \\
& 0.4707800 & -1.9901687 & -1.2441891 \\
\hline & 1.9505961 & -0.3242461 & -0.8129742
\end{tabular}




$\begin{array}{rrrr}\text { H } & 2.8039823 & -0.5641020 & 3.7135388 \\ \text { H } & 4.6097779 & -1.6503794 & 2.3563931 \\ \text { H } & 4.0355609 & -3.2229607 & 0.5068819 \\ \text { H } & 1.6487459 & -3.6213840 & -0.1355405 \\ \text { H } & 2.7094318 & 2.1436763 & -0.6402777 \\ \text { H } & 4.0330306 & 3.4123973 & -2.3350473 \\ \text { H } & 2.8777881 & 4.4746150 & -4.2993254 \\ \text { H } & 0.3902830 & 4.2708923 & -4.5425010 \\ \text { H } & -0.9378349 & 3.0119061 & -2.8434291 \\ \text { H } & -1.7534151 & 4.0744961 & -0.7445454 \\ \text { H } & -2.4681693 & 5.9261810 & 0.7699988 \\ \text { H } & -1.7727256 & 5.9221835 & 3.1797174 \\ \text { H } & -0.3475142 & 4.0572693 & 4.0670938 \\ \text { H } & 0.3741911 & 2.1939762 & 2.5292226 \\ \text { H } & -2.9077340 & 1.6040818 & 0.6402003 \\ \text { H } & -4.9858264 & 0.6135242 & -0.3269434 \\ \text { H } & -4.9211498 & -0.5039280 & -2.5744289 \\ \text { H } & -2.7562206 & -0.6339667 & -3.8362521 \\ \text { H } & -0.6793381 & 0.3323062 & -2.8657033 \\ \text { C } & 3.8368827 & -0.3720713 & -1.2072363 \\ \mathrm{O} & 4.7211528 & -0.9173935 & -2.0779544 \\ \mathrm{O} & 2.5718027 & -0.6656993 & -1.5628652 \\ \mathrm{H} & 4.2049422 & -1.4072688 & -2.7498466\end{array}$

\section{Optimized Geometries(TS-BXne-k)}

\begin{tabular}{lrrr} 
R3 & & & \\
79 & \multicolumn{4}{l}{} \\
SCF & Energy $=-4548.281800943$ & \\
C & 0.0694403 & -6.5292970 & 1.4698277 \\
C & -0.4811696 & -5.3643768 & 0.8836040 \\
C & -1.6055149 & -5.5109707 & 0.0334491 \\
C & -2.1330957 & -6.7758986 & -0.2500537 \\
C & -1.5578015 & -7.9275233 & 0.3177843 \\
C & -0.4585400 & -7.7967162 & 1.1816735 \\
C & 0.0484562 & -4.0297430 & 1.2142741 \\
Pd & -0.2090394 & 0.2751202 & -0.9184876 \\
P & -0.0700514 & 2.4206688 & 0.2365228 \\
C & 0.1993895 & -2.9246546 & 0.4162098 \\
Br & -0.3643153 & 1.6304226 & -3.1365083 \\
C & 0.1191619 & -2.8323937 & -1.0500865 \\
C & 0.4168019 & -3.9987120 & -1.8072271 \\
C & 0.2958163 & -4.0314189 & -3.1964919
\end{tabular}




\begin{tabular}{|c|c|c|c|}
\hline & -0.1307448 & -2.8772617 & -3.8713467 \\
\hline & -0.3641587 & -1.6963549 & -3.1506675 \\
\hline & -0.1958566 & -1.6264580 & -1.7494533 \\
\hline & 0.4813503 & -3.7193455 & 2.6476930 \\
\hline & -0.2382140 & -4.4014335 & 3.6635677 \\
\hline & 0.3701126 & -2.1818623 & 2.7036787 \\
\hline & 0.5412724 & -1.6862892 & 1.2373479 \\
\hline & -0.4823986 & -0.5931592 & 0.9406988 \\
\hline & 1.9887545 & -1.2215290 & 0.9759425 \\
\hline & 1.5398415 & -4.0385850 & 2.7953866 \\
\hline & 1.0860764 & -1.7065476 & 3.4027133 \\
\hline & -0.6530394 & -1.9460586 & 3.0658790 \\
\hline & -1.5103683 & -1.0047326 & 0.9827977 \\
\hline & -0.3978442 & 0.214 & 1.6 \\
\hline & 2.2422479 & -0.34 & 1.5 \\
\hline & 2.13 & -0.9 & -0.0 \\
\hline & 2.71 & -2.0 & 1.2 \\
\hline & -1.177 & -4.3 & 3.3 \\
\hline & -2.06 & -4.61 & -0.4 \\
\hline & 52891 & -6.8655005 & -0.9 \\
\hline & -1.9733809 & -8.9221233 & 0.0 \\
\hline & -0.0088338 & -8.6905726 & 1.6406220 \\
\hline & 0.9209904 & -6.4370955 & 2.1584218 \\
\hline & -1.5301356 & 0.5140099 & -1.6714570 \\
\hline & -0.6487869 & -0.7928773 & -3.7067454 \\
\hline & -0.2580196 & -2.8792771 & -4.9651880 \\
\hline & 0.5309872 & -4.9543782 & -3.7481186 \\
\hline & 0.7521397 & -4.8969118 & -1.2712926 \\
\hline & 0.0349907 & 4.0613145 & -0.5870838 \\
\hline & 1.4708586 & 2.296 & 1.21 \\
\hline & -1.4257436 & 2.741 & 1.4360537 \\
\hline & -1.3548705 & 3.84 & 2.31 \\
\hline & -2.4187820 & 4.11 & 3.1 \\
\hline & -3.5668064 & 3.2979341 & 3.1758251 \\
\hline & -3.6505357 & 2.2180237 & 2.2829580 \\
\hline & -2.5829611 & 1.9387275 & 1.4143934 \\
\hline & -0.4679767 & 4.4923762 & 2.3131347 \\
\hline & -2.3570075 & 4.9662777 & 3.8803060 \\
\hline & -4.4028144 & 3.5149303 & 3.8578275 \\
\hline & -4.5523383 & 1.5880665 & 2.2590435 \\
\hline & -2.6439159 & 1.0975830 & 0.7086042 \\
\hline & 1.1093757 & 4.9518890 & -0.4014406 \\
\hline & 1.0971431 & 6.2079876 & -1.0315353 \\
\hline & 0.0135685 & 6.5823836 & -1.8404926 \\
\hline & -1.0648415 & 5.6979618 & -2.0185914 \\
\hline & -1.0547820 & 4.4413855 & -1.4001415 \\
\hline
\end{tabular}




$\begin{array}{rrrr}\text { H } & 1.9572248 & 4.6720818 & 0.2397100 \\ \text { H } & 1.9416354 & 6.8978985 & -0.8827578 \\ \text { H } & 0.0072324 & 7.5660519 & -2.3338932 \\ \text { H } & -1.9168433 & 5.9846477 & -2.6529966 \\ \text { H } & -1.8943731 & 3.7480170 & -1.5519208 \\ \mathrm{C} & 1.4855797 & 2.1416574 & 2.6191505 \\ \mathrm{C} & 2.7002083 & 1.9145573 & 3.2883517 \\ \mathrm{C} & 3.9036134 & 1.8467546 & 2.5686695 \\ \mathrm{C} & 3.8924812 & 1.9944307 & 1.1700139 \\ \mathrm{C} & 2.6827368 & 2.2054579 & 0.4952286 \\ \mathrm{H} & 0.5462594 & 2.1914222 & 3.1884939 \\ \mathrm{H} & 2.7024675 & 1.7874994 & 4.3813327 \\ \mathrm{H} & 4.8524436 & 1.6680083 & 3.0962074 \\ \mathrm{H} & 4.8306354 & 1.9278539 & 0.5991934 \\ \mathrm{H} & 2.6719137 & 2.2867999 & -0.6034764\end{array}$

$\mathrm{R} 4$

79

SCF Energy $=-4548.285564658$

C $\quad-0.9247499 \quad 5.2606305$

C

C

$\mathrm{C}$

$\mathrm{Br} \quad-2.9532129$

C $\quad-0.8893975$

C $\quad-2.4254828$

C $\quad-0.2602077$

C $\quad-0.3537445$

C $\quad 0.8891663$

$0 \quad 0.9881371$

$\mathrm{H} \quad 1.8194924$

3.8981730

0.4139865

0.0910293

3.5067189

$-1.2687375$

$-2.3031178$

4.4403124

5.7970050

$-1.9908121$

$6.1905701-0.6483096$

$2.9271615 \quad 1.1391465$

$1.9953080 \quad 1.0798722$

$1.7474818-0.0151313$

$0.4445387-0.5350756$

0.2045966

$-1.5428899$

$\begin{array}{ll}1.2586084 & -2.0438767\end{array}$

$2.5571318-1.5310418$

$2.7998182-0.5257031$

$\begin{array}{ll}7.6758742 & 1.6681455\end{array}$

$3.0393535 \quad 2.5449477$

$3.0897128 \quad 2.5947988$

$4.2691209 \quad 3.2024764$

$1.7414804 \quad 3.2257172$

$1.3231140 \quad 2.4351173$

$-0.1008166 \quad 2.4062591$

2.8903138

$-0.1664920 \quad 1.8648758$

4.3106899

3.0994301

3.1802812

4.2538393

2.1303743 


$\begin{array}{lrrr}\text { H } & -2.8220700 & 3.9751110 & 2.0623169 \\ \mathrm{H} & -2.7746500 & 3.1434981 & 3.6471776 \\ \mathrm{H} & 1.8502385 & -0.3161204 & 2.0021722 \\ \mathrm{H} & 1.1945405 & -0.3714436 & -0.1602068 \\ \mathrm{H} & 2.9024129 & -0.8127009 & -1.9453829 \\ \mathrm{H} & 4.3006174 & 1.0706818 & -2.8353537 \\ \mathrm{H} & 3.9959511 & 3.3878853 & -1.9188153 \\ \mathrm{H} & 2.3025736 & 3.8144428 & -0.1236189 \\ \mathrm{H} & -0.4239763 & 2.4460262 & -1.5039936 \\ \mathrm{H} & -0.6596078 & 4.1093777 & -3.3514510 \\ \mathrm{H} & -0.9701158 & 6.5502620 & -2.7897448 \\ \mathrm{H} & -1.2146389 & 7.2482394 & -0.4229756 \\ \mathrm{H} & -2.2158877 & 6.0765177 & 1.3619822 \\ \mathrm{Pd} & -0.8503571 & 6.0551815 & 2.3544972 \\ \mathrm{P} & -0.4088049 & 7.1025355 & 4.4090496 \\ \mathrm{C} & -0.4742639 & 8.9292164 & 4.2683117 \\ \mathrm{C} & -0.1090911 & 9.5073571 & 3.0344632 \\ \mathrm{H} & 0.1610697 & 8.8575314 & 2.1872889 \\ \mathrm{C} & -0.1116547 & 10.9019221 & 2.8836923 \\ \mathrm{H} & 0.1714889 & 11.3470726 & 1.9182344 \\ \mathrm{C} & -0.4943338 & 11.7235521 & 3.9570164 \\ \mathrm{H} & -0.5076644 & 12.8172311 & 3.8351341 \\ \mathrm{C} & -0.8722908 & 11.1489821 & 5.1825295 \\ \mathrm{H} & -1.1825643 & 11.7909155 & 6.0208124 \\ \mathrm{C} & -0.8617544 & 9.7547455 & 5.3415844 \\ \mathrm{H} & -1.1671647 & 9.3067140 & 6.2986018 \\ \mathrm{C} & 1.2533565 & 6.7655945 & 5.1139340 \\ \mathrm{C} & 1.5064215 & 5.5478514 & 5.7855222 \\ \mathrm{H} & 0.6850927 & 4.8440176 & 5.9807309 \\ \mathrm{C} & 2.8034190 & 5.2375861 & 6.2200803 \\ \mathrm{H} & 2.9871312 & 4.2883407 & 6.7456655 \\ \mathrm{C} & 3.8611196 & 6.1328272 & 5.9884080 \\ \mathrm{H} & 4.8776815 & 5.8868503 & 6.3303293 \\ \mathrm{C} & 3.6142263 & 7.3451066 & 5.3236261 \\ \mathrm{H} & 4.4362133 & 8.0545653 & 5.1445488 \\ \mathrm{C} & 2.3186644 & 7.6623097 & 4.8875395 \\ \mathrm{H} & 2.1319335 & 8.6153590 & 4.3711056 \\ \mathrm{C} & -1.5875787 & 6.6583023 & 5.7383959 \\ \mathrm{C} & -1.2516718 & 6.7309506 & 7.1070173 \\ \mathrm{H} & -0.2378510 & 7.0331189 & 7.4095871 \\ \mathrm{C} & -2.2084431 & 6.4050682 & 8.0809196 \\ \mathrm{H} & -1.9423834 & 6.4597523 & 9.1474274 \\ & -3.5004098 & 6.0084779 & 7.6953839 \\ \mathrm{H} & -4.2470391 & 5.7496721 & 8.4614419 \\ & -4.8375092 & 5.9385222 & 6.3331077 \\ & & & \\ \mathrm{H} & & \end{array}$




\begin{tabular}{lrrr} 
C & -2.8852618 & 6.2593431 & 5.3541269 \\
H & -3.1410217 & 6.2119579 & 4.2836649 \\
& & & \\
S3 & & & \\
79 & \multicolumn{3}{r}{} \\
SCF & Energy $=-4548.291681654$ & \\
C & -1.9841859 & -0.2253298 & -2.0256696 \\
C & -1.3902658 & -1.1163982 & -2.9556410 \\
C & -1.9369997 & -1.3004562 & -4.2366693 \\
C & -3.1021564 & -0.6029870 & -4.5982921 \\
C & -3.7011494 & 0.2777958 & -3.6836094 \\
C & -3.1435695 & 0.4718481 & -2.4039811 \\
C & -0.1522861 & -1.7341284 & -2.4343344 \\
C & 1.1190209 & -1.2851551 & -2.6184804 \\
C & 1.5947462 & -0.2053113 & -3.5124170 \\
C & 0.8435781 & 0.9785046 & -3.7152555 \\
C & 1.3460222 & 2.0108237 & -4.5208826 \\
C & 2.5996161 & 1.8842998 & -5.1417982 \\
C & 3.3594933 & 0.7189310 & -4.9412692 \\
C & 2.8652768 & -0.3108105 & -4.1286060 \\
Br & -1.1669671 & 2.5750684 & -0.2628390 \\
C & -0.2769171 & -2.7089376 & -1.2519290 \\
C & -0.8779987 & -4.0540648 & -1.7110756 \\
C & -1.1997513 & -2.0824651 & -0.1834093 \\
C & 1.2074146 & -2.8661844 & -0.7993558 \\
C & 1.9776600 & -1.7114821 & -1.4414885 \\
O & 1.9895265 & -0.5723379 & -0.5329751 \\
H & 3.0309755 & -1.9711674 & -1.6884085 \\
H & 1.3305256 & -2.8711988 & 0.2993115 \\
H & 1.6154290 & -3.8221491 & -1.1840822 \\
H & -0.9474570 & -2.4532656 & 0.8266561 \\
H & -2.2640885 & -2.2936629 & -0.3944821 \\
H & -0.2402246 & -4.5310888 & -2.4839144 \\
H & -1.8882719 & -3.9019520 & -2.1416025 \\
H & -0.9691332 & -4.7529131 & -0.8538557 \\
H & 2.0040314 & 0.2284910 & -1.0986105 \\
H & -0.1288167 & 1.0971535 & -3.2186924 \\
H & 0.7509897 & 2.9267121 & -4.6552368 \\
H & 2.9889736 & 2.6959051 & -5.7751933 \\
H & 4.3446394 & 0.6124789 & -5.4207685 \\
H & 3.4635192 & -1.2225507 & -3.9763173 \\
H & -1.4394419 & -1.9768817 & -4.9485230 \\
H & -3.5381760 & -0.7419480 & -5.5992956 \\
H & -4.6106534 & 0.8302979 & -3.9677279 \\
& -3.6077549 & 1.1966961 & -1.7183159 \\
& -2.1345015 & 0.8922566 & 0.1721728
\end{tabular}




$\begin{array}{lrrr}\text { Pd } & -0.9467652 & -0.0141331 & -0.2494157 \\ \text { P } & 0.0671617 & 0.0916600 & 1.9537154 \\ \text { C } & -1.2110686 & 0.1769839 & 3.2765240 \\ \text { C } & -2.5783677 & 0.2150319 & 2.9469348 \\ \text { H } & -2.8779488 & 0.1982567 & 1.8879349 \\ \text { C } & -3.5520711 & 0.2570328 & 3.9592271 \\ \text { H } & -4.6182520 & 0.2862188 & 3.6891594 \\ \text { C } & -3.1635237 & 0.2577586 & 5.3074347 \\ \text { H } & -3.9254807 & 0.2904294 & 6.1009733 \\ \text { C } & -1.7982709 & 0.2102115 & 5.6448087 \\ \text { H } & -1.4905165 & 0.2046293 & 6.7014466 \\ \text { C } & -0.8264678 & 0.1660955 & 4.6364971 \\ \text { H } & 0.2410052 & 0.1202947 & 4.9007352 \\ \text { C } & 1.1108891 & 1.5776715 & 2.2433475 \\ \text { C } & 2.3142833 & 1.7423914 & 1.5210812 \\ \text { H } & 2.6568166 & 0.9504836 & 0.8412764 \\ \text { C } & 3.0633868 & 2.9203716 & 1.6530561 \\ \text { H } & 4.0017936 & 3.0313489 & 1.0887618 \\ \text { C } & 2.6124300 & 3.9586113 & 2.4844138 \\ \text { H } & 3.1965538 & 4.8865206 & 2.5781538 \\ \text { C } & 1.4037939 & 3.8113376 & 3.1821641 \\ \text { H } & 1.0312333 & 4.6260038 & 3.8214507 \\ \text { C } & 0.6545412 & 2.6309424 & 3.0628770 \\ \text { H } & -0.2991522 & 2.5381142 & 3.5997312 \\ \text { C } & 1.0304775 & -1.3710041 & 2.5283006 \\ \text { C } & 2.4350516 & -1.4511749 & 2.4563311 \\ \text { H } & 3.0222812 & -0.5793731 & 2.1444270 \\ \text { C } & 3.0940800 & -2.6463078 & 2.7830066 \\ \text { H } & 4.1921744 & -2.6909059 & 2.7250315 \\ \text { C } & 2.3627013 & -3.7784580 & 3.1775320 \\ \text { H } & 2.8830981 & -4.7160883 & 3.4242601 \\ \text { C } & 0.9631424 & -3.7024869 & 3.2663132 \\ \text { H } & 0.3793670 & -4.5778819 & 3.5886197 \\ \text { C } & 0.3009415 & -2.5071908 & 2.9490255 \\ \text { H } & -0.7934318 & -2.4514016 & 3.0460680 \\ & & & \\ \text { S4 } & & & \\ \text { 79 } & & & \\ \text { SCF } & \text { Energy }=-4548.273234355 & \\ \text { C } & 1.3518724 & 3.8001631 & -1.8728915 \\ \text { C } & 0.5506142 & 3.8974911 & -0.7010092 \\ \text { C } & -0.7131972 & 4.5466613 & -0.8009902 \\ \text { C } & -1.1352253 & 5.0175444 & -2.0671656 \\ \text { C } & -0.3376931 & 4.8741504 & -3.2133659 \\ \text { C } & 0.9149813 & 4.2507442 & -3.1168459 \\ & 1.1239543 & 3.3680159 & 0.5450862\end{array}$




\begin{tabular}{lrrr} 
C & 2.0035326 & 2.3196277 & 0.6697652 \\
$\mathrm{C}$ & 2.4098407 & 1.3170344 & -0.3390461 \\
$\mathrm{C}$ & 3.7459291 & 0.8489152 & -0.4022001 \\
$\mathrm{C}$ & 4.1234882 & -0.1209630 & -1.3456364 \\
$\mathrm{C}$ & 3.1786719 & -0.6498862 & -2.2398446 \\
$\mathrm{C}$ & 1.8455985 & -0.2042271 & -2.1789756 \\
$\mathrm{C}$ & 1.4650854 & 0.7615222 & -1.2391303 \\
$\mathrm{Br}$ & -4.0121531 & 5.7255016 & -0.7204922 \\
$\mathrm{C}$ & 0.8145872 & 4.0424571 & 1.8825715 \\
$\mathrm{C}$ & 1.3528854 & 5.4874615 & 1.9704686 \\
$\mathrm{C}$ & -0.6997122 & 4.0309281 & 2.1188996 \\
$\mathrm{C}$ & 1.5679914 & 3.1075219 & 2.8950118 \\
$\mathrm{C}$ & 2.6029637 & 2.3127866 & 2.0677976 \\
$\mathrm{O}$ & 3.9261831 & 2.8814508 & 2.0711956 \\
$\mathrm{H}$ & 2.7519698 & 1.2873884 & 2.4643277 \\
$\mathrm{H}$ & 2.0664036 & 3.6640600 & 3.7132290 \\
$\mathrm{H}$ & 0.8613092 & 2.3891357 & 3.3608079 \\
$\mathrm{H}$ & -1.0792713 & 2.9891728 & 2.0798656 \\
$\mathrm{H}$ & -0.9443253 & 4.4811923 & 3.1001889 \\
$\mathrm{H}$ & 2.4570807 & 5.5192265 & 1.8745045 \\
$\mathrm{H}$ & 1.0878783 & 5.9322627 & 2.9519208 \\
$\mathrm{H}$ & 0.9205853 & 6.1281687 & 1.1758773 \\
$\mathrm{H}$ & 3.8994381 & 3.6571270 & 1.4769384 \\
$\mathrm{H}$ & 4.4880353 & 1.2735824 & 0.2900821 \\
$\mathrm{H}$ & 5.1690885 & -0.4638071 & -1.3858954 \\
$\mathrm{H}$ & 3.4756660 & -1.4100124 & -2.9790208 \\
$\mathrm{H}$ & 1.0953743 & -0.6160142 & -2.8718258 \\
$\mathrm{H}$ & 0.4203721 & 1.1019223 & -1.1975113 \\
$\mathrm{H}$ & -2.5727967 & 3.7115480 & 0.3859961 \\
$\mathrm{H}$ & -2.1163866 & 5.4993195 & -2.1629266 \\
$\mathrm{H}$ & -0.7019842 & 5.2483985 & -4.1840002 \\
$\mathrm{H}$ & 1.5479924 & 4.1052662 & -4.0052491 \\
$\mathrm{H}$ & 2.3446057 & 3.3393819 & -1.7858945 \\
$\mathrm{Pd}$ & -1.9633676 & 5.0418184 & 0.7706462 \\
$\mathrm{P}$ & -3.2639139 & 6.0103567 & 2.8020128 \\
$\mathrm{C}$ & -2.0677386 & 6.5331608 & 4.1115052 \\
$\mathrm{C}$ & -1.9649866 & 5.8697246 & 5.3527765 \\
$\mathrm{H}$ & -2.6576937 & 5.0520114 & 5.5985086 \\
$\mathrm{C}$ & -0.9802493 & 6.2491398 & 6.2813628 \\
$\mathrm{H}$ & -0.9111633 & 5.7235926 & 7.2461220 \\
$\mathrm{C}$ & -0.0927050 & 7.2952620 & 5.9857067 \\
$\mathrm{H}$ & 0.6750592 & 7.5914621 & 6.7169069 \\
$\mathrm{H}$ & -0.1881661 & 7.9612622 & 4.7508523 \\
\hline
\end{tabular}




$\begin{array}{lrrr}\text { C } & -4.3068702 & 4.7549043 & 3.6482740 \\ \text { C } & -5.3282095 & 5.1858155 & 4.5212843 \\ \text { H } & -5.4955105 & 6.2625238 & 4.6691853 \\ \text { C } & -6.1343577 & 4.2492382 & 5.1823775 \\ \text { H } & -6.9299589 & 4.5987063 & 5.8574116 \\ \text { C } & -5.9293037 & 2.8749198 & 4.9702864 \\ \text { H } & -6.5736038 & 2.1409066 & 5.4773505 \\ \text { C } & -4.9127394 & 2.4402307 & 4.1039962 \\ \text { H } & -4.7568043 & 1.3644424 & 3.9329091 \\ \text { C } & -4.1005322 & 3.3759709 & 3.4409280 \\ \text { H } & -3.3102689 & 3.0363159 & 2.7562528 \\ \text { C } & -4.4365502 & 7.4414031 & 2.6647397 \\ \text { C } & -4.3584342 & 8.6275024 & 3.4308100 \\ \text { H } & -3.6405990 & 8.7209004 & 4.2595392 \\ \text { C } & -5.1995545 & 9.7147277 & 3.1242599 \\ \text { H } & -5.1777525 & 10.6212740 & 3.7483671 \\ \text { C } & -6.0425817 & 9.6666417 & 2.0005616 \\ \text { H } & -6.5935799 & 10.5649300 & 1.6802835 \\ \text { C } & -6.1705167 & 8.4682684 & 1.2824904 \\ \text { H } & -6.8136359 & 8.4134684 & 0.3896661 \\ \text { C } & -5.4298865 & 7.3408201 & 1.6635815 \\ \text { H } & -5.5801036 & 6.3894648 & 1.1410263\end{array}$

\section{Optimized Geometries(BXnk)}

$\begin{array}{lrrr}\text { R3 } & & & \\ 75 & & & \\ \text { SCF } & \text { Energy }=-4548.296931013 & \\ \text { C } & 0.5663550 & -6.7761834 & 0.7332637 \\ \text { C } & -0.0769977 & -5.5862358 & 0.3160774 \\ \text { C } & -1.1176754 & -5.6875814 & -0.6401963 \\ \text { C } & -1.4733862 & -6.9260729 & -1.1872025 \\ \text { C } & -0.8058068 & -8.0969562 & -0.7840694 \\ \text { C } & 0.2109180 & -8.0158595 & 0.1813145 \\ \text { C } & 0.2715981 & -4.2884483 & 0.9203049 \\ \text { Pd } & -0.2532659 & 0.2842652 & -0.4591179 \\ \text { P } & -0.1494962 & 2.3724357 & 0.6548743 \\ \text { C } & 0.3522478 & -3.0506457 & 0.3379387 \\ \text { Br } & -3.1886601 & 1.0679001 & -1.7500664 \\ \text { C } & 0.3419412 & -2.7009649 & -1.0917746 \\ \text { C } & 0.7706481 & -3.6295937 & -2.0767108 \\ \text { C } & 0.7311797 & -3.3150480 & -3.4386624 \\ \text { C } & 0.2516694 & -2.0615647 & -3.8595319\end{array}$




$\begin{array}{rrrr}\text { C } & -0.1465854 & -1.1191688 & -2.8969050 \\ \mathrm{C} & -0.0601666 & -1.4087735 & -1.5181847 \\ \mathrm{C} & 0.5643943 & -4.1985438 & 2.4174932 \\ \mathrm{O} & -0.1452389 & -5.1152206 & 3.2345791 \\ \mathrm{C} & 0.2818509 & -2.7126402 & 2.7230348 \\ \mathrm{C} & 0.5460585 & -1.9500975 & 1.3887745 \\ \mathrm{C} & -0.4867170 & -0.8407695 & 1.2403502 \\ \mathrm{C} & 1.9846052 & -1.3926337 & 1.3513372 \\ \mathrm{H} & 1.6387880 & -4.4325340 & 2.6041262 \\ \mathrm{H} & 0.8707954 & -2.3097352 & 3.5702402 \\ \mathrm{H} & -0.7923111 & -2.6386709 & 2.9958755 \\ \mathrm{H} & -1.5163549 & -1.2494917 & 1.1965092 \\ \mathrm{H} & -0.4020056 & -0.1128656 & 2.0736222 \\ \mathrm{H} & 2.1166584 & -0.5933079 & 2.1075300 \\ \mathrm{H} & 2.2089635 & -0.9597727 & 0.3553040 \\ \mathrm{H} & 2.7260603 & -2.1919904 & 1.5525596 \\ \mathrm{H} & -1.0599613 & -5.1473961 & 2.8929217 \\ \mathrm{H} & -1.6466367 & -4.7743691 & -0.9500605 \\ \mathrm{H} & -2.2837807 & -6.9803848 & -1.9301583 \\ \mathrm{H} & -1.0865375 & -9.0708140 & -1.2131810 \\ \mathrm{H} & 0.7304468 & -8.9280972 & 0.5125786 \\ \mathrm{H} & 1.3534840 & -6.7255442 & 1.4986636 \\ \mathrm{H} & -1.9381030 & 0.4688751 & -0.9737682 \\ \mathrm{H} & -0.5295791 & -0.1391422 & -3.2343320 \\ \mathrm{H} & 0.1927473 & -1.8151200 & -4.9310079 \\ \mathrm{H} & 1.0706275 & -4.0552275 & -4.1791534 \\ \mathrm{H} & 1.1467673 & -4.6113507 & -1.7570858 \\ \mathrm{C} & 0.1611218 & 3.8535541 & -0.3845335 \\ \mathrm{C} & 1.2814498 & 2.2748547 & 1.7980158 \\ \mathrm{C} & -1.5572407 & 2.8710855 & 1.7207353 \\ \mathrm{C} & -1.5649633 & 4.1258568 & 2.3665679 \\ \mathrm{C} & -2.6433068 & 4.4852452 & 3.1867409 \\ \mathrm{C} & -3.7211605 & 3.5983127 & 3.3619315 \\ \mathrm{C} & -3.7204143 & 2.3540264 & 2.7124369 \\ \mathrm{C} & -2.6419153 & 1.9892053 & 1.8902377 \\ \mathrm{H} & -0.7267098 & 4.8241352 & 2.2204407 \\ \mathrm{H} & -2.6470118 & 5.4650977 & 3.6877839 \\ \mathrm{H} & -4.5691268 & 3.8844569 & 4.0025317 \\ \mathrm{H} & -4.5680714 & 1.6636492 & 2.8358990 \\ \mathrm{H} & -2.6476888 & 1.0251153 & 1.3632864 \\ \mathrm{C} & 1.0925894 & 4.8554124 & -0.0420853 \\ \mathrm{C} & 1.2544888 & 5.9745109 & -0.8742927 \\ & 0.4888944 & 6.1008638 & -2.0454947 \\ \mathrm{C} & -0.4418417 & 5.1044798 & -2.3863342 \\ & 1.6959098 & 4.7571046 & 0.8726149\end{array}$




$\begin{array}{rrrr}\mathrm{H} & 1.9838868 & 6.7533642 & -0.6044266 \\ \mathrm{H} & 0.6197508 & 6.9788042 & -2.6961308 \\ \mathrm{H} & -1.0441759 & 5.1990553 & -3.3023517 \\ \mathrm{H} & -1.3416315 & 3.2019225 & -1.8272943 \\ \mathrm{C} & 1.1113547 & 2.1273835 & 3.1889132 \\ \mathrm{C} & 2.2236247 & 1.8814307 & 4.0122428 \\ \mathrm{C} & 3.5091249 & 1.7900182 & 3.4565922 \\ \mathrm{C} & 3.6830741 & 1.9371402 & 2.0684333 \\ \mathrm{C} & 2.5757513 & 2.1651750 & 1.2412684 \\ \mathrm{H} & 0.1062403 & 2.2036334 & 3.6289948 \\ \mathrm{H} & 2.0809925 & 1.7623859 & 5.0969101 \\ \mathrm{H} & 4.3781027 & 1.5984164 & 4.1037226 \\ \mathrm{H} & 4.6873822 & 1.8581871 & 1.6258901 \\ \mathrm{H} & 2.7125352 & 2.2490724 & 0.1517503\end{array}$

R4

75

SCF Energy $=-4548.295910824$

c

c

C

C

C

C

c

C

C

C

c

C

C

$\mathrm{H}$

$\mathrm{H}$
$-0.5597981$

$-0.4100134$

$-0.2364233$

$-0.2194280$

$-0.3804819$

$-0.5546571$

$-0.4512084$

0.3538648

1.5094362

1.6640666

2.7824873

3.7643397

3. 6210997

2. 5073085

$-3.3597939$

$-1.4204607$

$-2.8638086$

$-0.9001872$

$-1.2995672$

0.0451000

$-0.0757621$

0.8537641

$-1.4206958$

$-2.0881729$

0.0775884

$-1.6276142$

$-3.2288745$

$-2.9365441$
3. 9794052

3. 6362561

4.6303695

5.9806736

6.3305211

2.9703379

1.8887706

1.4841920

0.1439872

$-0.2405402$

0.7033620

2.0381505

2.4247633

7.7546121

3. 1805775

3.4554190

4.3119342

1.8237531

1. 2077512

$-0.2149905$

1.4843216

1.9242455

1.1343470

4.0565467

4.5914311

2.6456431

4.4135470
5.3403687
0.3150255

$-0.0455028$

$-1.4057995$

$-2.3942826$

$-2.0394011$

$-0.6869038$

1.0225121

1. 2069545

0.3825118

$-0.0461975$

$-0.8034463$

$-1.1446986$

$-0.7249192$

0.0316933

1.9115231

2. 1868188

1.7359585

3. 0685091

2. 9504344

2.5395384

2. 4962171

3.2633000

4.0471411

2.5849953

3. 5312717

3.8574633

1.0733487

1.1868436 


$\begin{array}{lrrr}\text { H } & -3.5362670 & 3.5159722 & 2.6163549 \\ \mathrm{H} & 0.8275748 & -0.5758639 & 2.4231504 \\ \mathrm{H} & 0.8748632 & -0.5832719 & 0.1940032 \\ \mathrm{H} & 2.8815538 & -1.2846188 & -1.1384982 \\ \mathrm{H} & 4.6391332 & 0.4016708 & -1.7406718 \\ \mathrm{H} & 4.3863844 & 2.7839280 & -0.9897558 \\ \mathrm{H} & 2.3923521 & 3.4682421 & 0.3610832 \\ \mathrm{H} & -0.1235119 & 2.5770549 & -1.6813954 \\ \mathrm{H} & -0.0943296 & 4.3489589 & -3.4509584 \\ \mathrm{H} & -0.3900518 & 6.7623334 & -2.8149239 \\ \mathrm{H} & -0.7074484 & 7.3927179 & -0.4236414 \\ \mathrm{H} & -2.1464365 & 6.7501468 & 2.0195513 \\ \mathrm{Pd} & -0.4801945 & 6.1130090 & 2.1666967 \\ \mathrm{P} & -0.1696179 & 7.2324979 & 4.2466841 \\ \mathrm{C} & -0.5727010 & 9.0194600 & 4.2190245 \\ \mathrm{C} & -0.2429449 & 9.7390649 & 3.0516486 \\ \mathrm{H} & 0.2283306 & 9.2182673 & 2.2021709 \\ \mathrm{C} & -0.5238149 & 11.1090766 & 2.9647378 \\ \mathrm{H} & -0.2697107 & 11.6629280 & 2.0487201 \\ \mathrm{C} & -1.1463984 & 11.7672310 & 4.0393871 \\ \mathrm{H} & -1.3779920 & 12.8405794 & 3.9669323 \\ \mathrm{C} & -1.4828536 & 11.0528039 & 5.2007792 \\ \mathrm{H} & -1.9765204 & 11.5652147 & 6.0403633 \\ \mathrm{C} & -1.1972727 & 9.6812269 & 5.2941907 \\ \mathrm{H} & -1.4726560 & 9.1187192 & 6.1986447 \\ \mathrm{C} & 1.5626266 & 7.1693745 & 4.8551572 \\ \mathrm{C} & 2.4141642 & 6.1673171 & 4.3455775 \\ \mathrm{H} & 2.0352771 & 5.4752145 & 3.5763877 \\ \mathrm{C} & 3.7366464 & 6.0645057 & 4.8057813 \\ \mathrm{H} & 4.3964024 & 5.2811106 & 4.4034855 \\ \mathrm{C} & 4.2161869 & 6.9669254 & 5.7693393 \\ \mathrm{H} & 5.2543385 & 6.8902477 & 6.1265082 \\ \mathrm{C} & 3.3736438 & 7.9750212 & 6.2710456 \\ \mathrm{H} & 3.7518371 & 8.6877672 & 7.0195140 \\ \mathrm{C} & 2.0507308 & 8.0788549 & 5.8162585 \\ \mathrm{H} & 1.3929583 & 8.8724552 & 6.2020906 \\ \mathrm{C} & -1.1644021 & 6.5466294 & 5.6244717 \\ \mathrm{C} & -0.5757484 & 5.9201419 & 6.7400889 \\ \mathrm{H} & 0.5188825 & 5.8998221 & 6.8451780 \\ \mathrm{C} & -1.3881344 & 5.3189733 & 7.7164532 \\ \mathrm{H} & -0.9225420 & 4.8298037 & 8.5854706 \\ \mathrm{C} & -2.7854829 & 5.3415046 & 7.5849018 \\ \mathrm{H} & -3.4189455 & 4.8688765 & 8.3507052 \\ & -3.3743329 & 5.9636504 & 6.4691553 \\ \mathrm{H} & -2.4689369 & 5.9788874 & 6.3570651 \\ & & & \\ \mathrm{H} & & \end{array}$




$\begin{array}{lrrr}\text { H } & -3.0342798 & 7.0259899 & 4.6008673 \\ & & & \\ \text { S3 } & & & \\ \text { 75 } & & & \\ \text { SCF } & \text { Energy }=-4548.301812355 & \\ \text { C } & -2.2931475 & 0.4072712 & -1.8873351 \\ \text { C } & -1.5346604 & -0.3033725 & -2.8508725 \\ \text { C } & -1.6790858 & -0.0222174 & -4.2254093 \\ \text { C } & -2.6093972 & 0.9405432 & -4.6453416 \\ \text { C } & -3.3843065 & 1.6275345 & -3.6949984 \\ \text { C } & -3.2199378 & 1.3709564 & -2.3203337 \\ \text { C } & -0.6265337 & -1.3448483 & -2.3315925 \\ \text { C } & 0.7222763 & -1.4731218 & -2.4618018 \\ \text { C } & 1.6692396 & -0.5085188 & -3.0395285 \\ \text { C } & 1.4300564 & 0.8858305 & -2.9567742 \\ \text { C } & 2.3782557 & 1.8042499 & -3.4210591 \\ \text { C } & 3.5964159 & 1.3595770 & -3.9671056 \\ \text { C } & 3.8542945 & -0.0182811 & -4.0493666 \\ \text { C } & 2.9011549 & -0.9424626 & -3.5912290 \\ \text { Br } & -1.7420792 & 2.7844548 & 0.6244301 \\ \text { C } & -1.2416062 & -2.4645023 & -1.4775358 \\ \text { C } & -2.0598956 & -3.4149540 & -2.3809907 \\ \text { C } & -2.1527926 & -1.8654775 & -0.3842569 \\ \text { C } & 0.0206337 & -3.1648215 & -0.9029020 \\ \text { C } & 1.2104976 & -2.7532102 & -1.7870419 \\ \text { O } & 2.3653445 & -2.5671496 & -0.9613664 \\ \text { H } & 1.4331602 & -3.5322153 & -2.5604685 \\ \text { H } & 0.2381135 & -2.7805263 & 0.1100981 \\ \text { H } & -0.1020093 & -4.2617453 & -0.8166662 \\ \text { H } & -2.0517672 & -2.4089836 & 0.5749036 \\ \text { H } & -3.2154499 & -1.8432414 & -0.6878778 \\ \text { H } & -1.4124858 & -3.8906600 & -3.1467427 \\ \text { H } & -2.8615645 & -2.8587056 & -2.9072845 \\ \text { H } & -2.5315638 & -4.2180533 & -1.7780293 \\ \text { H } & 3.0402935 & -2.1222840 & -1.5126603 \\ \text { H } & 0.5009725 & 1.2436878 & -2.4918210 \\ \text { H } & 2.1723557 & 2.8819557 & -3.3364569 \\ \text { H } & 4.3431646 & 2.0851452 & -4.3233986 \\ \text { H } & 4.8011962 & -0.3786424 & -4.4798796 \\ \text { H } & 3.1030061 & -2.0211398 & -3.6947707 \\ \text { H } & -1.0615462 & -0.5671165 & -4.9561258 \\ \text { H } & -2.7336313 & 1.1536563 & -5.7180681 \\ \text { H } & -4.1194205 & 2.3788041 & -4.0242824 \\ \text { H } & -3.8076353 & 1.9502233 & -1.5917883 \\ \text { H } & -2.5662399 & 1.3433492 & 0.5501137 \\ \text { Pd } & -1.5886256 & 0.1020316 & -0.0045411\end{array}$




$\begin{array}{rrrr}\text { P } & -0.2879332 & -0.1961561 & 1.9879906 \\ \text { C } & -0.8846922 & 0.4144649 & 3.6131371 \\ \text { C } & -2.1881450 & 0.9303439 & 3.7386948 \\ \text { H } & -2.8372652 & 0.9873929 & 2.8535258 \\ \text { C } & -2.6541707 & 1.3697770 & 4.9888077 \\ \text { H } & -3.6741172 & 1.7724024 & 5.0797766 \\ \text { C } & -1.8204795 & 1.2964819 & 6.1156100 \\ \text { H } & -2.1856673 & 1.6430919 & 7.0943208 \\ \text { C } & -0.5197874 & 0.7740222 & 5.9961778 \\ \text { H } & 0.1326210 & 0.7085877 & 6.8801148 \\ \text { C } & -0.0538357 & 0.3287608 & 4.7515251 \\ \text { H } & 0.9587687 & -0.0929244 & 4.6582392 \\ \text { C } & 1.2216985 & 0.7700545 & 1.6068690 \\ \text { C } & 1.9884636 & 0.3735590 & 0.4876232 \\ \text { H } & 1.7702737 & -0.5646399 & -0.0457585 \\ \text { C } & 3.0567996 & 1.1695386 & 0.0509481 \\ \text { H } & 3.6417012 & 0.8534573 & -0.8257237 \\ \text { C } & 3.3571380 & 2.3725470 & 0.7113882 \\ \text { H } & 4.1874281 & 3.0029073 & 0.3585570 \\ \text { C } & 2.5877460 & 2.7744572 & 1.8159868 \\ \text { H } & 2.8133177 & 3.7205244 & 2.3312551 \\ \text { C } & 1.5209290 & 1.9798277 & 2.2645673 \\ \text { H } & 0.9085723 & 2.3097411 & 3.1157796 \\ \mathrm{C} & 0.2737685 & -1.8885782 & 2.4283059 \\ \text { C } & 1.6070191 & -2.3276879 & 2.3246576 \\ \text { H } & 2.3951521 & -1.6305277 & 2.0112427 \\ \text { C } & 1.9263016 & -3.6682387 & 2.5937814 \\ \text { H } & 2.9685901 & -4.0055229 & 2.4937488 \\ \text { C } & 0.9242324 & -4.5742706 & 2.9736226 \\ \text { H } & 1.1782118 & -5.6257660 & 3.1756499 \\ \mathrm{C} & -0.4061841 & -4.1353665 & 3.0963950 \\ \text { H } & -1.1955120 & -4.8389454 & 3.4014273 \\ \text { C } & -0.7317477 & -2.8003385 & 2.8225479 \\ \text { H } & -1.7734537 & -2.4560261 & 2.9218220\end{array}$

S4

75

SCF Energy $=-4548.299602416$

$\begin{array}{lrrr}\text { C } & 1.2976302 & 4.0375188 & -1.7661649 \\ \text { C } & 0.5110921 & 3.9653785 & -0.5869444 \\ \text { C } & -0.7515330 & 4.6115029 & -0.5768036 \\ \text { C } & -1.2412314 & 5.2102983 & -1.7569140 \\ \text { C } & -0.4609907 & 5.2501988 & -2.9247284 \\ \text { C } & 0.8219241 & 4.6740488 & -2.9171935 \\ \text { C } & 1.0052041 & 3.2686805 & 0.6113643 \\ \text { C } & 1.8706296 & 2.2068944 & 0.6861480\end{array}$




$\begin{array}{lrrr}\text { C } & 2.3938490 & 1.3497875 & -0.3938003 \\ \mathrm{C} & 3.7313147 & 0.8866873 & -0.3582985 \\ \mathrm{C} & 4.2207895 & 0.0369271 & -1.3619522 \\ \mathrm{C} & 3.3845162 & -0.3754214 & -2.4121489 \\ \mathrm{C} & 2.0475620 & 0.0613575 & -2.4461679 \\ \mathrm{C} & 1.5558470 & 0.9111711 & -1.4479937 \\ \mathrm{Br} & -4.6623659 & 3.8590828 & -0.3881781 \\ \mathrm{C} & 0.5597142 & 3.7387454 & 1.9999249 \\ \mathrm{C} & 1.1064362 & 5.1413661 & 2.3379321 \\ \mathrm{C} & -0.9594574 & 3.6954245 & 2.1221470 \\ \mathrm{C} & 1.1753479 & 2.6497449 & 2.9375346 \\ \mathrm{C} & 2.3035596 & 1.9822915 & 2.1276845 \\ \mathrm{O} & 3.6067334 & 2.5432022 & 2.3697300 \\ \mathrm{H} & 2.4133461 & 0.9071382 & 2.3785520 \\ \mathrm{H} & 1.5481759 & 3.0692070 & 3.8924746 \\ \mathrm{H} & 0.4098731 & 1.8843778 & 3.1806921 \\ \mathrm{H} & -1.3629742 & 2.7127708 & 1.8046412 \\ \mathrm{H} & -1.2745570 & 3.9204116 & 3.1619024 \\ \mathrm{H} & 2.2149330 & 5.1393871 & 2.3654296 \\ \mathrm{H} & 0.7417312 & 5.4740252 & 3.3300163 \\ \mathrm{H} & 0.7785819 & 5.8861088 & 1.5848258 \\ \mathrm{H} & 3.6250239 & 3.3949314 & 1.8905873 \\ \mathrm{H} & 4.3802254 & 1.2170470 & 0.4663616 \\ \mathrm{H} & 5.2662353 & -0.3060293 & -1.3222471 \\ \mathrm{H} & 3.7692679 & -1.0423793 & -3.1986736 \\ \mathrm{H} & 1.3793838 & -0.2704170 & -3.2556587 \\ \mathrm{H} & 0.5072099 & 1.2417575 & -1.4711402 \\ \mathrm{H} & -3.2700952 & 4.1439093 & 0.3174529 \\ \mathrm{H} & -2.2524803 & 5.6555759 & -1.7741214 \\ \mathrm{H} & -0.8528428 & 5.7316562 & -3.8340659 \\ \mathrm{H} & 1.4529082 & 4.7156883 & -3.8179864 \\ \mathrm{H} & 2.3006845 & 3.5881290 & -1.7676431 \\ \mathrm{Pd} & -1.9269493 & 5.0970063 & 0.9758432 \\ \mathrm{P} & -3.1883975 & 6.0117162 & 2.7613774 \\ \mathrm{C} & -1.9714152 & 6.6063987 & 3.9977809 \\ \mathrm{C} & -1.7413214 & 5.9158366 & 5.2042030 \\ \mathrm{H} & -2.3922662 & 5.0754686 & 5.4867689 \\ \mathrm{C} & -0.6810713 & 6.2989295 & 6.0437500 \\ \mathrm{H} & -0.5048971 & 5.7505946 & 6.9814245 \\ \mathrm{C} & 0.1473652 & 7.3755345 & 5.6911475 \\ \mathrm{H} & 0.9758293 & 7.6738620 & 6.3508584 \\ \mathrm{H} & -0.0800955 & 8.0685466 & 4.4881519 \\ & 0.5708524 & 8.9084421 & 4.2026644 \\ \mathrm{C} & -1.1242406 & 7.6793326 & 3.6391031 \\ \mathrm{H} & -4.3048192 & 4.9045436 & 3.7064336\end{array}$




$\begin{array}{rrrr}\text { C } & -5.1247520 & 5.4122683 & 4.7366614 \\ \text { H } & -5.1103680 & 6.4876951 & 4.9708411 \\ \text { C } & -5.9632036 & 4.5483613 & 5.4546203 \\ \text { H } & -6.6030326 & 4.9474670 & 6.2561940 \\ \text { C } & -5.9922419 & 3.1762968 & 5.1452129 \\ \text { H } & -6.6552353 & 2.5008361 & 5.7068816 \\ \text { C } & -5.1847076 & 2.6706487 & 4.1145053 \\ \text { H } & -5.2150781 & 1.6006914 & 3.8604020 \\ \text { C } & -4.3427594 & 3.5321405 & 3.3926984 \\ \text { H } & -3.7247089 & 3.1437388 & 2.5714254 \\ \text { C } & -4.2355731 & 7.4683892 & 2.3731086 \\ \text { C } & -4.2875135 & 8.6194937 & 3.1861727 \\ \text { H } & -3.6601758 & 8.6860775 & 4.0875267 \\ \text { C } & -5.1352541 & 9.6835843 & 2.8394624 \\ \text { H } & -5.1717292 & 10.5808356 & 3.4759090 \\ \text { C } & -5.9342150 & 9.6038242 & 1.6866198 \\ \text { H } & -6.5964229 & 10.4405862 & 1.4173426 \\ \text { C } & -5.8852578 & 8.4560881 & 0.8769933 \\ \text { H } & -6.5094067 & 8.3886171 & -0.0267525 \\ \text { C } & -5.0371201 & 7.3906136 & 1.2130887 \\ \text { H } & -5.0047022 & 6.4866196 & 0.5816677\end{array}$

\section{Optimized Geometries(TS-BXnc-d-6)}

\begin{tabular}{lrrr} 
R4 & & & \\
75 & \multicolumn{4}{l}{} \\
SCF & Energy $=-4548.292553288$ & \\
C & 3.0976205 & 2.3606904 & -0.1368221 \\
C & 1.7782822 & 1.8620946 & -0.0031895 \\
C & 1.5361399 & 0.5039782 & -0.3202339 \\
C & 2.5723153 & -0.3257604 & -0.7677757 \\
C & 3.8741886 & 0.1845024 & -0.9090052 \\
C & 4.1312066 & 1.5289357 & -0.5901641 \\
C & 0.6674706 & 2.7405011 & 0.4457533 \\
Pd & -1.4369670 & 4.6731587 & 0.1332660 \\
P & -1.9536554 & 6.8980394 & 0.5477039 \\
C & -0.5170977 & 2.8014958 & -0.2448018 \\
Br & -0.5360131 & 5.5505453 & -2.0675337 \\
C & -0.8225033 & 2.1411833 & -1.5273202 \\
C & 0.0989432 & 2.2552771 & -2.5993032 \\
C & -0.1588216 & 1.6471877 & -3.8321236 \\
C & -1.3441306 & 0.9184400 & -4.0335214 \\
C & -2.2747474 & 0.8135701 & -2.9880467
\end{tabular}




\begin{tabular}{|c|c|c|c|}
\hline & -2.0199404 & 1.4243815 & -1.7507378 \\
\hline & 0.8945237 & 3.5896298 & 1.7045876 \\
\hline & 2.0716735 & 3.2452741 & 2.4225736 \\
\hline & -0.3234021 & 3.5632260 & 2.6809927 \\
\hline & -1.6978298 & 3.7419585 & 2.0613018 \\
\hline & -2.0841225 & 2.7431150 & 1.0881560 \\
\hline & -2.7561629 & 4.3262881 & 2.9666710 \\
\hline & 1.0480828 & 4.6432827 & 1.3866927 \\
\hline & -0.1463971 & 4.3430254 & 3.4503457 \\
\hline & -0.2769666 & 2.5880276 & 3.2170333 \\
\hline & -1.7400290 & 1.7018456 & 1.1810618 \\
\hline & -3.1251393 & 2.7950279 & 0.7186455 \\
\hline & -2.9458555 & 3.6245170 & 3.8108075 \\
\hline & -3.7165034 & 4.4857436 & 2.44 \\
\hline & -2.443 & 5.2906785 & 3.4 \\
\hline & 2.179 & 2.275 & 3210 \\
\hline & 0.516 & 0.10 & -0.2 \\
\hline & 2.3613807 & -1.378 & -1. \\
\hline & 4.6879965 & -0.465 & -1 . \\
\hline & 5.1474660 & 1.9370159 & -0.70 \\
\hline & 3.3016323 & 3.4118983 & 0.1073941 \\
\hline & -2.7653000 & 1.3365453 & -0.9487927 \\
\hline & -3.2118939 & 0.2552050 & -3.1354598 \\
\hline & -1.5464690 & 0.4443037 & -5.0059235 \\
\hline & 0.5694263 & 1.7542179 & -4.6502342 \\
\hline & 1.0134361 & 2.8431205 & -2.4514722 \\
\hline & -2.8969497 & 7.8281801 & -0.7257034 \\
\hline & -0.3256163 & 7.7182533 & 0.7389421 \\
\hline & -2.9192870 & 7.4218101 & 2.0345637 \\
\hline & -2.3629472 & 8.0687675 & 3.1543108 \\
\hline & -3.1711735 & 8.3880253 & 4.2596936 \\
\hline & -4.5378304 & 8.0701554 & 4.2544280 \\
\hline & -5.1018510 & 7.4394547 & 3.13 \\
\hline & -4.2985822 & 7.1169165 & 2.0298408 \\
\hline & -1.2967862 & 8.3356634 & 3.1634911 \\
\hline & -2.7255573 & 8.8957242 & 5.1287058 \\
\hline & -5.1674926 & 8.3205679 & 5.1214669 \\
\hline & -6.1754906 & 7.1979732 & 3.1133218 \\
\hline & -4.7437859 & 6.6275950 & 1.1488931 \\
\hline & -3.1079549 & 9.2136351 & -0.5581772 \\
\hline & -3.8838801 & 9.9232749 & -1.4861094 \\
\hline & -4.4717354 & 9.2499933 & -2.5713517 \\
\hline & -4.2805811 & 7.8675031 & -2.7266434 \\
\hline & -3.4904432 & 7.1552059 & -1.8102225 \\
\hline & -2.6693614 & 9.7360941 & 0.3057864 \\
\hline & -4.0366570 & 11.0055263 & -1.357078 \\
\hline
\end{tabular}




$\begin{array}{lrrr}\text { H } & -5.0845563 & 9.8067428 & -3.2967066 \\ \text { H } & -4.7408266 & 7.3369073 & -3.5736298 \\ \text { H } & -3.3085699 & 6.0792182 & -1.9419812 \\ \text { C } & 0.5113839 & 7.2705512 & 1.7851206 \\ \text { C } & 1.8146196 & 7.7712577 & 1.9141036 \\ \text { C } & 2.2974300 & 8.7133311 & 0.9895702 \\ \text { C } & 1.4732518 & 9.1472319 & -0.0613477 \\ \text { C } & 0.1666489 & 8.6506170 & -0.1926908 \\ \text { H } & 0.1455214 & 6.5118274 & 2.4936390 \\ \text { H } & 2.4590875 & 7.4098453 & 2.7294214 \\ \text { H } & 3.3237137 & 9.0995953 & 1.0818718 \\ \text { H } & 1.8545718 & 9.8698243 & -0.7985167 \\ \text { H } & -0.4630900 & 8.9663954 & -1.0358788\end{array}$

S4

75

SCF Energy $=-4548 \cdot 305081714$

$\begin{array}{lrrr}\text { C } & -1.9569223 & 3.4828322 & -1.5936960 \\ \text { C } & -1.0610529 & 2.5696816 & -0.9926324 \\ \text { C } & -0.7633267 & 1.3681640 & -1.6831170 \\ \text { C } & -1.3178926 & 1.1074448 & -2.9425020 \\ \text { C } & -2.1891059 & 2.0358621 & -3.5380877 \\ \text { C } & -2.5068400 & 3.2224803 & -2.8559192 \\ \text { C } & -0.5024615 & 2.7892431 & 0.3538142 \\ \text { C } & 0.7579613 & 2.4635903 & 0.7840470 \\ \text { C } & 1.8689939 & 2.2233144 & -0.1721403 \\ \text { C } & 2.7843621 & 1.1621834 & 0.0137816 \\ \text { C } & 3.8201298 & 0.9405818 & -0.9059331 \\ \text { C } & 3.9684757 & 1.7859640 & -2.0182159 \\ \text { C } & 3.0766827 & 2.8574294 & -2.2006122 \\ \text { C } & 2.0367208 & 3.0765243 & -1.2885134 \\ \text { Br } & -0.1923550 & 6.0325761 & 0.1628112 \\ \text { C } & -1.3984390 & 2.5571004 & 2.9728233 \\ \text { C } & -2.3090577 & 2.6448692 & 4.1707046 \\ \text { C } & -1.9248737 & 2.1012404 & 1.7109097 \\ \text { C } & 0.0408758 & 2.1405004 & 3.2687469 \\ \text { C } & 1.1338586 & 2.5749325 & 2.2581246 \\ \text { O } & 1.6005160 & 3.9031120 & 2.5413378 \\ \text { H } & 2.0082151 & 1.9128247 & 2.4377203 \\ \text { H } & 0.3466020 & 2.5390663 & 4.2570046 \\ \text { H } & 0.0689491 & 1.0283552 & 3.3530060 \\ \text { H } & -1.5823635 & 1.1566246 & 1.2627613 \\ \text { H } & -2.9911339 & 2.2942534 & 1.4936301 \\ \text { H } & -1.9211430 & 3.3358073 & 4.9426483 \\ \text { H } & -2.3835290 & 1.6371884 & 4.6397446 \\ \text { H } & -3.3325913 & 2.9628318 & 3.9000111\end{array}$




\begin{tabular}{|c|c|c|c|}
\hline $\mathrm{H}$ & 1.2166884 & 4.5087379 & 1.8625467 \\
\hline $\mathrm{H}$ & 2.6691931 & 0.4868316 & 0.8760813 \\
\hline & 4.5156318 & 0.1009855 & -0.7533600 \\
\hline & 4.7844547 & 1.6153138 & -2.7369262 \\
\hline & 3.1980573 & 3.5362606 & -3.0585100 \\
\hline & 1.3514675 & 3.9294251 & -1.4090532 \\
\hline & -0.0800228 & 0.6407128 & -1.2211061 \\
\hline & -1.0683825 & 0.1703184 & -3.4632369 \\
\hline & -2.6238668 & 1.8314400 & -4.5283383 \\
\hline & -3.1892103 & 3.9556922 & -3.3122799 \\
\hline & -2.1868621 & 4.4170854 & -1.0640632 \\
\hline $\mathrm{Pd}$ & -1.2668879 & 4.2637879 & 1.6759409 \\
\hline & -1.9819093 & 6.0716857 & 2.9755279 \\
\hline & -0.6157751 & 7.2345833 & 3.3734380 \\
\hline & 0.6110486 & 6.6737886 & 3.7867319 \\
\hline & 0.7472879 & 5.5806549 & 3.7913398 \\
\hline & 1.6807283 & 7.5101668 & 4.1377508 \\
\hline & 2.6365032 & 7.062 & 4.44 \\
\hline & 1.5398528 & 8.9060931 & 5607 \\
\hline & 2.3841311 & 9.561 & 66816 \\
\hline & 0.32469 & 9.463 & 3.6 \\
\hline & 0.21514 & 10.55 & 3.5 \\
\hline & -0.7536 & 8.6324366 & 3.285 \\
\hline & -1.69468 & 9.075 & 2.9 \\
\hline & -2.7652592 & $5.7 \mathrm{~s}$ & 4.6 \\
\hline & -2.0316025 & 5.900 & 5.8 \\
\hline & -0.9885571 & 6.2481227 & $5.7 \mathrm{~s}$ \\
\hline & -2.6298706 & 5.5742442 & 7.0489006 \\
\hline & -2.0471871 & 5.6669567 & 7.9780009 \\
\hline & -3.9641586 & 5.1398443 & 7.0942352 \\
\hline & -4.4312347 & 4.8867758 & 8.0579508 \\
\hline & -4.7037181 & 5.0371699 & 5.9028218 \\
\hline & -5.7523864 & 4.7040868 & 5.9305067 \\
\hline & -4.1080299 & 5.3556783 & 4.6748354 \\
\hline & -4.6912686 & 5.2718067 & 3.7447787 \\
\hline & -3.2850628 & 7.0743158 & 2.1443617 \\
\hline & -4.0277120 & 8.0290627 & 2.8755326 \\
\hline & -3.8383417 & 8.1664003 & 3.9504670 \\
\hline & -5.0166926 & 8.7933896 & 2.2390887 \\
\hline & -5.5866051 & 9.5370845 & 2.8164963 \\
\hline & -5.2806939 & 8.6065824 & 0.8711350 \\
\hline & -6.0594872 & 9.2045940 & 0.3738070 \\
\hline & -4.5512495 & 7.6535586 & 0.1428303 \\
\hline & -4.7532359 & 7.5013964 & -0.9280565 \\
\hline & -3.5557842 & 6.8913009 & 0.7742814 \\
\hline & -2.9645051 & 6.1614284 & 0.204457 \\
\hline
\end{tabular}




\section{Optimized Geometries(BXnd-6)}

$\begin{array}{lrrr}\text { R4 } & & & \\ \text { 75 } & & & \\ \text { SCF } & \text { Energy }=-4548.346498328 & \\ \text { C } & 1.4502956 & -2.8133518 & 2.3410726 \\ \text { C } & 0.3032353 & -3.3039358 & 1.6714069 \\ \text { C } & 0.1683804 & -4.7033644 & 1.5203184 \\ \text { C } & 1.1602572 & -5.5778919 & 1.9851081 \\ \text { C } & 2.3095761 & -5.0743597 & 2.6167680 \\ \text { C } & 2.4462248 & -3.6874363 & 2.7965649 \\ \text { C } & -0.7139650 & -2.3547471 & 1.1500073 \\ \text { Pd } & -0.8559851 & 0.3882988 & -0.9942017 \\ \text { P } & 0.0564305 & 2.0315488 & 0.1894472 \\ \text { C } & -1.2975765 & -2.4975378 & -0.0784961 \\ \text { Br } & 0.7574062 & 0.1749968 & -2.8941422 \\ \text { C } & -0.9075731 & -3.5517958 & -1.0570281 \\ \text { C } & 0.4070096 & -3.5794348 & -1.5700007 \\ \text { C } & 0.7770187 & -4.5383001 & -2.5224492 \\ \text { C } & -0.1598867 & -5.4809938 & -2.9786342 \\ \text { C } & -1.4756485 & -5.4503398 & -2.4876271 \\ \text { C } & -1.8494438 & -4.4848898 & -1.5397673 \\ \text { C } & -1.0013914 & -1.1337013 & 2.0174429 \\ \text { O } & -0.9399898 & -1.4104866 & 3.4143024 \\ \text { C } & -2.3151362 & -0.4500011 & 1.6472297 \\ \text { C } & -2.4815811 & -0.2217185 & 0.1621380 \\ \text { C } & -2.2532008 & -1.4713625 & -0.6530671 \\ \text { C } & -3.7120926 & 0.5846753 & -0.2066866 \\ \text { H } & -0.1780388 & -0.4069711 & 1.8382537 \\ \text { H } & -2.4066384 & 0.4948154 & 2.2142835 \\ \text { H } & -3.1534351 & -1.1025195 & 1.9968418 \\ \text { H } & -3.2046643 & -1.9350375 & -1.0098502 \\ \text { H } & -1.8289826 & -1.1862037 & -1.6981445 \\ \text { H } & -4.6399747 & 0.0790556 & 0.1513701 \\ \text { H } & -3.8074953 & 0.7270666 & -1.3023440 \\ \text { H } & -3.6822447 & 1.5819008 & 0.2738595 \\ \text { H } & -1.4250495 & -2.2439250 & 3.5651638 \\ \text { H } & -0.7278419 & -5.1073847 & 1.0303218 \\ \text { H } & 1.0329474 & -6.6630106 & 1.8521715 \\ \text { H } & 3.0906847 & -5.7607159 & 2.9774600 \\ \text { H } & 3.3356301 & -3.2798395 & 3.3012233 \\ \text { H } & 1.5673698 & -1.7332618 & 2.4980981 \\ \text { H } & -2.8798388 & -4.4674841 & -1.1502616 \\ \text { H } & -2.2161463 & -6.1821387 & -2.8450262\end{array}$




$\begin{array}{lrrr}\text { H } & 0.1328661 & -6.2343138 & -3.7256555 \\ \text { H } & 1.8034653 & -4.5422212 & -2.9186968 \\ \text { H } & 1.1313084 & -2.8282866 & -1.2251619 \\ \text { C } & 0.2298444 & 3.6555076 & -0.6552762 \\ \text { C } & 1.7653484 & 1.5642116 & 0.6846903 \\ \text { C } & -0.7571843 & 2.4530396 & 1.7783259 \\ \text { C } & -0.2675088 & 1.9680820 & 3.0072890 \\ \text { C } & -1.0120606 & 2.1479912 & 4.1838578 \\ \text { C } & -2.2377451 & 2.8306415 & 4.1463184 \\ \text { C } & -2.7199904 & 3.3388202 & 2.9260489 \\ \text { C } & -1.9898879 & 3.1436185 & 1.7458092 \\ \text { H } & 0.6908904 & 1.4312606 & 3.0442359 \\ \text { H } & -0.6322482 & 1.7396445 & 5.1315659 \\ \text { H } & -2.8211044 & 2.9687870 & 5.0690192 \\ \text { H } & -3.6770625 & 3.8810755 & 2.8927723 \\ \text { H } & -2.3735368 & 3.5300801 & 0.7890386 \\ \text { C } & 0.2394122 & 4.8644448 & 0.0750864 \\ \text { C } & 0.4334603 & 6.0845183 & -0.5908868 \\ \text { C } & 0.6192346 & 6.1061218 & -1.9833620 \\ \text { C } & 0.6111887 & 4.9033539 & -2.7092773 \\ \text { C } & 0.4147091 & 3.6787085 & -2.0531020 \\ \text { H } & 0.0868993 & 4.8545828 & 1.1644052 \\ \text { H } & 0.4377802 & 7.0235557 & -0.0167291 \\ \text { H } & 0.7683915 & 7.0646582 & -2.5035419 \\ \text { H } & 0.7566751 & 4.9137610 & -3.8000160 \\ \text { H } & 0.4182026 & 2.7295885 & -2.6158833 \\ \text { C } & 2.1935763 & 0.2324117 & 0.5219944 \\ \text { C } & 3.4756518 & -0.1509417 & 0.9459103 \\ \text { C } & 4.3368013 & 0.7954358 & 1.5235165 \\ \text { C } & 3.9166982 & 2.1292307 & 1.6750027 \\ \text { C } & 2.6350255 & 2.5155969 & 1.2566944 \\ \text { H } & 1.5183149 & -0.4934543 & 0.0433889 \\ \text { H } & 3.7983747 & -1.1947367 & 0.8178641 \\ \text { H } & 5.3442166 & 0.4965578 & 1.8509330 \\ \text { H } & 4.5940351 & 2.8749396 & 2.1180123 \\ \text { H } & 2.3120532 & 3.5613348 & 1.3699718 \\ & & & \\ \text { S4 } & & & \\ \text { 75 } & & & \\ \text { SCF } & \text { Energy }=-4548.350277218 & \\ \text { C } & -0.4901984 & -3.6114562 & -2.3962359 \\ \text { C } & -0.9875249 & -3.9149441 & -1.1075434 \\ \text { C } & -1.3193986 & -5.2528263 & -0.8016171 \\ \text { C } & -1.1551575 & -6.2647215 & -1.7573135 \\ & -0.6543310 & -5.9563962 & -3.0338984 \\ & -0.3210724 & -4.6284399 & -3.3481677\end{array}$




\begin{tabular}{|c|c|c|c|}
\hline & -1.1479832 & -2.8354699 & -0.0961607 \\
\hline & -0.5612419 & -2.8702619 & 1.1348583 \\
\hline & 0.3630377 & -3.9563576 & 1.5538890 \\
\hline & 0.1768064 & -4.6236939 & 2.7842198 \\
\hline & 1.0483457 & -5.6477464 & 3.1851974 \\
\hline & 2.1322921 & -6.0094285 & 2.3684062 \\
\hline & 2.3391648 & -5.3383518 & 1.1507863 \\
\hline & 1.4627125 & -4.3227995 & \\
\hline & 1.0786439 & 0.2923348 & -2.5587425 \\
\hline & -2.1857980 & -0.5034203 & 0.3964952 \\
\hline & -3.4693703 & 0.2744481 & 0.1737982 \\
\hline & -1.9495181 & -1.6434833 & -0.5635617 \\
\hline & -1.9095785 & -0.8360050 & 1.8491175 \\
\hline & -0.64361 & -1.66 & 731 \\
\hline & 0.55629 & -0.89 & 250 \\
\hline & -0.6 & -2.0 & 528 \\
\hline & -1.8 & 0.08 & \\
\hline & -2.7 & -1.4 & \\
\hline & -2.8 & -1.9 & -1. \\
\hline & -1.392 & -1.2 & -1 \\
\hline & -3.463 & 1.21 & 0.7 \\
\hline & -4.3511311 & -0.3158002 & 0.5 \\
\hline & -3.6267361 & 0.5283151 & -0.8935957 \\
\hline & 0.5373644 & -0.5990541 & 0.94 \\
\hline & -0.6760580 & -4.3504648 & 3.4 \\
\hline & 0.8792374 & -6.1670767 & 4.1 \\
\hline & 2.8189046 & -6.8099667 & 2.6 \\
\hline & 3.1936544 & -5.6063216 & 0.5 \\
\hline & 1.6265022 & -3.7947927 & -0.20 \\
\hline & -1.7032272 & -5.4922400 & 0.2 \\
\hline & -1.418 & -7.3028430 & -1.50 \\
\hline & -0.5215191 & -6.7521201 & -3.78 \\
\hline & 0.0849551 & -4.3792463 & -4.3403081 \\
\hline & -0.1820920 & -2.5811530 & -2.6427917 \\
\hline & -0.6063175 & 0.2715868 & -0.7196771 \\
\hline & -0.0735284 & 2.1874252 & 0.3061476 \\
\hline & 1.7401070 & 2.4545098 & 0.4651545 \\
\hline & 2.5458760 & 1.4755286 & 1.0876092 \\
\hline & 2.1020138 & 0.5608120 & 1.5042026 \\
\hline & 3.9324546 & 1.6627341 & 1.1785944 \\
\hline & 4.5488697 & 0.8937789 & 1.6678997 \\
\hline & 4.5319917 & 2.8102586 & 0.6340456 \\
\hline & 5.6222899 & 2.9465561 & 0.6956679 \\
\hline & 3.7358453 & 3.7747 & -0.0023738 \\
\hline & 4.1977365 & 4.6700634 & -0.4452442 \\
\hline & 2.3458506 & 3.6005511 & -0.0886557 \\
\hline
\end{tabular}




$\begin{array}{lrrr}\text { H } & 1.7347527 & 4.3550652 & -0.6024570 \\ \text { C } & -0.7802236 & 2.4575769 & 1.9802561 \\ \text { C } & -0.0807012 & 2.0665162 & 3.1398803 \\ \text { H } & 0.9485767 & 1.6938035 & 3.0641051 \\ \text { C } & -0.7027184 & 2.1380132 & 4.3957685 \\ \text { H } & -0.1455448 & 1.8293623 & 5.2930163 \\ \text { C } & -2.0257559 & 2.5956984 & 4.5095474 \\ \text { H } & -2.5106877 & 2.6466167 & 5.4960079 \\ \text { C } & -2.7256637 & 2.9933066 & 3.3580042 \\ \text { H } & -3.7598419 & 3.3611282 & 3.4371994 \\ \text { C } & -2.1088762 & 2.9232901 & 2.1000240 \\ \text { H } & -2.6595538 & 3.2441893 & 1.2037261 \\ \text { C } & -0.6699698 & 3.6250791 & -0.6666107 \\ \text { C } & -0.8110798 & 4.8989326 & -0.0731541 \\ \text { H } & -0.5951498 & 5.0350851 & 0.9969873 \\ \text { C } & -1.2238162 & 5.9918755 & -0.8493636 \\ \text { H } & -1.3344853 & 6.9820712 & -0.3819029 \\ \text { C } & -1.4912472 & 5.8230087 & -2.2193503 \\ \text { H } & -1.8161197 & 6.6822398 & -2.8256778 \\ \text { C } & -1.3393771 & 4.5595761 & -2.8132333 \\ \text { H } & -1.5380033 & 4.4245446 & -3.8870072 \\ \text { C } & -0.9300558 & 3.4600919 & -2.0421004 \\ \text { H } & -0.7841510 & 2.4696260 & -2.5030106\end{array}$

\section{Optimized Geometries(TS-BXnd-l)}

\begin{tabular}{lrrr} 
R4 & & & \\
75 & \multicolumn{4}{l}{} \\
SCF & Energy $=-4548.331360442$ & \\
C & 2.3678473 & 3.7166993 & 0.9671423 \\
C & 1.4625940 & 2.6357020 & 0.8227082 \\
C & 1.9966885 & 1.3480513 & 0.5791301 \\
C & 3.3780166 & 1.1532620 & 0.4480450 \\
C & 4.2609478 & 2.2403478 & 0.5604427 \\
C & 3.7479490 & 3.5216133 & 0.8248935 \\
C & 0.0035033 & 2.8733651 & 0.9142157 \\
Pd & -2.4568268 & 4.6343843 & -0.7525451 \\
P & -2.1198246 & 6.8225822 & 0.0788907 \\
C & -0.9036536 & 2.2889368 & 0.0636635 \\
Br & -1.9375400 & 5.3425062 & -3.0976223 \\
C & -0.5171954 & 1.4314938 & -1.0926075 \\
C & 0.2456565 & 1.9816869 & -2.1444664 \\
C & 0.5798561 & 1.2027656 & -3.2604032
\end{tabular}




\begin{tabular}{lrrr} 
C & 0.1549101 & -0.1341714 & -3.3424354 \\
C & -0.6204349 & -0.6847709 & -2.3084960 \\
C & -0.9626781 & 0.0971665 & -1.1939402 \\
C & -0.4632922 & 3.9366352 & 1.9042690 \\
O & 0.3441183 & 4.0197760 & 3.0745673 \\
C & -1.9391801 & 3.7962312 & 2.2743627 \\
C & -2.8607917 & 3.5322687 & 1.1027136 \\
$\mathrm{C}$ & -2.3504957 & 2.6312811 & 0.1145416 \\
$\mathrm{C}$ & -4.3391516 & 3.5461396 & 1.4170953 \\
$\mathrm{H}$ & -0.3286525 & 4.9209244 & 1.4022730 \\
$\mathrm{H}$ & -2.2530935 & 4.6815988 & 2.8555063 \\
$\mathrm{H}$ & -2.0369170 & 2.9221677 & 2.9650974 \\
$\mathrm{H}$ & -3.0295007 & 1.8836077 & -0.3286292 \\
$\mathrm{H}$ & -2.6372926 & 3.2858414 & -1.5452974 \\
$\mathrm{H}$ & -4.5746926 & 2.8070095 & 2.2170785 \\
$\mathrm{H}$ & -4.9547076 & 3.3038116 & 0.5288892 \\
$\mathrm{H}$ & -4.6501978 & 4.5389777 & 1.7971157 \\
$\mathrm{H}$ & 0.4841422 & 3.1080950 & 3.3942693 \\
$\mathrm{H}$ & 1.3154672 & 0.4907373 & 0.4941061 \\
$\mathrm{H}$ & 3.7677423 & 0.1419262 & 0.2568281 \\
$\mathrm{H}$ & 5.3456231 & 2.0879653 & 0.4530342 \\
$\mathrm{H}$ & 4.4295322 & 4.3800828 & 0.9263169 \\
$\mathrm{H}$ & 1.9844172 & 4.7219013 & 1.1840019 \\
$\mathrm{H}$ & -1.5658778 & -0.3359518 & -0.3802952 \\
$\mathrm{H}$ & -0.9618200 & -1.7293859 & -2.3701406 \\
$\mathrm{H}$ & 0.4193657 & -0.7451163 & -4.2187970 \\
$\mathrm{H}$ & 1.1680008 & 1.6474719 & -4.0770318 \\
$\mathrm{H}$ & 0.5586372 & 3.0336923 & -2.0849451 \\
$\mathrm{C}$ & -3.2391282 & 8.1583646 & -0.5172285 \\
$\mathrm{C}$ & -4.7200014 & 9.0356618 & -2.2403350 \\
$\mathrm{C}$ & -0.4154110 & 7.3761486 & -0.3415604 \\
$\mathrm{C}$ & -2.1863795 & 7.0469824 & 1.9023357 \\
$\mathrm{C}$ & -1.0181028 & 7.1051277 & 2.6870496 \\
$\mathrm{C}$ & -1.1065154 & 7.0902294 & 4.0886230 \\
$\mathrm{C}$ & -2.3601282 & 7.0426002 & 4.7171396 \\
$\mathrm{C}$ & -3.5323058 & 7.0112494 & 3.9391974 \\
$\mathrm{C}$ & -3.4471973 & 7.0032102 & 2.5403906 \\
$\mathrm{H}$ & -0.0324452 & 7.1456397 & 2.2024168 \\
$\mathrm{H}$ & -0.1849746 & 7.1067892 & 4.6883867 \\
$\mathrm{H}$ & -2.4273789 & 7.0308972 & 5.8153889 \\
$\mathrm{H}$ & -4.5186572 & 6.9818848 & 4.4264867 \\
& -4.3648196 & 6.9682526 & 1.9327836 \\
$\mathrm{C}$ & -3.4410624 & 9.3333140 & 0.2411791 \\
\hline
\end{tabular}




$\begin{array}{rrrr}\text { H } & -2.9568404 & 9.4463786 & 1.2225137 \\ \text { H } & -4.4266528 & 11.2635704 & 0.3521569 \\ \text { H } & -5.5715662 & 11.0034900 & -1.8660440 \\ \text { H } & -5.2200995 & 8.9138011 & -3.2130336 \\ \text { H } & -3.7126575 & 7.1033947 & -2.3581389 \\ \text { C } & 0.5361360 & 6.4054522 & -0.7149730 \\ \text { C } & 1.8658467 & 6.7784594 & -0.9638248 \\ \text { C } & 2.2486404 & 8.1253024 & -0.8568989 \\ \text { C } & 1.2988126 & 9.0992236 & -0.5017315 \\ \text { C } & -0.0293832 & 8.7277741 & -0.2431740 \\ \text { H } & 0.2211406 & 5.3571023 & -0.8287152 \\ \text { H } & 2.5993280 & 6.0101709 & -1.2502384 \\ \text { H } & 3.2893499 & 8.4204892 & -1.0599459 \\ \text { H } & 1.5932773 & 10.1571470 & -0.4288150 \\ \text { H } & -0.7701574 & 9.4942262 & 0.0286519\end{array}$

S4

75

SCF Energy $=-4548.336085906$

$\begin{array}{lrrr}\text { C } & -1.1117721 & 2.8775316 & -2.5189159 \\ \text { C } & -0.6785729 & 2.0274512 & -1.4761768 \\ \text { C } & -0.1597357 & 0.7564784 & -1.8129399 \\ \text { C } & -0.0647904 & 0.3534606 & -3.1510096 \\ \text { C } & -0.4865113 & 1.2125122 & -4.1807906 \\ \text { C } & -1.0105609 & 2.4750364 & -3.8592658 \\ \text { C } & -0.7806642 & 2.4494192 & -0.0532967 \\ \text { C } & 0.2594554 & 2.3525560 & 0.8364863 \\ \text { C } & 1.6387548 & 1.9784046 & 0.4423965 \\ \text { C } & 2.3700324 & 1.0270384 & 1.1885973 \\ \text { C } & 3.6776656 & 0.6758635 & 0.8214698 \\ \text { C } & 4.2849199 & 1.2845831 & -0.2891775 \\ \text { C } & 3.5756585 & 2.2481594 & -1.0273407 \\ \text { C } & 2.2661692 & 2.5903805 & -0.6673879 \\ \text { Br } & -1.5932215 & 7.2384900 & -0.3251482 \\ \text { C } & -2.4127182 & 3.1739726 & 1.7670826 \\ \text { C } & -3.8536502 & 3.0570022 & 2.2154232 \\ \text { C } & -2.0972228 & 2.9939331 & 0.3792190 \\ \text { C } & -1.3653369 & 2.7023883 & 2.7529370 \\ \text { C } & 0.0739384 & 2.8649179 & 2.2578764 \\ \text { O } & 0.5435467 & 4.2208384 & 2.3133124 \\ \text { H } & 0.7481085 & 2.3133413 & 2.9433076 \\ \text { H } & -1.4798876 & 3.1993555 & 3.7350874 \\ \text { H } & -1.5459760 & 1.6146270 & 2.9281325 \\ \text { H } & -2.9321369 & 2.7804731 & -0.3084260 \\ \text { H } & -2.0600857 & 4.5609079 & -0.5237440 \\ \text { H } & -4.0225715 & 3.6033899 & 3.1620398\end{array}$




$\begin{array}{lrrr}\text { H } & -4.1091414 & 1.9901834 & 2.4083476 \\ \mathrm{H} & -4.5583477 & 3.4462958 & 1.4552334 \\ \mathrm{H} & 0.0001455 & 4.7253629 & 1.6501537 \\ \mathrm{H} & 1.8977136 & 0.5380607 & 2.0549993 \\ \mathrm{H} & 4.2258561 & -0.0783382 & 1.4065022 \\ \mathrm{H} & 5.3125144 & 1.0136707 & -0.5753825 \\ \mathrm{H} & 4.0492810 & 2.7414614 & -1.8896338 \\ \mathrm{H} & 1.7149018 & 3.3492134 & -1.2406517 \\ \mathrm{H} & 0.1703840 & 0.0842622 & -1.0077903 \\ \mathrm{H} & 0.3402188 & -0.6409687 & -3.3929254 \\ \mathrm{H} & -0.4082509 & 0.8969163 & -5.2322217 \\ \mathrm{H} & -1.3376874 & 3.1583861 & -4.6575464 \\ \mathrm{H} & -1.4966386 & 3.8817745 & -2.2806332 \\ \mathrm{Pd} & -2.0285916 & 5.1436760 & 0.9369278 \\ \mathrm{P} & -2.1178227 & 6.3992017 & 2.9523821 \\ \mathrm{C} & -0.8428162 & 7.7228467 & 3.0433695 \\ \mathrm{C} & 0.5199507 & 7.3730910 & 2.9192594 \\ \mathrm{H} & 0.8182858 & 6.3226758 & 2.7899077 \\ \mathrm{C} & 1.5061789 & 8.3693546 & 2.9476809 \\ \mathrm{H} & 2.5645929 & 8.0839180 & 2.8518742 \\ \mathrm{C} & 1.1462558 & 9.7209692 & 3.0752594 \\ \mathrm{H} & 1.9221392 & 10.5014581 & 3.0845961 \\ \mathrm{C} & -0.2087818 & 10.0726084 & 3.1728747 \\ \mathrm{H} & -0.5018296 & 11.1303806 & 3.2535142 \\ \mathrm{C} & -1.2013554 & 9.0803419 & 3.1554897 \\ \mathrm{H} & -2.2596172 & 9.3694574 & 3.2105501 \\ \mathrm{C} & -2.0222247 & 5.5311255 & 4.5761905 \\ \mathrm{C} & -0.7790058 & 5.2729336 & 5.1902495 \\ \mathrm{H} & 0.1431914 & 5.6840256 & 4.7606115 \\ \mathrm{C} & -0.7104701 & 4.4784868 & 6.3447446 \\ \mathrm{H} & 0.2673460 & 4.2885195 & 6.8125250 \\ \mathrm{C} & -1.8770546 & 3.9258683 & 6.8988133 \\ \mathrm{H} & -1.8188050 & 3.2996966 & 7.8018256 \\ \mathrm{C} & -3.1194946 & 4.1842800 & 6.2972855 \\ \mathrm{H} & -4.0416552 & 3.7657389 & 6.7282526 \\ \mathrm{C} & -3.1932382 & 4.9820448 & 5.1450492 \\ \mathrm{H} & -4.1729622 & 5.1920101 & 4.6920042 \\ \mathrm{C} & -3.7279528 & 7.2752437 & 3.0628611 \\ \mathrm{C} & -4.1299798 & 7.9193236 & 4.2543815 \\ \mathrm{H} & -3.4785299 & 7.8895962 & 5.1407745 \\ \mathrm{C} & -5.3590832 & 8.5918593 & 4.3102453 \\ \mathrm{H} & -5.6661124 & 9.0925913 & 5.2409805 \\ \mathrm{C} & -6.1951550 & 8.6270741 & 3.1799056 \\ & -7.1600164 & 9.1547317 & 3.2262908 \\ & -6.7976368 & 7.9906808 & 1.9933875 \\ & & & \\ \mathrm{H} & & \end{array}$




$\begin{array}{llll}\mathrm{C} & -4.5672014 & 7.3166502 & 1.9319764 \\ \mathrm{H} & -4.2365621 & 6.8338180 & 0.9990671\end{array}$

\section{Optimized Geometries(BXnl)}

$\begin{array}{lrrr}\text { R4 } & & & \\ \text { 75 } & & & \\ \text { SCF } & \text { Energy }=-4548.331865813 & \\ \text { C } & 1.3415718 & -2.8039462 & 2.5036629 \\ \text { C } & 0.2389730 & -3.2765960 & 1.7482454 \\ \text { C } & 0.1241290 & -4.6726890 & 1.5434194 \\ \text { C } & 1.0915756 & -5.5569432 & 2.0382788 \\ \text { C } & 2.1977127 & -5.0695789 & 2.7547007 \\ \text { C } & 2.3137350 & -3.6888193 & 2.9883312 \\ \text { C } & -0.7457005 & -2.3209398 & 1.1916407 \\ \text { Pd } & -0.8396410 & 0.1963563 & -1.1818885 \\ \text { P } & 0.0919587 & 1.9661414 & 0.1556793 \\ \text { C } & -1.2785627 & -2.4396208 & -0.0712489 \\ \text { Br } & 1.0560109 & 0.4126074 & -2.7818304 \\ \text { C } & -0.8594974 & -3.4849822 & -1.0479671 \\ \text { C } & 0.4749136 & -3.5204732 & -1.5050591 \\ \text { C } & 0.8742585 & -4.4695943 & -2.4552883 \\ \text { C } & -0.0535450 & -5.3943718 & -2.9642972 \\ \text { C } & -1.3883112 & -5.3549203 & -2.5285405 \\ \text { C } & -1.7909368 & -4.3991162 & -1.5822550 \\ \text { C } & -1.0421438 & -1.0746678 & 2.0201919 \\ \text { O } & -1.0085492 & -1.3037429 & 3.4259803 \\ \text { C } & -2.3451993 & -0.3807261 & 1.6219399 \\ \text { C } & -2.5948796 & -0.2864500 & 0.1323894 \\ \text { C } & -2.1923705 & -1.4133497 & -0.6263966 \\ \text { C } & -3.7839926 & 0.5436596 & -0.2877661 \\ \text { H } & -0.2049108 & -0.3650920 & 1.8376710 \\ \text { H } & -2.3988325 & 0.6033723 & 2.1197565 \\ \text { H } & -3.1905170 & -0.9820113 & 2.0398626 \\ \text { H } & -2.7839129 & -1.7186216 & -1.5030311 \\ \text { H } & -1.1737012 & -0.7808760 & -2.3484039 \\ \text { H } & -4.7088081 & 0.1754618 & 0.2126860 \\ \text { H } & -3.9443471 & 0.5138970 & -1.3828570 \\ \text { H } & -3.6555823 & 1.6006457 & 0.0184307 \\ \text { H } & -1.5244917 & -2.1145530 & 3.5976255 \\ \text { H } & -0.7392522 & -5.0656440 & 0.9900884 \\ \text { H } & 0.9790151 & -6.6376220 & 1.8629054 \\ \text { H } & 2.9599957 & -5.7639713 & 3.1394036\end{array}$




$\begin{array}{lrrr}\text { H } & 3.1686847 & -3.2942381 & 3.5585783 \\ \text { H } & 1.4421471 & -1.7300965 & 2.7055363 \\ \text { H } & -2.8363102 & -4.3713434 & -1.2362885 \\ \text { H } & -2.1214731 & -6.0719925 & -2.9283774 \\ \text { H } & 0.2617401 & -6.1399473 & -3.7098919 \\ \text { H } & 1.9161886 & -4.4797964 & -2.8087425 \\ \text { H } & 1.1926294 & -2.7828940 & -1.1189632 \\ \text { C } & 0.1947817 & 3.6226232 & -0.6481715 \\ \text { C } & 1.8126676 & 1.5098713 & 0.6256334 \\ \text { C } & -0.6688892 & 2.4023362 & 1.7731858 \\ \text { C } & -0.1458100 & 1.9313798 & 2.9933308 \\ \text { C } & -0.8528087 & 2.1315310 & 4.1901235 \\ \text { C } & -2.0723639 & 2.8253876 & 4.1828294 \\ \text { C } & -2.5859475 & 3.3248749 & 2.9717599 \\ \text { C } & -1.8943578 & 3.1074490 & 1.7725231 \\ \text { H } & 0.8125249 & 1.3930559 & 3.0084183 \\ \text { H } & -0.4461882 & 1.7335400 & 5.1311173 \\ \text { H } & -2.6249002 & 2.9812177 & 5.1215681 \\ \text { H } & -3.5371312 & 3.8784188 & 2.9610237 \\ \text { H } & -2.3018404 & 3.4919118 & 0.8246280 \\ \text { C } & 0.3683856 & 4.7916922 & 0.1270290 \\ \text { C } & 0.4578209 & 6.0457436 & -0.4952406 \\ \text { C } & 0.3659337 & 6.1465151 & -1.8938302 \\ \text { C } & 0.1825663 & 4.9883948 & -2.6662165 \\ \text { C } & 0.0960333 & 3.7298715 & -2.0501376 \\ \text { H } & 0.4138894 & 4.7235938 & 1.2241222 \\ \text { H } & 0.5945730 & 6.9496733 & 0.1177084 \\ \text { H } & 0.4325138 & 7.1314356 & -2.3807573 \\ \text { H } & 0.1055530 & 5.0599647 & -3.7616591 \\ \text { H } & -0.0238284 & 2.8187961 & -2.6554212 \\ \text { C } & 2.1691186 & 0.1460358 & 0.5986692 \\ \text { C } & 3.4410746 & -0.2620761 & 1.0272274 \\ \text { C } & 4.3720862 & 0.6924855 & 1.4678254 \\ \text { C } & 4.0278276 & 2.0554164 & 1.4788175 \\ \text { C } & 2.7519909 & 2.4648090 & 1.0615377 \\ \text { H } & 1.4472985 & -0.5937007 & 0.2202032 \\ \text { H } & 3.7022740 & -1.3305078 & 1.0069820 \\ \text { H } & 5.3738183 & 0.3754906 & 1.7956673 \\ \text { H } & 4.7594823 & 2.8070531 & 1.8119847 \\ \text { H } & 2.4913139 & 3.5331454 & 1.0664645 \\ \text { S4 } & -1.0300436 & -3.9300590 & -1.1439057\end{array}$




\begin{tabular}{|c|c|c|c|}
\hline & -1.3764335 & -5.2307148 & -0.7103671 \\
\hline & -1.3837584 & -6.3069771 & -1.6063721 \\
\hline & -1.0458835 & -6.1073072 & -2.9564255 \\
\hline & -0.7106474 & -4.8188872 & -3.4027472 \\
\hline & -1.0331980 & -2.7892957 & -0.1889876 \\
\hline & -0.4582535 & -2.8506090 & 1.0578446 \\
\hline & 0.4006878 & -3.9695053 & 1.5117509 \\
\hline & 0.2330058 & -4.5381237 & 2.7945634 \\
\hline & 1.0515322 & -5.5920578 & 3.2272635 \\
\hline & 2.0660006 & -6.0855755 & 2.3906076 \\
\hline & 2.2572272 & -5.5145226 & 1.1201574 \\
\hline & 1.4334716 & -4.4692646 & 0.68 \\
\hline & 1.5800759 & 0.8390218 & -2.35 \\
\hline & -2.0640415 & -0.51 & 0.2 \\
\hline & -3.242 & 0.38 & -0.0 \\
\hline & -1.72 & -1.5 & -0.6 \\
\hline & -1.75 & -0.7 & 1.7 \\
\hline & -0.49 & -1.6 & 1.9 \\
\hline & 0.72 & -0.8 & 1.7 \\
\hline & -0.46 & -1.92 & 3.0 \\
\hline & -1.683 & 0.16 & 2.3 \\
\hline & -2.6200803 & -1.3371616 & 2.1 \\
\hline & -2.3094097 & -1.6512044 & -1.56 \\
\hline & -0.2665948 & -0.9367041 & -2.07 \\
\hline & -3.1235919 & 1.3799095 & 0.43 \\
\hline & -4.1720942 & -0.0593309 & 0.38 \\
\hline & -3.391 & 0.5228497 & -1.1 \\
\hline & 0.7832934 & -0.69 & $0.7 \varepsilon$ \\
\hline & -0.567 & -4.1 & 3.4 \\
\hline & 0.895 & -6.0 & 4.2 \\
\hline & 2.71 & -6.90 & 2.73 \\
\hline & 3.0598782 & -5.884 & 0.4 \\
\hline & 1.5886623 & $-4.01^{\circ}$ & -0.3057908 \\
\hline & -1.6440688 & -5.3886196 & 0.3442009 \\
\hline & -1.6585592 & -7.3110010 & -1.2488133 \\
\hline & -1.0481785 & -6.9541582 & -3.6593568 \\
\hline & -0.4413114 & -4.6507122 & -4.4565465 \\
\hline & -0.4233058 & -2.7363008 & -2.8605468 \\
\hline & -0.3151146 & 0.1267534 & -0.9400470 \\
\hline & 0.0067704 & 2.1571537 & 0.3496219 \\
\hline & 1.7896788 & 2.5612955 & 0.5717131 \\
\hline & 2.6213369 & 1.637 & 1.2419617 \\
\hline & 2.2061289 & 0.69 & 1.638349 \\
\hline & 3.9903661 & 1.90165 & 1.389674 \\
\hline & 4.6270273 & 1.1742458 & 1.9155196 \\
\hline & 4.5490111 & 3.0729172 & 0.852576 \\
\hline
\end{tabular}




$\begin{array}{lrrr}\text { H } & 5.6260607 & 3.2720269 & 0.9600447 \\ \mathrm{C} & 3.7297443 & 3.9787564 & 0.1615656 \\ \mathrm{H} & 4.1616843 & 4.8885659 & -0.2822288 \\ \mathrm{C} & 2.3566734 & 3.7256758 & 0.0181095 \\ \mathrm{H} & 1.7314458 & 4.4298765 & -0.5470934 \\ \mathrm{C} & -0.7344256 & 2.4305330 & 2.0195856 \\ \mathrm{C} & -0.0687695 & 1.9978172 & 3.1861251 \\ \mathrm{H} & 0.9443206 & 1.5823769 & 3.1194830 \\ \mathrm{C} & -0.7008254 & 2.0790556 & 4.4357518 \\ \mathrm{H} & -0.1652324 & 1.7389923 & 5.3349532 \\ \mathrm{C} & -2.0070155 & 2.5855414 & 4.5415039 \\ \mathrm{H} & -2.5017128 & 2.6428246 & 5.5227811 \\ \mathrm{C} & -2.6737301 & 3.0260419 & 3.3870432 \\ \mathrm{H} & -3.6927331 & 3.4358424 & 3.4576010 \\ \mathrm{C} & -2.0424962 & 2.9523020 & 2.1356127 \\ \mathrm{H} & -2.5689024 & 3.3184881 & 1.2425811 \\ \mathrm{C} & -0.6915800 & 3.5586877 & -0.6115753 \\ \mathrm{C} & -0.7439849 & 4.8641549 & -0.0732677 \\ \mathrm{H} & -0.3653727 & 5.0539973 & 0.9423859 \\ \mathrm{C} & -1.2834010 & 5.9158896 & -0.8274377 \\ \mathrm{H} & -1.3192407 & 6.9305111 & -0.4025666 \\ \mathrm{C} & -1.7768705 & 5.6742209 & -2.1221521 \\ \mathrm{H} & -2.2009174 & 6.5011132 & -2.7119910 \\ \mathrm{C} & -1.7276498 & 4.3788467 & -2.6607091 \\ \mathrm{H} & -2.1095186 & 4.1852005 & -3.6742518 \\ \mathrm{C} & -1.1855973 & 3.3233431 & -1.9096789 \\ \mathrm{H} & -1.1257766 & 2.3074897 & -2.3310208\end{array}$

\section{Optimized Geometries(MXn-TS-c-d-6)}

$\begin{array}{lcrl}\text { R4 } & & & \\ 75 & & & \\ \text { SCF } & \text { Energy }=-4548.304372085 & \\ \text { C } & 1.7758721 & 4.3543837 & -2.9994389 \\ \text { C } & 1.5881341 & 4.5724870 & -1.6093069 \\ \text { C } & 1.0396319 & 5.8176836 & -1.2174093 \\ \text { C } & 0.6496595 & 6.7761604 & -2.1640143 \\ \text { C } & 0.8260250 & 6.5335182 & -3.5345634 \\ \text { C } & 1.4055772 & 5.3192309 & -3.9428926 \\ \text { C } & 1.9833210 & 3.5396008 & -0.6187331 \\ \text { C } & 1.8418028 & 2.1721128 & -0.8037652 \\ \text { C } & 1.0683815 & 1.6009867 & -1.9306712 \\ \text { C } & -0.2074649 & 2.1259464 & -2.2549393\end{array}$




$\begin{array}{lrrr}\text { C } & -0.9469430 & 1.6131446 & -3.3275987 \\ \mathrm{C} & -0.4291350 & 0.5617149 & -4.1039325 \\ \mathrm{C} & 0.8260957 & 0.0168539 & -3.7842199 \\ \mathrm{C} & 1.5646096 & 0.5225977 & -2.7052600 \\ \mathrm{Br} & 5.4084591 & 4.0710603 & 2.3831917 \\ \mathrm{C} & 2.1086735 & 3.0717435 & 2.0343395 \\ \mathrm{C} & 2.3486858 & 3.4279577 & 3.4808483 \\ \mathrm{C} & 1.3890949 & 3.9912864 & 1.1788021 \\ \mathrm{C} & 1.9580116 & 1.6059805 & 1.7014138 \\ \mathrm{C} & 2.3887578 & 1.1984881 & 0.2522760 \\ \mathrm{O} & 2.0572313 & -0.1605036 & 0.0184807 \\ \mathrm{H} & 3.4984043 & 1.2255901 & 0.2308268 \\ \mathrm{H} & 2.5427760 & 0.9864486 & 2.4093973 \\ \mathrm{H} & 0.8923726 & 1.2967752 & 1.8074604 \\ \mathrm{H} & 0.3721235 & 3.7555241 & 0.8216407 \\ \mathrm{H} & 1.4517807 & 5.0493358 & 1.4933330 \\ \mathrm{H} & 3.2349096 & 2.9047239 & 3.8874457 \\ \mathrm{H} & 1.4594784 & 3.1286399 & 4.0834089 \\ \mathrm{H} & 2.5099981 & 4.5141558 & 3.6181786 \\ \mathrm{H} & 1.1057711 & -0.1998324 & -0.2012784 \\ \mathrm{H} & -0.6131429 & 2.9554541 & -1.6576161 \\ \mathrm{H} & -1.9346600 & 2.0385327 & -3.5614205 \\ \mathrm{H} & -1.0064091 & 0.1626970 & -4.9516527 \\ \mathrm{H} & 1.2353434 & -0.8118082 & -4.3821720 \\ \mathrm{H} & 2.5375347 & 0.0838209 & -2.4487669 \\ \mathrm{H} & 0.9178020 & 6.0524735 & -0.1534330 \\ \mathrm{H} & 0.2201596 & 7.7289626 & -1.8201503 \\ \mathrm{H} & 0.5271917 & 7.2875196 & -4.2782813 \\ \mathrm{H} & 1.5738993 & 5.1144582 & -5.0106850 \\ \mathrm{H} & 2.2186990 & 3.4137235 & -3.3437452 \\ \mathrm{Pd} & 3.6138250 & 3.9156302 & 0.6621804 \\ \mathrm{P} & 5.0635113 & 4.7420980 & -0.9574461 \\ \mathrm{C} & 4.8074360 & 6.5494103 & -1.0172418 \\ \mathrm{C} & 5.3638245 & 7.3117610 & 0.0371228 \\ \mathrm{H} & 5.9900511 & 6.8163126 & 0.7956081 \\ \mathrm{C} & 5.0926752 & 8.6830949 & 0.1282756 \\ \mathrm{H} & 5.5317177 & 9.2696001 & 0.9494905 \\ \mathrm{C} & 4.2558861 & 9.3051195 & -0.8167008 \\ \mathrm{H} & 4.0444485 & 10.3826530 & -0.7417361 \\ \mathrm{C} & 3.6810576 & 8.5437380 & -1.8455307 \\ \mathrm{H} & 3.0071729 & 9.0143824 & -2.5770765 \\ \mathrm{C} & 3.9476096 & 7.1686556 & -1.9455574 \\ & 3.4669744 & 6.5805745 & -2.7382509 \\ \mathrm{C} & 6.8793896 & 4.5172207 & -0.7645125 \\ & 6.3532418 & 3.2894091 & -0.2614192 \\ & & 2.5432924 & 0.1128254\end{array}$




$\begin{array}{rrrr}\text { C } & 8.7313763 & 3.0359411 & -0.2167889 \\ \text { H } & 9.0957244 & 2.0779544 & 0.1835685 \\ \text { C } & 9.6432609 & 4.0084359 & -0.6616101 \\ \text { H } & 10.7252071 & 3.8116839 & -0.6151060 \\ \text { C } & 9.1724562 & 5.2331589 & -1.1622735 \\ \text { H } & 9.8825856 & 5.9978117 & -1.5123209 \\ \text { C } & 7.7926592 & 5.4870047 & -1.2209455 \\ \text { H } & 7.4266193 & 6.4453392 & -1.6183041 \\ \text { C } & 4.8809290 & 4.0542167 & -2.6523552 \\ \text { C } & 4.6017385 & 2.6744689 & -2.7415328 \\ \text { H } & 4.4480983 & 2.0942567 & -1.8200596 \\ \text { C } & 4.4993554 & 2.0531276 & -3.9946952 \\ \text { H } & 4.2731467 & 0.9790383 & -4.0558385 \\ \text { C } & 4.6679786 & 2.8065329 & -5.1682707 \\ \text { H } & 4.5706989 & 2.3233272 & -6.1521396 \\ \text { C } & 4.9758412 & 4.1751027 & -5.0826150 \\ \text { H } & 5.1292140 & 4.7645426 & -5.9993644 \\ \text { C } & 5.0972615 & 4.7954892 & -3.8300364 \\ \text { H } & 5.3576866 & 5.8616967 & -3.7716355\end{array}$

S4

75

SCF Energy $=-4548.317001003$

$\begin{array}{lrrr}\text { C } & 1.6481853 & 3.7627017 & -3.1468911 \\ \text { C } & 0.9704067 & 4.1433655 & -1.9588460 \\ \text { C } & -0.2141100 & 4.9054829 & -2.1030814 \\ \text { C } & -0.7280492 & 5.2263368 & -3.3678614 \\ \text { C } & -0.0550624 & 4.8221384 & -4.5305663 \\ \text { C } & 1.1452985 & 4.1001503 & -4.4085043 \\ \text { C } & 1.5031114 & 3.7719532 & -0.6254954 \\ \text { C } & 2.1131239 & 2.5739753 & -0.3006455 \\ \text { C } & 2.2216283 & 1.4228829 & -1.2233840 \\ \text { C } & 1.1240261 & 0.9923539 & -2.0075602 \\ \text { C } & 1.2433996 & -0.1114108 & -2.8608430 \\ \text { C } & 2.4611925 & -0.8087365 & -2.9535048 \\ \text { C } & 3.5536102 & -0.4040161 & -2.1679780 \\ \text { C } & 3.4331731 & 0.6934350 & -1.3038881 \\ \text { Br } & 3.1885198 & 6.8378871 & 2.3549259 \\ \text { C } & 1.0665433 & 4.1231919 & 2.0364516 \\ \text { C } & 0.6846534 & 4.8842512 & 3.2813824 \\ \text { C } & 0.2605730 & 4.2462102 & 0.8436516 \\ \text { C } & 1.7399245 & 2.7779744 & 2.2358786 \\ \text { C } & 2.7070700 & 2.3542186 & 1.0963614 \\ \text { O } & 3.9987625 & 2.9541292 & 1.2493749 \\ \text { H } & 2.8948534 & 1.2690839 & 1.2113756 \\ \text { H } & 2.3219406 & 2.7794936 & 3.1788912\end{array}$




$\begin{array}{lrrr}\text { H } & 0.9608704 & 1.9869165 & 2.3363023 \\ \mathrm{H} & -0.3395290 & 3.3981326 & 0.4742421 \\ \mathrm{H} & -0.2993800 & 5.1956612 & 0.7558488 \\ \mathrm{H} & 1.5606877 & 5.0547670 & 3.9356208 \\ \mathrm{H} & -0.0663864 & 4.2900822 & 3.8525312 \\ \mathrm{H} & 0.2418201 & 5.8710920 & 3.0478879 \\ \mathrm{H} & 3.8369939 & 3.9358023 & 1.2328742 \\ \mathrm{H} & 0.1691458 & 1.5330780 & -1.9401335 \\ \mathrm{H} & 0.3769564 & -0.4335660 & -3.4582763 \\ \mathrm{H} & 2.5547597 & -1.6716851 & -3.6299719 \\ \mathrm{H} & 4.5082420 & -0.9488607 & -2.2279374 \\ \mathrm{H} & 4.2899325 & 1.0145609 & -0.6923445 \\ \mathrm{H} & -0.7476132 & 5.2617354 & -1.2137886 \\ \mathrm{H} & -1.6556665 & 5.8135303 & -3.4394683 \\ \mathrm{H} & -0.4535120 & 5.0784622 & -5.5237730 \\ \mathrm{H} & 1.7028432 & 3.7916683 & -5.3053706 \\ \mathrm{H} & 2.5810798 & 3.1936819 & -3.0787958 \\ \mathrm{Pd} & 2.2698270 & 5.3074380 & 0.6205354 \\ \mathrm{P} & 3.5310181 & 6.3898803 & -1.0230424 \\ \mathrm{C} & 5.0511425 & 7.3116996 & -0.5501397 \\ \mathrm{C} & 5.9371654 & 6.7132817 & 0.3678311 \\ \mathrm{H} & 5.6602271 & 5.7711923 & 0.8616333 \\ \mathrm{C} & 7.1613949 & 7.3280703 & 0.6633226 \\ \mathrm{H} & 7.8452767 & 6.8580034 & 1.3857564 \\ \mathrm{C} & 7.5045991 & 8.5473571 & 0.0541412 \\ \mathrm{H} & 8.4618405 & 9.0339885 & 0.2955841 \\ \mathrm{C} & 6.6232439 & 9.1448790 & -0.8609983 \\ \mathrm{H} & 6.8876932 & 10.0988920 & -1.3420940 \\ \mathrm{C} & 5.4004040 & 8.5269179 & -1.1692865 \\ \mathrm{H} & 4.7149046 & 8.9956189 & -1.8905842 \\ \mathrm{H} & 0.9790003 & 10.6036634 & -0.7841388 \\ \mathrm{C} & 4.2372708 & 5.3417969 & -2.3557496 \\ \mathrm{C} & 4.4248605 & 5.7929548 & -3.6772226 \\ \mathrm{H} & 4.1121709 & 6.8066085 & -3.9654751 \\ \mathrm{C} & 5.0093324 & 4.9464852 & -4.6309293 \\ \mathrm{H} & 5.1371370 & 5.2980143 & -5.6660894 \\ \mathrm{C} & 5.4376980 & 3.6585860 & -4.2651608 \\ \mathrm{H} & 5.8924588 & 2.9949335 & -5.0161187 \\ \mathrm{C} & 5.2970671 & 3.2260610 & -2.9363376 \\ \mathrm{H} & 5.6397067 & 2.2238443 & -2.6417990 \\ \mathrm{H} & 4.6966633 & 4.0611739 & -1.9817094 \\ \mathrm{H} & 2.5645533 & 3.7068063 & -0.9476703 \\ & 2.4205191 & 7.6417056 & -1.7561528 \\ \mathrm{H} & 2.6414190 & 8.7803274 & -0.9648735 \\ \mathrm{H} & 1.1923533 & 9.7192021 & -1.4032437 \\ \mathrm{H} & & \\ \mathrm{H} & & \end{array}$




$\begin{array}{rrrr}\text { C } & 0.5148232 & 9.5281601 & -2.6218374 \\ \text { H } & -0.2254626 & 10.2675957 & -2.9633085 \\ \text { C } & 0.7763664 & 8.3849573 & -3.3919119 \\ \text { H } & 0.2360103 & 8.2133633 & -4.3349040 \\ \text { C } & 1.7205982 & 7.4387539 & -2.9612311 \\ \text { H } & 1.8918228 & 6.5337805 & -3.5587553\end{array}$

\section{Optimized Geometries(MXnd-6)}

\begin{tabular}{lrrr} 
R4 & \multicolumn{3}{l}{} \\
75 & \multicolumn{4}{l}{} \\
SCF & Energy $=-4548.351903722$ & \\
C & -2.4354944 & 0.7332127 & -1.5687035 \\
C & -2.1123379 & 1.7640846 & -0.6558335 \\
C & -2.1030863 & 3.0924027 & -1.1466692 \\
C & -2.3889639 & 3.3721421 & -2.4915048 \\
C & -2.6826579 & 2.3304531 & -3.3861609 \\
C & -2.7082834 & 1.0068776 & -2.9125659 \\
C & -1.7534225 & 1.5037977 & 0.7600239 \\
C & -2.1655148 & 0.3790096 & 1.5436744 \\
C & -3.0192761 & -0.7370731 & 1.0385623 \\
C & -4.3356571 & -0.4973586 & 0.5930631 \\
C & -5.1471489 & -1.5616234 & 0.1745691 \\
C & -4.6613834 & -2.8801232 & 0.2110796 \\
C & -3.3635532 & -3.1307244 & 0.6873194 \\
C & -2.5497068 & -2.0653715 & 1.1037158 \\
Br & 1.8216503 & -0.8565876 & 2.4892988 \\
C & -0.2283069 & 1.8304029 & 2.5686830 \\
C & 1.0815586 & 2.4662953 & 2.9640641 \\
C & -1.0671552 & 2.6159444 & 1.5603145 \\
C & -1.0866697 & 1.2830907 & 3.6864455 \\
C & -2.3014044 & 0.5448960 & 3.0792906 \\
O & -3.5651536 & 1.1528232 & 3.3543194 \\
H & -2.3801088 & -0.4685240 & 3.5165159 \\
H & -0.4906263 & 0.5949128 & 4.3181433 \\
H & -1.4525062 & 2.1041224 & 4.3491370 \\
H & -1.7738067 & 3.3457431 & 2.0358841 \\
H & -0.4072500 & 3.1998678 & 0.8897627 \\
H & 1.6735561 & 1.8008024 & 3.6193070 \\
H & 0.8783288 & 3.4182878 & 3.5107875 \\
H & 1.6962774 & 2.7160298 & 2.0754559 \\
H & -3.5366590 & 2.0495123 & 2.9679143 \\
H & -4.7135530 & 0.5342923 & 0.5645973
\end{tabular}




\begin{tabular}{lrrr} 
H & -6.1689202 & -1.3602945 & -0.1813110 \\
$\mathrm{H}$ & -5.2998016 & -3.7133216 & -0.1196880 \\
$\mathrm{H}$ & -2.9802436 & -4.1614072 & 0.7345221 \\
$\mathrm{H}$ & -1.5274624 & -2.2466576 & 1.4720710 \\
$\mathrm{H}$ & -1.8630371 & 3.9244486 & -0.4710631 \\
$\mathrm{H}$ & -2.3726449 & 4.4150059 & -2.8432627 \\
$\mathrm{H}$ & -2.8888746 & 2.5473956 & -4.4449971 \\
$\mathrm{H}$ & -2.9181775 & 0.1704866 & -3.5956424 \\
$\mathrm{H}$ & -2.4380019 & -0.3051712 & -1.2309043 \\
$\mathrm{Pd}$ & -0.0301620 & 0.2188018 & 1.2158893 \\
$\mathrm{P}$ & 0.6796090 & -0.8570141 & -0.8634973 \\
$\mathrm{C}$ & 1.5149440 & 0.7365513 & -1.2540251 \\
$\mathrm{C}$ & 2.6998380 & 1.0651779 & -0.5525637 \\
$\mathrm{H}$ & 3.1770892 & 0.3126636 & 0.0931178 \\
$\mathrm{C}$ & 3.2343969 & 2.3582675 & -0.6394642 \\
$\mathrm{H}$ & 4.1539760 & 2.6014388 & -0.0860671 \\
$\mathrm{C}$ & 2.5937421 & 3.3406245 & -1.4150602 \\
$\mathrm{H}$ & 3.0114987 & 4.3571675 & -1.4743751 \\
$\mathrm{C}$ & 1.4170792 & 3.0193582 & -2.1114862 \\
$\mathrm{H}$ & 0.9024387 & 3.7799168 & -2.7174906 \\
$\mathrm{C}$ & 0.8769858 & 1.7267410 & -2.0339231 \\
$\mathrm{H}$ & -0.0549456 & 1.4978260 & -2.5677074 \\
$\mathrm{C}$ & 2.0159660 & -2.1180560 & -0.8410047 \\
$\mathrm{C}$ & 1.8617199 & -3.2297190 & 0.0099586 \\
$\mathrm{H}$ & 1.0034101 & -3.2718234 & 0.6958455 \\
$\mathrm{C}$ & 2.8141584 & -4.2597646 & -0.0016310 \\
$\mathrm{H}$ & 2.6919328 & -5.1235715 & 0.6689309 \\
$\mathrm{C}$ & 3.9309236 & -4.1765137 & -0.8495418 \\
$\mathrm{H}$ & 4.6833569 & -4.9798201 & -0.8497725 \\
$\mathrm{C}$ & 4.0913228 & -3.0639965 & -1.6934583 \\
$\mathrm{H}$ & 4.9668489 & -2.9951701 & -2.3569630 \\
$\mathrm{C}$ & 3.1338073 & -2.0383629 & -1.6945922 \\
$\mathrm{H}$ & 3.2594016 & -1.1681952 & -2.3564552 \\
$\mathrm{C}$ & -0.1897268 & -1.3637814 & -2.4106510 \\
$\mathrm{C}$ & -1.2875790 & -2.2388791 & -2.2817967 \\
$\mathrm{H}$ & -1.6232666 & -2.5489603 & -1.2809564 \\
$\mathrm{C}$ & -1.9728672 & -2.6897847 & -3.4193978 \\
$\mathrm{H}$ & -2.8366307 & -3.3614365 & -3.3024855 \\
$\mathrm{C}$ & -1.5631018 & -2.2742180 & -4.6980155 \\
$\mathrm{H}$ & -2.1043470 & -2.6204093 & -5.5915839 \\
$\mathrm{C}$ & -0.4564471 & -1.4183357 & -4.8323374 \\
$\mathrm{C}$ & -0.1250944 & -1.0979212 & -5.8318701 \\
& 0.2325207 & -0.9683158 & -3.6954708 \\
& 1.0984393 & -0.3003395 & -3.8101116 \\
& & & \\
\hline
\end{tabular}


75

SCF Energy $=-4548.357056489$

$\begin{array}{lll}\text { C } & -0.6760211 & -2.5840460\end{array}$

C $\quad-1.7585812 \quad-2.2618846$

$\begin{array}{lll}\text { C } & -3.0650479 & -2.3622415\end{array}$

$\begin{array}{lll}\text { C } & -3.2762530 & -2.7476877\end{array}$

C $\quad-2.1854016 \quad-3.0361196$

C $\quad-0.8825336 \quad-2.9563640$

$\begin{array}{lll}\text { C } & -1.5822626 & -1.7640924\end{array}$

$\begin{array}{lll}\text { C } & -0.4892779 & -2.0854509\end{array}$

C $\quad 0.6056264 \quad-3.0090839$

C $\quad 0.3083799$

C $\quad 1.3265619$

C $\quad 2.6570495$

C 2.9642990

C $\quad 1.9460947$

$\mathrm{Br} \quad 0.8551799$

C $\quad-2.0272299$

C $\quad-2.6488426$

C $\quad-2.7432195$

C $\quad-1.5956719$

C $\quad-0.5575507$

$0 \quad 0.7172976$

$\mathrm{H} \quad-0.8158920$

$\mathrm{H} \quad-1.1235945$

$\mathrm{H} \quad-2.4905344$

$\mathrm{H} \quad-3.5131440$

$\mathrm{H} \quad-3.2550393$

$\mathrm{H} \quad-2.0094809$

$\mathrm{H} \quad-3.6413454$

$\mathrm{H} \quad-2.8227300$

0.9170976

$-0.7363437$

$\mathrm{H} \quad 1.0784274$

$\mathrm{H} \quad 3.4560202$

$\mathrm{H} \quad 4.0066134$

$\mathrm{H} \quad 2.1737452$

$\mathrm{H} \quad-3.9346703$

$\mathrm{H} \quad-4.3039171$

$\mathrm{H} \quad-2.3480763$

$\mathrm{H} \quad-0.0103885$

$\mathrm{H} \quad 0.3474408$

$\mathrm{Pd} \quad-0.3264520$

P $\quad 0.8727472$

C $\quad 2.1374377$

C $\quad 3.1853873$
$-4.3544530$

$-5.2409395$

$-4.7956779$

$-3.4635800$

$-2.5766405$

1.6862074

$-0.0791284$

1. 2745673

$-0.9941128$

$-0.8661161$

$-1.9332400$

$-1.6368661$

$-2.9394834$

$-0.2139235$

$-1.3317523$

$-1.6472632$

$-0.3982009$

1. 9070451

1. 1494879

1. 8062812

$-0.7037742$

$-4.6977415$

$-6.2848626$

$-5.4897245$

$-3.1117041$

$-1.5297340$

$-2.1299708$

$-2.8145126$

$-3.3195181$

$-3.1609653$

$-2.5030995$

0.0191253

0.5985884

1. 9290473

1.8422934
$-1.4438257$

$-0.5919644$

$-1.1296214$

$-2.4622819$

$-3.2979434$

$-2.7759706$

0.7976967

1. 6644054

1. 2423942

0.9417627

0.5610922

0.4869403

0.8122841

1. 1935222

2. 5971669

2.4125047

2. 6421077

1. 4288698

3. 6297117

3. 2128679

3. 7502749

3. 6142163

4. 3895040

4.1069627

1.9119900

0.6475300

3. 2861655

3.1373900

1. 6846208

3. 4866336

0.9857417

0.3165118

0.1848278

0.7722907

1. 4371459

$-0.4998434$

$-2.8510456$

$-4.3486661$

$-3.4142528$

$-1.0698956$

1. 1390584

$-0.8870906$

$-0.8758477$

0.0610016 


$\begin{array}{rrrr}\text { H } & 3.1788562 & 1.0360462 & 0.8082268 \\ \text { C } & 4.2117251 & 2.8000748 & 0.0548396 \\ \text { H } & 5.0253912 & 2.7317457 & 0.7923049 \\ \text { C } & 4.1876552 & 3.8516573 & -0.8751869 \\ \text { H } & 4.9878853 & 4.6073667 & -0.8726112 \\ \text { C } & 3.1387777 & 3.9436793 & -1.8075509 \\ \text { H } & 3.1181717 & 4.7679532 & -2.5366593 \\ \text { C } & 2.1178878 & 2.9822224 & -1.8129239 \\ \text { H } & 1.2990421 & 3.0517355 & -2.5450687 \\ \text { C } & 1.3614628 & -0.3083456 & -2.4143650 \\ \text { C } & 0.9096394 & 0.0444607 & -3.7012973 \\ \text { H } & 0.2028567 & 0.8773154 & -3.8287817 \\ \text { C } & 1.3525581 & -0.6730403 & -4.8233316 \\ \text { H } & 0.9891380 & -0.3961986 & -5.8246757 \\ \text { C } & 2.2550496 & -1.7392966 & -4.6715299 \\ \text { H } & 2.5962494 & -2.3028783 & -5.5530636 \\ \text { C } & 2.7210299 & -2.0833658 & -3.3906298 \\ \text { H } & 3.4264030 & -2.9179901 & -3.2615430 \\ \text { C } & 2.2774507 & -1.3706543 & -2.2674127 \\ \text { H } & 2.6252105 & -1.6564592 & -1.2631871 \\ \text { C } & -0.7205758 & 1.4344503 & -1.2748909 \\ \text { C } & -1.0109585 & 2.6714106 & -0.6500622 \\ \text { H } & -0.2261110 & 3.1927029 & -0.0820355 \\ \text { C } & -2.3048391 & 3.2085710 & -0.7194565 \\ \text { H } & -2.5192114 & 4.1685418 & -0.2262646 \\ \text { C } & -3.3236928 & 2.5192690 & -1.3993834 \\ \text { H } & -4.3398598 & 2.9395143 & -1.4434540 \\ \text { C } & -3.0407790 & 1.2905052 & -2.0208676 \\ \text { H } & -3.8310840 & 0.7392837 & -2.5520346 \\ \text { C } & -1.7493066 & 0.7479100 & -1.9628335 \\ \text { H } & -1.5471815 & -0.2233980 & -2.4345287\end{array}$

\section{Optimized Product Geometries(Xn)}

\begin{tabular}{lrrr} 
R3 & & & \\
38 & \multicolumn{4}{l}{} \\
SCF & Energy $=-809.6157366451$ & \\
C & -2.4394944 & 4.1865518 & 0.9217375 \\
C & -1.1662954 & 3.7324715 & 0.4654712 \\
C & -0.6563585 & 4.1756099 & -0.7696322 \\
C & -1.4150534 & 5.0748330 & -1.5365083 \\
C & -2.6650931 & 5.5326955 & -1.0782907 \\
C & -3.1833077 & 5.0889691 & 0.1537083
\end{tabular}




\begin{tabular}{|c|c|c|c|}
\hline & -0.6451582 & 2.8079675 & 1.4741914 \\
\hline$C$ & 0.5559398 & 2.3883967 & 1.9717624 \\
\hline & 1.9225189 & 2.6756043 & 1.5083326 \\
\hline & 2.9527327 & 1.7231771 & 1.7085824 \\
\hline & 4.2652644 & 1.9902063 & 1.2935146 \\
\hline & 4.5817079 & 3.2144218 & 0.6815261 \\
\hline & 3.5729138 & 4.1766721 & 0.4968498 \\
\hline & 2.2594108 & 3.9121587 & 0.9061204 \\
\hline & -1.8330590 & 2.3292665 & 2.3200094 \\
\hline & -2.5450604 & 1.1355227 & 1.6443513 \\
\hline & -2.7726235 & 3.5700846 & 2.2718758 \\
\hline & -1.1426006 & 1.9121366 & 3.6347923 \\
\hline & 0.3033351 & 1.5235582 & 3.2114075 \\
\hline & 0.4924857 & 0.1226418 & 2.9518626 \\
\hline & 1.0317150 & 1.753 & 4.016 \\
\hline & -1.0879865 & 2.780 & 4.3 \\
\hline & -1.638 & 1.07 & 4.1 \\
\hline & -2.5097499 & 4.282 & 3.0 \\
\hline & -3.8477859 & 3.322 & 2.38 \\
\hline & -1.8742315 & 0.2567010 & 1.5688742 \\
\hline & -2.8853926 & 1.3965679 & 0.6219707 \\
\hline & -3.4302566 & 0.8288811 & 2.2383704 \\
\hline & 0.1199479 & -0.0482385 & 2.0655012 \\
\hline & 2.6974328 & 0.7627387 & 2.1804755 \\
\hline & 5.0493854 & 1.2334808 & 1.4500373 \\
\hline & 5.6124679 & 3.4229388 & 0.3569654 \\
\hline & 3.8149764 & 5.1479970 & 0.0385795 \\
\hline & 1.4839315 & 4.6821741 & 0.7875324 \\
\hline & 0.3132335 & 3.8094897 & -1.1360719 \\
\hline & -1.0313830 & 5.4200484 & -2.5085537 \\
\hline & -3.2462214 & 6.2370503 & -1.6928418 \\
\hline & -4.1652329 & 5.4442117 & 0.5033124 \\
\hline \\
\hline \\
\hline & Energy $=-809$ & 6147734832 & \\
\hline & 4.4336826 & 1.3998109 & -0.0605547 \\
\hline & 3.0374005 & 1.2285851 & -0.2337869 \\
\hline & 2.5937668 & 0.4130502 & -1.3039908 \\
\hline & 3.5062372 & -0.1763945 & -2.1882740 \\
\hline & 4.8874690 & 0.0278742 & -2.0214756 \\
\hline & 5.3446183 & 0.8133970 & -0.9502862 \\
\hline & 2.0885769 & 1.8478284 & 0.7019706 \\
\hline & 0.8102987 & 2.2794397 & 0.4944339 \\
\hline & -0.2145794 & 2.2938567 & -0.5497247 \\
\hline & -0.1373923 & 2.2708299 & -1.9559222 \\
\hline
\end{tabular}




$\begin{array}{rrrr}\text { C } & -1.3239419 & 2.3099946 & -2.7056849 \\ \text { C } & -2.5767463 & 2.3645942 & -2.0657107 \\ \text { C } & -2.6614427 & 2.3942588 & -0.6604865 \\ \text { C } & -1.4848611 & 2.3688064 & 0.0963905 \\ \text { C } & 2.4427699 & 2.0471756 & 2.1882649 \\ \text { O } & 3.2825988 & 1.0474176 & 2.7386002 \\ \text { C } & 1.0491238 & 2.1643487 & 2.8650876 \\ \text { C } & 0.1675630 & 2.8133528 & 1.7810874 \\ \text { C } & -1.3191410 & 2.3943117 & 1.6079519 \\ \text { C } & 0.2656843 & 4.3554878 & 1.8261783 \\ \text { H } & 3.0139933 & 2.9945518 & 2.3251854 \\ \text { H } & 1.0813976 & 2.7171112 & 3.8250446 \\ \text { H } & 0.6948445 & 1.1316953 & 3.0738354 \\ \text { H } & -1.4852801 & 1.3747638 & 2.0228421 \\ \text { H } & -2.0364428 & 3.0743587 & 2.1109612 \\ \text { H } & -0.2043428 & 4.7470534 & 2.7515935 \\ \text { H } & -0.2530015 & 4.8078030 & 0.9568577 \\ \text { H } & 1.3203661 & 4.6950329 & 1.8027742 \\ \text { H } & 2.9257504 & 0.1858069 & 2.4472598 \\ \text { H } & 1.5174714 & 0.2238666 & -1.4225164 \\ \text { H } & 3.1371196 & -0.8094177 & -3.0098766 \\ \text { H } & 5.6046931 & -0.4342741 & -2.7165946 \\ \text { H } & 6.4244429 & 0.9678859 & -0.8024138 \\ \text { H } & 4.8042186 & 1.9942264 & 0.7855314 \\ \text { H } & -3.6434257 & 2.4466164 & -0.1648074 \\ \text { H } & -3.4967947 & 2.3949599 & -2.6691077 \\ \text { H } & -1.2734062 & 2.3044664 & -3.8051248 \\ \text { H } & 0.8390111 & 2.2417674 & -2.4599121\end{array}$

S3

38

SCF Energy $=-809.6138763928$

$\begin{array}{lrrr}\text { C } & -2.0096176 & 3.0633477 & 1.7080276 \\ \text { C } & -1.1846378 & 3.1572011 & 0.5456128 \\ \text { C } & -1.7074414 & 3.7238738 & -0.6355935 \\ \text { C } & -3.0407057 & 4.1619789 & -0.6570113 \\ \text { C } & -3.8566254 & 4.0428391 & 0.4835745 \\ \text { C } & -3.3387756 & 3.4997106 & 1.6739449 \\ \text { C } & 0.1515277 & 2.6819129 & 0.9094138 \\ \text { C } & 1.4509350 & 2.8372164 & 0.5068563 \\ \text { C } & 1.9371178 & 3.2490907 & -0.8193537 \\ \text { C } & 1.2267597 & 2.8479378 & -1.9799133 \\ \text { C } & 1.6603594 & 3.2132507 & -3.2609308 \\ \text { C } & 2.8284133 & 3.9789232 & -3.4187700 \\ \text { C } & 3.5649004 & 4.3537984 & -2.2827084 \\ \text { C } & 3.1315307 & 3.9917343 & -0.9993508\end{array}$




\begin{tabular}{lrrr} 
C & 0.0483559 & 1.9395687 & 2.2447278 \\
C & -0.1611427 & 0.4329826 & 1.9685294 \\
C & -1.2254884 & 2.5358123 & 2.8973209 \\
C & 1.4337278 & 2.2008080 & 2.8663536 \\
C & 2.3868441 & 2.4210315 & 1.6578110 \\
O & 3.4177431 & 3.3657970 & 1.9481737 \\
H & 2.9395357 & 1.4925273 & 1.3919973 \\
H & 1.4089884 & 3.1485853 & 3.4462670 \\
H & 1.7847581 & 1.4026698 & 3.5501528 \\
H & -0.9666098 & 3.3809956 & 3.5738587 \\
H & -1.7973401 & 1.8028568 & 3.5024996 \\
H & 0.6808742 & 0.0138476 & 1.3818804 \\
H & -1.0926160 & 0.2632619 & 1.3912208 \\
H & -0.2373040 & -0.1297970 & 2.9219283 \\
H & 2.9628290 & 4.2044289 & 2.1634773 \\
H & 0.3432752 & 2.2036657 & -1.8632831 \\
H & 1.0912983 & 2.8842231 & -4.1440739 \\
H & 3.1713294 & 4.2674820 & -4.4239894 \\
H & 4.4920262 & 4.9365340 & -2.3962972 \\
H & 3.7206772 & 4.2650831 & -0.1146288 \\
H & -1.0803556 & 3.8253254 & -1.5310911 \\
H & -3.4531715 & 4.5977939 & -1.5796606 \\
H & -4.9037324 & 4.3799106 & 0.4453144 \\
H & -3.9718426 & 3.4251902 & 2.5720131 \\
& & & \\
S4 & & & \\
38 & & & \\
SCF & Energy $=-809.6157348489$ & \\
C & -0.1932313 & 3.3724852 & -1.5472439 \\
C & -0.0491248 & 2.7802067 & -0.2782736 \\
C & -1.1882905 & 2.2616693 & 0.4063073 \\
C & -2.4547787 & 2.3226776 & -0.1851351 \\
C & -2.5914755 & 2.9118880 & -1.4569434 \\
C & -1.4703276 & 3.4360686 & -2.1280677 \\
C & 1.1123644 & 2.5754624 & 0.5892187 \\
C & 2.4575478 & 2.3621078 & 0.4846286 \\
C & 3.3291009 & 2.3948494 & -0.7003167 \\
C & 4.6883110 & 2.7739176 & -0.5690884 \\
C & 5.5382007 & 2.7889563 & -1.6843324 \\
C & 5.0561583 & 2.4158587 & -2.9499615 \\
C & 3.7149487 & 2.0166356 & -3.0893804 \\
C & 2.8612616 & 2.0046778 & -1.9786006 \\
C & 0.5912611 & 2.3636710 & 2.0172048 \\
& 0.3472555 & 3.7173956 & 2.7218110 \\
& 1.7508652 & 1.5821916 & 2.6685004 \\
\hline
\end{tabular}




$\begin{array}{lrrr}\text { C } & 3.0135279 & 2.0185301 & 1.8707021 \\ \mathrm{O} & 3.7415697 & 3.1103689 & 2.4567817 \\ \mathrm{H} & 3.7553264 & 1.1947385 & 1.8133390 \\ \mathrm{H} & 1.8798887 & 1.7690824 & 3.7528157 \\ \mathrm{H} & 1.5996704 & 0.4926995 & 2.5221784 \\ \mathrm{H} & -0.6501988 & 0.5778430 & 1.6596060 \\ \mathrm{H} & -1.5326357 & 1.8563765 & 2.5481945 \\ \mathrm{H} & 1.2915077 & 4.2845041 & 2.8430398 \\ \mathrm{H} & -0.0752710 & 3.5536225 & 3.7342537 \\ \mathrm{H} & -0.3607169 & 4.3481605 & 2.1471377 \\ \mathrm{H} & 3.2454358 & 3.9231117 & 2.2401297 \\ \mathrm{H} & 5.0579315 & 3.0728338 & 0.4230399 \\ \mathrm{H} & 6.5886128 & 3.0954735 & -1.5632365 \\ \mathrm{H} & 5.7246273 & 2.4271910 & -3.8242197 \\ \mathrm{H} & 3.3332377 & 1.7009600 & -4.0726075 \\ \mathrm{H} & 1.8230615 & 1.6609486 & -2.0900056 \\ \mathrm{H} & -3.3365223 & 1.9215458 & 0.3385655 \\ \mathrm{H} & -3.5849733 & 2.9710287 & -1.9269881 \\ \mathrm{H} & -1.5968549 & 3.9065036 & -3.1149494 \\ \mathrm{H} & 0.6782573 & 3.7946883 & -2.0676832\end{array}$

\section{PPh3-Pd(H)Br}

$\begin{array}{lrrr}37 & & & \\ \text { SCF } & \text { Energy }=-3738.694650155 & \\ \text { Pd } & 0.9104044 & -0.2993020 & -4.3544411 \\ \text { P } & -0.5485020 & 1.3477324 & -4.7308459 \\ \text { C } & -0.7234497 & 2.6137654 & -3.4151195 \\ \text { C } & -0.0026431 & 2.3031185 & -6.1999752 \\ \text { C } & -2.2653263 & 0.8349643 & -5.1243907 \\ \text { C } & -0.6561171 & 2.2060289 & -2.0670507 \\ \text { C } & -0.8346898 & 3.1447512 & -1.0402705 \\ \text { C } & -1.0695421 & 4.4948473 & -1.3521249 \\ \text { C } & -1.1248763 & 4.9066187 & -2.6942568 \\ \text { C } & -0.9526226 & 3.9708228 & -3.7262788 \\ \text { H } & -0.4475965 & 1.1517227 & -1.8286156 \\ \text { H } & -0.7781016 & 2.8215585 & 0.0099488 \\ \text { H } & -1.2005062 & 5.2312755 & -0.5449348 \\ \text { H } & -1.2999453 & 5.9647419 & -2.9409293 \\ \text { H } & -0.9879787 & 4.2944854 & -4.7774516 \\ \text { C } & -0.8027458 & 2.4518571 & -7.3497984 \\ \text { C } & -0.3085045 & 3.1597223 & -8.4577941 \\ \text { C } & 0.9798083 & 3.7174612 & -8.4241855 \\ \text { C } & 1.7820323 & 3.5660575 & -7.2788643\end{array}$




$\begin{array}{lrrr}\text { C } & 1.2974624 & 2.8572333 & -6.1714425 \\ \text { H } & -1.8115508 & 2.0146201 & -7.3767478 \\ \text { H } & -0.9365039 & 3.2746834 & -9.3541461 \\ \text { H } & 1.3638592 & 4.2689374 & -9.2955259 \\ \text { H } & 2.7938217 & 3.9974872 & -7.2510106 \\ \text { H } & 1.9266776 & 2.7330509 & -5.2753323 \\ \text { C } & -3.3703441 & 1.6820503 & -4.9051304 \\ \text { C } & -4.6577420 & 1.2593180 & -5.2712564 \\ \text { C } & -4.8462880 & -0.0041982 & -5.8569830 \\ \text { C } & -3.7460048 & -0.8517120 & -6.0715522 \\ \text { C } & -2.4576397 & -0.4375087 & -5.7013674 \\ \text { H } & -3.2244959 & 2.6687643 & -4.4408361 \\ \text { H } & -5.5199005 & 1.9198408 & -5.0938895 \\ \text { H } & -5.8576066 & -0.3334174 & -6.1398995 \\ \text { H } & -3.8919497 & -1.8455911 & -6.5204943 \\ \text { H } & -1.5891936 & -1.1003128 & -5.8466025 \\ \text { Br } & 2.5848508 & -1.9987398 & -4.0729183 \\ \text { H } & -0.0956494 & -0.7262353 & -3.3055863\end{array}$

\section{H2CO3}

$\begin{array}{lrrr}6 & & & \\ \text { SCF } & \text { Energy }=-264.8348300398 & \\ \text { C } & 0.0402681 & 0.0000000 & -0.0453569 \\ \text { O } & -1.1650778 & 0.0000000 & -0.6458897 \\ 0 & -0.1071487 & -0.0000000 & 1.3143124 \\ \text { O } & 1.0938675 & -0.0000000 & -0.6331674 \\ \text { H } & -1.8564089 & -0.0000000 & 0.0467565 \\ \text { H } & 0.7992238 & 0.0000000 & 1.6844012\end{array}$

\section{$\mathrm{HBr}$}

2

SCF Energy $=-2574.735621963$

$\begin{array}{lllr}\mathrm{H} & 0.0000000 & 0.0000000 & -0.2138301 \\ \mathrm{Br} & 0.0000000 & 0.0000000 & 1.2218301\end{array}$

\section{HCO3-}

5

SCF Energy $=-264.2670446301$ 


$\begin{array}{lrrr}\text { C } & 0.0416374 & 0.0000000 & 0.0725983 \\ \text { O } & -0.1553027 & -0.0000000 & 1.3176595 \\ 0 & 1.0735796 & -0.0000000 & -0.6222903 \\ \text { O } & -1.2044528 & -0.0000000 & -0.7012735 \\ \text { H } & -1.8435775 & 0.0000000 & 0.0386950\end{array}$


